\title{
Piping Benchmark Problems
}

Dynamic Analysis Uniform Support Motion Response Spectrum Method

Prepared by P. Bezler, M. Hartzman, M. Reich

Brookhaven National Laboratory

Prepared for

U.S. Nuclear Regulatory

Commission

\section{DO NOT MICROFILM \\ COVER}




\section{DISCLAIMER}

This report was prepared as an account of work sponsored by an agency of the United States Government. Neither the United States Government nor any agency Thereof, nor any of their employees, makes any warranty, express or implied, or assumes any legal liability or responsibility for the accuracy, completeness, or usefulness of any information, apparatus, product, or process disclosed, or represents that its use would not infringe privately owned rights. Reference herein to any specific commercial product, process, or service by trade name, trademark, manufacturer, or otherwise does not necessarily constitute or imply its endorsement, recommendation, or favoring by the United States Government or any agency thereof. The views and opinions of authors expressed herein do not necessarily state or reflect those of the United States Government or any agency thereof. 


\section{DISCLAIMER}

Portions of this document may be illegible in electronic image products. Images are produced from the best available original document. 


\title{
NOTICE
}

This report was prepared as an account of work sponsored by an agency of the United States Government. Neither the United States Government nor any agency thereof, or any of their employees, makes any warranty, expressed or implied, or assumes any legal liability or responsibility for any third party's use, or the results of such use, of any information, apparatus product or process disclosed in this report, or represents that its use by such third party would not infringe privately owned rights.

\section{DO NOT MICROFILM
COVER}

\author{
Available from \\ GPO Sales Program \\ Division of Technical Information and Document Control \\ U. S. Nuclear Regulatory Commission \\ Washington, D. C. 20555 \\ Printed copy price: $\$ 8.00$ \\ and \\ National Technical Information Service \\ Springfield, Virginia 22161
}




\section{Piping Benchmark Problems}

NUREG/CR--1677-Vol .1

TI86 002698

\section{Dynamic Analysis Uniform Support Motion Response Spectrum Method}

Manuscript Completed: August 1980

Date Published: August 1980

Prepared by

P. Bezler, “M. Hartzman, M. Reich

Brookhaven National Laboratory

Upton, NY 11973

* Staff, U.S. Nuclear Regulatory Commission

Prepared for

Division of Systems Safety

Office of Nuclear Reactor Regulation

U.S. Nuclear Regulatory Commission

Washington, D.C. 20555

NRC FIN No. A3225

\section{DISCLAIMER}

This report was prepared as an account of work sponsored by an agency of the United States Government. Neither the United States Government nor any agency thereof, nor any of their employees, makes any warranty, express or implied, or assumes any legal liability or responsibility for the accuracy, completeness, or usefulness of any information, apparatus, product, or process disclosed, or represents that its use would not infringe privately owned rights. Reference herein to any specific commercial product, process, or service by trade name, trademark, manufacturer, or otherwise does not necessarily constitute or imply its endorsement, reconmendation, or favoring by the United States Government or any agency thereof. The views and opinions of authors expressed herein do not necessarily state or reflect those of the United States Government or any agency thereof. 


\section{THIS PAGE \\ WAS INTENTIONALLY \\ LEFT BLANK}




\section{ABSTRACT}

A set of benchmark problems and solutions have been developed for verifying the adequacy of computer programs used for dynamic analysis and design of nuclear piping systems by the Response Spectrum Method. The problems range from simple to complex configurations which are assumed to experience linear elastic behavior. The dynamic loading is represented by uniform support motion, assumed to be induced by seismic excitation in three spatial directions. The solutions consist of frequencies, participation factors, nodal displacement components and internal force and moment components. Solutions to associated anchor point motion static problems are not included. 


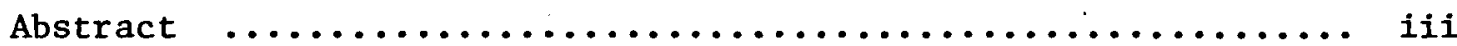

Table of Contents $\ldots \ldots \ldots \ldots \ldots \ldots \ldots \ldots \ldots \ldots \ldots \ldots \ldots \ldots \ldots$ iv

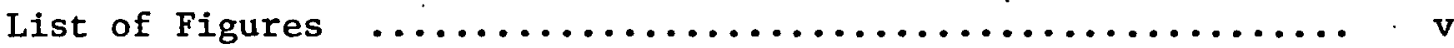

I. Introduction $\quad \ldots \ldots \ldots \ldots \ldots \ldots \ldots \ldots \ldots \ldots \ldots \ldots \ldots \ldots \ldots \ldots \ldots$

II. Background $\ldots \ldots \ldots \ldots \ldots \ldots \ldots \ldots \ldots \ldots \ldots \ldots \ldots \ldots \ldots \ldots \ldots \ldots \ldots \ldots$

II.1 Program Description $\ldots \ldots \ldots \ldots \ldots \ldots \ldots \ldots \ldots \ldots \ldots \ldots \ldots$

II.2 Input-Output $\ldots \ldots \ldots \ldots \ldots \ldots \ldots \ldots \ldots \ldots \ldots \ldots \ldots \ldots \ldots$

III. Description of Piping Benchmark Problems $\ldots \ldots \ldots \ldots \ldots \ldots \ldots \ldots$

III.1 Benchmark Problem 1 ....................... 15

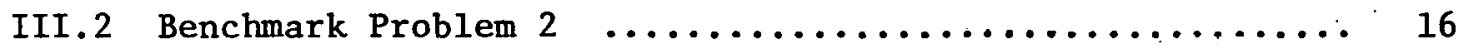

III.3 Benchmark Problem $3 \quad \ldots \ldots \ldots \ldots \ldots \ldots \ldots \ldots \ldots \ldots \ldots \ldots \ldots$

III.4 Benchmark Problem $4 \quad \ldots \ldots \ldots \ldots \ldots \ldots \ldots \ldots \ldots \ldots \ldots \ldots \ldots$

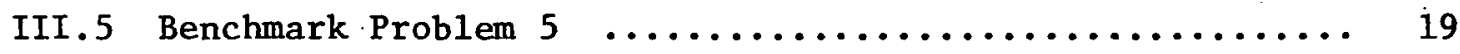

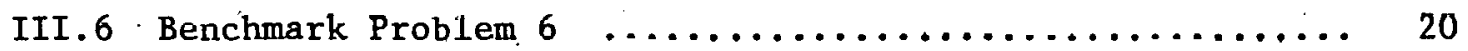

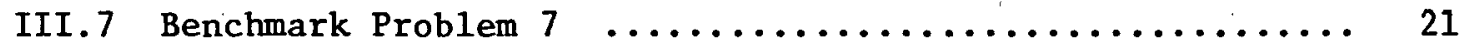

IV. Piping Benchmark Problems and Solutions $\ldots \ldots \ldots \ldots \ldots \ldots \ldots \ldots$

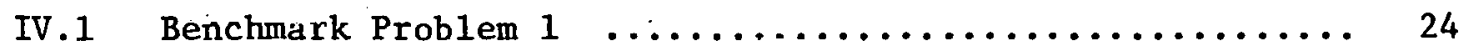

IV. 2 Benchmark Problem $2 \quad \ldots \ldots \ldots \ldots \ldots \ldots \ldots \ldots \ldots \ldots \ldots \ldots \ldots \ldots \ldots$

IV.3 Benchmark Problem $3 \ldots \ldots \ldots \ldots \ldots \ldots \ldots \ldots \ldots \ldots \ldots \ldots \ldots$

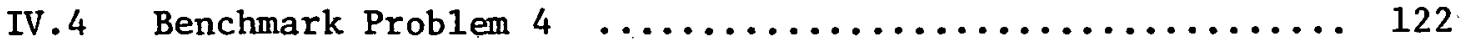

IV.5 Benchmark Problem $5 \quad \ldots \ldots \ldots \ldots \ldots \ldots \ldots \ldots \ldots \ldots \ldots \ldots \ldots .218$

IV.6 Benchmark Problem $6 \quad \ldots \ldots \ldots \ldots \ldots \ldots \ldots \ldots \ldots \ldots \ldots \ldots \ldots$

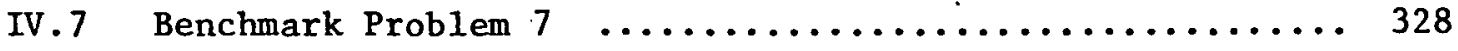

References $\quad \ldots \ldots \ldots \ldots \ldots \ldots \ldots \ldots \ldots \ldots \ldots \ldots \ldots \ldots \ldots \ldots \ldots \ldots \ldots$ 


\section{LIST OF FIGURES}

Figure

1

1.1

1.2

2.1

2.2

3.1

3.2

4.1

4.2

5.1

5.2

6.1

6.2

6.3

7.1

7.2

7.3
Title

Sign Convention for Forces and Moments

Benchmark Problem 1

Benchmark Problem 1 Spectrum

Benchmark Problem 2

48

Benchmark Problem 2 Spectrum

Benchmark Problem 3

81

Benchmark Problem 3 Spectrum

82

Benchmark Problem 4

Benchmark Problem 4 Spectrum

Benchmark Problem 5

218

Benchmark Problem 5 Spectrum

219

Benchmark Problem 6

263

Benchmark Problem 6 X-Z Spectrum

264

Beuchmark Problem 6 Y Spectrum.

Benchmark Problem 7

328

Benchmark Problem $7 \mathrm{X}-\mathrm{Z}$ Spectrum

329

Benchmark Problem 7 Y Spectrum 


\section{INTRODUCTION}

Dynamic structural analysis of piping systems is one of the most extensive engineering efforts required for the safety design of nuclear power plants. Such analysis is normally performed by using computer programs which can handle complex system geometries and various loading conditions, static or dynamic.

Applicants for nuclear power plant licenses are required to provide confirmation of the adequacy of their programs, as prescribed by the guidelines of the Standard Review Plant (NUREG75/087, Sept. 1975) and Appendix B, Section III, of 10CFR50. Of particular concern is the class of programs which are used by the industry to perform dynamic. structural analyses of complex piping systems using the response spectrum method. These are generally large programs, based on the finite element method, which consider the structures to be elastic over the entire deformation history and to experfence small displacements and rotations. Some of these programs are commonly available general purpose programs, while others are of a proprietary nature.

A need has been determined for additional verification of these structural computer programs beyond the level currently provided by applicants and licenses. The quality of these programs may be determined from a comparison of program generated solutions with known solutions of selected benchmark problems. A set of such problems and corresponding solutions are provided herein. The structures defined in these problems are assumed to experience linear elastic behavior and be subjected to dynamic loading induced by uniform earthquake 
type excitation in three directions. Dynamic solutions were determined by application of the response spectrum method of seismic analysis, based on the following combinations described in Regulatory Guide 1.92, Rev. 1, Feb. 1976: Interspatial combination SRSS (Section 2.1); Intermodal combination - Grouping method (Section 1.2.1).

The benchmark solutions were obtained by using the program EPIPE, which is a modification of the widely available program SAP IV. A brief outline and pertinent background of this program, and descriptions of its input-output formats are also included in this report. 


\section{BACKGROUND}

\section{II.1 Program Description}

The EPIPE program is a modified version of the general purpose program SAP $\mathrm{IV}^{4}$ specifically prepared to perform piping analyses. The piping system may consist of straight elements, elbows and tees with spring hangers and anchor restraints. The loads can consist of mechanical loads, dead weight loads, thermal loads, anchor point support movements, seismic loadings, or any combinations of these conditions.

Since elastic piping analysis is a well established procedure, only a brief outline of the theoretical considerations used in obtaining the dynamic solutions will be presented. The analysis of a piping system iscarried out by use of the stiffness matrix method, in which the piping is represented by a network of basic elements (straight and curved beams, and one-dimensional elements) interconnected at the nodes. The formulation of stiffness matrices for straight pipe (beam) and curved pipe may be found in several references such as (6) and (7). For a curved pipe or tee, the flexibility factor and stress intensification factor are considered in the formulation of the stiffness matrix and in the stress calculations, respectively. These faclurs are generally functions of pipe geometry and internal pressure. 5,8

The dynamic response of a piping system is described mathematically by the equations of motion

$$
\cdot[M]\{\ddot{u}\}+[C]\{\ddot{u}\}+[K]\{u\}=\{R(t)\} .
$$


Only the lumped mass approximation is allowed. Thus, In equation (1) M represents the diagonal mass matrix of the structure, $\ddot{u}$, the nodal acceleration vector; $\dot{u}$, the nodal velocity vector; $c$, a damping matrix, for which the Ragleigh damping is assumed, $1 . e ., C=\alpha M+\beta K$, where both $\alpha$ and $\beta$ are the damping coefficients; $K$, the stiffness matrix and $R(t)$, either a vector of arbitrary time-dependent loads or of effective loads resulting from earthquake motion.

The dynamic analysis may be performed by either the mode superposition method, the direct integration method, or by response spectrum analysis. All three methods are available in the EPIPE program, however only the response spectrum analysis procedure was employed in developing the solutions included herein.

The response spectrum analysis method is the primary method used to determine the dynamic response of a piping system to earthquake excitations. It is considered an acceptable, usually conservative, substitute for the more complex time history analysis methods.

The solution by this method closely parallels the solution by the modal superposition method. For ground motion excitation the system equations are expressed in terms of the relative deflection vector $u_{r}$;

$$
[M]\left\{\ddot{u}_{r}\right\}+[C]\left\{\dot{u}_{r}\right\}+[K]\left\{u_{r}\right\}=-[M]\left\{\ddot{u}_{g}\right\}
$$

where

$$
\begin{aligned}
& u_{r}=u-u_{g}, \text { system relative displacement } \\
& u_{g}=\text { ground displaicement } \\
& u=\text { system absolute displacement. }
\end{aligned}
$$


The equations are then expanded in terms of the system modal matrix and generalized coordinates;

$$
[M][\dot{\phi}]\{\ddot{q}\}+[c][\emptyset]\{\dot{q}\}+[K][\emptyset]\{q\}=-[M]\left\{\ddot{u}_{g}\right\}
$$

where

$$
\begin{aligned}
& {[\emptyset]=\text { normalized modal matrix, }\left[\emptyset^{\mathrm{T}}\right][\mathrm{M}][\emptyset]=\mathrm{I}} \\
& \{\mathrm{q}\}=\text {,generalized coordinates vector. }
\end{aligned}
$$$$
\text { Multiplication by the transpose of the modal matrix yields • }
$$

$$
\{\ddot{q}\}+[\Delta]\{\dot{q}\}+\left[\omega^{2}\right]\{q\}=-\left[\emptyset^{T}\right][M]\left\{\ddot{u}_{g}\right\}
$$

where

$$
\begin{aligned}
{\left[\omega^{2}\right]=} & \text { diagonal matrix of elgen-values } \\
{[\Delta]=} & \text { diagonal matrix of modal damping coefficients, where the } \\
& \text { damping is assumed to satisfy the modal orthognolity } \\
& \text { condition. }
\end{aligned}
$$

This results in $\mathrm{n}$ uncoupled equations for the generalized coordinates.

The equation set can be solved for the maximum modal response for each system degree of freedom corresponding to excitation in each spatial direction. That is the solution provides; 


$$
u_{m, n, i}=\frac{s_{n, i}}{; \dot{\omega}_{n}^{2}} \emptyset_{m, n} \Gamma_{n, i}
$$

where

$$
\begin{aligned}
& u_{m, n, i}=\text { maximum displacement of } m-t h \text { degree of freedom } \\
& \text { in the } n-t h \text { mode due to excitation in the } i-t h \\
& \text { spatial direction. } \\
& S_{n, i}=\text { value of' spectral acceleration corresponding } \\
& \text { to frequency } \omega_{n} \text {, and the } i-t h \text { spatial direction } \\
& \text { input response spectrum } \\
& \Gamma_{n, i}=\text { modal participation factor for mode } n \text { and } i-t h \\
& \text { spatial direction } \\
& \omega_{\mathrm{n}} \quad=\mathrm{n}-\mathrm{th} \text { system natural frequency } \\
& \emptyset_{\mathrm{m}, \mathrm{n}}=\text { modal deflection of degree of freedom } m \text { in } n \text {-th mode } \\
& \text { m . = degree of freedom index } \\
& \mathrm{n}=\text { modal index } \\
& \text { i }=\text { spatial index }
\end{aligned}
$$

For the general case where a three component selsmic excitation is considered the solution provides $3(\mathrm{~m})(\mathrm{n})$ component answers. For the final solution the component results must be combined over the $\mathrm{n}$ modes, intermodal combination, and over the 3 excitations, interspatial combination, to provide $\mathrm{m}$ resultant system degree of freedom displacements.

The EPIPE program has the facility to perform the spatial and modal combinations in several ways. The procedure adopted in a11 solutions except where noted is to perform the interspatial combination first in accordance with the SRSS rule followed by intermodal combinations using the grouping method as described in Section 1.2.1 
of the Regulatory Guide 1.92. However problems 2, 4, 6 and 7 also contain the solutions calculated by performing the intramodal combination first followed by the interspatial combination: 


\section{-II.2 Input-Output Format}

To facilitate proper use of the bench mark problems a brief summary of the pertinent EPIPE input-output data lists will be presented.

As with any piping analysis code the input data must fully define the geometry of the system under study, the calculational mode to be employed in the analysis and the forcing functions, if any, driving the system. The EPIPE data input format is almost identical to that of SAP IV, clearly labeled and should be easily interpreted. In the description to follow only those parameters for which misinterpretation may occur will be discussed.

The first page shows a table indicating the number of nodal points, element types, load cases, frequencies, etc.', followed by a listing of the nodal point coordinates. The number of load cases refers to static analysis runs and is not applicable to these problems. 'l'he number of frequencies is the number of frequencies solved for in the eigen-value routine and used in the subsequent response spectrum analysis. In the nodal point list, all-dimensions are in inches and for the boundary condition codes "1" signifies that that degree of freedom is completely restrained while "0" or "-0" signifies complete freedom. . As indicated, each node potentially has six degrees of freedom. "X" signifying displacement in the X global direction, "XX" signifying rotation about the $\mathrm{X}$ global axis.

The next blocks of input information are the input element data lists. Since the system may be subdivided into groups of elements 
this data is entered group by group. Only two types of elements exist, boundary or spring elements and pipe (either tangent or bend) elements. A single group is comprised of all pipe elements or all spring elements as intermixing element types within a group is not allowed.

For a typical pipe element data group, the first page lists control information for the group, i.e., type of element, number of. elements, etc. This is followed by a material properties table where on each line of this table are specified; the material identification number, the temperature for which the data applies and the corresponding modulus of elasticity, Poisson ratio and coefficient of thermal expansion. Following this is the section properties table where all data entries are clearly labeled. Next are listed entries entitled "Load Case Multipliers". These on1y apply to static analysis runs and may be ignored. The last and largest list in the group data is the element definition and connectivity table. Again the columns in this table are clearly labeled. For the bend elements, two lines are shown. The pressure indicated on the first line is the value of pressure used in the computation of the bend flexibility. The entries on the second line are the bend radius, third point declarer and the $X, Y$ and $Z$ coordinates of the third point. If the third point declarer is "TI" the coordinates of the bend tangent intercept are listed, if "CC" the coordinates of the bend center of curvature are listed. The columns entitled direction cosines, wall fraction and input tag may be ignored.

The group data list for boundary or spring elements is somewhat simpler. There is the element control information followed by 
element load case multipliers which again may be ignored. The element definition and connectivity list follows and is clearly labeled. The interpretation of the pertinent information in this table is; the spring spans between the piping system node $N$ and the fixed ground node $N I$, code $K D$ and $K R$ are spring type declarers where $K D=1$ signifies a linear spring while $\mathrm{KR}=1$ signifies a torsional spring; the last column lists the appropriate spring rates. All other information in this table may, be ignored.

The next table of input is labeled nodal loads. For the dynamic cases the table is a listing of all the concentrated masses, and the nodes at which they are located, acting on the system. The total mass used in each analysis is the sum of these quantities plus any distributed mass developed in the pipe elements. Distributed mass is developed in the pipe elements only if quantities appear in the columus entitled weight/unit length - mass/unit length in the pipe element section property tables.

The remaining input data describes the forcing function input spectra. The data is interspersed between the natural frequency mode shape - modal participation factor outputs. The information describing the type and number of input spectra, the spectra welghting factors, the combination rule declarer and the cluster factor employed are listed immediately following the output frequency list. The input spectrum definition list immediately follows the participation factor 
output. It must be noted that linear interpolation is used vis-a-vis the spectrum table.

The output includes listings of the' system natural frequencies, some of the corresponding mode shapes for both a mass normalization and a unity normalization, the global displacements/rotations of all nodal points and element force outputs (labeled stresses) presented group by group. The element force outputs are referred to local element coordinate systems, not the global system. Figure 1 shows the sign convention and coordinate interpretation of these element forces. All of the output tables are clearly labeled. 


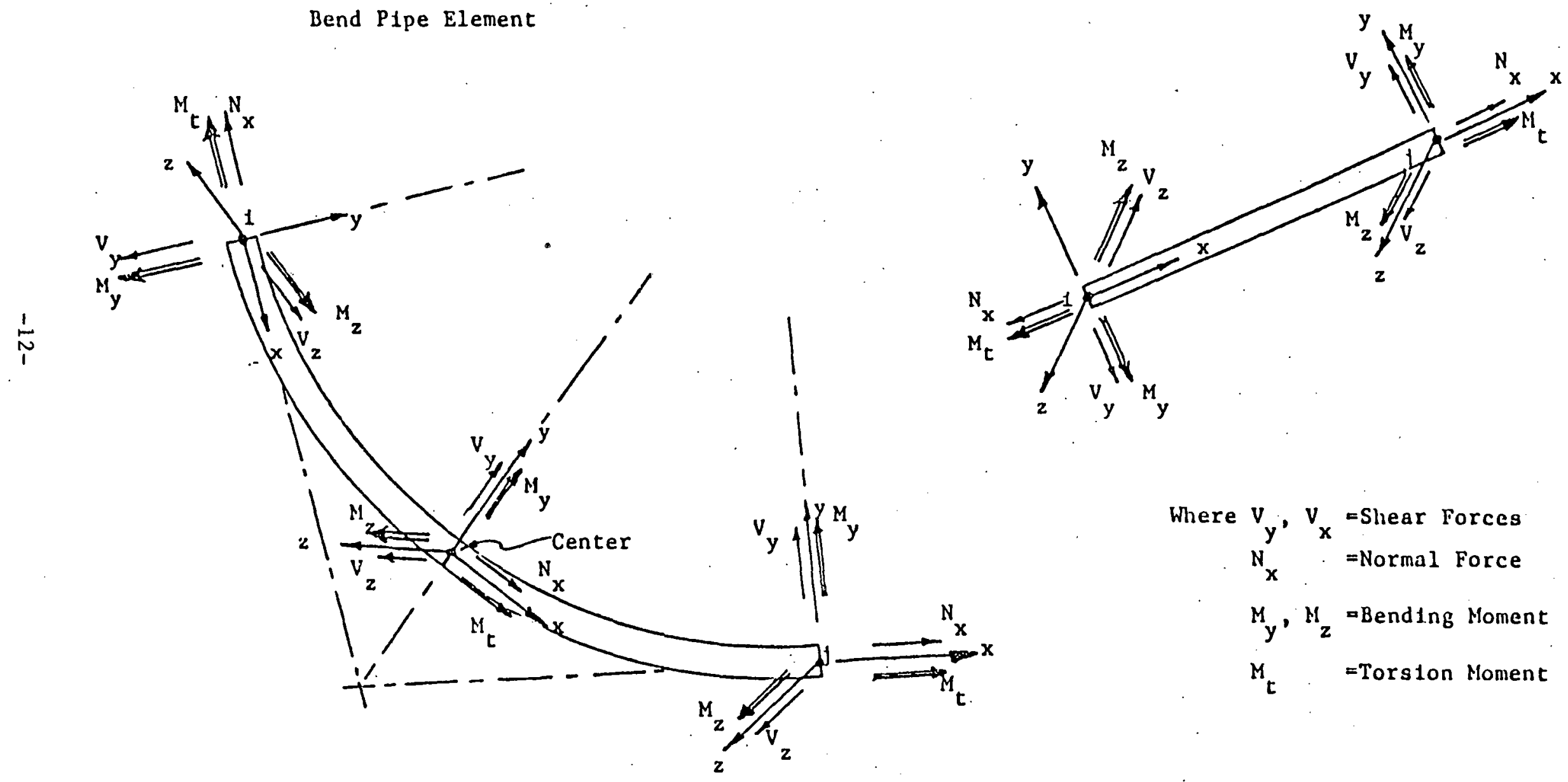

Fig. 1 


\section{DESCRIPTION OF PIPING BENCHMARK PROBLEMS}

Seven benchmark problems are included in this report, ranging from simple configurations to configurations taken from actual nuclear power plant piping systems. For each problem a schematic of the system, a complete listing of the input data and a complete listing of the output results for a response spectrum computation are included.

Although the response spectrum method was used throughout, the excitation function and computation options were not identical for all problems. The differences include: the combination sequence used, the use or ignorance of clustering, the number of input spectra and the corresponding weighting factors used and the frequency number approximation. A brief description of each problem delinating these differences is presented below; however the user is referred to the problem input data lists for the definitive description of each benchmark problem.

For benchmark problems $2,4,6$ and 7 clustering by the grouping method was employed. For each of these problems two solutions are included. The first solution, designated a, corresponds to the interspatial/intermodal combination sequence while the second solution, designated $b$, corresponds to the intermodal/interspatial combination sequence. The two solutions are provided to demonotrate that solutions with clustering are combination dependent, to quantify to a limited extent the resultant differences, and to accommodate those users' whose computer methods have one or the other combinational sequence preprogrammed. 
As mentioned the benchmark problems range from simple configurations, problems 1-3, to complex configurations problems 4-7. The simple configurations are included as they allow ready hand calculational checks of all pertinent results. However the solution of only these should not be construed as a suitable verification of a user code. To perform a complete verification solutions to all the problems should be developed. 
III.1 Benchmark Problem 1, Hovgaard Problem

This problem is a simple, three-dimensional piping bend made up of only in line straight and bend pipe elements between two. fixed anchors, Figure 1.1. The pertinent parameters are:

$$
\begin{array}{ll}
\text { pipe outer diameter } & -7.288^{\prime \prime} \\
\text { pipe wall thickness } & -.241^{\prime \prime} \\
\text { modulus of elasticity } & -24 \times 10^{6} \mathrm{psi} \\
\text { Poissons ratio } & -.3 \\
\text { reference pressure } & -0.0 \mathrm{psi} \\
\text { distributed mass } & - \text { no }
\end{array}
$$

where the reference pressure is the value of pressure ised in the computation of bend element flexibility factors and the no statement for distributed mass indicates that all the mass is input as concentrated loadings (i.e., no internal generation of element mass). For the solution a five frequency approximation was used with a single input spectrum, shown in Figure 1.2, having weighting factors of $1.0,0.667$ and 1.0 in the $X, Y$ and $\mathrm{Z}$ global directions respectively. As ali resultant system natural frequencies are spaced greater than $10 \%$ apart, clustering does not occur and the solution is therefore independent of the combination sequence employed.

This problem was selected as a benchmark because its simplicity allows the ready checking of key results. If lack of agreement between these and user generated results occurs, hand calculations can be used to resolve the differences. The problem is also one of the few piping problems for which partial analytical 
solutions for static loadings exist in the literature ${ }^{9,10}$. As such it was one of the problems used in the verification testing of the EPIPE code ${ }^{1}$.

III.2 Benchmark Problem 2, Coffee Table

The structure is a multibranched configuration resembling a table, Figure 2.1. The pertinent parameters are:

$$
\begin{array}{ll}
\text { pipe outer diameter } & -2.375^{\prime \prime} \\
\text { pipe wall thickness } & -.154 " \\
\text { modulus of elasticity } & -27.9 \times 10^{6} \mathrm{psi} \\
\text { Poissons ratio } & -.3 \\
\text { reference pressure } & -0.0 \mathrm{psi} . \\
\text { distributed mass } & - \text { no. }
\end{array}
$$

For the solutions included a five frequency approximation was used with a single input spectrum, Figure 2.2, having weighting factors of $1.0,0.667$ and 1.0 respectively. Clustering via the grouping method was employed with a cluster factor of 0.1 . Solutions were developed for both combination sequences. The solution corresponding to the interspatial/intermodal combination is designated $2 a$. Only the output listing for the second solution is included designated $2 \mathrm{~b}$. This problem is a modification of a problem which exists in the literature and for which both experimental and analytically estimates for frequencies were determined, references 12, 13, 14 . The original problem was modified so that its frequencies fell in the high amplification region of the prescribed response spectrum. The problem in its original form was used in the verification 
testing of EPIPE. The problem has symmetric and antisymmetric modes which permits a quick check on the symmetry of the deformatinn and clustering of frequencies which permits a hand check on various methods of modal combination.

\section{3 Benchmark Problem 3}

This structure, Figure 3.1, is an expanded version of the first benchmark problem. The pertinent parameters are:

$$
\begin{array}{ll}
\text { pipe outer diameter } & -7.288^{\prime \prime} \\
\text { pipe wall thickness } & -0.241^{\prime \prime} \\
\text { modulus of elasticity } & -24.0 \times 10^{6} \mathrm{psi} \\
\text { Poissons ratio } & -0.3 \\
\text { reference pressure } & -350 \mathrm{psi} \\
\text { distributed mass } & -
\end{array}
$$

For the solution a ten frequency approximation was used with a single input spectrum, Figure 3.2, having weighting factors of $1.0,0.6667$ and 1.0 respectively. A cluster factor of 0.1 was used but because of the natural frequency spacing clustering did not occur and the solution is insensitive to the combination sequence.

This problem was developed as an alternate to benchmark problem two. It contains several anchors and a branch connection, as with that problem, but in a configuration more nearly resembling a real piping system. It is a simple system which permits hand checklng of modal combination methods if necessary. It includes intermediate spring supports to simulate hangers and snubbers and a flexible anchor at nodal point 1 . The problem is designed to cvaluate the capacity of a users code to assess these features. 
The flexibility provision at anchor 1 allows a check on boundary or anchor force predictions.

\section{III.4 Benchmark Problem 4}

This structure, Figure 4.1 , simulates the primary system of a hypothetical two loop reactor plant. It consists of an elastically supported reactor vesse1, nodes 48-51, two steam generators, nodes 1-10 and 88-97 and four primary pumps connected by three and four foot diameter piping. The reactor, steam generators and pumps were modeled with massless pipe elements dimensioned to simulate the stiffness of these components. The pertinent parameters are:

$$
\begin{array}{ll}
\text { pipe outer diameter } & -36 \text { thru } 144^{\prime \prime} \\
\text { pipe wall thickness } & -0.38 \text { thru } 8^{\prime \prime} \\
\text { modulus of elasticity } & -29.0 \times 10^{6} \mathrm{psi} \\
\text { Poissons ratio } & -0.3 \\
\text { reference pressure } & -2400 \mathrm{psi} \\
\text { distributed mass } & - \text { yes }
\end{array}
$$

For the solutions a thirty frequency approximation was used with a single input spectrum, Figure 4.2, having weighting factors of 1.0 , 0.6667 and 1.0 respectively. Clustering, with a cluster factor of 0.1 , was used and was effective. Two solutions are included, that designated 4a corresponding to the interspatial/intermodal combination sequence while that labeled $4 \mathrm{~b}$ corresponding to the inverted sequence.

This problem was designed to be a definitive benchmark. It incorporates most of the features found in true piping systems in a realistic configuration. It is of a size or larger than the problems that would normally be considered in real plant evaluations. It is 
bisymmetric and exhibits some near equal eigen-values, frequency pairs $19 / 20,24 / 25$ and $26 / 27$. The large problem size, the symmetry and the equal eigen-value characteristies all pose significant tests of users codes. Any tendency towards round off error buildup will be magnified by the problem size and exhibited by a lack of symmetry in the solution results. Any sensitivity to the equal eigen-value condition, a common characteristic of many eigen-value solver routines, will result in completely distorted solution results. In short the problem is of sufficient complexity to fully exercise any computer code being used in the industry. This problem, in a slightly modified form, was the subject of an extensive study ${ }^{2}$. Solutions were developed for a response spectrum analysis, a time history analysis using the modal superposition solution method and a time history analysis using the direct integration solution method. The solution sensitivity to integration time step size, rotary inertia affects and the equal eigen-value condition were evaluated.

III.5 Benchmark Problem 5

The structure, Figure 5.1 , is an inline system between two fixed anchors. The pertinent parameters are:

$$
\begin{array}{ll}
\text { pipe outer diameter } & -12.75 \text { and } 14.0^{\prime \prime} \\
\text { pipe wall thickness } & -0.375 \text { thru } 1.312^{\prime \prime} \\
\text { modulus of elasticity } & -25.2 \times 10^{6} \text { and } 26.2 \times 10^{6} \mathrm{psi} \\
\text { Poissons ratio } & -0.3 \\
\text { reference pressure } & -0.0 \mathrm{psi} \\
\text { distributed mass } & - \text { no }
\end{array}
$$


For the solution an eleven frequency approximation was used with one spectrum, Figure 5.2, having weighting factors of $1.0, .6667$ and 1.0 respectively. As clustering was not used the solution is insensitive to the combination. sequence; however the interspatial/intermodal sequence was used for the solution included.

This problem, as well as the two problems to follow, were each taken from actual nuclear power plant piping systems. The problem is relatively straight forward with a simple geometry. It has two unique features; a transition between two materials and the inclusion of some valves. As regards the valves, they were modeled with thick walled, stiffened pipe elements where the stiffening was achieved by increasing the modulus of elasticity of the valve elements by a factor of three. This method of simulating valves is in accord with present industry practice. This problem is used to investigate the facility with which analysis methods treat the material transition and valve component.

\section{III. $6 \quad$ Benchmark Problem 6}

The structure, Figure 6.1, is essentially one large sweeping bend between two fixed points. The pertinent parameters are:

$$
\begin{array}{ll}
\text { pipe outer diameter } & -30 . \text { and } 32 . " \\
\text { pipe wall thickness } & -0.85 \text { and } 0.905^{\prime \prime} \\
\text { modulus of elasticity } & -29.9 \times 10^{6} \mathrm{psi} \\
\text { Poissons ratio } & -0.3 \\
\text { reference pressure } & -0.0 \mathrm{psi} \\
\text { distributed mass } & - \text { no }
\end{array}
$$


For the solutions a thirty-one frequency approximation was used with two excitation spectras, Figures 6.2 and 6.3. Clustering was employed $(C F=.10)$, and solutions for both combination sequences are included. In addition, a solution with no clustering is also included labeled $6 \mathrm{c}$.

This problem was selected from an actual piping system. It was chosen because of its unique, continuous curve geometry. For some of the analysis methods in use this type of geometry may pose a special problem as they require the introduction of imaginary tangent elements between bend elements. The problem can be used to investigate this characteristic. Additionally the problem includes independent spectra input allowing the investigation of the facility with which. independent inputs are treated by other analysis codes,

\section{7 Benchmark Problem 7.}

The structure, Figure 7.1 , is a multibranched configuration containing four anchor points. The pertinent parameters are:

$$
\begin{aligned}
& \text { pipe outer diameter - } 3.5 \text { and 4.5" } \\
& \text { pipe wa11 thickness - } 0.3 \text { and } 0.337 " \\
& \text { modulus of elasticity }-27.0 \times 10^{6} \text { poi } \\
& \text { Poissons ratio } \quad-0.3 \\
& \text { reference pressure } \quad-0.0 \mathrm{psi} \\
& \text { distributed mass - no }
\end{aligned}
$$

For the solution a twenty-two frequency approximation was used with two.independent input excitation spectra, Figure 7.1 and 7.2. Clustering was employed $\left(C F^{\prime}=0.1\right)$ and two solutions $7 a$ and $7 b$ are included for the two combination sequences. 
This problem was again selected from an actual piping system. It is the largest of the benchmark problems and permits checking analysis features which include multiple branches, multiple anchors intermediate supports and hangers, valves and multiple excitation. 
IV. PIPING BENCHMARK PROBLEMS

$-23-$ 

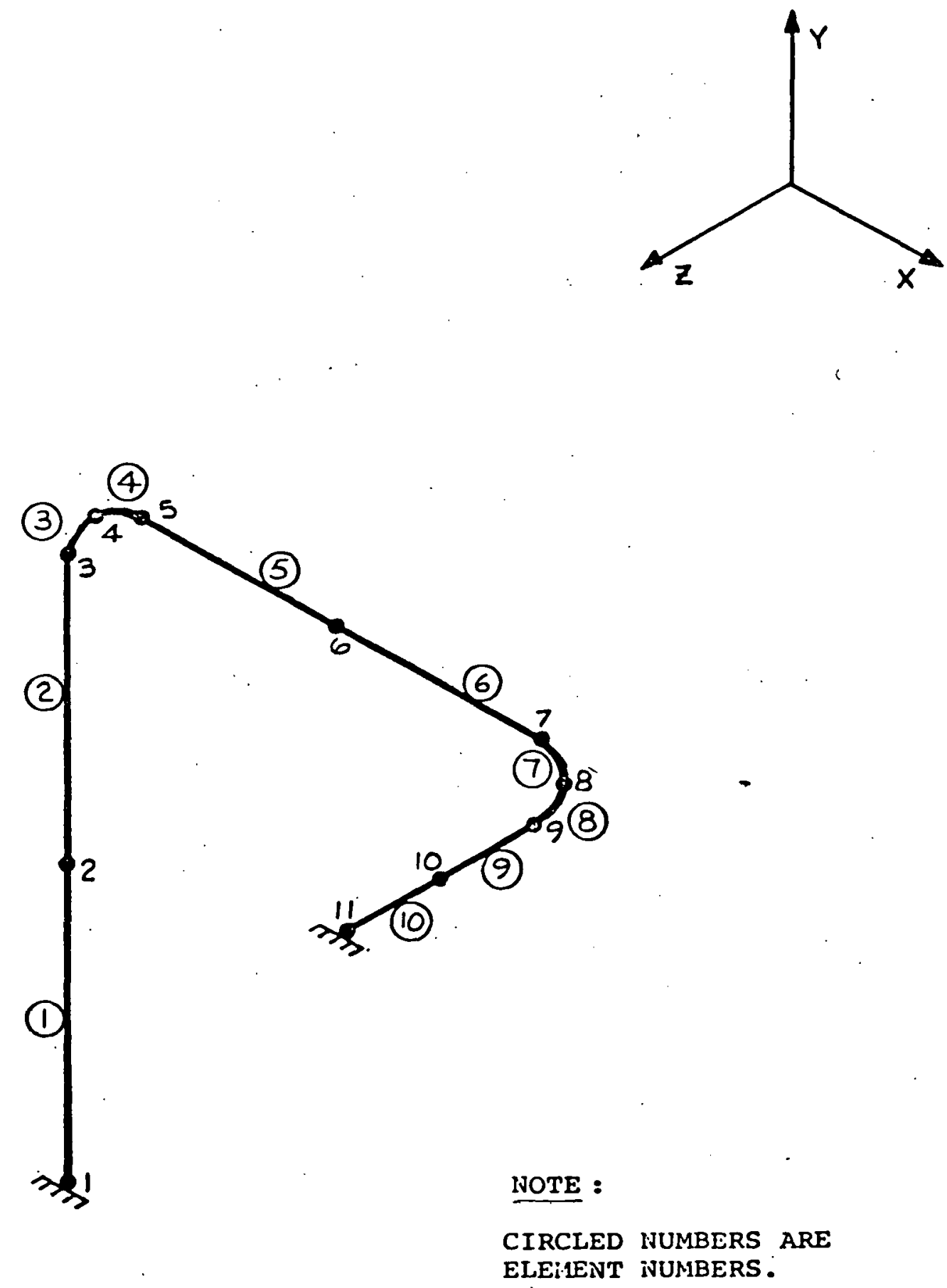

BENCHMARK PROBLEM NO.I

Figure 1.1 


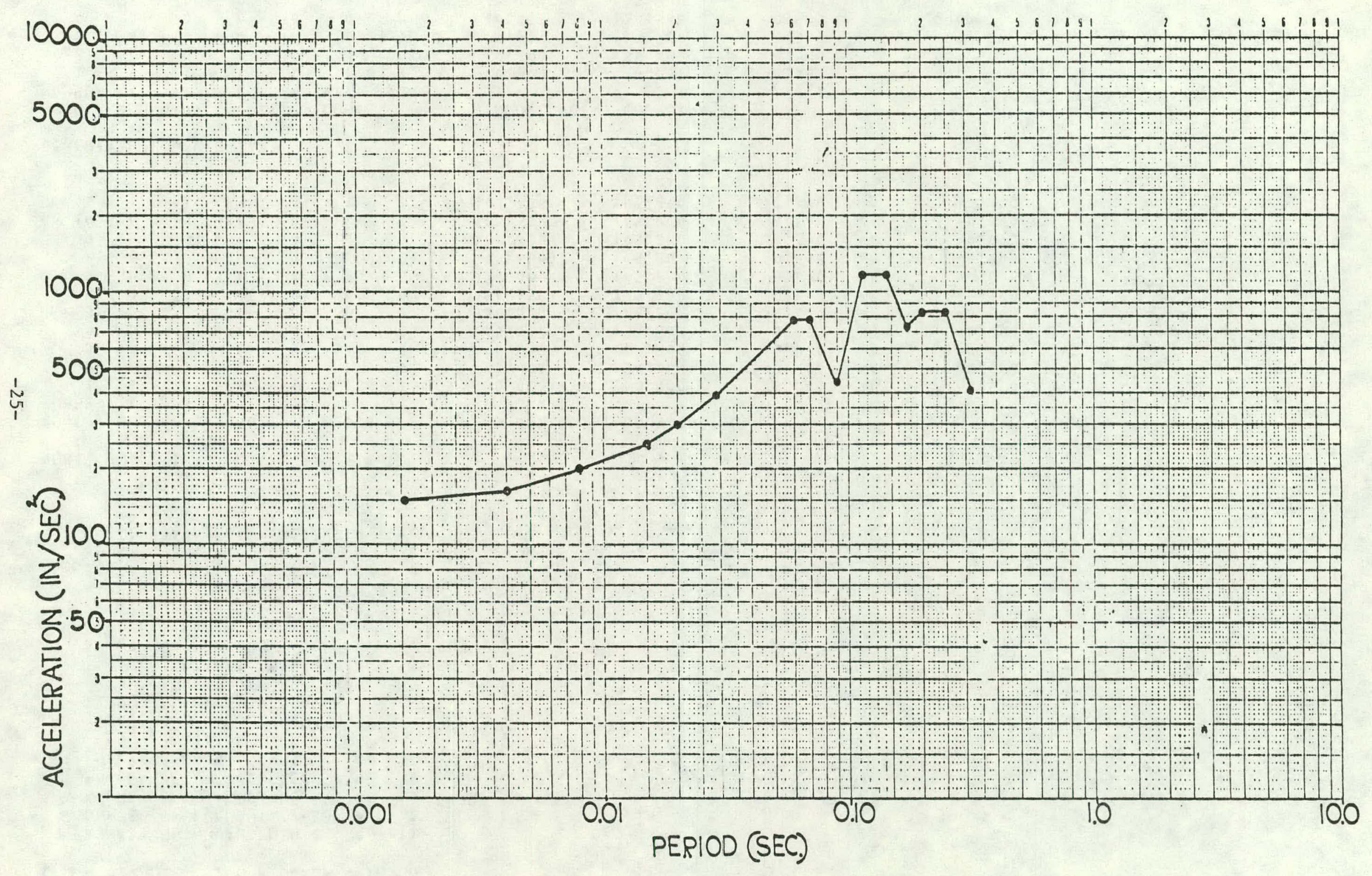

Figure 1.2 
MOVGAARD PRIJULFM (RESHITHSE BHECTHUM AHALYSIS)

CUNTHUL I NFUKMATIUN

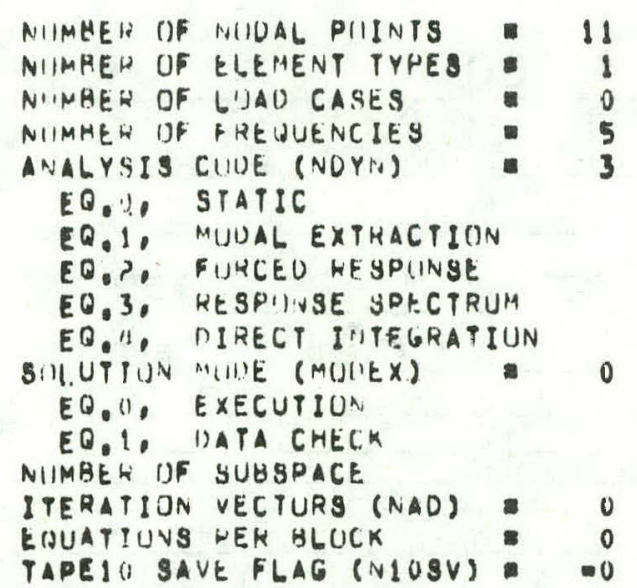

NODAL POINT INHUT DATA

\begin{tabular}{|c|c|c|c|c|c|}
\hline \multirow{2}{*}{$\begin{array}{l}\text { I NOUE } \\
\text { N NUMBER }\end{array}$} & \multicolumn{2}{|c|}{ HUUNDAHY } & \multicolumn{2}{|c|}{ CUNDIT IIIN } & \multirow{2}{*}{ COUES } \\
\hline & $x$ & Y & 2 & $x x$ & \\
\hline or & 1 & 1 & 1 & 1 & 1 \\
\hline 2 & 0 & $u$ & 0 & 0 & 0 \\
\hline 3 & 0 & 0 & 0 & 0 & v \\
\hline$\Delta$ & 0 & 0 & 0 & 0 & 0 \\
\hline 5 & 0 & 11 & 0 & 0 & 0 \\
\hline 6 & 0 & $n$ & 0 & 0 & () \\
\hline 7 & 0 & 11 & 0 & 0 & () \\
\hline 8 & 0 & (1) & 0 & 0 & 0 \\
\hline 9 & 0 & 0 & 0 & 0 & $v$ \\
\hline 10 & (1) & 1) & 0 & 0 & 0 \\
\hline 11 & 1 & 1 & 1 & 1 & 1 \\
\hline
\end{tabular}

\begin{tabular}{|c|c|c|c|c|}
\hline NUOAL & PUINT COURUINATES & 2 & & $T$ \\
\hline 0.000 & 0.000 & 0.0010 & $=0$ & -0.000 \\
\hline 0.000 & 54.450 & 0.0110 & $=1$ & $=0.000$ \\
\hline 0.000 & 108.900 & 0.000 & $-n$ & -0.000 \\
\hline 10.032 & 134.568 & 0.000 & $=0$ & -0.0100 \\
\hline 36.300 & 145.2110 & 0.000 & $=0$ & $=0.000$ \\
\hline 54.150 & 145.200 & 0.000 & $=1)$ & $-1) .000$ \\
\hline 72.000 & 145.200 & 0.0110 & $=0$ & $=0.000$ \\
\hline 97.608 & 145.200 & 10.032 & $=0$ & $=0.000$ \\
\hline 108.300 & 145.200 & 36.300 & $=0$ & -0.000 \\
\hline 108.300 & 145.200 & 56.5010 & $-n$ & $=0.000$ \\
\hline 108.300 & 145.200 & 77.5100 & $=0$ & $=0.0 \cap 0$ \\
\hline
\end{tabular}

GENERATEU NDOAL DATA

$\begin{array}{ccccccc}\text { NOUE } & \text { BUUNDARY } & \text { CUNDITIUIN } & \text { COUES } \\ \text { NUMBER } & X & Y & \mathbf{Z} & X X & Y Y & \mathbf{Z Z} \\ 1 & 1 & 1 & 1 & 1 & 1 & 1 \\ 2 & 0 & 0 & 0 & 0 & 0 & 0 \\ 3 & 0 & 0 & 0 & 0 & 0 & 0 \\ 4 & 0 & 0 & 0 & 0 & 0 & 0 \\ \mathbf{5} & 1 & 0 & 0 & 0 & 0 & 0 \\ 6 & 0 & 0 & 0 & 0 & 0 & 0 \\ 3 & 0 & 0 & 0 & 0 & 0 & 0 \\ & 0 & 0 & 0 & 0 & 0 & 0 \\ & 0 & 0 & 0 & 0 & 0 & 0 \\ 10 & 0 & 0 & 0 & 0 & 0 & 0\end{array}$

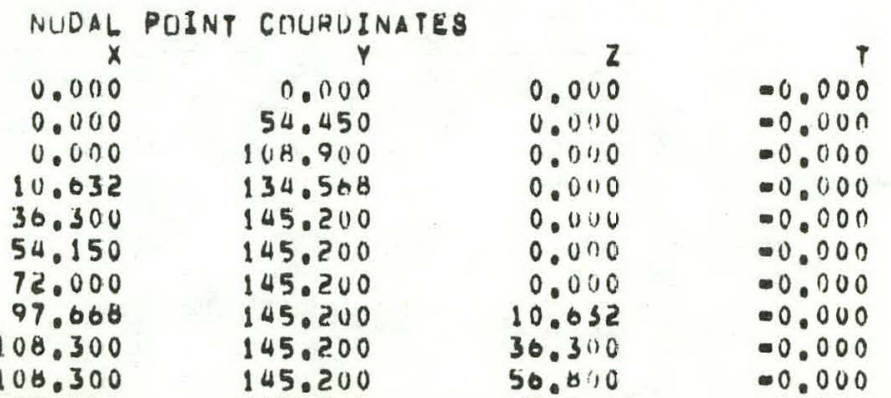


IRUL INFUKMATIUN

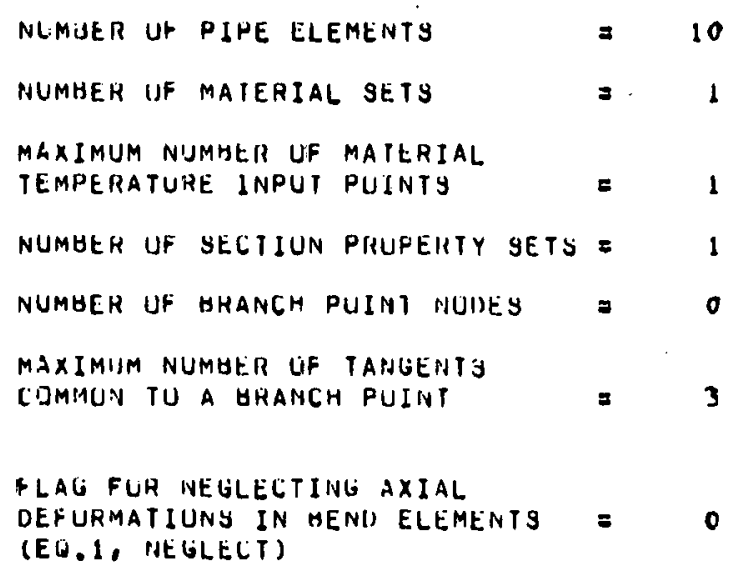

$N_{1}^{1}$

M. A TERI AL PRU UER TY TABLES

MATERIAL NUMBER $=(1)$

NUMHER UF

IEMPERATURE PULNTY a 6 (1)

IUENTIFICATIUN $\rightrightarrows($ ALLOY BTEEL

$-0.00<4000000.0$

$.300 \quad .760 t-U S$ 


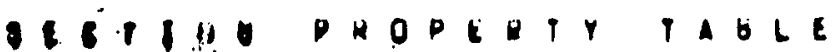

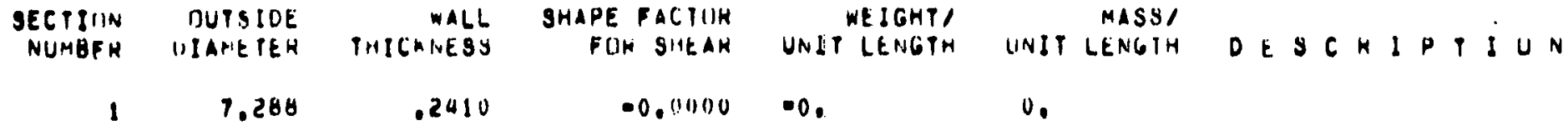

ELEMENT LUAIICASEMULIIPLIEKS

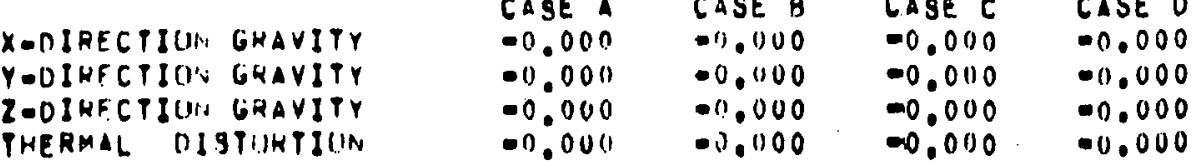

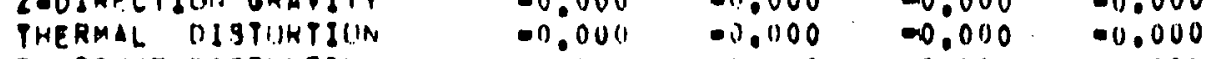

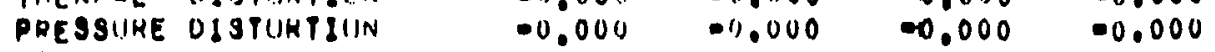


REFERENCE INTERNAL EMPTEATUNE PRT SSUHE (HENU CIHIHU

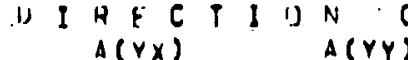
(X3- (Y)

$$
\text { UI I NES S N N INHUT }
$$
(23- A(Y-Z) INCAEM IHOINATE) (HI)INATE) (IREINATE) FHACTION)

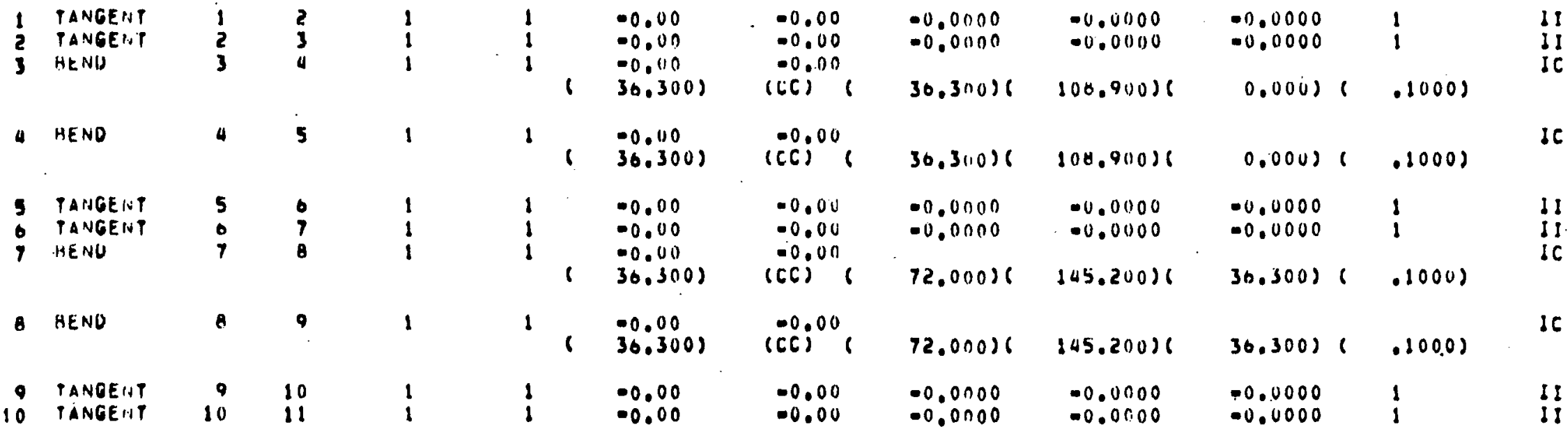


NUDAL LUADB (STATIC) UKRMASGES (OYNAMIC)

\begin{tabular}{|c|c|c|c|c|c|c|c|c|c|c|}
\hline $\begin{array}{r}\text { NUDE } \\
\text { NUMGER }\end{array}$ & $\begin{array}{l}\text { LIIAO } \\
\text { CASE }\end{array}$ & $\begin{array}{r}X \cap A X I S \\
\text { FUHCE }\end{array}$ & $\begin{array}{r}Y A A X I S \\
\text { FUNCE }\end{array}$ & $\begin{array}{r}2=A \times \text { IS } \\
\text { FUIRCE }\end{array}$ & & $\begin{array}{l}X \in A X \mid S \\
\text { MUMENT }\end{array}$ & & $\begin{array}{l}Y=A X \ S \\
\text { MUMENT }\end{array}$ & & $\begin{array}{l}Z-A \times \& 3 \\
\text { MUMENT }\end{array}$ \\
\hline $\begin{array}{r}2 \\
3 \\
4 \\
5 \\
-6 \\
7 \\
9 \\
0 \\
10\end{array}$ & $\begin{array}{l}0 \\
0 \\
0 \\
0 \\
0 \\
0 \\
0 \\
0 \\
0\end{array}$ & 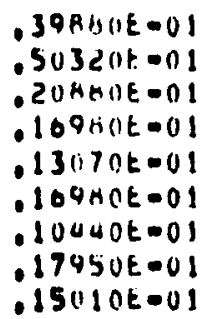 & 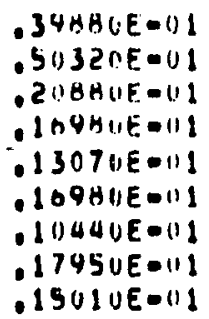 & $\begin{array}{l}.39480 E-01 \\
.50320 E-01 \\
.20880 E-01 \\
110900 E-01 \\
13070 E-01 \\
110980 E-01 \\
110440 E=01 \\
117950 E-01 \\
115010 E-01\end{array}$ & 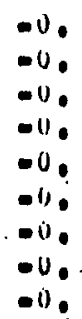 & . & 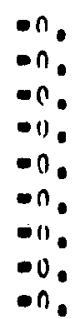 & & $\begin{array}{l}-0 . \\
-0 . \\
=0 . \\
00 . \\
-0 . \\
-0 . \\
0.0 \\
=0 . \\
-0 .\end{array}$ & \\
\hline
\end{tabular}

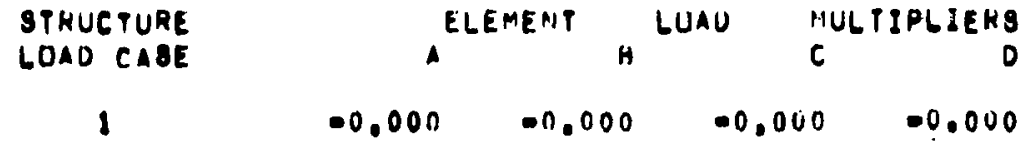


PRINT OF FREOUE NCIES

\begin{tabular}{|c|c|c|c|}
\hline $\begin{array}{l}\text { MOL } \\
\text { NUI }\end{array}$ & $\begin{array}{l}\text { CIHCULAH } \\
\text { FRE NUENCY } \\
\text { (HADISEC) }\end{array}$ & $\begin{array}{l}\text { PKE WUTENCY } \\
\text { (CYCLES/SEC) }\end{array}$ & $\begin{array}{l}\text { PERIIIU } \\
\text { (SEC) }\end{array}$ \\
\hline 1 & $.1793 E+03$ & $.2853 E+0 ?$ & $.3504 E=01$ \\
\hline$\dot{2}$ & $.35 \cup 4 E \mapsto 03$ & $.5577 E+02$ & $.1743 E-01$ \\
\hline $\mathbf{3}$ & $.5121 E+03$ & $.6150 E+02$ & $.1227 E-01$ \\
\hline$a$ & $.8900 E+03$ & $.1417 E+03$ & $.7055 E-02$ \\
\hline 5 & $.1023 E+04$ & - 102NE + 03 & $.6142 E-02$ \\
\hline
\end{tabular}

$\stackrel{\omega}{1}$ 
MODE NUMAER

FHE UUENCY $\Rightarrow 28.5350 \quad \mathrm{HZ}$.

EIGENVECTURS NURMALIZEU TO A UNIT MASY MATHIX

UISPLACEMENIS/RUTATIIJNS UF UNRESIHAINEI NLDEY

\begin{tabular}{|c|c|c|c|c|c|c|}
\hline $\begin{array}{r}\text { IUUE } \\
\text { IMUER } \\
2 \\
3 \\
4 \\
5 \\
0 \\
7 \\
0 \\
9 \\
10\end{array}$ & $\begin{array}{l}X ं- \\
\text { TKANYLATIUN } \\
3.42220 E-U 1 \\
1.01048 E+00 \\
1.30013 E+00 \\
1.30233 E+00 \\
1.30214 E+00 \\
1.30144 E+00 \\
9.40848 E-01 \\
3.57079 E-01 \\
9.54511 E-02\end{array}$ & $\begin{array}{r}Y= \\
\text { TRANSLATIUN } \\
-5.08445 F-05 \\
-1.13006 E-04 \\
-1.10545 E-01 \\
-2.90462 E-01 \\
-3.73009 E-01 \\
-4.34014 E-01 \\
-3.40124 E-U 1 \\
-1.24835 E-01 \\
-3.27549 E-02\end{array}$ & 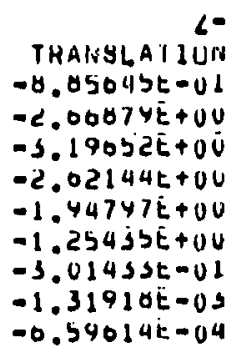 & $\begin{array}{r}x- \\
\text { HUTARIUN } \\
-2.74 .575 t-U 2 \\
-3.37031 t-U 2 \\
-2.651144 t-U 2 \\
-2.08340 E-02 \\
-1.85808 t-U 2 \\
-1.03271 t-U 2 \\
-1.2472 U t-U 2 \\
-5.78975 t-03 \\
-3.07754 t-03\end{array}$ & $\begin{array}{r}\text { HOTAIION } \\
\text { ROTAII } \\
-1.00245 t-122 \\
-2.00490 t-02 \\
-2.72333 t-02 \\
-3.07104 t-02 \\
-3.83704 t-02 \\
-3.68531 t-02 \\
-3.28633 t-02 \\
-1.01227 t-02 \\
-6.85114 t-03\end{array}$ & $\begin{array}{r}\text { KOTATICN } \\
-1.07313 E-02 \\
-1.25227 E-02 \\
-4.09107 E-05 \\
-3.17444 E-03 \\
=4.15046 E-03 \\
-9.20969 E-03 \\
-1.57579 E=03 \\
-1.36556 E-03 \\
-0.42776 E=04\end{array}$ \\
\hline
\end{tabular}


MUUE NIIMHEH 1

EIGENVECTOHS NURMALIZEU TU 1.000

UISPLACEMENIS/RUTATIUNY OF UNKESIKAINEU NUDEY

NLUE
INUMUER 10

द $-1.07000 E-01$

$3-3,14154 E-111$

$-4.00735 E-01$

$5 \quad-4,3<448 E-01$

$0 \quad-4.32401 E-0$

$7 \quad-4.323206-01$

$-3.12445 E-0$

4 -1.117UYE-O

$10-3.00174 E-02$

110
TRANSLATIUH 0.

1.77 H.5E-0S

$3.55593 E-05$

$3.45630 E-02$

$9.04081 E-02$

$1.10 B \square O E-01$

$1.37311 E-J 1$

1. 0 HOIE-O

3. $40535 \mathrm{E}-0 \mathrm{C}$

$1.02483 E-02$

0
TKANSLAIIUN 0 .

2.7700bt-U1 6. S44USE-01

1. OUUUUE + UU

$0.200425-01$

$0.094 U 2 E-01$

$3.42410 E-01$

$9.4300 S E-1)$

प. $12092 E=04$

$u$.
KUTATIUN

0.

$.740 d s t-03$

$1.05025 t-02$

$0.51740 t-03$

$5.812035-03$

$5010771-03$

5. $10777=03$

$1.011<75003$

$9.02743 E-0 a$

0 .
RLTAI IIIN

$3.13006 t-03$ $0.27212 t-03$

$0.51967 t-0 s$

$1.14465 t-02$

1.20113 HE -02

1.21 SUAE-U2

$1.02810 t-02$

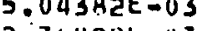

0 .
RUTATIEN

$935719 E-03$

$9.41754 t-03$

C. $84405 E-03$

$1.01677 E-03$

$1.30031 E-03$

$1.004125-03$

$4.92970 E=04$

$2.16724 t=04$

$v$. 
MUUE NIJMBEK 2

FREUUENCY $=55.7724 \quad H 2$

EIGENVELTURS NURMALIZEO TO A UNIT MASS MATHIX

UISPLACEMENTS/HOTATIUIAY UF UNHESIKALNEU NUUES

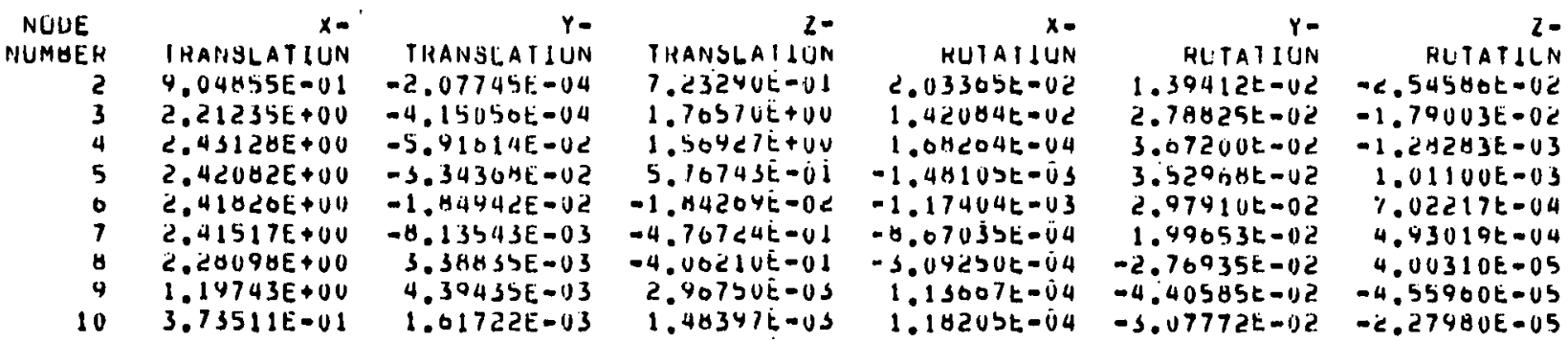


MUUE VIJMHER ¿

EIGENVEC TUHS NURMALIZED TU 1.000

UISPLACEMENISIRUTATIIJNY OF UNHESIKAINEU NUDEY

\begin{tabular}{|c|c|c|c|c|c|c|}
\hline $\begin{array}{l}\text { NOUE } \\
\text { NUMHEK }\end{array}$ & THANGLATIUN & THANSLAT IIUN & TRAHYLAIJUN & HUIATIUN & RUTAIIUN & HLTATILN \\
\hline $\begin{array}{l}1 \\
2 \\
3\end{array}$ & $\begin{array}{l}0.7 \\
3.72172 E-01 \\
9.0495 U E-01\end{array}$ & $\begin{array}{l}0.0 \\
-0.54405 E-05 \\
-1.70715 E-04\end{array}$ & $\begin{array}{l}0.97443 t-01 \\
2.9744201 \\
7.26241 E-01\end{array}$ & $\begin{array}{l}0.0 \\
0.3045 U t-03 \\
5.84348 t-03\end{array}$ & $\begin{array}{l}0 . \\
5.73411 t-03 \\
1.140 H 2 t-02\end{array}$ & $\begin{array}{l}0 . \\
-1.04713 E-02 \\
-1.36250 E=03\end{array}$ \\
\hline $\begin{array}{l}4 \\
5 \\
0 \\
7 \\
6 \\
9\end{array}$ & $\begin{array}{l}1.00000 E+00 \\
9.95045 E-01 \\
9.94046 E-01 \\
9.43374 E-01 \\
4.30179 E-01 \\
4.92509 E-01\end{array}$ & $\begin{array}{l}-2.43334 E-U 2 \\
-1.37527 E-112 \\
-7.6067 B E-03 \\
-3.34615 E-03 \\
1.30305 E-03 \\
1.80712 E-03\end{array}$ & $\begin{array}{r}0.45440 E-01 \\
2.37210 E-01 \\
-7.57410 E-0 S \\
-1.96074 E-01 \\
-1.07070 E-01 \\
1.220 S b E-03\end{array}$ & $\begin{array}{r}0.92077 t-05 \\
-0.04100 t-04 \\
=4.02841 t-04 \\
-3.50010 t-04 \\
-1.27190 E-04 \\
4.07510 t-05\end{array}$ & $\begin{array}{r}1.51032 t-02 \\
1.45176 t-02 \\
1.22532 t-02 \\
8.21183 t-03 \\
-1.13905 t-02 \\
-1.61215 t-02\end{array}$ & $\begin{array}{r}-3.27035 E-04 \\
4.15826 E-04 \\
2.44626 E-04 \\
6.027816-04 \\
1.04650 E-05 \\
-1.87534 E-05\end{array}$ \\
\hline 10 & $\begin{array}{l}1.53027 E-01 \\
0 .\end{array}$ & $\begin{array}{l}6.05172 E-04 \\
0 .\end{array}$ & $\begin{array}{l}0.10305 E-04 \\
0 .\end{array}$ & $\begin{array}{l}4.80104 t-05 \\
0 .\end{array}$ & $\begin{array}{c}-1.20588 t-02 \\
0 .\end{array}$ & $\begin{array}{l}-4.37094 E=0 D \\
U .\end{array}$ \\
\hline
\end{tabular}


MUUE NUMUER

FREQUENCY $\square 1.5000$

H2

EIGENVECTOKS NURMALLZEO TU A UIIT MASS MATHIX

IIISPLACEMENTS/RUTATIIINS OF UNHESIKAINEI NUUES

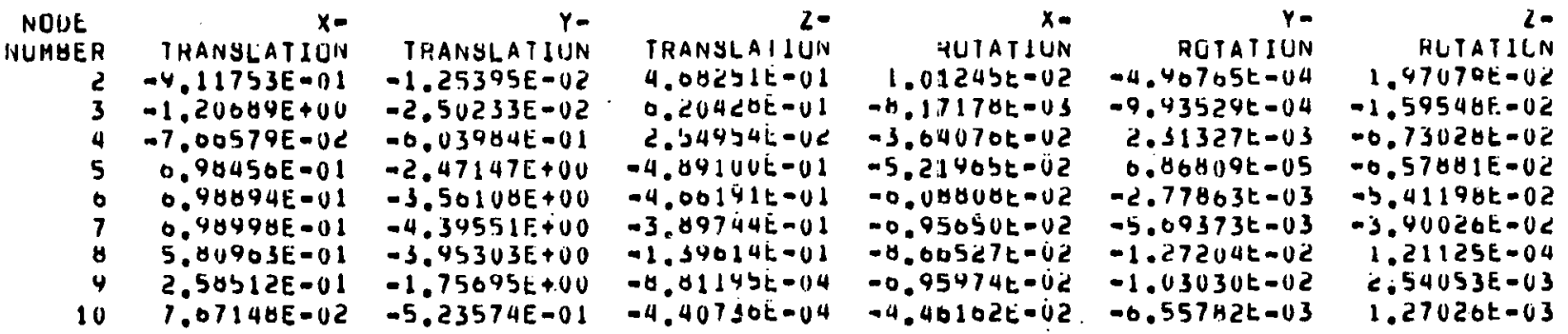


MUUE NUMHER 3

EIGEIVECTURS NURMALIZED TU 1.000

UISPLACEMENIS/RUTATIIJNY OF UNHESIITADNEU NIJUES

\begin{tabular}{|c|c|c|c|c|c|c|}
\hline $\begin{array}{l}\text { OOE } \\
\text { IMUEK }\end{array}$ & TIRANSLAT IUUN & $\begin{array}{r}\text { Y- } \\
\text { TRAHSLATIUN }\end{array}$ & TRANSLA I IJIN & $\begin{array}{r}x- \\
\text { HUTAIIUN }\end{array}$ & RUTAIIUN & RE. IATLLN \\
\hline $\begin{array}{l}1 \\
?\end{array}$ & $2.07420 E-01$ & $0.052 B 1 E-0.3$ & $-1.00 \geq 24 t-01$ & $-2.30337 t-03$ & & \\
\hline $\begin{array}{l}3 \\
4\end{array}$ & $\begin{array}{l}2.74574 E-U 1 \\
1.744 U O E-U C\end{array}$ & $\begin{array}{l}5.0 .2243 E-0.3 \\
1.37404 E-01\end{array}$ & $\begin{array}{l}-1.411 S U E-01 \\
-3.0 U C S S E-U S\end{array}$ & $\begin{array}{l}1.854 \\
0.242\end{array}$ & $\begin{array}{l}3 t-04 \\
j t=04\end{array}$ & $\begin{array}{l}\forall 0 E-03 \\
17 E-02\end{array}$ \\
\hline $\begin{array}{l}5 \\
6 \\
7 \\
8 \\
9\end{array}$ & $\begin{array}{l}-1.50402 E-01 \\
-1.59002 E-01 \\
-1.54025 E-01 \\
-1.32172 E-01 \\
-5.08128 E-02\end{array}$ & $\begin{array}{l}5.62273 E-01 \\
0.10102 E=01 \\
1.00000 E+00 \\
8.99334 E-01 \\
3.4471 .5 E-01\end{array}$ & $\begin{array}{l}1.11 C \bar{C} B E-01 \\
1.00601 E-01 \\
0.00<00 E-02 \\
5.17 E<0 E-02 \\
2.00410 E-04\end{array}$ & $\begin{array}{l}1.14750 E-02 \\
1.38507 t=02 \\
1.54204 t=02 \\
1.47139 t-02 \\
1.58338 t-02\end{array}$ & $\begin{array}{r}-1.50252 t-05 \\
0.32151 t-04 \\
1.29535 t-113 \\
2.09390 t-0.3 \\
2.34349 t-03\end{array}$ & $\begin{array}{l}71 t-02 \\
25 E-02 \\
26 t-03 \\
05 t=05 \\
03 E-04\end{array}$ \\
\hline $\begin{array}{l}10 \\
11\end{array}$ & $\begin{array}{l}-1.14530 E=02 \\
0 .\end{array}$ & $\begin{array}{l}1.19116 E-01 \\
0 .\end{array}$ & $\begin{array}{l}\text { 1. DOE } 7 U E-04 \\
\text { U. }\end{array}$ & $\begin{array}{l}1.015 \cup 4 t=02 \\
0 .\end{array}$ & $\begin{array}{l}1.49144 t-03 \\
0 .\end{array}$ & $\begin{array}{l}-4.88992 t-04 \\
v .\end{array}$ \\
\hline
\end{tabular}


MUDE NUMHER 4

FREUUENCY $\Rightarrow 141.742 \quad \mathrm{HZ}$

EIGENVECTORS NURMALLZLU TO A UNIT MASY MAIKIX

DIYHLACEMENTS/HOTATIUNY UF UNKESIKAINEU NUUES

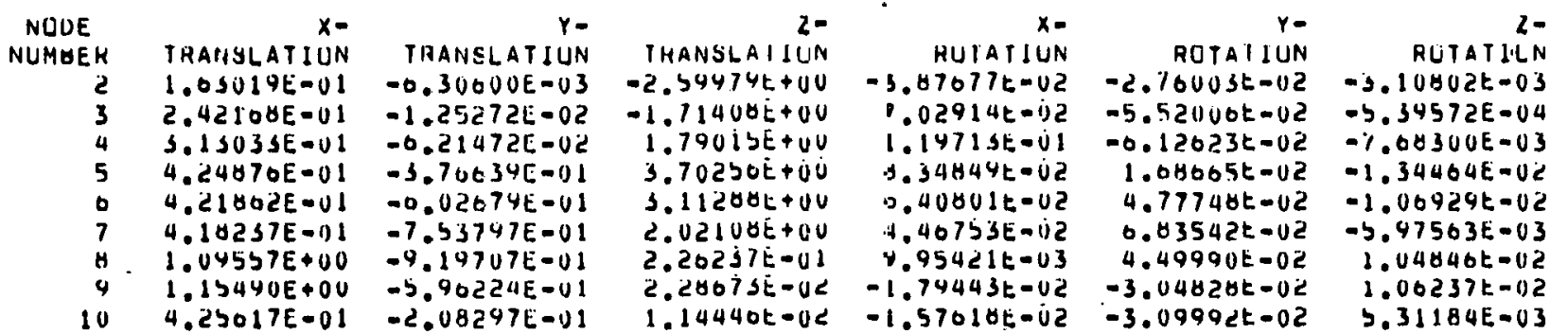


MUUE NUIAHER

EIGENVECTURS NURMALJZEIS TU 1.000

II SPLACEMENIS/ROTATIUNS OF UVKESIRAINEU NUUES

\begin{tabular}{|c|c|c|c|c|c|c|}
\hline $\begin{array}{l}\text { NUUE } \\
\text { NUMGER }\end{array}$ & TRANSLATIIJN & TRANSLATIUN & THANSLA! HUN & HUTAIIUN & ROTATIUN & $\begin{array}{r}\text { 2- } \\
\text { RUTATILN }\end{array}$ \\
\hline $\begin{array}{l}1 \\
2 \\
3\end{array}$ & $\begin{array}{l}0.4008 E-1) 2 \\
4.402 \triangle 57 E-02 \\
0.5405\end{array}$ & $\begin{array}{l}0 . \\
-1.7031 S E-03 \\
-3.34339 E-03\end{array}$ & $\begin{array}{l}0.0 \\
-7.02100 t-01 \\
-4.02440 i-01\end{array}$ & $\begin{array}{r}0 . \\
-1.047 \cup b t-02 \\
1.89840 t-02\end{array}$ & $\begin{array}{l}0 . \\
-7.45434 t-03 \\
-1.490 B 4 t-02\end{array}$ & $\begin{array}{l}0.0 \\
-0.39425 t-04 \\
-1.45730 E-04\end{array}$ \\
\hline $\begin{array}{l}4 \\
5 \\
6 \\
7 \\
5 \\
9\end{array}$ & $\begin{array}{l}8.4543 U E-02 \\
1.14752 E=01 \\
1.13438 E=01 \\
1.14439 E-01 \\
2.95895 E-01 \\
3.11919 E-111\end{array}$ & $\begin{array}{l}-1.07849 E-02 \\
-1.01724 E-01 \\
-1.02774 E-01 \\
-2.035134 E-01 \\
-2.48344 E-01 \\
-1.01030 E-01\end{array}$ & $\begin{array}{l}4.03441 E-01 \\
1.00000 E+00 \\
0.407 j 0 E-01 \\
5.45001 E-01 \\
0.11024 E-00 \\
0.17004 E-0 S\end{array}$ & 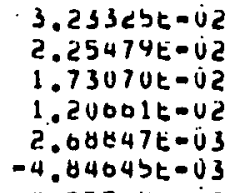 & $\begin{array}{r}-1.05454 t-02 \\
4.55537 t-03 \\
1.29032 t-02 \\
1.04014 t-02 \\
1.21535 t-02 \\
-8.23291 t-03\end{array}$ & $\begin{array}{r}-2.07505 E-03 \\
-3.03105 E-03 \\
-2.04798 E-03 \\
-1.01392 E-03 \\
2.03172 E-03 \\
2.06424 E-03\end{array}$ \\
\hline $\begin{array}{l}10 \\
11\end{array}$ & $\begin{array}{l}1.14452 E-01 \\
0 .\end{array}$ & $\begin{array}{l}-5.02577[-02 \\
0 .\end{array}$ & $\begin{array}{l}3.040445-05 \\
0 .\end{array}$ & $\begin{array}{c}-4.257 \cup \angle t-0.33 \\
U .\end{array}$ & $\begin{array}{c}-8.37237 t-03 \\
0 .\end{array}$ & $1.43464 E-03$ \\
\hline
\end{tabular}


MUIJE NIJMHEH 5

FREUUENCY a 102.820

M2

EIGENVECTURS NUHMALIZED TO A UNIT MASS MATKIX

UISPLACEMENIS/ROTATIUNY OF UINRESTKAINED NUDES

\begin{tabular}{|c|c|c|c|c|c|c|}
\hline $\begin{array}{r}\text { NOUE } \\
\text { NUMBEER } \\
2 \\
3 \\
4 \\
5 \\
0 \\
7 \\
0 \\
4 \\
10\end{array}$ & $\begin{array}{r}X- \\
\text { TRANSLATIUN } \\
-3.26447 E+U U \\
-1.54153 E+00 \\
1.14521 E+00 \\
1.44734 E+U 0 \\
1.50400 E+00 \\
1.50911 E+U U \\
1.44024 E+00 \\
6.20320 E=01 \\
2.73838 E-01\end{array}$ & $\begin{array}{l}Y- \\
10 N \\
-03 \\
-03 \\
+00 \\
+00 \\
-01 \\
+00 \\
+00 \\
+00 \\
-01\end{array}$ & 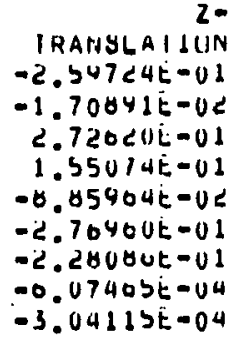 & 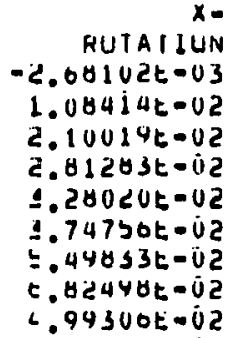 & 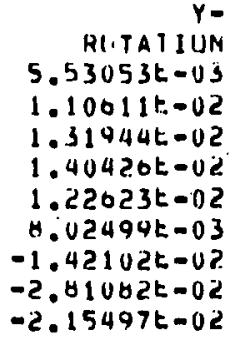 & $\begin{array}{r}\text { HLTATICN } \\
4.04174 E-02 \\
-0.77539 E-02 \\
-1.53784 E-02 \\
3.91981 E-02 \\
0.34935 E-02 \\
0.801615-02 \\
6.48752 E-02 \\
2.72084 E-02 \\
1.30042 E-02\end{array}$ \\
\hline
\end{tabular}


MUDE NUMHEH

EIGENVELTURY NUHMALIZED TO 1.000

UIYPLACEMENTSIROTATIUNY OF UNKESTHALNLU NUUEY

\begin{tabular}{|c|c|c|c|c|c|c|}
\hline $\begin{array}{l}\text { ROUE } \\
\text { NUMUER }\end{array}$ & THANSLATIUN & TRANBLATIIIN & THANSLAI ZUN & HUIATIUN & RUTAIIUN & HUTATICEN \\
\hline 1 & u. & 0 . & 0 . & 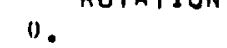 & HUTA DIUN & RUIAIDLI \\
\hline $\begin{array}{l}2 \\
3\end{array}$ & $\begin{array}{l}1.00000 E+U U \\
.71494 E-U 1\end{array}$ & $\begin{array}{l}-5.04043 E-114 \\
-1.12000 E-03\end{array}$ & $\begin{array}{l}7.44 S 4 S t-U E \\
3.22004 E-U S\end{array}$ & $\begin{array}{r}0.20017 t-044 \\
-3.31540 t=03\end{array}$ & $\begin{array}{l}-1.09157 t-03 \\
-3.18314 t-03\end{array}$ & $\begin{array}{r}-1.23021 t-02 \\
2.08404 E-02\end{array}$ \\
\hline 4 & $3.5 U 275 E-01$ & $3.27 \cup 14 E-U 1$ & $.6 .33851 t=0 c$ & $=0.42304 t-03$ & $-4.03504 t-113$ & $2.30554 E-02$ \\
\hline $\begin{array}{l}5 \\
0\end{array}$ & $\begin{array}{l}=4.57475 E-01 \\
=4.00214 E-01\end{array}$ & $\begin{array}{r}3.07540 E-01 \\
-4.16370 E-02\end{array}$ & $\begin{array}{r}-4.74310 E-0 C \\
2.70901 E-0 C\end{array}$ & $\begin{array}{l}-0.003 S S t-03 \\
-1.00320 E-02\end{array}$ & $\begin{array}{l}-4.29508 t-03 \\
-3.75054 t-03\end{array}$ & $\begin{array}{l}-1.01063 E-02 \\
-2.55373 E=02\end{array}$ \\
\hline $\begin{array}{l}7 \\
8 \\
4\end{array}$ & $\begin{array}{l}-4.01570 E-U 1 \\
-4.42340 E-01 \\
-2.53352 E-01\end{array}$ & $\begin{array}{l}-5.30418 E-01 \\
-9.74478 E-U 1 \\
-5.90534 E-01\end{array}$ & $\begin{array}{l}0.471 U Y E-U E \\
0.47625 E-0 E \\
1.05744 E-04\end{array}$ & $\begin{array}{l}-1.14023 t-U z \\
-1.08172 t-02 \\
-2.08744 t-02\end{array}$ & $\begin{array}{r}-2.45452 t-U 3 \\
4.34634 t-U 3 \\
8.59710 t-03\end{array}$ & $\begin{array}{l}-2.71041 E-02 \\
=1.07641 E-02 \\
-6.32196 E-03\end{array}$ \\
\hline 10 & $-H .37562 E-0 C$ & $-1.908 B 3 F_{0}-01$ & $4.30105 E-05$ & $-1.52710 t-02$ & $0.50119 t-03$ & $-4.10099 t=03$ \\
\hline 11 & 0. & 11 & 0. & 0 . & 0 . & v. \\
\hline
\end{tabular}


CASE 1

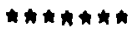

DIRECTIUN FALTURS
$x=$
1.0000
$Y=$
0007
$2=$
1.0000

INUICATUR FUH UISPLACEMENT OR ACLELEKATIUN SPECINUM =

EU.U UISPLACEMENT

EU. 1 ACCELERATIUN IN IN. ISEC.?

EU.? ACCELERATIUA IN IS

1 gPECtra are entereu fuh case

1. KINUE 2

CLUSTEH FACTUR, CF $=.10000$ 
MOO

$$
\text { DIJIRECTIUN Y-GIRECTION L-DIPECTIUN }
$$

$\begin{array}{lrrr}1 & .175 C E+00 & -.2593 E-01 & -.3308 E+U 0 \\ 2 & .3024 E+00 & -.2074 E-02 & .1478 F+00 \\ 3 & -.5347 E-01 & -.2582 E+00 & .2743 E-01 \\ 4 & .0501 E-01 & -.5208 E-01 & -.1174 E-01 \\ 5 & -.7925 E-01 & -.0005 E-01 & -.1115 t-01\end{array}$

SPECTHUM TAHLE CIMPEHIAL VALLEY E.H. ELCENTHU 144.J SE NUMUEK UF PUINTS $=11$
SCALE FACTUK SCALE FACTUH $=.10000 T+01$

\begin{tabular}{|c|c|c|}
\hline $\begin{array}{l}\text { INPINT } \\
\text { PUINT }\end{array}$ & PER I UU & $\begin{array}{l}\text { YHEC TRUM } \\
\text { VALIJE }\end{array}$ \\
\hline 1 & $.1040 E-02$ & $.1450 E+193$ \\
\hline 2 & . $2 B \cup \cup E=01$ & $.3800 E+03$ \\
\hline 3 & $.5800 E=01$ & $.7750 r+03$ \\
\hline 4 & $.71 \cup \cup E-01$ & $.7750 E+03$ \\
\hline 5 & $. \cup 1 \cup 0 E-01$ & $.4400 E+U 3$ \\
\hline 6 & $.1140 E+U O$ & $.1188 E+04$ \\
\hline 7 & $.141 U E+U U$ & $.1184 t+04$ \\
\hline$\theta$ & $.1720 E+O O O$ & .7 OUUE +OS \\
\hline 9 & $. \angle O U U E+U U$ & $.4710 E+03$ \\
\hline 10 & - $\angle S O O E+O O$ & $.9710 E+U 3$ \\
\hline 11. & $.32 S 0 E+00$ & $. \triangle 0 O O E+U 3$ \\
\hline
\end{tabular}

CLUSTEZING. 11 MEANY MUUE I CLDSE IU I+1, -1 , NUI. $-1 .-1,-1,-1,0$. 


\begin{tabular}{|c|c|c|c|c|c|c|c|c|}
\hline & & $\begin{array}{c}\text { OI } \\
\text { MUUE } \\
\text { NUMUER }\end{array}$ & $\begin{array}{l}P L A C C E \\
\text { TKANYLATIIIN }\end{array}$ & THANSLATIUN & THANSLAIIUN & $\begin{array}{r}x= \\
\text { RUTATIUN }\end{array}$ & MUTAI YUN & RUIAIILN \\
\hline & 1 & TUTAL... & u. & 0 . & 0. & 0. & 0 . & 3. \\
\hline & 2 & TOTAL... & $2.04357 E-03$ & $2.10823 E=00$ & $4.00 s 78 t=0 \mathrm{~s}$ & $1.53<24 E=04$ & $5.00049 t=05$ & $0.24377 t-03$ \\
\hline & 3 & TUTAL.. & 5.8941 SE.03 & $4.20722 t=00$ & $1.40073 t-0 c$ & $1.0413 \cup E-04$ & $1.12 \cup 10 E-04$ & $7.01230 t-u b$ \\
\hline & 4 & TUTAL... & $7.41387 E-03$ & $0.12141 E-04$ & $1.74471 t-1 \mathrm{JC}$ & $1.44572 E=04$ & 1.51 YUQE- $\cup 4$ & $5.07153 t=05$ \\
\hline & 5 & TUTAL.. & $7.83907 E-03$ & $1.03<35 t-03$ & $1.4<049 t-0<$ & $1.13084 E-04$ & $2.02521 E-04$ & $3.02036 E-05$ \\
\hline & 0 & TOTAL.. & $7.83 \forall 22 E-\cup 3$ & $2.11587 E-0 S$ & 1. USYOHE $=0 \mathrm{C}$ & 1.U1 SOYE - U4 & $2.10497 E-04$ & $2.43003 t-05$ \\
\hline & 7 & TUTAL.. & $7.83021 E-03$ & $2.49029 E-0 \mathrm{~S}$ & 0.0 SISTt-Us & O. Y505 CE-US & $2.12142 t-v 4$ & $1.06710 t-05$ \\
\hline & H & TOTAL.. & $5.62150 E-03$ & $1.99302 E-03$ & $1.00145 t-01$ & 0.4343 YE 03 & $1.80506 E-04$ & $0.02004 t-00$ \\
\hline & 9 & TOTAL.. & $2.23003 t-03$ & $7.34284 E-04$ & $7.04032 t-03$ & $3.55482 E-05$ & $9.65 S 49 E-U S$ & $7.56499 t-00$ \\
\hline & 10 & TUTAL.. & $0.24073 E-04$ & $1.4 B 397 E=04$ & $3.04 \supset 37 t=00$ & $1.0313 \mathrm{SE}=0 \mathrm{~S}$ & 5.57 Y & $3.70250 E-110$ \\
\hline & 11 & TOTAL. & v. & U. & 0. & 0. & 0. & 0. \\
\hline
\end{tabular}


RESPUNSE SPELTRUM STHESS LUMPGNENTSS (KINR Z 2$)$

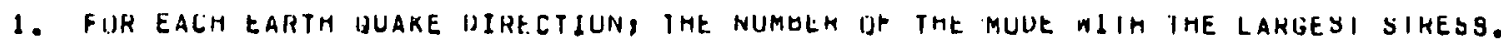

2. THE VALUE of THAT STRESS

3. IF REIUUESTLU, THE MUUE HY MUUE gTKESSEY rUR EALH EAHIM WUAKE UIKECTIUN.

4. THE REYULTAHT FUK EACH EAHTH IJUAKE UIREGIIUN.

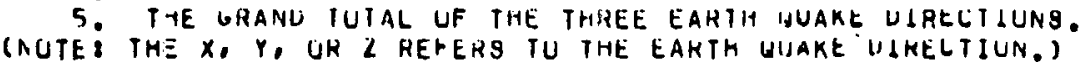

ELEMENT TYPE (3/U P I P E ,, 1 LLEMENT NUMAEH $(1)$

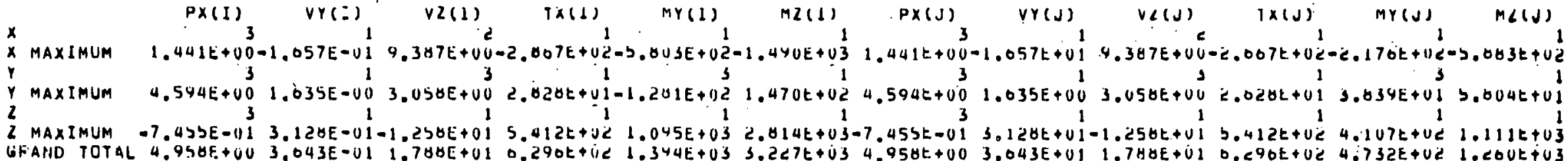

ELEMENT TYPE ( $3 / U$ P I PE $>, ;$; ELEMENT NUMGEK 1 C)

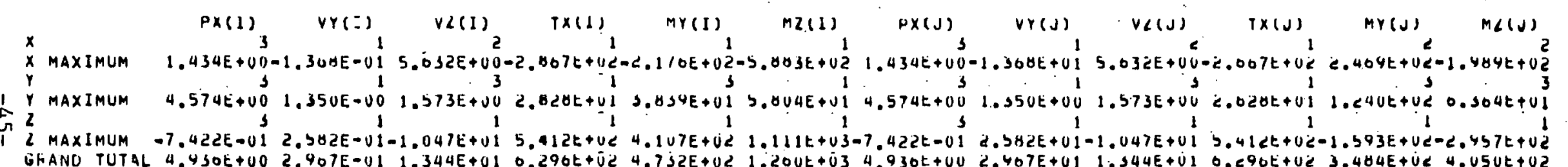

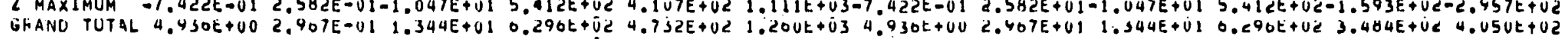
ELEMENT TRPE ( $3 / 0$ P I HE ,, 1 ELEMENI NUMUEH 1 ( $)$

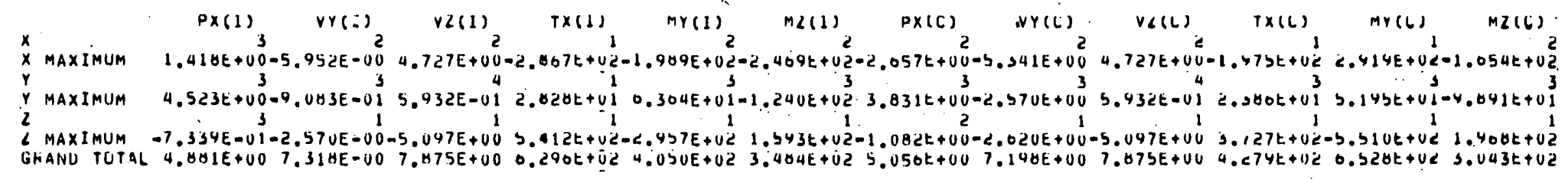

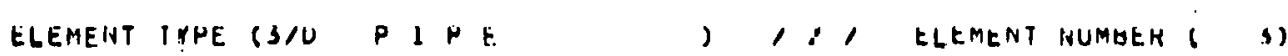

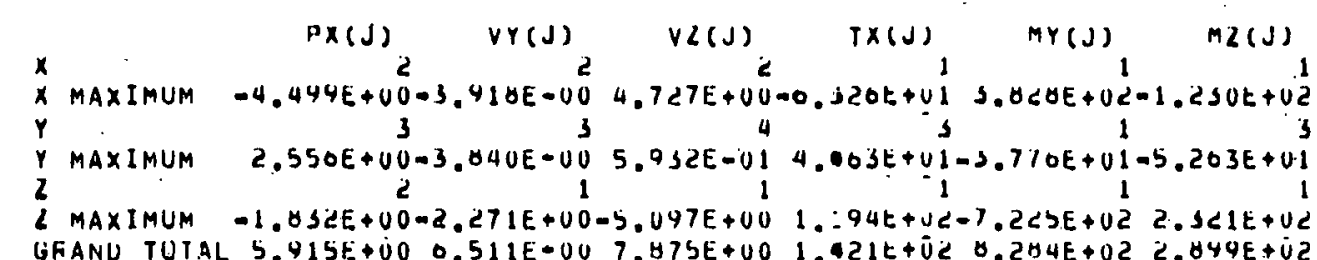

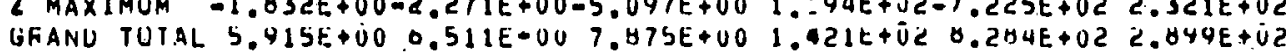

ELEMENT TRPE (3/U P I P : ) , I GLEMENI NUMUEH 1 4)

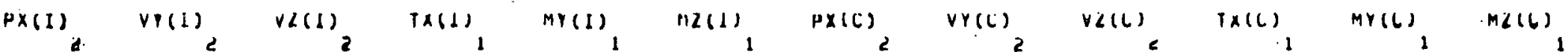

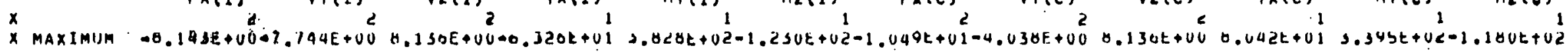




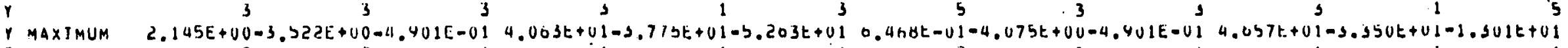

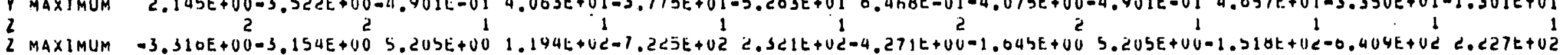

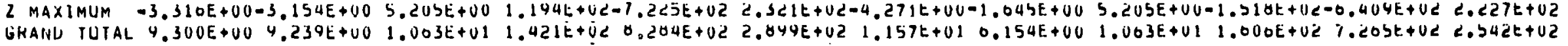
ELEMEHT TYPE (3/0 P I P E ) 1,1 ELEMENT NUMUEK 14 )

\begin{tabular}{|c|c|c|c|c|c|c|}
\hline$x$ & $H \times(J)_{2}$ & $\operatorname{VY}(J)_{3}$ & $V Z(J)_{2}$ & $T \times(J){ }_{1}$ & $\operatorname{Mr}(J)$ & $M 2(J)$ \\
\hline MAXIMUM & $-1.123 E+v 1-$ & $1.257 \mathrm{E}+\cup 0$ & $0.130 E+00$ & $1.400 t+02$ & $2.440 E+02$ & $-1.177 \dot{E}+02$ \\
\hline 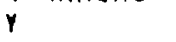 & s & $3^{\circ}$ & 3 & s & 1 & 3 \\
\hline HAXIMUM & & & & & 11 & 0.0 \\
\hline & 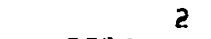 & 3 & 1 & 1 & 1 & \\
\hline $\begin{array}{l}\text { MAXIMUM } \\
\text { HANE TUTA }\end{array}$ & $\begin{array}{r}-4.575 E+00 \\
15 \Delta E+111\end{array}$ & $\begin{array}{l}0.3028 \\
4.3051\end{array}$ & 0 & 2 & 12 & $\begin{array}{l}2.2 \\
2.0\end{array}$ \\
\hline
\end{tabular}

ELEMENT TYPE ( $3 / 0$ P I H E $, 1,1$ LLEMENT NUMUEH 1 b)

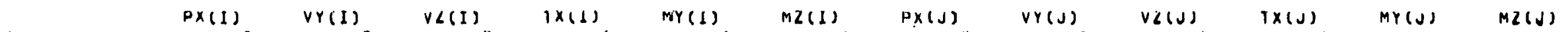

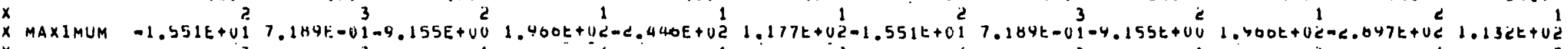

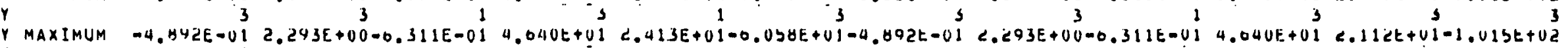

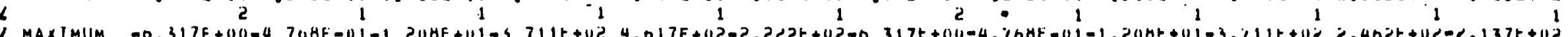

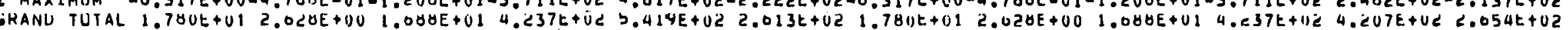




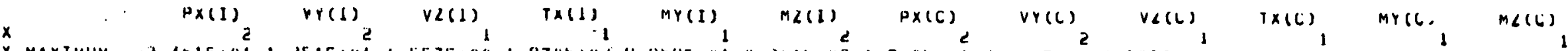

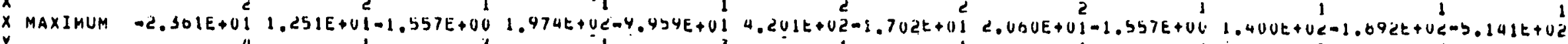

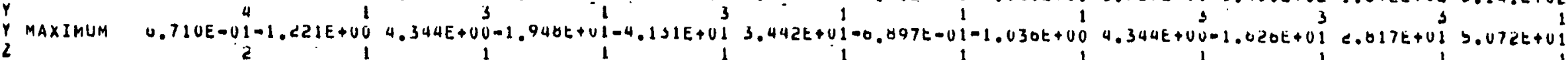

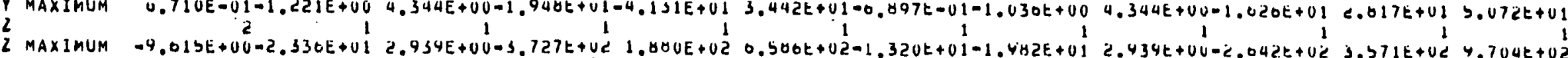

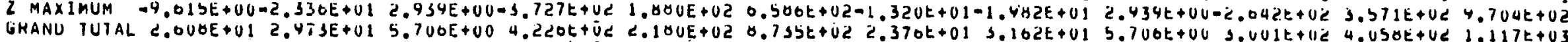
ELEMENT TYPE $13 / 0$ P I PE

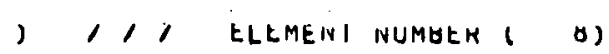

$x \quad P X(J), \quad P Y(J), \quad V L(J), T X(J), \quad M Y(J), \quad M L(J)$

$x$ MAXIMUM $1.040 E+012.554 E+01-1.557 E+005.202 E+U 1-C .5 U U E+02-0.4 U 7 E+02$

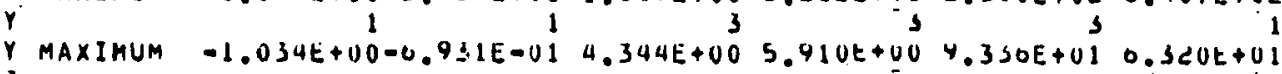

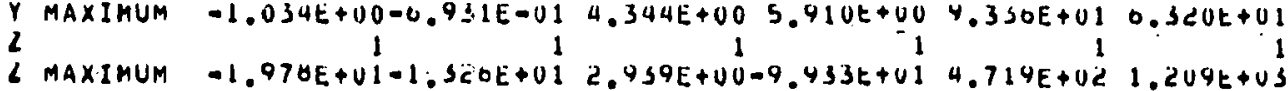

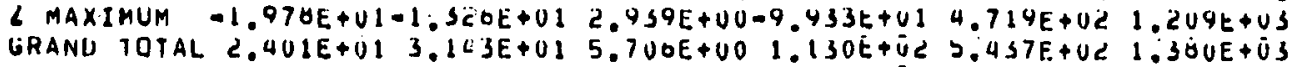

ELEMENT TYPE $3 / U$ P 1 HE

1,1, ELEMENT NUMUER $(4)$

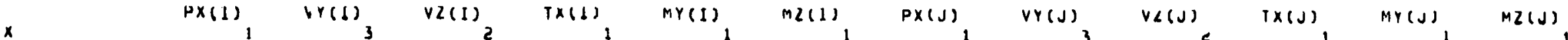

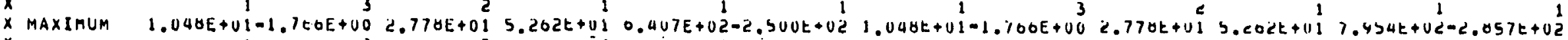

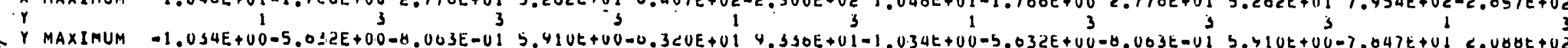

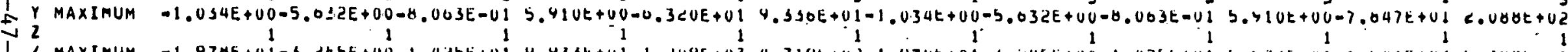

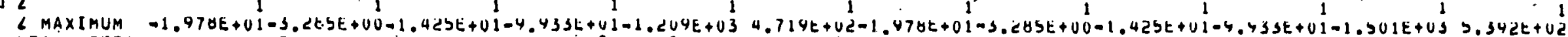

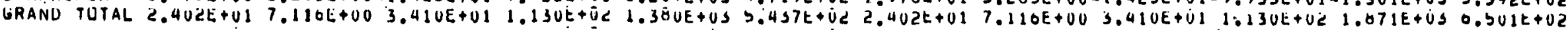
ELEMENT TYPE $(3 / U$ P 1 PE

, 1, ELEMENT NUMOEH (10)

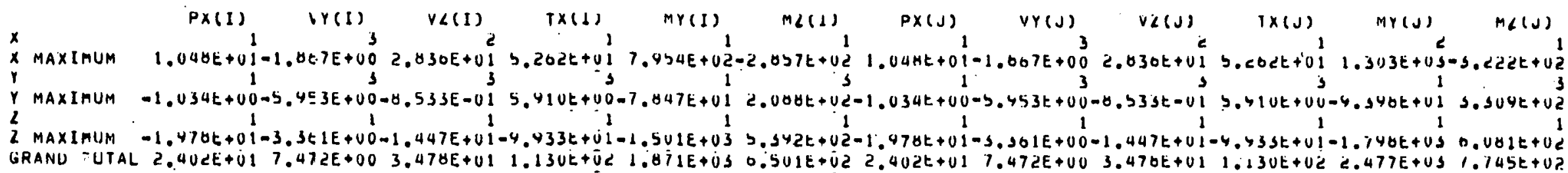



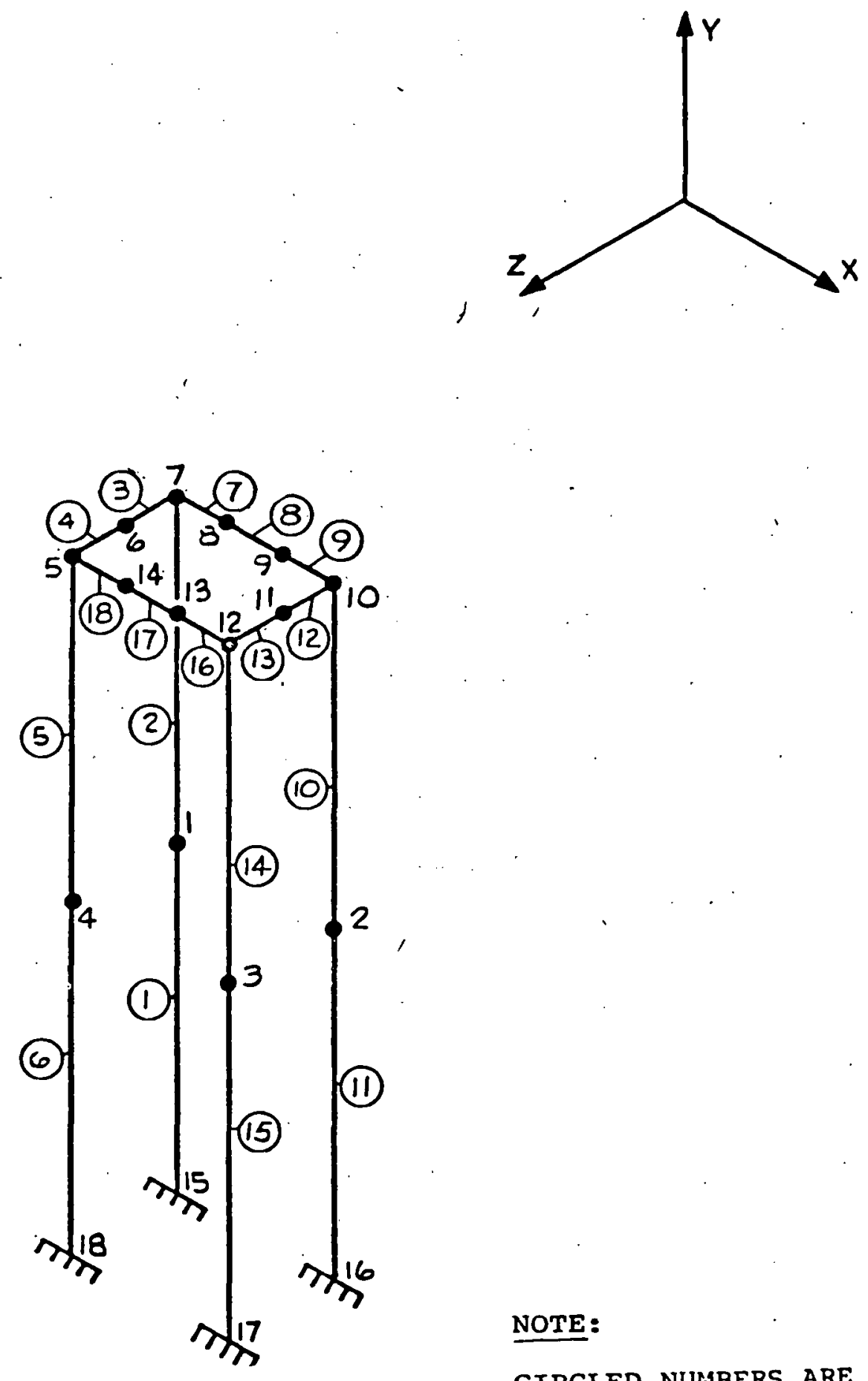

NOTE:

CIRCLED NUMBERS ARE ELE:IEITT INUMBERS.

BENCHMARK PROBLEM NO. 2

Figure 2.1 
BENCHMARK PROBLEM NO. 2

10000

5000

1000

500

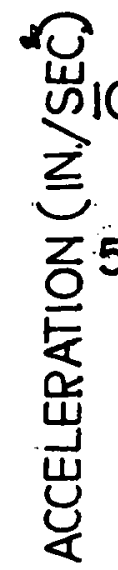

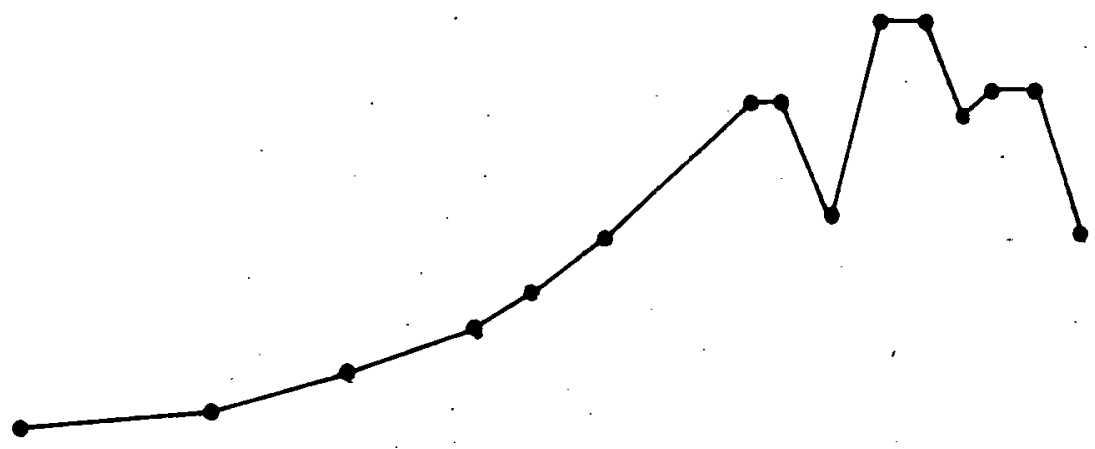

0.0்ol

O.ól PERIOD (SEC)

1.6

10.0

Figure 2.2 
CONIROL. INFORMAIION

mumaca of moOAL Prints - 18

MUMBER OF ELEMCNI IYPCS -

NUMBTR OO LOAD CASES

MUMER Or FRC QR NCIES

ANALYSIS CODC INDYNI

Co.0. Siafic

rj.1. MOOAL ExiAaCilion

Co.2. rORCEO RESDONSE

60.3. RESPONSE SPECIRUH

Solvition moOE ImOOTXI

SOLvilion moOr inoor $x$

c0.0. Excculion

CQ.1. Daia CHECK

mureca or suespace

IICAAIION VECIOAS IMADI

COUATIONS PTA BLCK

iAPEIO SAVT rLag INIOSVI: -0

moll poINT INPUT Data

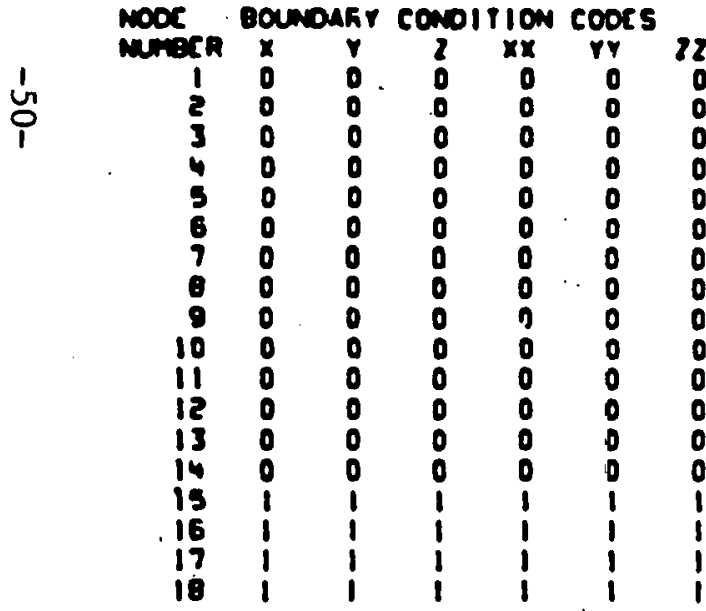

CEmenatro nOOAL DATA

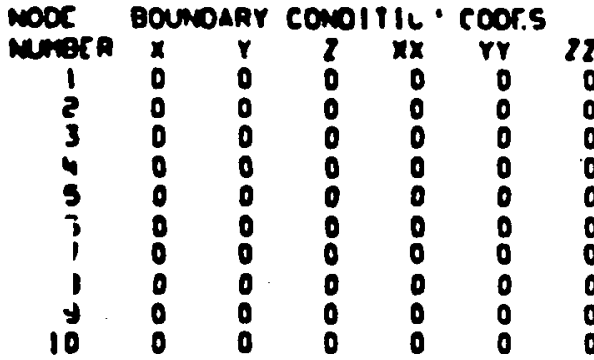

\section{MODAL PiJINI COCAOIMATES}

\begin{tabular}{|c|c|c|}
\hline $\begin{array}{r}0.000 \\
0.000 \\
27.250 \\
P 7.250 \\
0.000 \\
0.000 \\
0.000 \\
0.000 \\
A .625 \\
18.625 \\
27.250 \\
27.250 \\
27.250 \\
18.625 \\
8.625 \\
0.000 \\
27.050 \\
27.250 \\
0.010\end{array}$ & $\begin{array}{r}- \\
-30.000 \\
-30.000 \\
-30.000 \\
-30.000 \\
18.625 \\
18.625 \\
18.625 \\
18.655 \\
18.625 \\
18.625 \\
18.625 \\
18.625 \\
18.625 \\
18.625 \\
-80.009 \\
-80.000 \\
-80.000 \\
-80.000\end{array}$ & $\begin{array}{r}02 \\
0.000 \\
0.000 \\
17.250 \\
17.250 \\
17.250 \\
0.625 \\
0.000 \\
0.000 \\
0.000 \\
0.000 \\
8.625 \\
17.250 \\
17.250 \\
17.250 \\
0.000 \\
0.000 \\
17.250 \\
17.250\end{array}$ \\
\hline
\end{tabular}

1

-0.000
-0.000 $-0.000$ $-0.000$

$-0.000$

$-0.000$

$-0.000$

$-0.000$

$-0.000$

$-0.000$

$-0.000$

$-0.000$

$-0.000$

$-0.000$

$-0.000$

$-0.000$

$-0.000$

\section{nODAL PRINT COOAOInatrs}

\begin{tabular}{|c|c|c|c|}
\hline $\begin{array}{r}x \\
0.000 \\
27.250 \\
27.250 \\
0.000 \\
0.000 \\
0.000 \\
0.000 \\
9.625 \\
18.625 \\
27.250\end{array}$ & $\begin{array}{r}y \\
-30.000 \\
.30 .000 \\
-30.000 \\
\cdot 30.000 \\
18.625 \\
18.625 \\
18.625 \\
18.625 \\
18.625 \\
18.625\end{array}$ & $\begin{array}{r}2 \\
0.000 \\
0.000 \\
17.250 \\
17.250 \\
17.250 \\
0.625 \\
0.000 \\
0.000 \\
0.000 \\
0.000\end{array}$ & $\begin{array}{r}1 \\
-0.000 \\
-0.000 \\
-0.000 \\
-0.000 \\
-6.000 \\
-0.000 \\
-0.000 \\
-0.000 \\
-0.000 \\
-0.000\end{array}$ \\
\hline
\end{tabular}




\begin{tabular}{|c|c|c|c|c|c|c|c|c|c|}
\hline $\begin{array}{l}0 \\
i \\
i\end{array}$ & $\begin{array}{l}0 \\
i \\
i\end{array}$ & $\begin{array}{l}0 \\
i \\
i\end{array}$ & $\begin{array}{l}0 \\
1 \\
1 \\
1\end{array}$ & $\begin{array}{l}0 \\
1 \\
1 \\
1 \\
1\end{array}$ & $\begin{array}{l}0 \\
i \\
\vdots \\
1\end{array}$ & $\begin{array}{r}8.625 \\
0.000 \\
27.250 \\
27.250 \\
0.000\end{array}$ & $\begin{array}{r}18.623 \\
-60.000 \\
-60.000 \\
-00.000 \\
-80.000\end{array}$ & $\begin{array}{r}17.250 \\
0.000 \\
0.000 \\
17.250 \\
17.250\end{array}$ & $\begin{array}{l}-0.000 \\
-0.000 \\
-0.000 \\
-0.000 \\
-0.000\end{array}$ \\
\hline
\end{tabular}


P IEELENENI INPUT DAIA

CONTEL INTOAMATINN

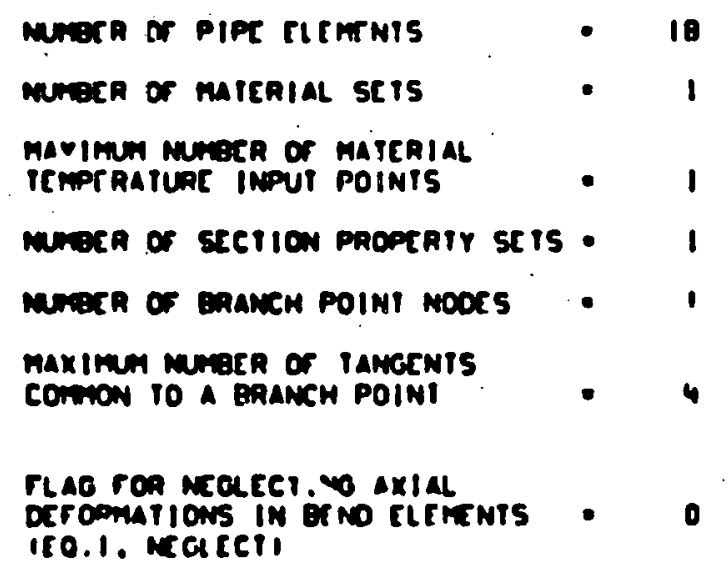

nATEIAL PAOPEATY TAOLES

mienic muse -111

merech or

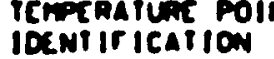

0.00 '

.3000. 


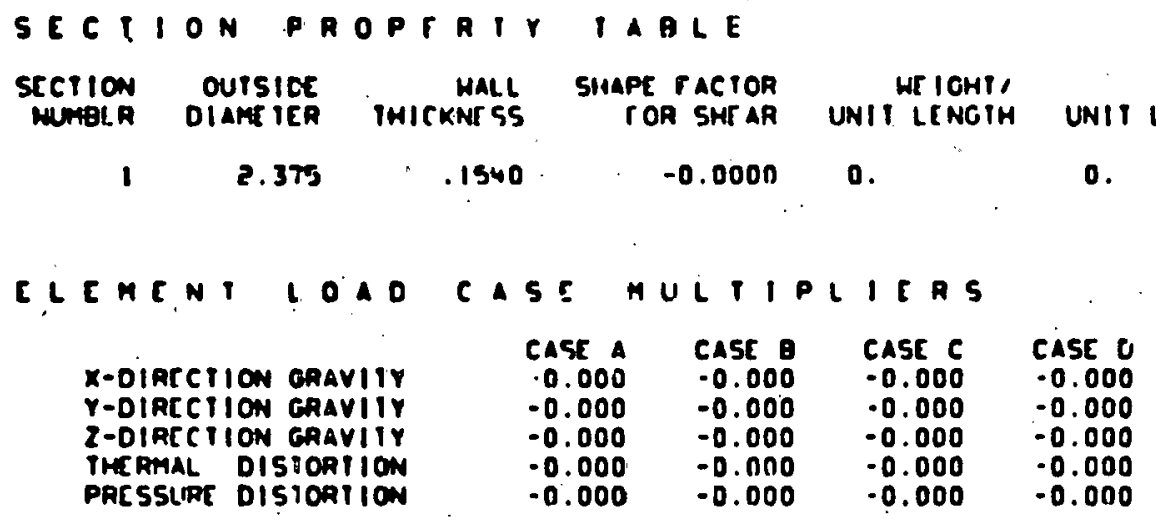


PIPE ELENENT INPUI DATA

CLEKENT CLEMENI NOOK MOD MAIL. SECTION numaca irrf. - I -J numara numbra

PETERENCE INIERNAL MPERAIUAC PHESSURC BCND IIHIRD POINTI -6.00
-0.00 $-0.00$ $-0.00$ 0.00 $-0.00$

$-0.00$ $-0.00$ $-0.00$ $-0.00$

$-0.00$

$-0.00$

$-0.00$ $-0.00$ $-0.00$

.0 .00

$-0.00$

.0 .00

$$
\begin{aligned}
& -0.00 \\
& -0.00 \\
& -0.00 \\
& -0.00 \\
& -0.00 \\
& -0.00 \\
& -0.00 \\
& -0.00 \\
& -0.00 \\
& -0.00 \\
& -0.00 \\
& -0.00 \\
& -0.00 \\
& -0.00 \\
& -0.00 \\
& -0.00 \\
& -0.00 \\
& -0.00
\end{aligned}
$$

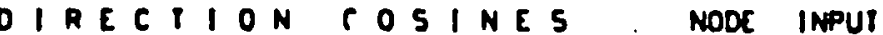
(XX3airzi I I 0 n t IY3AIYYI 1. 4 I I INCRT MIN IAC OROINATE.) ORDINATEI ORDINATEI TRACIIONI

$\begin{array}{ll}-0.0000 & -0.0000 \\ -0.0000 & -0.0000 \\ -0.0000 & -0.0000 \\ 0.0000 & -0.0000 \\ -0.0000 & -0.0000 \\ -0.0000 & -0.0000 \\ -0.0000 & -0.0000 \\ -0.0000 & -0.0000 \\ -0.0000 & -0.0000 \\ -0.0000 & -0.0000 \\ -0.0000 & -0.0000 \\ -0.0000 & -0.0000 \\ -0.0000 & -0.0000 \\ -0.0000 & -0.0000 \\ -0.0000 & -0.0000 \\ -0.0000 & -0.0000 \\ -0.0000 & -0.0000 \\ -0.0000 & -0.0000\end{array}$

-0.0000
-0.0000
-0.0000
-0.0000
-0.0000
-0.0000
-0.0000
-0.0000
-0.0000
-0.0000
-0.0000
-0.0000
-0.0000
-0.0000
-0.0000
-0.0000
-0.0000
-0.0000




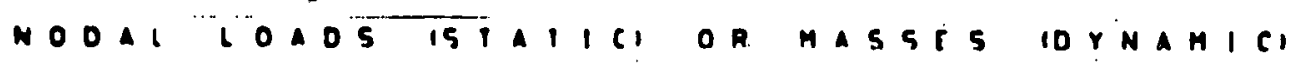

\begin{tabular}{|c|c|c|c|c|c|c|c|c|}
\hline $\begin{array}{l}\text { MOoc } \\
\text { MumarR }\end{array}$ & $\begin{array}{l}\text { LOAD } \\
\text { CASE }\end{array}$ & $\begin{array}{r}x-\Delta x 15 \\
\text { rOACr }\end{array}$ & $\begin{array}{l}r-A \times 15 \\
\text { rORCE }\end{array}$ & $\begin{array}{r}2-A \times 15 \\
\text { rORCE }\end{array}$ & & $\begin{array}{l}X-A \times 15 \\
\text { MOHENI }\end{array}$ & & $\begin{array}{l}\text { Y-AXIS } \\
\text { MOMENI }\end{array}$ \\
\hline $\begin{array}{c}1 \\
2 \\
3 \\
4 \\
5 \\
6 \\
7 \\
9 \\
5 \\
10 \\
11 \\
12 \\
13 \\
11\end{array}$ & $\begin{array}{l}-0 \\
-0 \\
-0 \\
-0 \\
-0 \\
-0 \\
-0 \\
-0 \\
-0 \\
-0 \\
-0 \\
-0 \\
-0 \\
-0\end{array}$ & $\begin{array}{l}.447005-01 \\
.447005-01 \\
.447005-01 \\
.477005-01 \\
.432706-01 \\
.974005-02 \\
.432706-01 \\
.894005-02 \\
.894005-02 \\
.43770[-01 \\
.894005-02 \\
.432705-01 \\
.894005-02 \\
.894005-02\end{array}$ & $\begin{array}{l}.44700 E-01 \\
.44700 E-01 \\
.44700 E-01 \\
.44700 E-01 \\
.43270 E-01 \\
.89400 E-02 \\
.43770 E-01 \\
.89400 E-02 \\
.894 .10 E-07 \\
.43270 E-01 \\
.89400 E-0 E \\
.43270 E-01 \\
.89400 E-02 \\
.894005-02\end{array}$ & $\begin{array}{l}.44700 E-01 \\
.44700 E-01 \\
.44700 E-01 \\
.44700 E-01 \\
.43270 E-01 \\
.89400 E-02 \\
.43270 E-01 \\
.89400 E-02 \\
.89400 E-02 \\
.43270 E-01 \\
.89400 E-02 \\
.43270 E-01 \\
.89400 E-02 \\
.894005-02\end{array}$ & $\begin{array}{l}-0 . \\
-0 . \\
-0 . \\
-0 . \\
-0 . \\
-0 . \\
-0 . \\
-0 . \\
-0 . \\
-0 . \\
-0 . \\
-0 . \\
-0 . \\
-0 .\end{array}$ & & $\begin{array}{l}-0 . \\
-0 . \\
-0 . \\
-0 . \\
-0 . \\
-0 . \\
-0 . \\
-0 . \\
-0 . \\
-0 . \\
-0 . \\
-0 . \\
-0 . \\
-0 .\end{array}$ & \\
\hline
\end{tabular}

STRuctune

in LOAD CASE

1

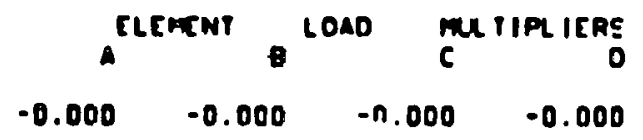


PRINT OP FAEOUEM IES

\begin{tabular}{|c|c|c|c|}
\hline $\begin{array}{l}\text { moor } \\
\text { mumbers }\end{array}$ & $\begin{array}{l}\text { CIACUCUAA } \\
\text { rREOUKMY } \\
\text { IRABISECI }\end{array}$ & $\begin{array}{l}\text { Facourner } \\
\text { erel Esisfel }\end{array}$ & $\begin{array}{l}\text { PEAIDO } \\
\text { IST.CI }\end{array}$ \\
\hline 1 & $.34746 \cdot 02$ & $.07126 \cdot 0 \%$ & $.1148 \pi \cdot 00$ \\
\hline 2 & $.553 x \cdot 02$ & $.08068 \cdot 01$ & $.11365 \cdot 00$ \\
\hline 3 & $.1100[\bullet 0]$ & $.17518 \cdot 02$ & $.57126-01$ \\
\hline 4 & . 55 ser 03 & $.4037 \% .02$ & .247ז-01 \\
\hline 5 & $.26155 \cdot 03$ & $.4163 E \cdot 02$ & $.24072-01$ \\
\hline
\end{tabular}




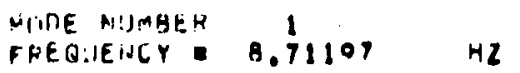

ElGEAVELTUNG NLIKMALIZELI IU A UHIT MASS MAIKIX

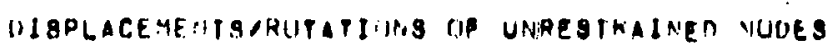

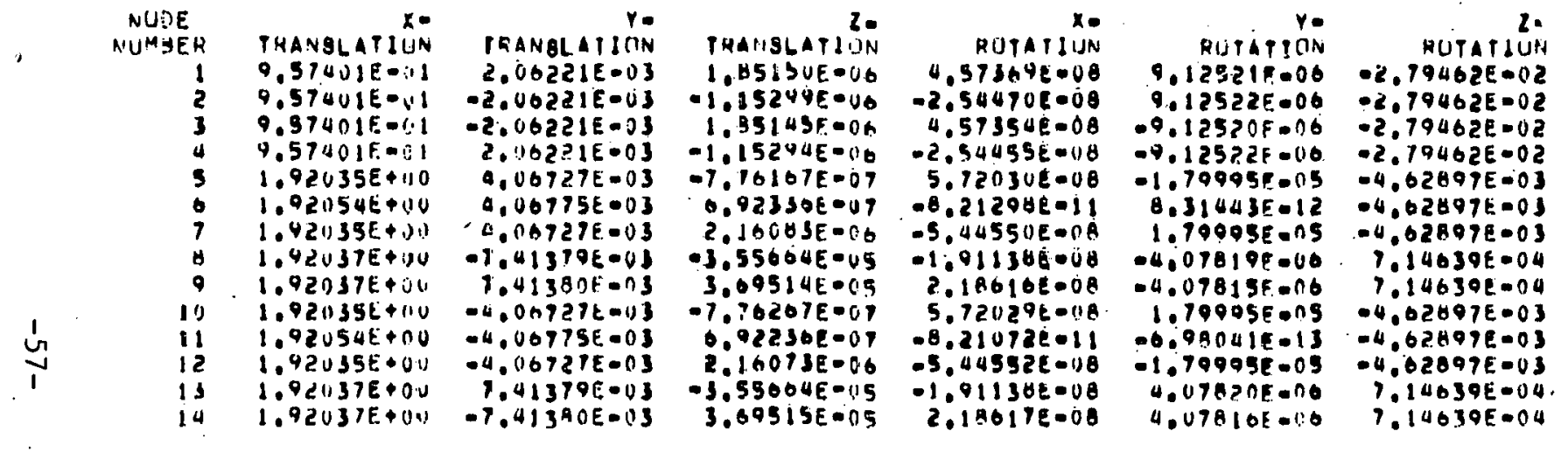


MIOE NUMHEH

FIGEHVECTINA WIJHALIZED, PIS 1.000

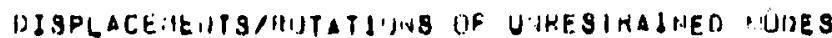

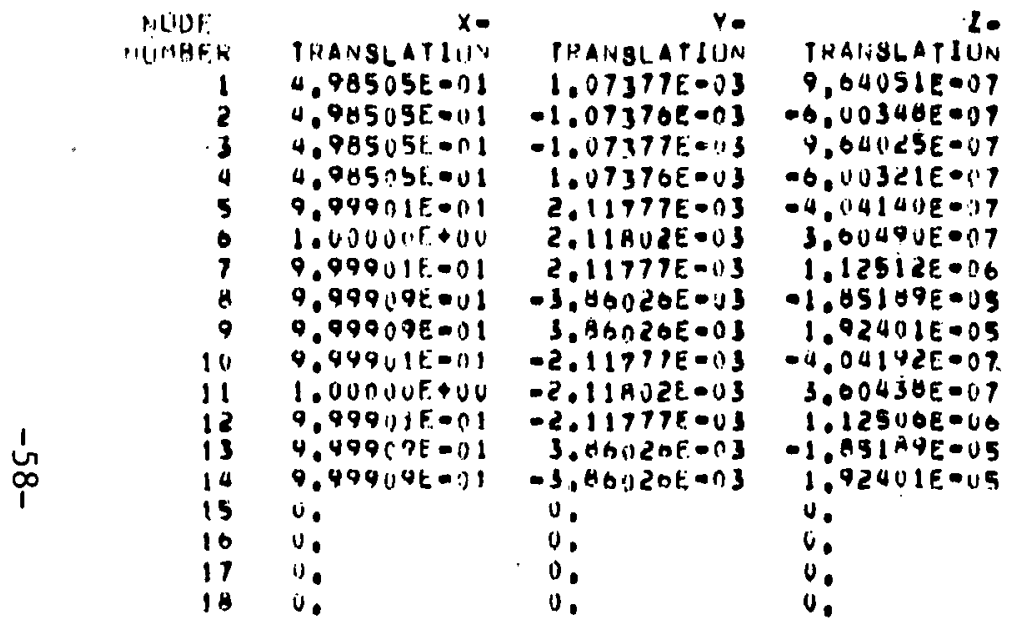

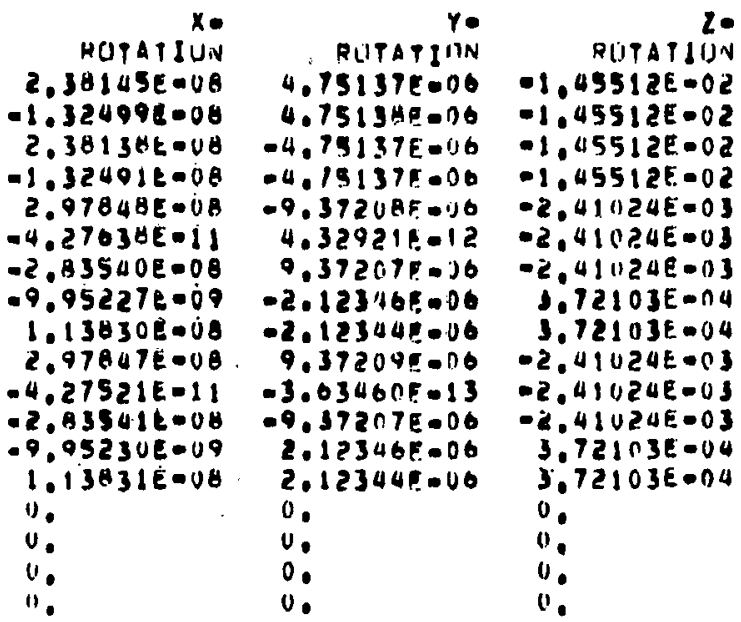


"IILE NUMALER 2

CREDIETCY O.80024 H2

E. GEMVELTURS NIJAMALIZE! TU a UHIT MASS MATHIX

DI SPLACEMENTSARUTATIIIHS JF UTHESTHALNED NUUES

\begin{tabular}{|c|c|c|c|c|c|c|}
\hline $\begin{array}{r}\text { NUDDE } \\
\text { IIIHEER } \\
1 \\
2 \\
3 \\
4 \\
5 \\
6 \\
7 \\
9 \\
9 \\
10 \\
11 \\
12 \\
13 \\
14\end{array}$ & 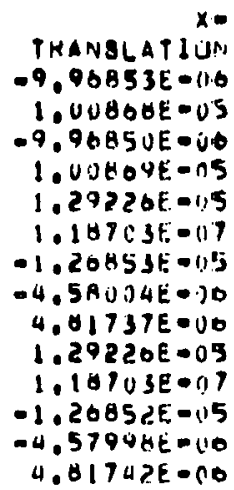 & 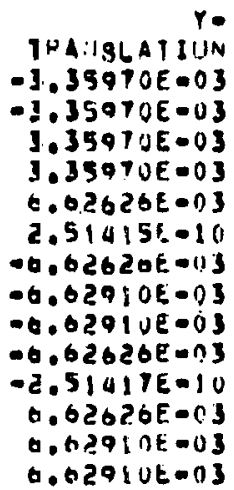 & 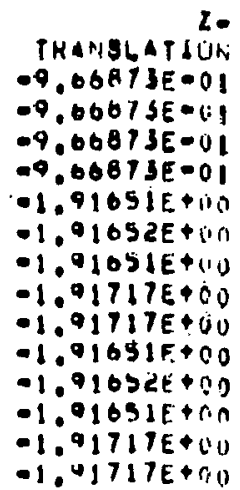 & 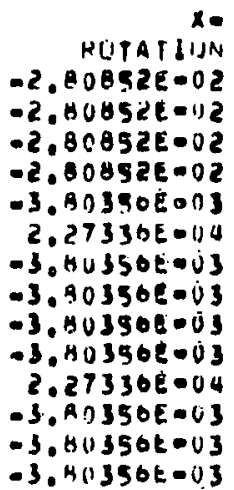 & 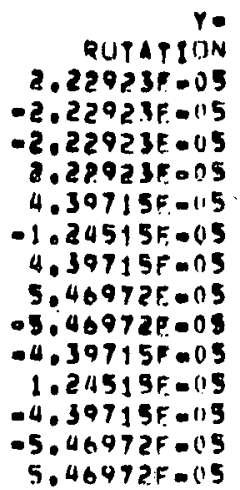 & 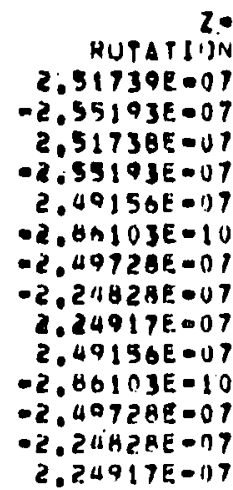 \\
\hline
\end{tabular}


MTOEE PUMHFEH

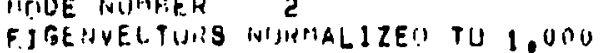

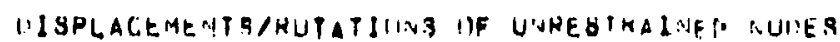

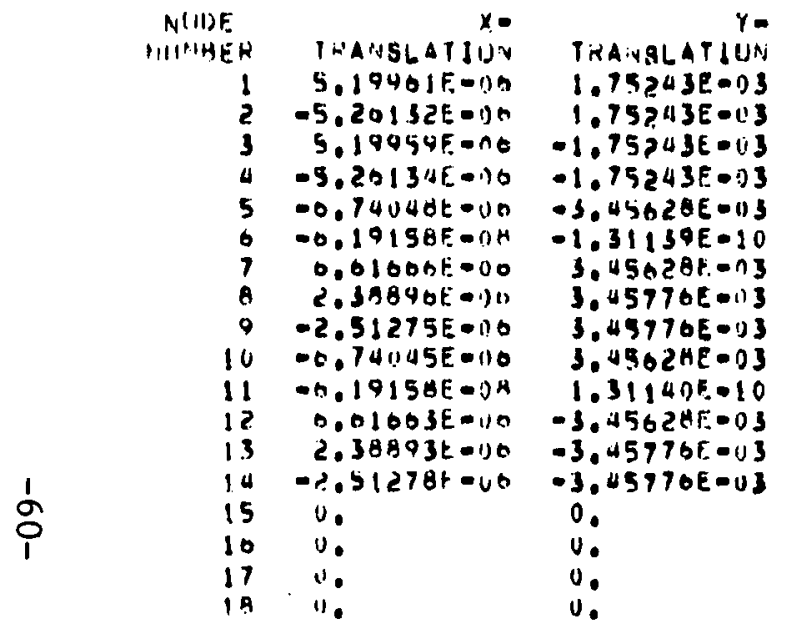

\begin{tabular}{|c|c|c|c|}
\hline 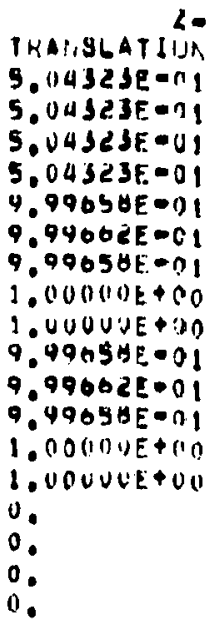 & 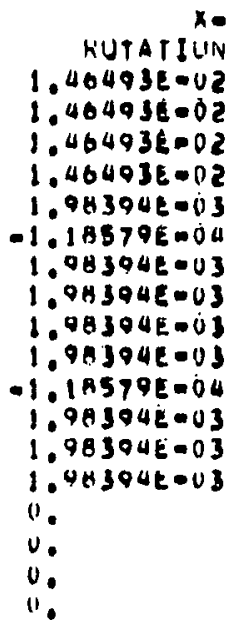 & 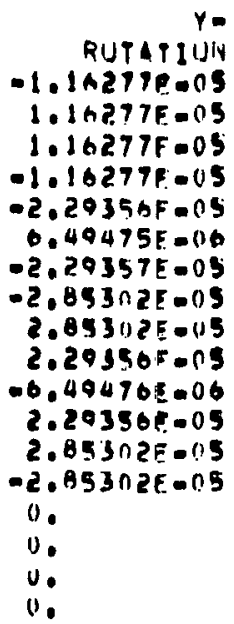 & 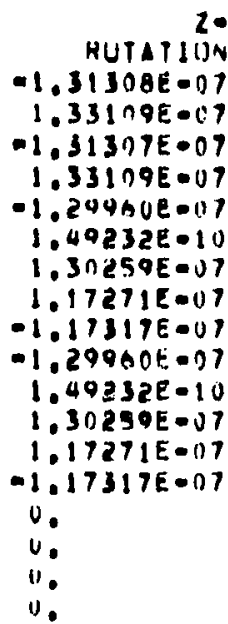 \\
\hline
\end{tabular}


WOEE NUMHER

FRE JUEDULY 17,5074

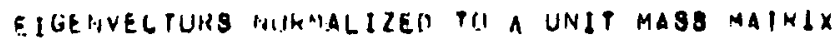

DISPLACEMEITS/RUTATIIINS OF GNHESIRAINFR NUDEB

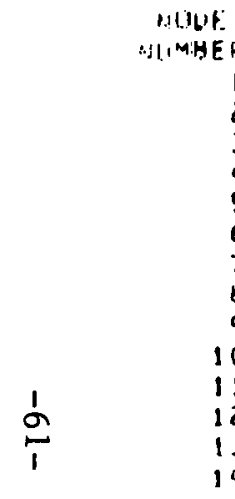

MOL:

\begin{tabular}{|c|}
\hline 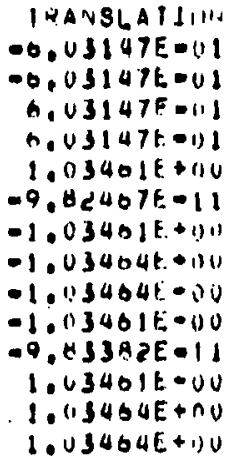 \\
\hline \\
\hline
\end{tabular}

$x \rightarrow$

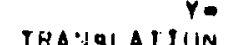

N

TEANBLATIIIA RUTATIUN

$1.11960 E-03 \quad 9.70100 E-01 \quad 2.46567 E-02$

$-1.14969 E-U 3$ - $4.701 U U E-01 \quad-2.46567 E-02$

$1.149+9(-1) 3 \quad-9.70100 \mathrm{C}-61 \quad-2.46567 E-02$

$-1.14909+-03$

9.7010 UECOI

$1.06517 t+00$

$2.405 \mathrm{~h} 7 \mathrm{C} 0 \mathrm{02}$

$1.855016 \bullet 0$

$-1.48672 \mathrm{E}-13$

$1.06520 \mathrm{E}+00$

3.4RASAE-03 O.170AdE-U1 G.BOT30E-UA

$-3.49884 \%, 003-6.176885=01$

$-2.26675 E-03-1.00517 E+00$

$-6,8073 \Delta E=00$

$-1.05501 E=113$

$5.594416=04$

2. B5501000

$2.20675 E-03$ - $1.00511 E 000$

-3. 4AR.SLE-U3

-6.

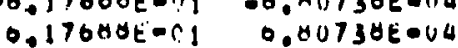

PIITATIUN $0.03597 E-02$ $0.03507 \mathrm{~F}=012$

$6.03507 F-112$

$0.03597 E=11$

$1,190 A D F=U 1$

1.20248500

$1.19060 F=01$

$1.22958 F-01$

$1.1906 C$ C 0

$1.202080=01$

$1.100 \mathrm{OE}=0$

1.

$1.52058 \%-01$
NUTA 20

$1.53263 E=0.2$

$1.53263 \mathrm{E}=6 \mathrm{~T}$

$-1.53263 t-02$

-1.532634 -n?

$-1.12523 E-113$

$1.64052 k-13$

1.125232003

$-4.645752-04$

$1.12523 E-03$

$1.64965 E-13$

$4.68575 E=04$ 
MULE NUMAER 3

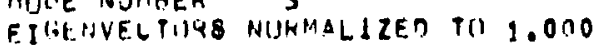

DISHLACEMENTS/RUTATIONS IJF UIRESTKAINFE NLIIES

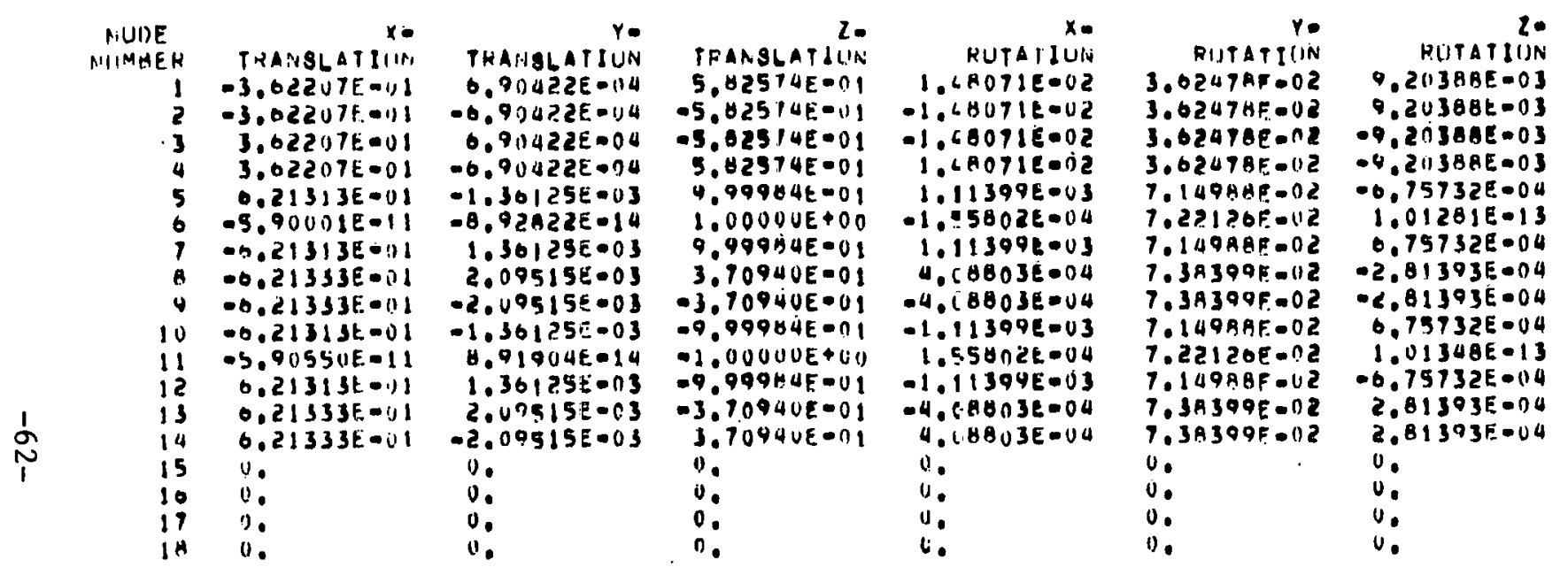


MODE NUMGER

FREUIJEACY A U.3OHO ME

TEENVECTURB NIJMALAZEH IIS A UNIT MASS MATRLX

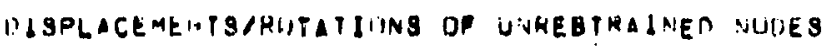

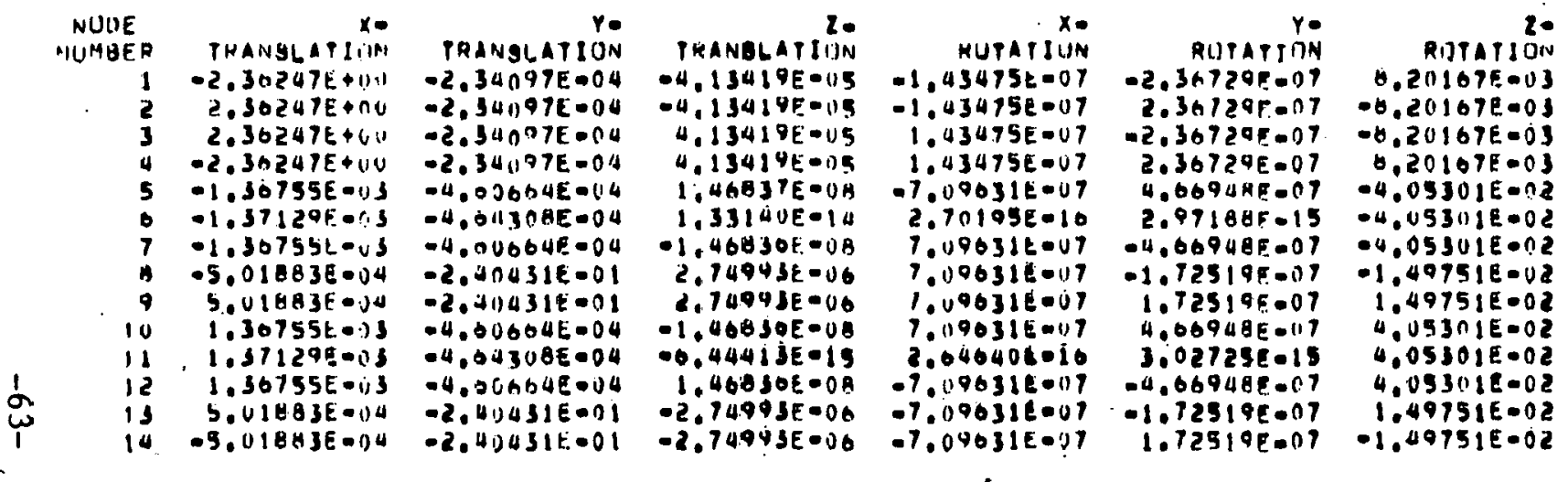


MIDALANALY Y I I

MUDE NUMBER

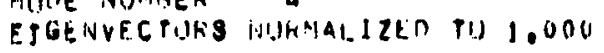

DISPLACEMENISINUTATIIINS GF. UINREOTRAINEO NUDES

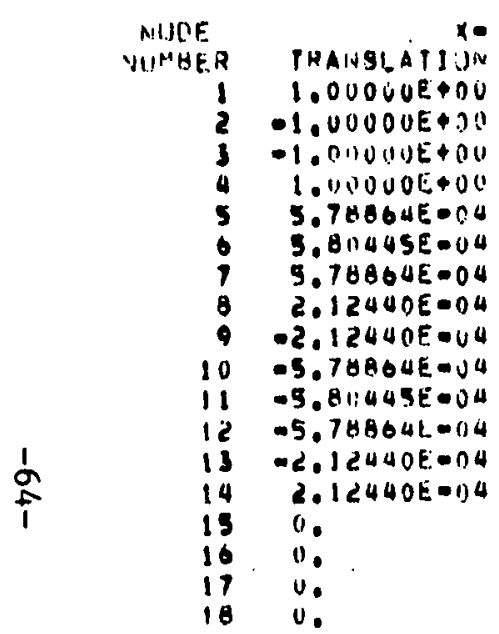

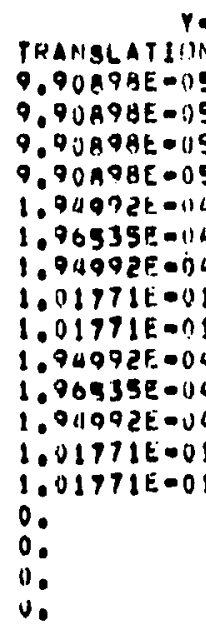

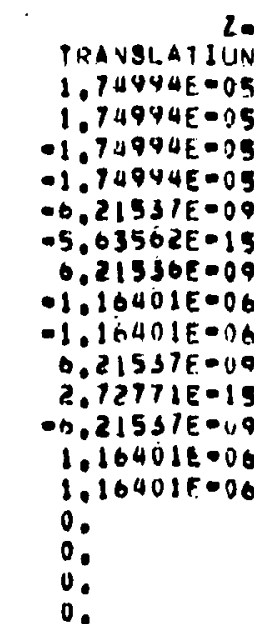

\begin{tabular}{|c|c|c|}
\hline 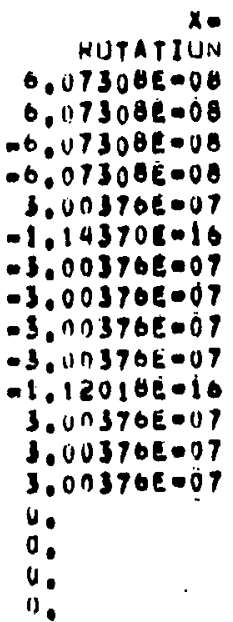 & 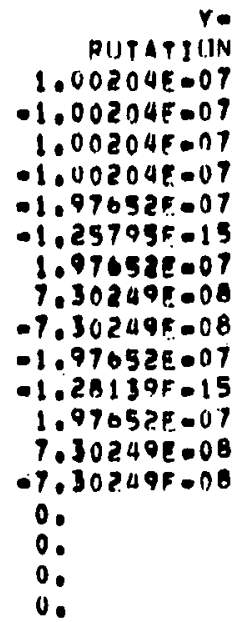 & 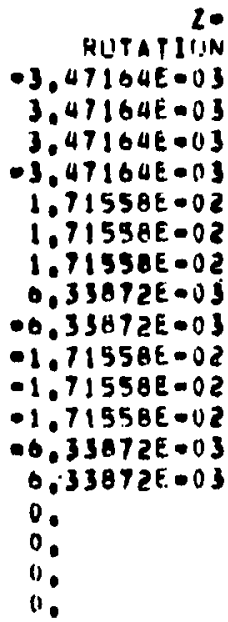 \\
\hline
\end{tabular}


MTICE NUMAER 5

PREUULNCY 41.6264 HZ

EITENVELTURB NUKMALIZEO TU A UHIT MASB MATHIX

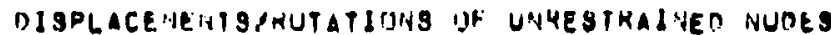

\begin{tabular}{|c|c|c|c|c|c|c|}
\hline $\begin{array}{r}\text { NODE } \\
\text { HIINHER } \\
1 \\
2 \\
3 \\
4 \\
5 \\
6 \\
7 \\
8 \\
9 \\
10 \\
11 \\
12 \\
13 \\
14\end{array}$ & 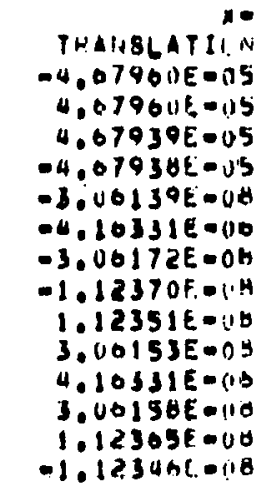 & 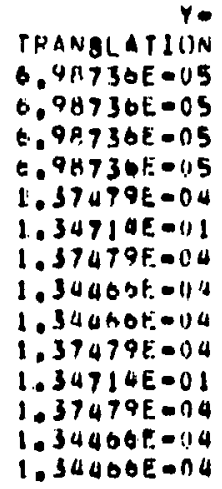 & 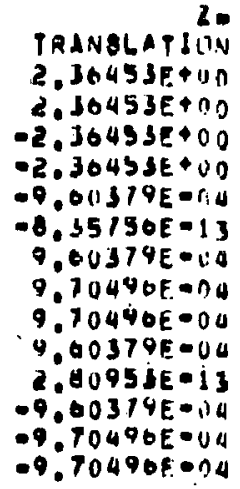 & $\begin{array}{l}\text { ROTATIUN } \\
849211003 \\
849218-03 \\
849218-03 \\
84921 E-03 \\
11645 E-02 \\
21915 E-19 \\
11645 E=02 \\
11645 E-02 \\
11045 E-02 \\
11045 E-02 \\
690815-14 \\
11645 E-02 \\
11045 E-02 \\
11645 E-02\end{array}$ & 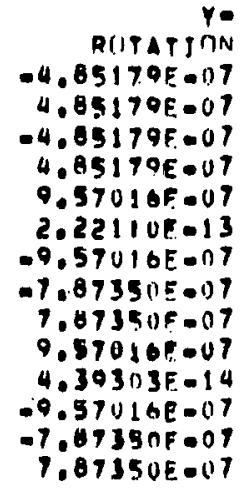 & 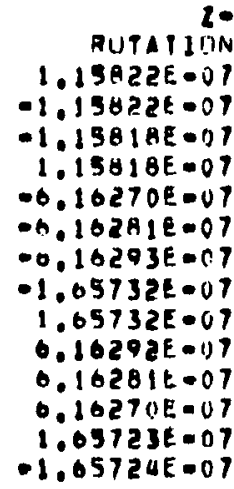 \\
\hline
\end{tabular}


MOOE NUMHER $S$

F. I GENVECTUNG NGIKMALIZEOT TO 1.000

DISPLACEMENIG/RLTATIUNG IIF LNNESTKAJNEU WUDES

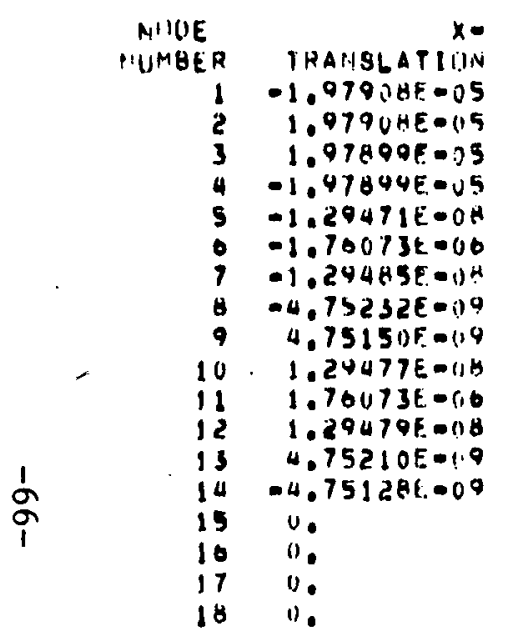

E IGENSLLUTIUN

EIGENSULUTIUN

DRINIINIT

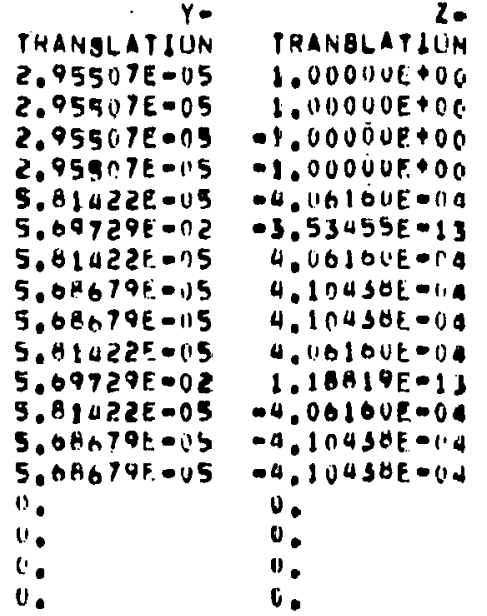

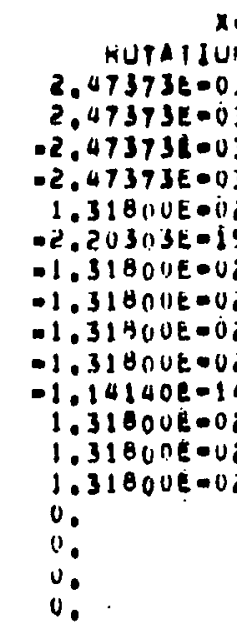

RIITATTOSN $-2.05100 E-07$

4,898208000 $2.05190 E-07 \quad-4.89629 E-0 B$ -2.0519 กE.07 $\quad-4.89813 E-08$ $2.05190 F=07 \quad 4.89 H 13 E=08$ $4.1043 A 5007-2.670306-07$ $9.39339 \mathrm{E}-14 \quad-2,00035 \mathrm{E} \cdot 07$ $-4.047375007 \quad-2.00640 E-07$ $-3.324 A 3 E-07$-7.0C407E-0B 3. $32993 F=07 \quad 7.019 \cup 7 E=0$. 4.0473 HF $=07 \quad 2.011640 E=0$ ? $1.85788 E-14$ 2.6nO35E-C? $-4.047385-07 \quad 2.00631 E-07$ - $.04738 E-07$ 2.60631E-C $-3.329031007 \quad 7.00671 E=00$ J. $29,3 \mathrm{E}-07$ 0. 0.

0
0.

0.

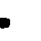


CASE

D: ION FAC PIIHS
$x=1.04000$
.6667
2.1 .110011

INIICAYOH FIJR IISPLACEMENT IIR ACCELEHATLLN SPECTHUM

EU." UISILLAEMENT

EQ.I ACCELERATIUN III IN, /SEC, 2

EU. 2 ACCELERAPIUA IN GS

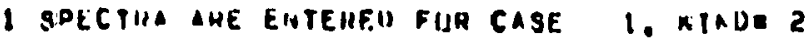

CLUSTF. FACTUH, CF: 10NUN

$\frac{1}{1}$ 
muidal pahticipatiun Facturs

\begin{tabular}{|c|c|c|c|}
\hline MUUE & $x$-1IIHECTIU: & $Y=I I D E C T I U U$ & $2-1)$ IREC IIUN \\
\hline 1 & $.0066 F+U U$ & . URI UE-ID & $.2194 t=00$ \\
\hline 2 & $.374 T E \bullet U 7$ & $\therefore 4541 E-17$ & $0.0074 E \$ 111)$ \\
\hline 3 & -.323 SE-10 & . SWUHE-II & . ODHOE-11 \\
\hline 4 & $-.2741 t-13$ & $-.872 \mathrm{HE}-02$ & $-.95476-15$ \\
\hline 5 & $.21+2 E-14$ & .245UE - & $.9,142 E-13$ \\
\hline
\end{tabular}

SPECTRIM TAILE RIAPERIAL VALLFP F.P. F.LCERTHIS 144U SE

\begin{tabular}{|c|c|c|}
\hline & $\begin{array}{l}\text { IJF PUIlW19 } \\
\text { FC PUA }\end{array}$ & 11.101101 \\
\hline & & YOECIFIIM \\
\hline & PE. $\|[1\})$ & VALIIE \\
\hline I & D 16UAE-UL & $.145 U F+U 3$ \\
\hline 2 & EAoull-111 & $.36 \cup U E+U 3$ \\
\hline 3 & .5411 .UE-11 & $.7750 \mathrm{~F}+. .33$ \\
\hline む & $.711105-11$ & $.7750 E+13$ \\
\hline 5 & .71 IIE-UI & $.11400 E+1) .3$ \\
\hline b & $.1110 t+011$ & . IIHQE +IIA \\
\hline 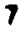 & $.1410 E+\eta 11$ & .11 . \\
\hline r & $.1720 E+110$ & $.70110 E+113$ \\
\hline & $.20 u n E+v 0$ & . $A>1 O E+1) 3$ \\
\hline & $25(1).) F_{0}+(1 n$ & $.5710 E+113$ \\
\hline & $.3230 E+111)$ & $. n(n, 1) E+n]$ \\
\hline
\end{tabular}

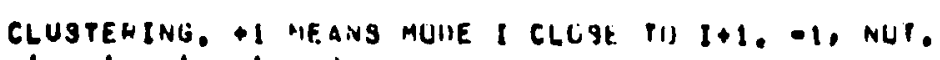
1. 1.1 .1 .1 .1 . 
Solution 2a

Interspatial/intermodal combination

$$
-69-
$$


NDUE DI YOLACEMENT SINGTATIUNS

NIJUE MIIISE $X$ O Y Y

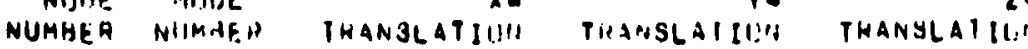

Mistaigin

RUIATIU'

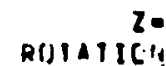

1 TIITAL.. 2.SU252t-11! $1.274211=03 \quad 2.251216-01$

$0.5342 \cup E-113$

$7.38496 E-116$

$6.121197 E-03$

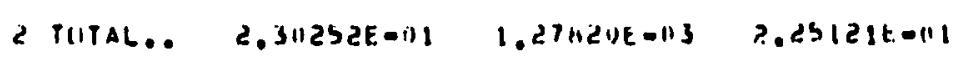

$6.5392 \cup E=03$

$7.3+496 E-06$

$0.72097 t \cdot 03$

3 TITAL.. 2.30252E-111 1.2111?.1E-113 2.25121E-111

$6.53920 E \cdot 0.3$

7.3ค496E- 06

6.720976 .013

4 TUTAL.. 2.3125?t-1)1

1.RTH2UE-IIS $2.2512 \mid k-111$

$0.53420 E=11.5$

7.394965006

$6.721) 97 E-03$

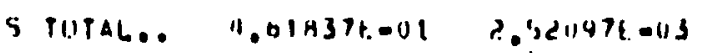

$4.110231, t=111$

H. ASOI UE - 114

$1.115 U A R E-115$

1.113300 .03

a TUTAL.. 4.D19H:DE. -U1

$7.7 \times 271 k-014$

$4.40231 t-01$

$5.29310 E \cdot 115$

2. $A Q 414 E-U 6$

$1.11324 E-03$

7 TITAL.. 4.01837E-1II 2.52J47E-03 4.4623 U1E-01

$H .0501$ UE 0104

$1.1456 B E-115 \quad 1.11330 E-03$

A TUTAG.. 4.61H3OE-11

3.3204 AE $03 \quad 1.40543 E-11$

$H .85001 E-114$

$1.37102 E-05$

$1.71920 E-04$

- TIITAL.. 4.0IA3OE. HI

$3.320475-113 \quad 4.40391 E-111$

$8.056026=04$

$1.371616005 \quad 1.71920 E-044$

IU PITAL

$4.131837 E-111$

$2.52 .147 t-05$

4.4023 IIJE-11

0.850106 .04

$1.0560 B E-05$

$1.11330 E-03$

11 PIIPAL.. 4.013HIE-111

$9.7 n \geq|1 E-111 \quad .4 .4023| t-111$

$5.24510 E=115$

2. $R 94$ | $4 E-115$

$1.11324 E=03$

12 IIITAL.. 4.01A37E-11)

P. T21197E-11S $4.40230 t-111$

$0.850108-114$

$1.4500 R E-05 \quad 1.11330 E=03$

13 PIJTAL.. 4.01939E-113 3.3204UK-113 - 4.40341t-111

$H .85601 E \cdot 04$

$1.371026-015$

1.719206004

11 TUTAL.. 4.0143 .21 .01

$3.32(345-1) .1$

4.405416 .01

$8.85002 E 004$

1.371016005

$1.71920 E-114$

15 IUTAL.. H.

".

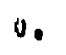

$n$.

0.

).

in HUTAL.. U.

11

ง.

0.

0.

17 TUTAL.. N.

n.

0.

11

TAL.. N.

a.
11

n.


RESPUNGESPECTRUM STRESS CUMPUNENTS (KIND! 2$)$

OR E LE IEIT, THE FLLLUMINIS INFURMATILH IS PKINTEUI

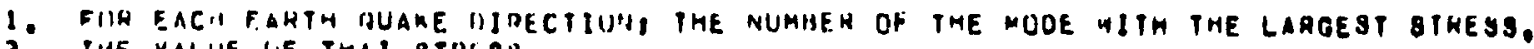

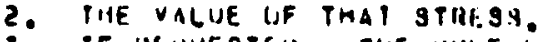

3. IF hEITESTEU, PHE IUUF. HY MHIDE STHEBSES FUA EACH EARTH GUAKE UIRECTIUN.

4. PIIE HESIJLTANT FDA FACH EARIH IUUAKE IIRECTIUN.

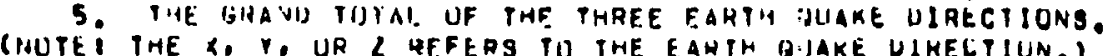

ELEMENT TYPE ISIU DI DE , 1, ELEMENT NUMUER $($ i)

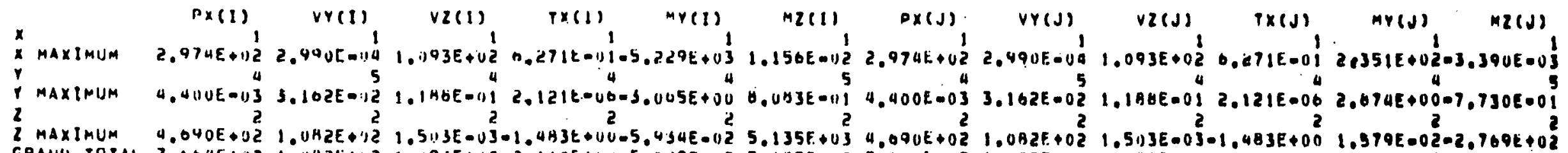

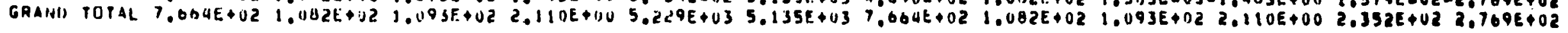

ELEMEIT TYPE (3M P I PE $1,1,1$ ELEHENT NUMHEH $($ द)

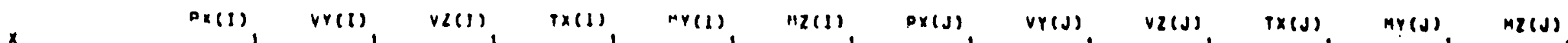

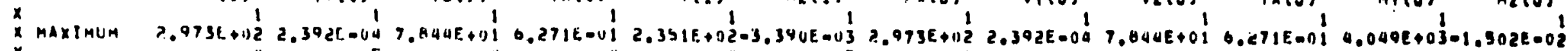

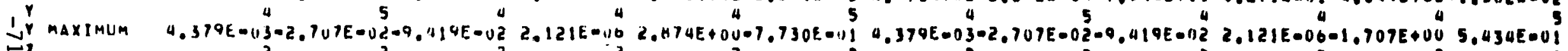

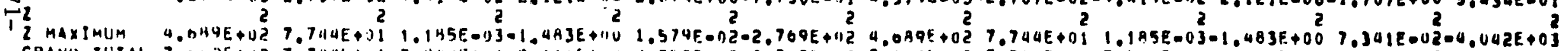

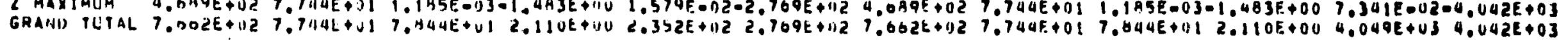
ELEMEHT IYPE (\$M P 1 P $:$, 1,1 ELEHENT NUMHER 13 (3)

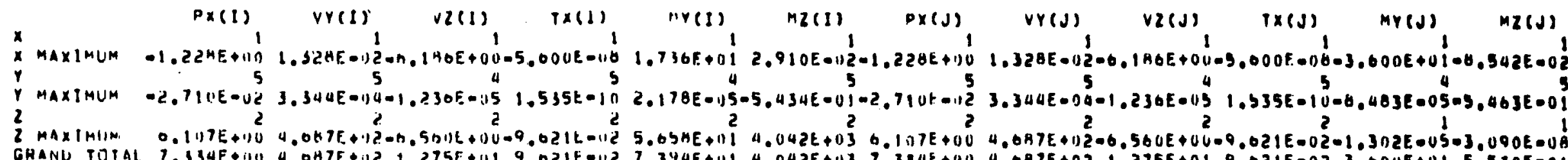

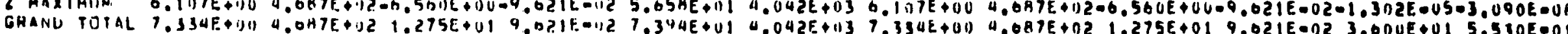

ELEMEIT TYPE (3/N P I P E

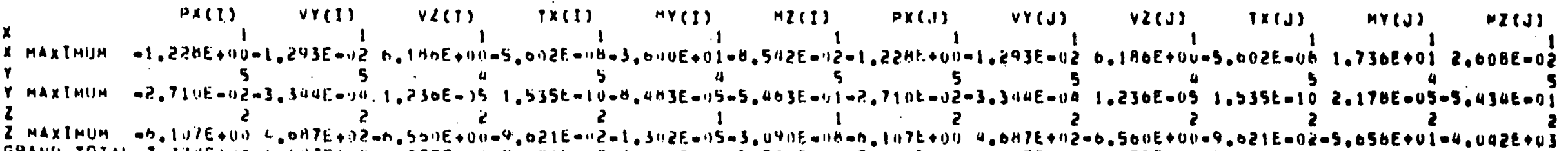

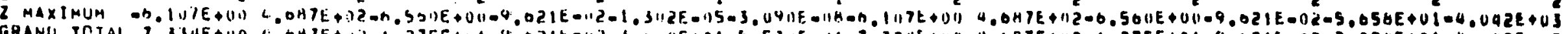

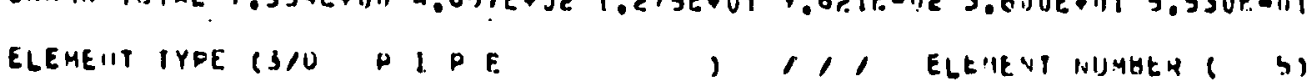

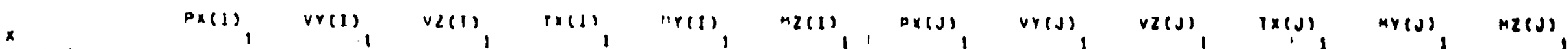

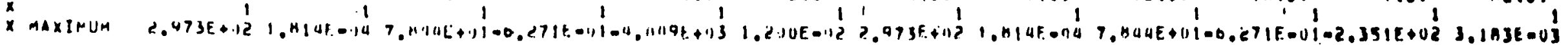




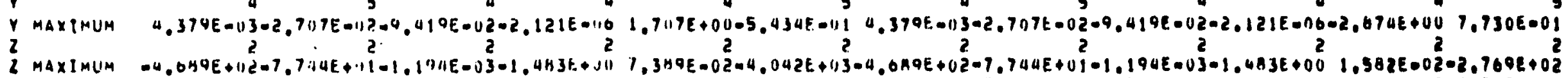

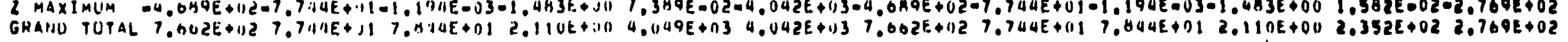
ELEHEiT TYPE (3/1) D I.PE ,, 1 ELLAENT NUMAER $(D)$

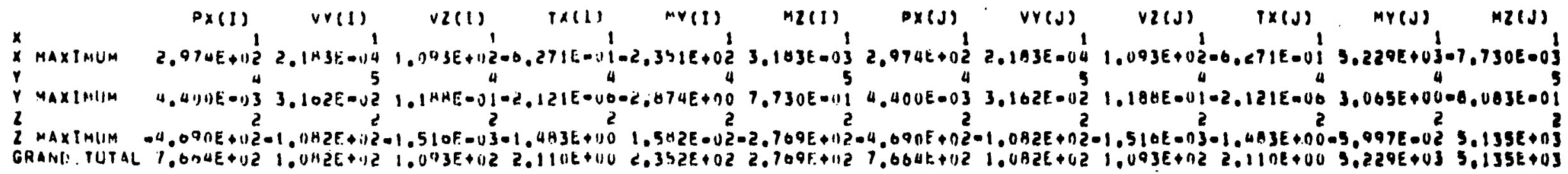

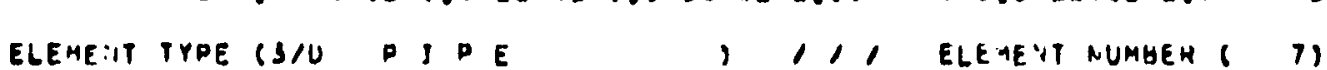

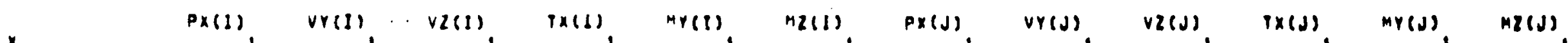
$x$
$X$
$y A x I M U M$
$Y$

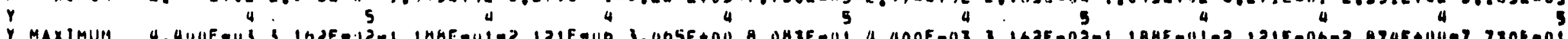

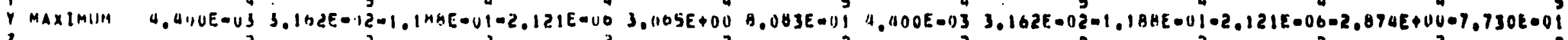

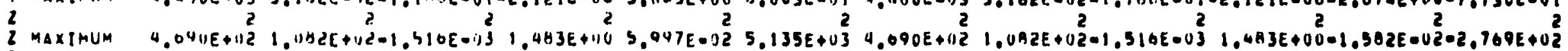

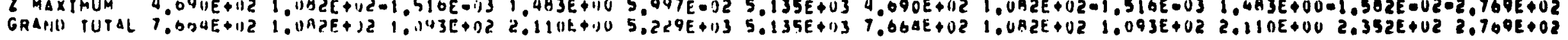
ELEMENI TYPE (S/U P D E , , , ELE TENT AUMUER ( H)

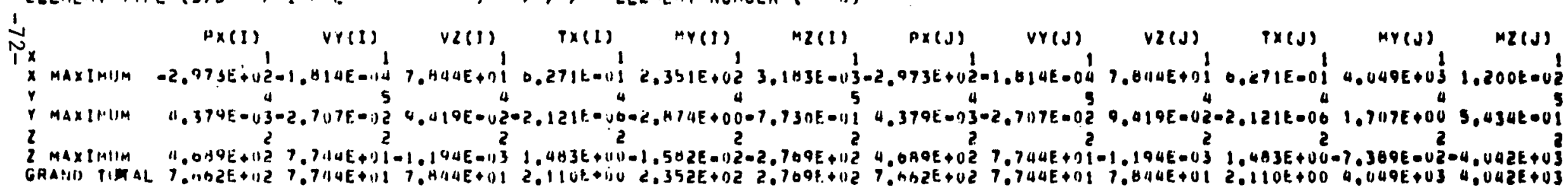
ELEHE:HT PYOE (3/D P I DE ,, 1 ELE. IENT PIJHAEN $($ 4)

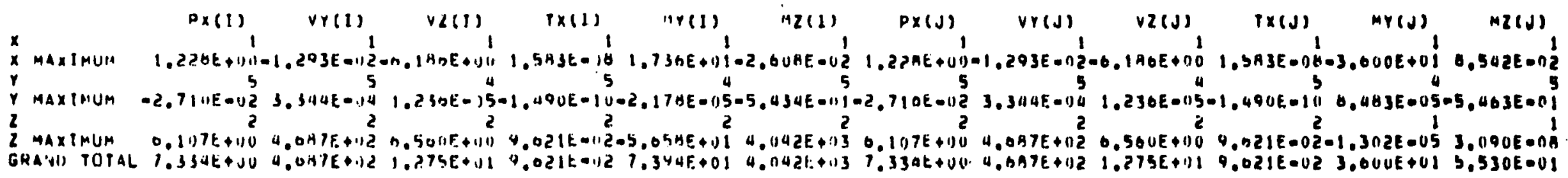
ELEMENT TYPE (3) D I PE , 1, , ELE IENT NUMGER 6 (ii)

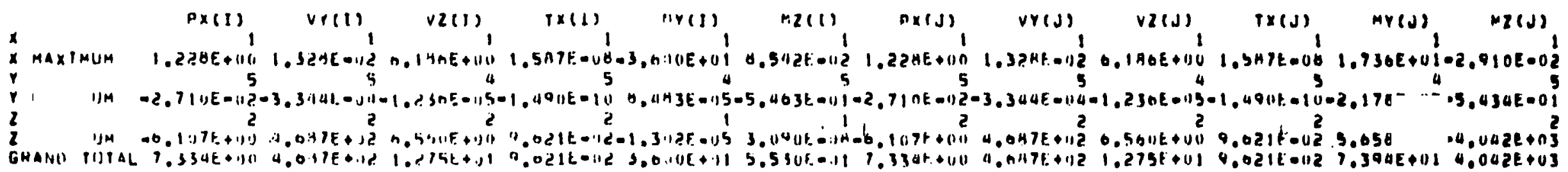




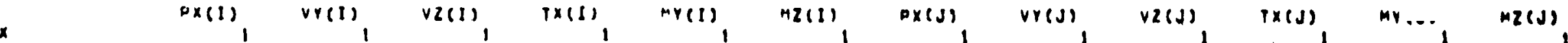

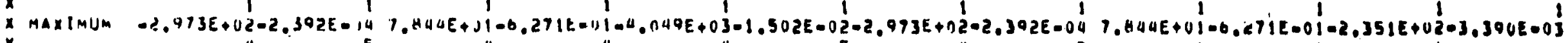

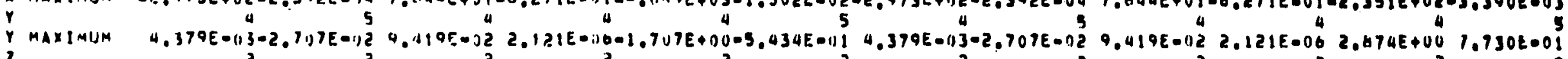

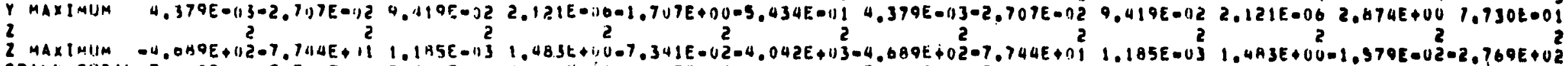

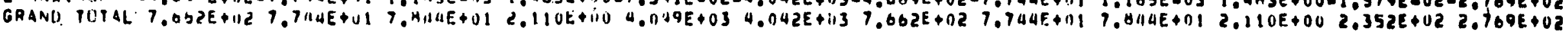
ELEMEIT TYPE (M/1) P I DE , , ELEMENT NUMHEH 1 12)

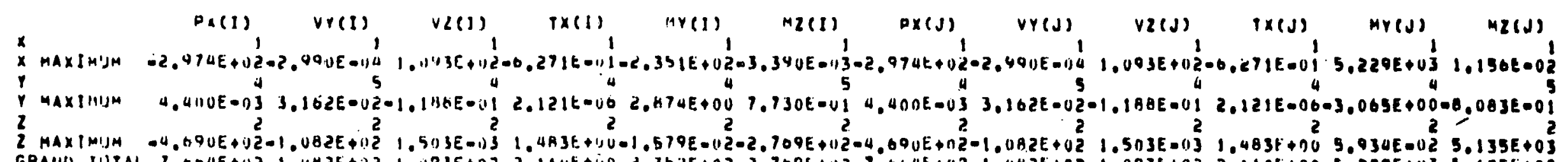

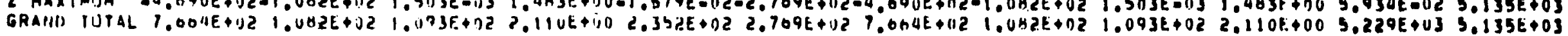

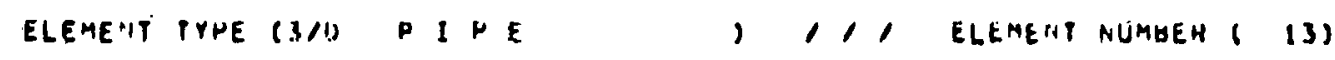

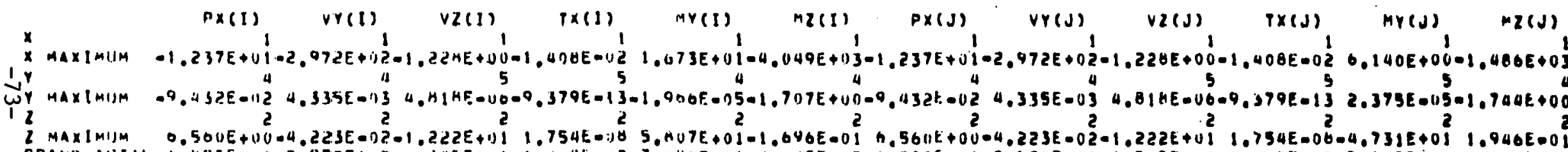

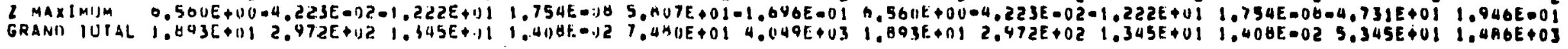

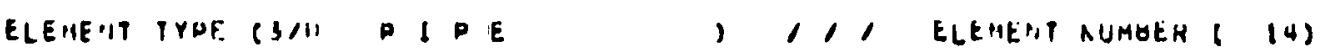

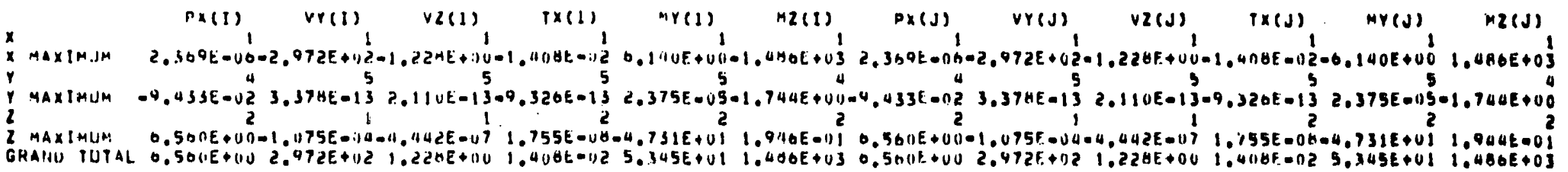
ELEAE.IT TYPE C.TU DIDE

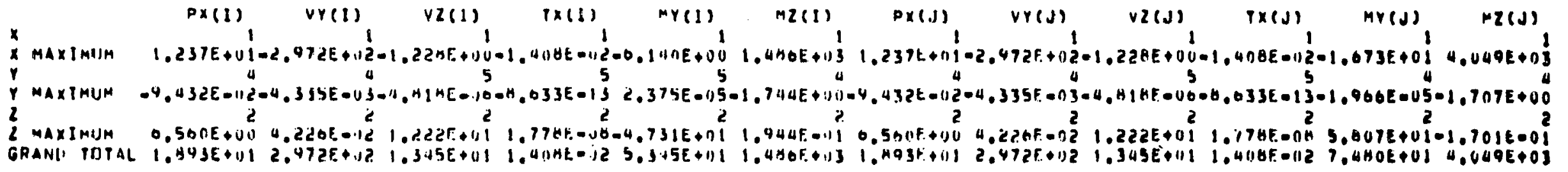
ELEMEIT TYPE (3/U $P I$ H

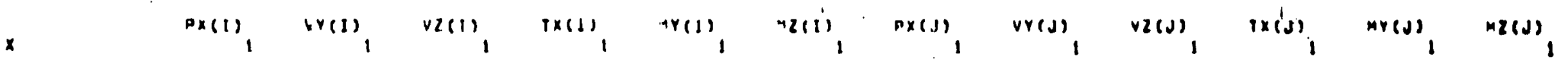




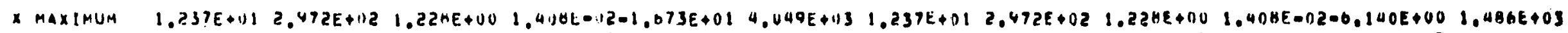

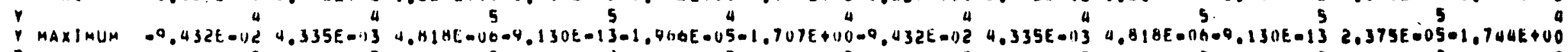

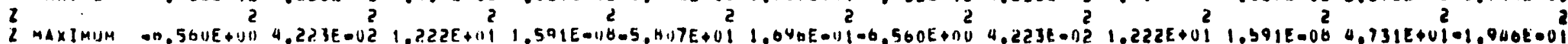

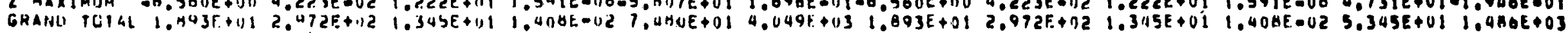

ELEMEHT TYPE (3/1) 1 PE , , , ELEMETS NUMHEH 1 17)

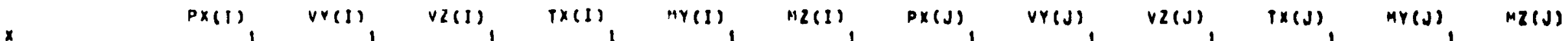

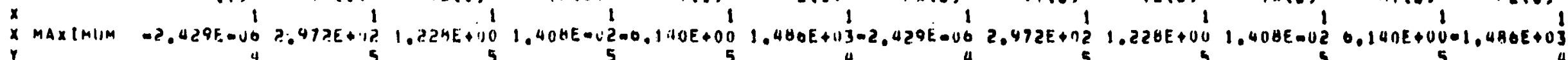

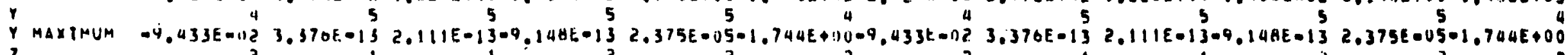

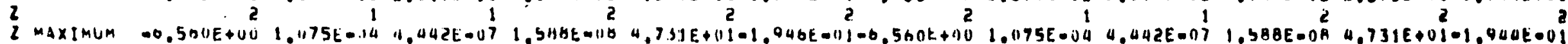

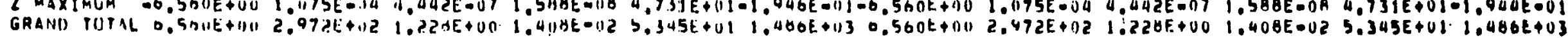

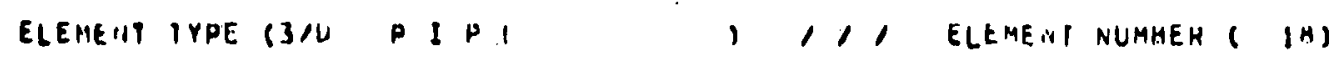

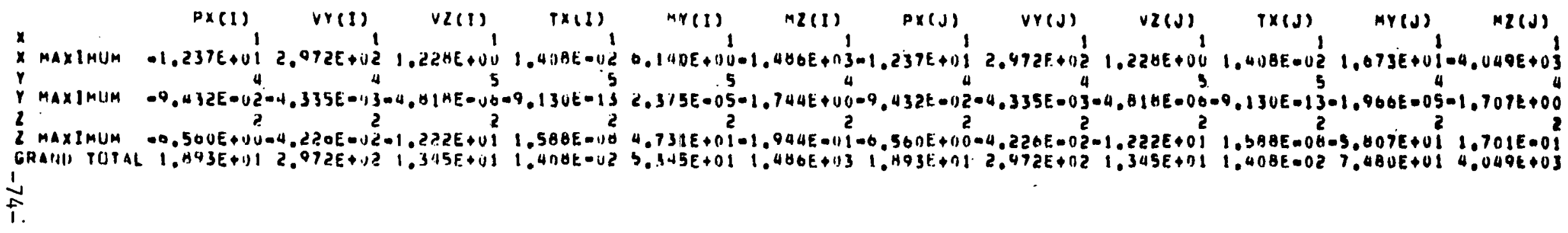


Solution $2 b$

Intermodal/Interspatial Combination 


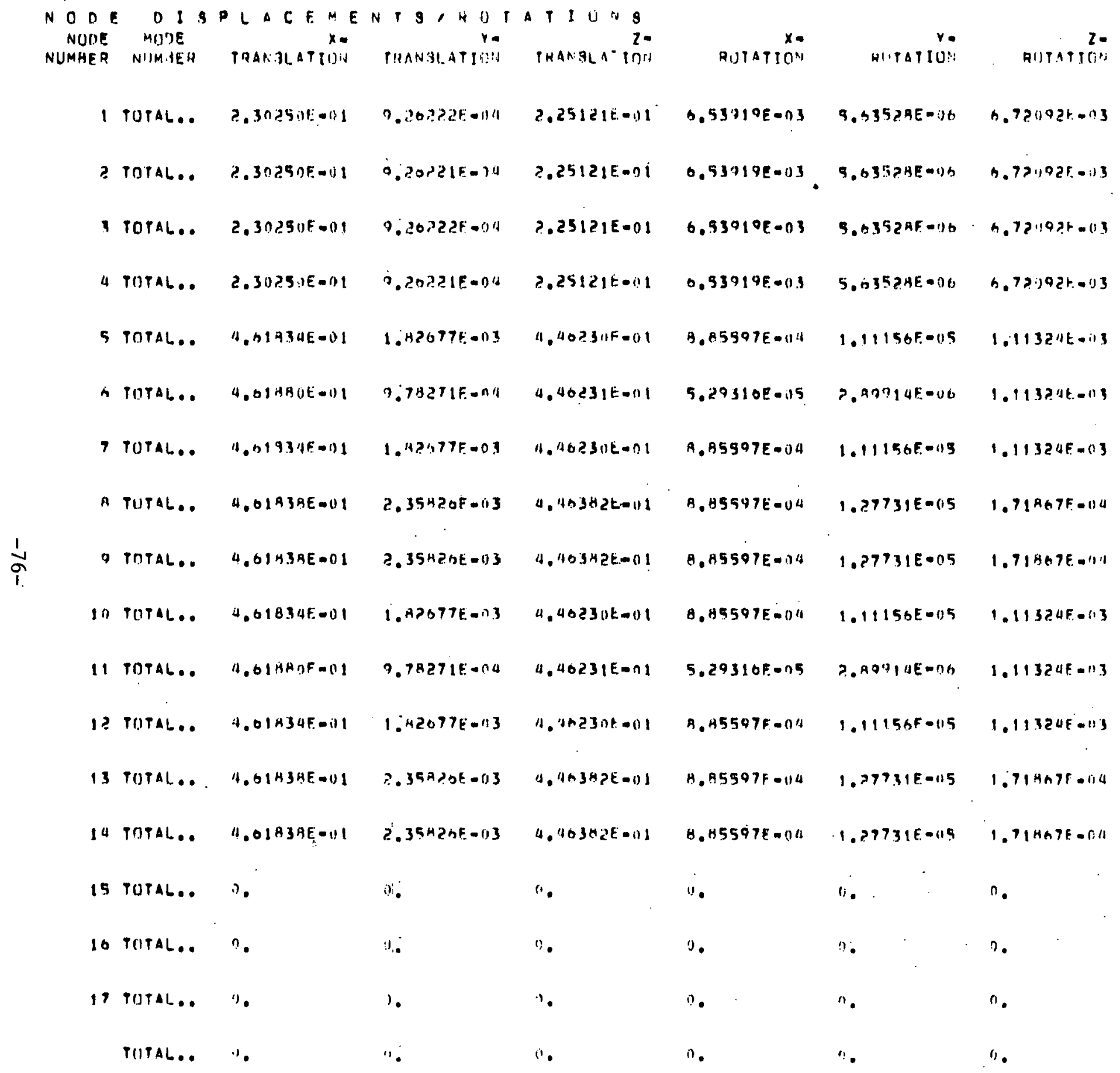




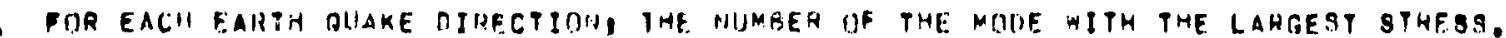

- the valile lif that sthesa

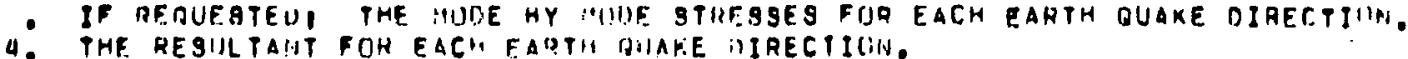

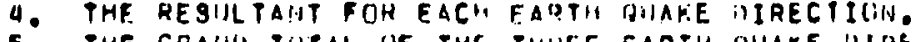

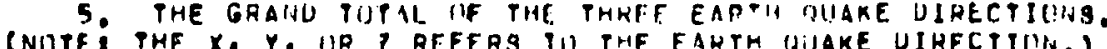

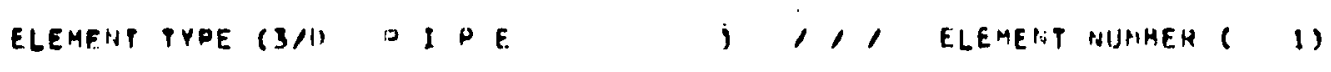

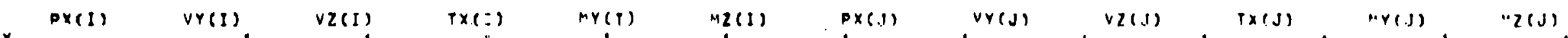

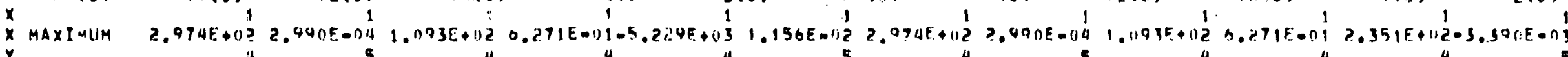

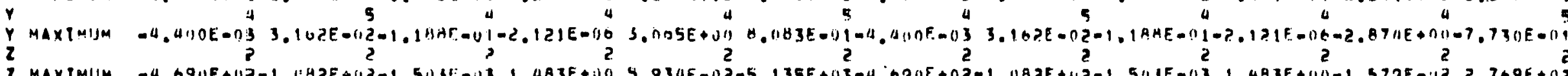

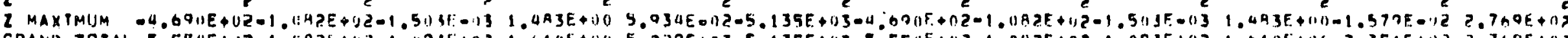

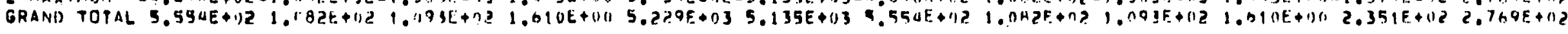

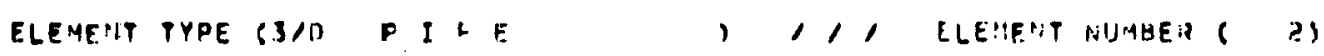

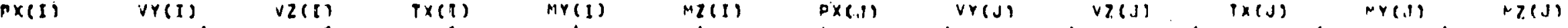

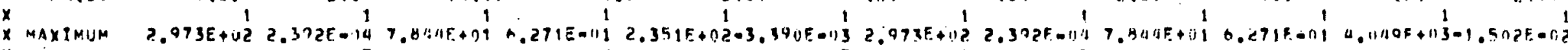

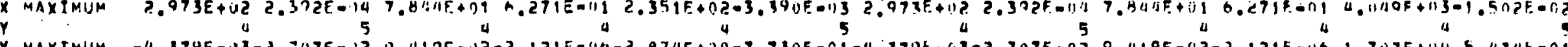

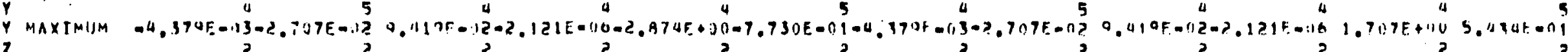

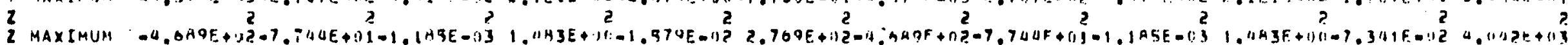

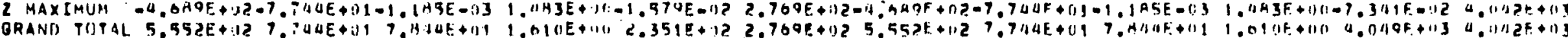

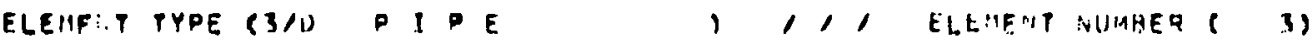

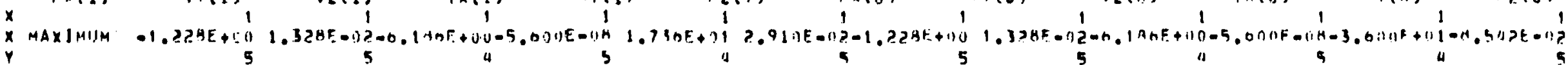

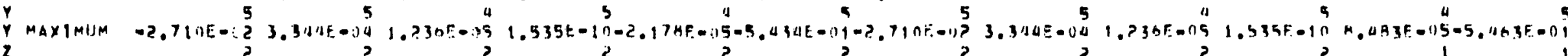

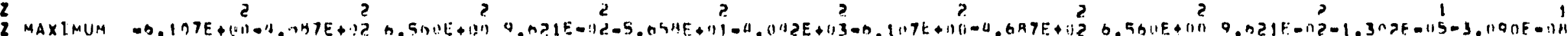

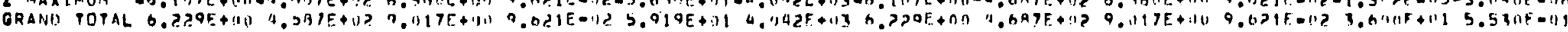

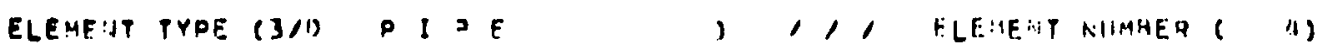

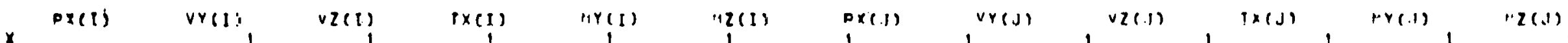

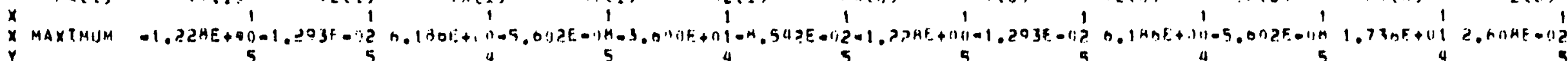

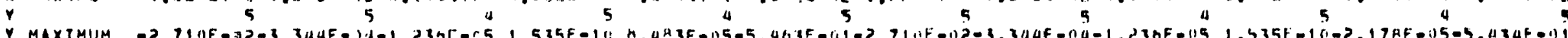

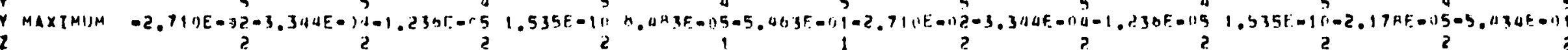

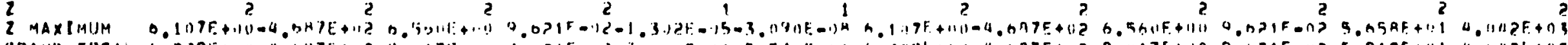

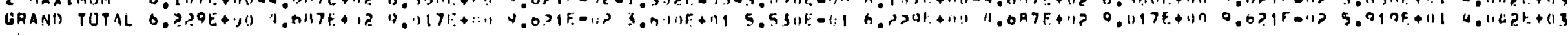

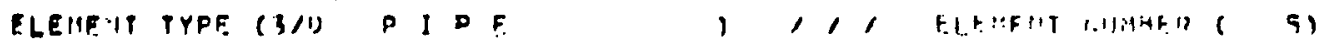

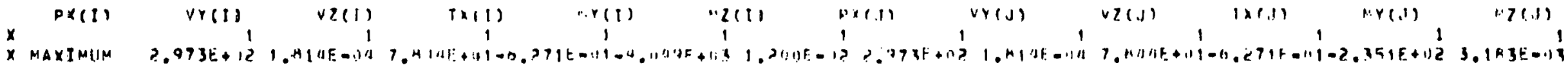




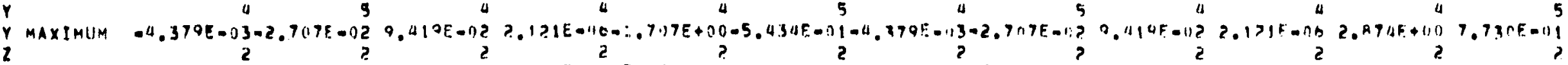

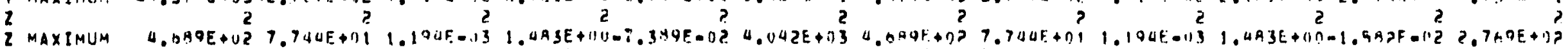

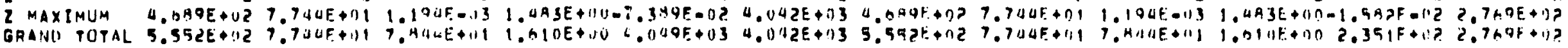

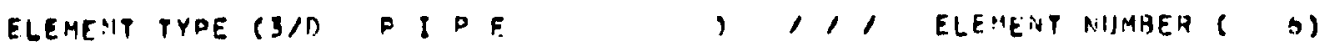

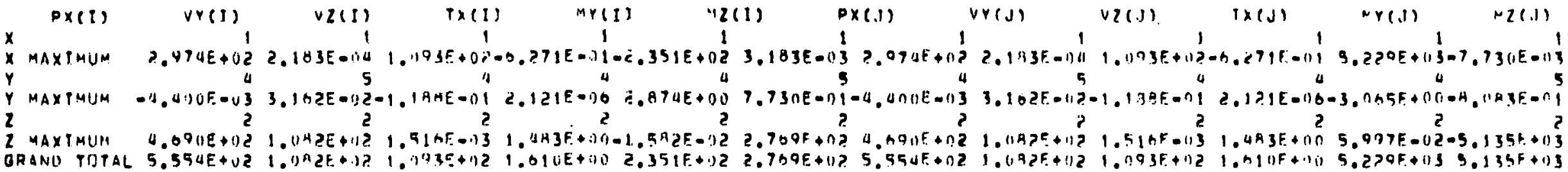
ELEHEUT TYPE (3/1) P I PE , , , ELE"ENT NUMGER $(7)$

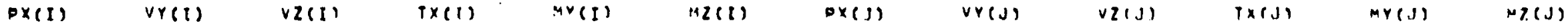

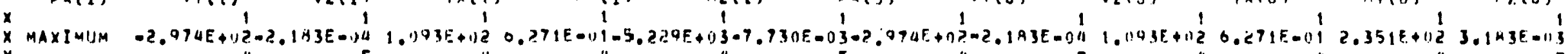

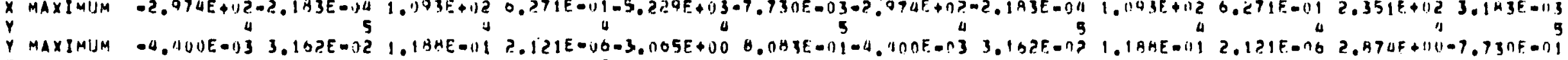

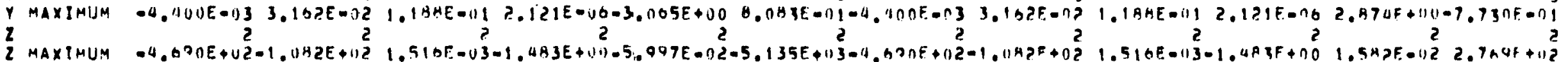

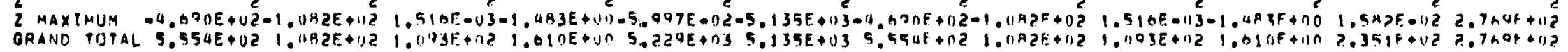

ELEMENT TYME (3/1) D I P E

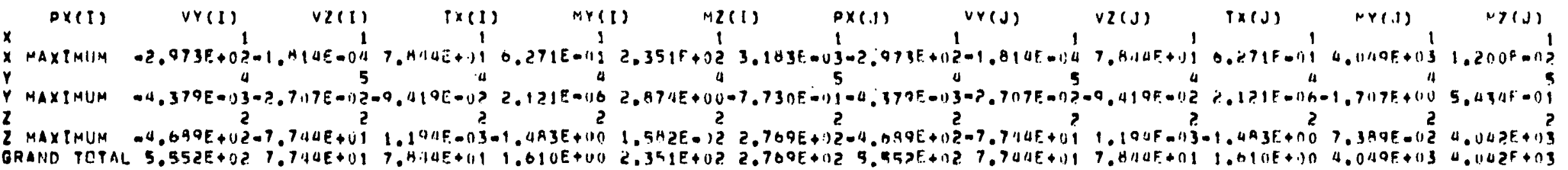

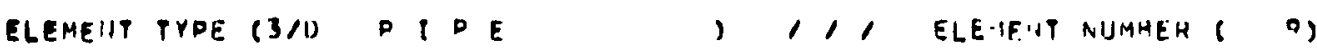

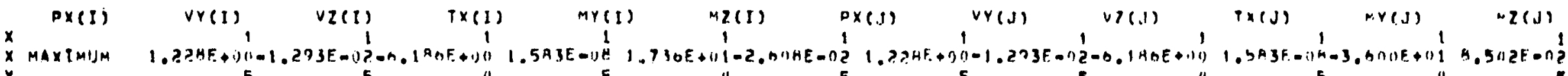

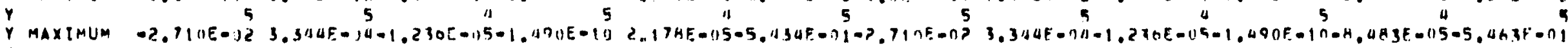

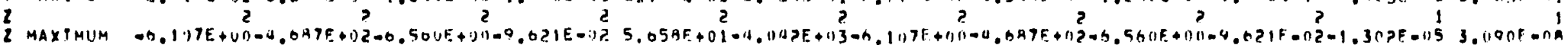

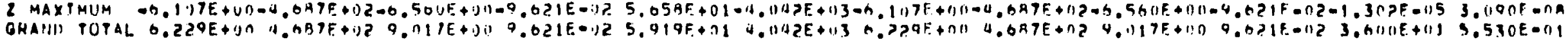
Elemeyt TYPE (XI) D I

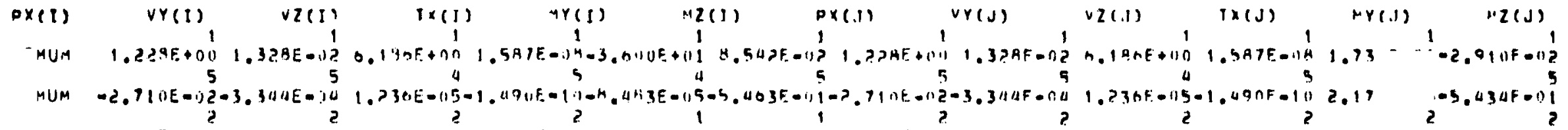




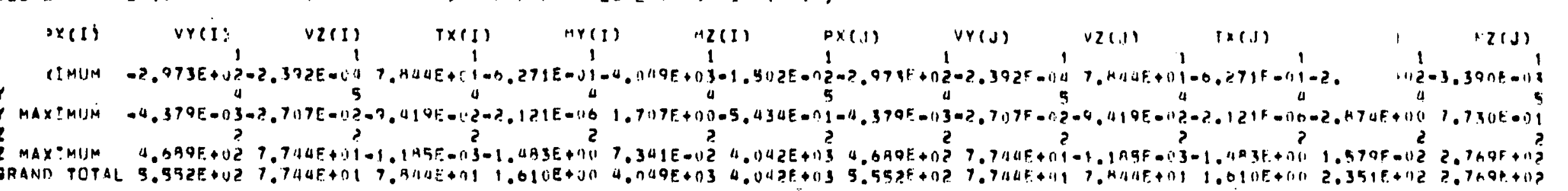

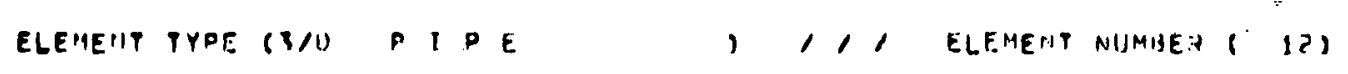

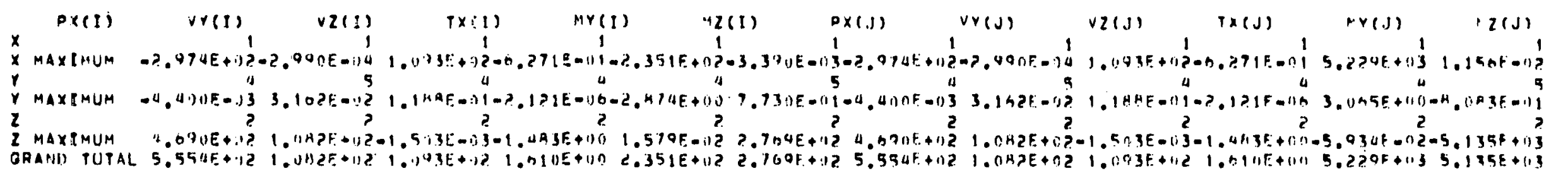

ELEMFNT TYOE (3/0 D 1 DE

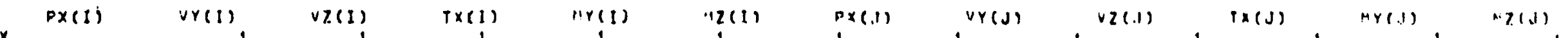

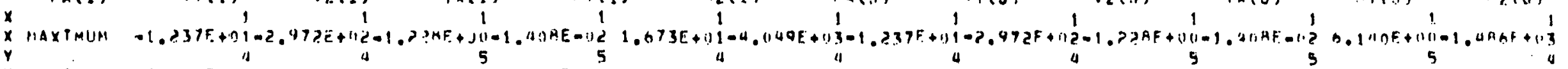

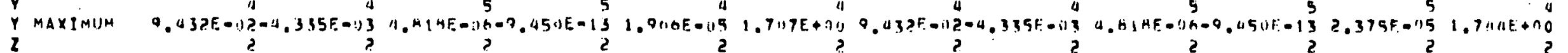

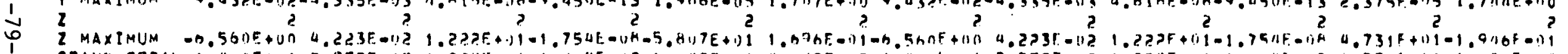

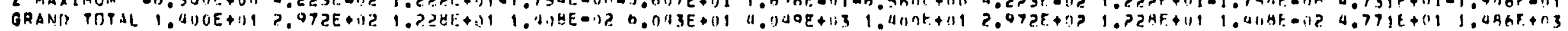

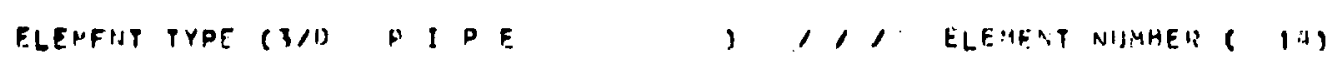

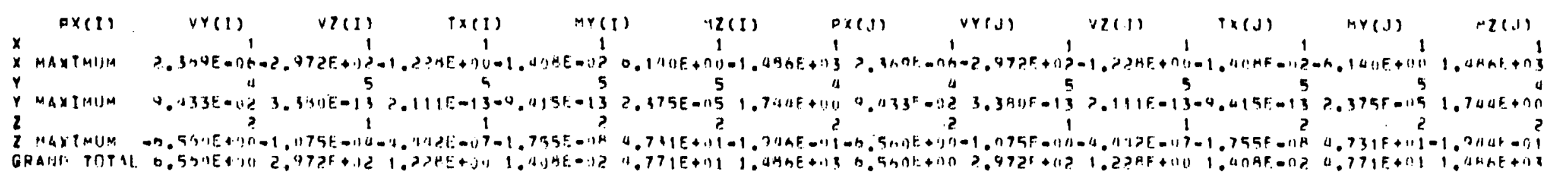

ELEMFIT PYPL (3/) P $t$ a

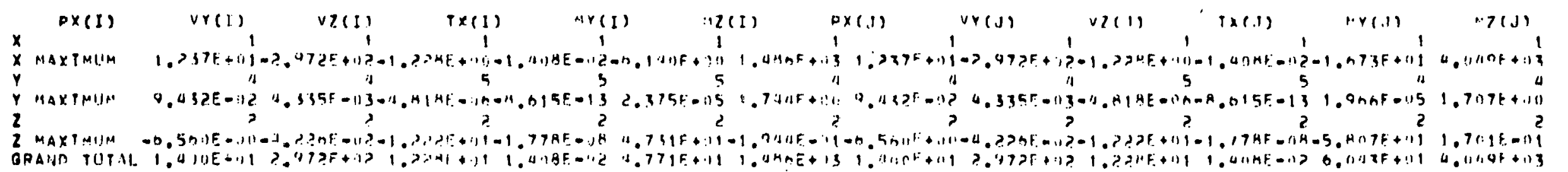

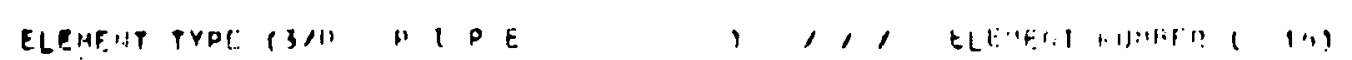

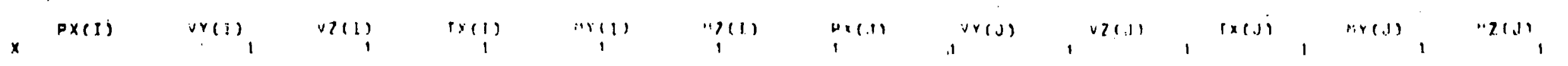




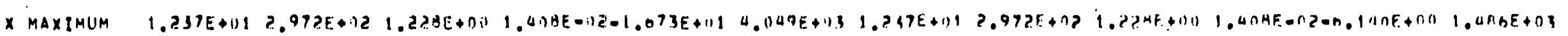

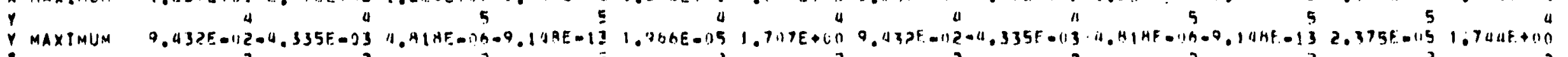

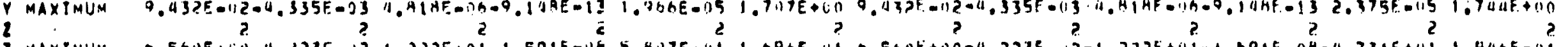

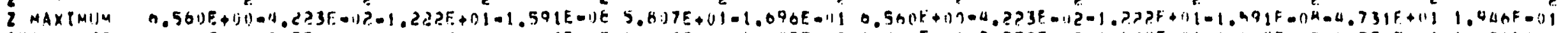

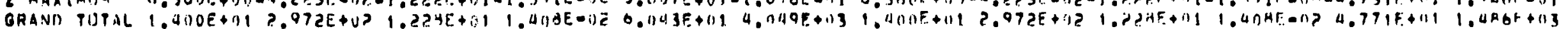

\section{ELEMENT TYPE (3/D P I WE}

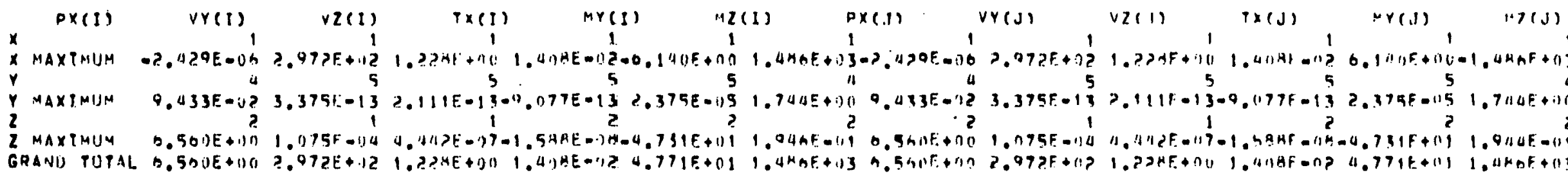
ELEME:IT TYPE (3/D P I P E

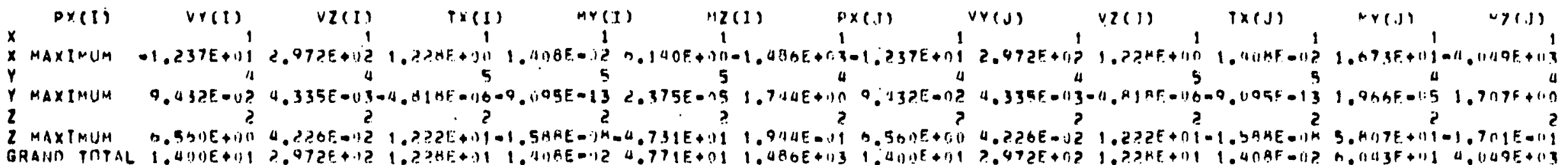



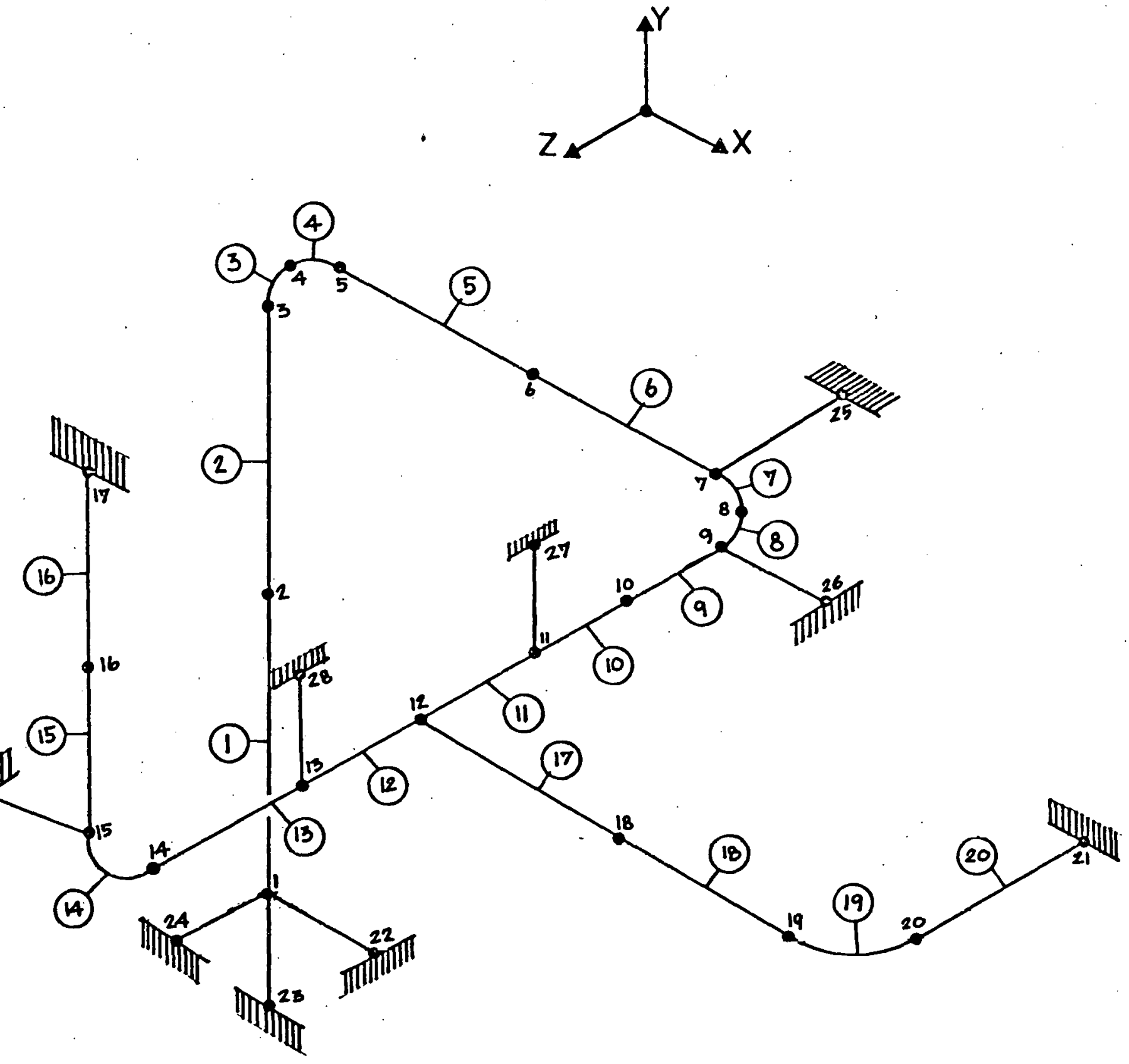

BENCHMARK PROBLEM NO. 3

Figure. 3.1 
BENCHMARK PROBLEM NO. 3

10000

5000
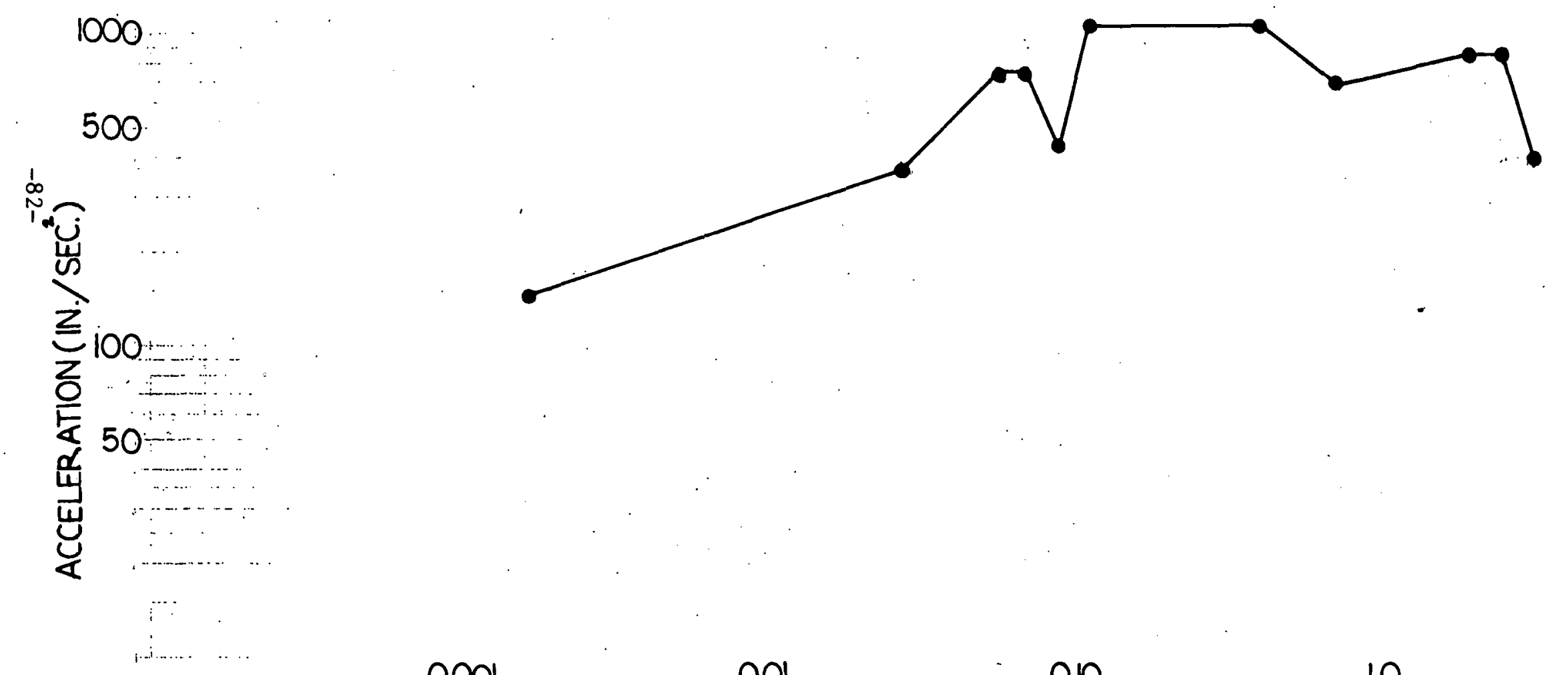

0.001

0.01

1.0

10.0

PERIOD (SEC)

Figure 3.2 
MUNAER OF NODAL POINTS

NUMIEP CIF LUMENT TYPES a

NUMiJER CIF LUAU CASES

NUMJER UF FRE QUENCIES
ANASYSIS COUE (NOYN)

E. O, STAIIC

D.1, MUDAL EXTRACTIUN

20.5. FORCEO FESPOVSE

EQ.3, RESPUNSE SPEETRUM

GO.4, DIRECT INTEGPAIIUN

SOLUTION HUUE (MDDEX)

PO.0, EXECUTIUN

ED.1. DATA CHECK

MUABEA OF GUYSPACE

ITFRATION VECTURB (NAD)

EDIIATIUNS PER HLOCK

TaPElo save FLAG (NIOSV). -

NODAL POINT JNPUT DATA

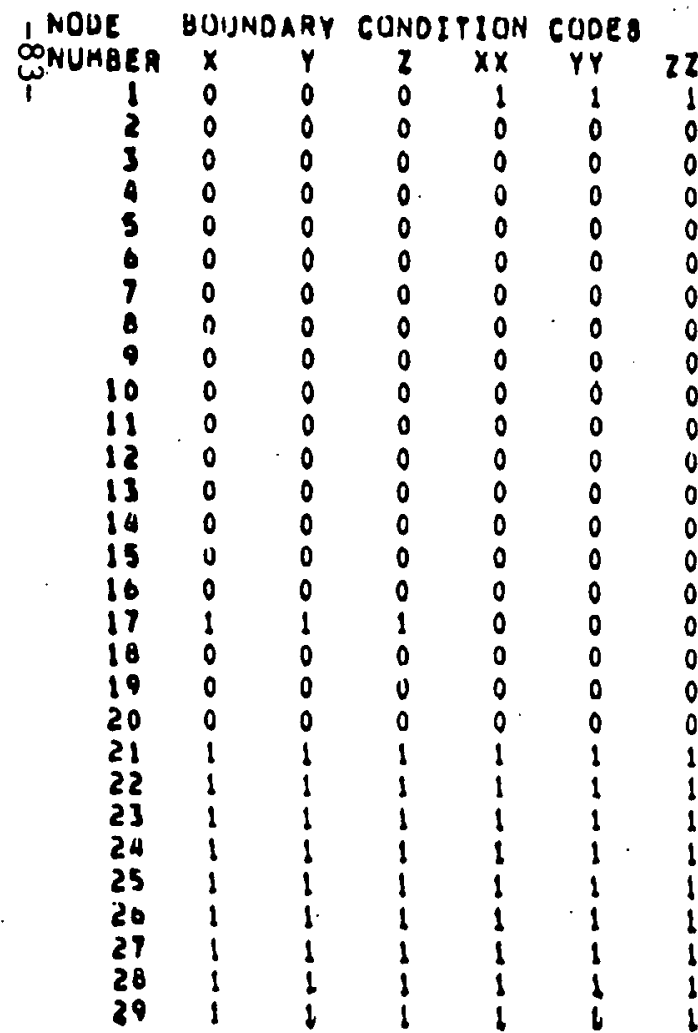

NOOAL POINT CUDROINATES

\begin{tabular}{|c|c|c|c|c|}
\hline$x$ & y & 7 & & 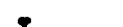 \\
\hline 0.000 & 0.000 & $0.000^{6}$ & $=0$ & I \\
\hline 0.000 & 54450 & 0 & & -0.001 \\
\hline 0.000 & 10800 & 0.00 & $\bullet$ & $-0,000$ \\
\hline 10.032 & 345 & 0.000 & -0 & $=0.000$ \\
\hline 36.300 & $145 ? 00$ & 0.000 & -0 & $=0.000$ \\
\hline 54.150 & 145.200 & 0.000 & -0 & -0.000 \\
\hline 72.000 & Jas 200 & $\begin{array}{l}0.000 \\
0.000\end{array}$ & -0 & $=0.000$ \\
\hline 91.608 & 145,200 & 10.632 & $=0$ & 0.000 \\
\hline 08.300 & 145.200 & 36.300 & -0 & $=0.000$ \\
\hline 08.300 & 145.200 & 56.800 & -0 & -0.000 \\
\hline 08.300 & 145.200 & 77.300 & .0 & -0.000 \\
\hline 108,300 & 145.200 & 97.800 & .0 & -0.000 \\
\hline 108.300 & 145.200 & $11 \mathrm{H} .300$ & -0 & .0 .000 \\
\hline 08.300 & 145.200 & 188.800 & -0 & .0 .000 \\
\hline 108.300 & 181.500 & 225.100 & $=0$ & $=0.000$ \\
\hline 100.300 & 236.000 & 225.100 & -0 & $-0,000$ \\
\hline 108.300 & 290.000 & 225.100 & -0 & $\therefore 0,000$ \\
\hline 300 & 145.200 & 97.800 & -0 & $=0.0 n_{0}$ \\
\hline 300 & 145.200 & 97.800 & -0 & .0 .000 \\
\hline 224.600 & .200 & 01.500 & -0 & -0.000 \\
\hline 224.000 & 145.2010 & 20.000 & -0 & $-0,0 \cap 0$ \\
\hline 1.000 & 0.000 & 0.000 & -0 & $-0,000$ \\
\hline 0.000 & 1.000 & 0.000 & -0 & $-0,000$ \\
\hline 0.000 & 0.000 & 1.000 & -0 & $-0,000$ \\
\hline 72.000 & 145.200 & -1.000 & -0 & $-0,000$ \\
\hline 109.300 & 145.200 & 36.300 & -0 & $=0.000$ \\
\hline 106.300 & 146.200 & 77.300 & -0 & -0.000 \\
\hline 00 & 140.200 & 1.18 .300 & -0 & -0.000 \\
\hline & & .500 & -0 & $=0.000$ \\
\hline
\end{tabular}


GENERATED NOOAL DATA

NOOE BUIJNOARY CONDITIIJN COUES

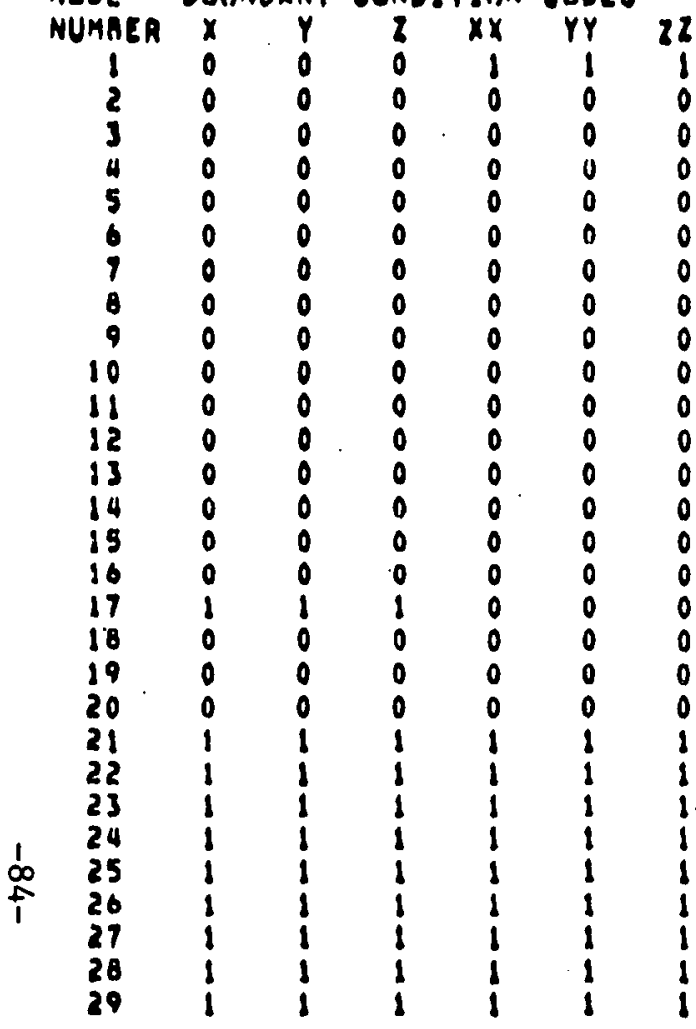

NODAL POINT COUROINATES

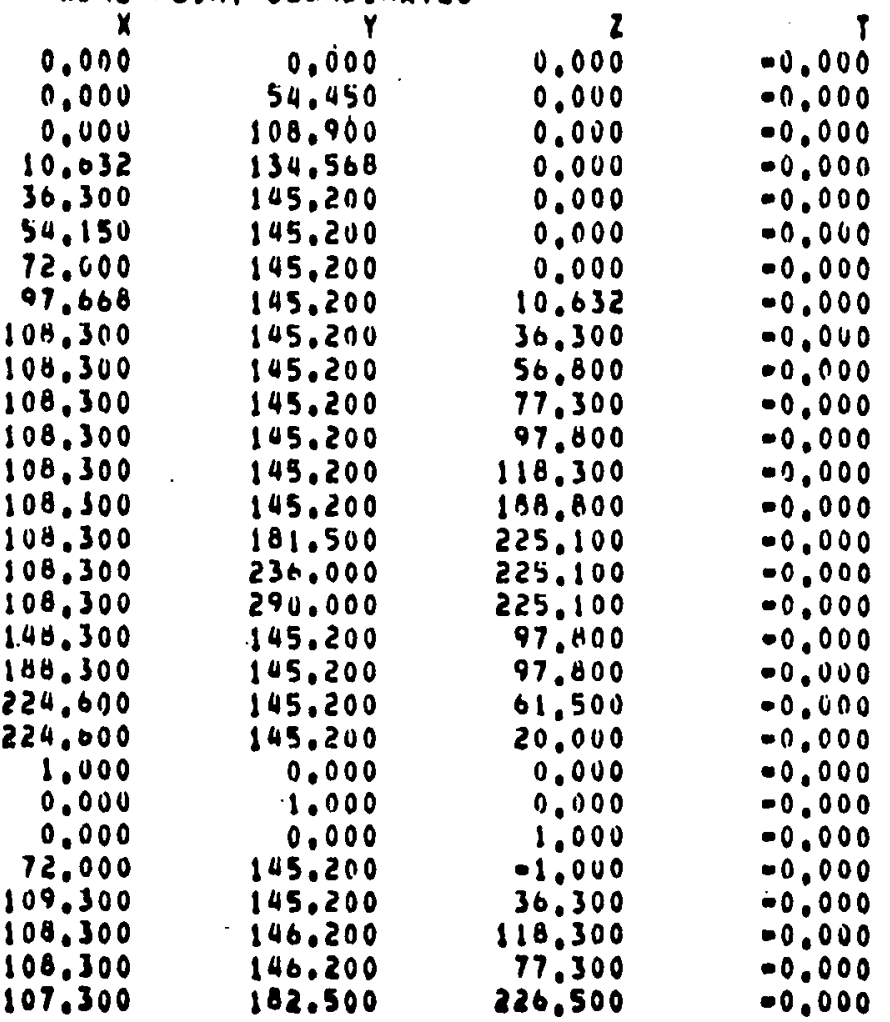


CLI TYPE

ELI TYPE

ELEMENT LUAD CASE MULTIPLIERS

$\begin{array}{rrrr}\text { CASE(A) } & \text { CASE(B) } & \text { CASEC(C) } & \text { CASE(D) } \\ 1.0000 & 1.0000 & 1.0000 & 1.0000\end{array}$

ELEMENT NUDE NUDES DEFINING CONSTRAINT DIRECTIUN NUMAE

CODE

CUDE GENERATION

CODE (KM) DISPLACEMENT

SPECIR IEO

BPRING

$\begin{array}{rrrr} & \text { (N) } & \text { (NI) } & \text { (NJ) } \\ 1 & 1 & 22 & =0 \\ 2 & 1 & 23 & =0 \\ 3 & 1 & 24 & =0 \\ 4 & 1 & 25 & =0 \\ 5 & 9 & 26 & =0 \\ 0 & 11 & 27 & =0 \\ 7 & 13 & 28 & =0 \\ 8 & 15 & 29 & -0\end{array}$

$\begin{array}{ll}-0 & =0 \\ =0 & =0 \\ =0 & =0 \\ =0 & =0 \\ =0 & =0 \\ =0 & =0 \\ =0 & -0 \\ =0 & =0\end{array}$

$=0$
$=0$
$=0$
$=0$
$=0$
$=0$
$=0$
$=0$

$=0$
$=0$
$=0$
$=0$
$=0$
$=0$
$=0$
$=0$

-0
-0
-0
-0
-0
-0
-0
-0

$\begin{array}{ll}=0 & 0, \\ =0 & 0 \\ =0 & 0 \\ =0 & 0 \\ =0 & 0 \\ =0 & 0 \\ =0 & 0 \\ =0 & 0 .\end{array}$

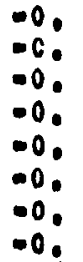

$.1000 E+11$

$1000 E+11$

IONOE+11

$.1000 E+09$

$1000 E+09$

$.1000 E \$ 05$

$1000 E+05$

$\underbrace{\infty}_{1}$

$\stackrel{1}{\infty}$ 
PIPE ELEMENT INPUT DATA

CONTROL INFONMATION

NUMAER OF PIPE ELEMENTS

- 20

NUMBER OP MATERTAL BETS

- 1

MAXIMUM NUMBER OF MATERIAL

TEMPERATURE INPUT POINTS

NUMBEA OP BECTIUN PROPERTY BETS " 1

nUmber of branch poINT nLOES - 0

maXjMUM NUMBER UF TANGENTS

COIMMON TO A BRANCH POINT

- 3

mLag fOR neOLECTING aXIAL

DEFURMATIONS IN GEND ELEMENTS : 0

(EO,1, NEQLECT)

Ó

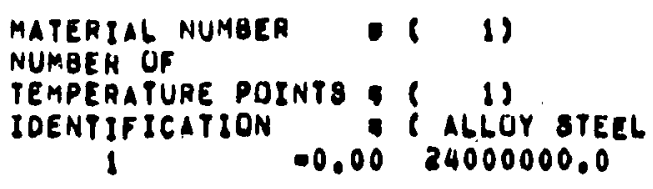


SECIIUN PROPEATY TABLE

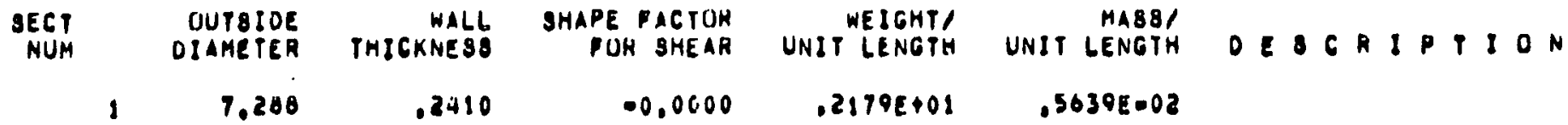

ELEMENT LUAO CABE MULIIPLIERS

\begin{tabular}{|c|c|c|c|}
\hline $\begin{array}{l}\text { X-DIRECTION GRAVITY } \\
\text { YODIRECTION GRAVITY } \\
\text { Z-DIRECTION GRAVITY } \\
\text { THEKMAL DIQTUKTION } \\
\text { PRESSURE DISTURTIUN }\end{array}$ & $\begin{array}{l}0.000 \\
1.000 \\
0.000 \\
1.000 \\
1.000\end{array}$ & $\begin{array}{l}-0.000 \\
=0.000 \\
-0.000 \\
-0.000 \\
-0.000\end{array}$ & $\begin{array}{l}-0.000 \\
-0.000 \\
-0.000 \\
-0.000 \\
-0.000\end{array}$ \\
\hline
\end{tabular}


PIDE ELEMENT INPUT DATA

ELEMENT ELEMENT NOOE NDDE MATL. OFCTION NUMBER PYPE $-I$ G NUMBER NUMEER

REFERENCE INIEKNAL
TEMPERATURE PRESSUHE (BEND PRESSUHE

DIRECIION C

N COOINES NODE INPUT RAOIUS) PUINT)

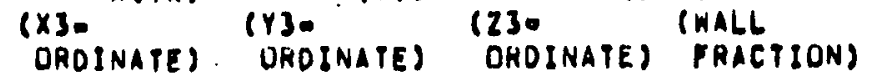
$A(r y)$

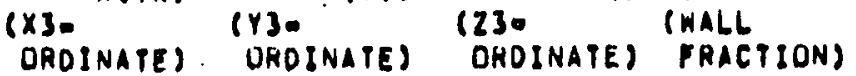

Paction)

2 TANGENT

3 BEND

4 HENO

5 TANGENT

- tANGEnT

BEND

- BEND

8

$\underset{1}{1}$

9 TANGENT

10 TANGENT

il TANOENT

12 TAIGENT

13 TANGENT

I4 BENO

$\begin{array}{lllll}15 & \text { TANGENT } & 15 & 16 & 1 \\ 16 & \text { TANGENT } & 16 & 19 & 1 \\ 19 & \text { TANGENT } & 12 & 18 & 1 \\ 18 & \text { TANGENT } & 10 & 19 & 1 \\ 19 & \text { OEND } & 19 & 20 & 1\end{array}$

20

TANGENT

$\begin{array}{lll}9 & 10 & 1 \\ 10 & 11 & 1 \\ 11 & 12 & 1 \\ 12 & 13 & 1 \\ 13 & 14 & 1 \\ 14 & 15 & 1\end{array}$

20
$-0.00$

$-0.00$

350.00

350.00

0.00000

0.0000
.0 .0000

0.00000

( 30.300$)$

-0.00
36.300

(C) 1

350.00

(CC)

$1 \quad 0.000$

-0.00
-0.00

350.00

350.00

350,00

$(36.300)$

(CC) 1

$1 \quad-0.00$

-0.00
36.3005

350,00

$=0.00$

$=0.00$

$-0.00$

$-0.00$

$-0.00$

$-0.00$

$\left(\begin{array}{l}-0.00 \\ 36.300)\end{array}\right.$

350.00

350.00

350.00

350.00

350.00

350.00

$-0,0000$

.0000

$=0.0000$

1

$0.000)(.1000)$

$36,30016 \quad 100,90036$

$30.3100) 8 \quad 108.90018$

0.00021 .010008

0.00000

$.0,0000$

$-0.0000$

$-0,0000$

$-0,0000$

$-0,0000$

1

$72.000)(145.200) 1$

$36.3003(.1000)$

$12.00018 \quad 145.20018$

36.30081 .10008

-0.0000
.0 .0000

$-0,0000$

00.0000

$-0.0000$

.0 .0000

.0 .0000

$0.0,0000$

20000

$-0.0000$

108,30018

181.5001

350.00

350.00

$=0.00$

$-0.00$

$-0.00$

$-0.00$

350.00

350.00

350.00

( 36.3008

(CC)

$-0.0000$

$-0.0000$

$-0.0000$

00,0000

$188.300) 1$

0.0000

.0 .0000

$-0.0000$

$-0.0000$

350.00

$-0,0000$

$(45.200)$

.0 .0000

$-0.00$
$-0.0000$

$-0.0000$

$-0,0000$

$-0.0000$

$.0,0000$

$(88.000)(.1000)$

$-0.0000$

$-0.0000$

$-0.0000$

$-0.0000$

$-0,0000 \quad 1$

$$
i
$$

!

ic

16

It

If

IC

II

I!

II

ic

I!

II

II

II 
CIRCIULAR

(AAD/SEC) (CYCLES/SEC) (BEC)

$\begin{array}{llll}1 & .4472 E+02 & .7118 E+01 & .1405 E+00 \\ 2 & .1945 E+02 & .1265 E+02 & .1908 E-01 \\ 3 & .0920 E+02 & .1580 E+02 & .0328 E-01 \\ 4 & .1000 E+03 & .1719 E+02 & .5819 E-01 \\ 5 & .1344 E+03 & .2139 E+02 & .04074 E-01 \\ 6 & .1572 E+03 & .2501 E+02 & .3098 E-01 \\ 7 & .1796 E+03 & .2859 E+02 & .3498 E-01 \\ 8 & .2248 E+03 & .3648 E+02 & .2747 E-01 \\ 0 & .2555 E+03 & .4067 E+02 & .2459 E-01 \\ 10 & .3062 E+03 & .4873 E+02 & .2052 E-01\end{array}$




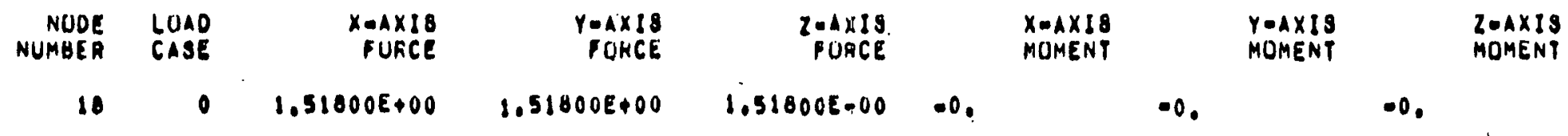

STRUCTURE
LOAOCASE ELMENT LOAD MULTJPLIERg

1

$\begin{array}{llll}-0.000 & -0.000 & -0.000 & -0.000\end{array}$

1
$\stackrel{0}{0}$ 
MODE NIJMBER

PAEQUENCY 7,11809

Hz

EIGEUVECTORS NURMALIZEO TO A UNIT MASS MATKIX

OISPLACEMENTS/RUTATIONS OF UNREBTHAINED NUDES

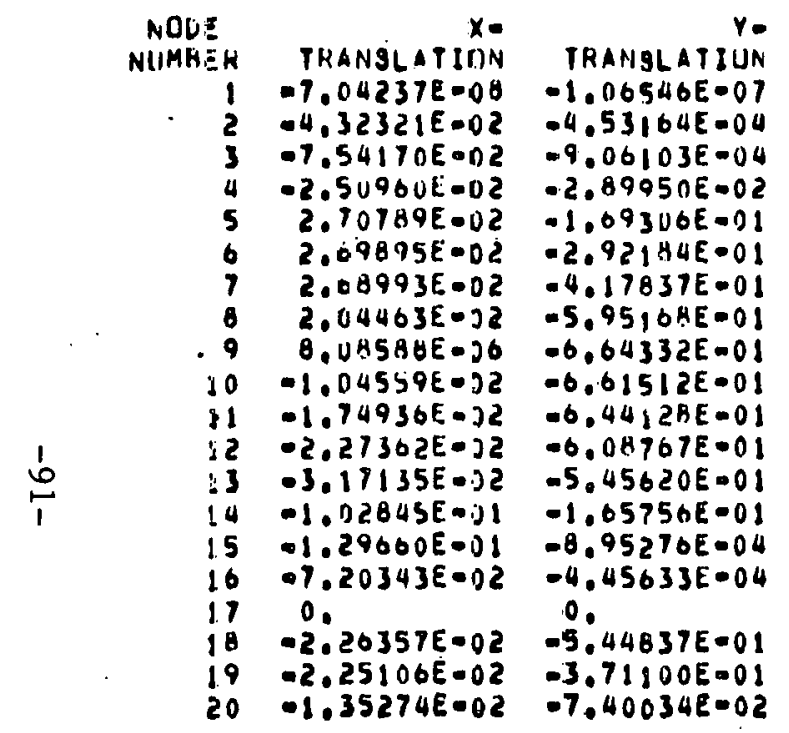

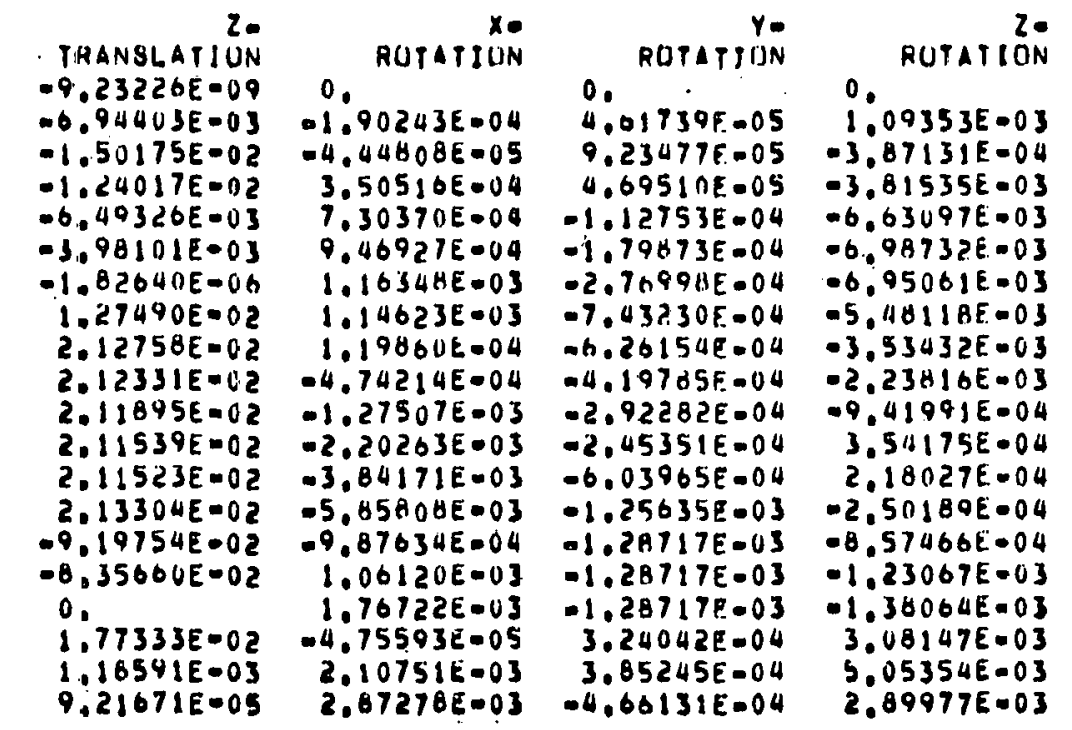


MOUE NUMBER I

EIGENVECTORS NOHMALIZED TO 1.000

DISPLACEMENTS/RUTATIUNS OF UNRESTKAINED NODES

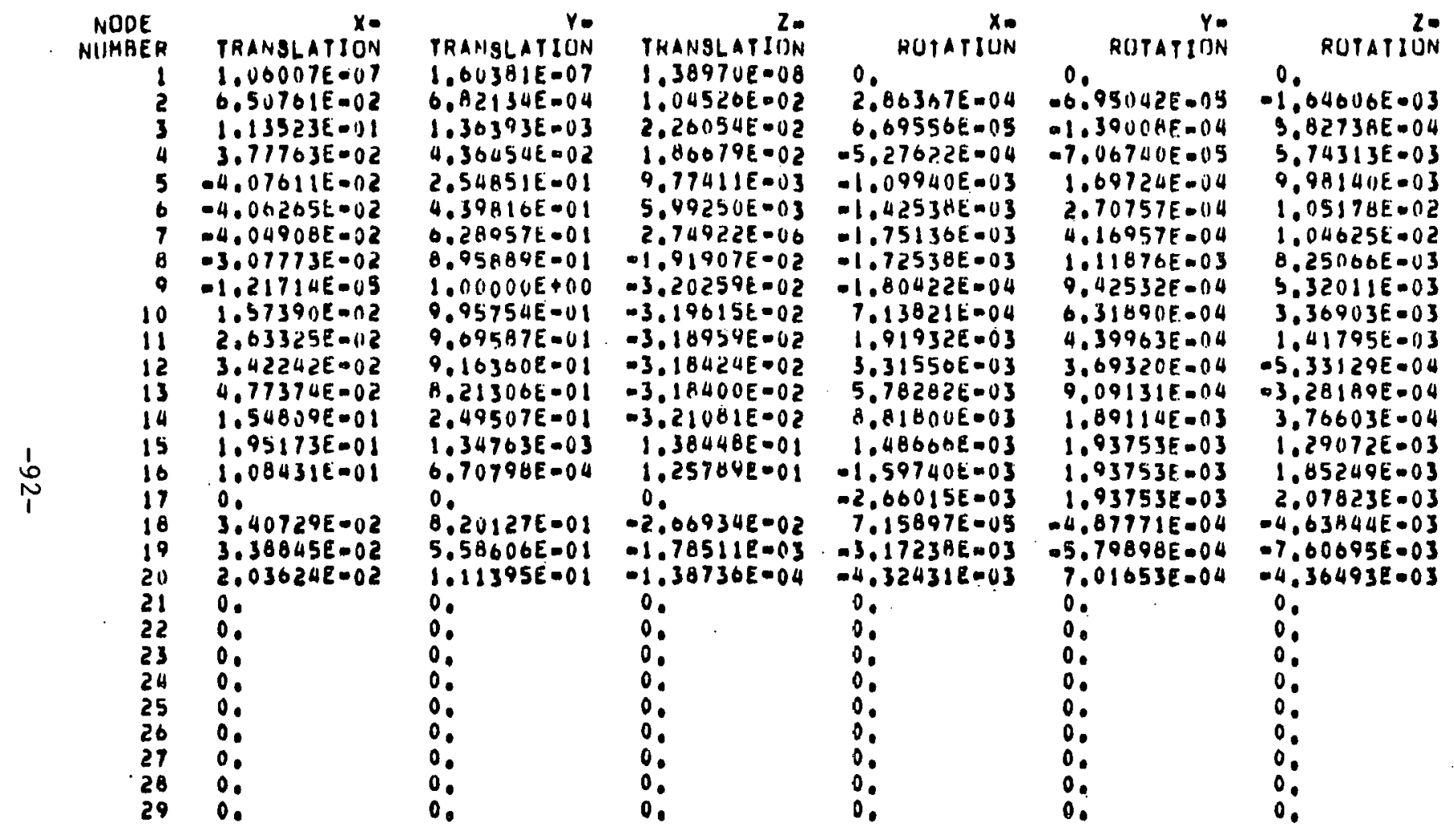


MUDE NUMAER 2

PREQUUENCY 12.6452

42

EJGENYECTORS NURMALJZEO TO I UNIT MASS MATHIX

DISPLACEMENTS/RUTATIOHS OF UNREBTRAINED NUDES

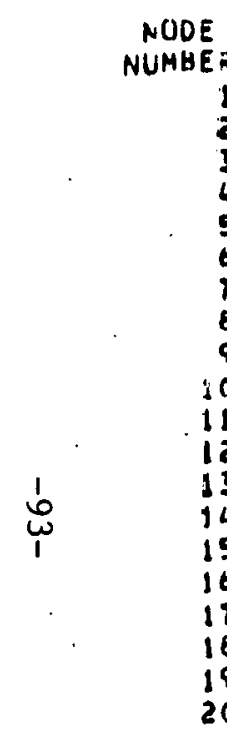

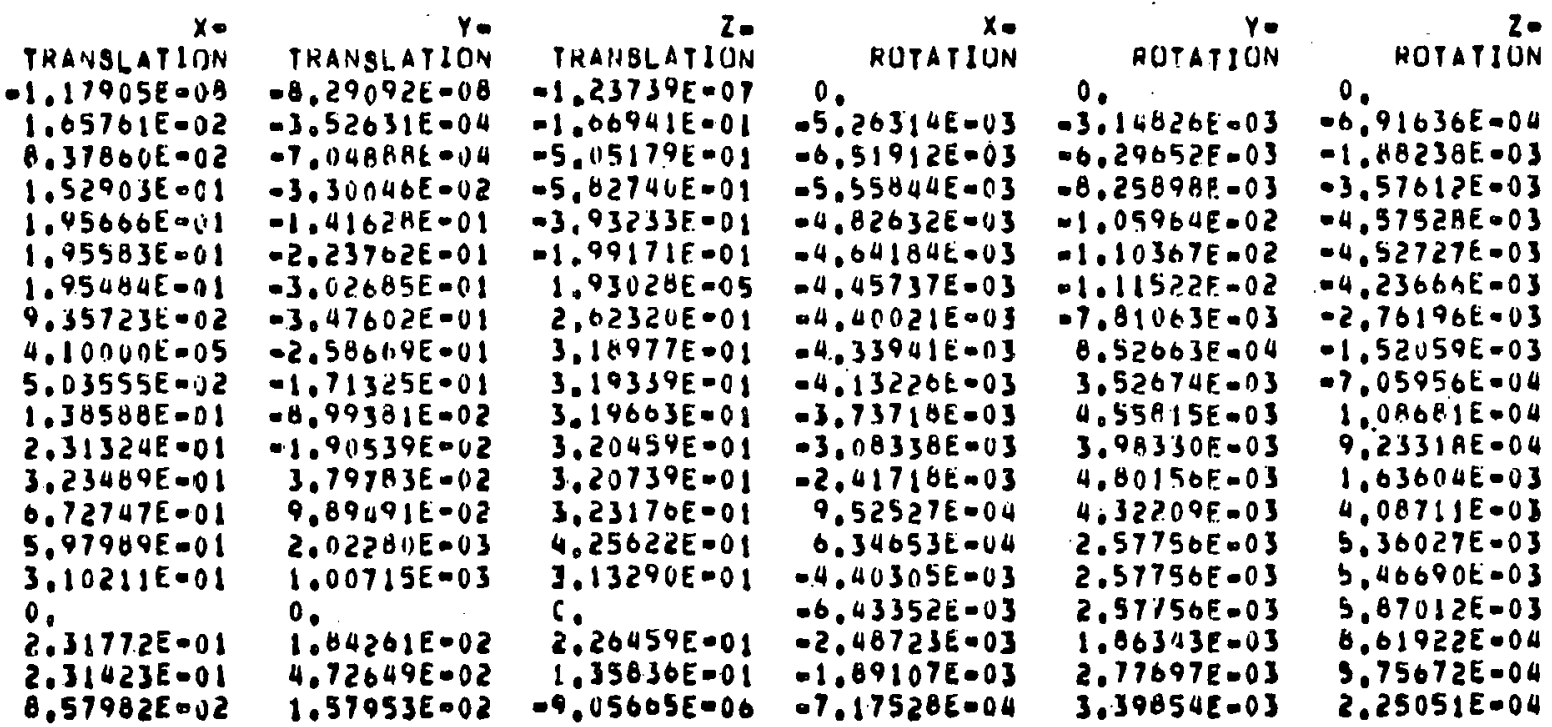


MODE NUHEER

EIGENVECTORS NORHALIZEO TO 1.000

DISPLACEMENTSIRUTATIUNS OF UNRESTRAINED NUDEO

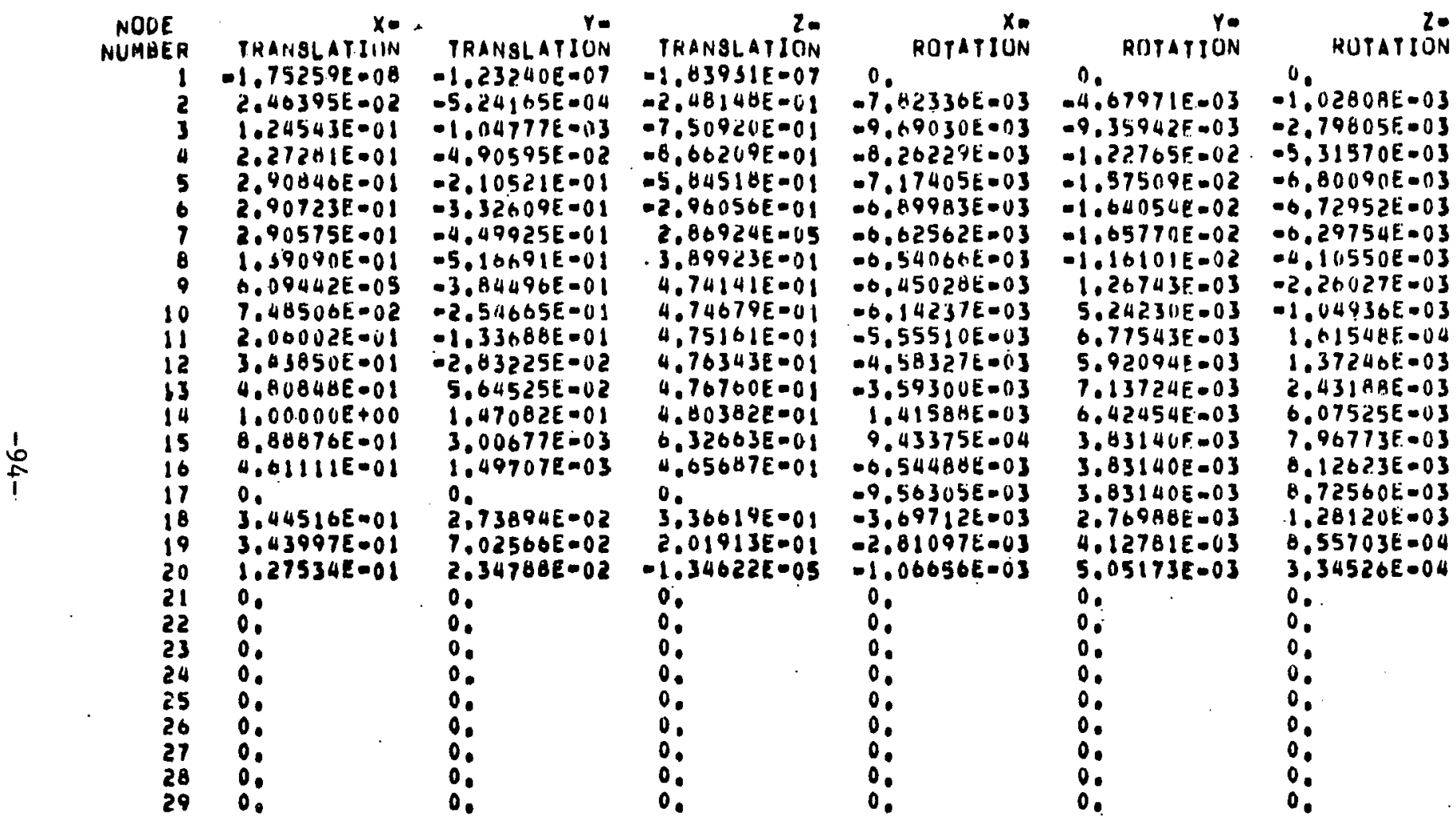


MUDE. NUMGER 3

PREQIENCY $15.8032 \quad H 2$

EIGENVECTORB NUAMALIZED TO A UNIT MASS YATHIX

DISPLACEMENTSIRUTATIOHS OF UNRESTRAINEO NORE

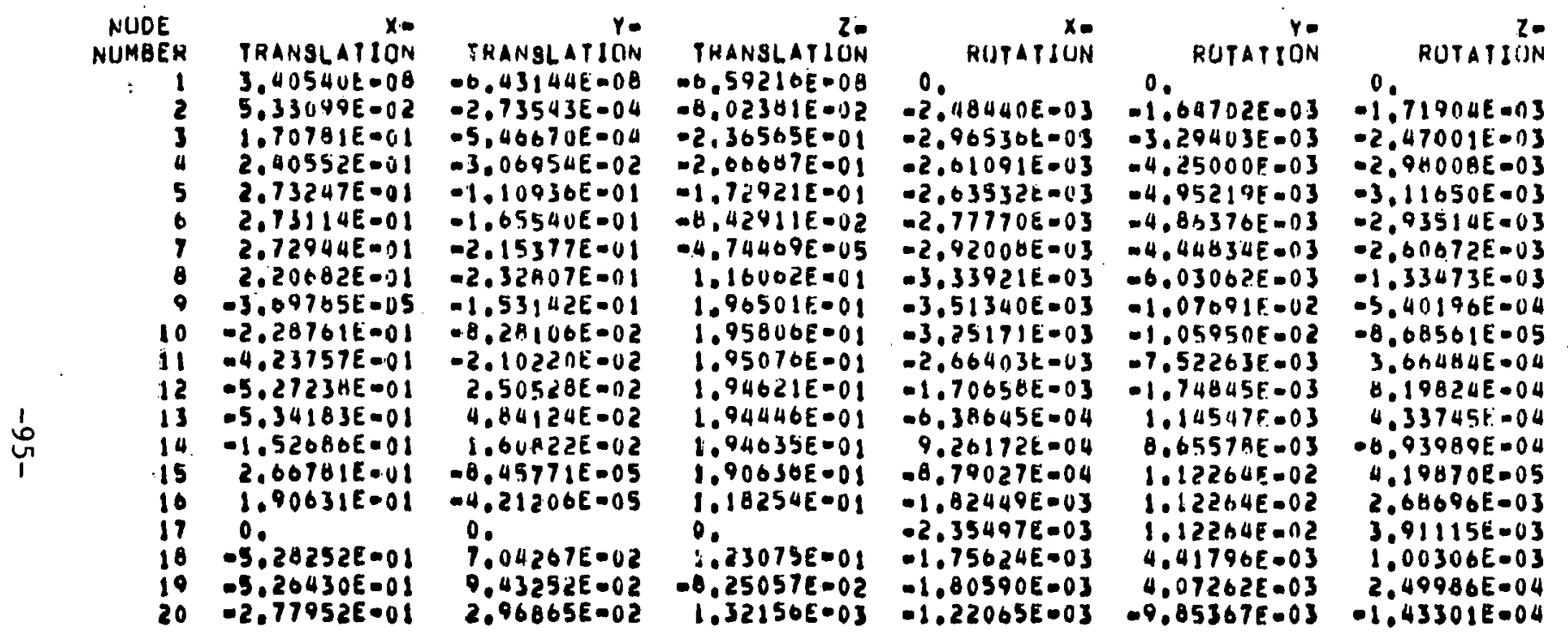


MUDE NUMBEA 3

EIGENVECTURS NURMALIZEO TO 1.000

DISPLACEMENTS/RUTATIUNS OF UNAESTRAINED NUDES

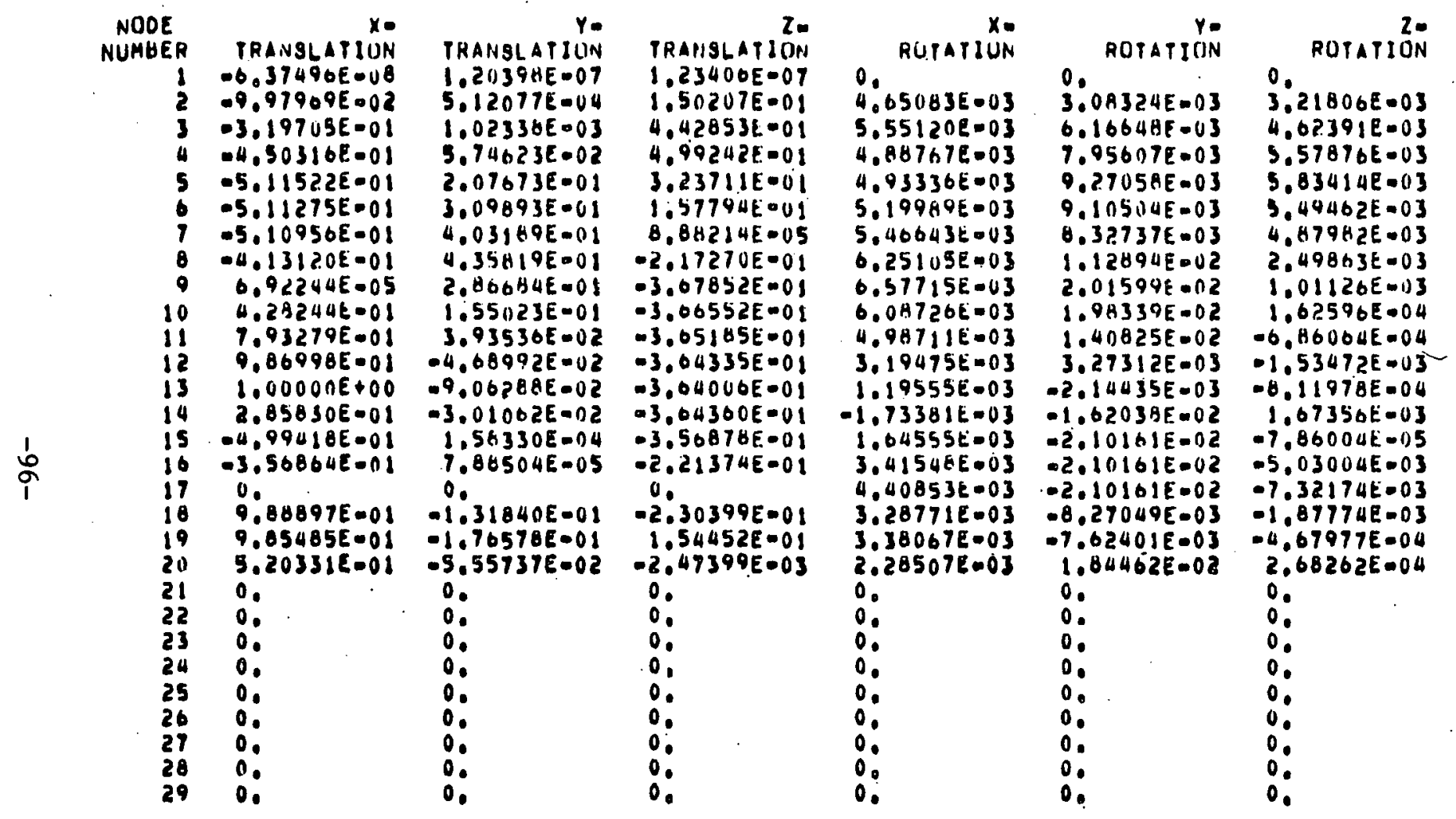


MODE NUMBEN 4

PAEQUENGY $17.1855 \quad \mathrm{HZ}$

EIGENVECTORS NOHMALIZEO TU A UNIT MASS MATHIX

DISPLACEMENTS/ROTATIUNS OF UINHESTHAINED NUDES

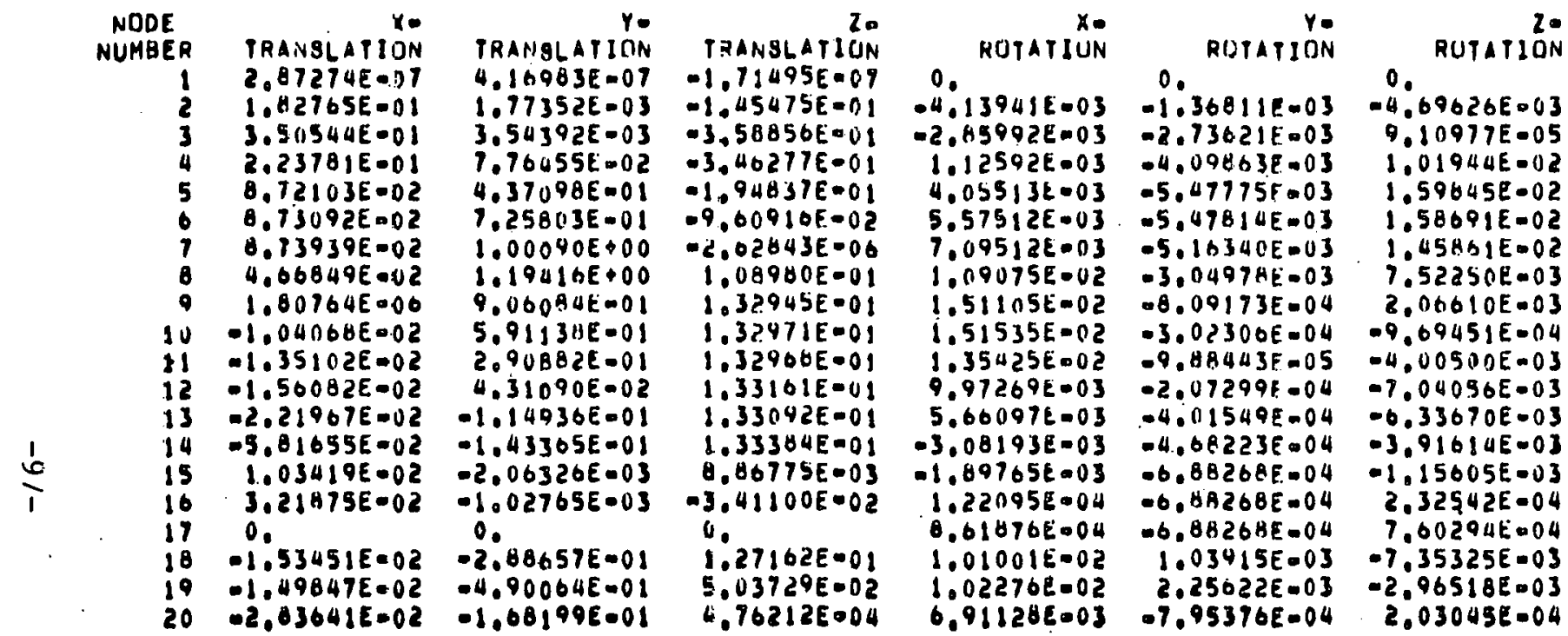


MCIOE NUMBER

EIGENVECTORS NURMALIZEO TO 1,000

DISPLACEMENTSIRUTATIONS OF UNRESTRAINED NIJOES

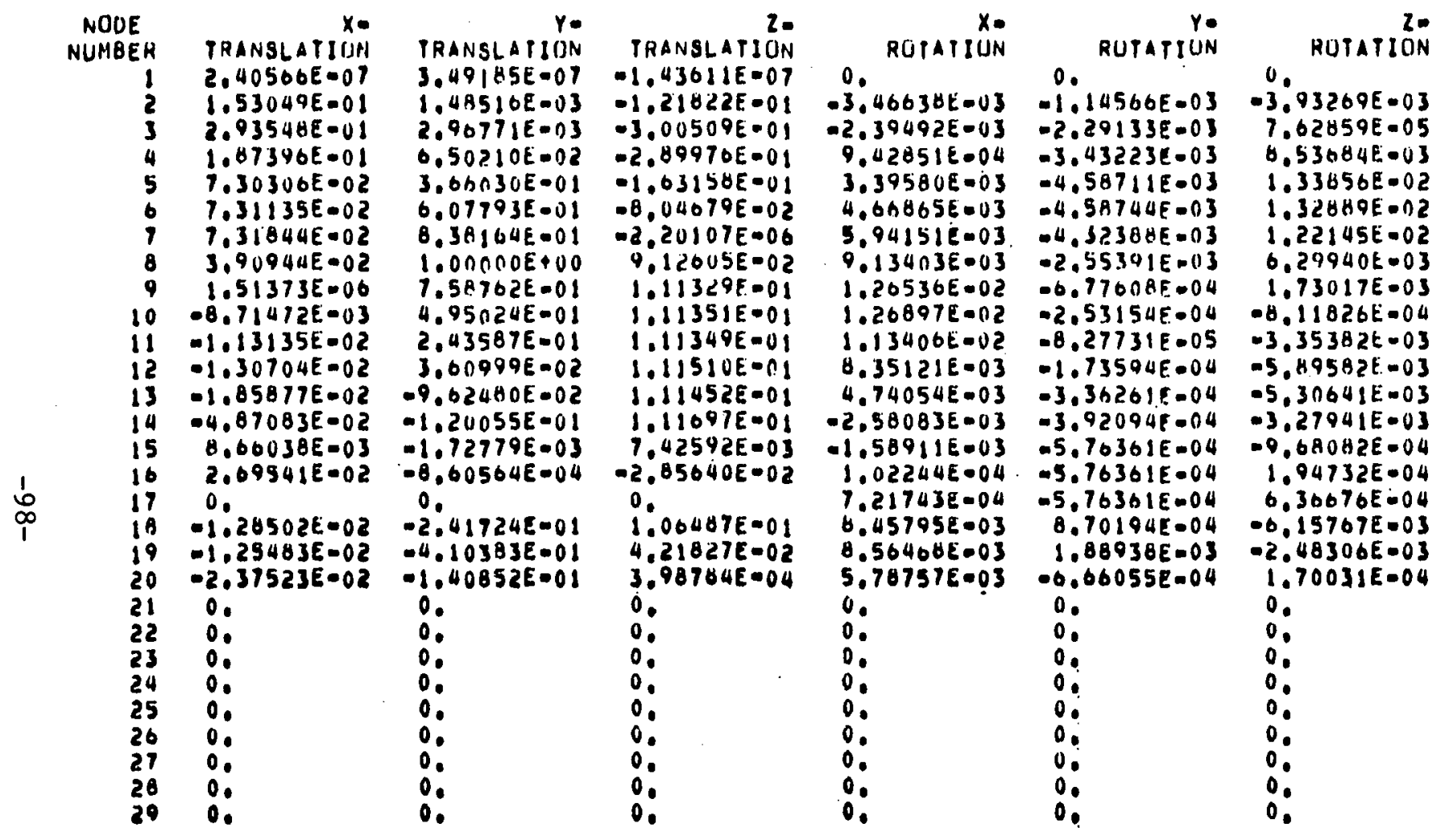


MODE NUMGER

PREQIIENCY

5

CIGENVECTORS NURMALIZEO TO L UNIT MASS MATRIX

DISPLACEMENTSIRUTATIONS OF UNRESTAAINED NODES

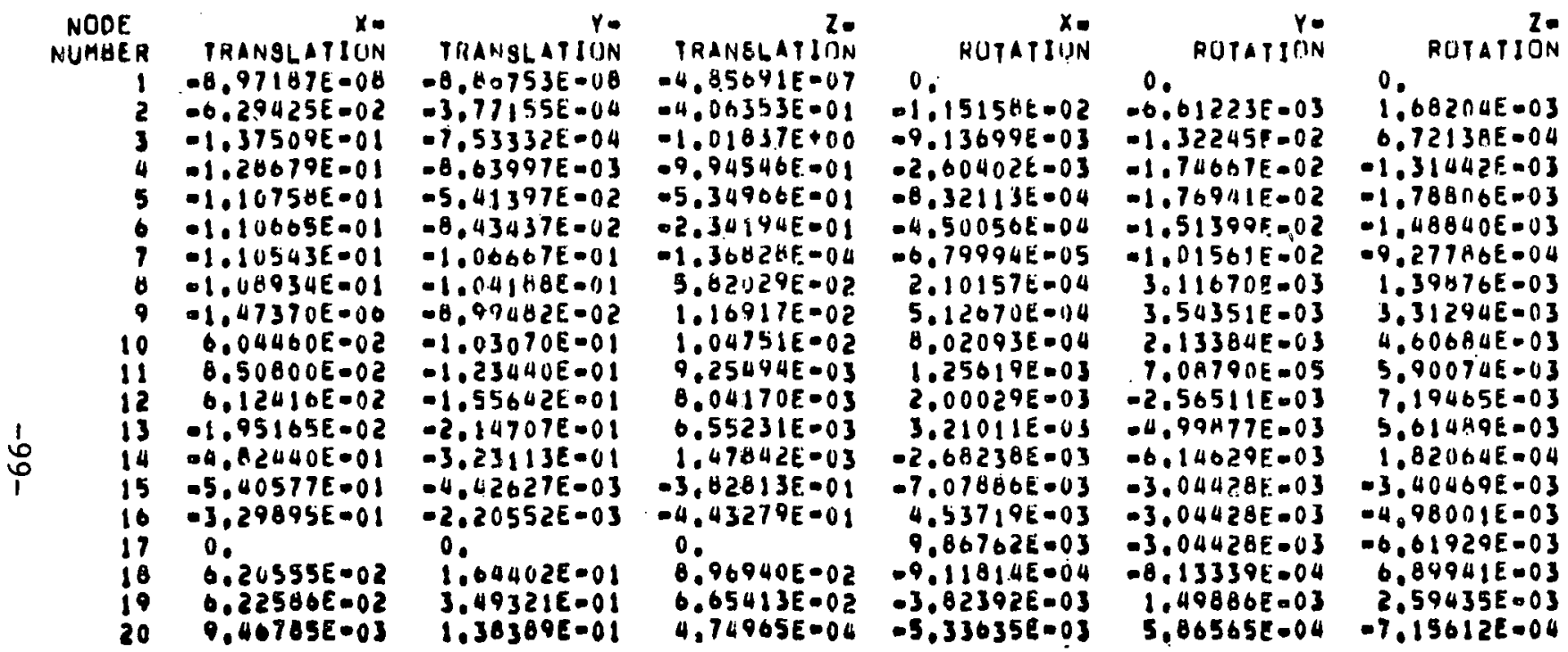


MOOE NIJMAER 5

CIGENVECTORS NORMALIZEO TU 1.000

DISPLACEMENTS/ROTATIONS OF UNRESTRAINED NUDES

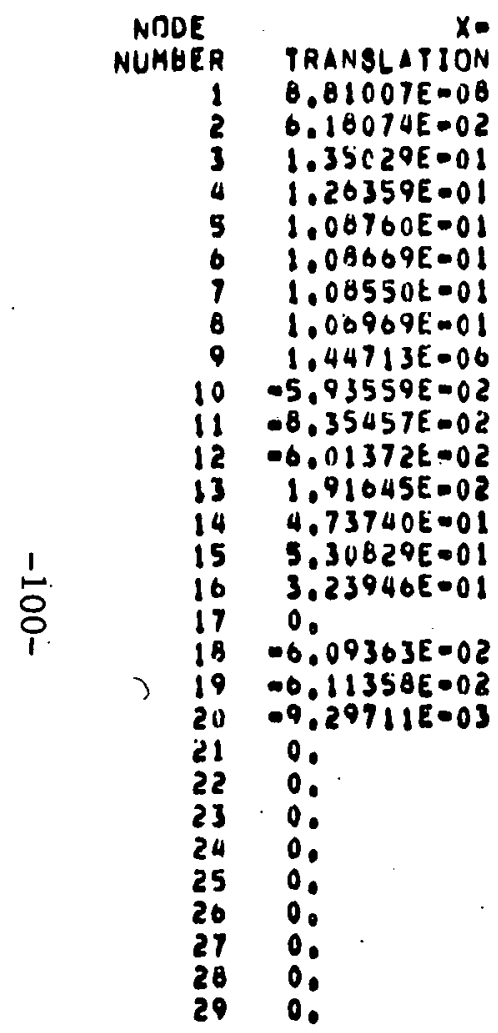

\begin{tabular}{|c|c|}
\hline 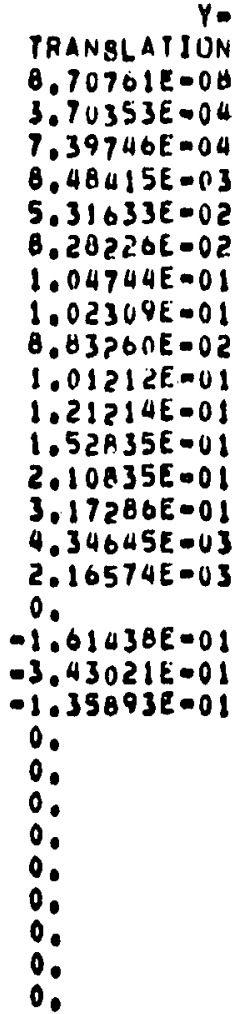 & $\begin{array}{l}-1.02862 E \\
-9.08804 E \\
-7.09607 E= \\
=0.43415 E \\
-1.45176 E= \\
3.75910 E= \\
4.35285 E= \\
0 . \\
-8.80764 E= \\
=0.53413 E= \\
=0.06390 E= \\
0 . \\
0 .\end{array}$ \\
\hline
\end{tabular}

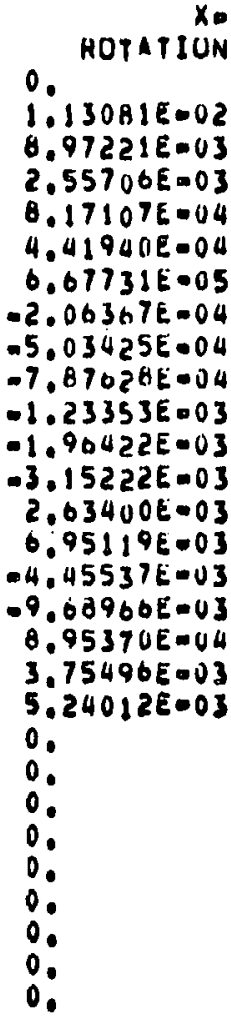

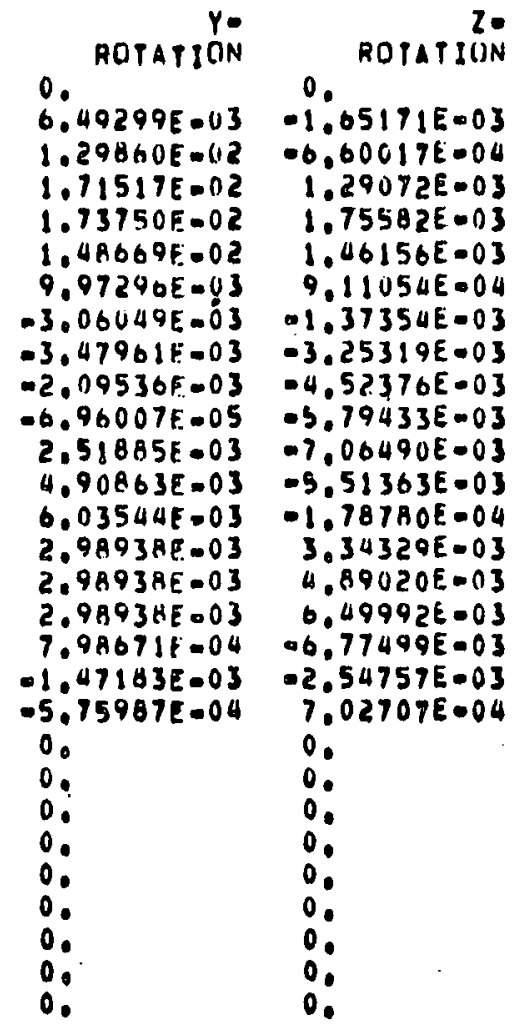


MODE NUMBER

FREOUENCY 25.0137

$\mathrm{HZ}$

ETOENVECTORS NORMALIZEO TU \& UNIT MABS MATAIX

DISPLACEMENTA/RUTATIONS OF UNRESTHAINED NODEB

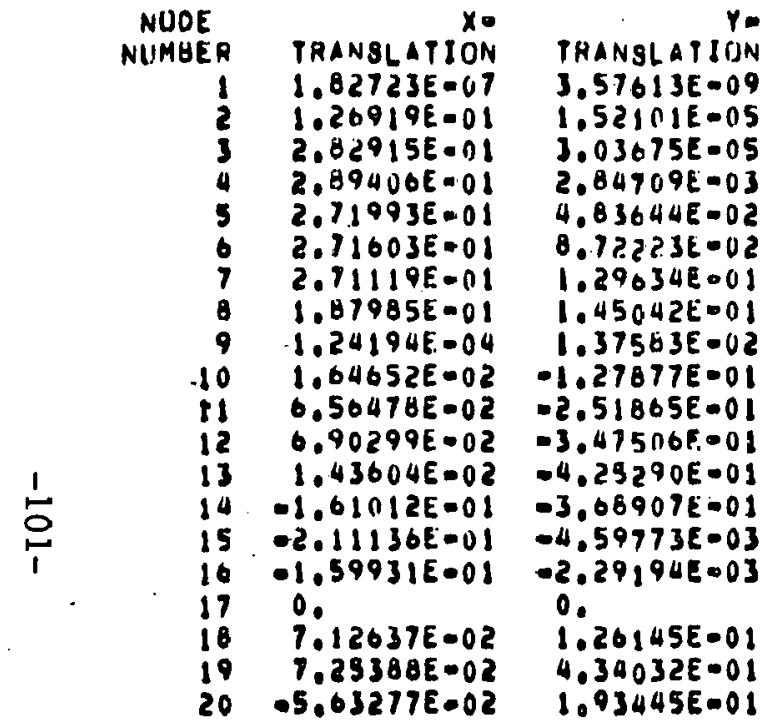

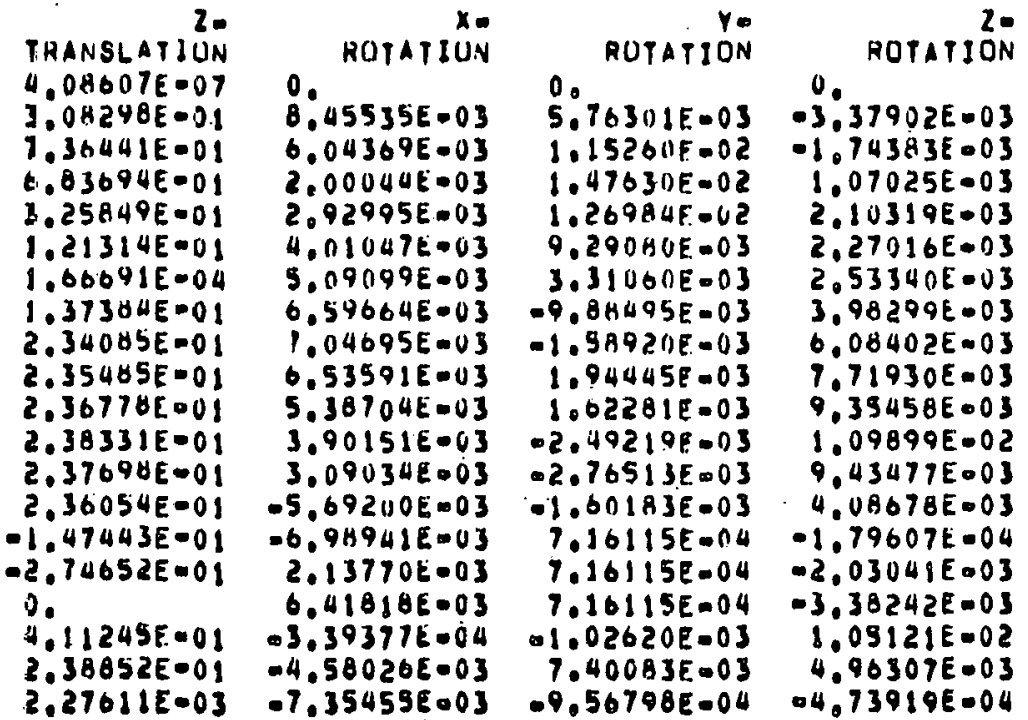


MUUE NUMBER

EIGENVECTURS NURMALIZED TO 1,000

DISPLACEMENTS/ROTATIONS OF UNREBTKAIHED NODES

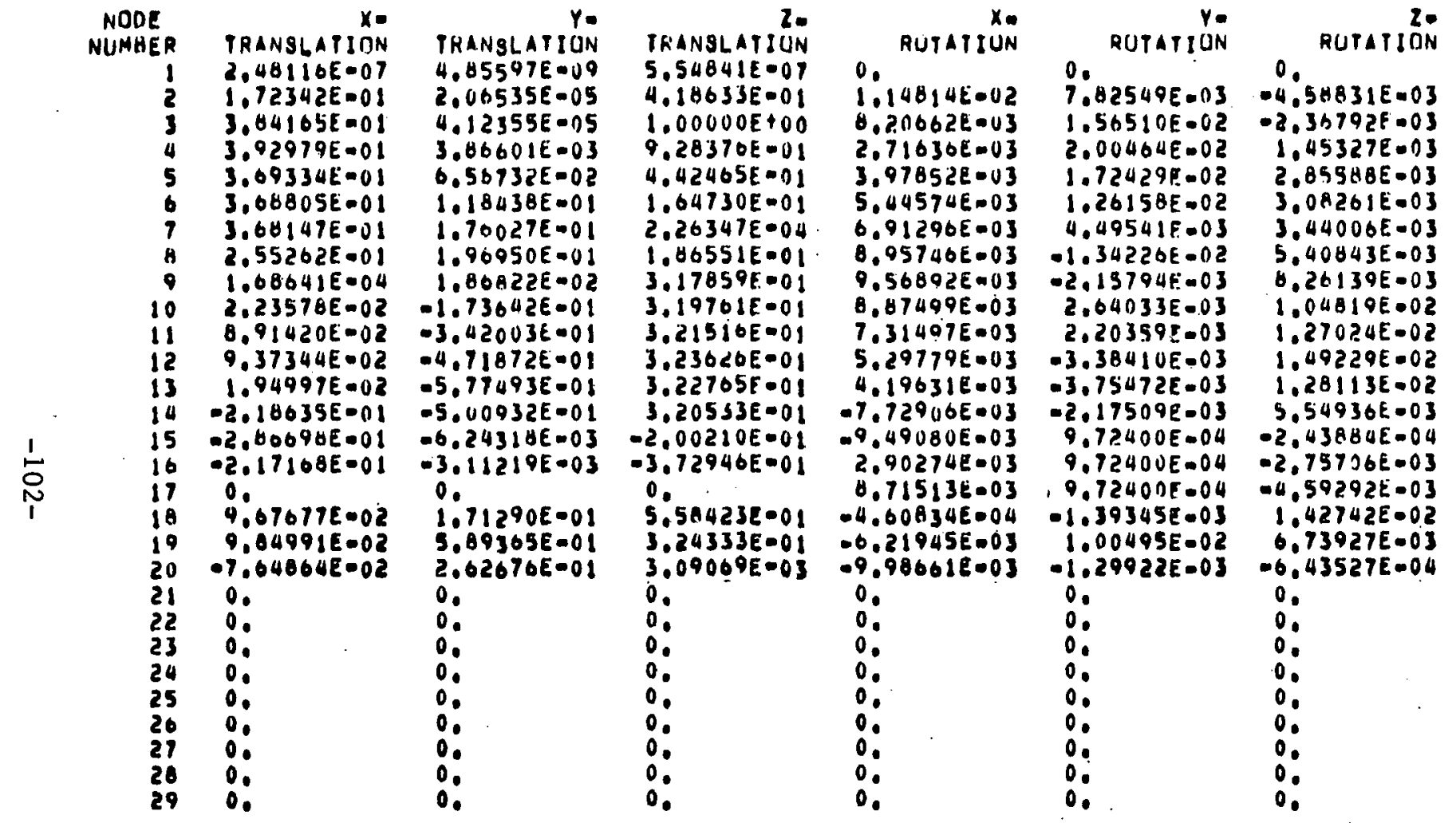


MCOE NUMGEH

PRE RUENCY

28.5917

Hz

EIGENVEGTORS NUGMALIZED PU A UNIT MASS MATHJX

DISPLACEMENTS/ZOTATIUNS DF UNRESTRAINFD NUDES

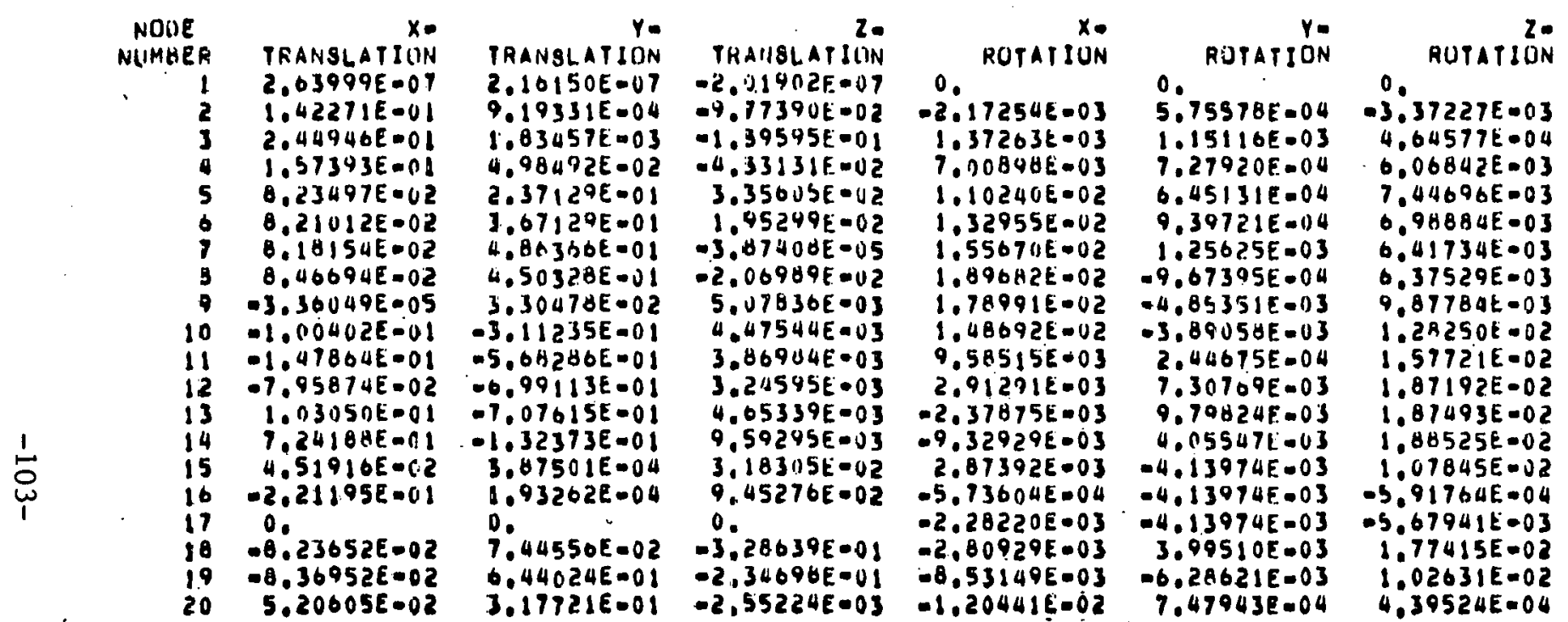


MODE NUMBER ?

EIGENVECTOR NURMALIZEO TO 1.000

OISPLACEMENTB/ROTATIUNS OF UNRESIRAINED NODES

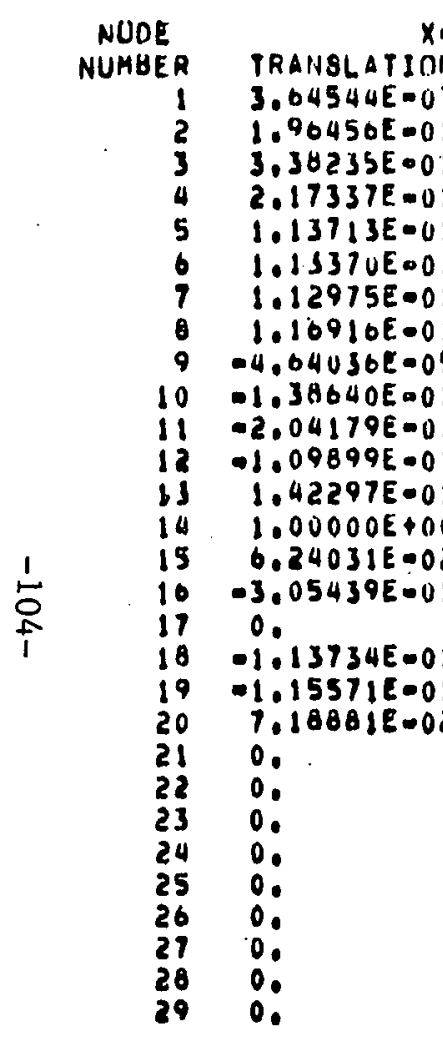

\begin{tabular}{|c|c|}
\hline 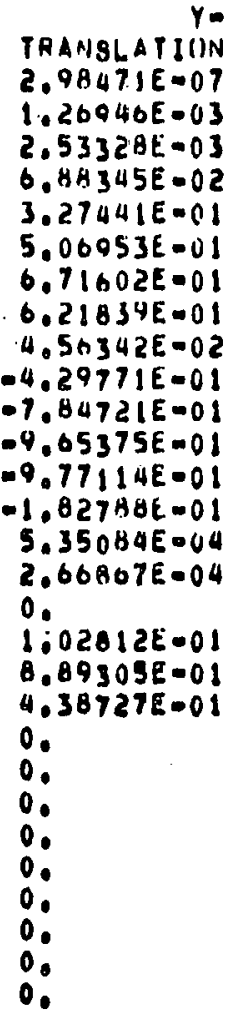 & $\begin{array}{l}-2.65822 E= \\
7.41249 E= \\
0.17944 E=1 \\
5.34370 E= \\
4.48219 E= \\
0.42566 E= \\
1.32405 E= \\
4.39533 E= \\
1.30529 E= \\
0 . \\
-4.53603 E= \\
-3.24084 E= \\
-3.52428 E= \\
0 . \\
0 . \\
0 . \\
0 . \\
0 . \\
0 . \\
0 .\end{array}$ \\
\hline
\end{tabular}

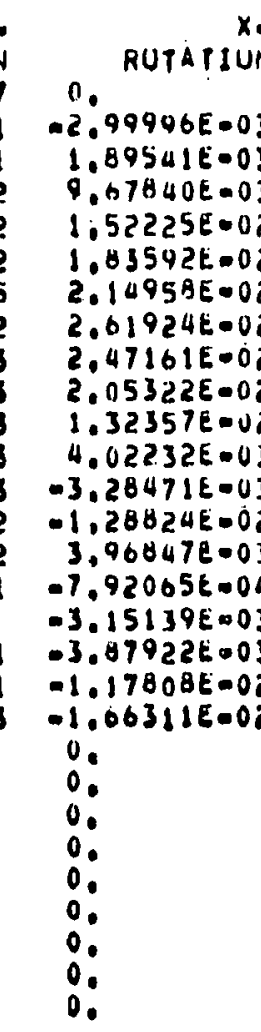

\begin{tabular}{|c|c|}
\hline RUTATIUN & RUTATION \\
\hline $\begin{array}{l}0 . \\
7.94790 E=04 \\
1.58958 E=03 \\
1.00515 E=03 \\
8.90833 F=04 \\
1.29762 E=03 \\
1.73470 E=03 \\
-1.33583 E=03 \\
-6.70200 E=03 \\
-5.37233 E=03 \\
3.37861 E=04 \\
1.00909 E=02 \\
1.39300 E=02 \\
5.00002 E=03 \\
-5.71639 E=03 \\
-5.71639 E=03 \\
-5.71639 E=03 \\
5.51666 E=03 \\
-8.08035 E=03 \\
1.03260 E=03 \\
0 . \\
0 .\end{array}$ & 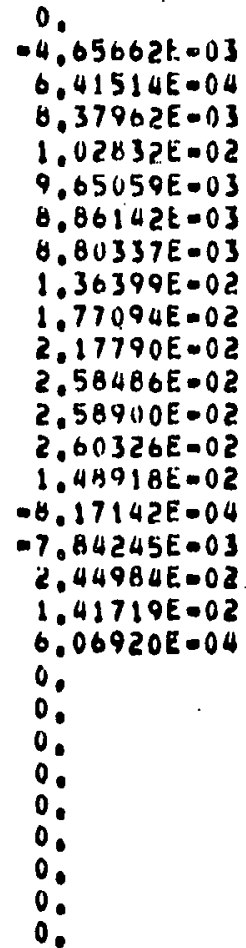 \\
\hline
\end{tabular}


MODE NUMBER

PHEQUENCY $36.4075 \quad \mathrm{HZ}$

EIGENVECTORS NURHALIZEO PO A UNIT MASS MATKIX

OISPLACEMENTS/RUTATIONS OF UNHESTRAINED NUDES

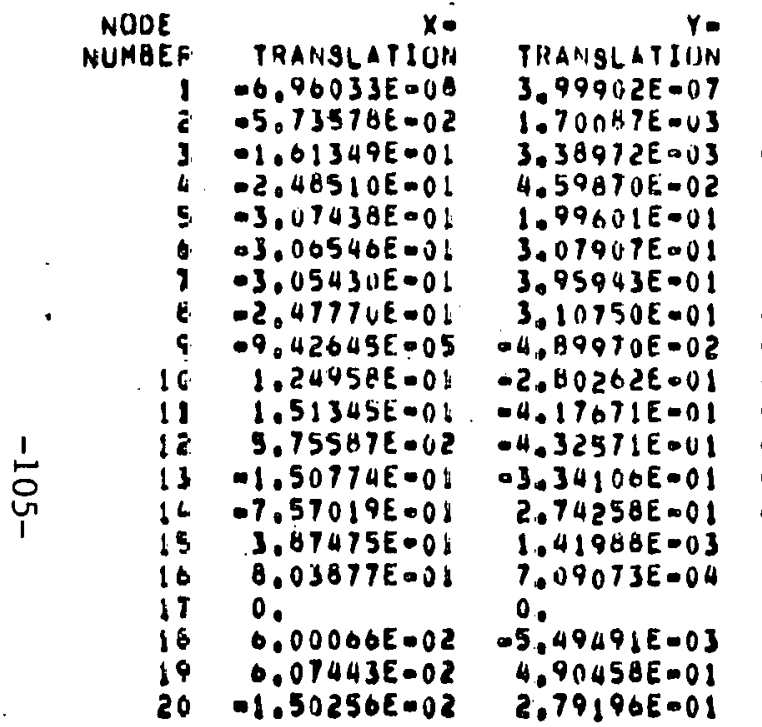

0.

0.

$-1.554026003$

$1.609158-03$

$6.14585 E=03$

$0.97925 E-03$

1.057816002

1.217702002

$1.48330 E=02$

$1.26544 E=0$

$9.161140-0$

$3.784068-03$

$-2.372064-03$

$-7.0192 \forall E=0$

$03.72904 E-03$

$1.28394 E-02$

.3 .371756003

$-1.46138 E-02$

$.5 .51182 E-03$

$.8 .65158 \mathrm{E}=\mathrm{U}$

$-1.03978 \mathrm{E}=02$
5.303655 .04

$1.06073 E=03$

$8.66205 E 004$

$1.30907 E .03$

$1.62818 E-03$

$1.98947 \varepsilon-03$

7.67 OSBE-03

$7.92733 F=03$

$3.80646 E=03$

$-1.57067 E-03$

.7 .762816 .03

- 1.130395002

$1.80779 E=04$

$1.71403 \mathrm{~F}=0 \mathrm{Z}$

$1.71403 E=02$

$1.71403 E=0$ ?

$-4.20438 E-03$

$2.01671 E .03$

$3.15736 E-05$
ROTATION 0 .

$1.61814 E-01$ $2.35995 E-03$ $4.84347 E=03$ $6.27751 E .03$ $5.53552 \mathrm{E}-0 \mathrm{~J}$ $4.24620 E-03$

$1.396501-03$

$2.77380 E-03$

$4.22942 t=03$

$5.66584 E=03$

7.14186E-03

$-1,36777 E-02$

$-1.657645=02$

$4.63015 E-03$

$1.95202 E=02$

$1.20891 E=02$

1.037702 .02

$1.71766 E=03$ 
MUDE NUMBER 6

EIGENVECTURS NOHMALIZED TO 1.000

DIBPLACEMENTS/ROTATIONS OF UNRESTRAINEO NUDES

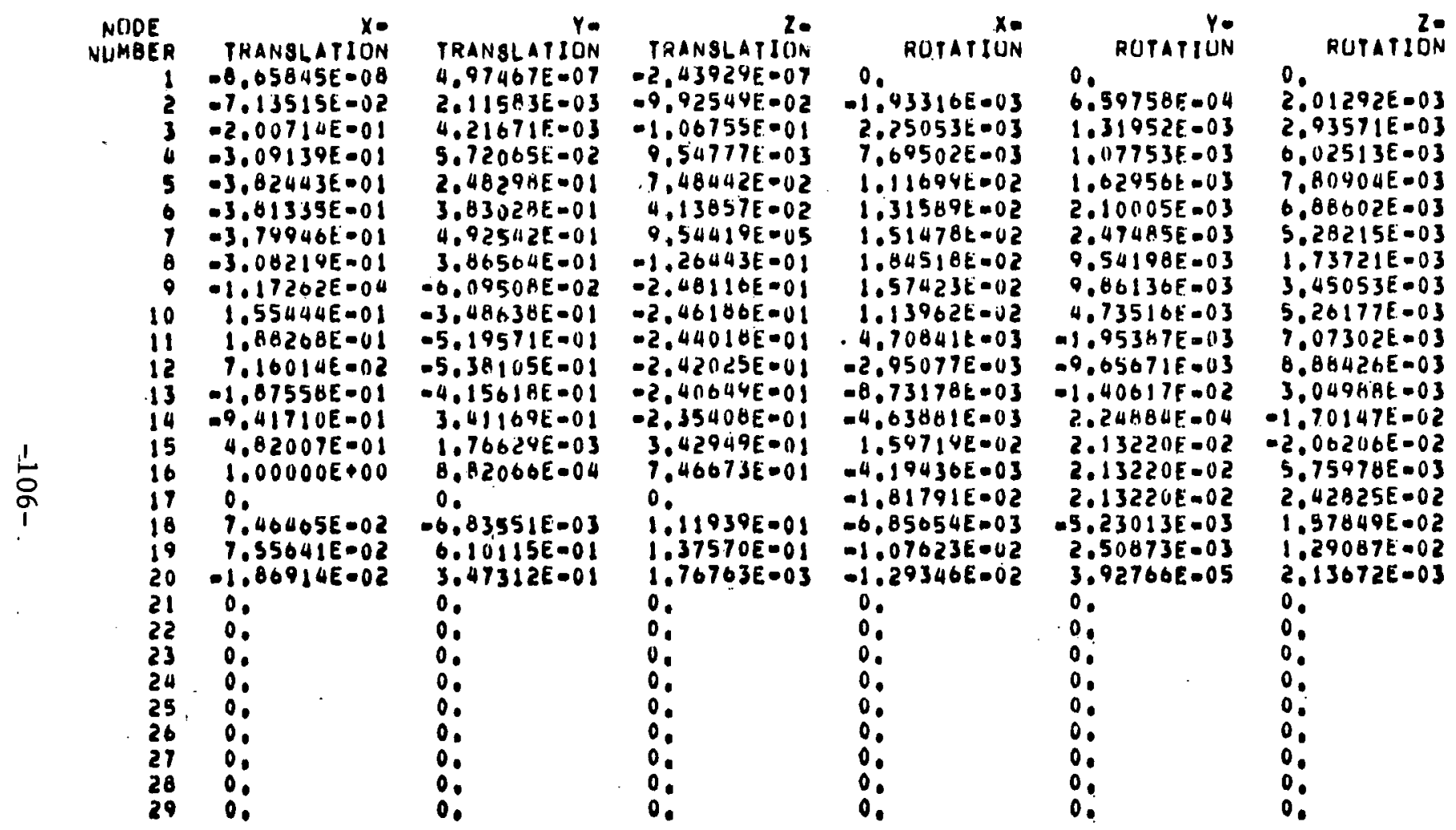


MOOE NUMBER

FREQUENCY $40.6675 \quad \mathrm{HZ}$

EIGENYECTORS NURMALIIEO TO A UNIT MA88 MATKIX

OISPLACEHEVTS/RUTATIGINS OF UNRESIRAINEO NOUES

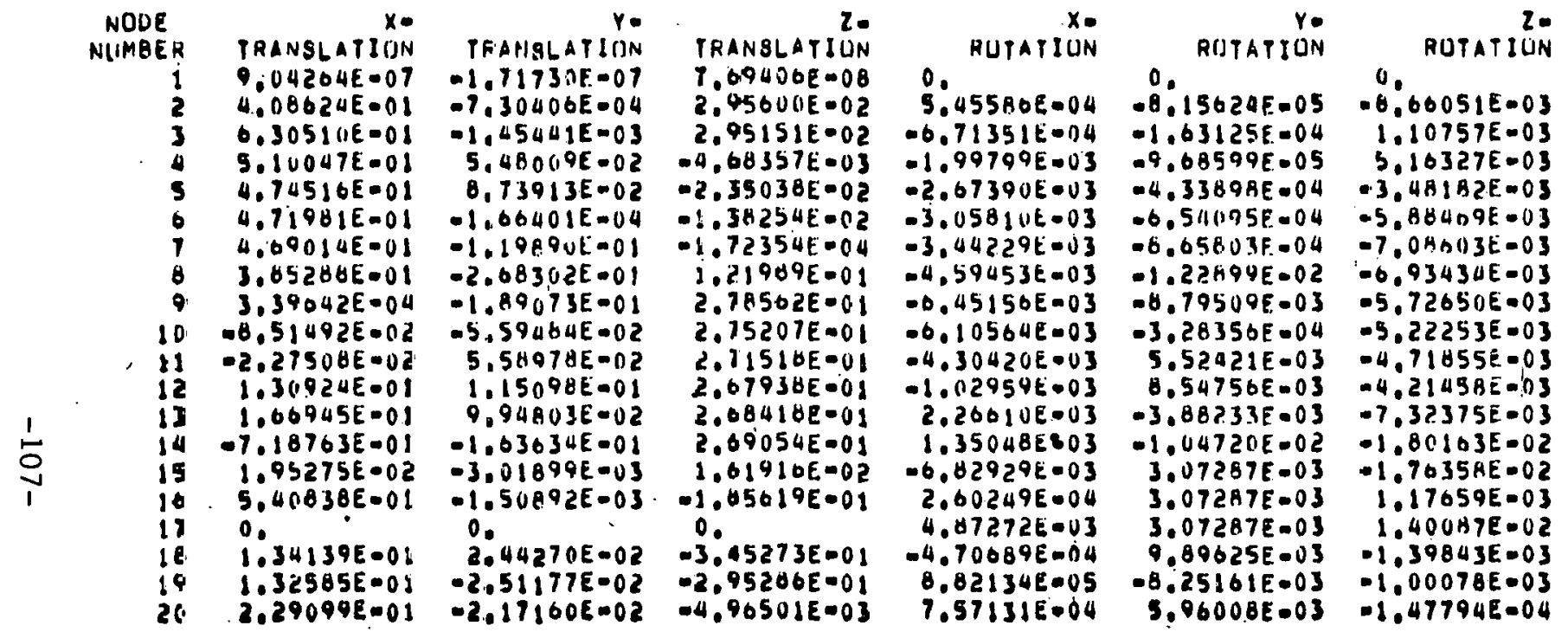


MODE NUMBER 9

CIGENVECTORB NURMALIZEO TO 1,000

DISPLACEMENTS/RUTATIONS DF UNRESTRAINED NODES

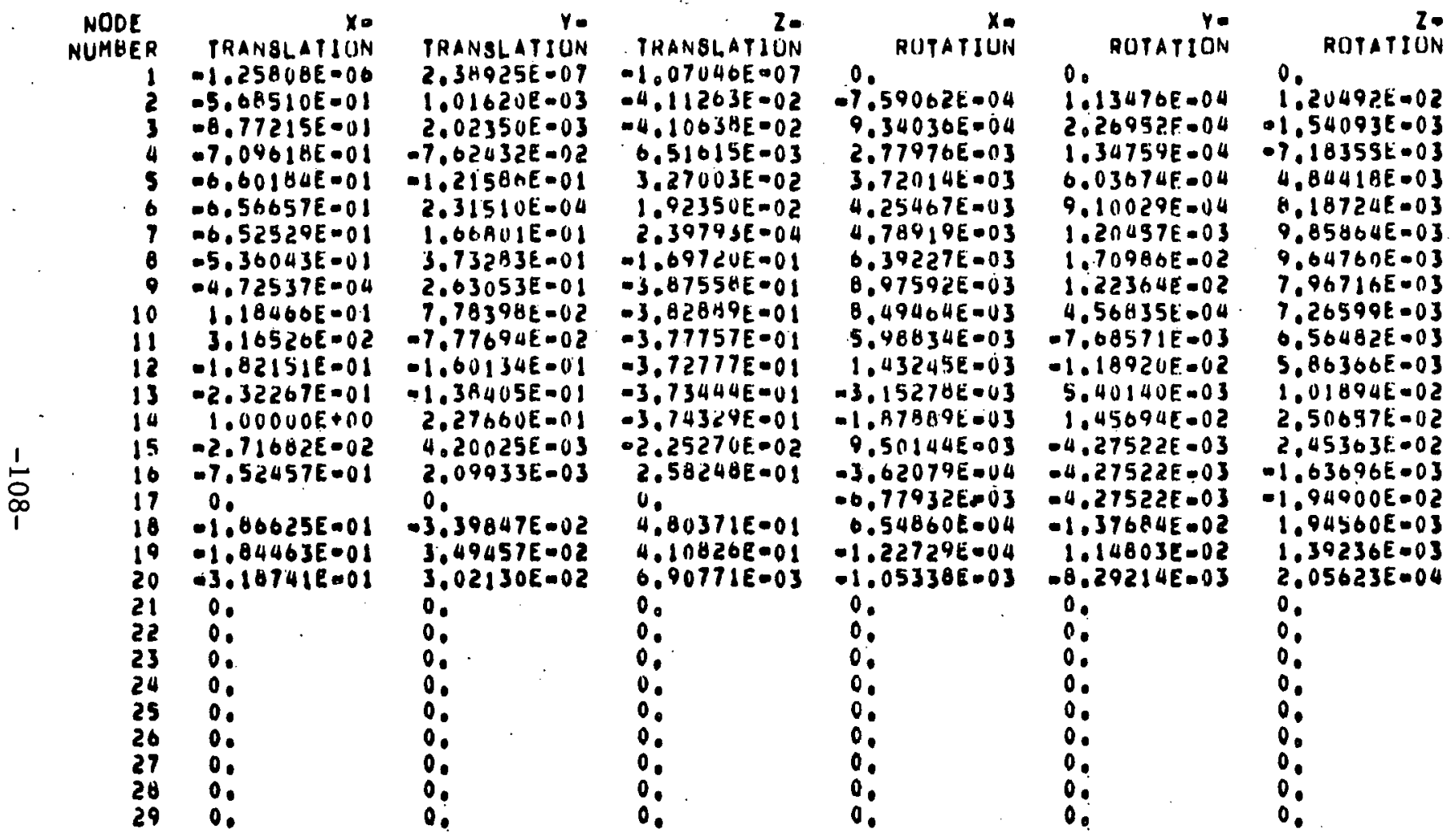


MODE AUMBER 10

PREDUENCY U8,7209

18

EIGENVECTORS NURMALIZEO TO A UNIT MABS HATHIX

UISPLACEMENTS/RUTATIONS OF UNAESTRAINED NUDES

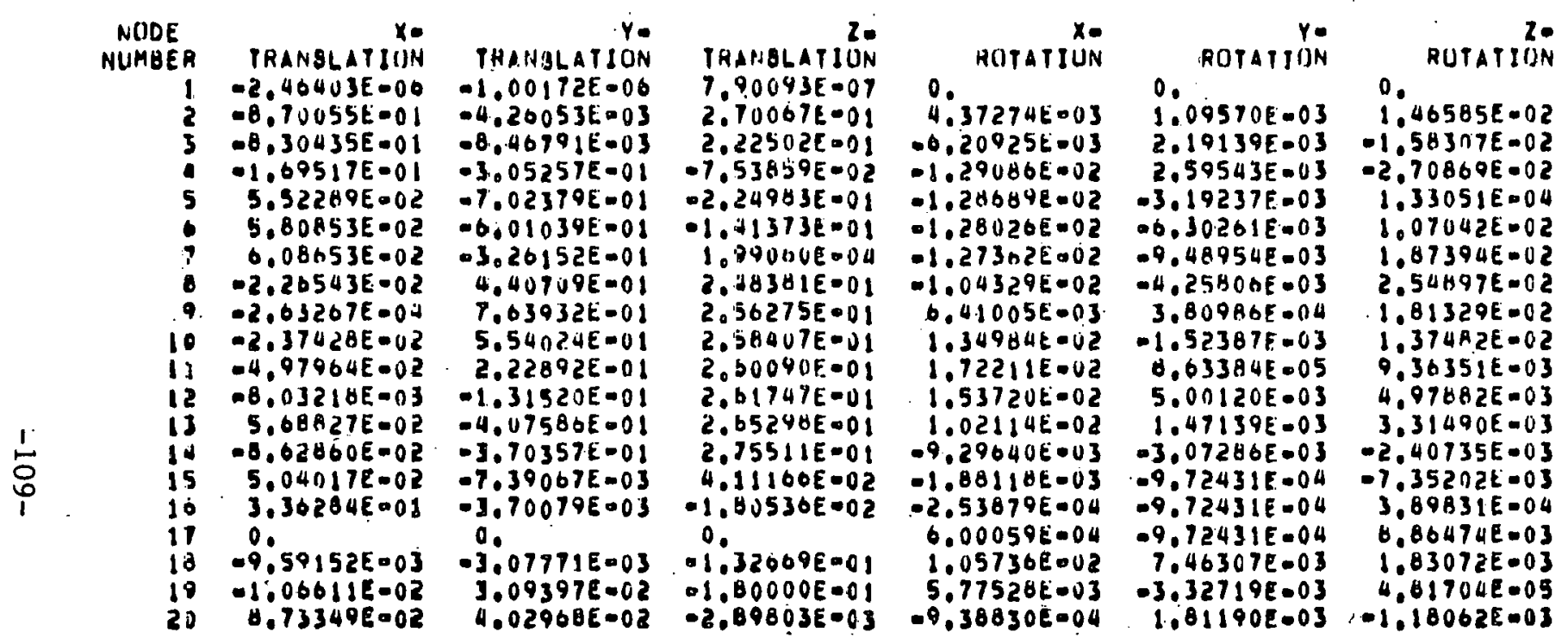


MUDE NUMGER 10

EJGENVECTURS NIJAMALIZED TO 1.000

- DIBPLACEMENTS/RUTATIONS OF UNRESTRAINED NODES

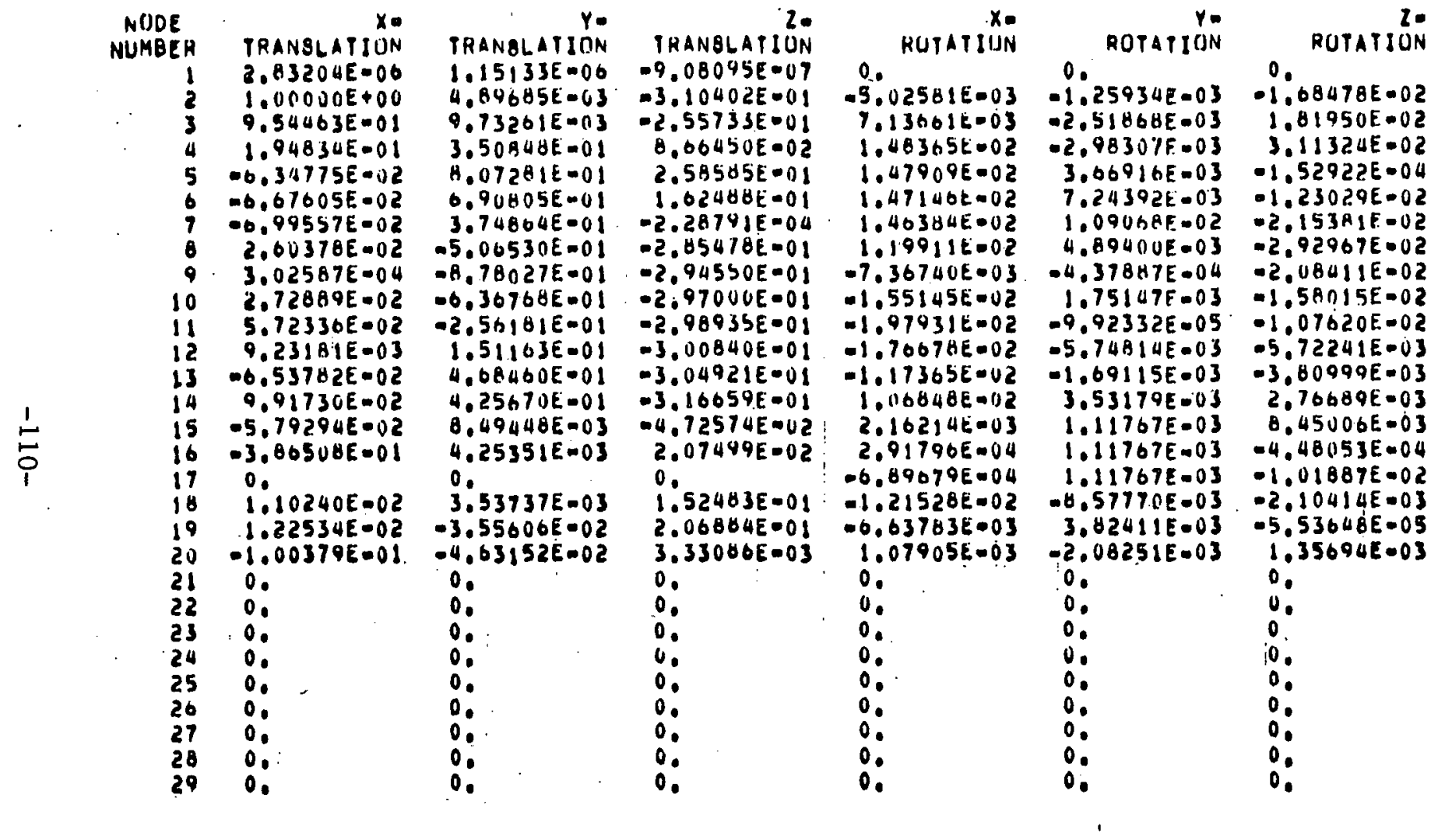

E I GENBOLU.TION TINE LOG

EIGENSULUTIUN: 1.12
PRINTINO 
utuntut

CTION FACTUIRS
$x=1 .(10) 0$
$.13 \operatorname{sen} 7$
2.1 .0000

INDICATUH FIJK UISPLACEHENT IJA ACCELERATICN HPEGTHUM :

EO.U IISPLLCEMENT

EO.I ACCELEIIATION LIA IN. J3EC.S

EO.Z ACCELERATLUIN : A GS

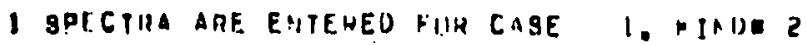

CLUSTEK FACTUR, CF. InIIJU

$\stackrel{5}{\varpi}$ 
MUIIAL. PARTICIPATICN FACPUISY

\begin{tabular}{|c|c|c|c|}
\hline MODE & $x=11$ IRt.CIIIJN & $Y=1) I I E C I I O U$ & $Z-1)[$ HEC $[(1)]$, \\
\hline 1 & .1 IIIE + UI & $-11024 t=01$ & $.0734 E+U 0$ \\
\hline 2 & $-.25245 .+1010$ &.$-|P .12 E+11|$ & $.44222=01$ \\
\hline 3 & $-.1427 E+01$ & $.2045 E+(1: 3$ & $.2254 E+U 0$ \\
\hline 4 & $-, 2,13 F .011)$ & $. .2250 E+U .1$ & $-.500 .5 t-v 1$ \\
\hline 5 & $\because .1105 ? .5 .0 \mathrm{~V}$ & -.27 ADE $\triangle 11$ & $\left.-.7775 E+U^{\prime}\right)$ \\
\hline - & $0.131+E$ W110 & $.9327 E-111$ & $-.1337 E+111$ \\
\hline 7 & $0.22548+110$ & $.23 \operatorname{nn} 3 E+11: 1$ & $.0093 E+100$ \\
\hline H & $.110(t+111)$ & $.1737 E=11 ?$ & $.1192 E+00$ \\
\hline$?$ & $-.4223 E$ +UII & - INOIE-nI & $.4207 E+u \|$ \\
\hline 10 & $=04552.2+111)$ & -.21 HHE $\triangle 0 \mathrm{~J}$ & $.1001 E+011$ \\
\hline
\end{tabular}

SPECTHIII" TAISLE (IMPERIAL VALLEY F. H. ELCENTRIS 1941 SL

\begin{tabular}{|c|c|c|}
\hline & & SHECTHIM \\
\hline & PEHTUL. & VALIIE \\
\hline & $.102 \mathrm{KF}-112 \mathrm{Z}$ & $.1450 E+13$ \\
\hline & . $2 H$ BILL-II) & - SUUUEL +1) 3 \\
\hline & - उHIDE=:1 & $5,116+.13$ \\
\hline & $.71111 E_{-}-111$ & $.77508+0.3$ \\
\hline & . TIUUE-U1 & 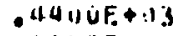 \\
\hline & $.11+11[+11)$ & $0115 \theta F_{-}+1 / 4$ \\
\hline & - IOIOELQU0 & $.1148 C+114$ \\
\hline & $.1720 F_{0}+1110$ & $.7190[+1 / 3$ \\
\hline & $.2\left(1,11 D_{0}+11 v\right.$ & .371 Uf +13 \\
\hline & $\begin{array}{l}-25.10 E+00 \\
32310 E+U 11\end{array}$ & 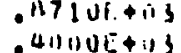 \\
\hline
\end{tabular}

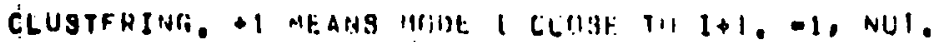
1. - $1,-1,-1,-1,-1,-1,1,-1,1$. 


\begin{tabular}{|c|c|c|c|c|c|c|c|}
\hline 1 & POTAL.. & $2.00177 F-11 \mathrm{H}$ & $1.5 \Delta U \| 2 E=U H$ & $4.03522 \mathrm{E}-1, \mathrm{H}$ & 0. & 0. & 0. \\
\hline$?$ & TIJTAL... & $2.74424 E-U E$ & $n .567117 E-n 5$ & $0.54521 t=02$ & $2.10534 E=03$ & $1.2001 A E-113$ & B. $74451 E=04$ \\
\hline 3 & THTAL.. & B. $47772 E=112$ & $1.312 .46 t-114$ & $2.03504 k=111$ & $2.71020 E-.03$ & $2.411035 E-03$ & 1.157 HE -03 \\
\hline 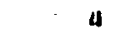 & TI:PAL.. & 1.1372AE-C: & $1.20359 E-112$ & $2.3949 .16-31$ & $2.371144 E=03$ & $3.171975-43$ & $1.22 B 19 E-03$ \\
\hline 5 & THPAL... & $1.25322 E=01$ & $11.111,901) E-(12$ & $1.0040 \mathrm{HE}=11$ & $1.94 M O 4 E-0 S$ & $4.31735 E-03$ & $1.24172 E=03$ \\
\hline$"$ & InTAL.. & $1.25311 E-01$ & $1 . .59707 E=02$ & $9.62+22 t=112$ & $1.79118 B E=03$ & $0.66 .347 E-133$ & $1.20837 E=03$ \\
\hline 7 & TDPAL... & $1.25294 t-1) 1$ & A.BSBIDE-OL & $1.4549 A E=05$ & $1.03010 E=03$ & 4.993 DEEDUS & $1.10503 E-03$ \\
\hline A. & TnTAL.. & $7.40440 E-172$ & T. HADSAE- 02 & $1.24572 E-111$ & $1.42107 \varepsilon-03$ & $0.37353 E=03$ & $9.52113 E=114$ \\
\hline 9 & TIITAL... & $1.1353 .1 t=115$ & $7.4237146-132$ & $1.60269 t-111$ & $1.095 H 1 E-133$ & 1. . . OOUIE-03 & B. $.22116 E-04$ \\
\hline 1) & TOTAL.. & 2. ค९४।मE = 112 & 10.263597 .112 & $1,36533 t-111$ & $9.251194 E=014$ & $1.36044 E-03$ & B. $76 \geq 65 E=114$ \\
\hline 11 & TJTAL.. & $5.4147 .35-0.2$ & $5.1322 \cup E-112$ & $1.00100 E-111$ & $6.40 \times 9 \mathrm{bE}=0 \mathrm{~A}$ & $1.44345 E-03$ & $0.06201 k=04$ \\
\hline 12 & PIITAL... & 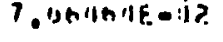 & $1.50 \cap 7$ BE -112 & $1.30487 t-111$ & $4.42+5 \forall E-1) 4$ & $1.510103 E=1) 3$ & $9.68045 E-114$ \\
\hline 13 & TOTAL.. & 9.4 ain $^{\prime} \mathrm{F}-1: ?$ & $1.10 ? .1416=02$ & $1.07132 t=01$ & $4.55129 \mathrm{E}=114$ & $1.84177 E-03$ & $9.502 .20 t=04$ \\
\hline 14. & Matal.. & $2.2+13, E=-11$ & 2. $154211 E-112$ & $1.07543 t-i 11$ & $4.41202 t-011$ & $2,35 \cdot 172 E-43$ & $1.34465 E=03$ \\
\hline 1.5 & TUTAL.. & 2.3550 . 01 & $14.2^{411716-114}$ & $1.6 \mathrm{H}: 1 \mathrm{CAt}=111$ & $7.204645-104$ & $2.79732 E-103$ & $1.901195 E=03$ \\
\hline in & IUTALE. & $1.25643 E=.11$ & $2.11373 t=114$ & 1.1158 $72 k-111$ & $1.65 .592 E-03$ & $2.20732 .2 E=113$ & $2.19279 E=013$ \\
\hline 17 & $T U T A L \ldots$ & 11. & 1. & 11. & 2.1 UR OHE-0S & $2 .>9732 . t-113$ & $2.39 n 113 k=113$ \\
\hline IA & . ICITAL... & $7.114150 \mathrm{~F}=11 ?$ & $7.117 n+2 F-0 द$ & $1.11 .073 t-01$ & 4.755516 .014 & $1.5 H 1159 E-113$ & $5.551 \mathrm{~A} 2 \mathrm{E}-\mathrm{U} 4$ \\
\hline in & RDPAL.. & 7.0645 ItE $=11$ ? & $0.17353 \mathrm{E}=11 \mathrm{c}$ & $10.162115 t-11 ?$ & B. 11452 IE- $=(1)$ & $1 . n ? \ln 3 E=1,3$ & $2.991) 37 t=(11)$ \\
\hline
\end{tabular}




\begin{tabular}{|c|c|c|c|c|c|c|}
\hline zO PUTAL... & $3,53321 F=132$ & ?. 35 म4.11F $=02$ & $.20155242 t .-114$ & $0.291007 E=-114$ & 1.2 HSUAE $=03$ & $3.881165 E=04$ \\
\hline I Tutal.. & $0 . \cdots$ & $"$ & ". & o. & o. . & n. \\
\hline PP TUTAL.. & ". & ". & $n$. & 0. & 0. & 0. \\
\hline 2.3 rintal.. & ". & \% & $n$. & 0. & 0. & 0. \\
\hline 2" PIITAL.. & ". & ". & 1. & ग. & 0. & 0. \\
\hline QT MTAL.. & i. & ". & ". & i. & ". & 0. \\
\hline 2" TUTAL.. & $\therefore$ & $\because$. & 0 & 0. & ". & $n$ \\
\hline 27 TITAL.. & $\because$ & י. & ". & 0. & 0. & 0. \\
\hline 2a TIITAL.. & ". & a. & 0. & 0. & 0. & 0. \\
\hline 20 PUTAL.. & "• & י. & ". & 0. & $n$. & 0. \\
\hline
\end{tabular}




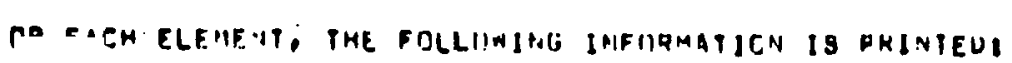

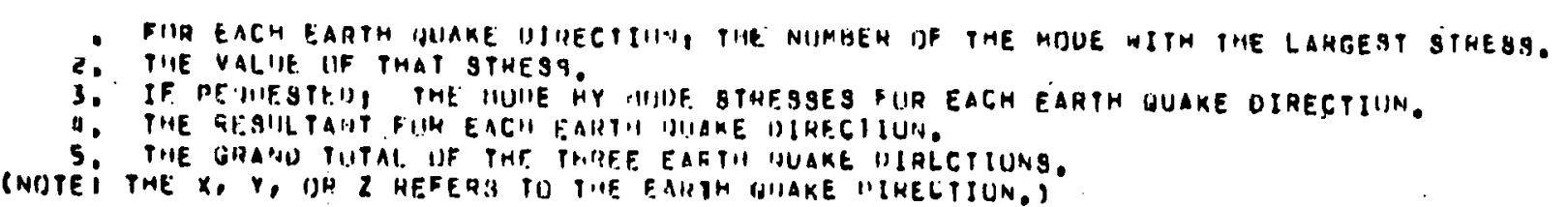

ELEME'IT IYPE SH OUN N II A Y

1,1, element number 6

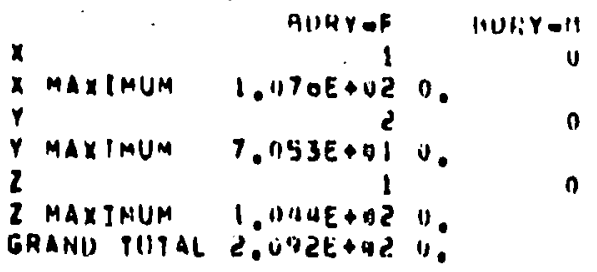

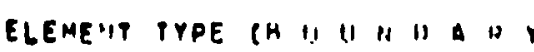

1,11 element nUMHER 1 a)

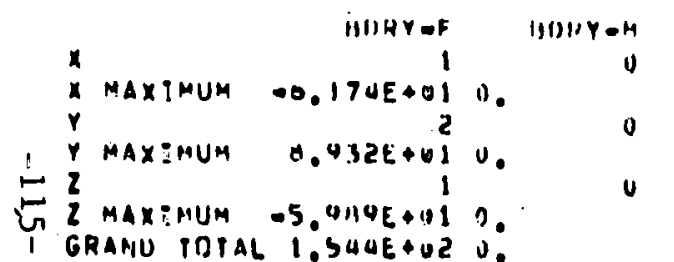

ELEMFPI PYPE (H II U N U) A H Y

, 1, ELEMFN NUMHER ( 3)

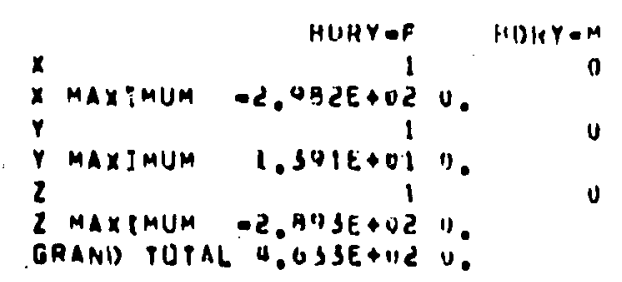

ELEMEIIT TYOE (H U U N D A $K$ Y

, 1, P.LENENT MHMHER (4)

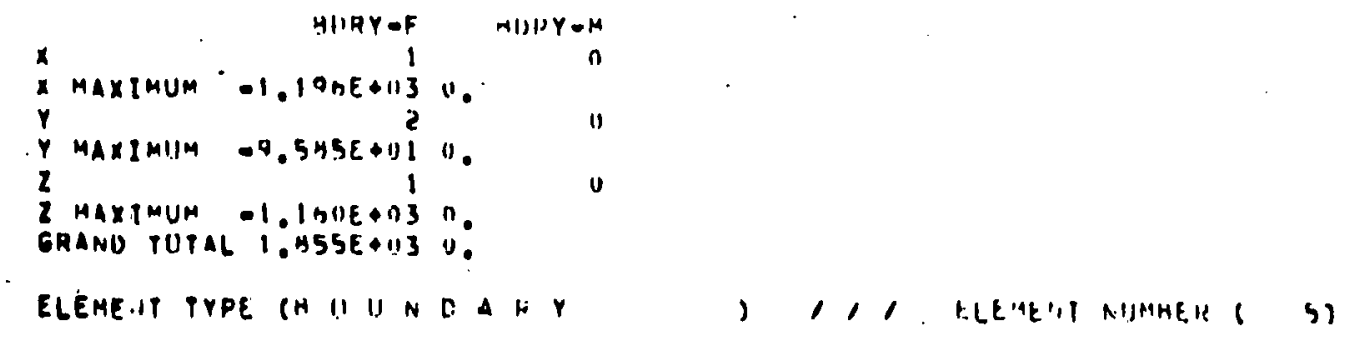

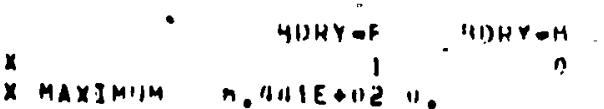




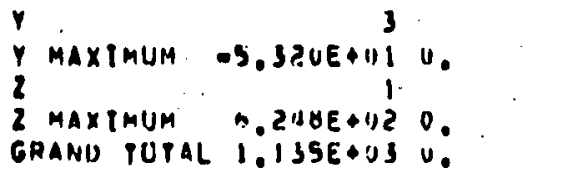

ELEMEII TYPF. CH O II N

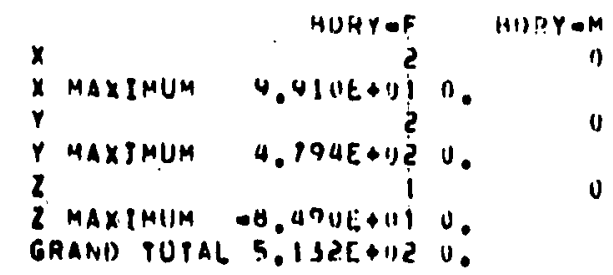

Element PrPe (H U IJ N D A K Y

) , , ELEMEMT NUMHEH ( 1)

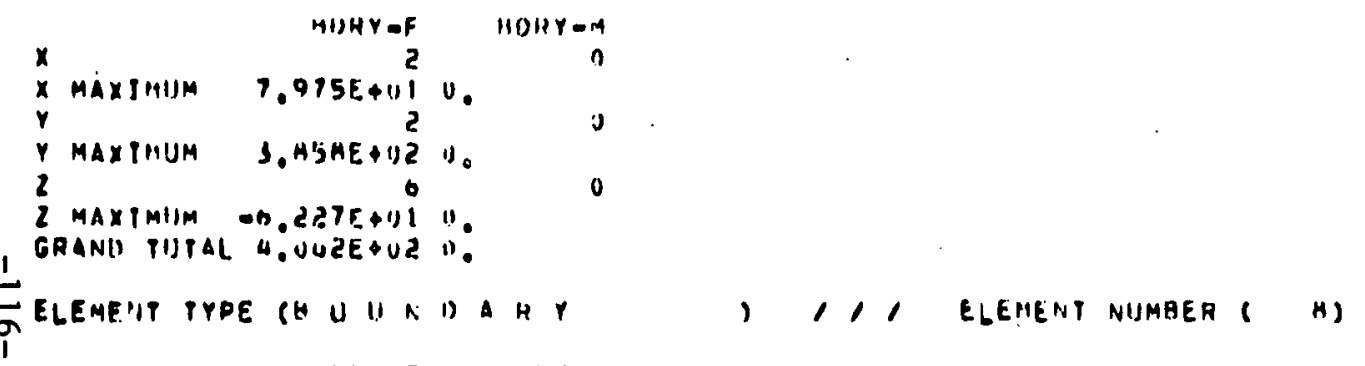

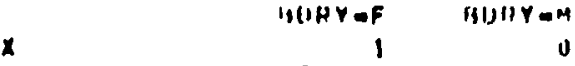

M MAXIMIJM -5.157E*1) $U_{0}$ j j

4 maximum $-1.001 E+112^{2} u$.

2 MAXI14UM $-5,003 E+11231$

GRANI) TOIAL $0.01,4 E+15 \mathrm{~V}$,

ELEME.19 TYOE (3/T) D I P E

1,1, ELEHH'I NUHHEH (1)

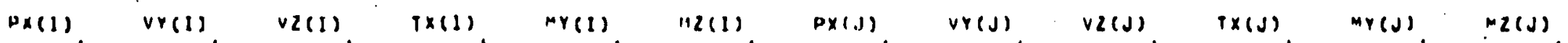

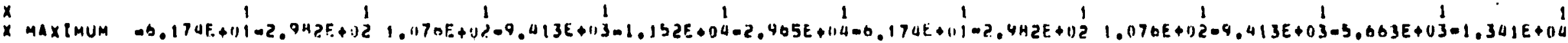

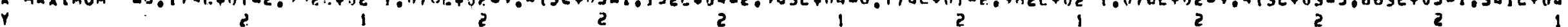

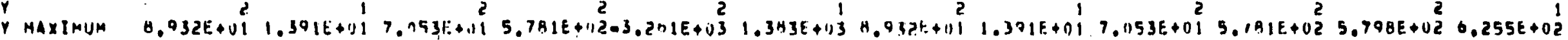

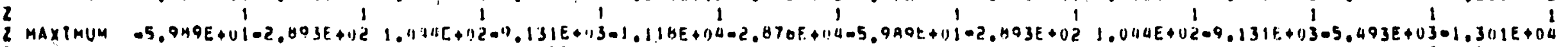

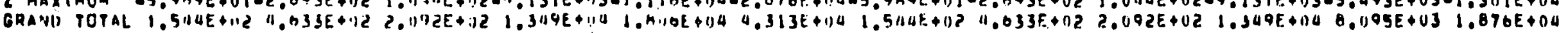

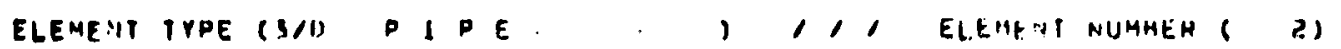

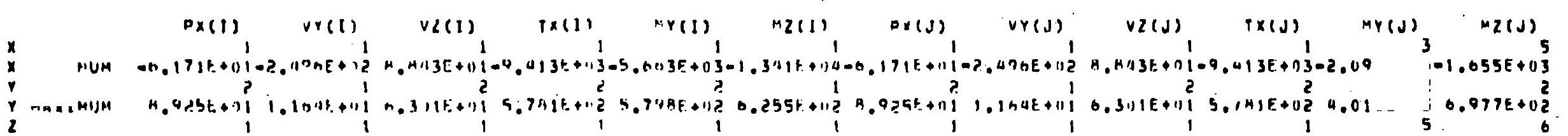




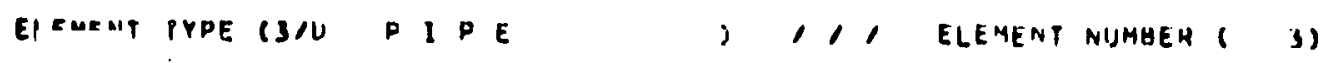

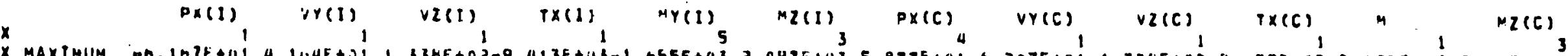

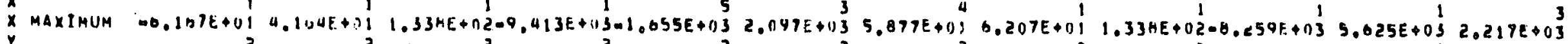

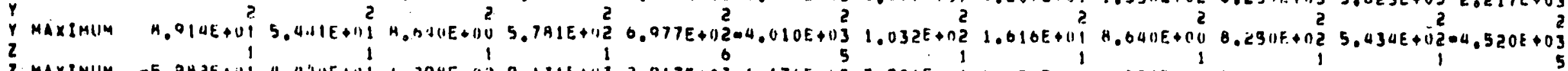

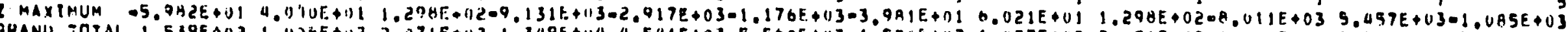

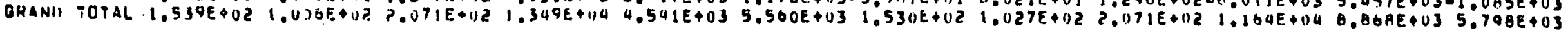

ELEMEIIT: TYPE (3/0 DI P E ; 11, ELEMENT NUMHER ( 3)

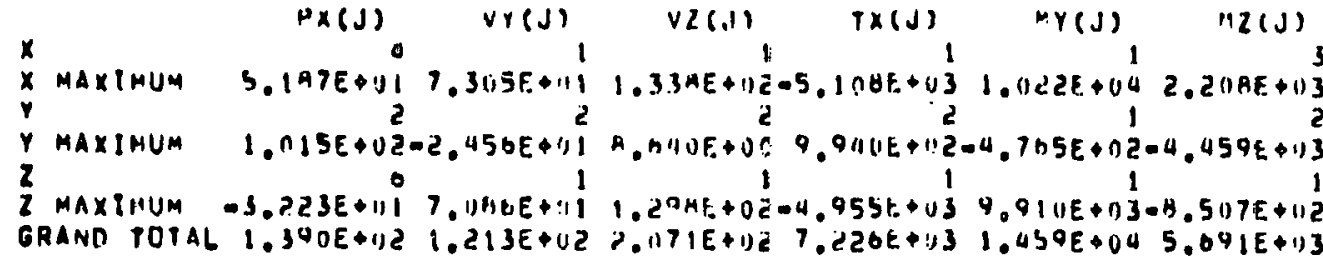

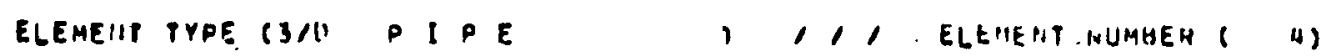

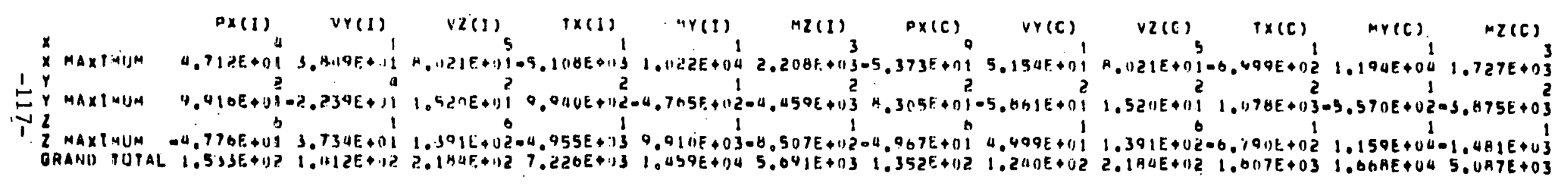
ELEMEHT TYPL (3) D P E 1,1, ELEMEUT NIJMHEH $($ a)

$D \times(J)$
$x$ MAXMUM

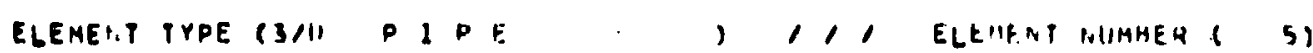

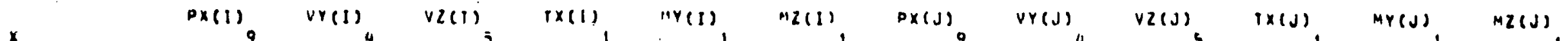

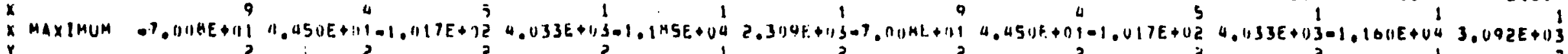

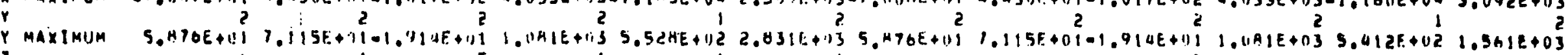

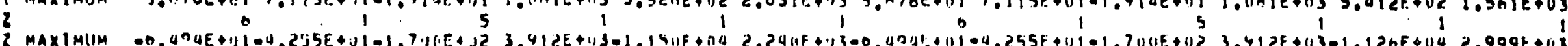

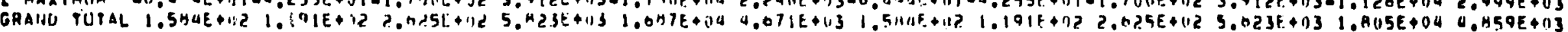

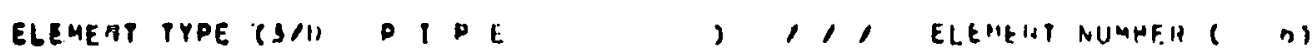

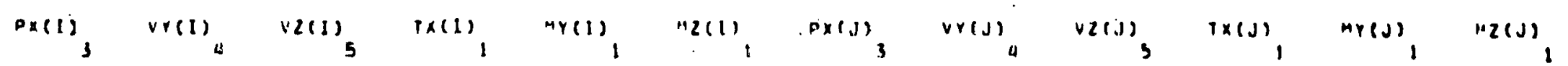




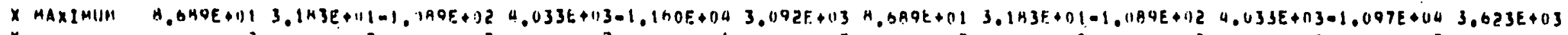

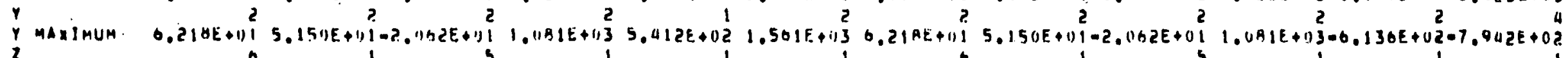

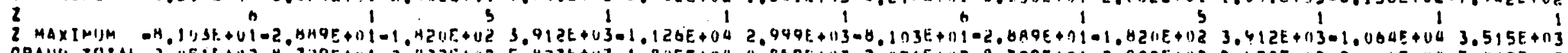

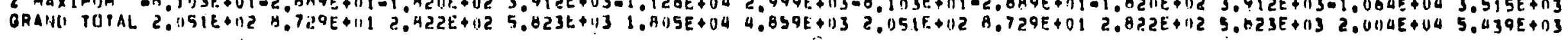

ELEMEIIT TYPE (3/N P 1 P E , 1,1 ELEMENT NUMBEH $(7)$

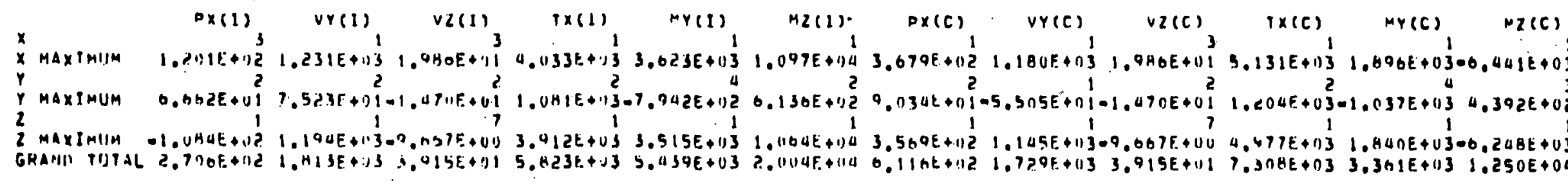

ELEMEAT PYPE (3/1) P 1 P E

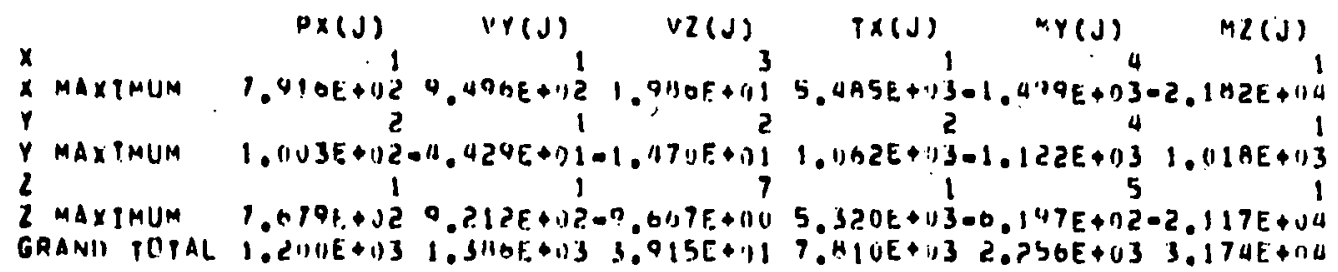

IELEmFit TYPE (3/U D 1 DE

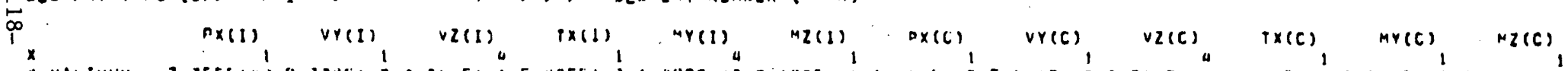

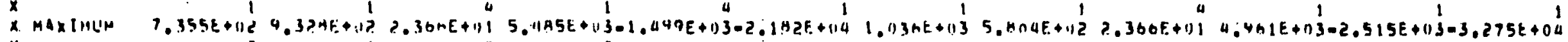

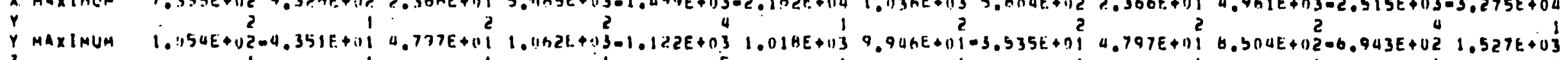

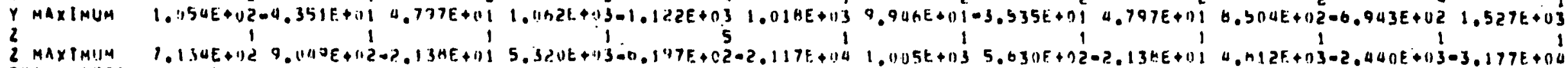

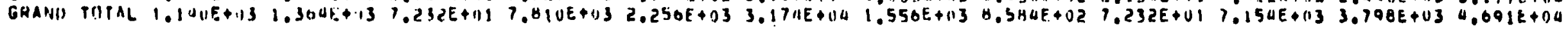

ELEMFIT TYPE (3/1) P I E $1,1 /$ ELEHENT NUMHER $(H)$

$0 \times(J)$
$x$

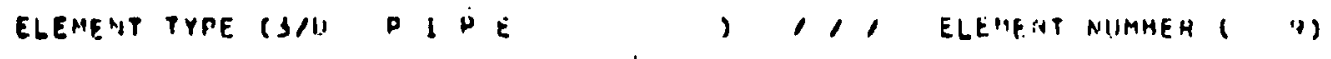

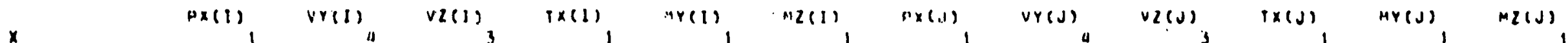

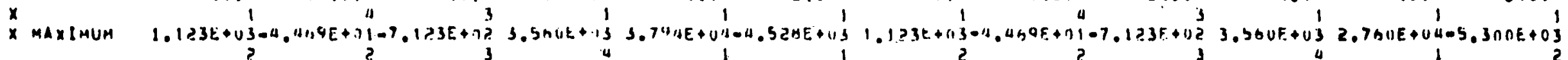

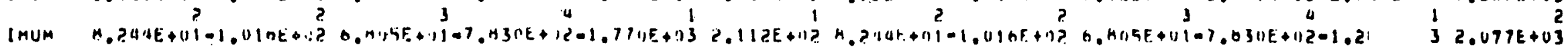

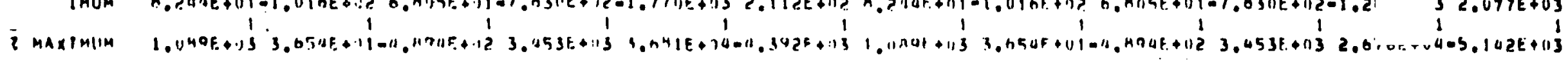




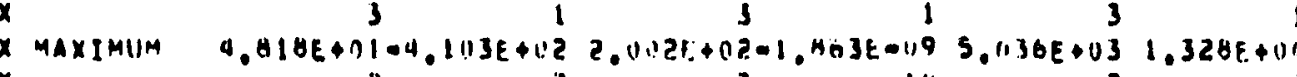

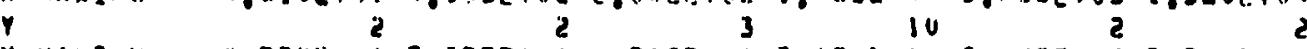

MAXIMUM -4.5ANE+UI-7.355E+UI-1.0135.111 3.10UE-1U 3.112.2E+113 3.9nOE+113

3

2 MAXIMUM $-2.5311 E+U 1-3.118 J F+112-5.710 E+1) 1-1.030 E-119-2.540 E+1131.248 E+114$

GRANI) POPAL $9.434 E+1117.460 \mathrm{E}+1127.375 F_{0}+1122.840 E-1197.313 E+032.072 E+110$

ELEMENT TYPE (3/1 P I P F , , ELEHENT NUMHEH $(15)$

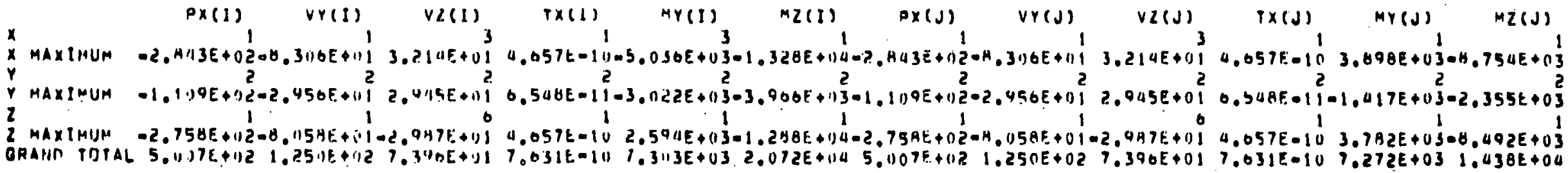

ELEMENT TYPE (3/0 DI PF $, 1,1$ ELEMENT NUMHEH $(10)$

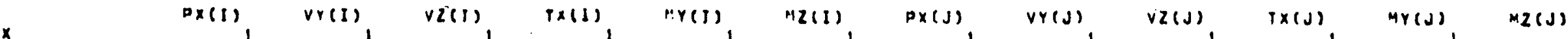

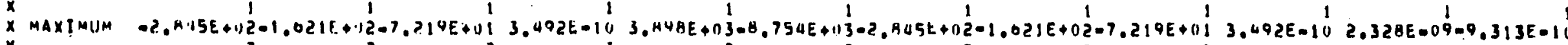

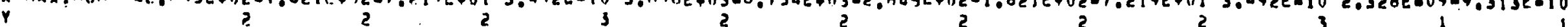

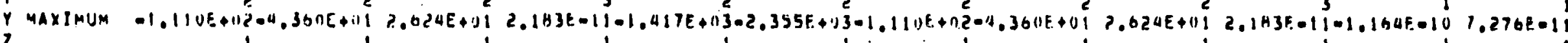

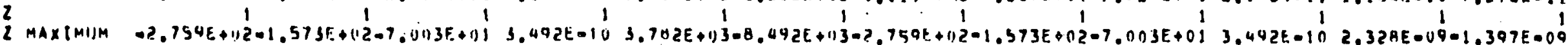

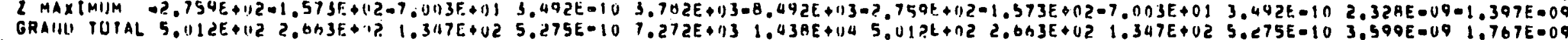

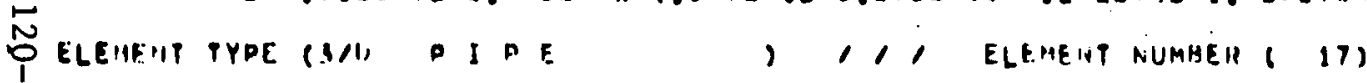

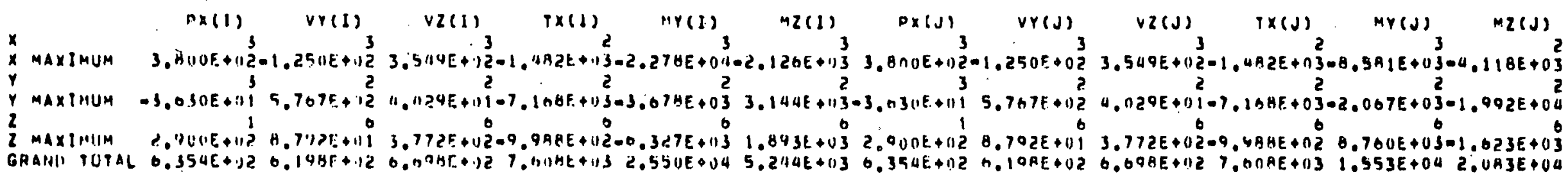

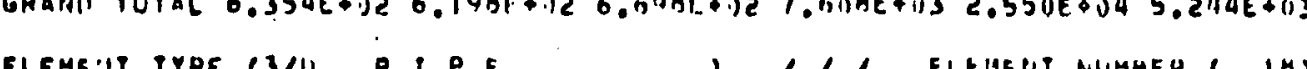

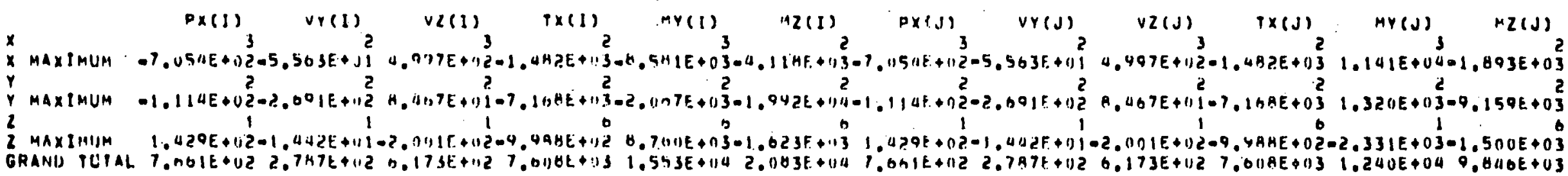

ELEMENT TYOE (SAD) $P$ I P E, 1, ELENENT NIJMHEH 1 I4,

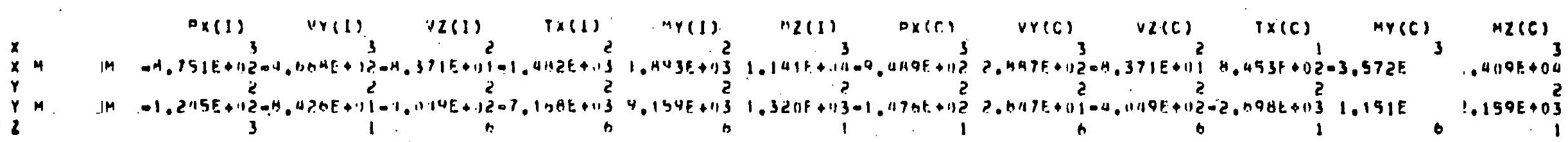




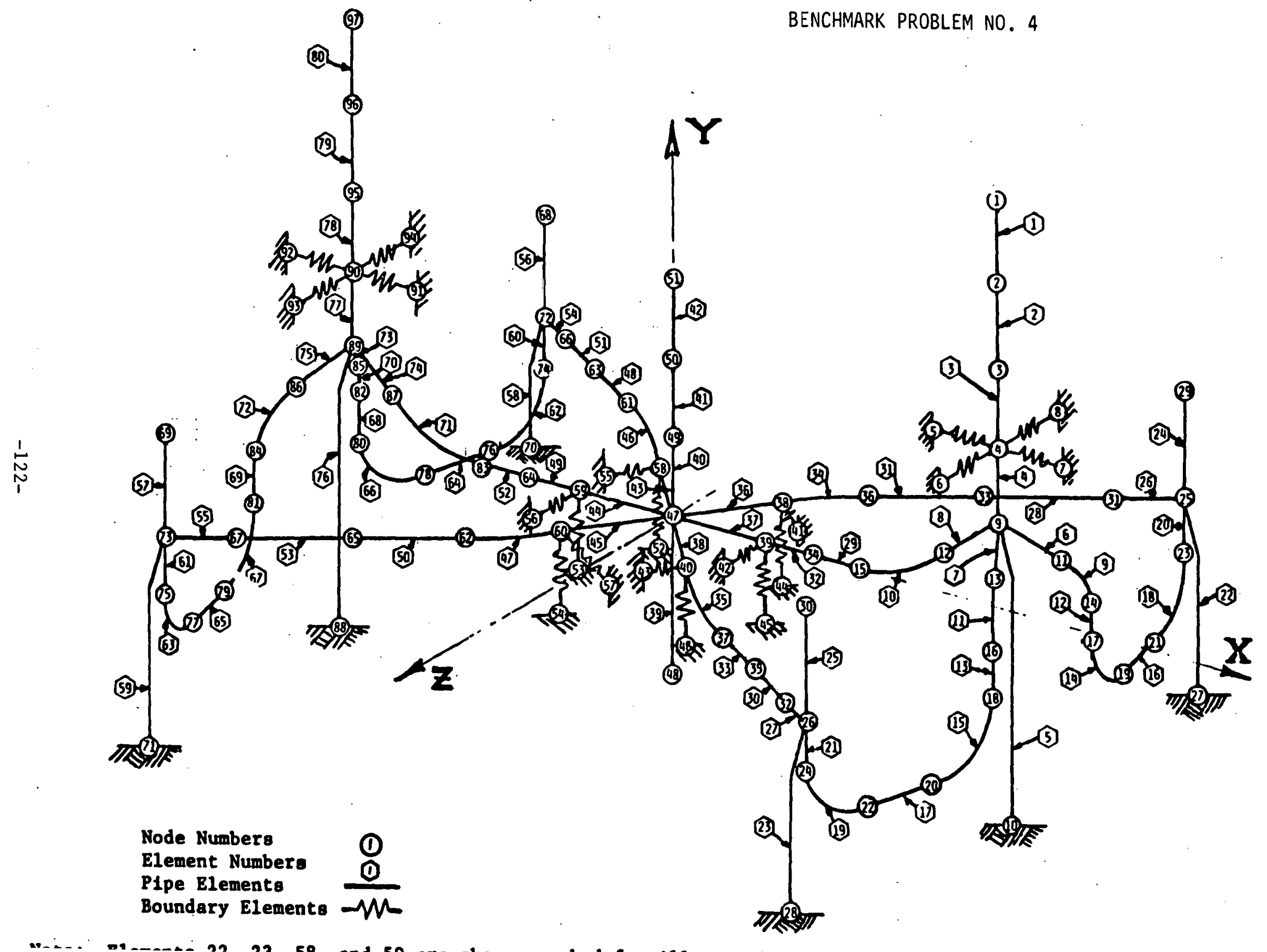


BENCHMARK PROBLEM NO.4.

10000

5000
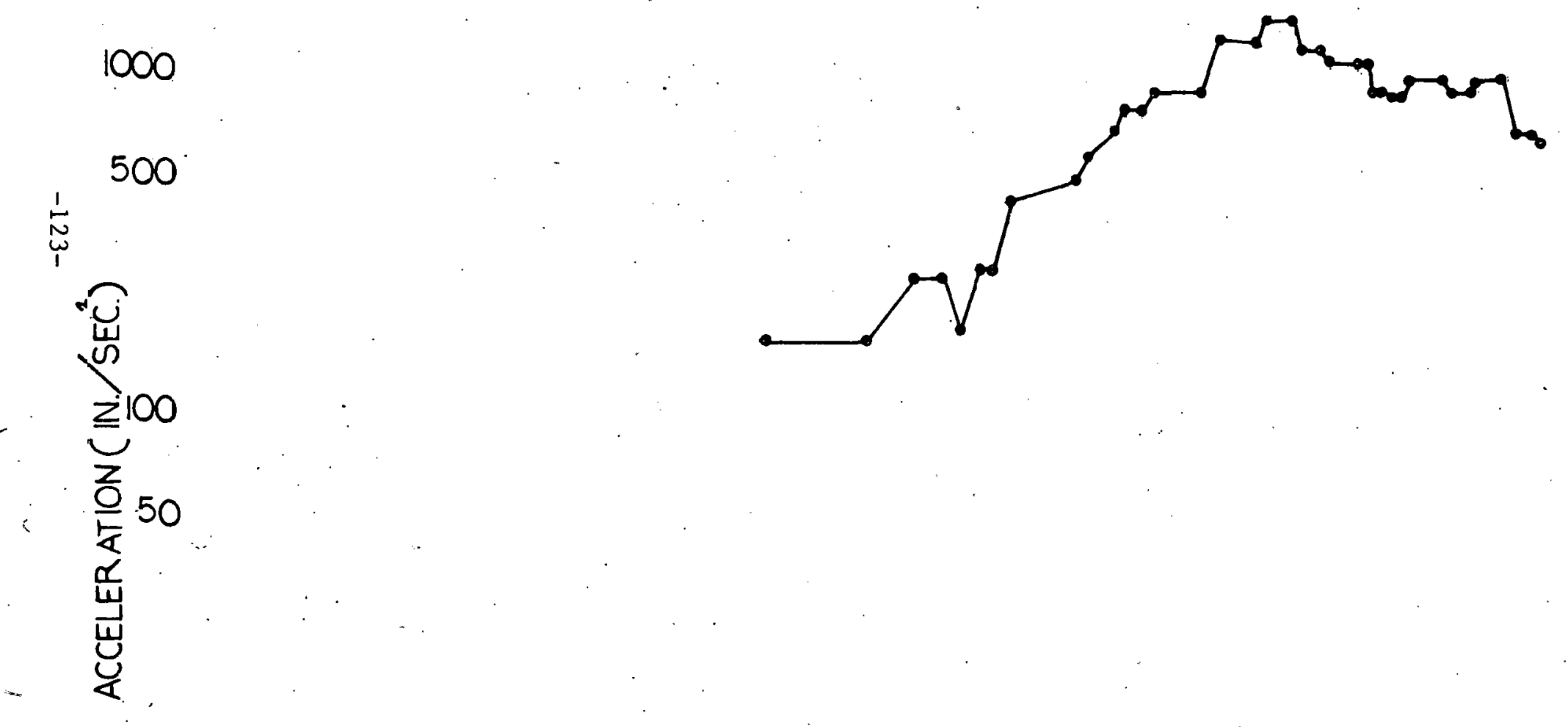

0.001

0.01 PERIOD(SEC)

10

10.0

Figure 4.2 
CONTROL. INFORMATTON

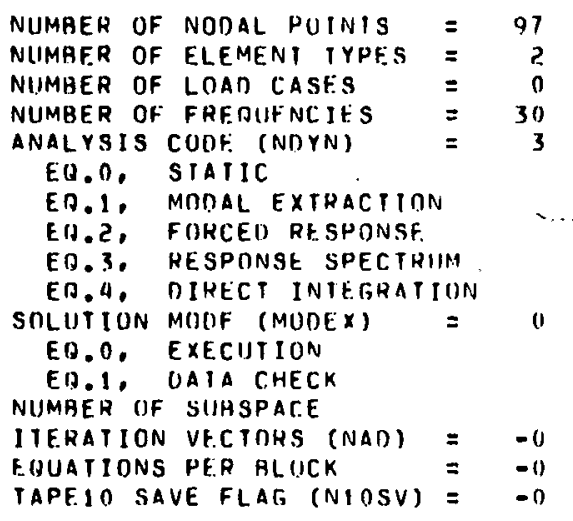

NUDAL POINT INPIIT DAIA

NUDE HIUUNISARY CONDITION CODFS

\begin{tabular}{|c|c|c|c|c|c|c|}
\hline NUMRER & $x$ & 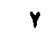 & 2 & $x x$ & $r y$ & 77 \\
\hline 1 & -0 & -0 & -0 & -0 & -0 & -0 \\
\hline 2 & -0 & -0. & -0 & -11 & -0 & -0 \\
\hline 3 & -0 & -0 & $-n$ & -0 & -0 & $-n$ \\
\hline 4 & -0 & -0 & -0 & $=0$ & -0 & $-n$ \\
\hline 5 & 1 & 1 & 1 & 1 & 1 & 1 \\
\hline 6 & 1 & 1 & 1 & 1 & 1 & 1 \\
\hline 7 & 1 & 1 & 1 & 1 & 1 & 1 \\
\hline 8 & 1 & 1 & 1 & 1 & 1 & 1 \\
\hline 9 & -0 & -0 & -0 & -0 & -0 & $-1)$ \\
\hline 10 & 1 & 1 & 1. & 1 & 1 & 1 \\
\hline 11 & -0 & -0 & -0 & -0 & -1 & -0 \\
\hline 12 & -0 & -0 & -0 & -0 & -1 & -0 \\
\hline 13 & -0 & -0 & -0 & -0 & -11 & $-1)$ \\
\hline 14 & -0 & -0 & -0 & -0 & $-n$ & -0 \\
\hline 15 & -0 & -0 & -0 & -0 & $=n$ & -0 \\
\hline 16 & -0 & -0 & -0 & -0 & -1 & -0 \\
\hline 17 & -0 & -0 & -0 & $=0$ & -0 & -1 \\
\hline 18 & -0 & -0 & -0 & $-n$ & $-n$ & $=0$ \\
\hline 19 & -0 & -0 & -0 & -0 & -0 & -0 \\
\hline 20 & -0 & -0 & $-n$ & $-1)$ & $-1)$ & -0 \\
\hline 21 & -0 & -0 & -0 & -0 & $-n$ & $-n$ \\
\hline 22 & -0 & -0 & -0 & -0 & -0 & -11 \\
\hline 23 & $-n$ & -0 & -0 & -0 & $-n$ & -0 \\
\hline 24 & -0 & -0 & -0 & -0 & -0 & -0 \\
\hline 25 & -0 & -0 & -0 & $-n$ & $\because 0$ & $-n$ \\
\hline 26 & -0 & -0 & $-n$ & -0 & -0 & -0 \\
\hline 27 & 1 & 1 & 1 & 1 & I & 1 \\
\hline 28 & 1 & 1 & 1 & 1 & 1 & 1 \\
\hline 29 & -0 & -0 & -11 & -0 & -0 & -0 \\
\hline 30 & $=0$ & -0 & -0 & $-n$ & -1 & -0 \\
\hline & 0 & -0 & -0 & -0 & -1 & -0 \\
\hline & 0 & -0 & -0 & $-n$ & -1 & $-n$ \\
\hline & 0 & -0 & $-n$ & -11 & $-r$ & $=0$ \\
\hline & & & & & & \\
\hline
\end{tabular}

TUUAL POINT C.ODRDIUATES

\begin{tabular}{|c|c|c|c|c|}
\hline & $r$ & 2 & & $T$ \\
\hline 3A4. 1000 & 696.0000 & 0.000 & -0 & $-0.00 n$ \\
\hline 384.000 & 552.0190 & 0.000 & -11 & -0.000 \\
\hline 384.000 & 456.1100 & 0.000 & -0 & -0.000 \\
\hline 384.000 & 276.000 & 0.000 & -0 & -0.000 \\
\hline 383.000 & ?76.0100 & 0.000 & -0 & -0.000 \\
\hline 3811.000 & 276.0000 & 1.1100 & $-n$ & $-1) .000$ \\
\hline 385.000 & 216.000 & $0.0 \cap 0$ & $-1)$ & -0.000 \\
\hline SA4.0ON & 276.000 & -1.000 & $-n$ & -0.000 \\
\hline 384.0010 & 96.000 & 0.000 & -0 & -0.000 \\
\hline 384.000 & -180.000 & $0.0 n n$ & -0 & - \\
\hline 399.000 & $26.9 n n$ & $-4 \pi .0000$ & $-n$ & -0.0011 \\
\hline $3{ }^{A} .8 \| 0$ & व?. . 101 & 0.000 & -0 & - \\
\hline 399.000 & ?h.9110 & Sh.000 & -0 & -0.000 \\
\hline 402.6010 & -11.600 & -69.500 & -0 & $=0.000$ \\
\hline 248.400 & 0.000 & $0.00 n$ & -0 & $-0.0 \cap 0$ \\
\hline 402.600 & -11.600 & 69.5011 & -0 & -0.0100 \\
\hline $40 ? .600$ & -12.000 & -69.5011 & -0 & -0.0100 \\
\hline $402 . h 00$ & $-1 P .000$ & 09.500 & -0 & $=0.000$ \\
\hline 354.700 & -133.000 & -105.600 & -0 & -0.000 \\
\hline 354.700 & -132.000 & 105.600 & -0 & -0.000 \\
\hline 335.9011 & -132.000 & -119.900 & -11 & $-0.0 \cap 0$ \\
\hline 335.9011 & -132.000 & 119.000 & -0 & $-0.0 n n$ \\
\hline 234.000 & -12.0011 & $-156.00 n$ & -0 & $-0.0 \cap 0$ \\
\hline ग्A.,01011 & - & $15 \mathrm{~h} .000$ & -0 & $-0.00 n$ \\
\hline 288.000 & 0.1000 & -1 Sh.0000 & $-n$ & -0.001 \\
\hline 288.000 & 0.000 & 154.000 & $-n$ & -0.000 \\
\hline 28R. 010 & -180.000 & - I 5 . . Oחn & -0 & -0.000 \\
\hline ZRA. OOU! & - I31, 0ก0 & $15 \mathrm{~h} .1100$ & -0 & -0.000 \\
\hline रBष. & $1 \geq n .000$ & - ISh.01011 & -11 & -11.000 \\
\hline 288.0110 & 126.000 & $15 \mathrm{~A} .0011$ & $-n$ & -0.0100 \\
\hline 253.2011 & 0.0 (n) & -146.700 & $=0$ & -0.000 \\
\hline 253.200 & 0.000 & 146.700 & $-n$ & -0.01100 \\
\hline 187.300 & 0.000 & - & -0 & - \\
\hline 117,000 & 0.000 & 0.000 & -0 & $-11-000$ \\
\hline
\end{tabular}




\begin{tabular}{|c|c|c|c|c|c|c|c|c|c|c|c|}
\hline 35 & -0 & -0 & -0 & -0 & -0 & -0 & 187.300 & 0.000 & 129.500 & -0 & $=0.000$ \\
\hline 36 & $-n$ & -0 & -0 & -0 & -0 & -0 & 121.400 & 0.000 & -110.200 & -0 & -0.010 \\
\hline 37 & & -0 & -0 & -0 & -0 & -0 & 121.400 & 0.000 & 110.200 & -0 & -0.000 \\
\hline 38 & & $=0$ & -0 & -11 & -11 & -0 & 94.600 & 0.000 & -94.600 & -0 & -0.000 \\
\hline 39 & $-\mathbf{v}$ & -0 & -0 & -0 & $-n$ & $-n$ & 96.0011 & $0.00 n$ & 0.000 & -0 & -0.0100 \\
\hline 40 & -0 & -0 & -0 & -11 & -0 & $-n$ & 94.606 & $0.00 n$ & 94.600 & -0 & -0.000 \\
\hline 41 & 1 & 1 & 1 & 1 & 1 & 1 & 95.605. & 0.000 & $\begin{array}{l}-93.600 \\
03.00\end{array}$ & -0 & $\begin{array}{l}-10.0100 \\
-0.0110\end{array}$ \\
\hline 42 & $i$ & $i$ & $i$ & $i$ & $i$ & 1 & 96.000 & 0.000 & 1.000 & -11 & -0.0010 \\
\hline 43 & $i$ & $i$ & $i$ & $i$ & $i$ & $i$ & $93.60 r$ & 0.0110 & 95.600 & -0 & -0.0110 \\
\hline & $i$ & & & & & & & & & & \\
\hline $\begin{array}{l}45 \\
-15\end{array}$ & 1 & 1 & $\frac{1}{1}$ & 1 & 1 & 1 & 96.006 & -1.0000 & $\begin{array}{r}-94.600 \\
n .000\end{array}$ & $\begin{array}{l}-11 \\
-0\end{array}$ & $\begin{array}{l}-10.000 \\
-0.000\end{array}$ \\
\hline 46 & $i$ & $i$ & $i$ & $i$ & $i$ & $i$ & 90.6018 & -1.000 & 94.600 & -0 & -0.000 \\
\hline 47 & -0 & -0 & $\begin{array}{l}1 \\
-0\end{array}$ & $\begin{array}{c}1 \\
-0\end{array}$ & -0 & -0 & $0.0 n c$ & $\begin{array}{r}-1.0000 \\
0.000\end{array}$ & $\begin{array}{l}0.0001 \\
0.000\end{array}$ & $\begin{array}{l}-11 \\
-0\end{array}$ & $\begin{array}{l}-10.0100 \\
-0.000\end{array}$ \\
\hline 48 & -0 & $\begin{array}{l}-0 \\
-0\end{array}$ & $\begin{array}{l}-0 \\
-0\end{array}$ & $\begin{array}{l}-1 \\
-0 \\
-0\end{array}$ & 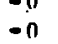 & $\begin{array}{l}-11 \\
-0 \\
-0\end{array}$ & 0.006 & -192.000 & 0.000 & $\begin{array}{l}-0 \\
-0\end{array}$ & $\begin{array}{l}-0.0100 \\
-0.0100\end{array}$ \\
\hline 49 & -0 & $\begin{array}{l}-1 \\
-0\end{array}$ & $\begin{array}{l}-0 \\
-n\end{array}$ & $\begin{array}{l}-1 \\
-0\end{array}$ & - & $\begin{array}{l}-10 \\
-0\end{array}$ & $0.00 r$ & $\begin{array}{r}-195.001 \\
\text { A } 4.0 n 0\end{array}$ & 0 & $\begin{array}{l}-10 \\
-0\end{array}$ & $\begin{array}{l}-0.0100 \\
-0.000\end{array}$ \\
\hline bo & -0 & $\begin{array}{l}-0 \\
-0\end{array}$ & -0 & -0 & $\begin{array}{l}-01 \\
-0\end{array}$ & $\begin{array}{l}-0 \\
-0\end{array}$ & $\begin{array}{l}0.001 \\
0.006\end{array}$ & $\begin{array}{l}24.000 \\
156.000\end{array}$ & $\begin{array}{l}0.000 \\
0.000\end{array}$ & $\begin{array}{l}-10 \\
-0\end{array}$ & $\begin{array}{l}-0.000 \\
-0.0110\end{array}$ \\
\hline 51 & -0 & $\begin{array}{l}-11 \\
-0\end{array}$ & $=0$ & -0 & $\begin{array}{l}-0 \\
-0\end{array}$ & -0 & 0.006 & $\begin{array}{l}x=000 \\
P .000\end{array}$ & 0 & $\begin{array}{l}-0 \\
-0\end{array}$ & $\begin{array}{l}-0.01011 \\
-0.0110\end{array}$ \\
\hline $5 ?$ & 1 & 1 & 1 & 1 & 1 & 1 & $\begin{array}{l}-94.600 ! \\
-94.60\end{array}$ & -1,000 & $\begin{array}{l}-94.600 \\
-94.600\end{array}$ & $\begin{array}{l}-11 \\
-0\end{array}$ & $\begin{array}{l}-0.0101 \\
-0.010\end{array}$ \\
\hline 53 & $i$ & 1 & 1 & 1 & 1 & 1 & -96.0011 & $\begin{array}{l}-1.000 \\
-1.000\end{array}$ & $\begin{array}{r}-94.000 \\
0.000\end{array}$ & $\begin{array}{l}-0 \\
-0\end{array}$ & $\begin{array}{l}-10.01000 \\
-0.000\end{array}$ \\
\hline 54 & 1 & $i$ & 1 & $i$ & 1 & 1 & $\begin{array}{l}-911, \text { sna } \\
-1\end{array}$ & -1.000 & $\begin{array}{l}91.0000 \\
9.600\end{array}$ & -0 & $\begin{array}{l}-0.10101 \\
-0.000\end{array}$ \\
\hline 55 & 1 & 1 & 1 & 1 & 1 & 1 & $\begin{array}{l}-95.601 \\
-95.601\end{array}$ & $\begin{array}{r}-1.00100 \\
0.0100\end{array}$ & $\begin{array}{r}93.6000 \\
-93.600\end{array}$ & $\begin{array}{l}-0 \\
-0\end{array}$ & $\begin{array}{l}-0.0001 \\
-0.000\end{array}$ \\
\hline 56 & $i$ & $i$ & $i$ & $i$ & $i$ & $i$ & -94.009 & 0.000 & 1.000 & -0 & $-0.0 n 0$ \\
\hline 51 & $i$ & $i$ & $i$ & $i$ & $i$ & 1 & -93.600 & 10.000 & 95.500 & -0 & -0.000 \\
\hline 58 & -0 & -0 & -0 & -0 & -0 & $-n$ & -94.600 & 0.000 & -94.600 & -0 & -0.000 \\
\hline 59 & -0 & -0 & $-n$ & -0 & -0 & -0 & -96.000 & 0.000 & 0.000 & -0 & $-0.0 n n$ \\
\hline 60 & -0. & -0 & -0 & -0 & -0 & -0 & -94.400 & 0.000 & 411.600 & -0 & -0.0010 \\
\hline hi & -0 & -0 & -0 & $-n$ & -0 & -11 & -121.400 & 10.000 & -110.200 & $-n$ & $-0.0 n 0$ \\
\hline 62 & -0 & -0 & -0 & -0 & -0 & -0 & -121.400 & a.onn & 110.200 & -0 & -0.000 \\
\hline 63 & -0 & -0 & -0 & $-n$ & $=0$ & -0 & -181.300 & 0.000 & $-1 \geqslant 4.5 n 0$ & -0 & -0.1100 \\
\hline $\begin{array}{l}63 \\
64\end{array}$ & $\begin{array}{l}-0 \\
-0\end{array}$ & $\begin{array}{l}-0 \\
-0\end{array}$ & $\begin{array}{l}-0 \\
-0\end{array}$ & $\begin{array}{l}-11 \\
-0\end{array}$ & $\begin{array}{l}-01 \\
-0 \\
-0\end{array}$ & $\begin{array}{l}-10 \\
-0\end{array}$ & $\begin{array}{l}-187.500 \\
-177.1100\end{array}$ & $\begin{array}{l}0.000 \\
0.000\end{array}$ & 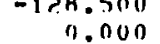 & $\begin{array}{l}-0 \\
-0\end{array}$ & $\begin{array}{l}-0.000 \\
-0.0100\end{array}$ \\
\hline $\begin{array}{l}64 \\
45\end{array}$ & $\begin{array}{l}-0 \\
-0\end{array}$ & $\begin{array}{l}-0 \\
-0\end{array}$ & $\begin{array}{l}-1 \\
-0 \\
-0\end{array}$ & $\begin{array}{l}-0 \\
-0\end{array}$ & $\begin{array}{l}-0 \\
-0\end{array}$ & $\begin{array}{l}-11 \\
-0\end{array}$ & -187.300 & $\begin{array}{l}0.100 \\
0.000\end{array}$ & 129.500 & $\begin{array}{l}-0 \\
-0\end{array}$ & $\begin{array}{l}-10.000 \\
-0.000\end{array}$ \\
\hline $\begin{array}{l}\text { nd } \\
56\end{array}$ & -0 & $\begin{array}{l}-0 \\
-0\end{array}$ & $\begin{array}{l}-0 \\
-0 \\
-0\end{array}$ & $\begin{array}{l}-11 \\
-0\end{array}$ & $\begin{array}{l}-10 \\
-0 \\
-i\end{array}$ & $\begin{array}{l}-0 \\
-0\end{array}$ & -253.2011 & $\begin{array}{l}0.1000 \\
0.000\end{array}$ & $\begin{array}{r}146.700 \\
-1460\end{array}$ & $\begin{array}{l}-1) \\
-0\end{array}$ & $\begin{array}{l}-0.000 \\
-0.0101\end{array}$ \\
\hline $\begin{array}{l}50 \\
67\end{array}$ & $\begin{array}{l}-0 \\
-0\end{array}$ & $\begin{array}{l}-10 \\
-0\end{array}$ & $\begin{array}{l}-11 \\
-0 \\
-n\end{array}$ & $\begin{array}{l}-0 \\
-1)\end{array}$ & $\begin{array}{l}-1 \\
-0 \\
-0\end{array}$ & $\begin{array}{l}-1 \\
-0 \\
-1\end{array}$ & $\begin{array}{r}-253.20100 \\
-253.200\end{array}$ & $\begin{array}{l}0.000 \\
0.000\end{array}$ & $\begin{array}{r}-146.700 \\
1 / 16.700\end{array}$ & $\begin{array}{l}-1 \\
-0\end{array}$ & $\begin{array}{l}-0.0001 \\
-0.000\end{array}$ \\
\hline 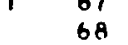 & $\begin{array}{l}-0 \\
-0\end{array}$ & $\begin{array}{l}-0 \\
-0 \\
-0\end{array}$ & $\begin{array}{l}-11 \\
-0\end{array}$ & $\begin{array}{l}-11 \\
-0\end{array}$ & $\begin{array}{l}-11 \\
-0 \\
-0\end{array}$ & $\begin{array}{l}-1 \\
-n\end{array}$ & $\begin{array}{l}-58 B .0 ก 0 \\
-535.500\end{array}$ & $12 h .000$ & $\begin{array}{r}-156.000 \\
-156.00\end{array}$ & $\begin{array}{l}-1 \\
-0\end{array}$ & $\begin{array}{l}-0.0100 \\
-0.000\end{array}$ \\
\hline $\begin{array}{l}60 \\
69\end{array}$ & $\begin{array}{l}-0 \\
-0\end{array}$ & $\begin{array}{l}-0 \\
-0\end{array}$ & $\begin{array}{l}-0 \\
-0\end{array}$ & $\begin{array}{l}-0 \\
-0\end{array}$ & $\begin{array}{l}-61 \\
-0 \\
-1\end{array}$ & -6 & $\begin{array}{l}-58 R .001000 \\
-58 R\end{array}$ & $\begin{array}{l}12 h .000 \\
126.0100\end{array}$ & $\begin{array}{r}-154.000 \\
154.000\end{array}$ & $\begin{array}{l}-0 \\
-0\end{array}$ & $\begin{array}{l}-0.000 \\
-0.000\end{array}$ \\
\hline 10 & 1 & 1 & $i$ & $i$ & 1 & 1 & -588.000 & $-1 \times 0.000$ & -156.000 & -0 & -0.000 \\
\hline 11 & $i$ & $i$ & 1 & $i$ & 1 & 1 & 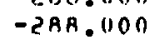 & -150.000 & 156.000 & -0. & -0.000 \\
\hline 72 & -0 & -0 & -0 & -0 & -0 & -0 & -PAB.0ก0 & 0.100 & -156.000 & -0 & -0.000 \\
\hline 73 & -0 & -0 & -0 & $-n$ & -1 & -0 & - DRA.กOก & 0.000 & 154.000 & $-n$ & $=0.1100$ \\
\hline 74 & -0 & -0 & $=0$ & -0 & -0 & -0 & - $2 A 4.000$ & $-1 ? .000$ & -159.000 & -0 & -0.000 \\
\hline 75 & -0 & -0 & -0 & -0 & -11 & -0 & -PBA.000 & $-12.00 n$ & 156.000 & -0 & -0.000 \\
\hline 16 & -0 & -0 & -0 & $=0$ & -0 & $=0$ & -335.900 & $-1<0.000)$ & -119.9010 & -11 & -0.000 \\
\hline 17 & -0 & -0 & -0 & -0 & $-n$ & $-n$ & -335.900 & $-192.0 n n$ & 119.900 & -0 & -0.000 \\
\hline 18 & -0 & -0 & -0 & -0 & -0 & -0 & -354.700 & -132.000 & -105.600 & -0 & -0.1000 \\
\hline 19 & -0 & $-n$ & -0 & $-n$ & -0 & -0 & -354.700 & -192.000 & 105.600 & -0 & -11.0 กn \\
\hline BO & -0 & -0 & -0 & -0 & -0 & -0 & -402.600 & $\begin{array}{l}-19000 \\
-190\end{array}$ & -69.500 & -0 & -0.000 \\
\hline A1 & -0 & -0 & -0 & -0 & -0 & -0 & -40?.6010 & $-12.00 n$ & 69.500 & -0 & -0.000 \\
\hline 82 & -0 & -0 & -0 & -0 & -0 & -11 & $-40 ? .600$ & -11.600 & -69.500 & -11 & -0.000 \\
\hline 83 & -0 & -0 & -0 & -0 & -0 & -0 & -P.4H.101 & 11.0 no & 0.000 & -0 & $-0.0 \mathrm{nn}$ \\
\hline 84 & -0 & $-n$ & -0 & -0 & -0 & -0 & -402.6000 & -11.6110 & 69.500 & $=0$ & -0.000 \\
\hline A5 & -0 & -0 & -0 & -0 & -0 & -11 & $-394 . n(11)$ & $26.911 n$ & $-5 h .00 n$ & -0 & -0.000 \\
\hline B6 & -0 & -0 & -0 & $-n$ & $-n$ & $-1)$ & -399.10011 & 20.900 & $5 h .000$ & -0 & -0.0110 \\
\hline A7 & -0 & -0 & $-n$ & -0 & -0 & - -1) & -334.4011 & 42.100 & 0.000 & -0 & -0.0010 \\
\hline BA & 1 & 1 & 1 & 1 & 1 & 1 & -384.0000 & -140.000 & 10.000 & -0 & $-0,0110$ \\
\hline B9 & -0 & -0 & -0 & -0 & -0 & -0 & -384.00010 & 96.000 & 10.000 & -0 & -11.000 \\
\hline 90 & -0 & -0 & -0 & -0 & -0 & -0 & $-384.000)$ & 276.000 & 0.0010 & -0 & -0.000 \\
\hline 91 & 1 & 1 & 1 & 1 & 1 & 1 & $-3 A 3.0000$ & $? 7 t .000$ & 0.000 & $-1)$ & -0.000 \\
\hline 92 & $i$ & $i$ & $i$ & $i$ & $i$ & $i$ & -385.0110 & 276.1100 & 0.0000 & -0 & -0.000 \\
\hline 93 & $i$ & 1 & $i$ & $i$ & $i$ & $i$ & -384.000 & 216.0100 & 1.000 & $-1)$ & -0.0100 \\
\hline 94 & 1 & 1 & $i$ & 1 & $i$ & 1 & -384.000 & $\sin 000$ & -1.1010 & -0 & $-0,010$ \\
\hline 95 & -0 & -0 & $-n$ & -0 & -0 & -11 & - TaA.0OIi & 155.000 & 0.0110 & $-n$ & -0.000 \\
\hline 96 & -0 & -0 & -0 & -0 & -0 & -0 & $-3 H 4.0100$ & חס & 1.0110 & -11 & -0.000 \\
\hline 97 & -0 & $-n$ & -0 & -0 & -0 & -11 & -3R4.1100 & 6045.0000 & vi.01010 & -11 & -0.0110 \\
\hline
\end{tabular}


GENERATED NODAL DATA

\begin{tabular}{|c|c|c|c|c|c|c|c|c|c|c|}
\hline NOUE & 801 & JARY & $\cos$ & $10 \mathrm{~N}$ & COD & & NOIIAI. & Pl[ [D, & & \\
\hline NUMAER & $x$ & $r$ & 2 & $x \times$ & YY & 77 & $x$ & $r$ & 7 & 1 \\
\hline 1 & -0 & -0 & -0 & $-1)$ & -0 & -0 & 384.000 & h⿻h.0n0 & 0.000 & -0.000 \\
\hline 2 & -0 & -0 & -0 & -0 & -0 & -0 & 384.000 & $55 ? .000$ & 0.000 & -0.000 \\
\hline 3. & -0 & -0 & -0 & -0 & -0 & -0 & 384.000 & 456.000 & 0.000 & -0.000 \\
\hline 4 & -0 & -0 & -0 & -0 & -0 & -0 & 384.000 & 276.000 & 0.000 & -0.000 \\
\hline 5 & 1 & 1 & 1 & 1 & 1 & 1 & 383.0110 & 276.000 & 0.000 & -0.008 \\
\hline$h$ & 1 & 1 & 1 & 1 & 1 & 1 & 384.000 & 276.000 & 1.000 & -0.008 \\
\hline 7 & 1 & 1 & 1 & 1 & 1 & 1 & $385.00 n$ & $27 h .000$ & 0.000 & -0.000 \\
\hline 8 & 1 & 1 & 1 & 1 & 1 & 1 & 384.000 & 276.000 & -1.000 & $=0.001$ \\
\hline 9 & -0 & -0 & -0 & -0 & -0 & -0 & 384.000 & $\forall 6.000$ & 0.000 & -0.000 \\
\hline 10 & 1 & 1 & 1 & 1 & 1 & 1 & 384.000 & -190.000 & 0.000 & -0.000 \\
\hline 11 & -0 & -0 & -0 & -0 & -0 & -0 & 399.000 & 26.900 & -56.000 & -0.000 \\
\hline 12 & -0 & -0 & -0 & -0 & -0 & -0 & 338.800 & 42.100 & 0.000 & -0.000 \\
\hline 13 & -0 & -0 & -0 & -0 & -0 & -0 & 399.000 & 26.900 & 56.000 & -0.008 \\
\hline 14 & -0 & -0. & -0 & -0 & -0 & -0 & 402.6011 & -11.600 & -69.500 & $-11.00 r$ \\
\hline 15 & -0 & -0 & -0 & -0 & $-n$ & -0 & 248.4011 & $1) .000$ & 0.000 & -0.000 \\
\hline 16 & -0 & -0 & -0 & -0 & -0 & -0 & 402.600 & $-11 \cdot+600$ & 69.500 & -0.000 \\
\hline 17 & -0 & -0 & -0 & -0 & -0 & -0 & $402.6 \cap 0)$ & $-7 ? .000$ & -59.500 & -0.008 \\
\hline IA & -0 & -0 & -0 & $-n$ & -0 & $-n$ & 402.6011 & $-7 ? .000$ & 69.500 & -0.008 \\
\hline 19 & -0 & -0 & -0 & -0 & -0 & $-n$ & 354.700 & -132.000 & -105.600 & -0.000 \\
\hline$? .0$ & -0 & -0 & -0 & -0 & -0 & -0 & 354.100 & -132.001 & 105.600 & -11.000 \\
\hline ?. 1 & $=0$ & -0 & -0 & -0 & -0 & -0 & 335.900 & -152.000 & -119.900 & -0.000 \\
\hline 22 & -0 & $-n$ & -0 & -0 & -0 & $-n$ & 355.900 & -132.000 & 119.901 & -0.000 \\
\hline 23 & -0 & $-c$ & -0 & -0 & -0 & -0 & 288.00 .11 & -12.000 & $-15 h .00 n$ & -0.000 \\
\hline 24 & -0 & -0 & -0 & -0 & -0 & -0 & 288.000 & $-7 ? .000$ & 155.000 & $-0.00 c$ \\
\hline 25 & -0 & -0 & -0 & -0 & -0 & -0 & 288.000 & 0.000 & -156.000 & -0.008 \\
\hline 26 & -0 & -0 & -0 & $-1)$ & -0 & -0 & 2AR.000 & 0.000 & 156.000 & -0.001 \\
\hline 27 & 1 & 1 & $\mathrm{t}$ & 1 & 1 & 1 & 288.0011 & -1.80 .000 & -156.000 & -0.000 \\
\hline 28 & 1 & 1 & 1 & 1 & 1 & 1 & 288.000 & -180.000 & 156.000 & -0.000 \\
\hline 29 & -0 & -0 & -0 & -0 & -0 & -0 & 289.0110 & 1.26 .000 & -156.000 & -0.000 \\
\hline 30 & -0 & -0 & -0 & -0 & -0 & -0 & 2AH.000 & 126.000 & 156.000 & -0.000 \\
\hline 31 & -0 & -0 & -0 & -0 & -0 & -0 & 253.200 & 0.000 & -146.700 & -0.000 \\
\hline 32 & -0 & -0 & -0 & -0 & -0 & -0 & 253.200 & 0.000 & 146.7 .00 & -0.000 \\
\hline 33 & -0 & -0 & -0 & -0 & -0 & -0 & 187.300 & 0.000 & $-12 A .500$ & -0.000 \\
\hline 34 & -0 & -0 & -0 & -0 & -0 & -0 & 177.000 & 0.000 & 0.000 & -0.000 \\
\hline 35 & -0 & -0 & -0 & -0 & -0 & -11 & 187.300 & 0.000 & 124.500 & -0.000 \\
\hline 36 & -0 & -0 & -0 & -0 & -0 & -0 & 121.400 & 0.000 & -110.200 & -0.008 \\
\hline 37 & $=0$ & $\sim 0$ & -0 & -0 & -0 & -0 & 121.4011 & 0.000 & 110.200 & -0.001 \\
\hline $3 \mathrm{~B}$ & -0 & -0 & -0 & -1 & $-n$ & -0 & $94.601)$ & 0.000 & -94.600 & -0.001 \\
\hline 39 & -0 & -0 & -0 & -0 & -0 & -11 & 96.000 & 0.000 & 0.000 & -0.000 \\
\hline 40 & -0 & -0 & -0 & -0 & -0 & -0 & 94.6011 & 0.1100 & 911.6010 & -0.008 \\
\hline 41 & 1 & 1 & $i$ & 1 & 1 & 1 & $95.60 n$ & 0.000 & -93.600 & -0.1100 \\
\hline 42 & 1 & 1. & 1 & 1 & 1 & 1 & 96.000 & 0.000 & 1.000 & -0.000 \\
\hline 43 & 1 & 1 & 1 & 1 & 1 & 1 & 93.600 & n.000 & 95.600 & -0.001 \\
\hline 44 & 1 & 1 & 1 & 1 & 1 & 1 & 94.6010 & -1.000 & -94.600 & -0.000 \\
\hline 45 & 1 & 1 & 1 & 1 & 1 & 1 & $9 h .001)$ & $-1.00 n$ & 0.000 & -0.000 \\
\hline 46 & 1 & 1 & 1 & 1 & 1 & 1 & $94 . n 00$ & -1.000 & 94.6010 & -0.000 \\
\hline 47 & $=0$ & -0 & -0 & -0 & -0 & -0 & 0.000 & 0.000 & 0.001 & -0.000 \\
\hline 48 & -0 & -0 & -0 & -0 & $-1)$ & -11 & 0.000 & $-19 ? .000$ & 0.0101 & -0.000 \\
\hline 49 & -0 & -0 & -0 & -0 & -0 & -11 & 0.000 & 84.0100 & 0.000 & $=0.000$ \\
\hline 50 & -0 & -0 & -0 & -0 & -0 & -0 & 1.0110 & 154.000 & $0.0 n 0$ & -0.000 \\
\hline 51 & -0 & -0 & -0 & -0 & -0 & -0 & $n .00 n$ & $=A H .000$ & 0.000 & -0.001 \\
\hline 5 & 1 & 1 & 1 & 1 & 1 & 1 & -94.600 & -1.000 & -94.600 & -11.0100 \\
\hline 5 & 1 & 1 & 1 & 1. & 1 & 1 & -96.0110 & $-1.00 i$ & 0.000 & -0.1101 \\
\hline 5 & 1 & 1 & 1 & 1 & 1 & 1 & -911.600 & -1.000 & 94.6110 & -1.000 \\
\hline 55 & 1 & 1 & 1 & 1 & 1 & 1 & -95.600 & 0.000 & -93.600 & -0.000 \\
\hline
\end{tabular}




\begin{tabular}{|c|c|c|c|c|c|c|c|c|c|c|}
\hline 60 & $=0$ & -0 & -0 & -0 & $-n$ & -11 & $-9 a, 600$ & 0.010 & 94.600 & $-0,000$ \\
\hline 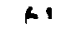 & -0 & -0 & -0 & $-n$ & -0 & $-n$ & -121.4110 & 0.000 & -110.2011 & -0.0110. \\
\hline 1 & -0 & -0 & -0 & -0 & -0 & -0 & -121.401 & 0.000 & 110.200 & -0.000 \\
\hline 1 & -0 & -0 & -0 & $-1)$ & -0 & $-n$ & -187.300 & 0.000 & -124.500 & -0.070 \\
\hline hu & -0 & -0 & -0 & -0 & -0 & -0 & -177.000 & 0.000 & 0.000 & -0.010 \\
\hline 65 & -0 & -0 & -0 & $-n$ & -11 & -0 & -187.301 & 0.000 & $12 A .500$ & -0.000 \\
\hline $6 h$ & -0 & -0 & -0 & -0 & -0 & $-n$ & -253.200 & 0.1000 & $-14 h .700$ & $\begin{array}{l}-0.0011 \\
-0.0011\end{array}$ \\
\hline 6.7 & -0 & -0 & -0 & -0 & -0 & -0 & -253.200 & $0 . n \cap 0$ & $14 \mathrm{~h} .700$ & -0.000 \\
\hline h⿻ & -0 & -0 & -0 & -0 & -0 & -0 & - РАA.Оก & 1 ?.h.ก०0 & - ish.oun & $-n .000$ \\
\hline 69 & -0 & -0 & -0 & -0 & $-n$ & -10 & -PAR. חOU & 126.000 & 156.000 & -0.0011 \\
\hline 10 & 1 & 1 & 1 & 1 & 1 & 1 & $=P A H . O O O$ & -190.000 & $-15 \mathrm{~h} .000$ & -0.000 \\
\hline 71 & 1 & 1 & 1 & 1 & 1 & 1 & -PAH.OON & -140.000 & 156.000 & $\begin{array}{l}-.0100 \\
-0.0110\end{array}$ \\
\hline 72 & -0 & -0 & -0 & -0 & $-n$ & $-n$ & $-884.00 n$ & 0.000 & -156.0100 & -0.000 \\
\hline 73 & -0 & -0 & -0 & -0 & -0 & -11 & $-\angle A R . O 110$ & 0.000 & 156.000 & -0.000 \\
\hline 74 & -0 & -0 & -0 & -0 & -0 & -0 & $-\bar{C} B A .000$ & -12.1100 & -156.000 & -0.0001 \\
\hline 15 & -0 & -0 & $-n$ & -0 & -0 & -0 & -DBR.กOU & -72.000 & ISh.0ก0 & -0.000 \\
\hline 76 & -0 & $-n$ & -0 & -0 & -0 & -11 & -335.9010 & -132.000 & -119.901 & -0.000 \\
\hline 77 & -0 & -0 & -0 & $-n$ & -0 & -0 & $-335.90 n$ & $-13 ? .000$ & 119.900 & -0.000 \\
\hline 7A & -0 & -0 & -0 & -0 & -0 & $-n$ & -354.700 & -132.000 & -105.600 & $-0.00 n$ \\
\hline 79 & -0 & -0 & -0 & -0 & $-1)$ & -0 & -354.700 & -132.000 & 105.600 & -0.000 \\
\hline AO & -0 & -1 & -0 & -0 & -0 & -0 & -4.02 .600 & -73.000 & $-69.5 n 0$ & -0.000 \\
\hline AI & -0 & -0 & -0 & -0 & -0 & $-n$ & -4R?.600 & -72.000 & 69.500 & -0.000 \\
\hline ค? & -0 & -0 & -0 & -0 & -0 & -0 & -4012. की०ก & -11.600 & -69.500 & $-(1.000$ \\
\hline ค 3 & -0 & $-n$ & -0 & -0 & $-n$ & -0 & $-2 \Delta R .400$ & 0.000 & 0.000 & -11.000 \\
\hline 84 & -0 & -0 & -0 & $-n$ & -0 & -0 & - 40 ? . & $-11: 500$ & h9.500 & -0.000 \\
\hline 85 & -0 & -0 & -0 & -0 & -0 & -0 & -390.000 & 26.900 & -36.000 & -0.000 \\
\hline B6 & -0 & -0 & -0 & -0 & -0 & -0 & -379.1100 & 26.900 & $5 h .0 n 0$ & -0.0100 \\
\hline A7 & -0 & -0 & -0 & -0 & $-n$ & -0 & $-33 A . A 00$ & $11 ? .100$ & 10.010 & -0.000 \\
\hline BA & 1 & 1 & 1 & 1 & 1 & 1 & -3.34 .0110 & - IHO.OחO & 0.000 & -0.000 \\
\hline B9 & -0 & -0 & -0 & -0 & -0 & $-n$ & $-3 \times 4.0 n 11$ & 96.0110 & 0.0110 & -0.000 \\
\hline 90 & -0 & -0 & $-n$ & -0 & -0 & $-n$ & -3114.000 & 276.000 & 0.1100 & -0.000 \\
\hline 91 & 1 & 1 & 1 & 1 & 1 & 1 & $-3 R 3.001)$ & 27h. חก0 & 0.000 & -0.000 \\
\hline $9 ?$ & 1 & 1 & 1 & 1 & 1 & 1 & $-385.00 n$ & 276.000 & 0.000 & -0.000 \\
\hline 93. & 1 & 1 & 1 & 1 & 1 & 1 & $-38 n .0011$ & 276.000 & 1.000 & $-0.0 n 0$ \\
\hline 94 & 1 & 1 & 1 & 1 & 1 & 1 & -384.0011 & 216.000 & -1.000 & -11.0001 \\
\hline 95 & -0 & -0 & -0 & -0 & $-n$ & -0 & -3811.0010 & 195.000 & 0.000 & -0.000 \\
\hline 96 & -0 & $-n$ & -0 & -0 & -0 & -0 & $-35 \pi .0 \cap n$ & 557.000 & $0.0 .0 n$ & -11.000 \\
\hline 91 & -0 & -0 & -0 & $-n$ & $=0$ & $-1)$ & $-35: 0.000$ & 696.1100 & 0.000 & -0.000 \\
\hline
\end{tabular}


ELEMENT TYPE

NUMBER OF ELEMENTS =

20

ELEMENT LOAD CASE MULTIPLIERS

$\begin{array}{cccc}\text { CASE (A) } & \text { CASE (B) } & \text { CASE(C) } & \text { CASE(D) } \\ 1.0000 & -1) .0000 & -0.0000 & -0.0000\end{array}$

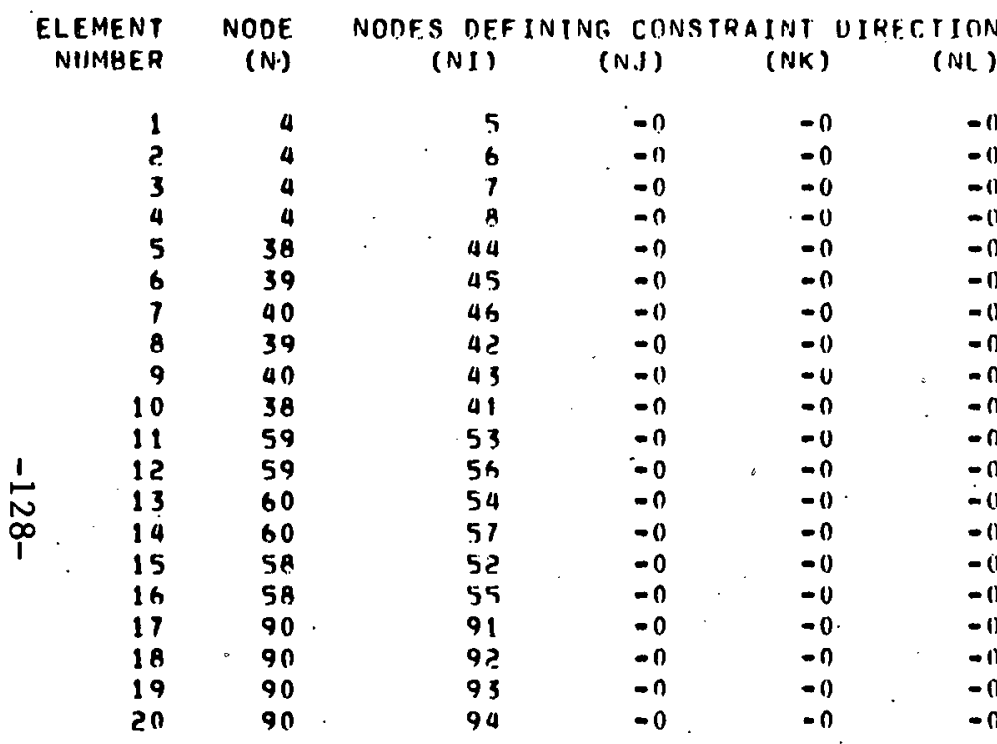

CONE

COIDE GENERATIIIN CONE (KN)

SPLACEMENT

SPFCIFIED

SPRING;

LI

-0
-0
-0
-0
-0
-0
-0
-0
-0
-0
-0
-0
-0
-0
-0
-0
-0
-0
-0
-0

-0
-0
-0
-0
-0
-0
-0
-0
-0
-0
-0
-0
-1
-0
-1
-0
-0
-0
-0
-0

-0.
-0.
-0.
-0.
-0.
-0.
-0.
-0.
-0.
-0.
-0.
-0.
-0.
-0.
-0.
-0.
-0.
-0.
-0.
-0.

-0 .

-0 .

$-n$

$-0$.

$-n$.

$-n$.

$-n$.

$-0$.

$-n$

$-0$

-0
-0
-0
-0
-0

-0 .

:

$.1000 E+11$ $-100 \mathrm{OE}+11$

$10 D U F+11$

IONOE+11

- Somprato

D. + HA

$5000 E+O A$

.1000E+ 118

$.10 \cap 1) F+0)$ ?

1 IOODE +OB

$.5000 \mathrm{E}+0$ A

$1000 F+11 B$

$5001)+$ +OA

inODE + Da

. 50onf t to

$1000 F+n A$

$-1000 E+11$

1000E + 11

- IONOE+11

. IOOOE + 11 
- NTROL I NFURMATIUN

NUMBER OF PIPE ELEMENIS

NUMBER OF MATERIAL SEIS

MAXIMUM. NUMAER OF MATERIAL

TEMPERATURE INPIIT POTNTS

$=t$

NIMMBER OF SFCTION PRAPERIY SETS $=7$

NUMBER OF BRANCH POINT NODES

MAXIMUM NUMBER OF TANGENTS

COMMON TO A BRANEH POINT

FLAG FOR NEGLECTING AXIAL

DEFORMATIONS IN BEND ELEMENTS (EQ.1. NF.GLECT)

MATEH JAL PROPERIY TA ALES

$\stackrel{1}{\stackrel{1}{N}}$

MATERIAL NUMAER = (i)

NUMBER OF

TEMPERATURE POINTS $=(1)$

IDENTIFICATION $=1$ SIATNLFSS SIEEL

1

10.00 29000000.0

.300

$.500 E-\cap a$ 
SEC TI ON PR OPERTY TAB.L.E

SECTION OUTSIDE NUMBER DIAMETER THICKNFSS FUR SHEAR

WEIGHT/ MASS/

$3.0000 \quad 0.0000$

144.000 36.000 48.000

12.000

$\begin{array}{ll}3.5000 & -0.0000 \\ 3.7500 & -0.0000\end{array}$

-0.0000
-0.0000

$-0.0000$

192.000

135.000

-0.00000
-0.0000

-0.0000
-0.0000

UNIT I:ENGIH

D E S C R I P T I O N

100.000

.4000
$.38 \cap 0$

$-n$.

. 200 OE +02

. 1 KAOE+0.3

0.

$.21 ? 2 \mathrm{t}+100$

S I

-0 .

-0.
-0.

$.4 ? \Delta 4 E+10$

0.

0 .

PI

PII

0 . 
BRANCH NODE
POINT NUMBER

$\begin{array}{ll}1 & 9 \\ 2 & 25 \\ 3 & 26 \\ 4 & 47 \\ 5 & 72 \\ 6 & 73 \\ 7 & 89\end{array}$

E L E Y E N T

$X$-DIRECTION GHAVITY $Y$ OIRECTION GRAVITY Z DIRECTION GRAVITY THERMAL OISTORTION PRESSURE DISTOETIUN C A S E M IJ L T I PL I E R S

$\begin{array}{rlll}\text { CASE A } & \text { CASE B } & \text { CASE C } & \text { CASE D } \\ 1.000 & -0.000 & -0.000 & -0.000 \\ 1.000 & -0.000 & -0.000 & -0.000 \\ 1.000 & -0.000 & -0.000 & -0.000 \\ -0.001 & -0.000 & -0.000 & -0.000 \\ 1.000 & -0.000 & -0.000 & -0.000\end{array}$

它. 
PI PE ELEMENT INPITT DATA

ELEMENT ELEMENT NODE NODE MATL. SECTION

RFFERENCE INTERNAI. TENPFKATURE PRFSSILF RADTUS) (1HTRO PUth

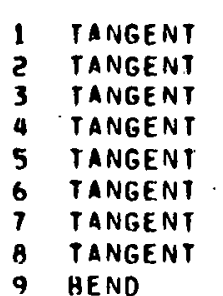

( 60.0011

10 GEND

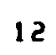

11 BENO

12 TANGENT

13 TANGFNT

14 BENO

15 BENO

16 TANGENT

17 TANGENT

1 A BENO

19 REND

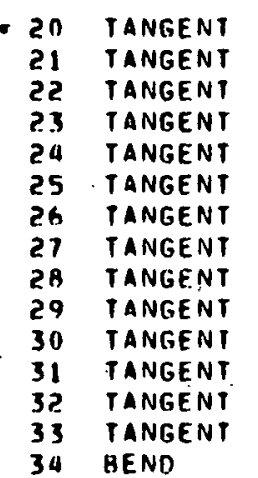

$-0.001$

2$$
\begin{aligned}
& 2 \\
& 2 \\
& 2
\end{aligned}
$$$$
2
$$

$19 \quad 21$

$\begin{array}{ll}51 & 53 \\ 50 & 39\end{array}$

$2.2 \quad 21$

23

24

25

$-0.00$

$-11.00$

$-0.00$

$-0.00$

-1).0
$-0.00$ $-0.1)$ n

$-1.00$

$-0.00$

$-0.00$

$-0.00$

-0.00
-0.010

$-0.00$

$? 400.00$ 2400.110 2400.00 2400.011

$$
-0.00
$$

2400.01

? 400.00

$2400.0 n$

2400.00

(II)

?400.00

(TI) 1

$240 n .00$

$61.000)$

(TI) (

2400.00

2400.00

60.0001

$24000^{n n}$

(II)

(I) 1

$402.600)(-132.000)($

2400.00

5400.00

جunn

60.000)

(TI)

$-0.0000$

$-0.0000$

$-0.01100$

$284.000)(-132.000)($

$.2400 .0 n$

(II) (

$00.000)$

24.00 .00

2400.00

$-0.00$

2400.00

24011.00

2401.00

2400.00

2400.00

2400.00

5400.00

500.10

?

?.400.011

3400.110

2400.00

(TI)

$-0.0000$

$-0.0000$

.0000

$-0.0000$

$403.600)($

$-0.0000$

$-0.0010$

$-0.0000$

$-0.000 n$

$-0.00110$

$-0.0000$

$0.000)($

$-0.0000$

$-0.00000$

$-0.0000$

$-11.0000$

$-0.0000$

$-0.0000$

$-0.0100$

$-1) .0000$

$-0.0000$

$-0.0000$

$-0.0000$

$-0.0000$

$-0.0000$

$\operatorname{lng} .0(10)($

$-0.000 n$

$-0.0(100)$

$-0.0000$

$\therefore 0.0000$

$-0.00110$

$-0.00110$

$-0.0001$

$-0.01000$

$-0.0000$

$-0.0000$

$-0.0000$

$-0.0000$

$-0.00110$

$-0 . \operatorname{col} 00$

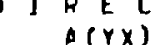

(I)

UHDINATE)

C)!

$I N+S$

N(Di)E INPIIT 23- (WAILL

$-0.0000$

$-0.0001)$

-0.01100
-0.0000

$-0.1100$

$-0.0000$

$-0.0000$

$-0.0000$

$-69.500)(.1000)$

$0.000)(.1000)$

IC

$69.5(10)(.1000)$

IC.

$-0.0000$

$-0.00110$

II

11

$-0.9 .500)(.1000)$

$69.5001(.1000)$

Ir.

$-132.0001)($

$-0.0000$

$-0.00110$

$-156.000) \quad 1 . .10(10)$

$-0.0001$

$-0.0000$

$-0.0000$

$-0.00(10)$

$-0.0000$

$-0.0 n \pi n$

$-0.0000$

$-0.000$

$-0.0000$

$-0.01100$

$-0.0 n n i$

$-0.000)(-106.000)(.1000)$ 


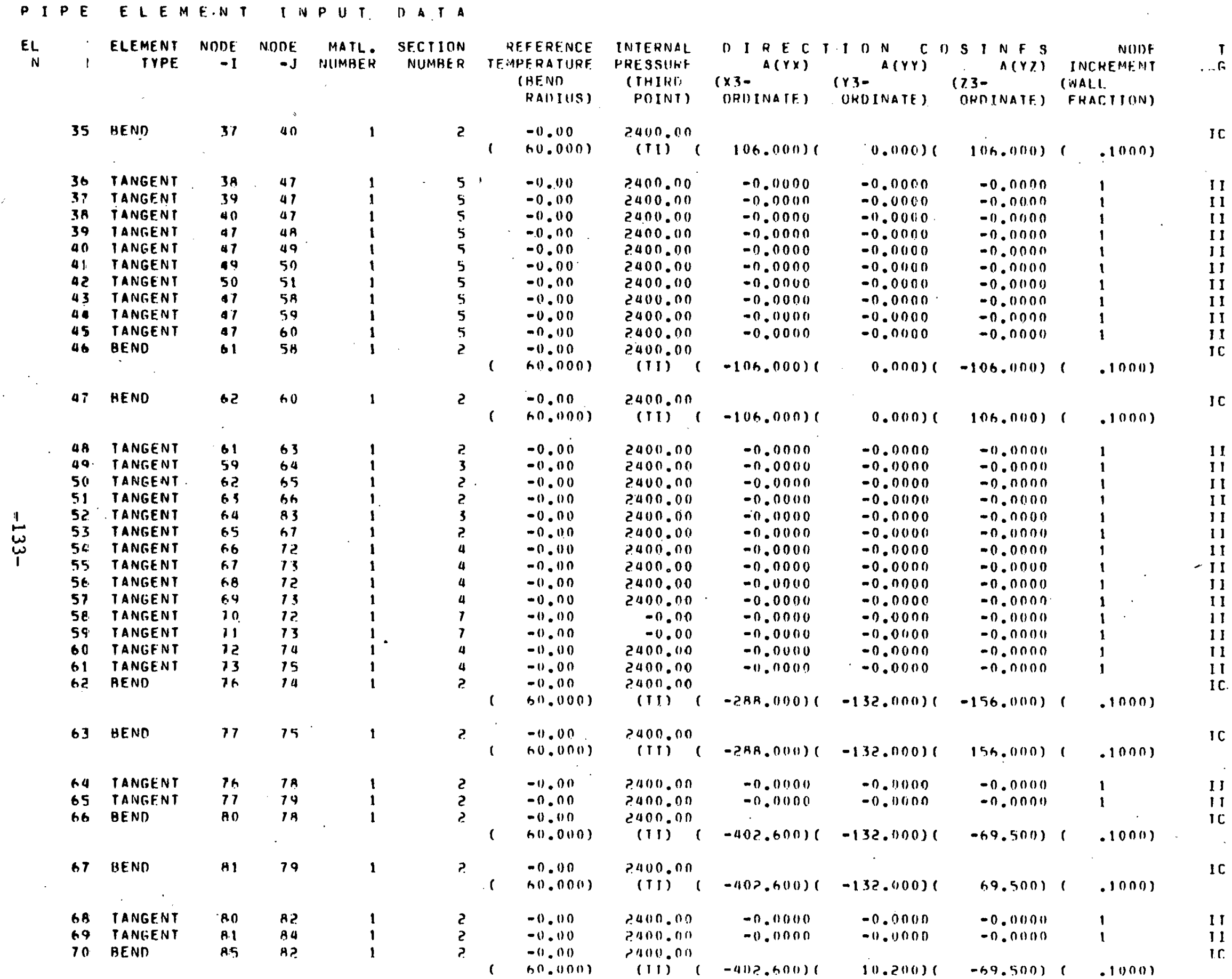


PIPE ELEHENT INPUT DAIA $\begin{array}{rrrrrr}\text { ELEMENT } & \text { ELEMENT } & \text { NOOE } & \text { NODE } & \text { MATL. } & \text { SECTION } \\ \text { NUMBER } & \text { TYPE } & -I & -J & \text { NIMMEER } & \text { NIMMHF. }\end{array}$ TYPE

-I
NIIMHF.

DEFERENCE. (HENI) PRESSIJRF (IHTRN

QAD IUS)

PUINT)

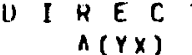

$\begin{array}{llll}E C T H & 0 & C \\ (Y X) & & A(Y Y)\end{array}$ $(x 3-$

$3 \quad-0.010$

$(117.900)$

2400.00

$(11),-303.4001)$

ORDINATE)

(i) I NE S

NOI)E

A Y T) INCHFMFN (WAI.L.

INPIIT

$\begin{array}{llll}71 \text { BEND } & \text { BT } & \text { BY } & 1 . \\ 72 \text { BEND } & \text { Bh BA } & 1 .\end{array}$

-0.000
$010.000)$

2400.00

(IT) $(-402.600)(10.200)($

$0.000)($

a. 000$)($

$.1000)$

$-0.00$

24011.00

2400.00

2.400.00

$-0.00$

2400.00

3400.00

ว400

2400.00

$-0.0000$

$-0.0000$

$-0.0000$

$-0.0000$

$-0.0000$

$-0.0000$

-0.0ura

$-0.0000$

$-0.01100$

$-0.0000$

$-0.0000$

$-0.0000$

$-0.0000$

$-0.0000$

- 0.0ua

$-0.0000$

$69.500)(.01000)$

$-0.0000$

$-0.0000$

$-0.0000$

$-0.0000$

$-0.0000$

$-0.0000$

$-0.0000$

$-0.0000$
Ir

Al:

I I

II

$\stackrel{1}{\omega}$ 
C ONNECTI INS...

5
3
4
5
6
7

$\begin{array}{ll}9 & -4 A T \\ 25 & -20 A T \\ 26 & -21 A T \mathrm{~J} \\ 47 & -36 A T \mathrm{~J} \\ 72 & -54 A T \mathrm{~J} \\ 73 & -55 A T \mathrm{~J} \\ 89 & -73 A T \mathrm{~J}\end{array}$

SAT I
$2.2 A T$ I
$23 A T$ I
$-37 A T J$
$-5 A A T J$
$-57 A T J$
$-74 A T J$

GAT
SAAT I

5.5AT I

- BAAT J
-ShAT J

-soat j

-75atj
$7 \mathrm{AT} I$
5GAT I
$27 \wedge \mathrm{I}, \mathrm{J}$
39ATI
GOAT 1
GIA] I
GIATI

A A T I

ONONE

MOAT 1

DNONE

ONONE

77 ATI

\section{ONONE}

ONINEF

ONONE

$43 A$ I 1

ONIINE

ONONE

ONONE

ONINF

DNONF

ONONE

प MAT I

ONONE

ONINE

ONONE

$45 A T I$
ONIINF

UNONE ONOTEE 


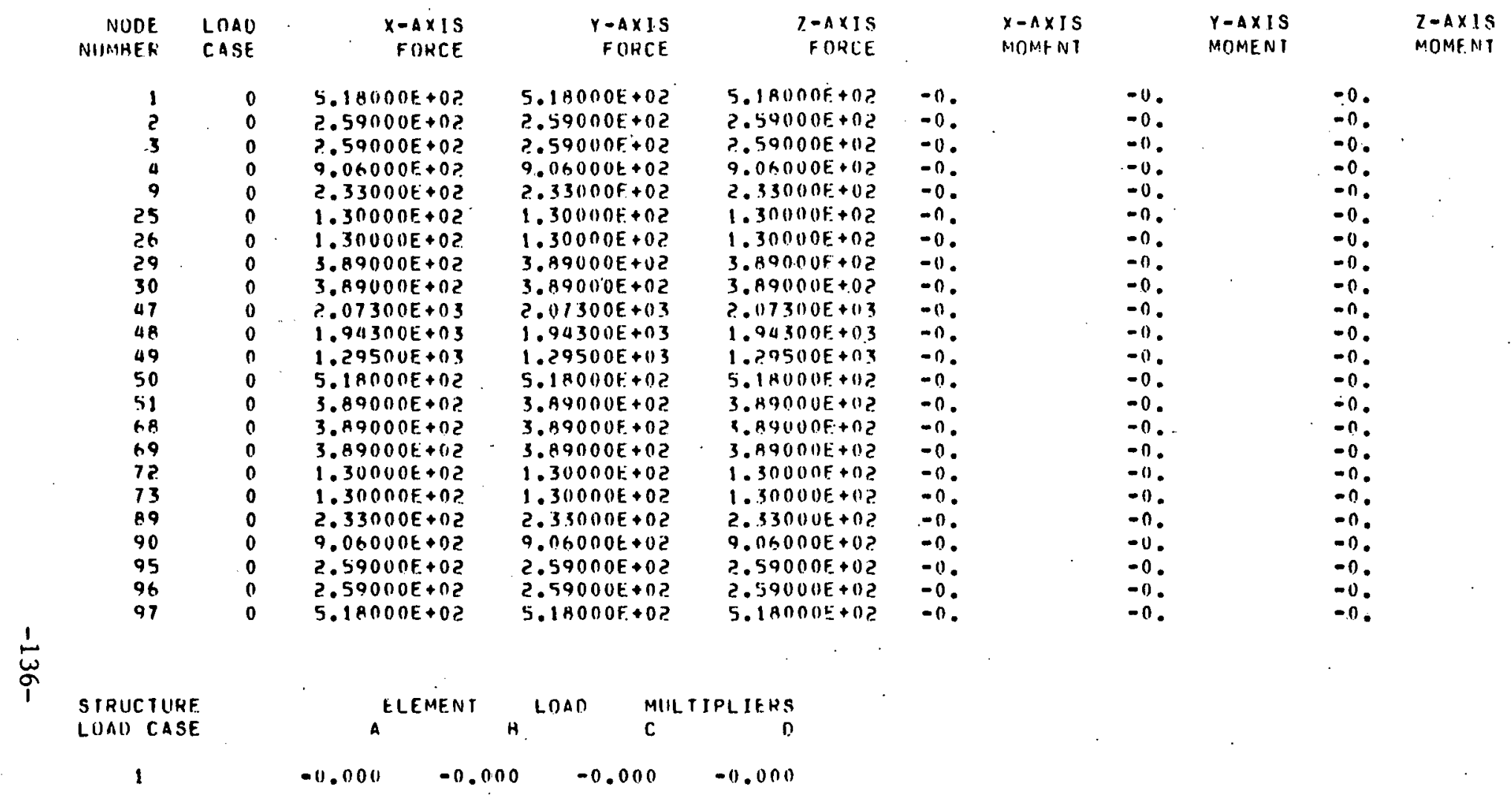


PRINT OF FREQUUENCIES

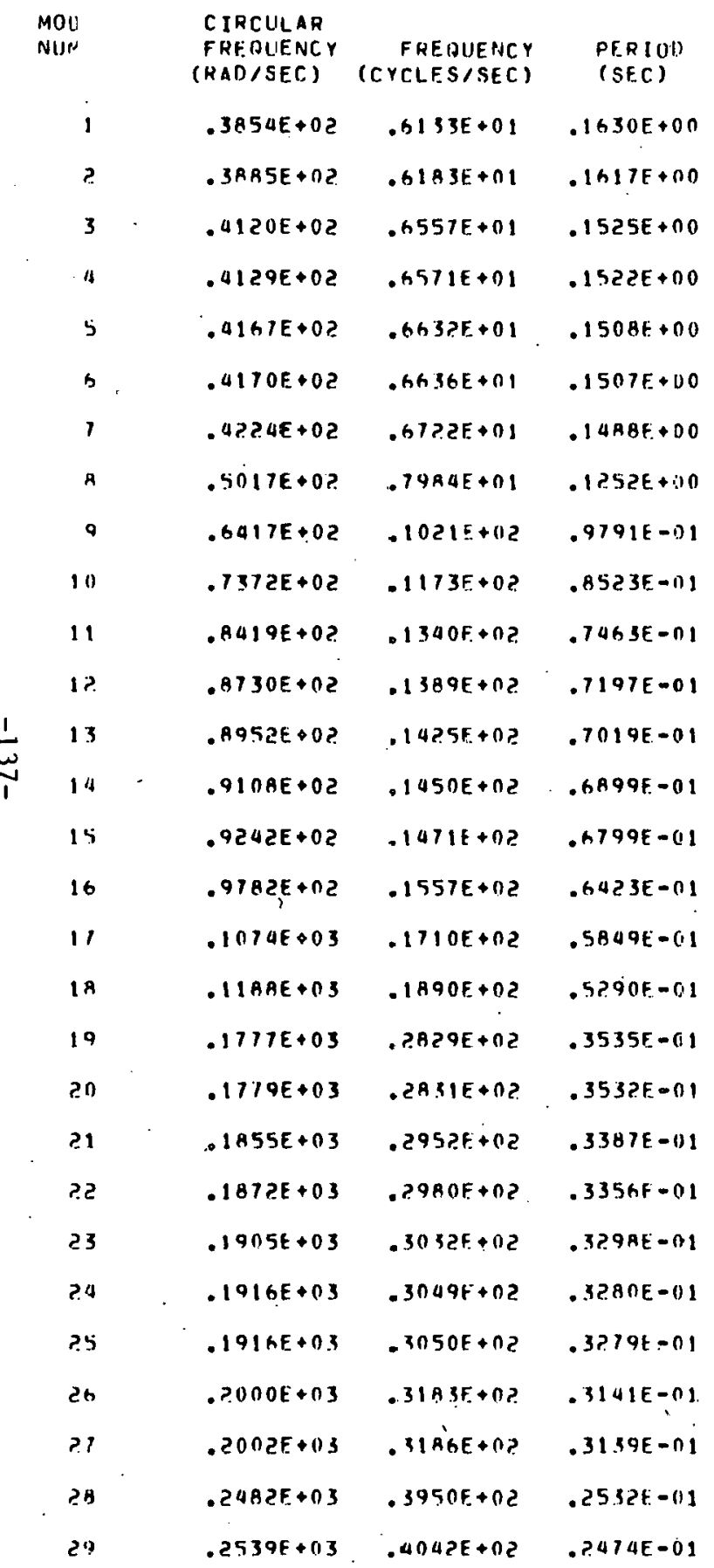


EIGENSOLUTION TIME L OG

E.IGENSOLUTION $=$ AR.60 PRINTING = 03 
"OUE AMIALER 1

FRE HIV HCY O 0.13318 . HZ

EIGEIUECTIIRS WUHMALIZEH IO A JIIT MASS MAIHIX

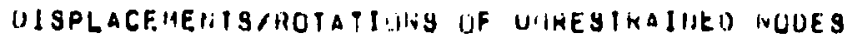

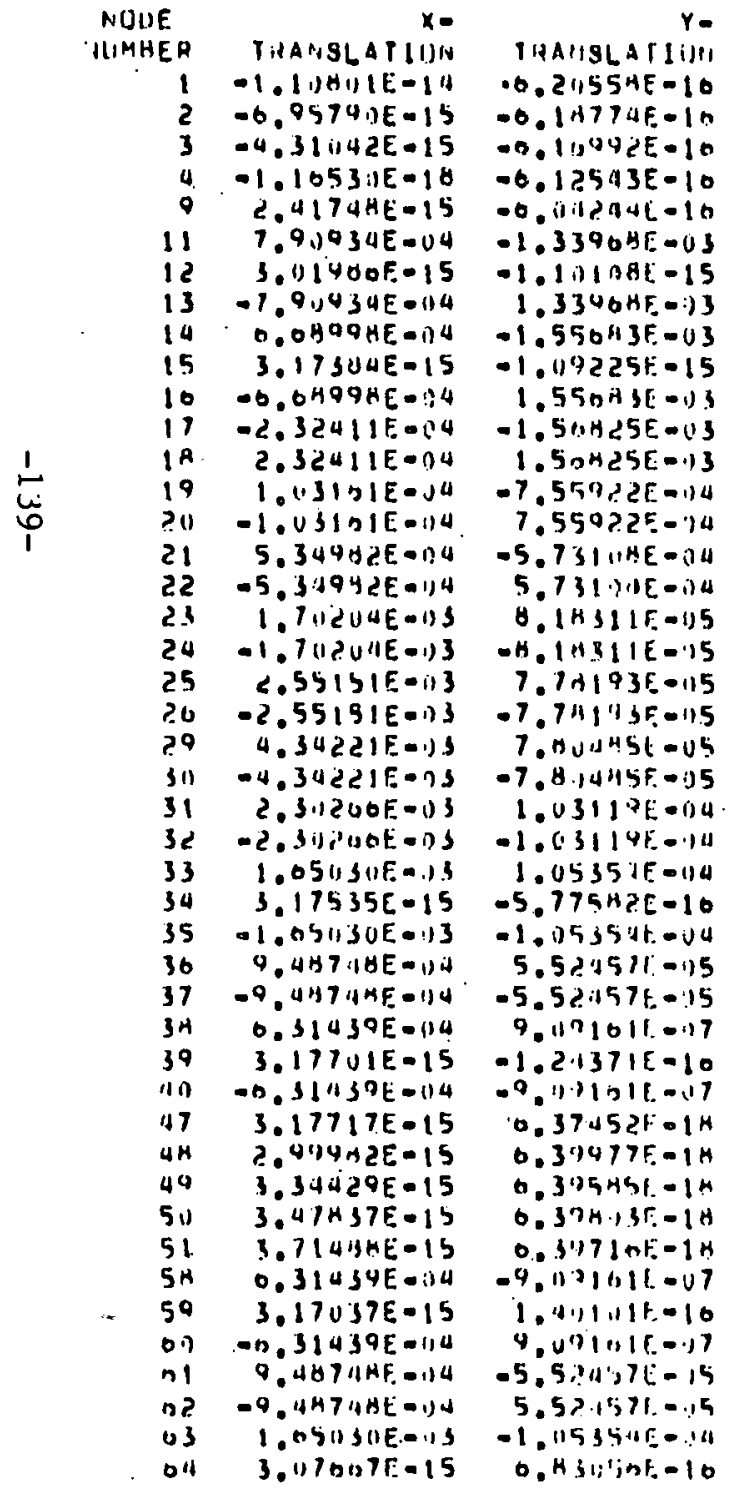

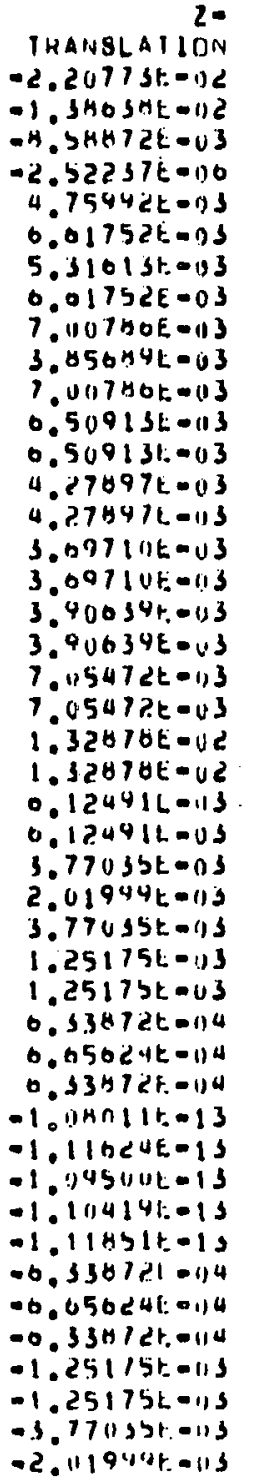

\section{hutATSUn} $-5.53590 E-U 5$ $-5.35219 \mathrm{E}-05$ $0.99945 E \cdot 05$ $-3.70104 E-05$ $-2.340456-05$ $2.39043 E-05$ $-2.36945 E-05$ $-2.35584 E=115$ $-2.30945 E-05$ $2.23070 E=116$ $-1.13269 \mathrm{E}-05$ $2.2367 \cup t=116$ $1.05299 E-05$ $1.052996=05$ $1.6254116=05$ $1.6254 U E=05$
$1.70071) E=05$ $1.70071) E=05$
$1.70070 E=05$ $4.35250 \mathrm{k}=05$ $4.35256 E=05$ $4.5035 \mathrm{HE}=0 \mathrm{~S}$ . $5035 \mathrm{AE}=0$ $4.5035 B E=05$ $4.927526=05$ $4.92752 E=U 5$ $4.433 K 8 E=05$ $4.4330 \mathrm{HE}-115$ $2.0723 \mathrm{HE}-115$ $-0.04200 E-116$ $2.67238 \mathrm{E}-115$ $9.15875 E-116$ - 15 t5t-vo $9.15 B 15 E-0$ 5. H63U3E-11H $-4.05717 E-U A$ $5.86363 E=00$ -7.3749 ILE-19 4.7 HSSUE- 18 - 5 . 4 H $39 \%$ E 18 $-7.00525 E-1 \mathrm{H}$ - BDSLSE- 1 H *.057lut:- I $-5.803 n 3 E=110$ $9.057176-115$ - S. HO3HSE-UA -4. 15H75t-VO $-9.15 \Delta 75 E-110$ $-2.07258 \mathrm{t}=05$

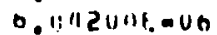

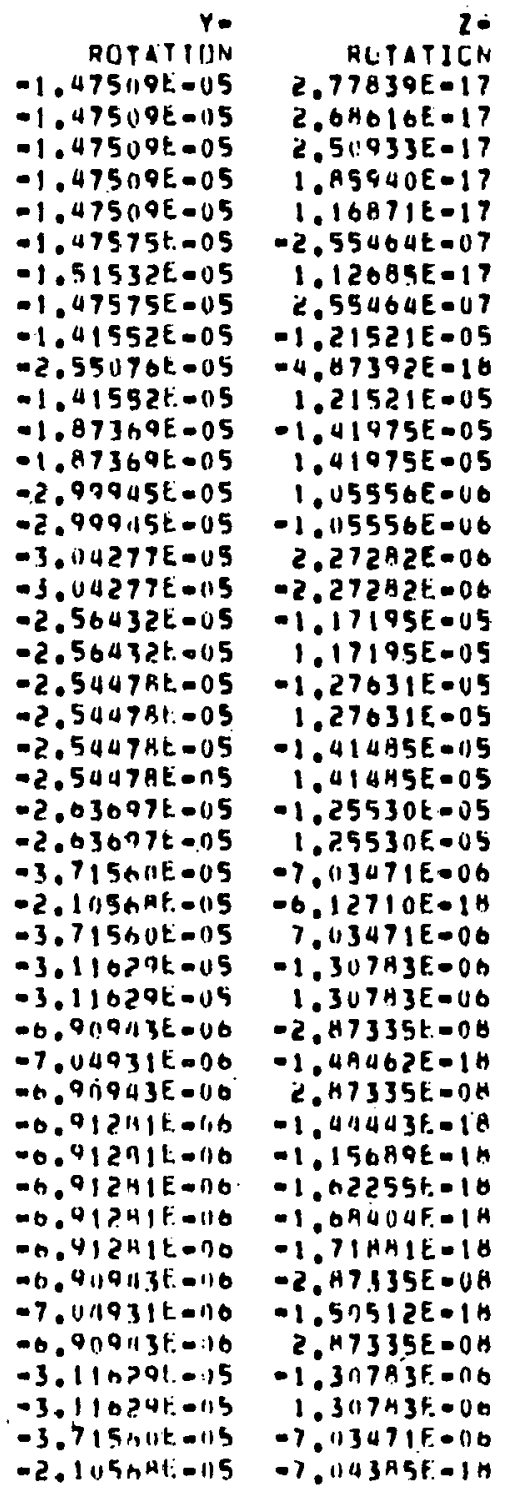




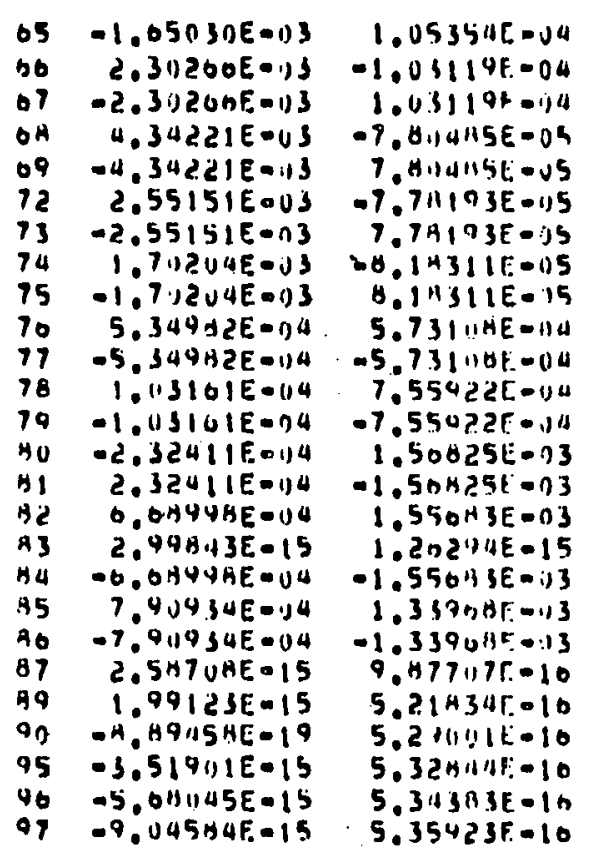

$-3.77035 t .013$ 00.124916005

$0.12441 t .0 \mathrm{~s}$

$-1.1267 \mathrm{dE}-02$

$-1.3207 \mathrm{ar}-102$ $-7.115472 \mathrm{t}-0 \mathrm{~J}$ $-7.115472 E-03$ $-3.90059 t-03$ $-3.40634 \mathrm{E}-0 \mathrm{~S}$ 3.071 ucous 3.0971ut. $-3.69710 \mathrm{r} .03$ - $4.27847-103$ $-4.27097 t-03$ $-0.5 .1913 t-1) 3$ $-0.50913 \mathrm{E}-1,3$ $01.190700 \mathrm{t}=0 \mathrm{~s}$ $-3.65004 \mathrm{k}-11 \mathrm{~s}$ -? - viginctions - .017521 .010 - $0.0175 \mathrm{de}-11 \mathrm{~s}$ $-5.31013 t=0 \mathrm{~s}$ $-4.75992 E-U S$ $2.52237 E-10$ $A .58872 \mathrm{E}-U 3$

$1.39030 t-112$ $2020773 t=02$
$-2.07238 E=05 \quad-3.71560 E=05$ $0.43385 E-U 5$ $-4.4334$ $-4.02752 E-U 5$ $-4.92752 E-U S$ $0.5035 B E=05$ $-4.50358 E-05$ $-4.35250 \mathrm{k}-0 \mathrm{~S}$ $0 ., 35250 E=01$ $-1.70070 E 0 U 5$ $-1.700705005$ -1.7025 - 1.025 UDE-05 $-1.05294 .005$ $-2.23670 E-0 h$ $1.13209 k-05$ $-2.230701000$ 2.36945E $=05$ $2.36945 E=05$ $2.35504 t=115$ 2.390435 .005 $3.7004 U E=1) 5$ $4.49985 E=115$

$5.35219 E-U 5$ $5.53590 E=05$ - $1,25530 E=05$ $-2.63697 E=05 \quad 1.25330 E=05$ $-2.54478 E-05-1.41485 E-05$ $-2.54478 E-05$ 1.41 $4 A 5 E-05$ $-2.54478 E-05 \quad-1.27631 E=05$ $-2.54 A 7 A E 005 \quad 1.27631 E=05$ $-2.56432 E-05-1.17105 E-05$ $-2.56432 E-05 \quad 1.17195 E-105$ - 3.04a77E-05 2. -3.04277E-05 Ca. - 30 . $-2.999115 E-115 \quad 1.015556-110$ $-2.09945 E=05 \quad-1.05350 E=00$ $-1.473698-115$ - $1.41975 E=05$ -1.873692005 1.41975E-05 $-1.41552 E-05$ - $1.21521 E-05$ $-2.55070 \mathrm{0} 05 \mathrm{~S}-4.70955 \mathrm{E}-10$ -1.41552E-05 l.21521E005

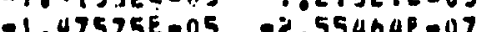
a $-1.47575 k=05 \quad 2.55404 E=07$ $-1.51532 E-05$ Q.53689E-18 $-1.47500 E-115 \quad 4.33228 E-18$ $-1.47509 E-1,1.51804 E-17$ $-1.47509 E-05 \quad 2.04865 E-17$ $-1.49509 E-05 \quad 2.19302 E-17$ $-1.475119 E-115$ 2.20831E-17 
"(i)E NI:MHEH 1

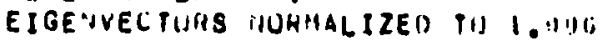

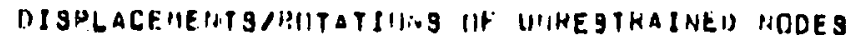

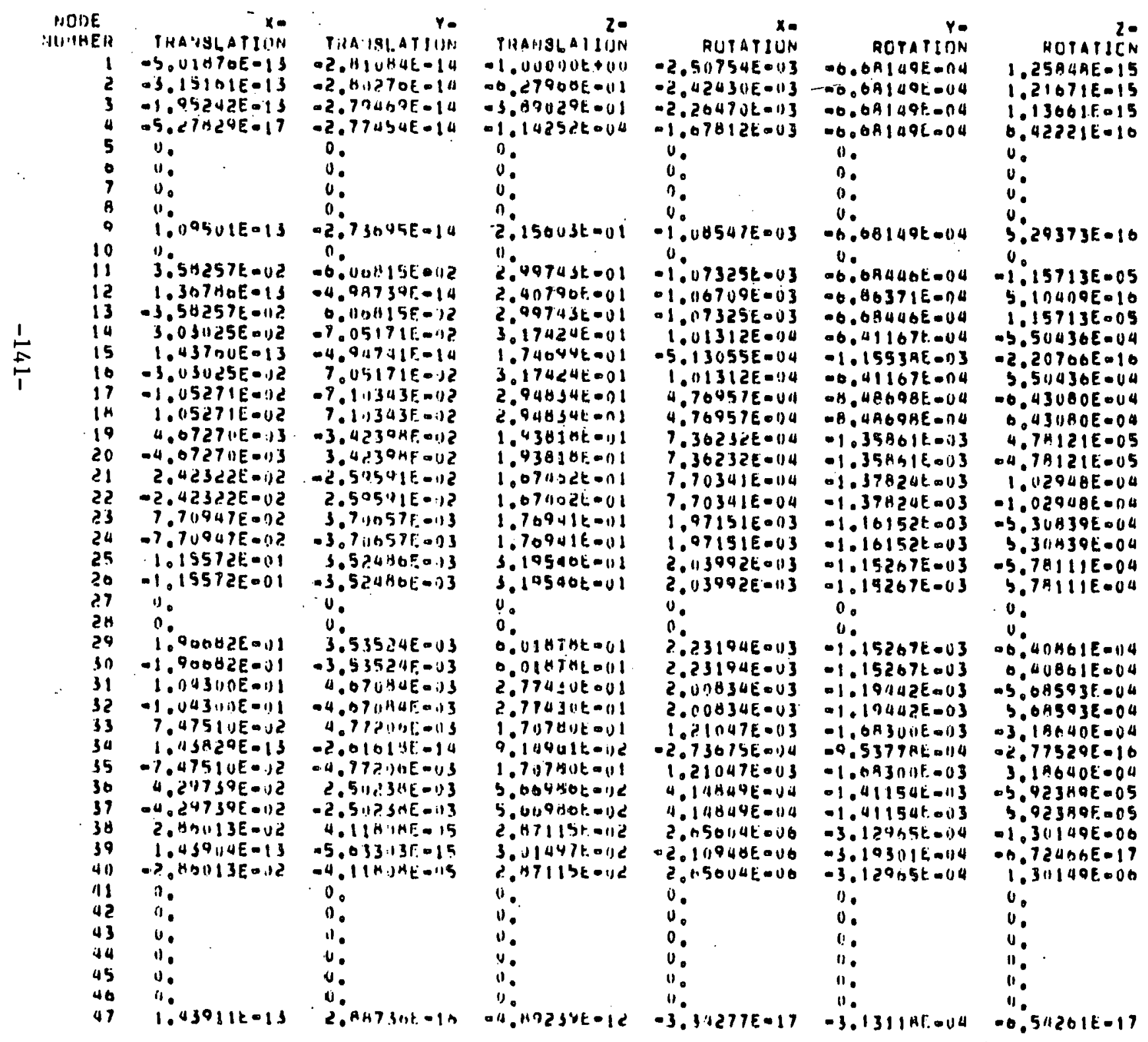




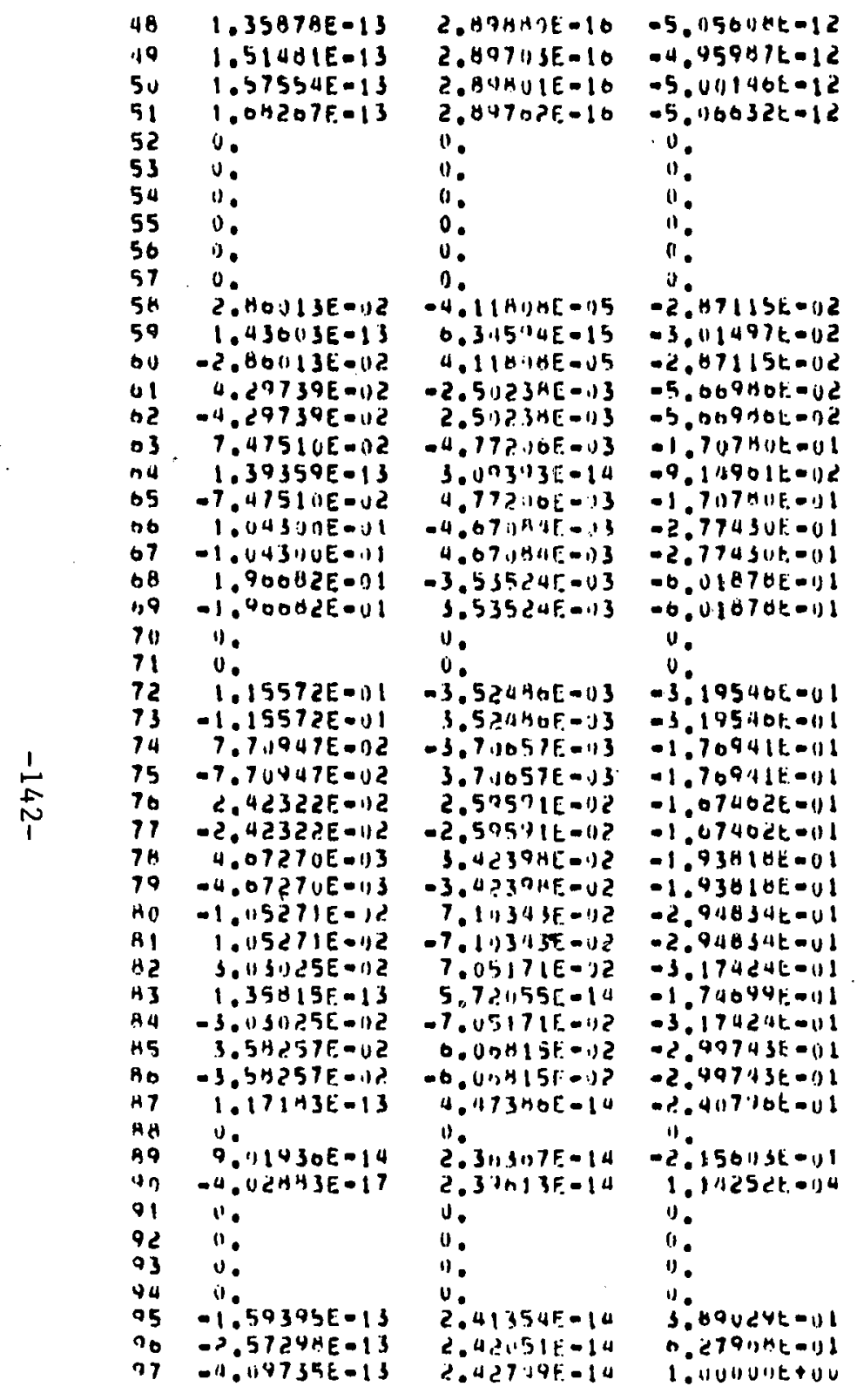

\begin{tabular}{|c|c|c|}
\hline U UEE $=6$ & 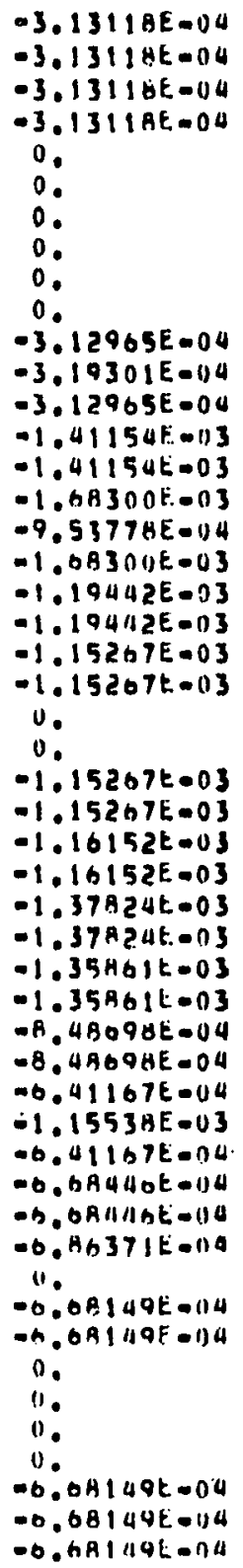 & $\begin{array}{r}-1.30149 E=06 \\
-6.01751 E=-17 \\
1.30140 E-00 \\
-3.92389 E=05 \\
3.92389 E=05 \\
-3.18040 E=04 \\
-3.19054 E=16 \\
3.18640 E=04 \\
-5.68593 E=04 \\
5.68593 E=04 \\
-0.40861 E=04 \\
0.40861 E=04\end{array}$ \\
\hline
\end{tabular}


TIJISE. N JMLIER

FHEIUIE ICY O.1B341 HZ

E.JGE'NVECTOIIS NIJHMALIZEIS TU A WIIT HASS MAIHIX

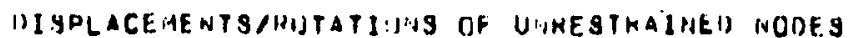

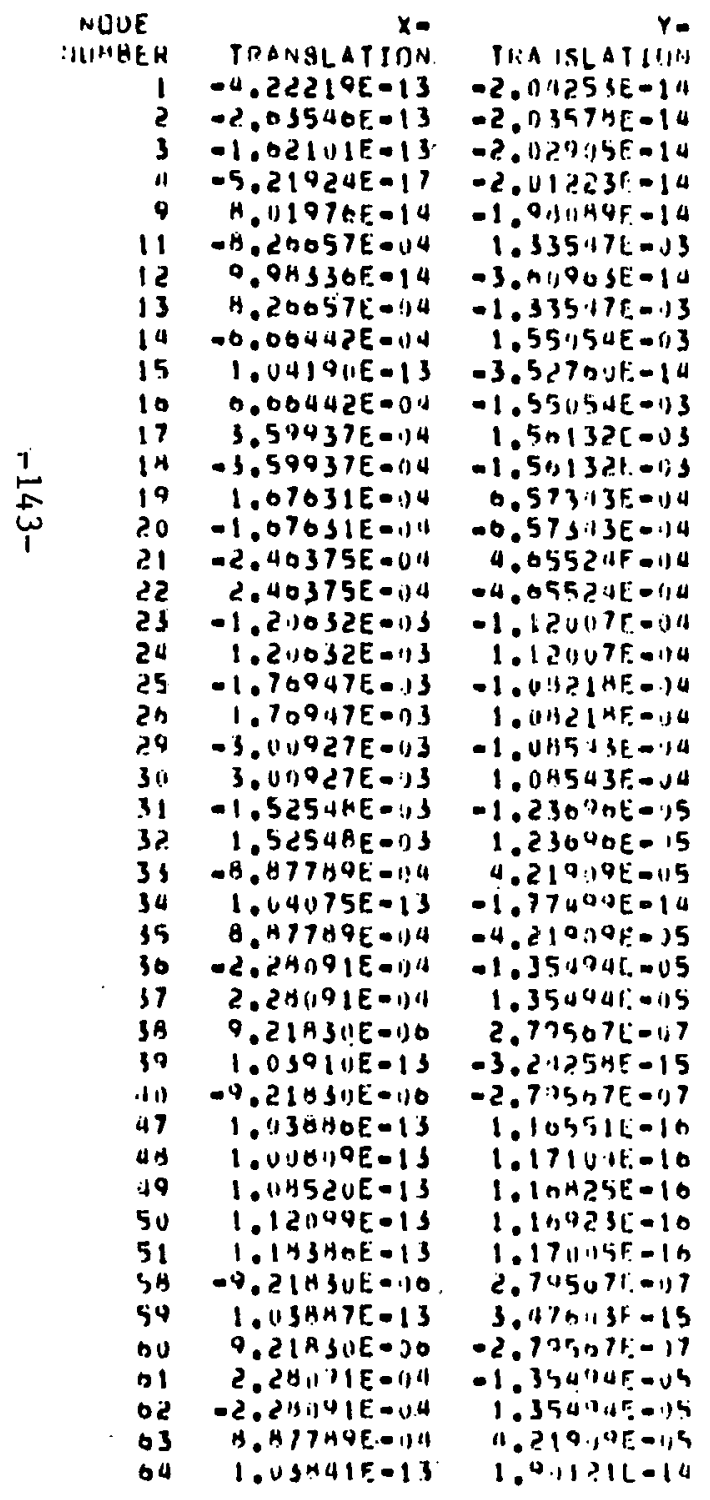

THANSLAIJUNA RUIATIUN

2.24141t-02 5.62509E-115

$1.110045 \mathrm{~L}-0 \mathrm{C} \quad 5.43545 E=05$

$0.7 n 515 L-113$

$2.01351 t=100$

$.4 .75170 t-113$

-0.014 ibtals

$-5.26037 t-03$

-0.014 ust-os

$-6.98848 k-113$

$-3.70425 k-103$

$-0.9864 \mathrm{HE}=11 \mathrm{~S}$

-0.44 (4I,E-IIS

$0.441406-0 S$

- 4.1084 tiE- 113

$-4.10846 E-113$
$-4.19 H C Q E-113$

-5.0347 Ut-us

-3.0397 iut $-u 3$

-3.082 i15t-11

$-0.470511=03$

- 0.970511 .003

$-1.3117116-n E$

$-1.311711 L-n 2$
$-1.31170 L=02$

$-1.3117 U L=12$
-6.067 UIL $=03$

-6.00711 Ut-113

$-3.79754 E-1 ! 3$

$-1.44640 \mathrm{OL}-1,3$

-3.77754 LeUs

$-1.421 \cup 7 t-05$

$-1.421 \mathrm{~V} / \mathrm{L}-1) \mathrm{S}$

$-4.345 \cdot 10 f-1.14$

$-9.383536=04$

$-4,311540 t=04$

$-9.410 j 0 t=114$

-9. BH $2006-n 4$

-4.504 sul- 14

$0.06445 t-114$

4. M2905t.-14

$-4.3115406-116$

$-9.343133 t .014$

$-4.315 .106-1] 4$

- 1 c

-3.7775 ist.e1s

$-1.440101-115$
$5.07167 E-05$

$3.73572 E=05$

$3.73572 E-05$

301 THE- 05

$2.34412 E=15$

$2.30178 \mathrm{E}-05$

$1.09263 E=05$

0.413318006

$1.14348 \mathrm{E}=05$

I $14348 E-05$

1.

$-1.77091 E-05$

$1.17041 E-U 5$

$\left.0.27880^{\circ} \mathrm{L}=1\right) 5$

$4.27884 E-05$

$0.42459 E-05$

C4. $42459 E=05$

$405397 E-115$

TE-

A. AS397E-US

- . $35497 E-U 5$

$167 E-05$

S.81310E-1)6

$-2.55007 E-05$

$-6.25324500$

- 4.2532450 .00

. 1)38 L UE OO

I.25857E-UE

(I)

$24724 E-1) B$

$5.958 \cup 2 E \cdot U$

$-7.77847 E=U$

$-4.350075-28$

$-1.11 ? 352 E-U 7$

- H. IISBIUE-UE

I.25HS7E-11A

- $H$. IISBIOE=IJ

- H. $25324 \mathrm{CE}=110$

$4.25324 E=110$

- 2.95110 It- 15

S. MIBISt) In
RIjThI IIIN $1.54534 E-05$ $1.54534 E_{-05}$ $1.54534 E-115$ $1.54534 E-115$ $1.54534 k-1) 5$
$1.54460 E-05$ $1.5 A 839 E-05$ $1.511464 E-05$ $1.40371 E-05$ $2.51204 E .05$ $1.40371 E=05$ $1.80923 \mathrm{E}-1) 5$ $23 E-0$ 2. GAUAIE-115 2.8 A A I E. 115 2. 9 ? $A 31 t=05$ $2.92831 E-05$ $2.49 B A 1 E=05$ 2. $498 A 1 E-U 5$ 2. 49ARIE-US 2. 1 SA 2. 483 BSE- 05 2. WA3A5E - 0 $2.4 B 3$ ASE 05 $2.57418 E-05$ $2.57416 E=05$ $3.54529 E-115$ $1.03779 t=115$ $3.54520 \varepsilon-U 5$ $3.545298-05$ $2.75942 k-0$ $2.75942 t .05$ $4.9 n 917 \mathrm{~K}-116$ $1.02656 t-117$ 4.969174 .116 4. Uก732E- 16 $4.00730 t-16$ $4.00751 t-10$ 4.0075at-10 . 0075pte-16 allosatiolo $0.96917+$ mil 1. h $2 n 56 t-07$ 4.4 पी 1760138 -2.759 11 2E- -115 $-2.759112 t-115$ $-3.545291-115$ $-1.437700-115$
ROTATICN 1.065 h6E-15 $1.02598 E-15$ $4.499405-16$ $0.70723 E-16$ 3. 81 पA 3E- 10 $2.79810 E-07$ $3.66997 E-16$ $-2.79810 E=07$ 1.3881 UE-U5 $-1.7878 A E-16$ -1.3840 LE=05 $1.02242 E-05$ $1.62242 E=05$ $4.67629 E=07$ $0.67629 E 007$ $-2.59067 E-06$ $2.59667 E 006$ $7.79551 E-00$ $-7.79551 E=06$ - OIOIOE-OD - O19 $4.79506 E-00$ $-4.79506 E-06$ 4.83 B $6 E=00$ $6.83 B 16 E=00$ $7.09931 E-06$ -2.04425E-16 $\rightarrow 09931 E-06$ 305915006 - $2.44591 E-06$ $4.02572 E-O B$ $-3.76030 E-17$ - $4.02572 E-0 R$ $-3.54453 E-17$ - $252023 E-17$ $4017510 E-17$ - 1 H 단 $-4.5112375-17$ $4.112572 \mathrm{E}-1) \mathrm{A}$ $3.77733 E-17$ $4.02572 E=48$ - ट 4 H5915:-00 2. 4 H59 It 00 $-2.2 \cdot 224 F-1 n$ 
$-0.677 H 9 E-1 A \quad-4.21909 E-115$ $\left.1.52546 E-03-1.23 n^{4}\right) F_{0}-115$ $-1.52546 E-U S$

3.0114 $7 E=113$ -

$-3.77754 t-03 \quad-2.55007 E-05$ $-4.35497 E-U 5$

$1135=00$

0.007 UUt-0 0.354978005

$-3.011021 E-03$ I.11RG43E-JU

$1.3117 U t-02 \quad-4.85347 t-05$

$-1.10047 E-03$

- D. DB? IHE-11G

-1.3117 UE-UE

$1.206325 \cdot 113$

$-1.120170=0$

$-4.42859 E 005$

$-1.23 h 32 E-03$

$-3.40 \$ 7550114$

$-1.07631 E-04$

1. 12 VI $78=114$

I.: 03

$-4.42059 E-09$

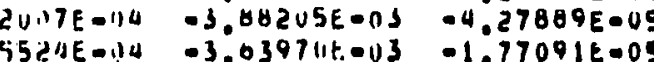

$-4.65521 \mathrm{ME}-? 0$

$-3.039701-03$

$-1.770918$

$0.57313 E-34$. $1.19840 \mathrm{H}-03$

$-1.77091 E-05$

$1.07031 \varepsilon=i)$

$-3.59437 E-U 4$
$3.54937 E-V 4$

$0.57343 E=114$

1003248045

$3.54937 E-U 4$

$1.511 .32 E-1\}$

- 1 . 2 DUMr. OS

$-1.69324 E-U 5$

$0.00442 E-114$

$1.551154 E-03$

-1.1434 HE 0

$-1.1434 \mathrm{HE}-\mathrm{US}$

$-2.91331 E-06$

$-0,001142 E=04$

1.

$-3.70425 \mathrm{~L}=03$

$4.200575=114$

$-6.20057 E-114$

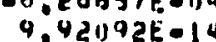

$1,9544 \forall E-14$

$-5.1111420-11$

$-1.011112 E-13$

$1.09263 E-U 5$

$-2.913316 .116$

$2.36176 E \cdot 05$

$2.3617 \mathrm{BE} \cdot 05$
$2.34412 \mathrm{0} .05$

$3.937996-11$

$001405 t^{0}$

$-0.014115 t-05$

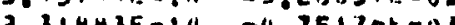

$-4.75170 \mathrm{t}=13$

2. $344656=05$

3.735726005

2.01351

5.1171876005

$2.37533 E-11$ O. $711515 t-03$

$5.3 H 3 ? 4 E-10$

$1.40045 t-112$

$5.43545 E-05$

$-4.145+2 E-13$

2.3ग115E-14

$2.241416=02$

$-3.545296 .05$

S7OIBE-115 -6.83816E-06

$0.83816 E-06$

-2.483 HE.05 - $4.79506 E-00$

-2.483 ASE-05 प.79506E-06

$-2.483 A 5 E-05 \quad 0.81910 E-00$

-2.433 A5E $=15 \quad \square .81919 E=06$

$-2.09 A$ AIE-O5 $-7.79551 E 006$

$2.49 B A I E=05 \quad 7.79551 E=00$

$0.92331 E=05 \quad 2.59667 E=00$

$-2092831 E-05$ - $2059667 E-00$

- 0 OOUAIE-OS

200ARIE-05

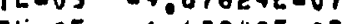

- 1 .

$-1.011923 E-05 \quad 1.022112 E-05$

$-1.00371 E-05$ - $1.38810 E-05$

$-2.502 n O E=05-1.94297 E-16$

$-1.40371 E-05$ I.3H8IOE-05

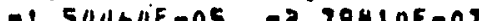

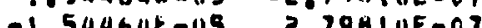

- I.

1. 1 AajoE-05 $3.03378 E-10$

$-1.54534 k=05 \quad 3.76991 E-16$

$-1.54534 E .05 \quad 0.04906 E-10$

$-1.54534 E=115 \quad 4.41751 E-10$

$1.54534 \mathrm{E}=115 \quad 1.01713 E-15$

$1.05049 E-15$ 
MOIJE N'JMIIER

E]GEIVECTHAS NURMGLIZEN TU 1., UU:I

i) SPLACFINENTSAQITATIIINY OF WIKESTHAIHEI) NODES

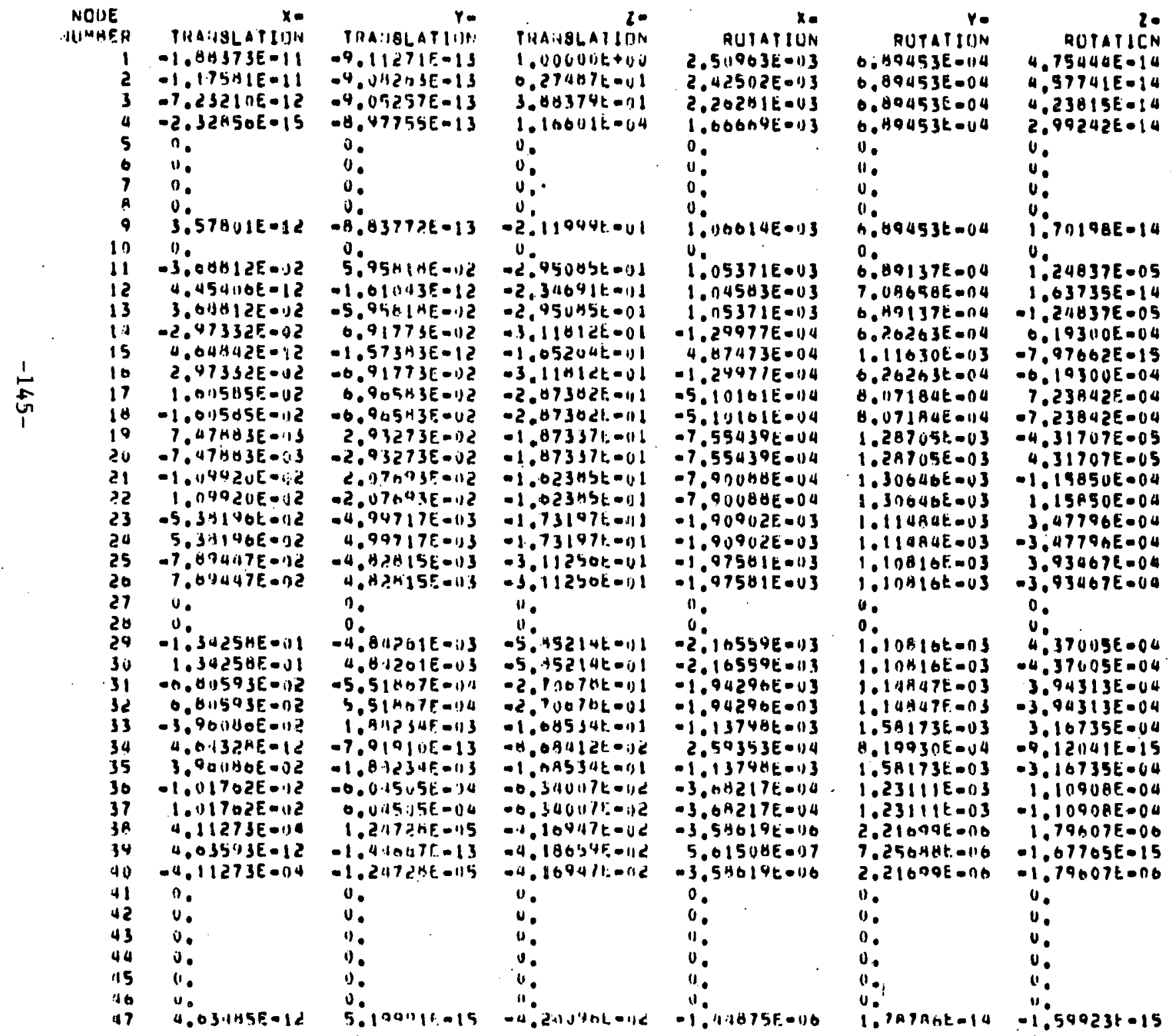




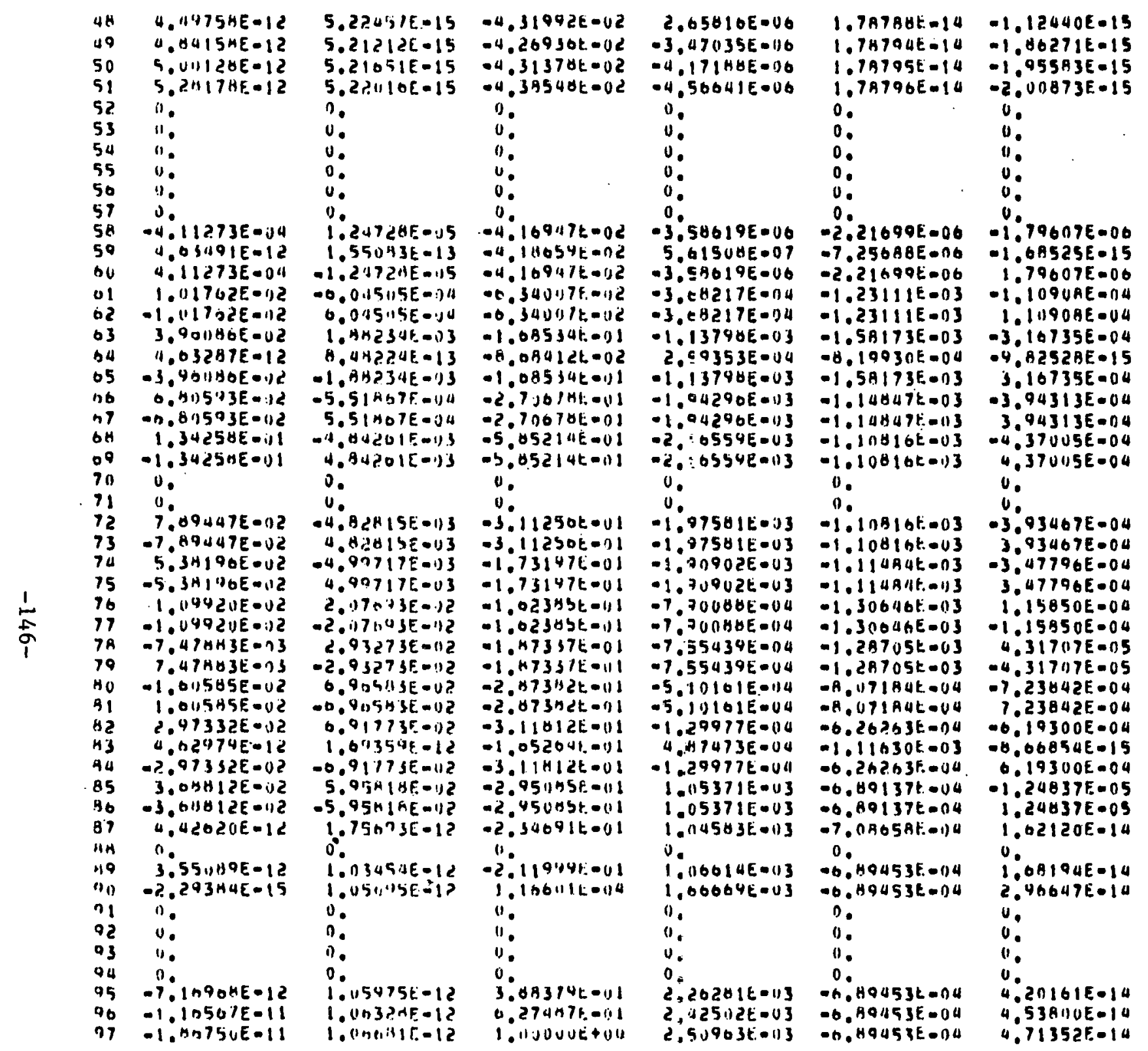


MULEE NIJMHET

FHEDIIEIICY a $6.55734 \quad \mathrm{HZ}$

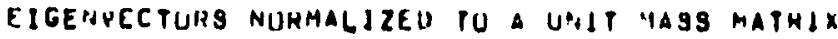

IISPLACEMENISIRITATIJUS UP UINESIKALIHEI) RUDES

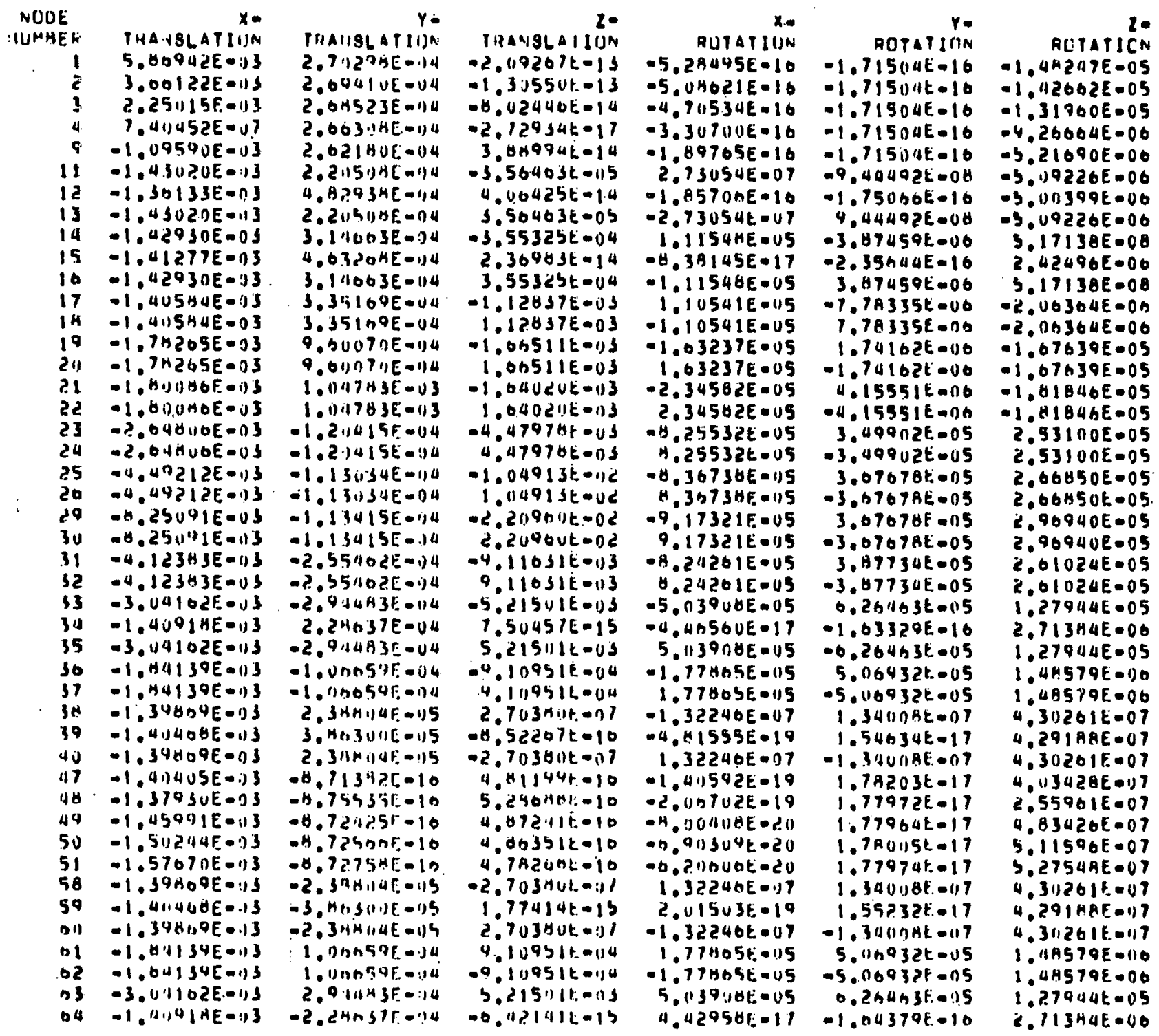


$65-3.114102 E-43$

$00 \quad-4.12383 E-1) 3$

01 - $42383 E-113$

of $-4.25091 E-1)$

कY -4.250916003

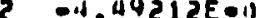

$3-4.44$ ? (2E-1)

$4-2.014800 E-013$

$15-2.0181) 6 E-13$

In -1.4 INBOE-T3

$7-1.8110 \mathrm{HOE}-113$

$-1.74265 E-i 13$

$-1.74265 E-03$

0 -1.405H4E-.13

$1-1.40584 E-J 3$

$-1.4 C 950 E-113$

- $1.41277 E-13$

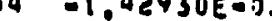

$5-1.431120 E-n S$

- $1.431120 E-43$

$7-1.50133 E-13$

$-1.0459 .11=113$

$7.41452 \mathrm{CE}-17$

$2.25 U 15 E-113$

$3.00122 E=13$
$5.00942 E=113$
$5.9 .34 H 3 E-114$

2.55u02E-.)

2.55\%1, 2E-1)

-1.5015E-ma

$1.13031 \mathrm{JE}-134$

1.13113at-114

I. 2.INISE-JA

$1.2115150-14$

$1.0+7435-113$

$-1.011783[0.1)$

$0.61117 \mathrm{JE}-110$

$9.61070 E=04$

$0.35169 E-114$

$\left.-3.35169 F_{0}-1\right)$

$-3.140035-1) 4$

.0S2 THE. DO

3.Juhbse-1)

-2.2I5IHE-OL

$-2.21511 H E=114$

$-4.0 \geq 93 H E=14$

- .0? INUE-1)

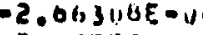

$-2.011523 E-11$

-2.01111 IIE- J4

-2.7I) PHE-IJ
$9.21501 E-03$

$.11651 t=u s$

.116511 .03

-

$1.04913 t .02$

$1.0491 S k-U 2$

$4.47976 t-03$

.4. $4791 \mathrm{BE}=93$

$1.0402 U E=03$

$1.06511 t=0 S$

1.065115 .003

$1.12437 t=03$

$-1.12437 E-03$

$3.55325 E-04$

-2.2744 पLE- 14

3. $55325 L 004$

$3.56403 E-0 S$

$-3.5 n 403 t=0 S$

$0.05554 t-14$

$3.911111=14$

$2.75450 t-17$

$9.09735 E-14$

$1.31707 E-13$
$-5.03904 E-115$ $0.24201 E-05$ - $1.24261 E .05$ 9.173216 .05 $0.17321 E-05$ $4.36738 E 005$ $-0.36734 E-05$ Q. $25532 E-05$ $-H .25532 E-05$ ?. $34562 E=05$ $-2.34582 E-05$

$1.03237 E-05$

$-1.03237 E 005$

$-1.111542 E 015$

$1.10541 E-05$

- $1.1154 A E-05$

$0.11723 E-17$

1.115UAE-US

$-2.73054 E-07$

$3.73 \cup 54 E 007$

$1.958918-10$

$1.90051 E-16$

$3.33224 E-16$

$4.74914 E-10$

$5.13300 E-16$

$5.33647 E-10$
.26463E-0S

3.877345 .05

$-3.87734 \mathrm{E}-0 \mathrm{~S}$

3.676786 .05

$-3.6767 \mathrm{BE} 005$

$3.67678 E=05$

3.6767AE-05

$3.49902 E-05$

$-3.49902 E-05$

$4.15551 E=00$

$15551 E=00$

1.741625 .00

$-1.74162 E-10$

$-7.7 A 335 t-110$

$7.7 A 335 E-90$

$-3.87459 E-16$

$.43459 E=16$

$3.87459 E-06$

$0.444925=08$

0 पaUOak-0B

$-1.412006-10$

$-1.7739 n E-10$

$-1.77396 E-16$

$1.77306 E-10$

$-1.77396 E-10$
$1.27944 t-05$

6.611245005

2.610245005

$\therefore 66450 E-05$

$0.0650 E 0$ D

5310

$2.53100 E-115$

$2.53130 E-05$

$1.81846 E-05$

.81846E-75

$1.67639 E=05$

$1.67639 E=05$

$2.06364 E 000$

$2.06360 E=00$

$042496 E-06$

5.171305008

- $3.09226 E 000$

$-5.09226 E-0 B$

-3. $00390 E=00$

- $.21690 E=06$

1. $420025=05$

$-1.40247 E 005$ 
MUIDE NIJMHER 3

EIGEIIVECTUAS NUKMALIZEO IO 1.000

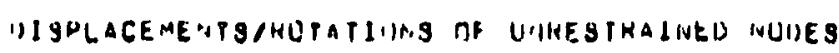

NDDE

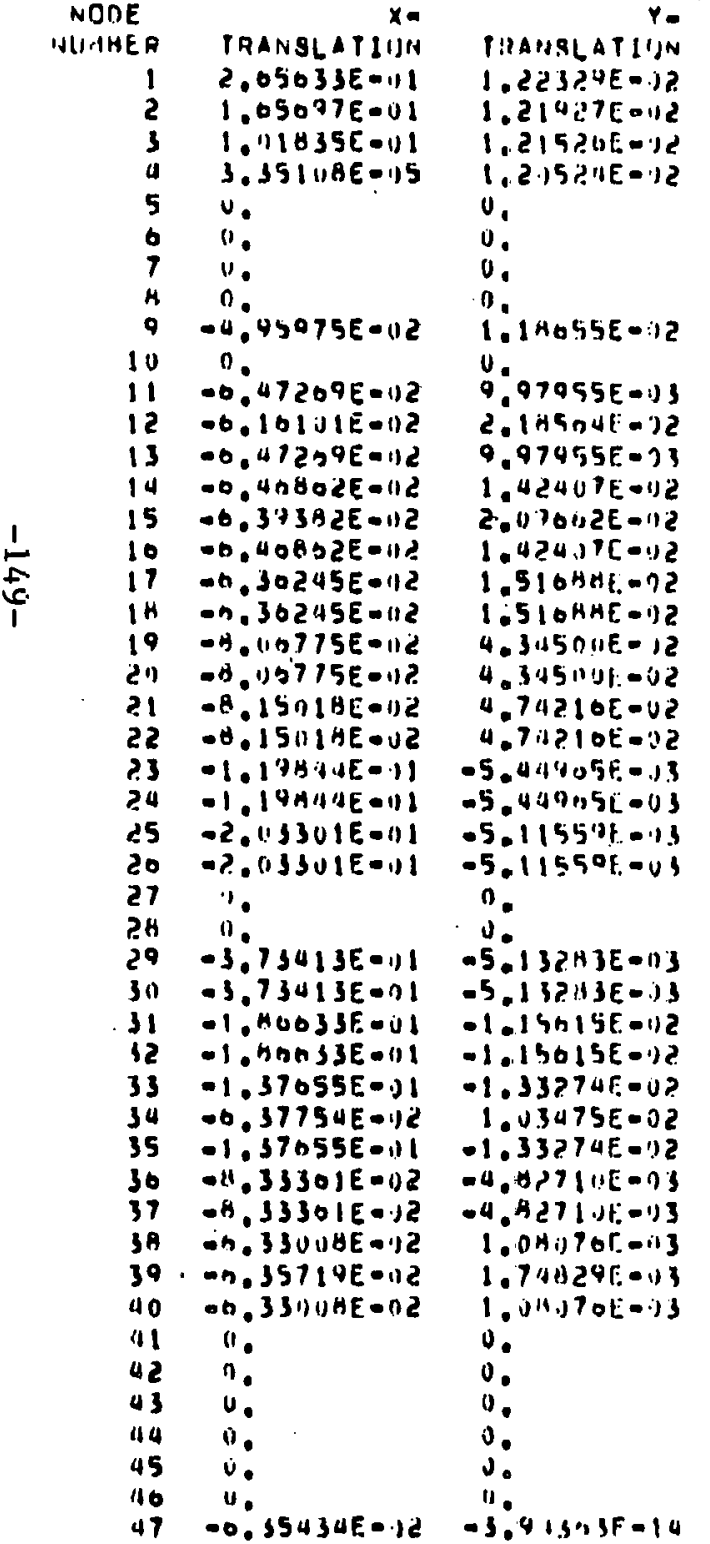

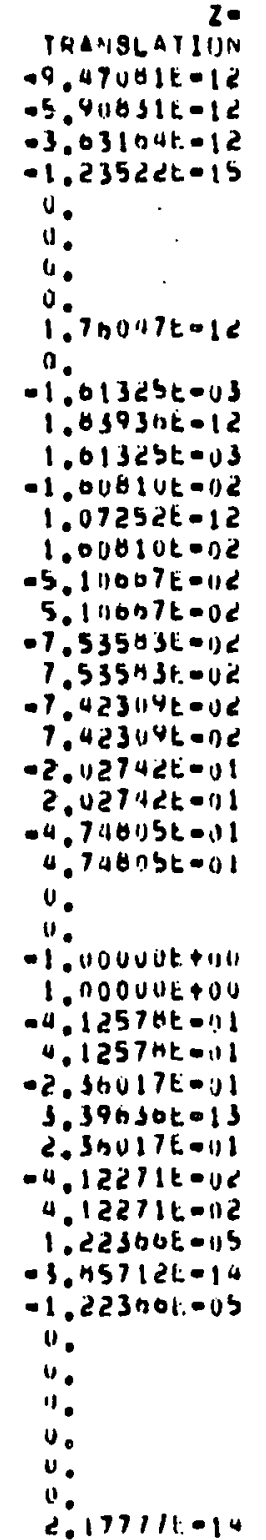

1AMASLATIUN $-5.911631 E-12$ 3.031 nat. -12

$1.70007 t-12$ a.

0725ac-12

500071100112

5.1110B7t-Od

$-7.53503 E-1) 2$

$7.423114 k=02$

$2.027+2 t=11$

$4.7401156-11$

. 74 abt $=01$

1. 10uvitt +1111

1. NOOUUE $\$ 0$

4.1257 HE $=11$

-2. SGUITE-UI

3.39 sotel

$4.12371 k-0$

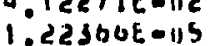

3.h57 $12 k-14$

1

"..

0

$2.17711 t-14-0.302 E 1 t=14$ $5.945 \cup 4 \mathrm{t}-\mathrm{JNh}$
RITATIUN

$-2.39142 E-14$ $-2.3 \cap 167 E-14$ $2.12450 E-14$ $1.49065 E-14$ 0.

0

0.5 ค 25E- 15 10.

$1.23577 \mathrm{E}-05$ $0.40451 E-15$ $-1.23577 E-05$

$5.111833 E-114$ $0.79321 E-15$

5 . 0270 E 04 5.002795004 $-7.34703 E=04$ 7. $349036=04$ $01.06105 \mathrm{E}=03$ 1. 11h165E-U3 $-3.73612 E-03$ $3.73012 E-113$ -3.7 BOUUE-1) 3.7AGHAE-U3 0.

0.0

$4.15154 k=03$ $4.15154 E-13$ $-3.731) J 7 E=03$ $3.73037 E-03$ $-2.2 B \cap 54 E=\cap 3$ -2.0 1 IUUE-15 2.2HU54E-U. -H. $114904 E-114$ $8.114904 t-114$ $-5.945114 E 006$ - 2.1793 UE- 17$$
\text { I. }
$$$$
11 .
$$$$
\text { U. }
$$$$
\text { 11. }
$$

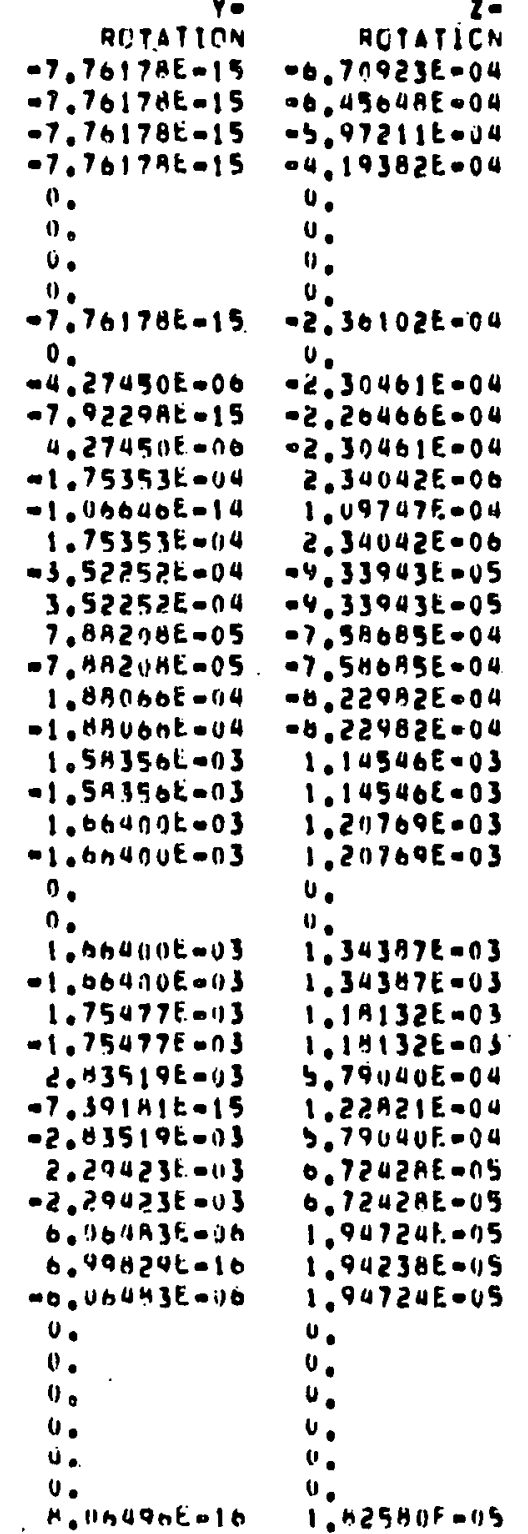




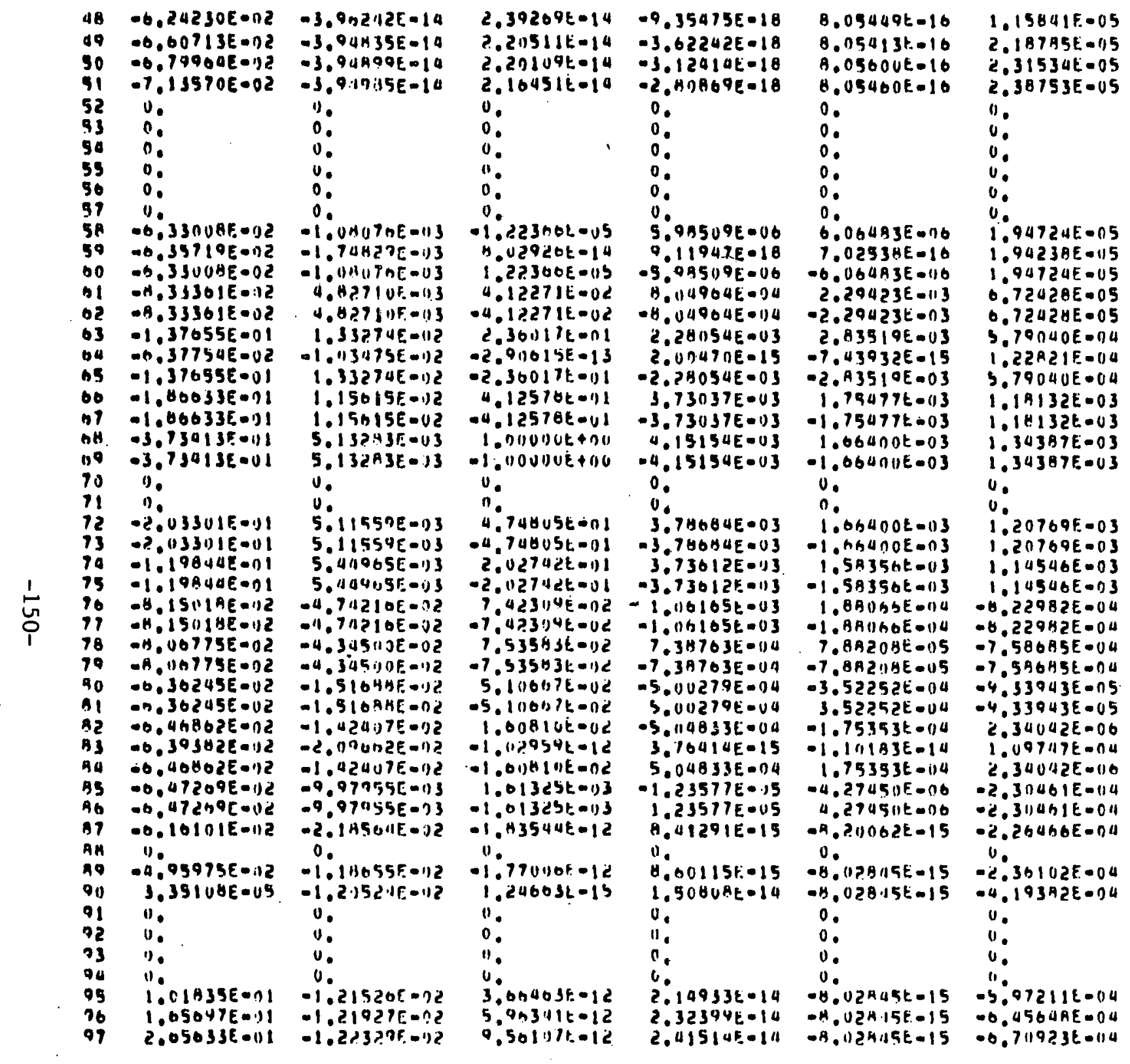


PIIIE N.JMIIE" FREIUIE ICY

. 6.571

H2

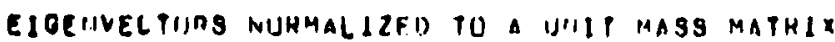

DI GPLACENENTSRIOTATIINS OF UHRESIMAIHED NUOES

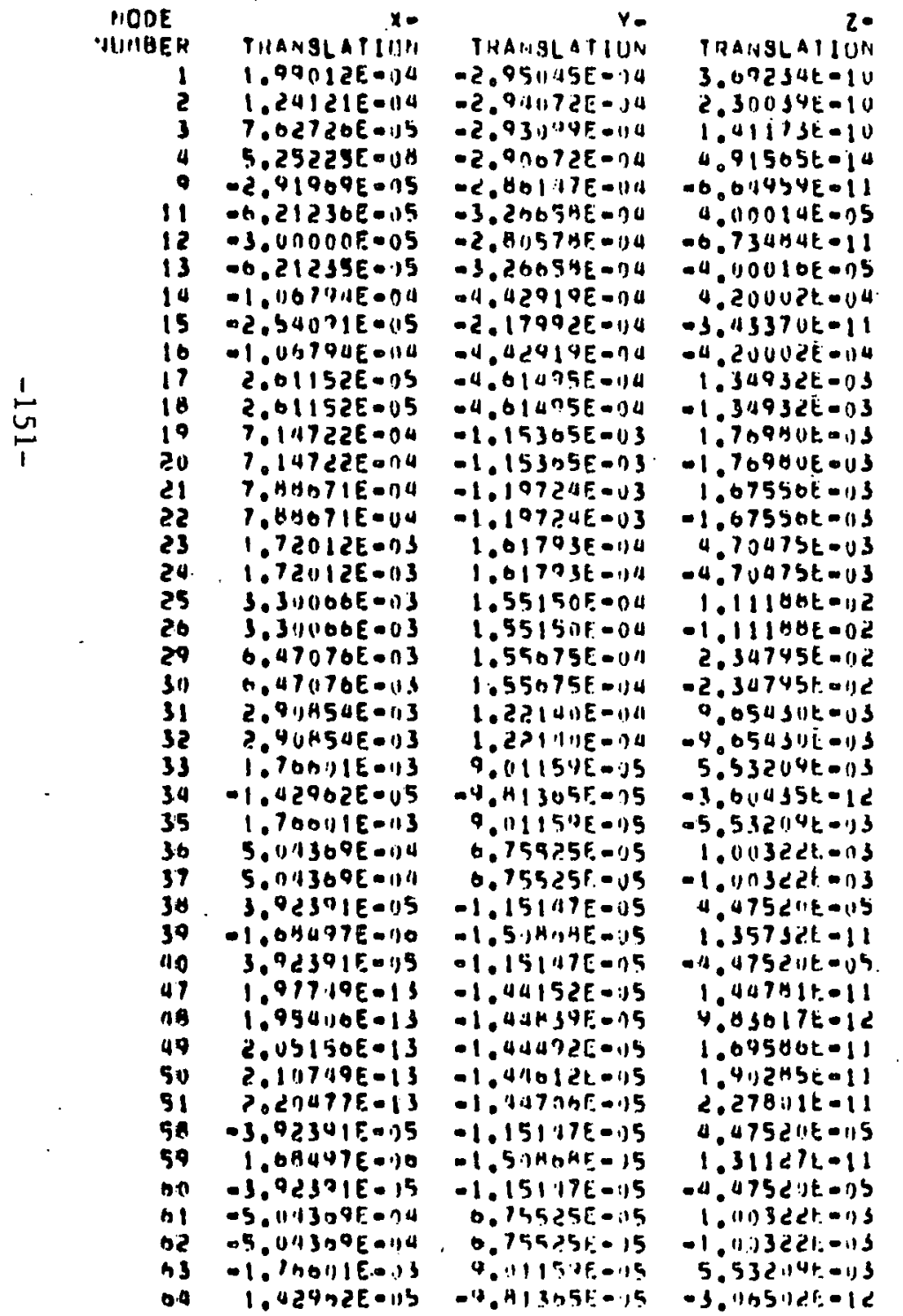

RUIATIUN $0.33 A 15 E-13$ M. 47 YHIE- 13 $4.59023 E-13$ $5.762705-13$ 3.209H5E-13 $-3.06021 E-07$ 3. 1422 IE- 13 $3.00022 E-117$ $-1.33325 E-05$ $1.51437 \mathrm{E}-13$ $1.33325 E-U 5$ $1.325 B 4 E-115$ $1.325 B 4 E-05$ $1.46200 E-05$ $-1.9620 \mathrm{HE}-115$ $2.73890 \mathrm{E}-05$ 2. 73090 - 05 -2.73899E-1) 6.810 OEE-05 - A.81062t.uS $0.910^{\circ} 1 \mathrm{E}-115$ $0.911191 E-U 5$ $9.77081 E-05$ $4.771141 E=015$ $0.76930 E=05$ $0.76936 E-05$ . $76936 E-115$ $5.232 \cup 5 E-U$ $9.27951 E-14$ $-5.232 \cup 5 E-U S$ $1.757 \cup 2 t-1) 5$ $-1.7 .5702 E-15$ $1.101036-117$ $2.00940 t-10$ $-1.10103 E-117$ $2.017911 E=10$ $2.510 S Q E-10$ $2.72 h 2$ bt- 14 2. $76661 t-14$ $2.791138 t=1 a$ $1.161036-017$ $2.6651111 \mathrm{E}-14$ $1.101113 \mathrm{E}-117$ 1.157 112t-115 -1.757 Uदt-115 $5 . ? 32115 E-115$ H.72HOHE-114
RUTATIIIS 3.351B1E-13 $3.3511615-13$ $3.35061 E-13$ $3.351161 E-13$ $3.35061 t-13$ $7.07605 t=08$ $3.414 A 7 E-13$ -7.0159HE-กB $2.113956 E=06$ $4.46124 t .-13$ $-2.113956 E=06$ 5.5 ? $720 E-n b$ $-5.52720 E-1) 6$ $-5.43554 E-116$ $9.43554 t-110$ $-7.87374 E-06$ 7. 0737 UE - 1) $-3.74750 E=05$ $3.74790 E-135$ $-3.92180 E-115$ 3.921 AUK-05 -3.921 anE-nS 3. 921 AOE-OS $-4.1306 U E-115$ $4.13(160) E-1) 5$ $6.0 n 414 t-i 15$ $3.19373 t-13$ 0.6 inll पtal) 5 $-5.33423 F=115$ $5.334 ? 3 t .05$ $-2.02252 k-07$ 2. उ6คOOLt -15 $2.42252 E-117$ $-? .75452 t .15$ $-2.75452 E-15$ $-2.75453 t-15$ $-2.751153 E=15$ $-2.15453 E-15$ 2. $2252 \mathrm{E}=07$ $-7.51)+35 t-15$ -2.4 2) 5 ?t.-117 $5.33123 E=115$ $-5.334>35.115$ D.hiा य) 4t-25 $-2.970115 t-13$

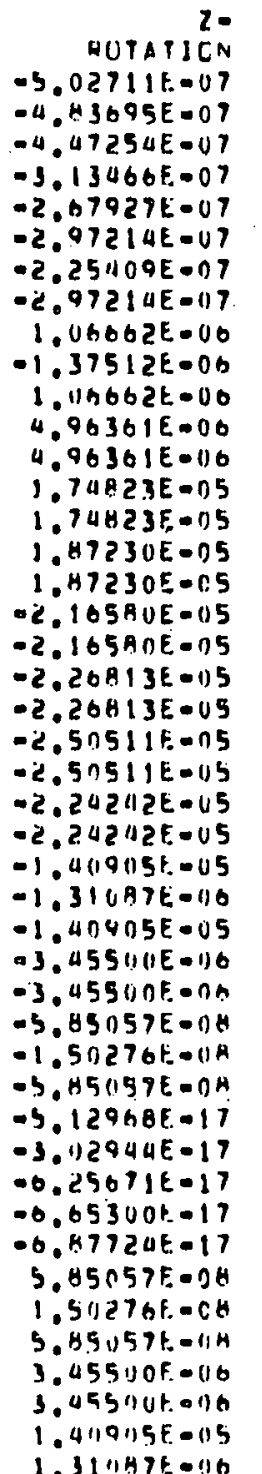




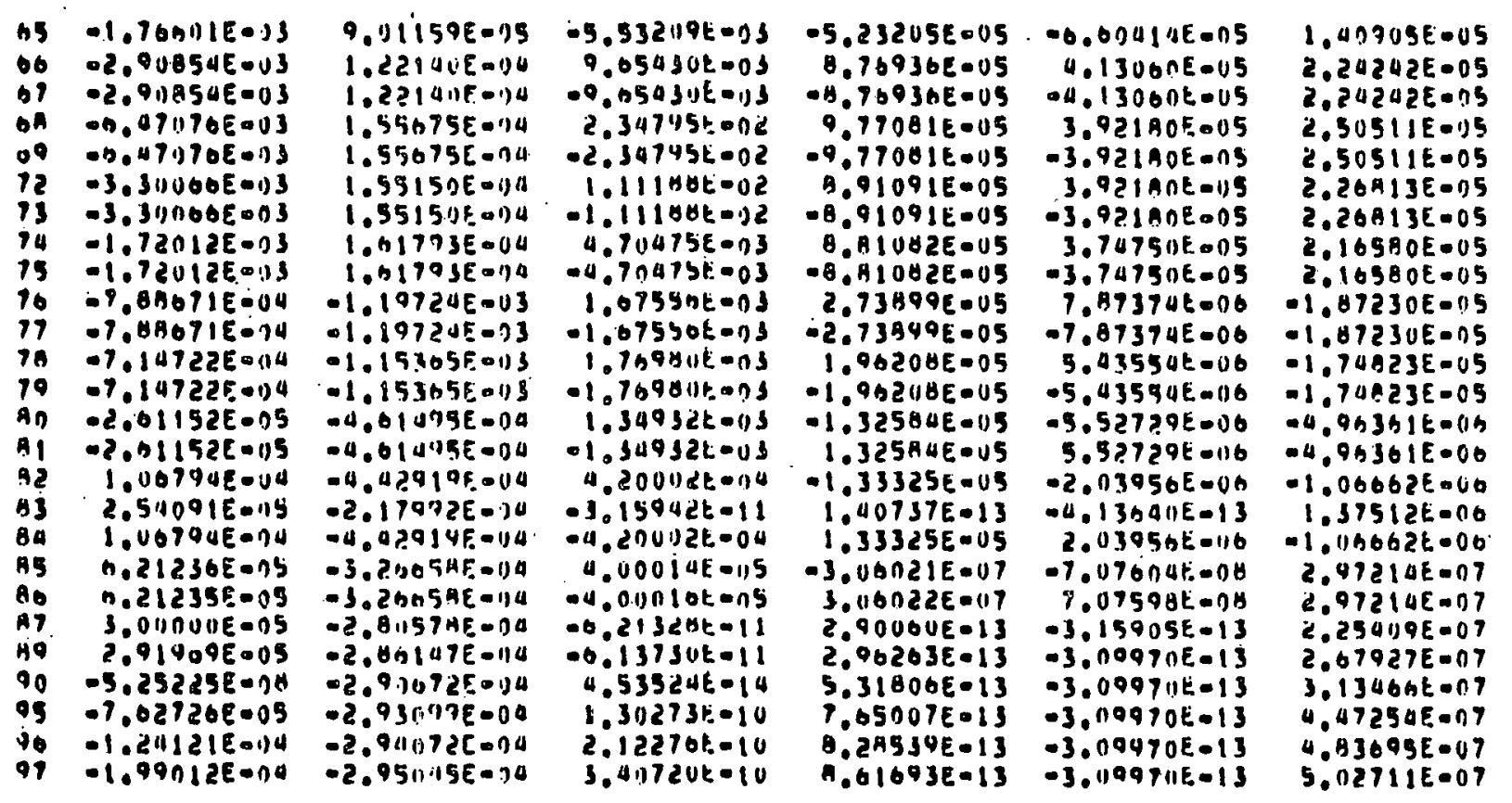


900E NIIMHER O

ETOE VVECTIIRS NUHMALIZED TO 1.1110

HISPLACEMENISARUTAPIIMIS JPF UTHESIKAINED NODES

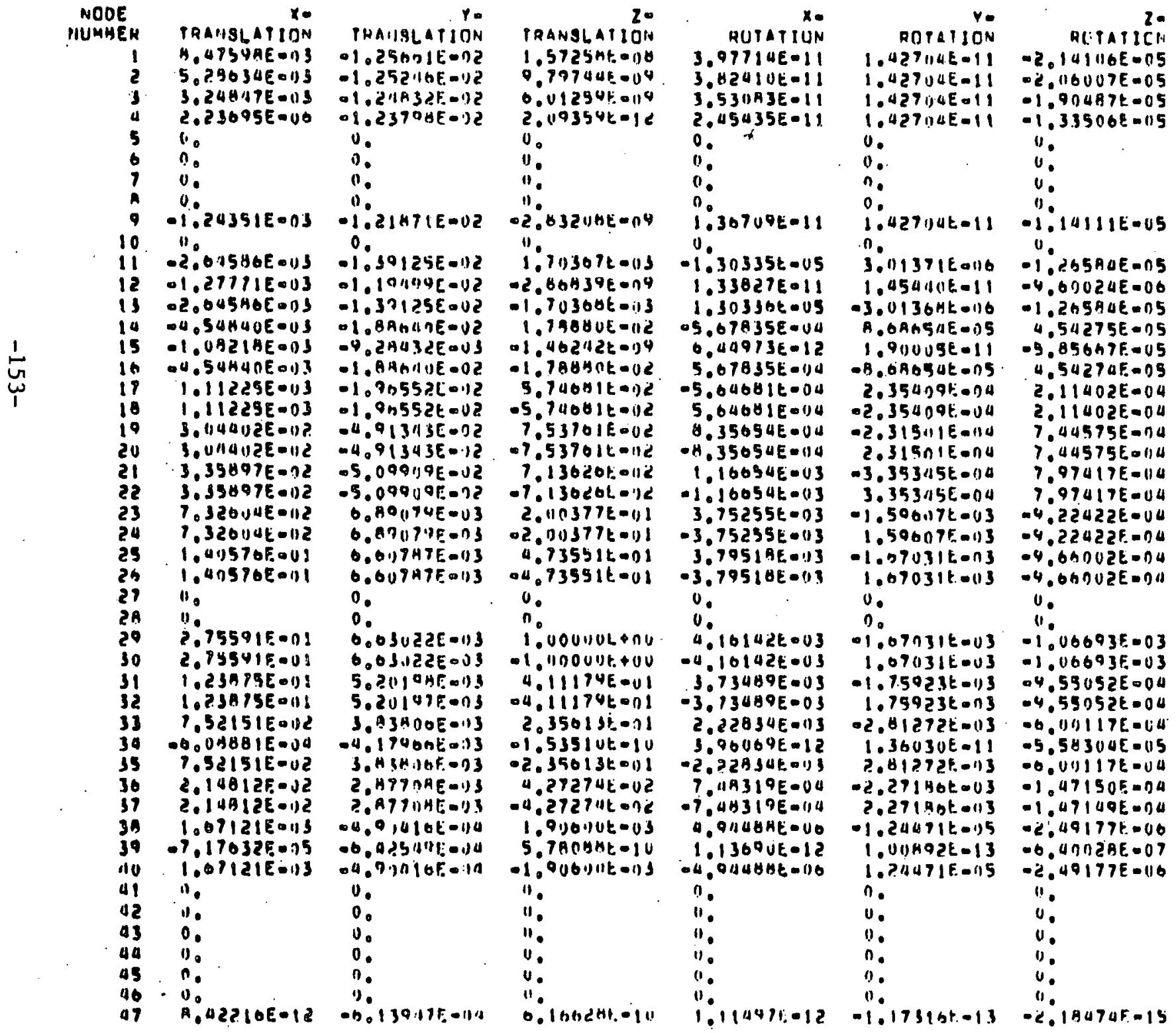




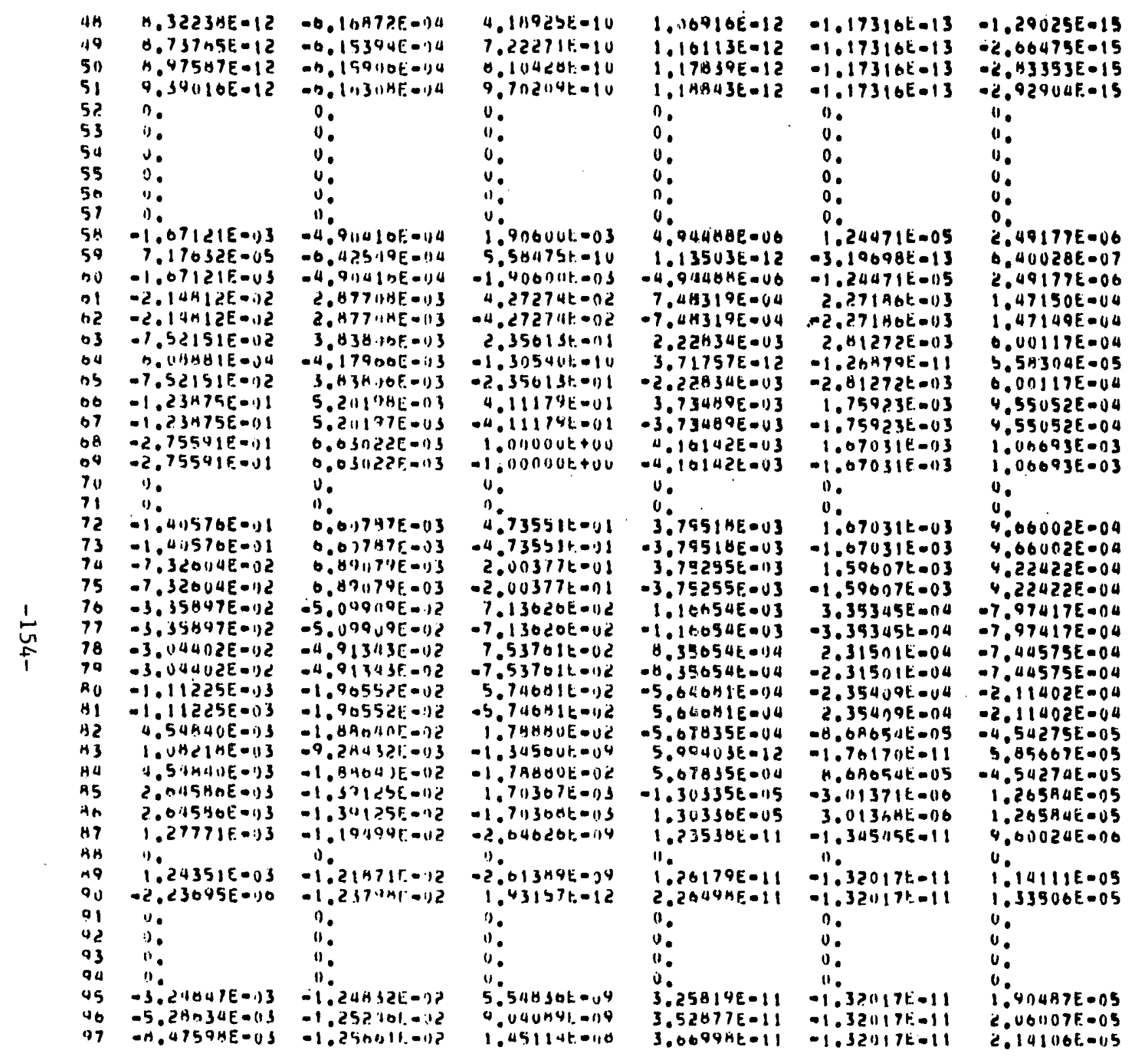


MIJIE NIINCIEN 3

FHE JIL VCY $0.63198 \quad \%$.

E IOENVECTMIIS NURMALIZE'S TO A UIIT MASS MATRIX

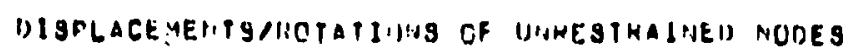

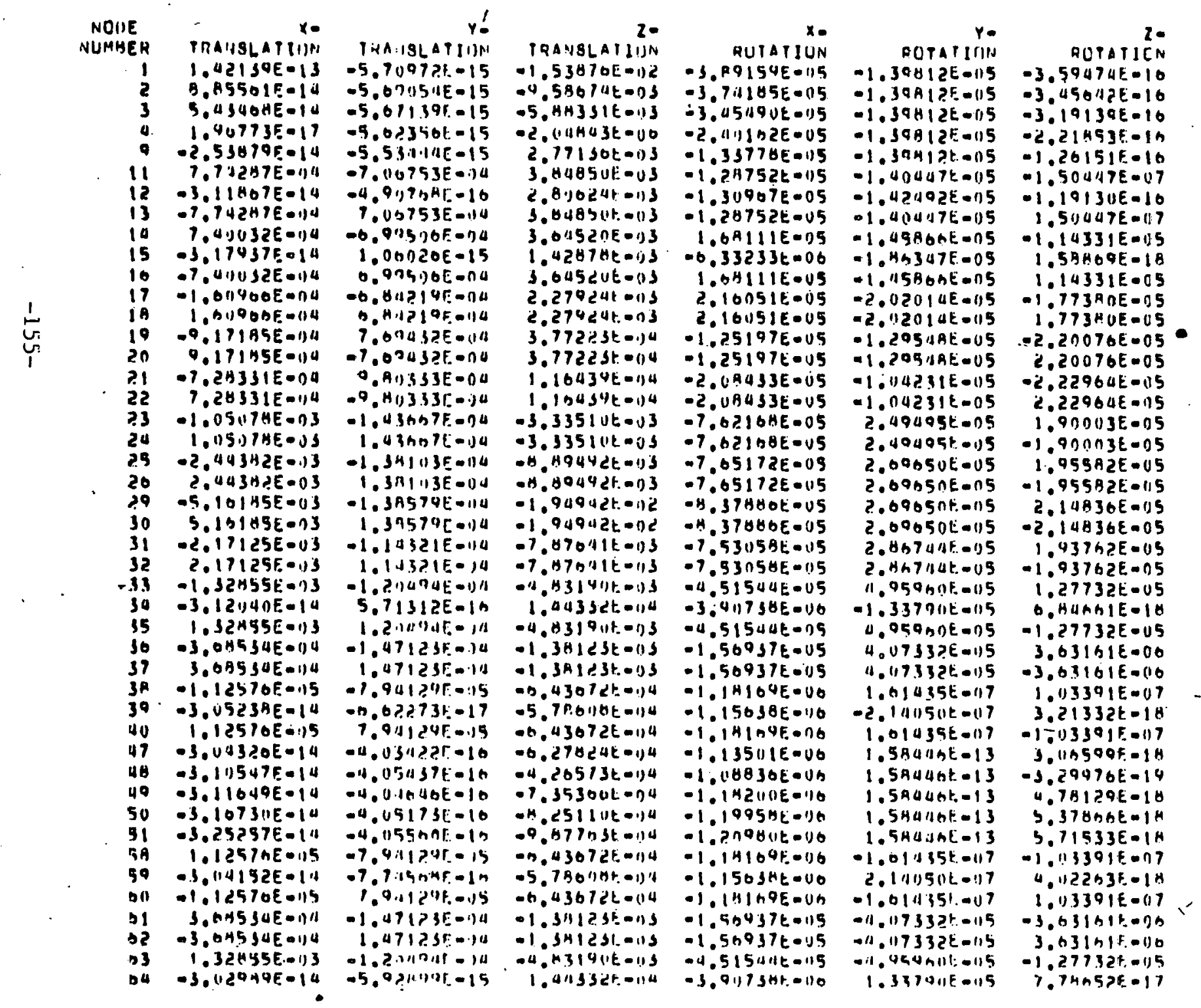




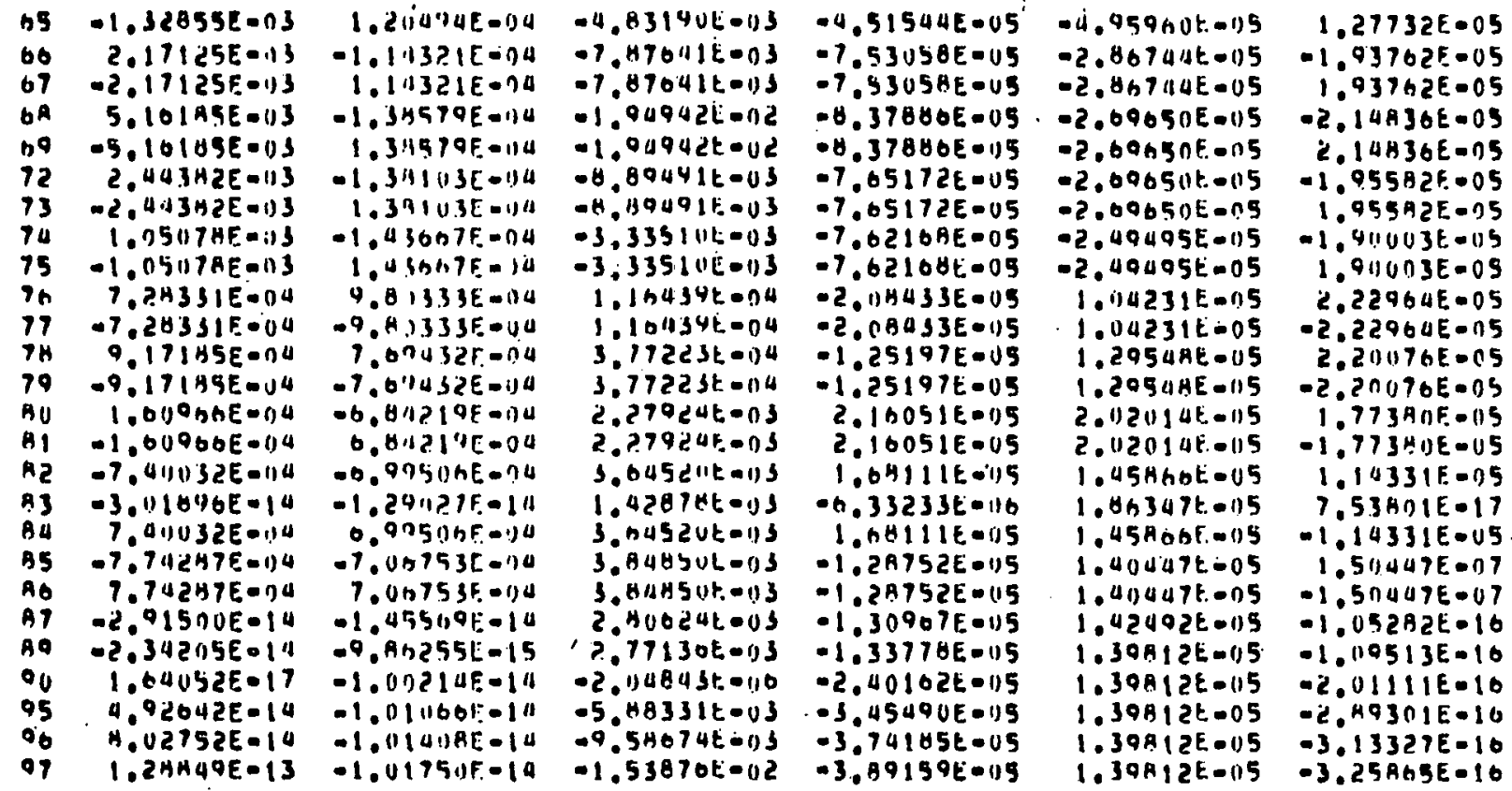




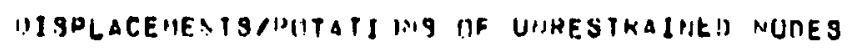

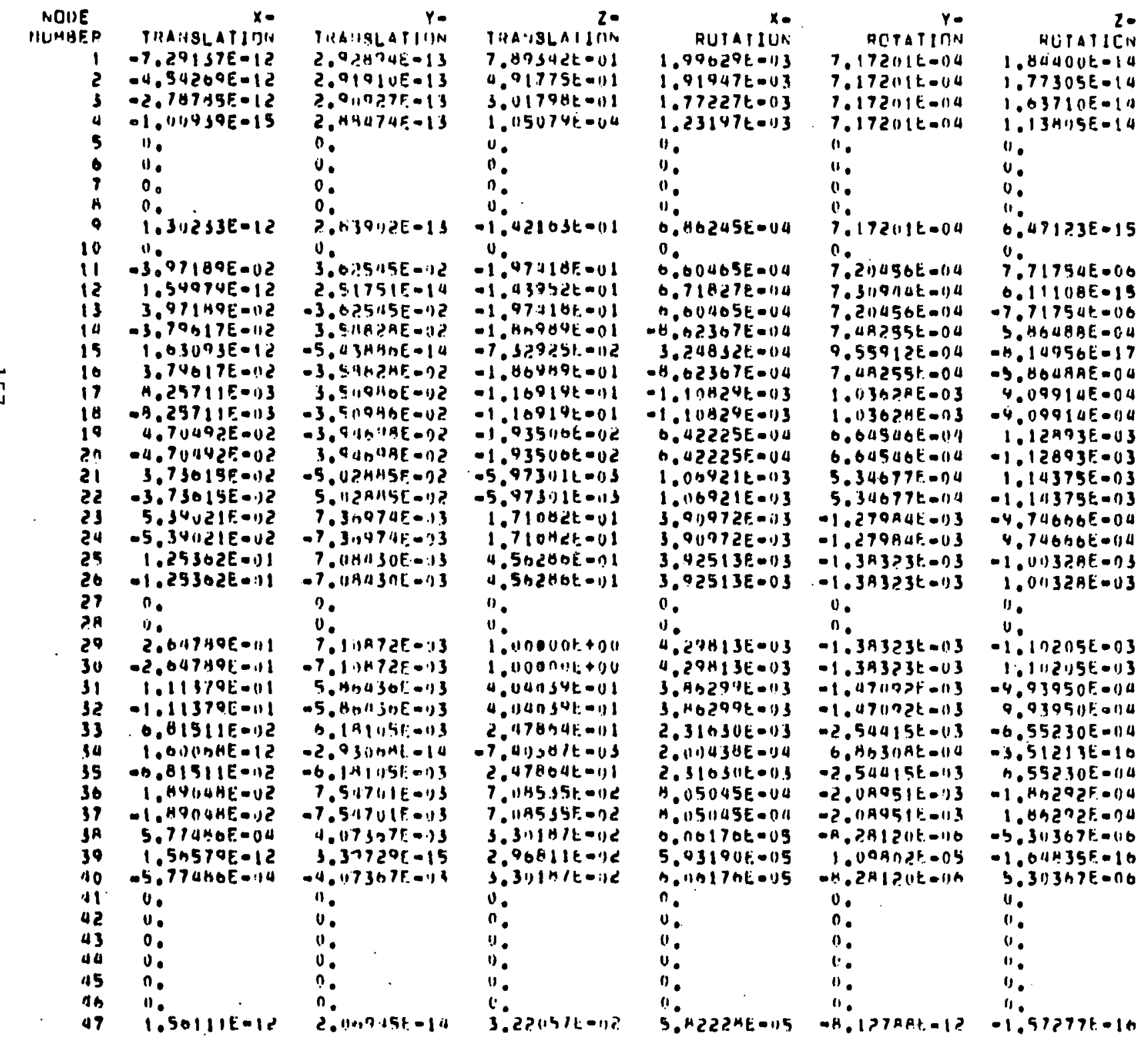




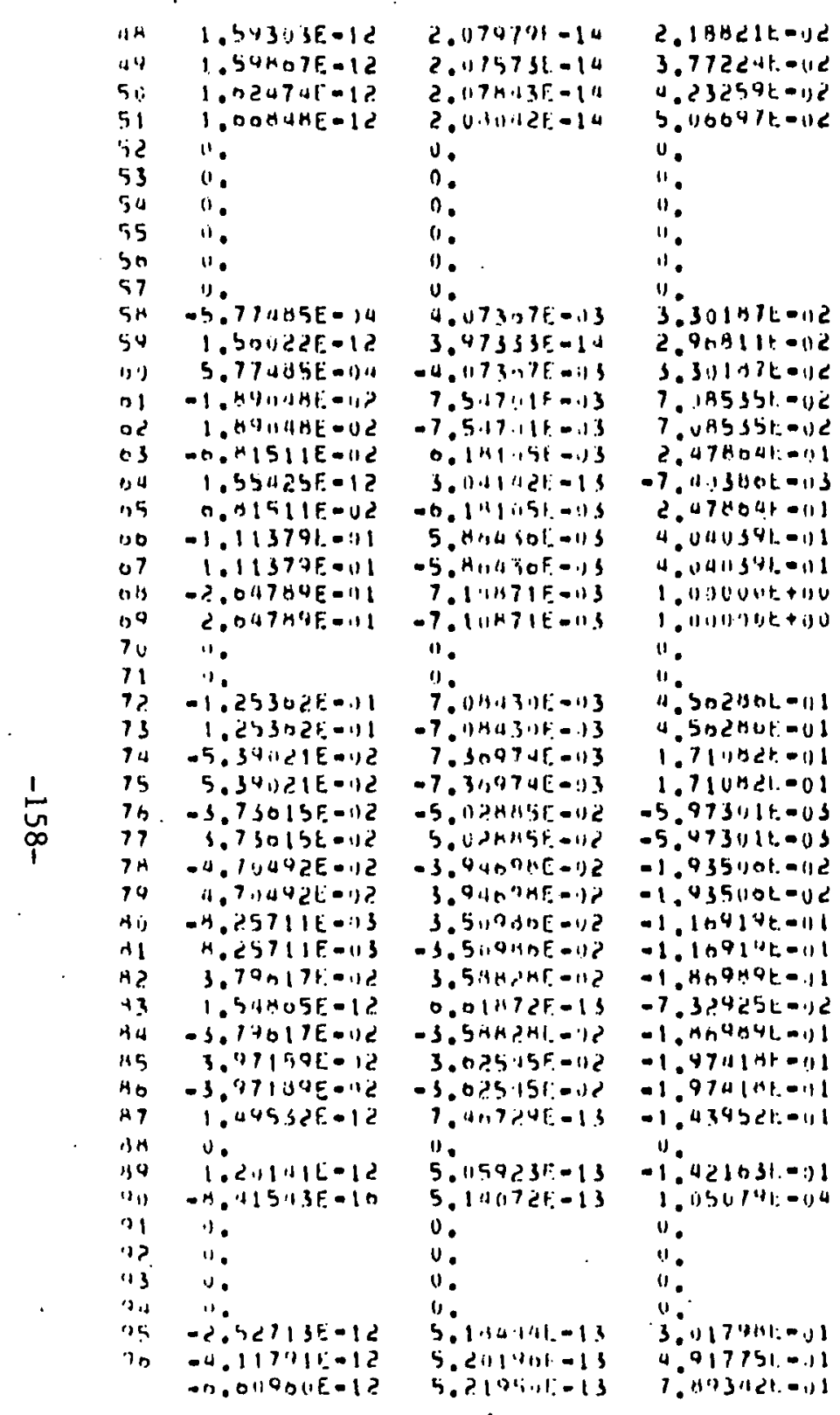

$5.5 n 3110 E=015$ $0.06337 k=05$ $0.15352 E=U 5$ $0.20595 E-115$ 11.

o.

u.

i.

0.

$6.116110 t-15$

$5.031911 E-U S$

$0.06170 E-015$

$0.115045 t-04$

H. $115045 E-04$

. $115045 E-U A$

2.
$2.016330 E=U 4$

$2.310 S S_{E}-113$

3. Mम244E-11S

3. $H \cap 294 E-113$

$4.29813 F-03$

$4.29813 t-113$

".

4.

$3.42513 t-113$

3.9251350113

3.919972t-u

$3.411972 \mathrm{E}=113$

$1.01642\} E-113$

1. $110421 E-113$

$0.42225 E=010$

$4.112225 t-114$

$-1.11) 829 E-03$

$-1.111629 \mathrm{E}-\mathrm{U}$

-11.02307t-11

3. $24432 t-114$

$=\mathrm{H} .02307 \mathrm{E}-114$

h. B)1105E-1)1

$0.611405 \mathrm{r} .114$

$0.718<7$ Eara

".

ninn245E-JA

$1.23147 k-03$

"1.

i.

$1.77227 t=113$

$1.01447 t-U 3$

$1.94024 t-113$
H. 1 1 . 7 AAE - I?

-8.127 AAt- 12

- 127 HAE- 12

A. 1 2 THAE-12

0 .

0.

i. 0

0 o 0

B. 2811AL-0. $3.30367 E-00$

1.1108112L-05-C.06351)E-16

H.2RIIRT.-1)O -3.30367E-110

2.04941K-0.3 1.6A2925-00

2. $484511-03-1.00202 \mathrm{E}-011$

?.54UI5E =113 $0.55230 E-110$

0.

Q. 9 gasot-0

$1.47092 t=03 \quad 4.93951) F .114$

$1.471192 k-1.3 \quad-4.93950 E-04$

$1.3 A 323 E-03 \quad 1.10205 E-03$

$1.3 A 3 ? 3 E-(13-1.11) 205 E=03$

$1.3 A 325 t .03 \quad 1.01328 E-03$

$1.3 A 323 t-113-1.00328 E-01$

$1.299 A 4 E-113$ 4.74606E-nA

$1.279 \mathrm{~A} A \mathrm{AE}-113 \quad-4.74066 \mathrm{H}-04$

$-5.3407 n t-04-1.14375 E-03$

$-5.34 n 7 n E-1) 4 \quad 1.14375 E-(13$

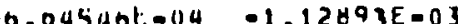

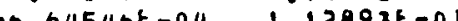

व.

-

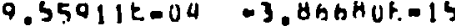

7.4A255E-1) S.HOAHAE-1)a

$07.20456 t-114$ - $7.71754 E=00$

7 7) 70117545010

7. Biladatena S. un)

0.

5. $\triangle 1111 B A E-15$

0.

$1.03165 \mathrm{E}-14$

.

".

$-1.1721116-14$

- 7 - $172015-14$

-1.17zante-ina v.

1. WA IIIIE- 14

$1.011720 E-14$

$1.071005-14$ 


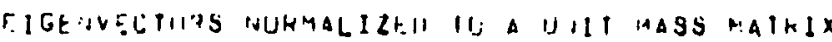

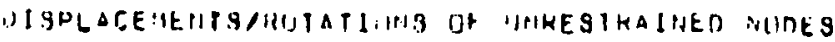

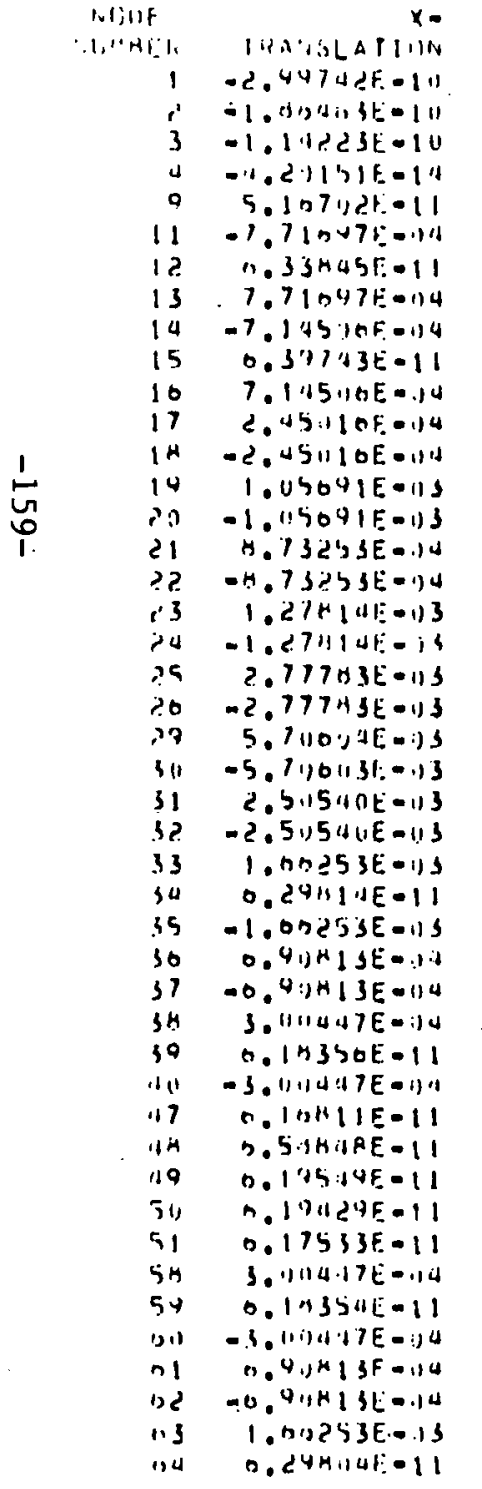

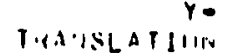
$1.711 ; 5 F .12$

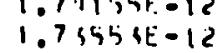
$1.75556 E-12$
$1.7,79531:-12$ $1.714531-18$
$1.7,39531-15$ $1.7145 .31-18$
$1.0400,15-12$

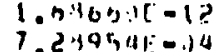
$-4.32,1021-12$ $-1.340505-111$ 1.211905t-114 $-4.523015-17$

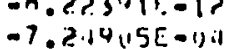

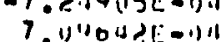

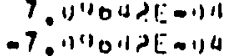
$-7.8751125-111$ 1.9 तु.ाटf $=74$ -1.0115 पe-13 1.11)11591:-.13 i. linativer-o)

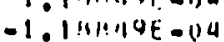

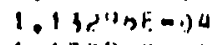
$-1.132 \%$ OE-1) $1.110476=14$ -1.1 , nith - : In 1. "W11135E-114 $-1.4 n 9.14 \times-114$ 1.5 5 . $-3.014051-12$ $-1.525 .155:-111$ H.OSU1, पe 015 $\left.-13.0 . j 404 r_{0}-i\right) s$ $1.1117 n F-110$

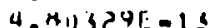
$-1.111740-10$ A. $075515-11$ H. $113>35.17$ $0.103 \times 71=17$ H. (116) H. $11157115-17$ $-1.1117 n i-a, 1 n$ $-4.4 .11550-13$ 1.1117 bi-un - 0 त. $53+1,31-15$ $-1.4,5.145-1: 1$
5.111
T!KAIISLA I IIIO 1. SHAUBLEIS

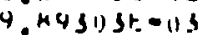
n. $11711,41-113$ 2.11H11t.-11n - P. O5.4 4t. $=115$ -3.9574 at-lls $-2.41) 0<1$ tolis $-3.95146 t-113$ $-3.75427 t=113$ $-1.52427 t-115$ $-3.75427[-1) 3$ -2 sbuciut.-us $-2.36101 j 4 t=11$ - $4.27 b S I t=04$ $-4.276 J 1 t=0 a$ $-1.73134 t-116$ - 1.73154 E $-1.73154 t=114$ 3. ?II $152 \mathrm{~L}=13$ 3. दा1 152t-.)s H.OnIACl-1)S 0.001 HEt-1)S $1.41,64.35-13 \mathrm{c}$ $1.411043 t-1$, ? $7.0131141:=05$ $7.013114 t-0$ 7 . GOSIJEt-is $-3.023110 t-00$
$11.533112 t=05$ 1. UगS72E-11S $1.010572 t-0 S$ $3.1150 .145-114$ 2.5119MUL-13d 3.1)51134t-is $-0.3374 a t-12$ - 4 .311DSE- 1 -7. 4 2ubel:- 16 $-\pi .3$ ?asur. -12 -17.9nटJut:-12 -3.113114 पF. -14

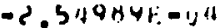
- $1.19 \mathrm{~g} / 2 t-115$ $-1.1195121 .113$

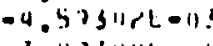
3. 19 31141-114 $x=$

RUIATIIJN A. IOSGE-115 $3.1161+3 E=115$ 1.505201 .0115 2. $47067 E-1) 5$ $1.37752 E-1) 5$ $1.32054 E-115$ $1.311619 E-05$ $1.306140-055$ i. 3205 स. $-1.711436-05$ $-5.51 \mathrm{OAJE}-\mathrm{US}$ $-2.216435 .015$ $1.15751 k=115$ $1.15751 E=05$ 1.9A1ट2E-115 $1.94122 E=115$

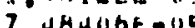
7. 4 HUUOE-US $1.51\left(2.4 F_{0}-1\right) 5$ $7.51124 \mathrm{E}-115$ औ. 223 SHE-1)5 H.223SHE- 115 $7.34437 E-U 5$ 7 उ०पड है-U5 4.447435015 S. $7904 t=v 0$ $4.44743 E=115$ $1.51734 E-115$ 1.5173 WE-115 $9.64725 E-1) \theta$ 2. $\triangle 51112 E-11 \mathrm{H}$ 9 $4725 E-0)$ - inA 2 se - 1 11200E- 1 $-1.119571 E-14$ $-1.1402 n E-19$ -1.207 गUE-11 - I. PIAJUE- 14 - Q oA l cOF-11B $-2.45 ! 12()$. - . . DA $20 E-114$ - - 517 sateng -1.517 SAt.-115 -11
$-3.1791136=.15$
R(:TAI) YIIP 1.3971 UE $=05$ $1.39714 t=.15$ 1.3971 idt.-115 1.3971 al $=115$ $1.39714 E-115$ $1.40295 E=15$ $1.420135 E-115$ $1.40205 k=115$ $1.17555 t=05$ 1. AOHOQE-II 5 $1.42555 t-05$ $1.97399 t=115$ $1.97300 \mathrm{O}=015$ 1.2 h $43 E-115$ $1.2 n 3113 t-05$ 1.U1179t. 15 1. $1175 t-05$ 1. $11175 E-05$ $-2.5011976-115$ $-2.6200(1 t .0115$ $-2.699011 \mathrm{k}=115$ - ?. . म999तל-115 $-2.0049116-115$ - 2.07 UhlF-105 2. B7unitas - $2.07061 \mathrm{l}=155$ 1.10 OJ11. 115 $-4.494119 t-115$ $-4,2$ 1) ? I ? -115 $-4.2112126-115$ $-3.210>21.0116$ - Hasant=110 c. Hus - 3 - य5inat 3. -3.1151h?ẗ-11h -3.1151 का 21.110 - 3.11511,2t- $-11 n$ $-3.151 \mathrm{~h} 2 \mathrm{t}-116$ -3. ? 111 ? $21=110$ - 2.045ubt-an $-3.2111>31:-110$ - a. zuziat-as - 4 .21? $12 t=$ ils $-4.0741191=115$ 1.10n:14t-115
2HITIATICN $7.59290 E-13$ $7.29317 F .-13$ 6.71 ARQE. -13 4.011 प5E-1S 2.51 HC3E- 13 $1.03+191.007$ $2.37345 t-13$ - $1038105=07$ 1.2243 IE-U5 $-5.56273 E-14$ $-1.22431 E-05$ 1. HAS 5 BE- 05 - ?. R652AE-O5 2. 1793 UE-15 $-2.17934 \mathrm{f}-05$ - 1 HO35E-05 - 144020 -05 E. C. UINB? QE-US - $2.1010 B E-05$ C. 101 ABt. 05 k. $31423 k-115$ - 2. OGHUAE-115 . ObHado - 1 1 $5557=05$ - 5 . SR ZUAF.-1A 1.1A55TE-115 - P. $371911=00$ C.37101E=00 $-4.93511 E-0 H$ O. Hr. U9Qk-15 $4.035118 .-114$ $0.11525 t-15$ $1.55179 E-14$ 4. 11) $549 F .-15$ $3.53335 \%-15$ 2. A7ด $77 E-15$ -4.93511 t. - 11 H 0. HAUTAE-IS 4.03511 t. UH -c.37ITIE-Un c.37191t.-1)n $-1.14557 \mathrm{E}-1 \mathrm{JS}$
$-4.54420 \mathrm{~F}-14$ 


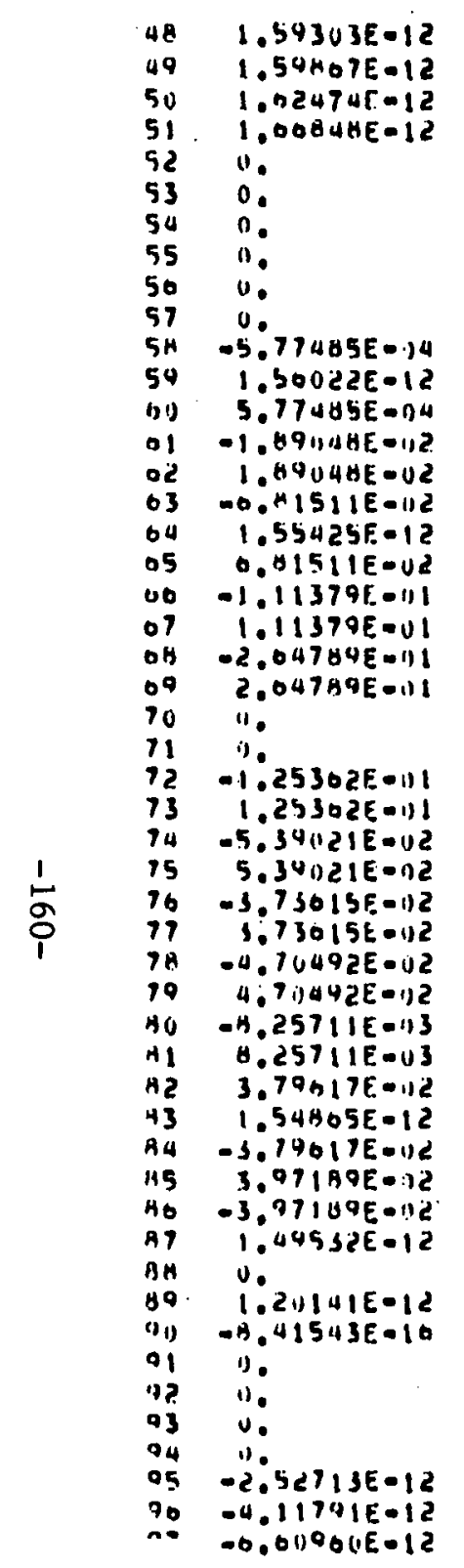

$2.07074 f-14 \quad 2.18021 t-v 2$ $2.115735-14$ $2.07443 E-1$

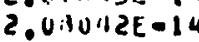

0.

0.

0.

0.

$4.07307 E-.13$ $3.97333 E-10$

$-4.073 \cap 7 E-015$

7.547015 .03

$-7.5+7,11 E-i)$

- int.use =jos

3. 1) $4112 \mathrm{I}-1$

$-6.14105 t-113$

5. $4,4,50[-1)]$

$-5.40430 \mathrm{~F}-0) 3$

7. $1: 16715-113$

-7.11147 IE- 13

$7.0 \mathrm{OH} 30 \mathrm{OE}-113$

$-7.1) \mathrm{A}(3) \mathrm{W}-\mathrm{O})$

$7.5697+E-113$

$-7.31,97$ पE -03

$-5.024+5[-1) 2$

$5.02 H 115 E-11 ?$

$-3.9400 N E-1) 2$

3. $9402 H E-112$

3. 519 गमE -

$-3.5110 \mathrm{HAE}-112$

3. 5AHZHE $=n 2$

$0.0141725-13$

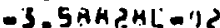

$3.0 ? 545 F-11$ ?

$-3.02515 \mathrm{~F}=02$

7. 4n? $24 E-13$

$5.059235-13$

$5.14072 F .-13$

$v$.

..

$5.134+4 E-13$

5. 21119ut-1

5. ?.194iir.-13
$3.77224 \mathrm{~F} .0112$

$4.23259 t-1) 2$ 5. 06097 t-UC

11.

".

$3.30157 t-02$ 2. $90 B 11 \mathrm{~L} 0 \mathrm{C}$

S. JUInTt-UC

7.3A5 J5t-UC

7.UASSSE - U 2.47804t:-1) 7. 1), 360t-113 2.4740UF-11 $4.04054 \mathrm{~L}-1) 1$ $4.04(1$ SUF. 0111 1. 1) OUUUt +1) I. II (III) Ut +0

$4.50250 t-111$

$4.502 \mathrm{BUE}=\mathrm{U}$

$1.7105<t-111$

$-5.97311 \mathrm{LE} 03$

$-5.97301 t=0$
$-1.93500 t-11$

-

I. Hh9BOt-il

1.3 . $925 \mathrm{E}=\mathrm{U}$

- 1.97 alat-o

1.974 lat-1)

$1.43952 t=11$

$u$

$1.421631:=01$

$1.05014 t .04$

$U$.

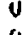

.

3.0174At-01

$4.917751 .-i 1$

1.013 $32 \mathrm{ta}=\mathrm{il}$
$5,583 \cup U E=05$

$0.00337 E-05$

$0.15352 E-05$ $0.20595 E-U 5$

0.

0

0.0

U. 0

1). 0 .

$0.00110 k=05$

$5.03190 E-U 5$

$0.06170 E 005$

$8.450456-04$

$H .05045 E-04$

$2.31030 E=03$

$2.00438 E-04$

2. $31630 E=03$

3. $H 5249 E-U J$

3:A $2299 E-U 3$

$4.278135=03$

$4,278\{3 E=0\}$

0.

$3.42513 E=113$

$3.92513 E=113$

3. $9: 1972 E=03$

$3.911972 E-03$

$1.116421 E-03$

$1.110421 E=113$

$6.42225 E=04$

$0.42225 E=04$

1.1)B29E-0

$-1,10629 E-03$

$-13.02307 E-04$

3.2UA32E-UU

列

$0.00465 E=04$

0.6 i) $405 t-(14$

$0.71827 \mathrm{ENOH}$

0.

h. HS2 $45 E-04$

$1.23147 t-03$

(1.)

0 .

$1.77227 E-113$

$1,91447 E-03$

$1.44024 E-113$
- 9.127 ABE-12

0.127 AAt- 12

$127 B B E-12$

. 127 BAE-1

-

B. 2811 1AE 00

$1.170802 t=05$

0.2811 At. -06

$2.08951 E=03$

$2.08951 E-03$

2.54/15E-0I

$6.8 B 3 \cap A E-D A$

2.5UA \{Stinns

$1.47002 \mathrm{E}=0 \mathrm{~S}$

$1.471192 \mathrm{2E}-03$

$1.3 A 323 E-03$

1.3 B $23 t=03$

1.3A323t-03

1.3 A3 $3 E-03$

$1.279 A 4 E=113$

$1.279 B$ UE-113

$-5.34076 E-04$

$5.3467 n E-04$

$-6.04540 t-04$

$-0.64540 t-04$

$-1.03628 E-03$

-1.0362 AE - 13

-7.4 A255t-04

$-9.55011 t=04$

7. 4 A $25 E-1) \Delta$

$-7.20456 t$ - 114

$-7.20456 t-1) 4$

$-7.311004 t=n a$

0.

$.7 .172016-04$ $-7.17201 t=04$ 0.

0.

0.

$-7.17201 k=00$

-7.1720160 ada

$-7.17 \partial_{11} 1.0114$
$1.69269 E-17$

$-2.452672-16$

- $2.799118-16$

0.

0

0.

$3,30367 E-00$

13036000

1.462925 .04

1.00292500

-

.994 दBE- 15

$.55230 t=04$

.93950 EULA

$.93950 E \cdot 04$

$1.10205 E .03$

$-1.10205 E-03$

0.

$1.00328 E \cdot 03$

$-1.00328 E-03$

$4.74660 E=n L$

1.14375E.03

$1.14375 E-03$

$-1.12493 E-03$

1. $12003 E-01$

-3. HAUBQEOOH

3. $\triangle 6 B H O E-15$

$3.804 \mathrm{HBE}=110$

$-7.71754 E=00$

$7.71754 E .06$

(.)

o.

$3.61772 E-15$

$1.03165 E-14$

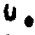

0

I. UAUALE-14

$1.01120 E-14$

1.07100 - 14 
CASE 1

O ION FACTORS
$x=1.0100$
$r=$
. 6 6ht7.
$2=1.0000$

INI)ICATIR FOR DISPLACEMFNT OR ACRFLERATION SPECTRIIPI =

EO." DI SPLACEMENT

EO. I ACCELERATION IN IN, / SEC.?

EO.? ACEELERATION IN ISS

1 SPESTRA ARE ENTERED FUR CASE 1. KIND $=2$

Q: CLIISIER FACTOR, CF $=.10000$ 
MUDAL PARIICIPATION FACTURS

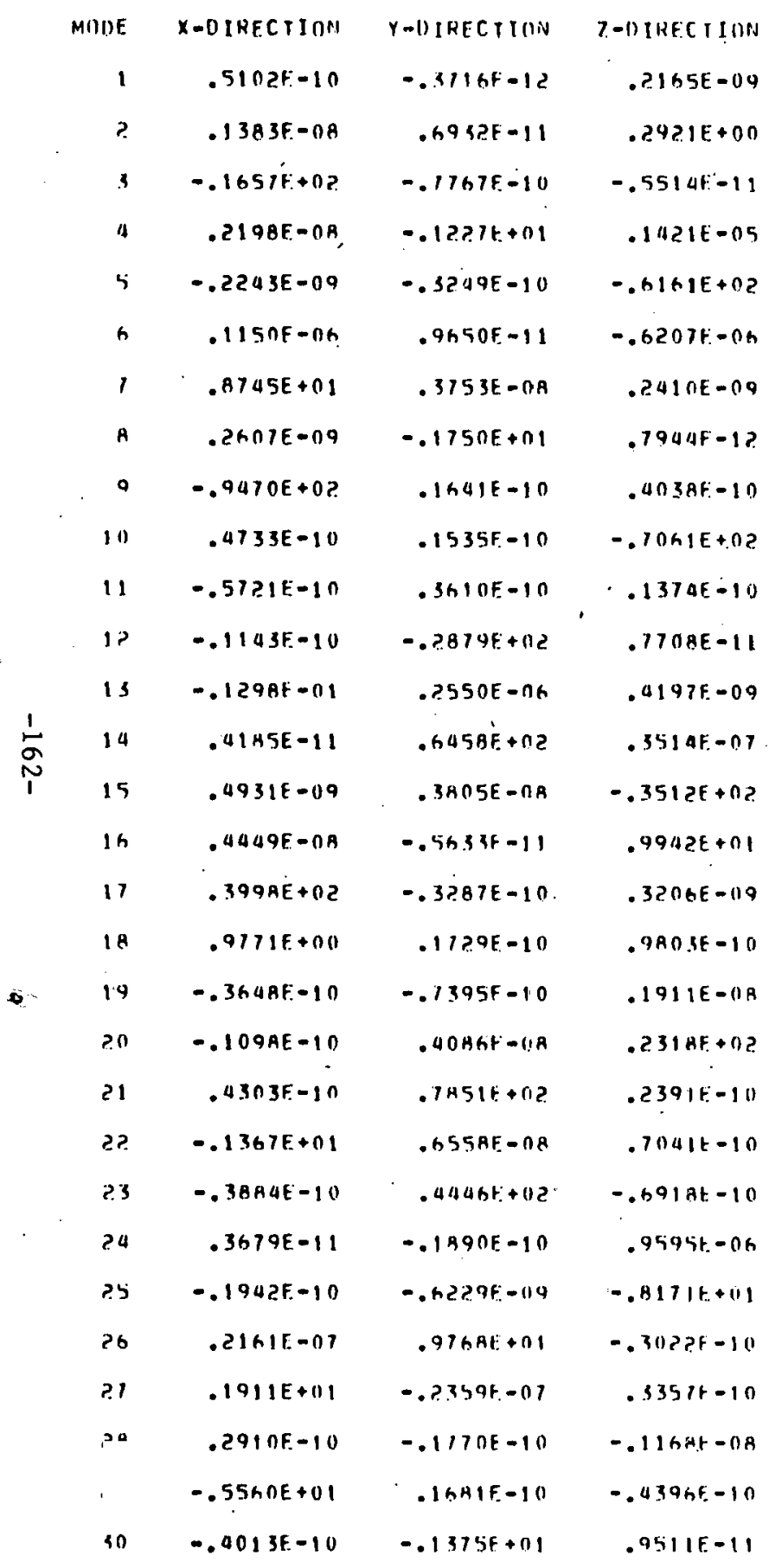


SPECTRIIM TAALE ( BENCHMARK PRORLEM RESPIONSE SPFCTRLIM.

NUMBER OF POINTS $=38$

SCALE FACTUR = $10000 F+O 1$

\begin{tabular}{|c|c|c|}
\hline INPIIIT & & SPFCTRIIM \\
\hline POINT & PERIOD & VAI.UFE: \\
\hline 1 & $.5000 E-02$ & $.1600 E+03$ \\
\hline 2 & $.1020 E-01$ & $.1600 E+03$ \\
\hline 3 & $.1391 E-01$ & $.2430 E+03$ \\
\hline$\Delta$ & $.1700 E-01$ & $.20430 E+03$ \\
\hline 5 & $.19 \geq 3 E-01$ & $. J T \cap O E+03$ \\
\hline 6 & $.2164 E-11 i$ & - $? \div 50 E+\cap 3$ \\
\hline 7 & $.2439 E-111$ & $.2<50 E+03$ \\
\hline A. & $.2667 E-01$ & $.46 .70 E+03$ \\
\hline 9 & $.4202 E-01$ & $.475 \cap E_{-}+03$ \\
\hline 10 & $.4609 E-01$ & $.55,5 \cap F+03$ \\
\hline 11 & $.552 A E-01$ & . $A 5 T O D E+03$ \\
\hline 12 & $-5 A B 2 E-01$ & $.7550 E+03$ \\
\hline 13. & $.6711 E-01$ & $.7 .550 \mathrm{~F}+103$ \\
\hline 14 & $.7110 E-01$ & . $A G S O F+03$ \\
\hline 15 & $.1000 E+O N$ & . AB5OE +03 \\
\hline 16 & $.1156 E+00$ & .12 2?E + n4 \\
\hline 17 & $.1413 E+00$ & $.12225+04$ \\
\hline $1 H$ & - I $\triangle A Z E+O D$ & $.1150 E+n 4$ \\
\hline 19 & $.1534 E+00$ & $.1399 E+04$ \\
\hline 20 & - IATGE+0O & $.13995+104$ \\
\hline 21 & $.1923 E+00$ & $.1140 E+04$ \\
\hline ?2 & $.2 ? .6 B E+00$ & $.1140 E+04$ \\
\hline 2.3 & $.2392 E+00$ & $.1 .757 E+n A$ \\
\hline 24 & $.2924 E+00$ & $.1357 F+104$ \\
\hline 25 & $.3049 E+00$ & $.1323 \mathrm{~F}+0.4$ \\
\hline ?ִh & $.3175 E+00$ & $.8550 E+1 j 3$ \\
\hline 27 & $.346 O E+00$ & . HSSOE + U3 \\
\hline 28 & $.3571 E+00$ & $.812 \cap F+n 3$ \\
\hline 29 & $.3922 E+00$ & $.8120 t+03$ \\
\hline 30 & $.4167 E+00$ & $.91 \angle \cap 0+113$ \\
\hline 31 & $.520 B E+00$ & $.9140 E+0 \%$ \\
\hline 32 & $.5263 E+00$ & . An5OE + 03 \\
\hline 33 & $.6173 E+00$ & - RASOF.+O3 \\
\hline 34 & $.6250 E+00$ & $.9050 E+03$ \\
\hline 35 & $.7813 \mathrm{~F}+100$ & $.9050 E+03$ \\
\hline 36 & $.8696 E+00$ & $.6620 E+03$ \\
\hline 37 & $.9524 E+00$ & . HG2OE + 03 \\
\hline 38 & $.10 \cap 0 E+01$ & . $6000 E+03$ \\
\hline
\end{tabular}

CLUSTERING. + 1 MEANS MHOE I CLDSF TO $1+1,-1$, HOT

1. 1. 1. 1. 1. 1. 1. 1. 1.-1.-1.-1.-1.1.1.1.1.1.-1.1.-1.-1.1.1.1.1.1.1.1.-1. 
Solution $4 a$

Interspatial/Intermodal Combination 


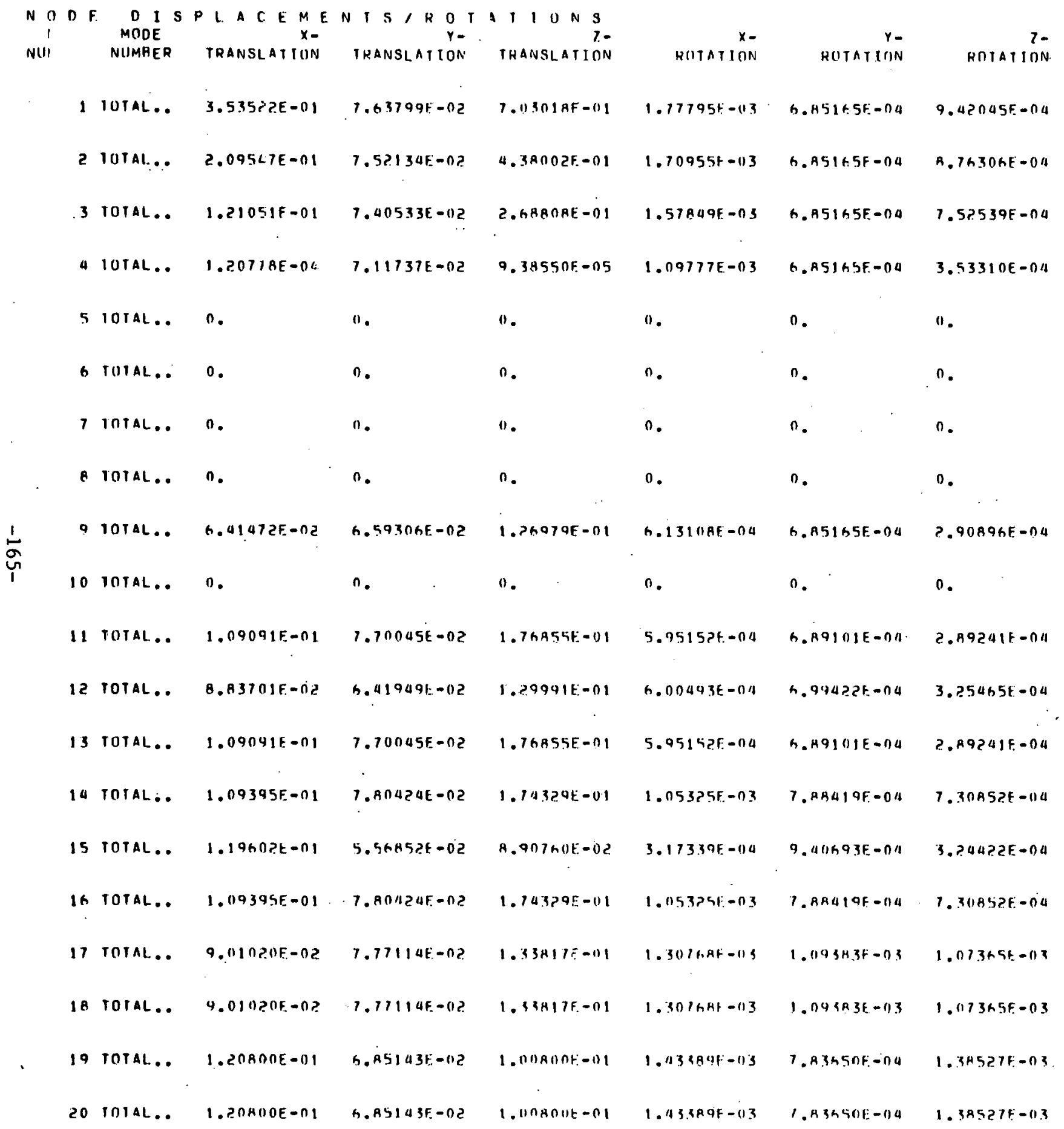




\begin{tabular}{|c|c|c|c|c|c|c|c|c|}
\hline . & 21 & TOIAL.. & $1.14317 E-01$ & $7.12521 E-02$ & 9. HU4GGAE-312 & 1. $A \cap 49$ ?E $=03$ & 7.17 ป $1 F-04$ & $1.41851 f-03$ \\
\hline & 22 & TOTAL... & $1.14317 E-01$ & $7.12521 E-02$ & $9.6414 h A F-112$ & 1. $.40492 F=03$ & $7.174 A 1 E-04$ & $1.41851 \mathrm{E}-0.7$ \\
\hline & 23 & TOTAL... & $1.2451 A E-01$ & $2.29123 E-02$ & $2.26213 E-01$ & $4.83634 E-03$ & $1.74657 E-03$ & $1.97270 E-03$ \\
\hline & 24 & TOTAL.. & 1.2451 AE $=01$ & $2.29123 F_{-}-02$ & $? .2 h ? 13 E-01$ & A. $336311 F-1) 3$ & $1.74657 E=03$ & $1.97270 F=03$ \\
\hline & 25 & TOTAL.. & $2.38773 E-01$ & 2.2AG52E $=02$ & $5.73150 E-01$ & 1. ABO $0.2 \cup E-03$ & $1.46211 E-03$ & 2. . $0457 h E-03$ \\
\hline & 26 & TOIAL... & $2.38773 E-n 1$ & ?. TRASTE-0? & $5.73150 E-01$ & $4.8 A 020 E-03$ & 1. . $65211 E-03$ & $2.04576 F=03$ \\
\hline & $? 7$ & TOTAL.. & 0. & 0 & $n$ & 0. & $n$. & 0 \\
\hline & 28 & TOTAL.. & 0 & $n$ & 0. & 0. & 0. & $n$ \\
\hline & 29 & TOTAL.. & $5.35547 E-01$ & $2.39140 E=02$ & $1.24560 E+00$ & 5.33 ?.4E -03 & 1.Aค? 1 IE-ก3 & $2.47273 F-03$ \\
\hline & 30 & TOIAL.. & $5.35547 E-n 1$ & $2.39140 E-02$ & $1.24560 E+00$ & $5.332445-03$ & $1.36211 E-0.3$ & $2.47273 F .-03$ \\
\hline & 31 & TOTAL... & $2.24894 E-01$ & $4.78673 E-02$ & 5. OAAOOE -01 & $4.78029 t-03$ & $1.97228 E-03$ & $1.95717 F-03$ \\
\hline & 32 & TOTAL.. & ว.?.४QपE-01 & $4.78673 E-0.2$ & $5.048001-101$ & $4.7802 .9 F .-03$ & $1.9722 B E-03$ & $1.95717 E-03$ \\
\hline & 33 & TOTAL.. & $1.832 .45 E-01$ & $1.47348 \mathrm{AF}-0$ ? & $3.014565 F-01$ & $2.84233 E-03$ & $3.30590 F-113$ & $8.12854 E-04$ \\
\hline & 34. & TOTAL.. & $1.26534 E-01$ & $2.52305 E-02$ & A.A $\{A 93 F=02$ & $2.29164 f-04$ & h.76? UOE-OI & $3.50185 E-044$ \\
\hline & 35 & TOIAL.. & $1.83 ? 45 E=01$ & $7.47348 \mathrm{~F}=02$ & $3.0 .8565 F_{-}-01$ & $2.8423 \times f-0.3$ & $3.30590 E-03$ & B. $12854 F=04$ \\
\hline & 36 & IOTAL... & $1.45296 E-n 1$ & $3.18595 E_{-}-02$ & $1.22921 t=n 1$ & $1.021 .291:-113$ & $2.70194 t=-0.3$ & $5.9 A G ? 2 E-04$ \\
\hline & 37 & TUTAL.. & $1.45296 E-01$ & $3.18595 E-02$ & 1. Р.2)? IE-01 & $1.0 ? 129 \mathrm{E}-03$ & $2.70194 E-03$ & $5.986 ? 2 E-114$ \\
\hline & 38 & TOTAL... & $1.33095 t-01$ & $1.54223 E-02$ & $1.0 H S \cap R F-n 1$ & 1. HGPAAF-(1) 4 & $2.35 .3119 F-05^{\circ}$ & 4. 1997 A6E -05 \\
\hline & 39 & TOTAL.. & $1.34420 E-01$ & $7.1 A 936 F=03$ & $1.0 h>2.3 F-1) 1$ & $1.70155 t-04$ & $1.523 \cap h F-05$ & $3.47639 E-115$ \\
\hline & & TOTAL... & $1.3 .3095 E-01$ & $1.54 ? ? 3 F-0 ?$. & $1.0450 A F=01$ & $1.6 A 2 A R F-011$ & $\therefore .353113 F-05$ & $4.49766 F-115$ \\
\hline
\end{tabular}




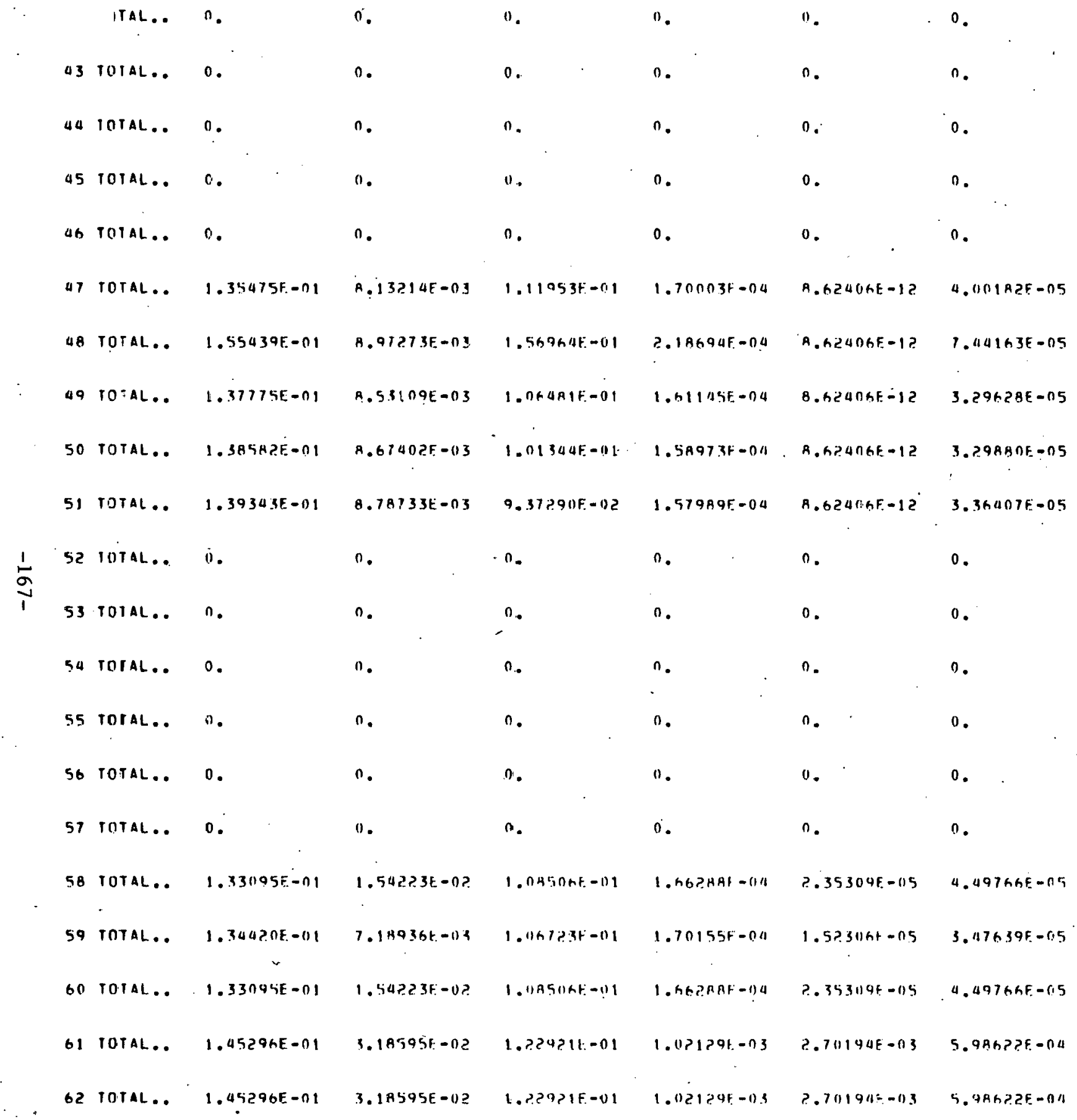




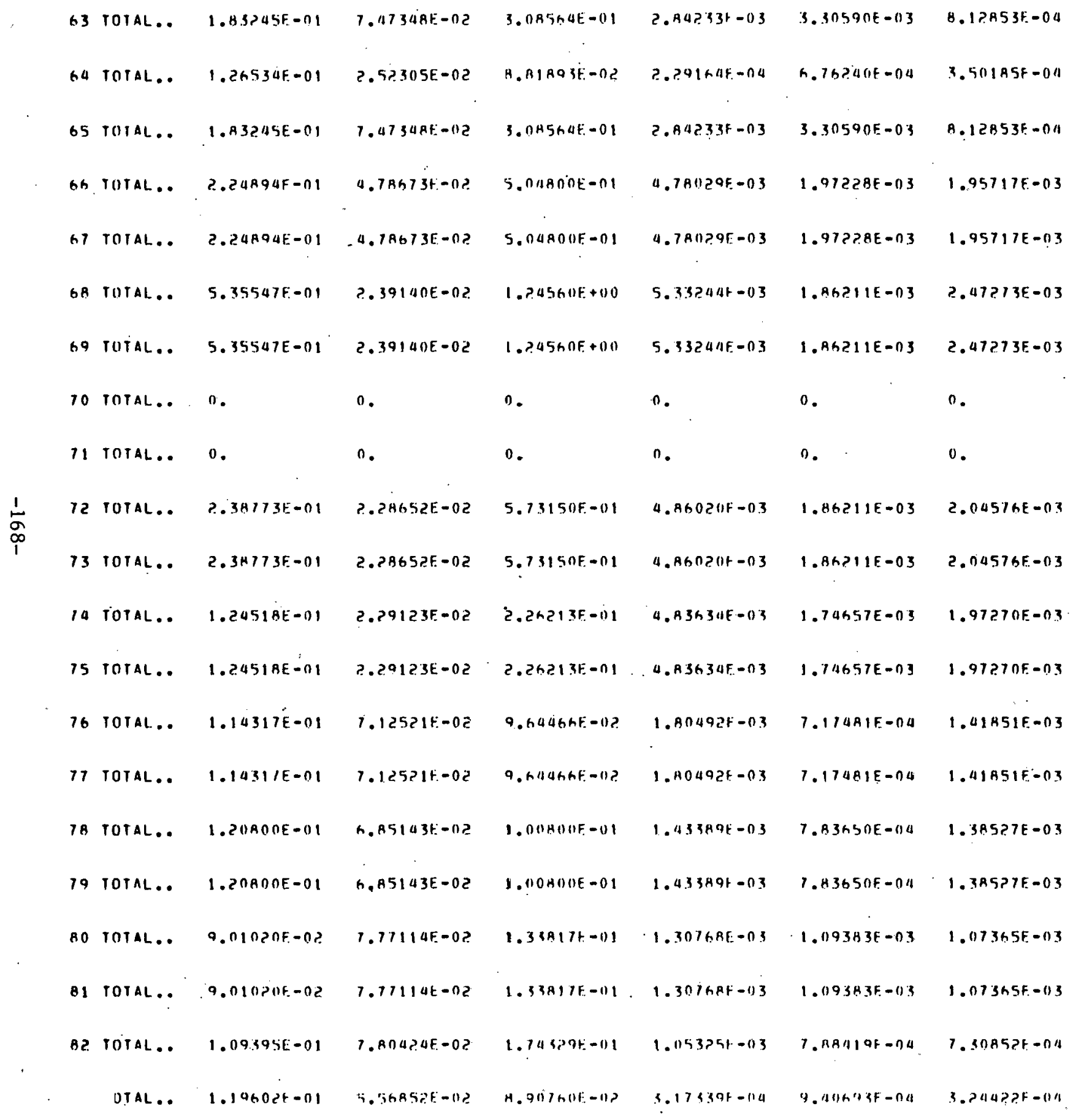




\begin{tabular}{|c|c|c|c|c|c|c|c|}
\hline$\cdots$ & TOTAL.. & $1.09395 E-01$ & $7.8 \cap 424 F-012$ & $1.711329 t-61$ & $1.010325 t-013$ & 7.RAQ1 GF -04 & 7.3085 2t -04 \\
\hline 85 & TOTAL.. & $1.09(1 Y \mid E-1) 1$ & $1.70045 t-0 \bar{c}$ & $1.76855 E-111$ & 5.9515 P. -04 & $\because A \cap \mid \cap 1 E-0) 4$ & ?.R9?41F-011 \\
\hline Bh & TOTAL.. & $1.09091 E-01$ & $1.70045 E-0 \tilde{E}$ & -1.7 hA5SF -01 & $5.4515 P F-04$ & R.A9101E-04 & 2.89241E-04 \\
\hline $8 ?$ & FOTAL.. & A.B3:OIE-O? & $6.41949 E-0 \bar{c}$ & $1 . ? 9991 E-01$ & $6.00493 F-144$ & $6.9947 .2 E-04$ & $3.25465 E-04$ \\
\hline 88 & TOTAL.. & $n$. & n. & $n$. & 0 . & $\because$. & 0 . \\
\hline 89 & TOTAL.. & 6.41472E -0? & h.5930hF -02 & $1 . ? 6979 F-01$ & $6.1310 \mathrm{AF}-04$ & G.A51RSE-04 & $2.90896 E=04$ \\
\hline 90 & $\begin{array}{c}\text { TOTAL.. } \\
\text {. }\end{array}$ & $1.20 P 78 E=04$ & $7.11737 \mathrm{E}-02$ & $9.38550 F-05$ & $1.097775=03$ & 6.AS165E-0a & $3.53310 E-04$ \\
\hline 91 & TOTAL.. & $n$. & 0 . & 0 . & 0. & n. & 0 . \\
\hline $9 ?$ & TOYAL.. & 0. & 0 . & 0 . & 0 . & 0 . & 0. \\
\hline 93 & TOTAL.. & 0. & 0 . & 0 . & 0 . & 0. & 0 . \\
\hline 94 & IOTAL.. & $n_{0}$. & 0. & 0 . & $n$. & D. & 0 . \\
\hline 95 & IOTAL... & $1.21051 E-01$ & $1.40533 E-02$ & 2. KAAกAE - OI & $1.57 \times 408-013$ & 6.A5165E-04 & $7.52539 E-114$ \\
\hline 96 & TOTAL.. & $2.09547 \mathrm{~F}=01$ & $7.52134 \mathrm{t}-02$ & 4. 3Aก1)IE-n1 & $1.70455 F-03$ & B. ASIBSE-04 & $8.76306 E-114$ \\
\hline 97 & TOTA & 3 & $99 E=02$ & $7 E-01$ & $5 F-1) 3$ & 6.A5165F-04 & $9.42045 E$ \\
\hline
\end{tabular}


RESPONSE SPECTRIM STRESS C OHPONEN.TS (KINO I ?)

OH EACH ELEMENT, THE FOLLOWING INFORMATION IS PRITITED:

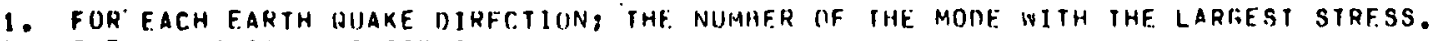

2. THE VALIHE OF THAT STRFSS.

3. IF REUUESTED): THE MODE aY MOUE STRESSES FIR EACH EARTH WILAKE DIRECTIUN.

4. THE RESIILTANT FDR EACH EARTH JILAKE DIRECTION.

5. THE GRAND TOTAL OF THF. THREE FARTH DIIAKE DIHE.CTIUNS.

(NOTE THE $X$, $Y$, OR $Z$ REFEHS IO THE FARIH DUAKF. DIHECTION.)

ELEMENT TYPE (A O UIN D A R $Y$

), 1, ELEMENT NUMAFE,

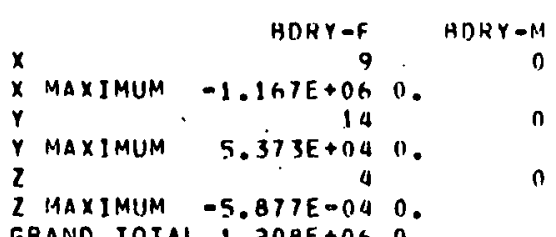

GRAND TOTAL I. $20 B E+06$ O

ELEMENT TYPE (A U II N D A R

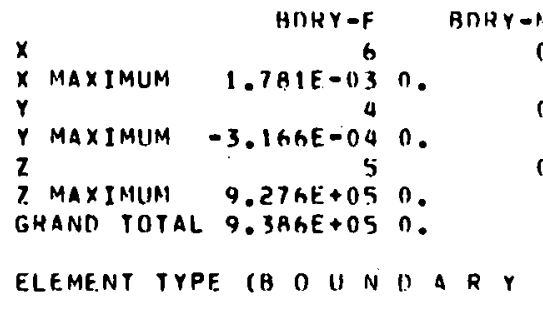

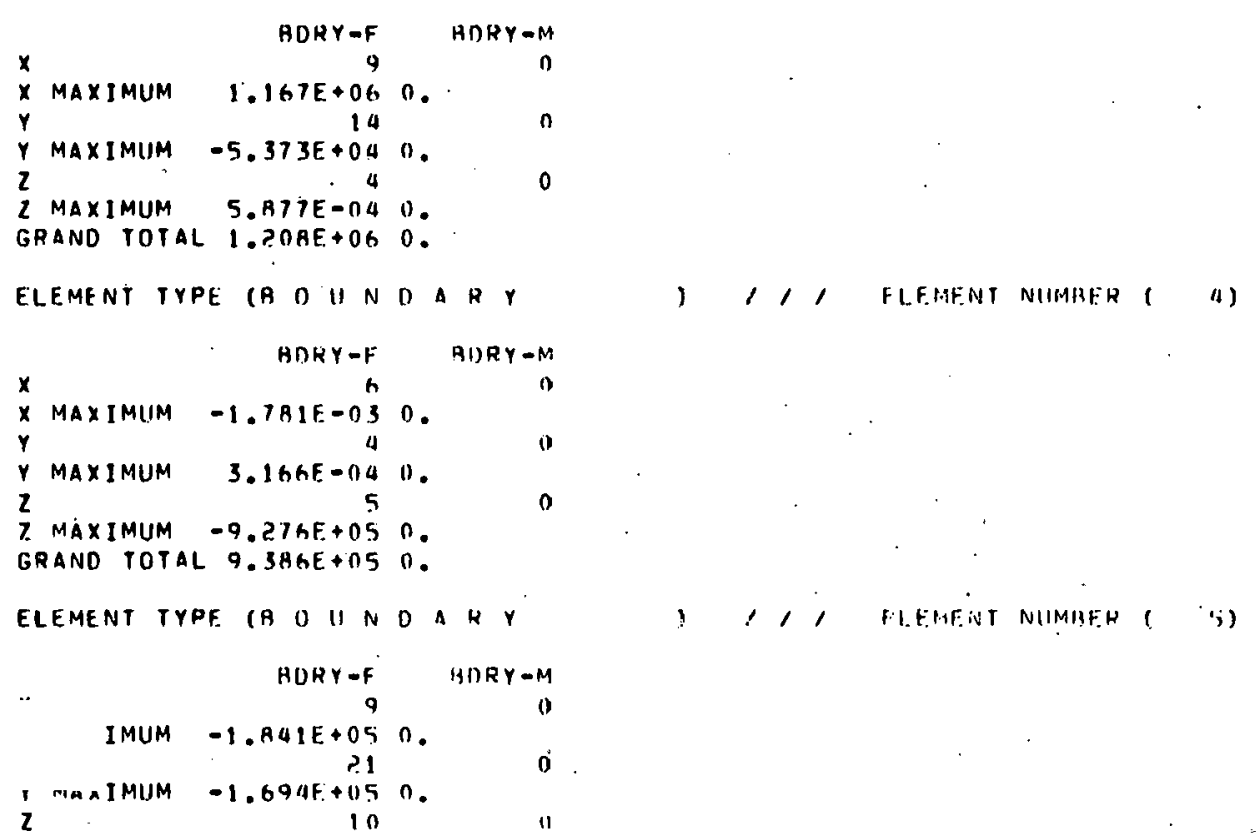

) 1,1 FI.EMENT NIIMRFR (2)

10 
ELEMENT IYPE (A I) U N N D A A 
$Y$ MAXIMUM -1.A15E+05 0 .

2 MAXIMUM

GRAND TOTAL $3.595 E+050$.

ELEMENT TYPE I $U$ U UNDAR $Y$

, 1,1 ELEMENT IJUMAFR $(1 \geq)$

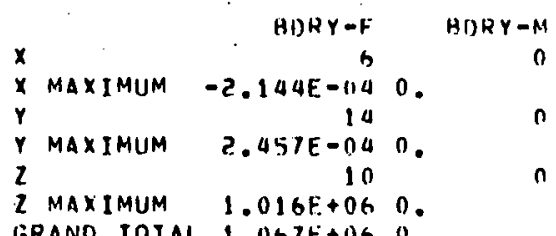

GRANO TOIAL $1.067 t+060$

ELEMENT TYPE (H OU U N I) A R Y

) 1,1 ELEMENT NUMHER ( 13)

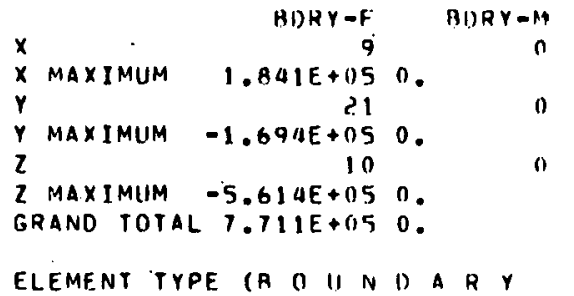

ELEMENT TYPE (A O UN

), 1, FLEMENT NUMARER (14)

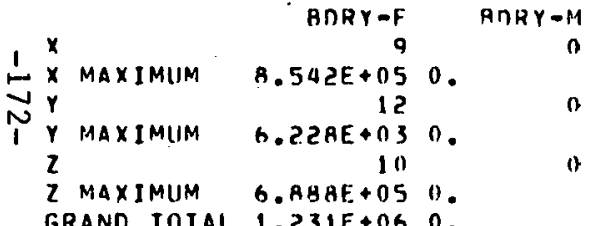

GRAND TOTAL 1.231E+06 0 .

ELEMENT TYPE CH U U N

, 1, FLEMENT NIMMAR ( 15)

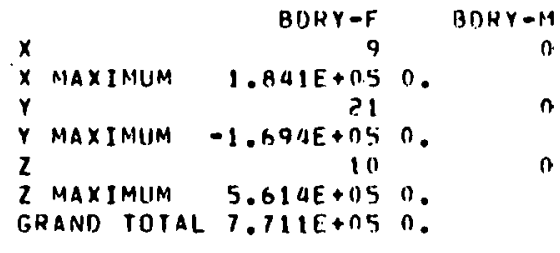

ELEMENT TYPE (A O UIN N) A R Y

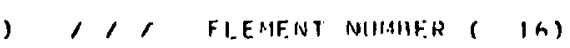

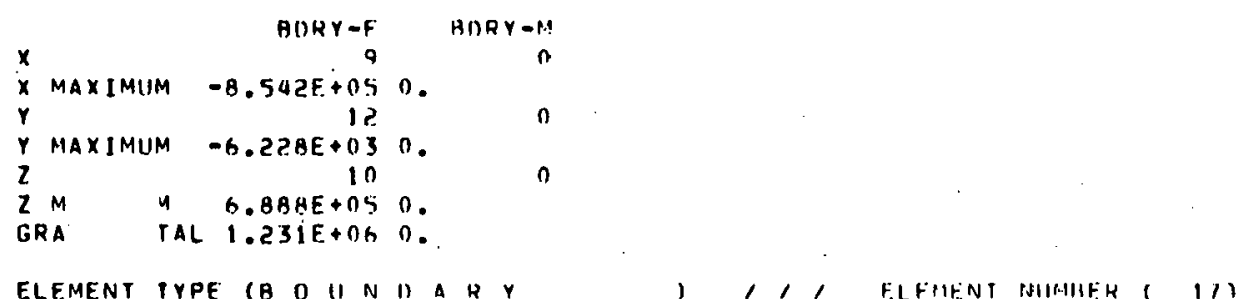




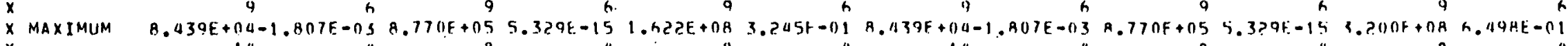

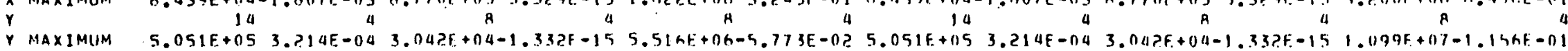

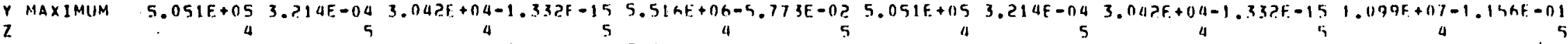

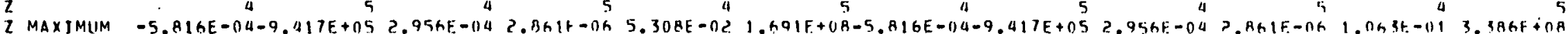

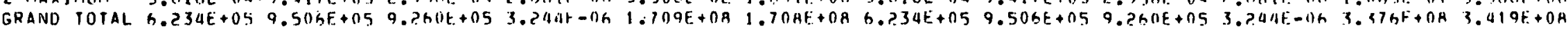

ELEMENT IYPE (3/1) P I P FE,, 1, FLEMENT NIAMHEK $($ (1)

$x$ MAXIMUM

Y MAXIMUM

V2(i) $1 \times(1)$

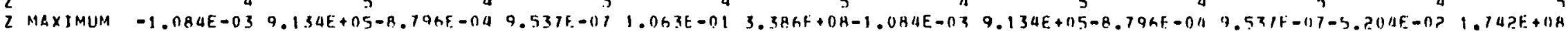

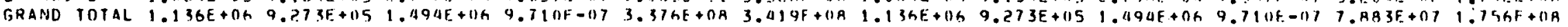

ELEMENT TYPE (3/1) P I P E , 1, F.LEMFINT NIJHHER $(5)$

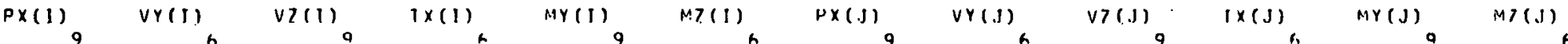

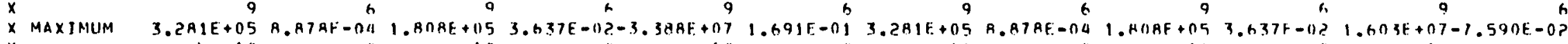

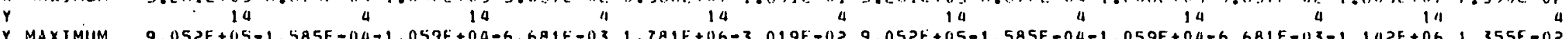

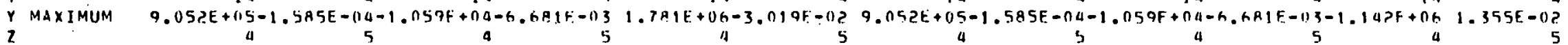

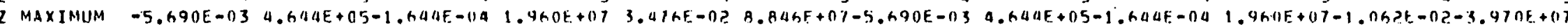

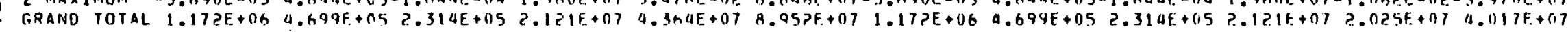

ELEMENT TYPE $(3 / D$ P I P E

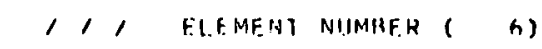

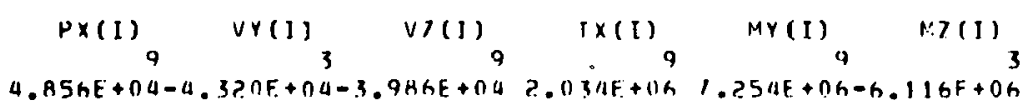

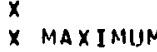

$P \times(J)$

$V Y(J)$

$v 7($.

$1 \times(1)$

MY(I) MZ(J)

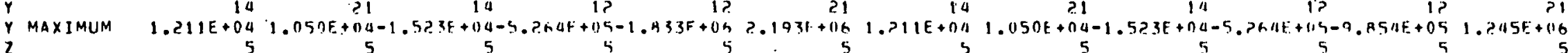
$Y$
$z$ D. BSAE + OA $04-4.320 E+014-3$.

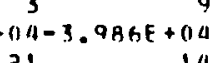

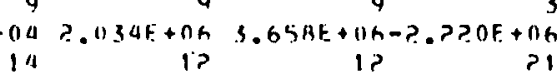

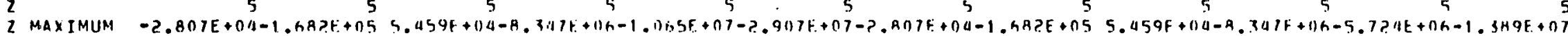

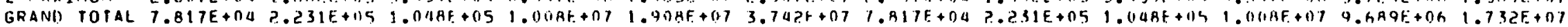

ELEMENT TYPE (3/0, P P E 1,1 , ELFHFNT MIIMAER $(7)$

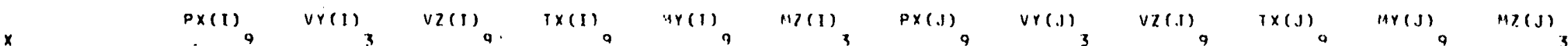

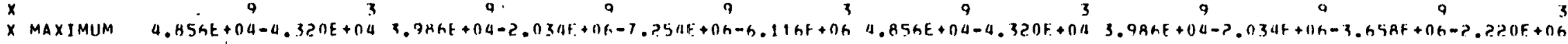
$X$
$Y$

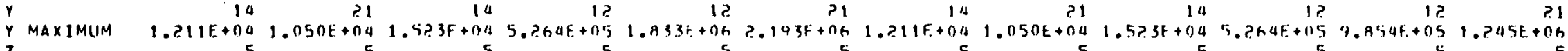

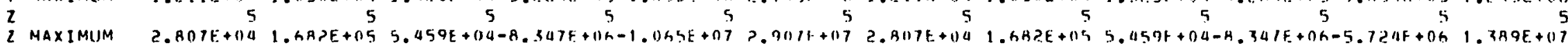

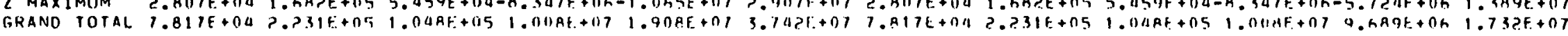
ELEMENT IYPE $(3 / 0$ PIPF
$x$
$x$
$y$
$y$
PX(I) $\quad \operatorname{vr}(1)$

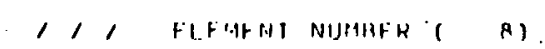


ELCMENT TYPE (3/D P I PE ), 1,1 FLEMENT NUMHFN ( 9)

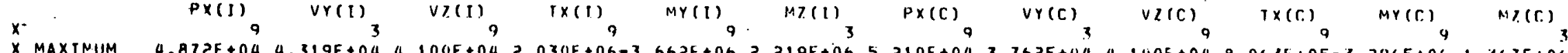

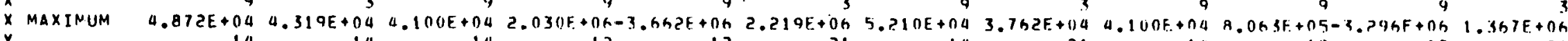

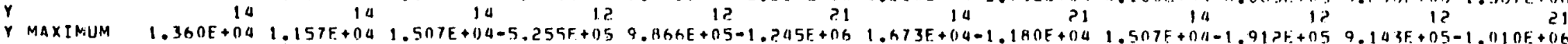

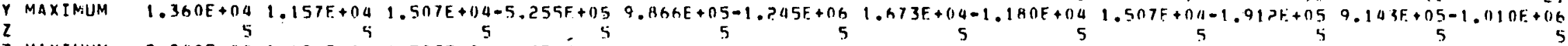

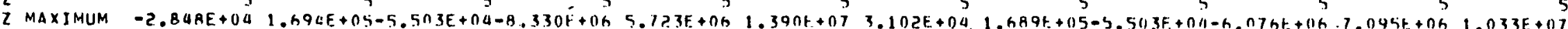

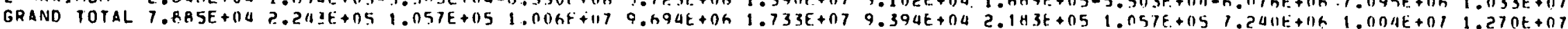

ELEMENT TYPE $(3 / 0$ P I P E $, 1,1$ EI.FMENT NUNBER $(9)$

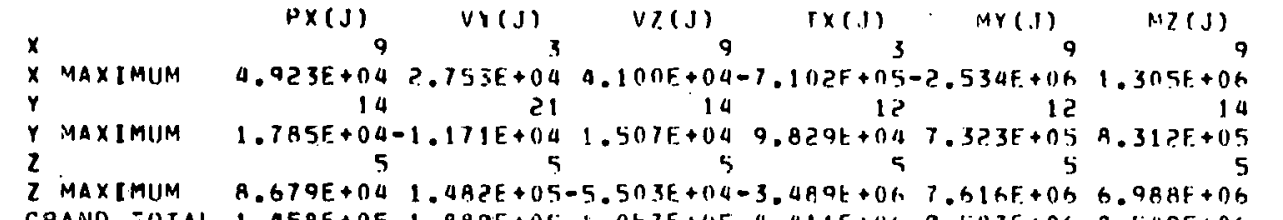

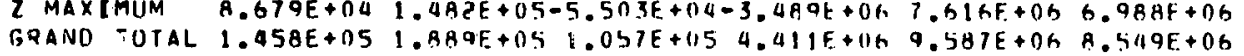

ELEMEN TYPE $13 / 0$ P I P E $, 1,1$ E.LEMENT NUHAER $(10)$

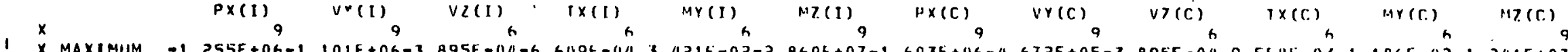

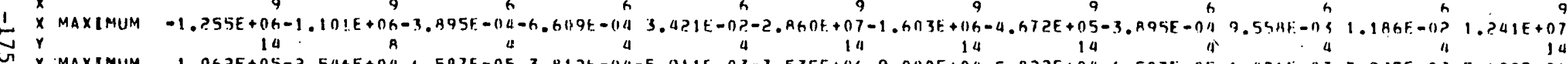

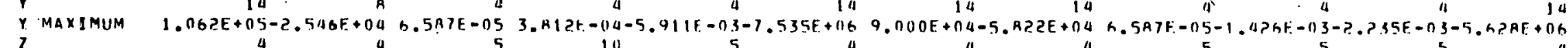

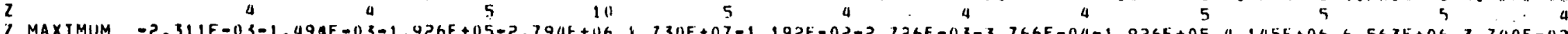

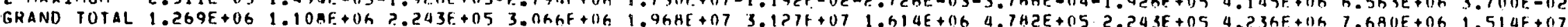

ELEMENT TY.PE (3/0 P I P E , 1,1 FI.FIAENT NIJMBF.R $(10)$

PX(J)
$X$

ELEMENT TYPE $(3 / n$ P IPE ,, 1, ELFNENT NIJ:AHFR $(11)$

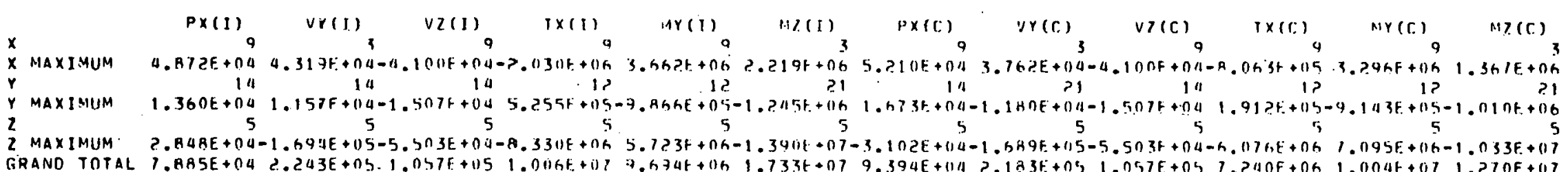

ELEMENT TYPE $(3 / 1) \cdot P$ I $P$,, 1, F.LFMENT NIHAHFR $(11)$

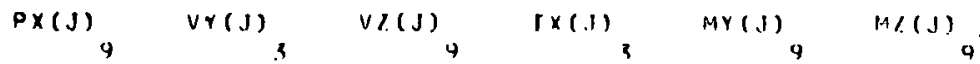




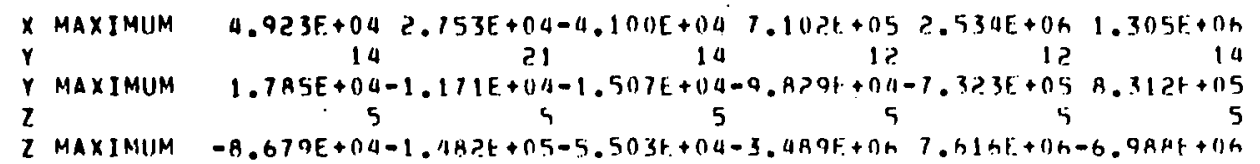

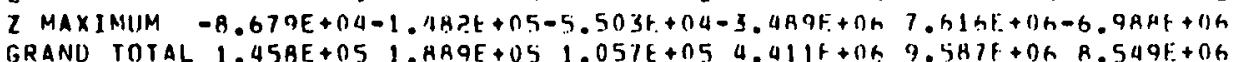

ELEMENT TYPE (3/0 P I P E , 1,1 HLEENT MIJMAER $(12)$

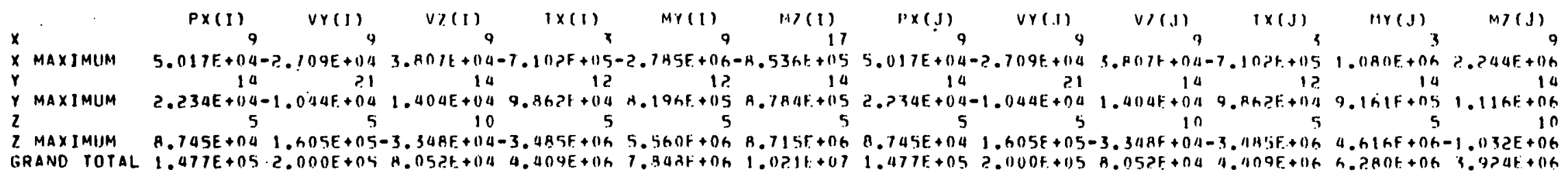

ELEMENT TYPE (3/0 P I PE , , , FELNFNT NURAREK 1 13)

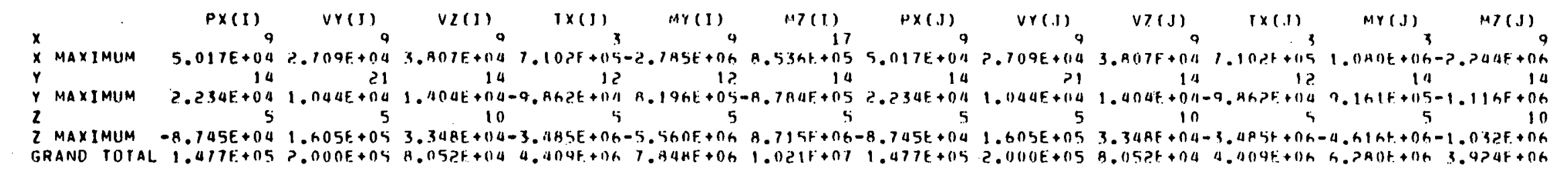

ELEMENT TYPE (3/0) P I PE , 1,1 T.LEMENT NIJMHFR ( 14)

尚

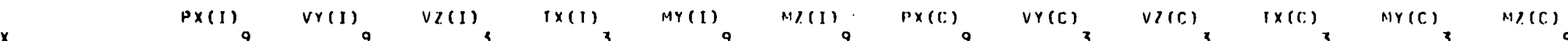

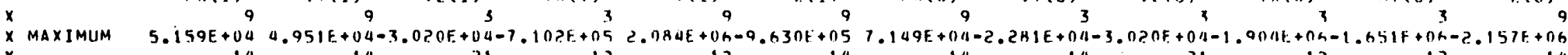

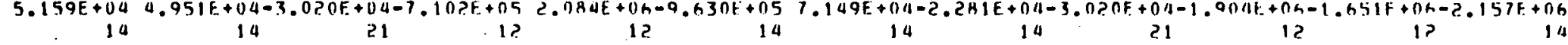

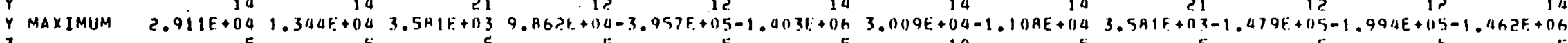

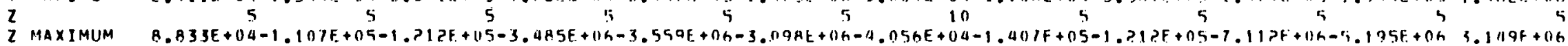

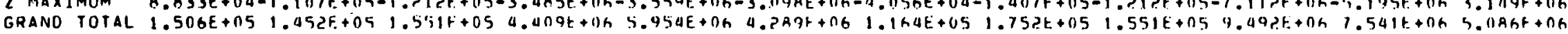
ELEMENT TYPE $13 / 0$ P I P E $, 1,1$ ELEMENT NIMARER $(14)$

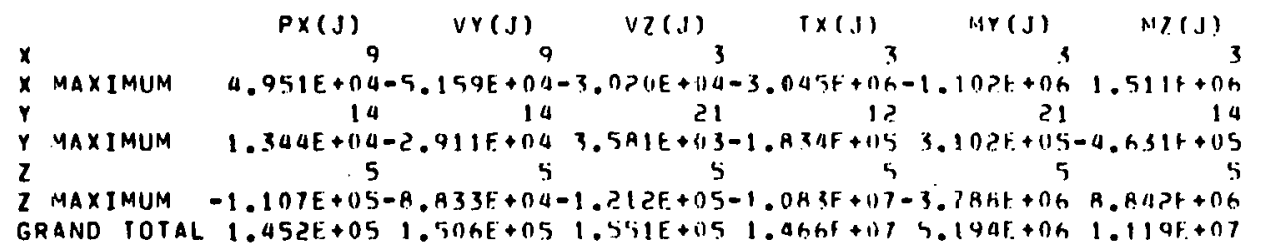

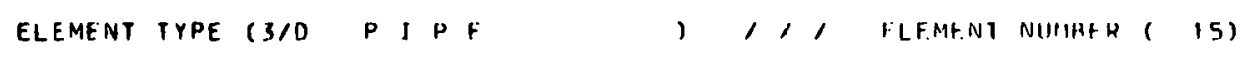

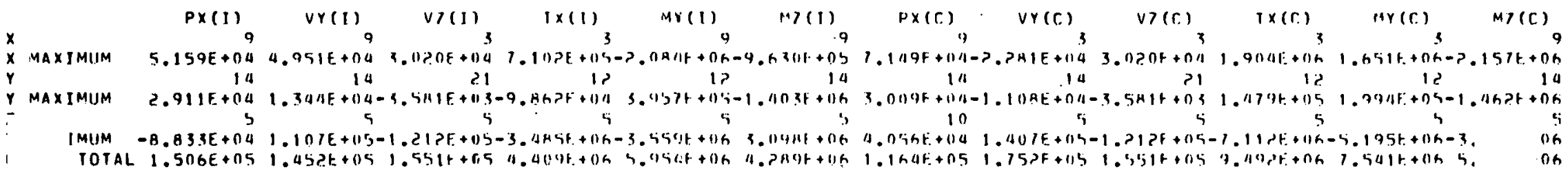




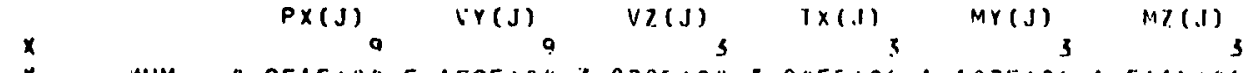

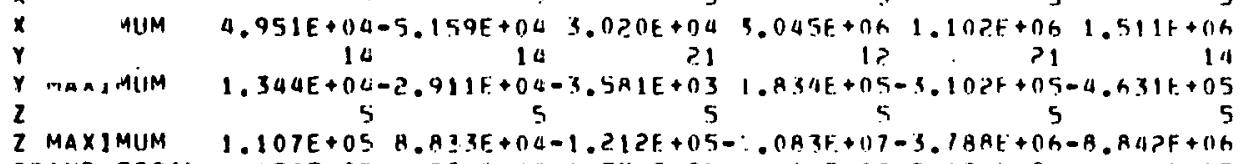

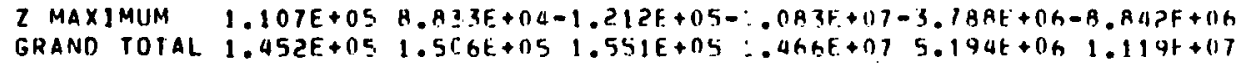
ELEMEAT TYPE (3/D F I P F ) $;,$ F.F.MENT NIMHER $(16)$

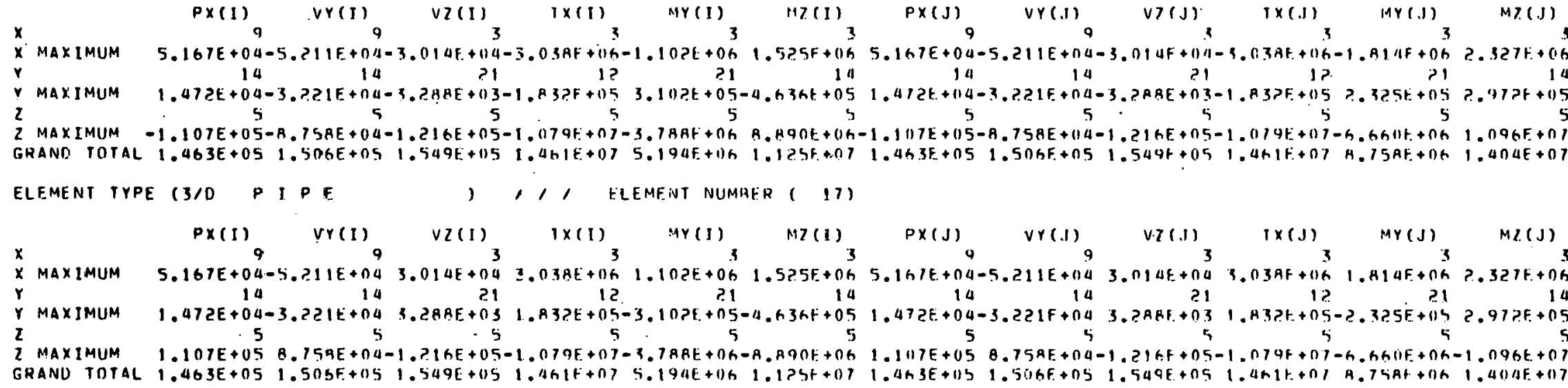
I ELEMENT TYPE $(310$ P I P $\begin{array}{lccccc}x & 9 & 9 & 3 & 3 & 3 \\ x\end{array}$

PX(I) VY(I) VZII) TX(I) MY(I) NiZ(I)

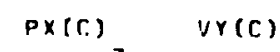

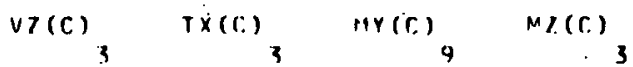

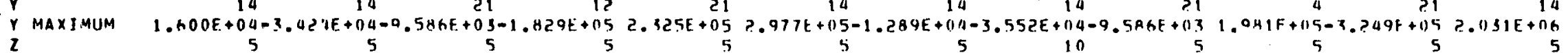

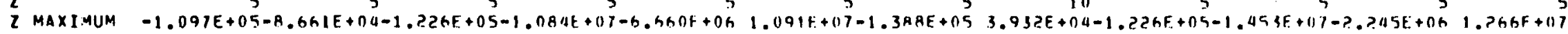

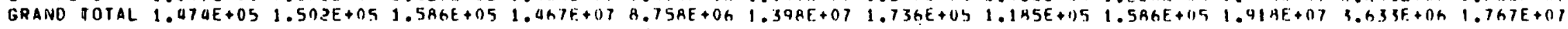

ELEMENT TYPE (3/0 P I P E ), 1, HLEMENT NIIHAFH $($ IA)

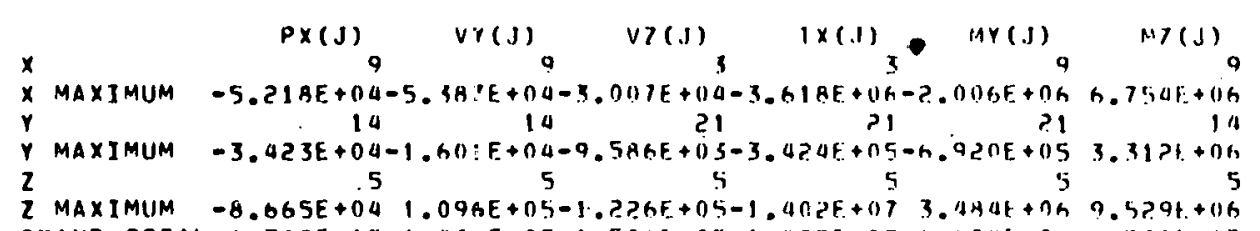

2 MAXIMUM $-8.065 E+041.096 E+05-1.0 ? 26 E+05-1.002 E+1173.9444+9 h-9.5 ? 9 t+06$

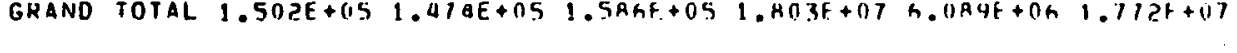

ELEMENT TYPE $(3 / 1)$ P I P $f, 1$, FIEMENT NUMAFE $(19)$

$x$
$x$ MAXINIIM

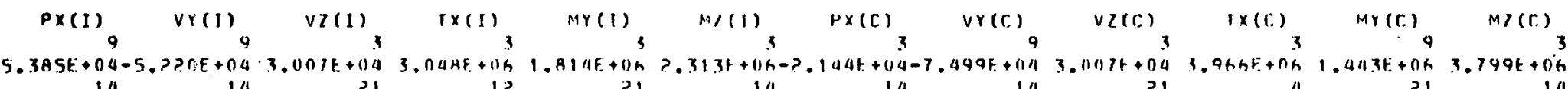

Y MAXINUM

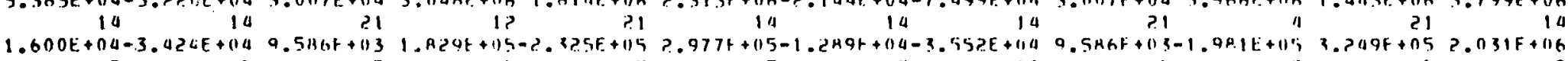




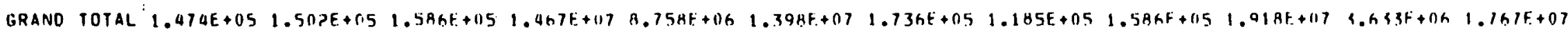
ELEMENT TYPE $(3 / 0$ P I P E

1,1 E.LEMENT NIIMHER $(19)$

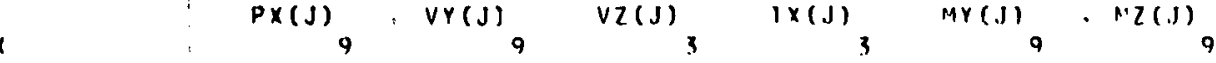

$\times$ MAXIMIIM - -5.218E+04-5. $3 A 7 E+043.007 E+043.61 R E+0 h \quad 2.006 F+06 \quad 6.754 F+06$

$Y$ MAXIMUM $-3.423 E+04-1.601 E+04$
2

2 MAXIMUM 50555 CRAND TOTAL $1.50250051 .474 E+05$

ELEMENT TYPE (3/D P I P E , , , FLEMENT NUMHER $(20)$

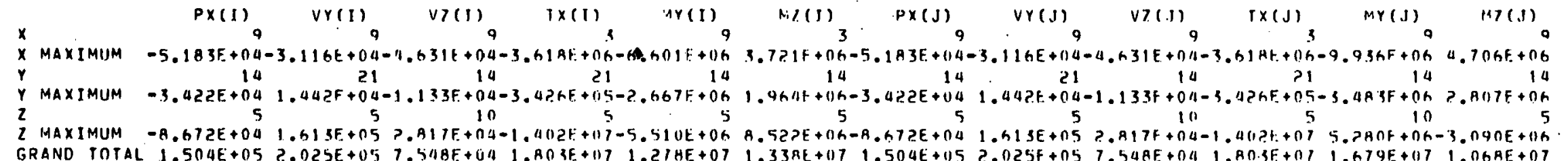

ELEMENT TYPE (3/0 P I PE , , , FLFMENT NUMHFR $(21)$

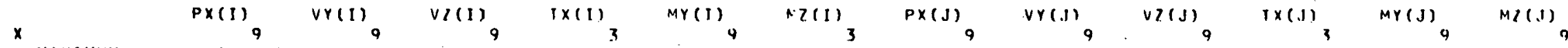

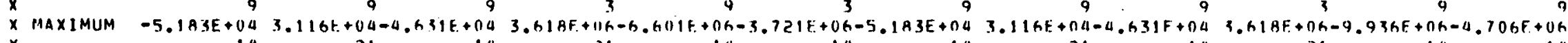

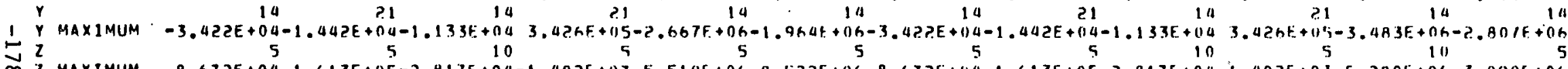

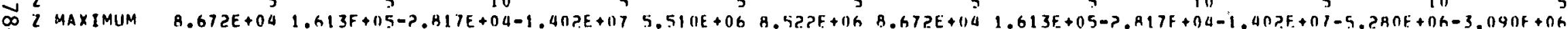

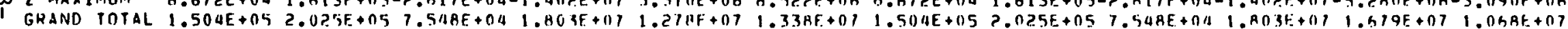

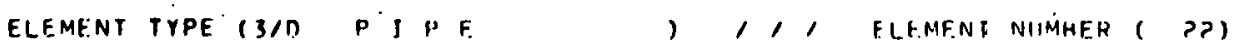

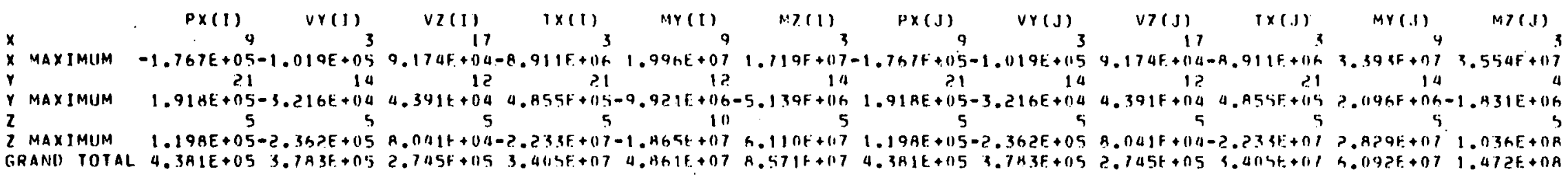

ELEMENT TYPE (3/0) PI PE,, 1, ELEMFINT NUMHER 1 ?

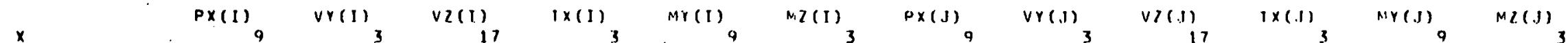

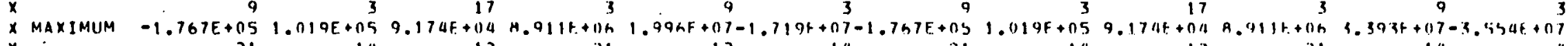

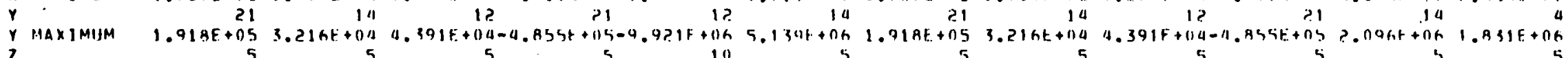

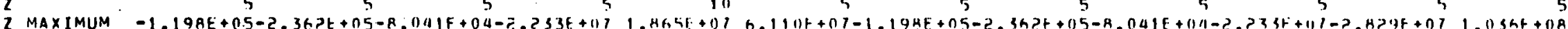

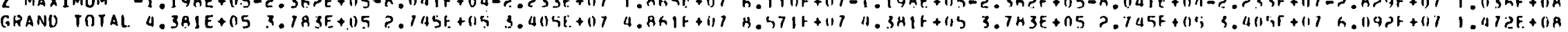

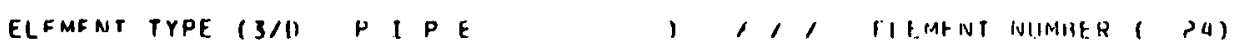

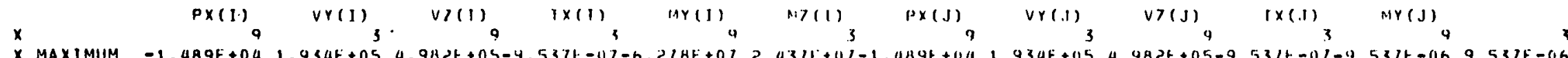

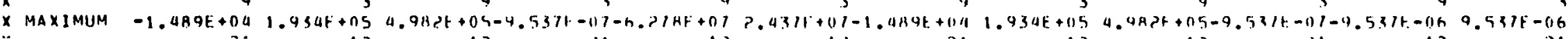


$Y$
$Z$

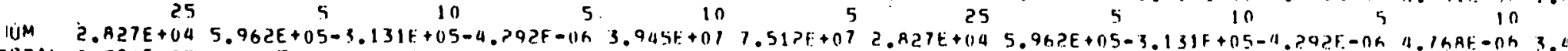

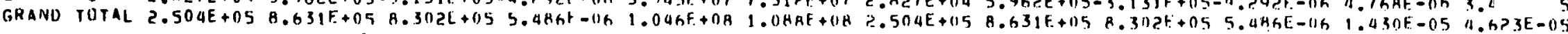

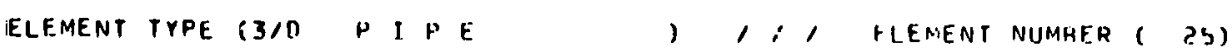

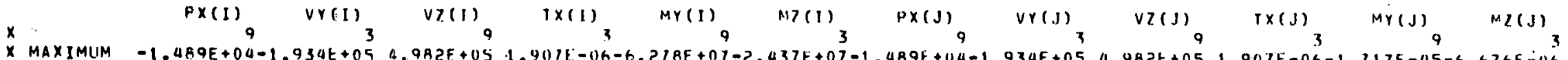

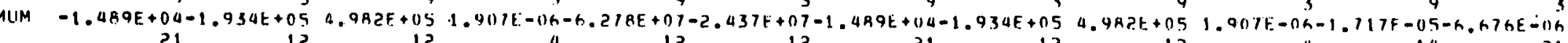

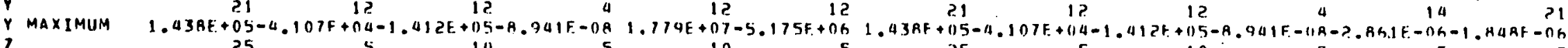

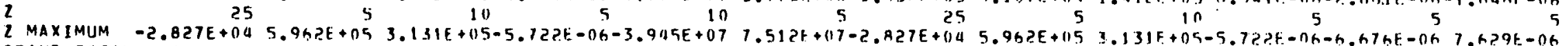

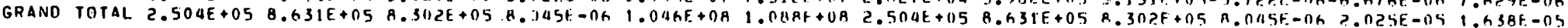

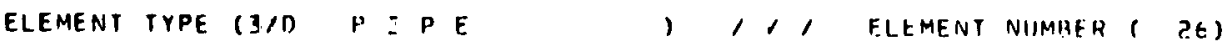

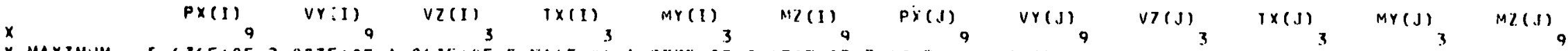

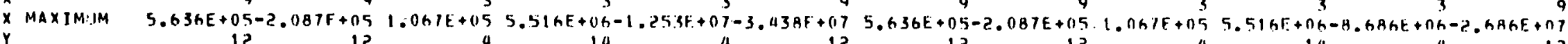

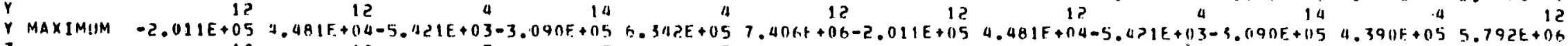

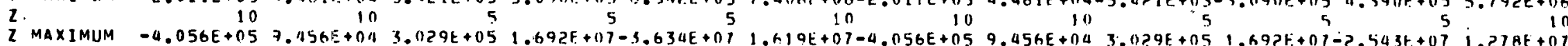

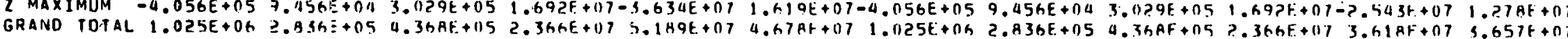
ELEMENT TYPE (3/0 F I PE , , ELLMENT NUHHER ( ? ?)

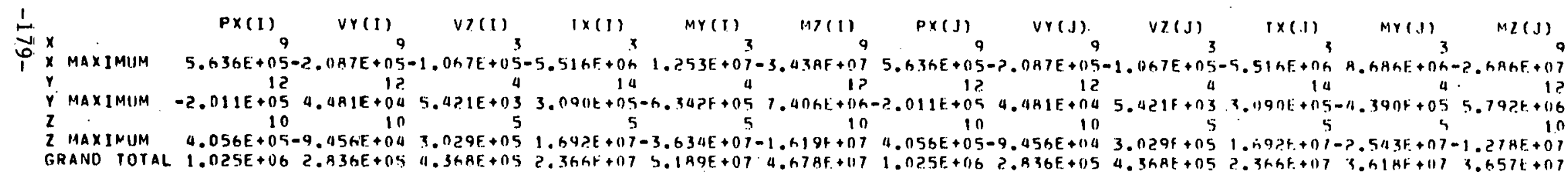

ELEMENT TYPE (3/D P I PE , , , FLEMENT NUMHFR ( 2 H)

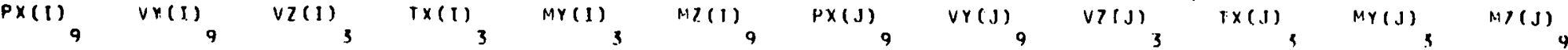

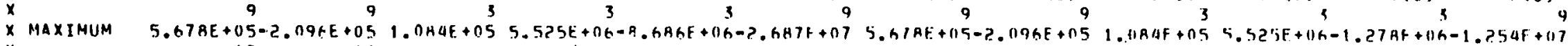

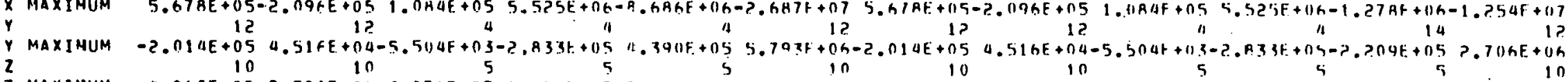

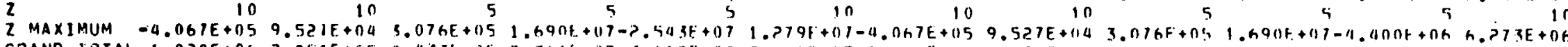

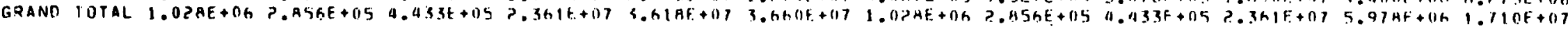

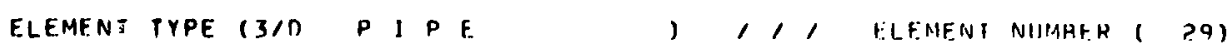

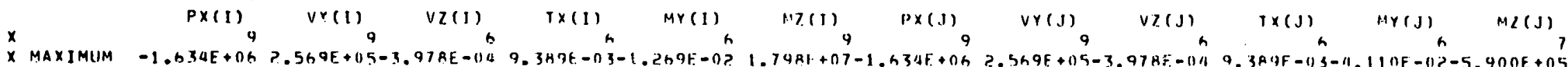

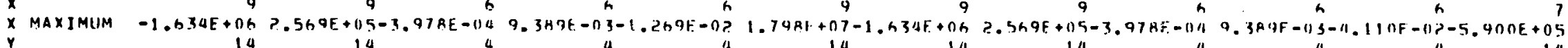

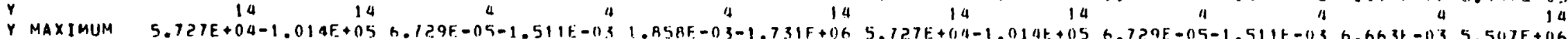

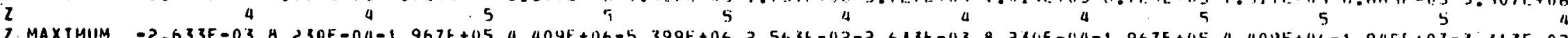

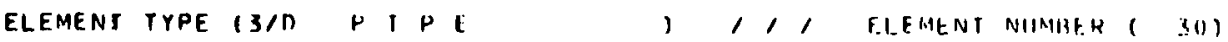

\begin{tabular}{|c|c|c|c|c|}
\hline$P \times(I)$ & $\operatorname{vr}(1)$ & V)(I) & $1 x$ & ) \\
\hline
\end{tabular}




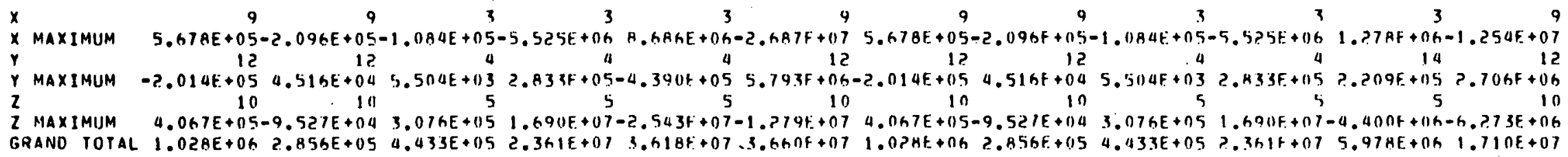

ELEMENT TYPE ( $3 / 0$ P I P E , , , FIEMENT NIMAHER ' 31 )

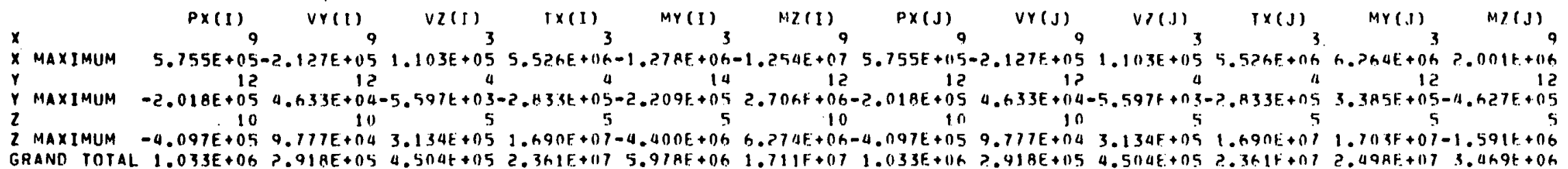
ELEMENT TYPE $13 / 0$ P I P E ,, 1, ELEMENT NUHAHR $(3 ?)$

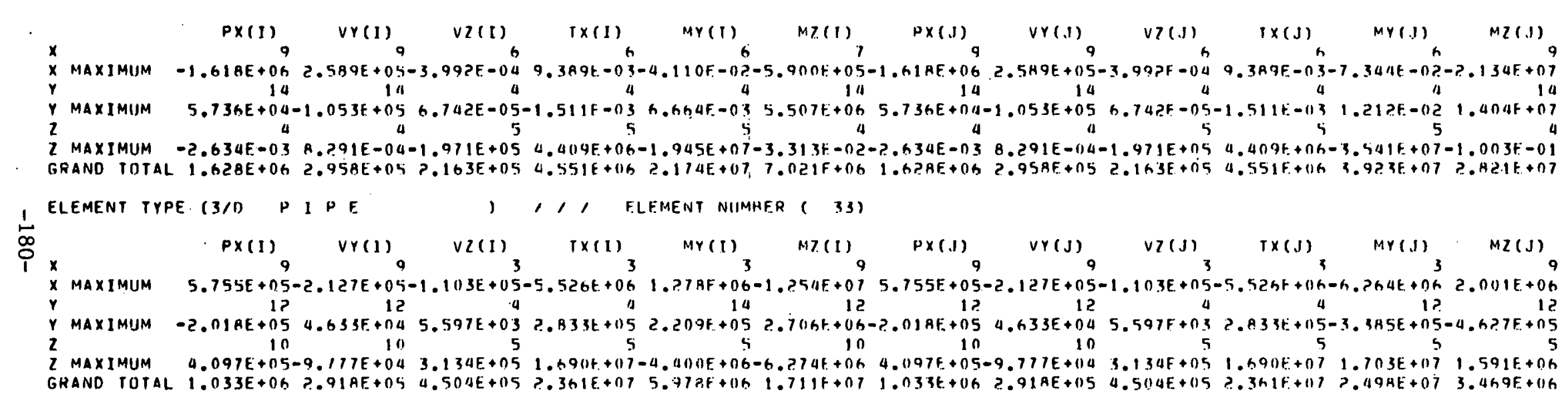

ELEMFNT TYPE (3/0 P I.PE , 1,1 ELEMENT NUMHER 134 )

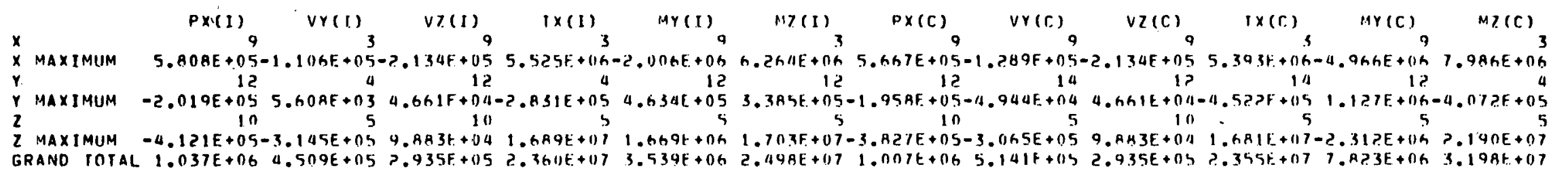
ELEMENT TYPE $(3 / 0$ P I PE , , , HIEMFNT NIJHAFR $(39)$

\begin{tabular}{|c|c|c|c|c|c|c|c|}
\hline & & $P \times(J)$ & $\operatorname{Vr}(.1)$ & $V<(J)$ & $1 \times(. I)$ & $\operatorname{cy}(.1)$ & (J) \\
\hline & & 9 & 9 & 9 & 3 & 9 & 3 \\
\hline & MAXIMUM & $5.145 E+05$ & $-2.10 S E+115$ & $-P .134 E+115$ & A. $A 7$ 가 +06- & $1.534 t+0 \mathrm{~h}$ & $9.4211 F+116$ \\
\hline$y$ & & 12 & t2 & 10 & 14 & i? & 14 \\
\hline & MAXIMUM & $1.765 E+05$ & A1IE+OA & 4. AGIL+OLA- & -7. BIIIF +05 & 1. $115 t+0 h$ & $1.34 \mathrm{st}+0 \mathrm{~h}$ \\
\hline 2 & & 10 & 5 & 10 & 5 & 5 & 5 \\
\hline & MUJM & $6 E+05$ & $-2.18 \cap E+O S$ & $9 . A H 3 F+04$ & $1.511++117-$ & $1 \mathrm{t}+0 \mathrm{~h}$ & $4 t+07$ \\
\hline & TOTAL & $23 A E+0 S$ & 11 it +105 & $2.955 E+05$ & $2 .+37 f+07$ & $1.3951+07$ & \{. 92 $25+01$ \\
\hline
\end{tabular}




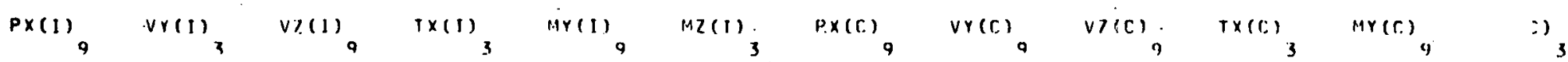

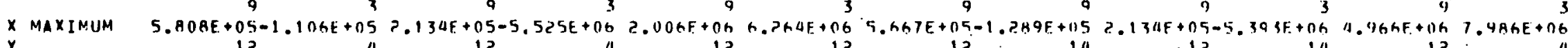

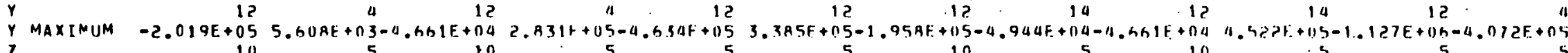

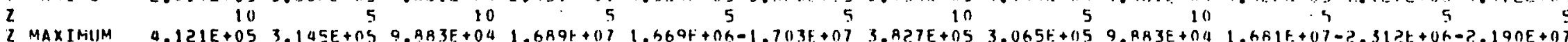

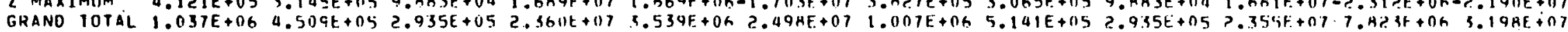

ELEMENT TYPE (3/0 P 1 PE , , , ELEMENT NUMAFH ( 35)

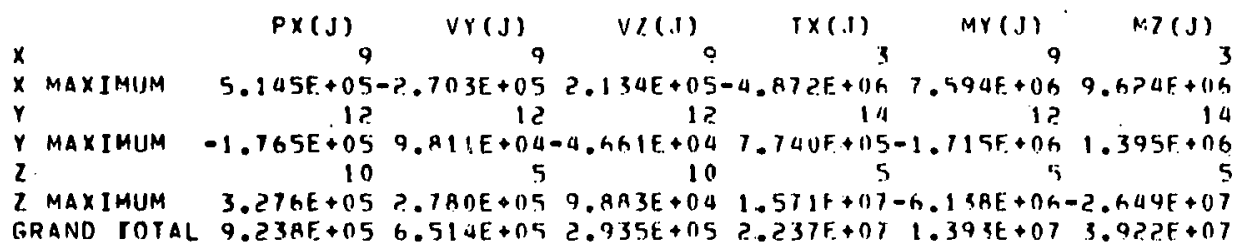

ELEMENT TYPE (3/0 PI PE , $1 \%$ ELEMENT NUMAFR $(36)$

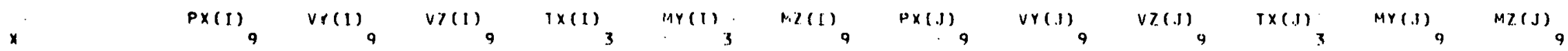

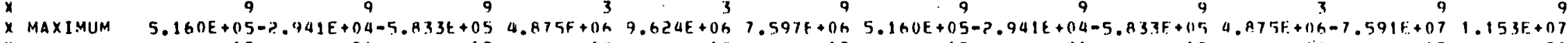

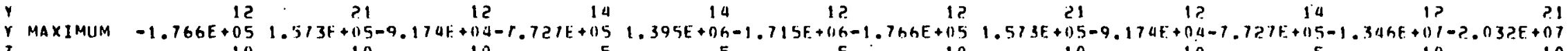

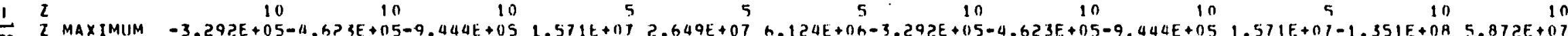

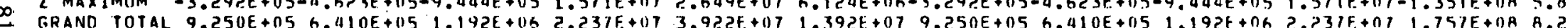

ELEMENT TYPE (3/0 P I PE ; 1,1 FI.FMENT NIJMAER ( 37)

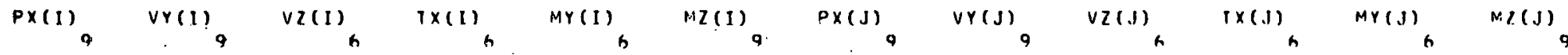

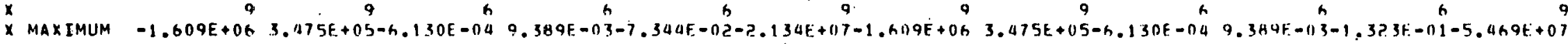
$X$
$Y$

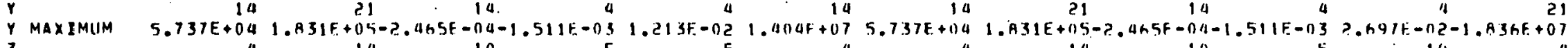

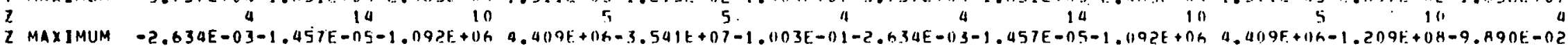

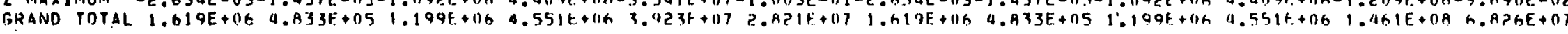
ELEMENT TYPE (3/0 P I PE $;: 1$, EILFMFNT NIIMAER ( $3 A)$

\begin{tabular}{|c|c|c|c|c|c|c|c|c|c|c|c|c|}
\hline & $P \times(I)$ & $\operatorname{vr}(1)$ & $V Z(I)$ & $1 \times(1)$ & $\operatorname{Mr}(I)$ & $\operatorname{mz}(1)$ & $p \times(, J)$ & VYं (J) & $V Z(J)$ & $1 \times(.1)$ & $.1 .4(.1)$ & M7. (J) \\
\hline$x$ & 9 & 9 & 9 & 3 & 3 & 9 & 9 & 9 & 9 & 3 & 4 & \\
\hline$X$ MAX:MUIM & $16 \cap E+05$ & $-2.941 E+04$ & $5.833 t+05$ & $-4.87 \mathrm{SF}+\mathrm{Oh}-$ & $-9.62 H E+0 h$ & $7.597 t+06$ & $5.160 f+05-$ & $-? .941 E+04$ & S.HS3E+ & $=11.875 t+116$ & $1.541 f+07$ & $1.153 E+07$ \\
\hline$\gamma$ & 12 & 21 & 12 & 14 & 14 & 12 & 1? & ?.1 & 12 & 14 & i? & 1 \\
\hline$Y$ MAXIMUUM & $1.766 E+05$ & $1.573 E+05$ & $9.174 t+104$ & $7.127 \mathrm{t}+105-$ & $-1.395 F+n h-$ & $-1.715++10 h-$ & -1. IGAE + US & $1.573 E+015$ & $9.174 F+04$ & $7.7 ? 7 t+n 5$ & $1.346 t+07=$ & $-2.03 ? F+07$ \\
\hline 2 & 10 & 10 & 10 & 5 & 5 & 5 & 10 & 10 & $\ln$ & 5 & 10 & ( \\
\hline$Z$ MAXIMUM & $3.292 E+05$ & 4. he $3 F_{-}+05-$ & $-9.1144 E+05$ & $1.571++07$ & $2.6498+07=$ & $-n .1245+0 h$ & 3. 2.92E+115 & 4. $R ? ? E+05-$ & $-9.1144 t+05$ & $71 F+117=$ & $+O A-$ & +07 \\
\hline GRAND TOTAL & $9.250 \mathrm{~F}+0 \mathrm{~S}$ & $0.410 F+05$ & $1.192 E+66$ & $? .23 \mid t+1) 7$ & $3.92 ? 6+07$ & $1.39 ? f+(i)$ & $9.251 E_{-}+05$ & 6.410 & $1.192 f+r i h$ & +67 & $1.737 t+08$ & H. 2 \\
\hline ELEME VT TYP & E $(3 / 1)$ & I PE & ) & 1,1 & FHE NS NIUMH & HEN 1 & & & & & & \\
\hline & $p x^{\prime}(1)$ & $\operatorname{VY}(1)$ & V711) & $1 \times(1)$ & $\ln Y(I)$ & 1:7 (1) & $P:(J)$ & VY (.J) & V7 (J) & $7 \times(3)$ & HY $(. J)$ & $M 7(J)$ \\
\hline$x$ & $2 n$ & in & 9 & 9 & 9 & in & 2h & 16 & 3 & 9 & $\varphi$ & \\
\hline MAXIMUJM & $E-195$ & $-05-$ & $173 t+06$ & $5.5311-06$ & 2.25Pt +0 A- & $-1 . A 11+-02$ & A 1 BE - $115-$ & $1316-05-$ & $-1.1735+n s$ & $5.6371-11 h$ & . $6 ?$ AF. - OSF- & $.435 F-106$ \\
\hline $\mathbf{r}$ & I ج & 14 & 13 & $1 ?$ & 13 & 14 & 21 & 14 & 13 & 12 & 14 & \\
\hline MAXIMUM & $E+0 S$ & 4.1A $4 F-n i-$ & $-7.551 F-014$ & $3.3104=01$ & $1.1505-01$ & A. $1344-0)=$ & $-3.206 ? t+n i$ & $4.1 A \triangle E-114=$ & $-7.551 f-04$ & $\{.310 E-1) 8$ & $5.16 / 4 F-01$ & $.055 E-07$ \\
\hline 7 & 4 & 10 & 16 & 5 & in & in & a & 10 & in & 5 & 4 & in \\
\hline
\end{tabular}


2 MAXIMUM 5.368E-05 1.62AE+06-1.563E-05 5.732E-07 3.001E-03 3.125F+0H 5.36AE-05 1.62AE+06-1.563F-05 5.73PE-117 1.033E-01) 7.629E-06

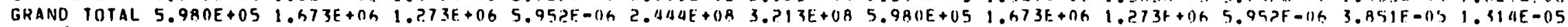
ELEMENT TYPE (3/0 P I PE 1,1, HIEMENT NUMHE. 1 an)

\begin{tabular}{|c|c|c|c|c|c|c|c|c|c|c|c|c|c|}
\hline & & $P \times(1)$ & $\operatorname{vr}(1)$ & $V I(I)$ & $I X(I)$ & $\operatorname{MY}(I)$ & $M Z(1)$ & $P \times(J)$ & $\operatorname{VY}(J)$ & $v z(.1)$ & $1 \times(3)$ & $M Y(.1)$ & $M Z(J)$ \\
\hline$x$ & & $\because 2 h$ & in & 9 & 9 & 9 & is & 26 & in & 9 & 9 & 9 & 16 \\
\hline & MAXIMUM & $-4.115 E-05$ & $1.067 F-04$ & $1.158 E+06-$ & $-2.530 E-0 S-$ & $1.5 R G E+0 R$ & 1. 8 Р ВF $-112-$ & $-4.115 E-05$ & $1.067 \mathrm{E}-04$ & $1.15 H F+0 F=$ & ?. $53 \cap E-\cap S-$ & $-6.128 .8 F+07$ & Q. 31 AE -03 \\
\hline$Y$ & & 21 & 14 & 13 & ? 1 & 13 & 14 & 21 & 14 & 13 & ?. 3 & 13 & in \\
\hline & MAXIMLIM & +05 & h. $2075-04=$ & $5 E-04$ & $A 1 E=\cap A$ & 4.5AhE - 02 & $9.025 F-02$ & $544 E+05$ & $6.207 E-04-$ & -04 & $(E-O) A$ & A09F-0? & $3.911 E-n ?$ \\
\hline$z$ & & & 10 & 16 & 10 & is & 10 & 4 & 10 & $1 \mathrm{~h}$ & 10 & 16 & 10 \\
\hline & $\begin{array}{l}\text { MAXIMUM } \\
\text { RAND TOTAL }\end{array}$ & $\begin{array}{l}05 \\
05\end{array}$ & $\begin{array}{l}1.097 E+0 h \\
1.227 E+06\end{array}$ & $\begin{array}{l}S 2 E-05- \\
23 E+06\end{array}$ & $\begin{array}{l}77 E-1) 6- \\
47 E-05\end{array}$ & $\begin{array}{l}8 E-03 \\
P E+O B\end{array}$ & $\begin{array}{l}41 R E+O R- \\
642 E+O B\end{array}$ & $\begin{array}{l}072 E-05 \\
491 E+05\end{array}$ & $1.097 E+0 h$ & $=052 E-05$ & $\begin{array}{l}077-06- \\
747 E-05\end{array}$ & $\begin{array}{l}.265 E-013 \\
-260 E+07\end{array}$ & +07 \\
\hline
\end{tabular}
ELEMENT TYPE (3/0 P I PE $, 1,1$ ELEMENT NIIMHER $(41)$

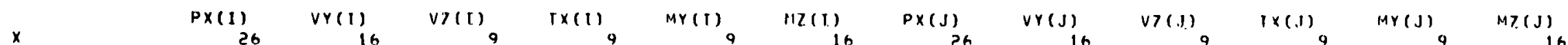

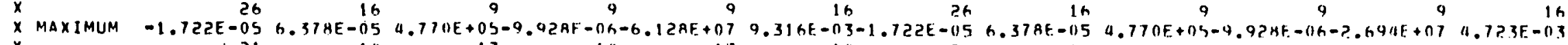

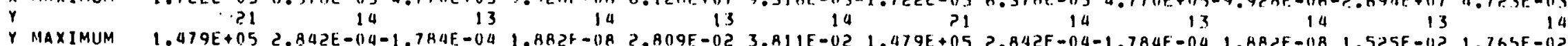

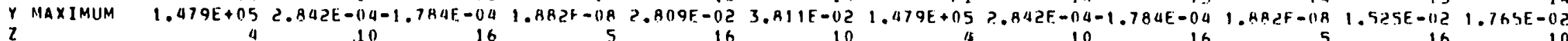

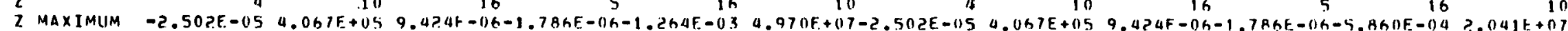

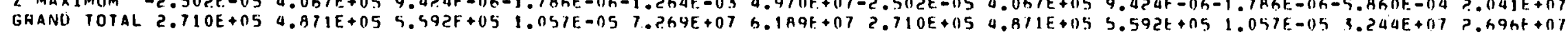
ELEMENT TYPE (3/0 P P $P$, , , ELFHF.NT NUMBER $(4 ?)$

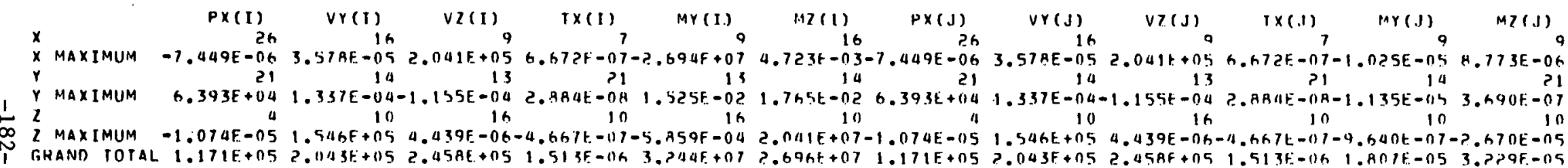
ELEMENT TYPE ( $3 / 0$ P I P F , , , FLEMENT NIIMBER ( 43 )

$\times$
$\times$

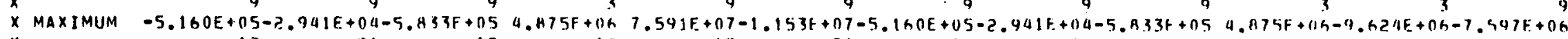

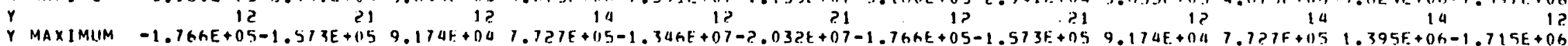
Y MAXIMUM -1.76hE+05-1.573E+05 9.174F+00 $1.727 E+05-1.346 E+07-2.032 E+07-1.76 h E+05-1.573 E+059-174 E+0117.7 ? 7 F+115 \quad 1.395 E+06-1.715 E+06$

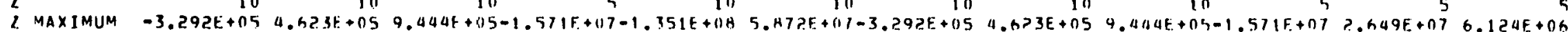

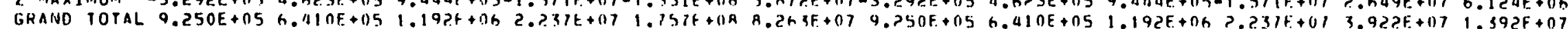

ELEMENT IYPE (3/D P 1 P E , , , FLENHET NUMRER ( 44 )

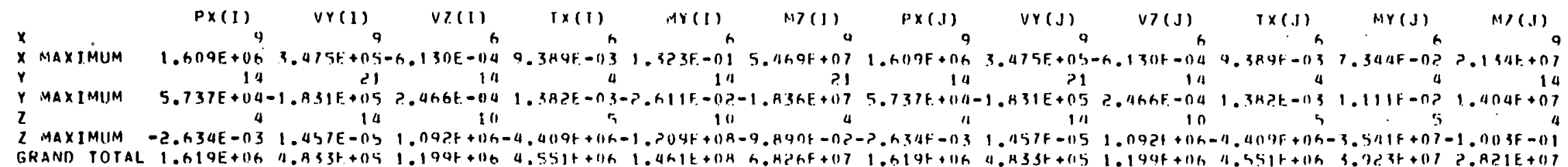

ELEMENT TYPE (3/0 P I PE , , , FLFHFNT NIIHFE ( 45$)$

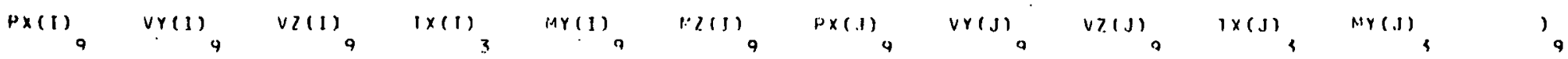

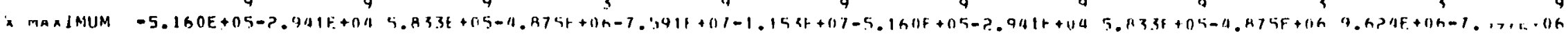




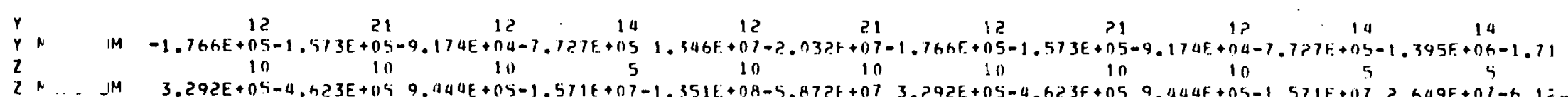

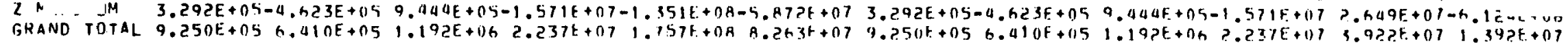
ELEMENT TYPE (3/0 P P PE, , , HLFMENT NUMHFE $(46)$

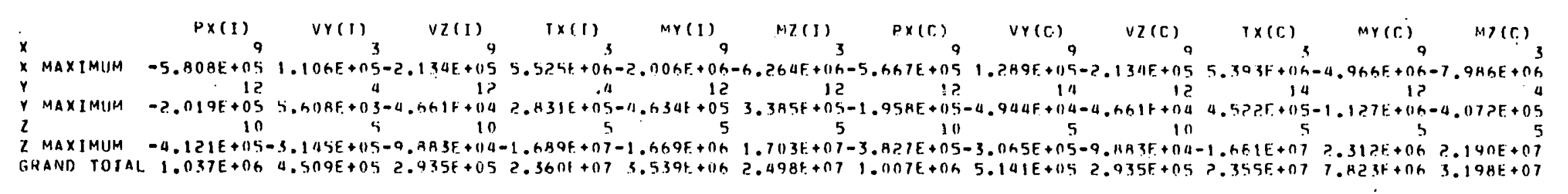

FLEMENT IYPE (3/0) P I P F. , , , E.LFMENT NIJHAER $(46)$

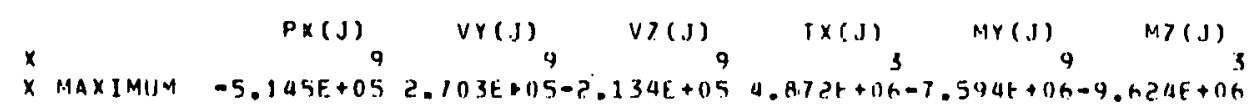

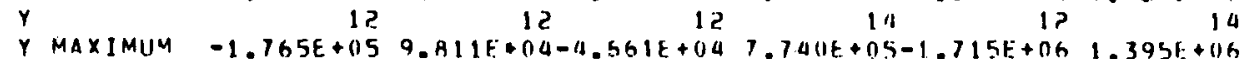

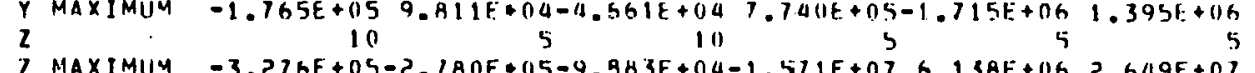
GRAND TOTAL $9.23 A E+056.5114+052.935 F+052.231 F+1171.393 F+073.93 ? F+07$

I ELEMENT TYPE (3/1) $P 1 . P$ E $, 1,1$ FLEMENT NIIMHER $(47)$

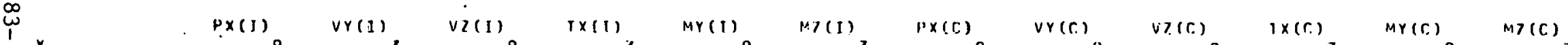

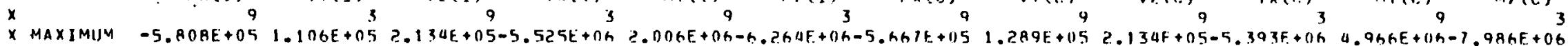

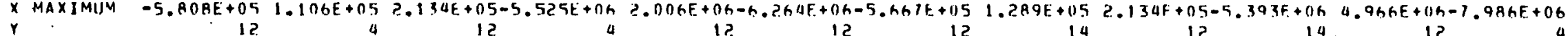

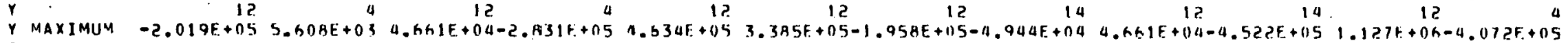

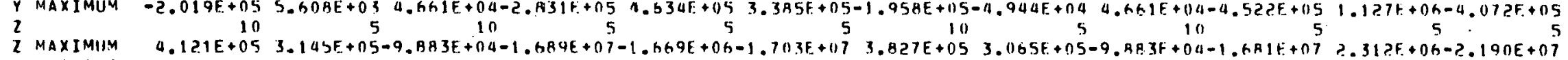

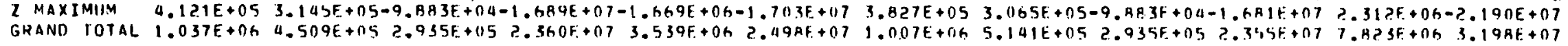
ELEMENT TYPE (3/D) P I $P$,,$r$, HLEMENT NIJMHER $(47)$

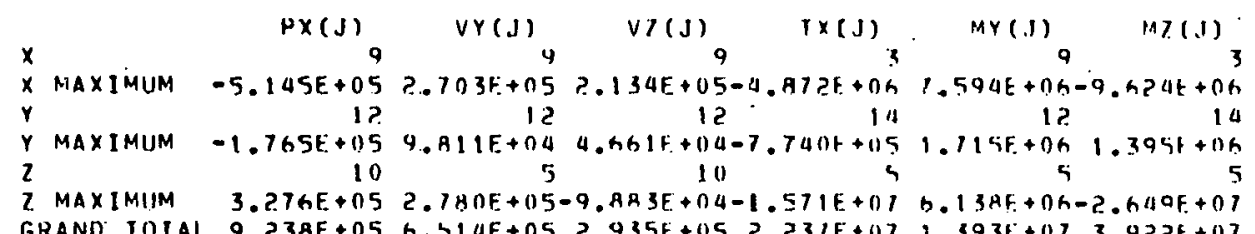

ELEMENT TYPE (3/D PIPF , , , FLFMFNI NIJMHFR, 48$)$

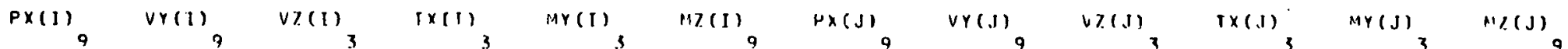

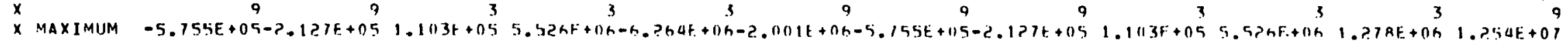

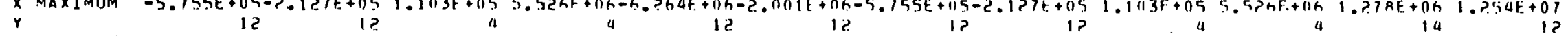
$Y$ MAXIMIJM $-2.01 A E+05-4.633 E+045$
7

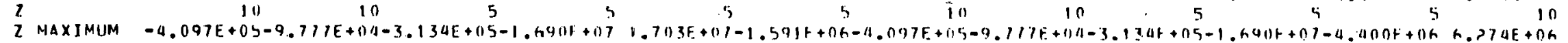

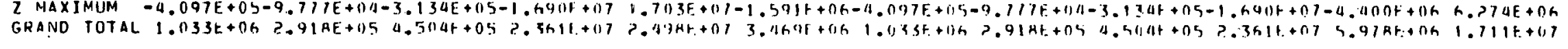
ELEMENT TYPE (3/D) P P 


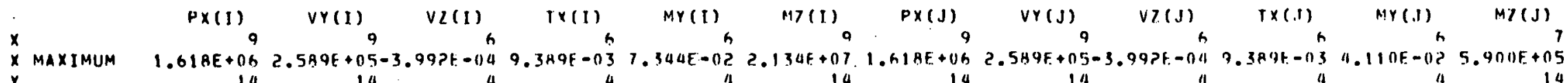
$r$ MAXIMUM $5.736 E+04$
5

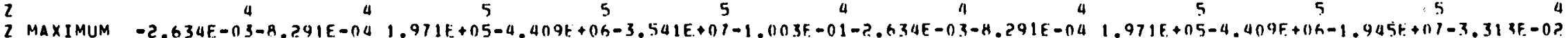

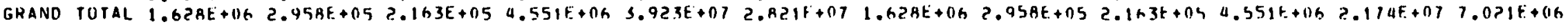
ELEMENT TYPE (3/D P I PE , 1,1 EI.EHENT NIJHHER $(50)$

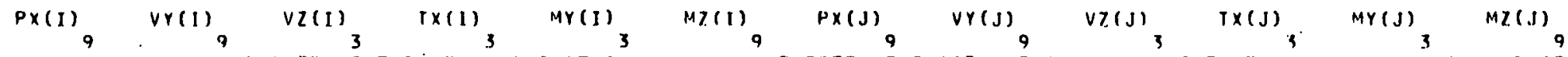

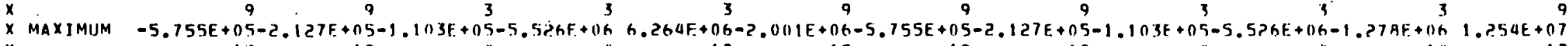

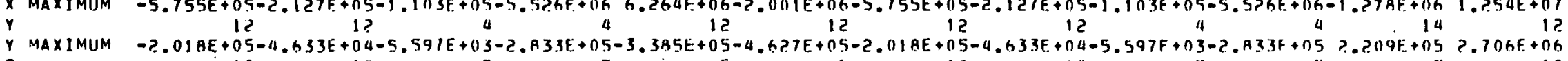

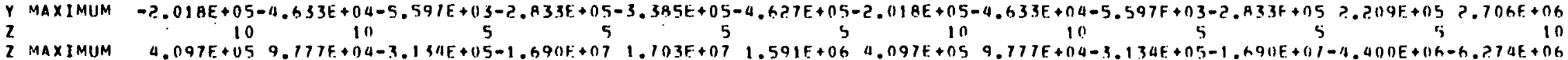

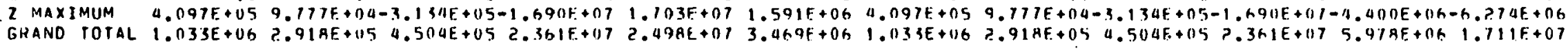
ELEMENT TYPE (3/D) PIPE , , FLE.MENT NIMMHER $(51)$

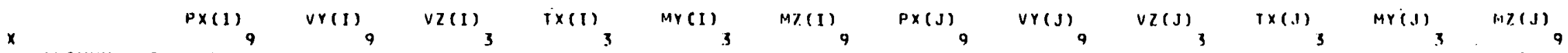

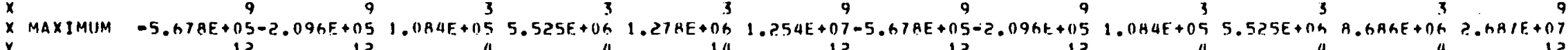

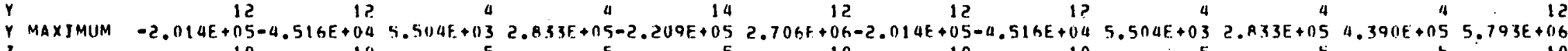

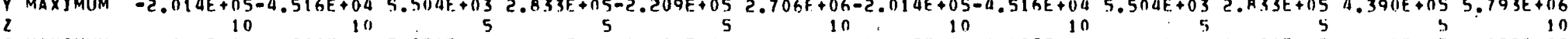

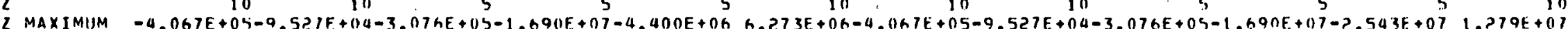

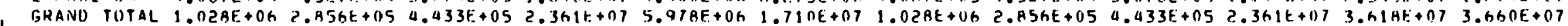

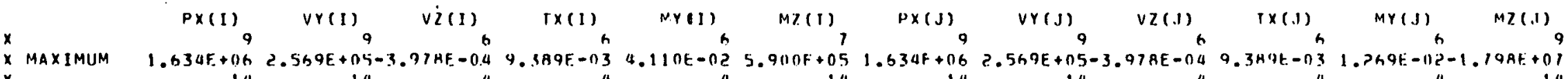

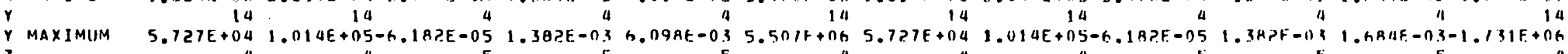

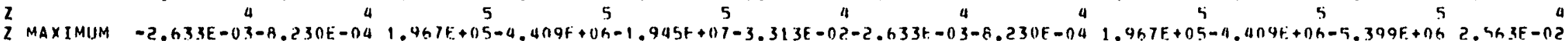

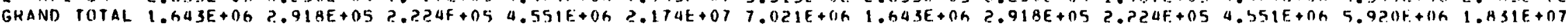

ELEMENT TYPE $13 \%$ P I P E 1,1 FLFMENT NUMHFR ( 53)

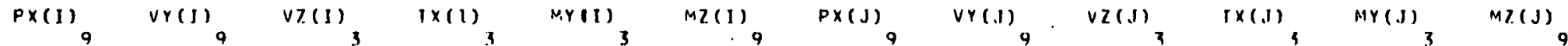

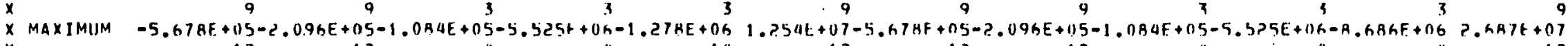

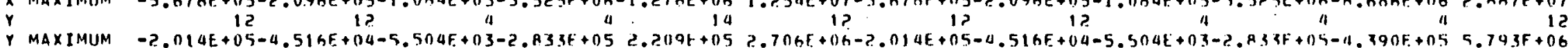

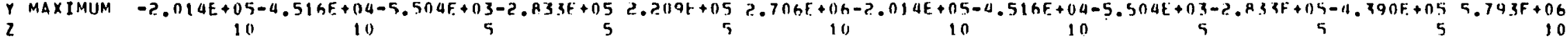

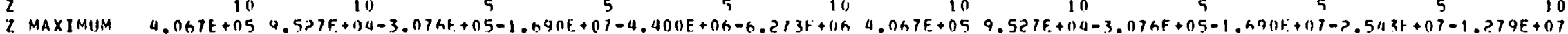

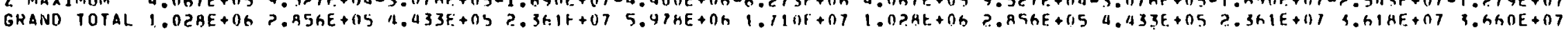
ELEMENT TYPE (3/D P 1 P E , , , FLFMFNI MIILAMEH $(54)$ 


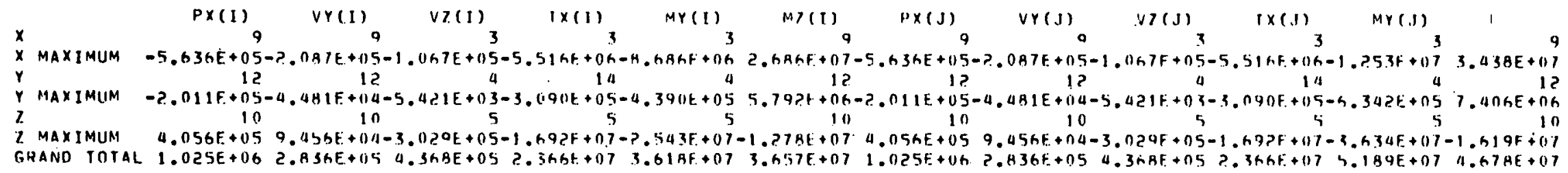
ELEMENT TYPE (3/0 PIPF. , , , LLEMENT NIIMHER 1 5G)

\begin{tabular}{|c|c|c|c|c|c|c|c|c|c|c|c|c|c|c|}
\hline & $P \times(I)$ & VY $(t)$ & V? (I) & & $1 \times(1)$ & MY (I) & $M 2(1)$ & $P \times(J)$ & $r(J)$ & $v 7(.1)$ & & $I \times(. J)$ & $Y(. J)$ & $197(J)$ \\
\hline$x$ & 9 & 3 & 9 & & 0 & 9 & 3 & 9 & 3 & 9 & & $n$ & 9 & 3 \\
\hline MAXIMUM & $1.489 E+04$ & $1.93 \mathrm{JE}+05$ & $4.9 B 2 E+05$ & 0 . & & ?.444E-116 & $2.384 \mathrm{t}-0 \mathrm{~h}$ & 1. $4 R 9 E+\cap \triangle$ & $1.9 .54 E+05$ & 4. 9A2F +05 & ( & & h. 27 AF. $+07-$ & $-? .037 E+07$ \\
\hline & 21 & 12 & $1 ?$ & & 1 & 14 & 12 & 21 & 12 & 1? & & $n$ & 12 & 12 \\
\hline MAXINUM & $38 E+05-$ & $I E+114$ & $1.412 E+05$ & 0 & & $E=01$ & $1.5>7 F=07$ & $1.43 A E+05-$ & $107 E+04$ & $1.112 E+04$ & 0. & & $1.1795+07$ & $5.115 E+06$ \\
\hline & 25 & $s$ & 10 & & $n$ & 5 & 15 & 25 & $\mathbf{s}$ & 10 & & 0 & & \\
\hline XIMUM & $27 E+104-$ & +05 & $3.131 E+05$ & 0. & & 3.1 & -07 & $2.827 E+04=$ & +115 & $3.131 E+05$ & 0 & & $3.945 F+07$ & +07 \\
\hline RAND TOTAL & $4 E_{n}+05$ & +115 & $302 E+115$ & 0 & & -06 & fif $=06$ & $4 E+(1) 5$ & $31 E+05$ & B. $30>0$ + 5 & 1) & & 1. $\cap A \cap E+\cap B$ & 1. OABE + OB \\
\hline
\end{tabular}

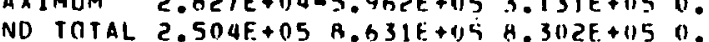

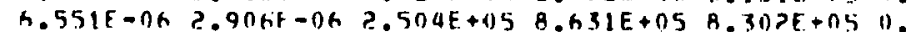

ELEMENT TYPE (3/0 P I P F. , , F.LFMENT NUMARER ( 57$)$

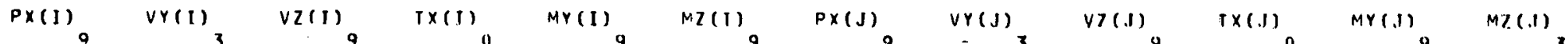

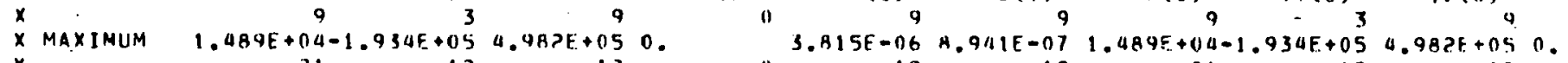
$Y$ MAXIMUM $1.438 E+05$
$2.107 E+011$
$2.412 E+050$. $0^{-1.11 B F-06} 1 . P A 7 F-07 \quad 1.43 A E+(15 \quad 4.107 E+114 \quad 1.412 F+05 n$.

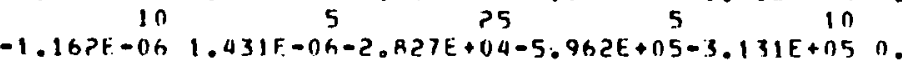

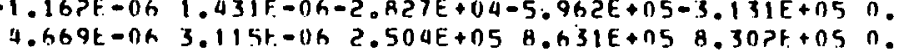

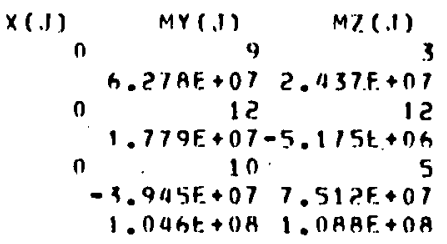
ELEMENT TYPE (3/1) P I P E , , , FLEMENT NUMHER ( 58$)$

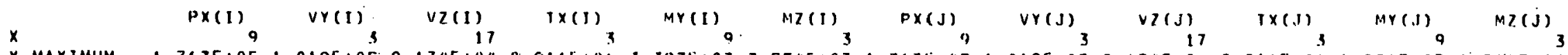
$x$ MAXIMUM $1.767 E+05-1.019 E+05$
$y$

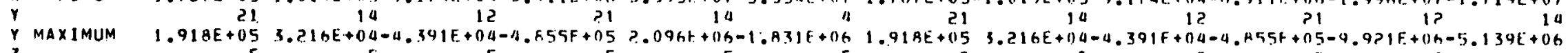

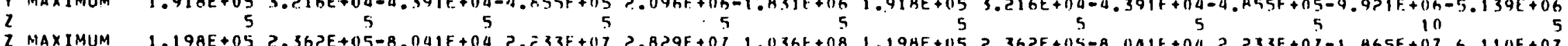

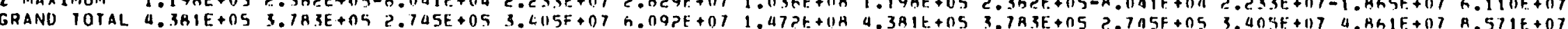
ELEMENT IYPE (3/D P I PE , , , FI.FMEHT NUMHER $($ S 9$)$

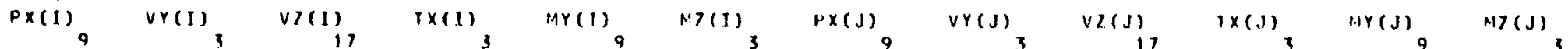

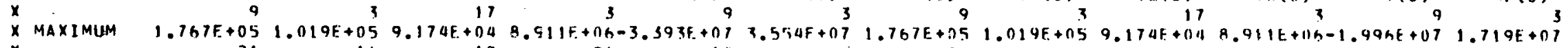

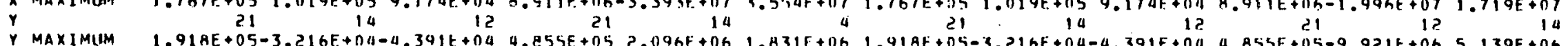
Y MAXIMLIM $1.91 A E+05-3.216 E+011-4.341 t+04 \quad 4.255 E+052.096 E+061.431 E+061.91 A E+05-3.216 E+04-4.391 F+0114 . A 55 E+115-9.92 .1 F+065.139 E+06$

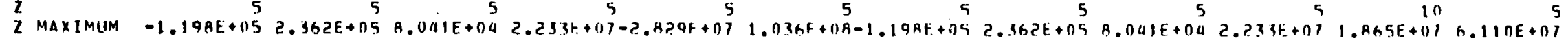

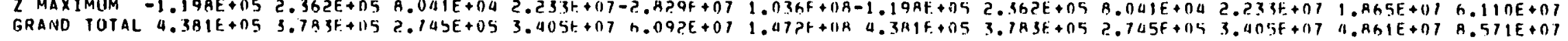
ELEMENT TYPE $(3 / 0)$ PIPH:, , ELFHFNT NIIMIIER $($ hol)

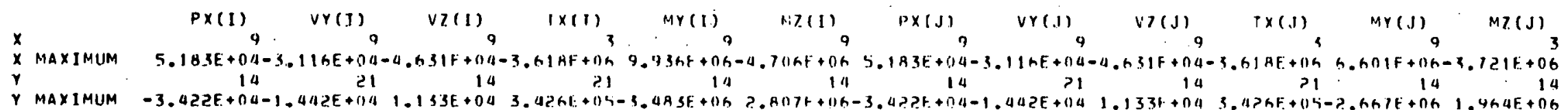




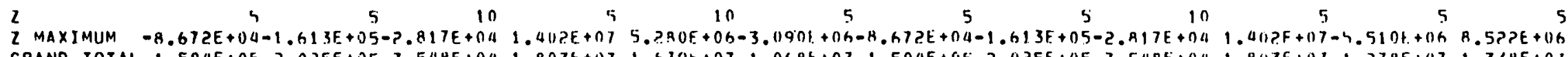
GRAND TOTAL $1.504 E+052.025 E+057.54 A E+041.003++1071.519 E+071.06 A T+071.504 E+052.025 E+057.54 A E+041.0113 E+011.27 A E+1171.354 E+01$ ELEMENT TYPE (3/D) P I $P+1$, HI.ENENT NUMHER $($ bI)

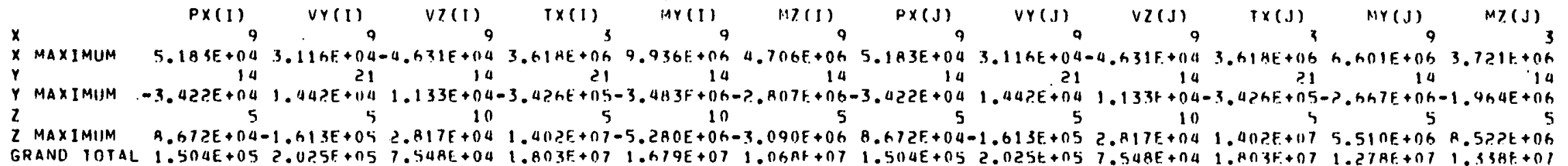
ELFMENT TYPE (3/D PI PE ), , ELFMENT NIIMHFR ( 62)

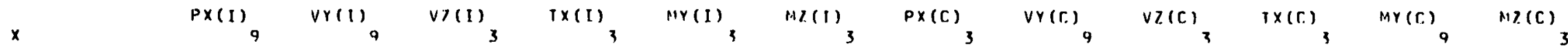

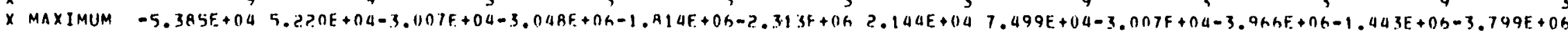

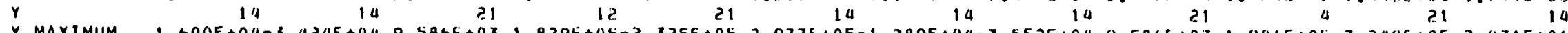

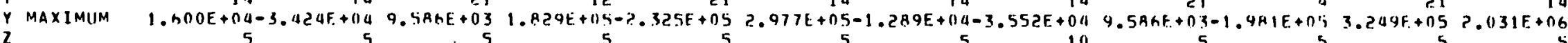

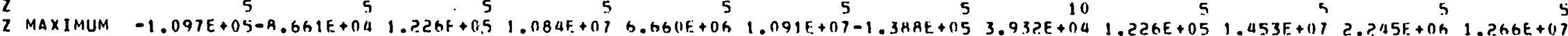

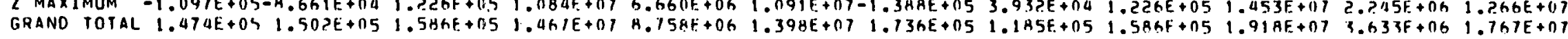
ELEMENT TYPE 1310 P I PE , 1,1 ELEMENT NUMHER ( A2)

\begin{tabular}{|c|c|c|c|c|c|c|c|}
\hline & & $P \times(J)$ & $\operatorname{VY}(J)$ & $V 7(J)$ & $T \times(.8)$ & $\operatorname{MY}(J)$ & Ml (.J) \\
\hline$x$ & & 9 & 9 & 3 & 3 & 9 & \\
\hline$x$ & MAXIMUM & 5.2.1AE + П4 & $5.38 / E+04-$ & $-3.007 t+04-$ & $-3.618++11 h-$ & $-2.0106 E+0 h-$ & \\
\hline$r$ & & 14 & 14 & ? 1 & 21 & 21 & \\
\hline$y$ & MAXIMUM & $-3.423 E+04-$ & $1.601 E+04$ & $9.58 h t+03$ & $3.424 f+05$ & $6.920 t+05$ & $3.31>t+1$ \\
\hline & $\begin{array}{l}\text { MAXIMUM } \\
\text { TAND TOIA }\end{array}$ & $\begin{array}{r}-8.665 E+04 \\
1.50 P E+05\end{array}$ & $\begin{array}{l}1.09 G E+05 \\
1.17 / 4 E+115\end{array}$ & $\begin{array}{r}526 E+115 \\
.2286 F+05\end{array}$ & $403 F+07-$ & $\begin{array}{r}5 \\
O H Q E+O B \\
O B Q F+O G\end{array}$ & 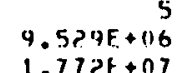 \\
\hline
\end{tabular}

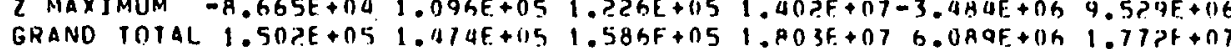
ELEMENT IYPE (3/1) P I P $F$, , , FLEMFNT NIJHAFR ( a 3 )

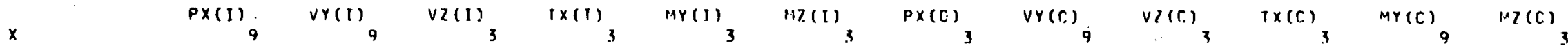

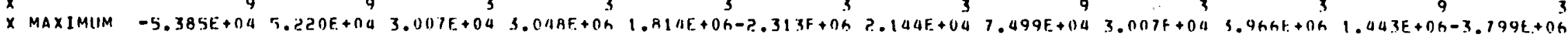

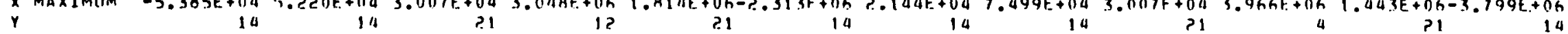

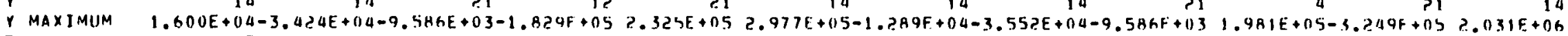

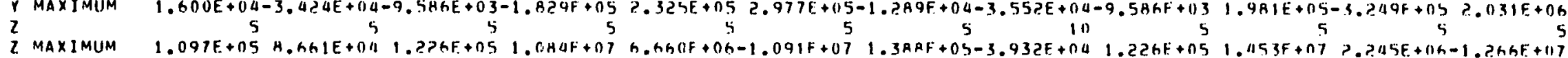

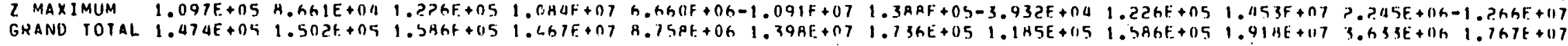

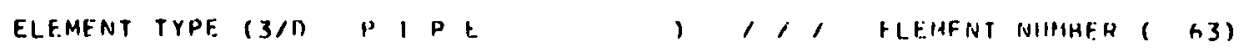

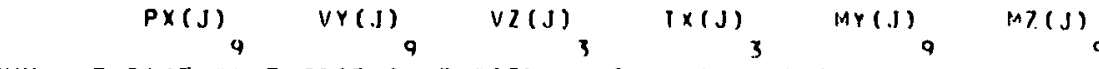

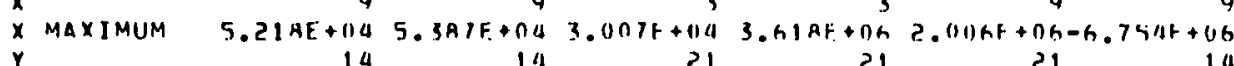
$Y$ MAXIMUM $-3.423 E+04-1.601 E+014-9.5 A H E+03-3.424 F+05-h$. 9? $01++0133.312 t+n h$

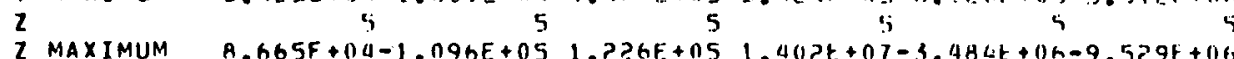

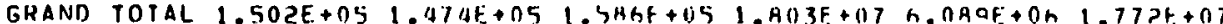

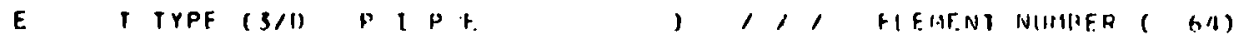

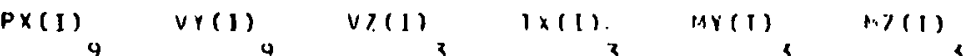


$X$ MAXIHUM $-5.167 E+04-5.211 E+04-3.014 E+04-3.03 H E+06 \quad 1.0111 E+06-2.32 .7 F+06-5.167 E+04-5.211 E+04-3.014 E+011-3.03 H E+06 \quad 1.102 F+116-1.525 E+06$

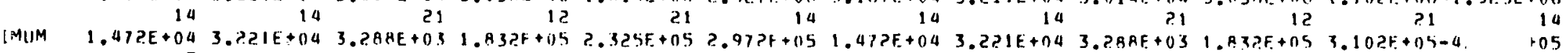

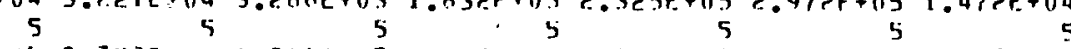

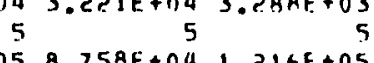

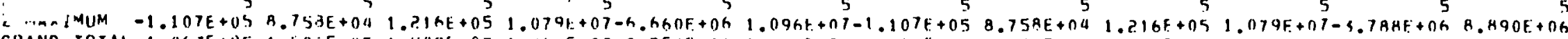

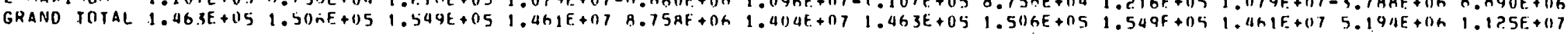
ELEMENT TYPE $(3 / 0$ P I P E

, 1, EI.EMENT NIMHAFR ( 65)

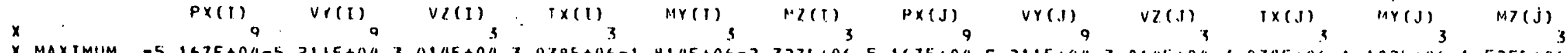

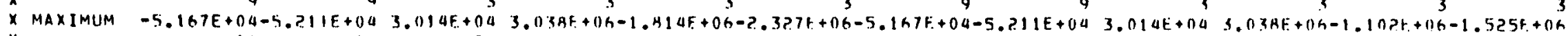

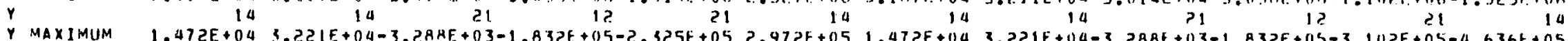

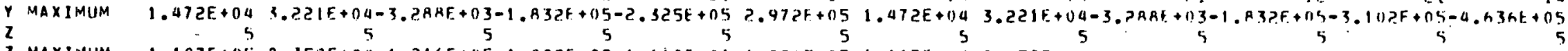

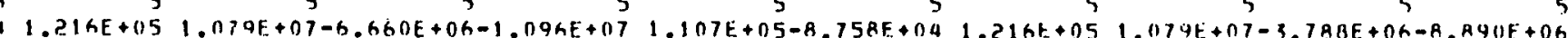

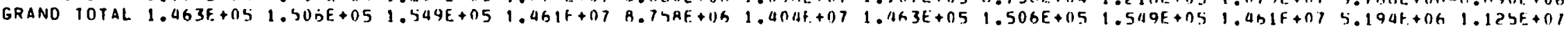
ELEMENT IYPE $(3 / 1)$ P I P E , 1, ELEMENT HUPHER 1 GR)

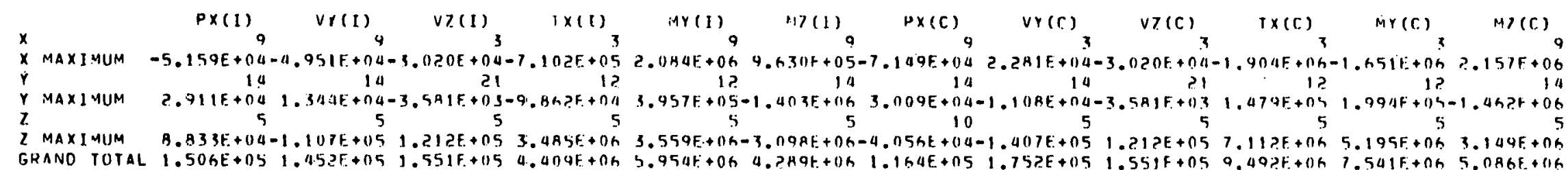
$\frac{1}{\infty}$

ELEMENT TYPE (3/D P I PE ), , GLEMFNT NUMAEH ( 66$)$

\begin{tabular}{|c|c|c|c|c|c|c|c|}
\hline & & $P x$ & ver & 1) & i) & J) & I) \\
\hline & & 9 & 9 & 3 & 3 & 3 & \\
\hline & $\operatorname{MAX} 1=16$ & $4.951 E+04$ & $1577+011=$ & $1.0201+00-$ & $-3.045 t+n t=1$ & $+0 n=1$ & +0 \\
\hline & & 14 & 14 & 21. & 1 ? & 21 & 4 \\
\hline $\begin{array}{r}y \\
z\end{array}$ & & $4 E+04-$ & $+04-$ & $t+03$ & $E+05-$ & $5-4$ & $n 5$ \\
\hline & $x I M U M$ & $1.107 E+115-$ & $935 E+1 \cap 4$ & $1.21 ? F+05$ & $36+07$ & THAE + OK & $+n 6$ \\
\hline
\end{tabular}

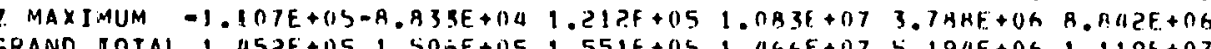

ELEMENT TYPE $(3 / 1)$ P I PE , , , FIEMENT NIIMHER $(67)$

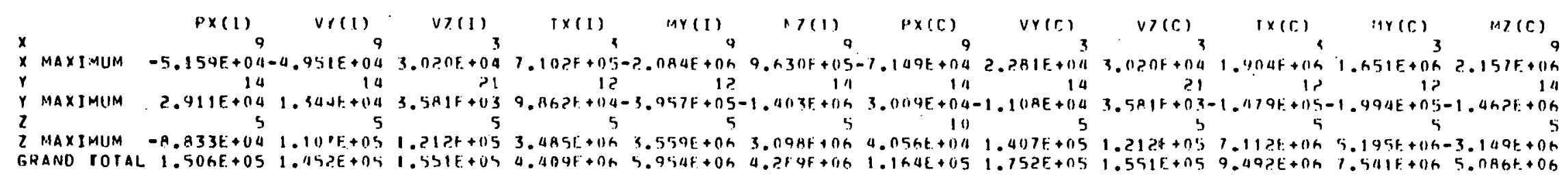
ELEMENT IYPE (3/D) P I P

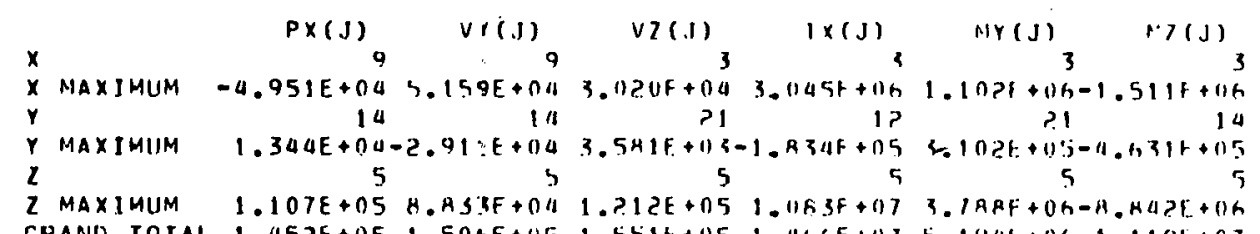

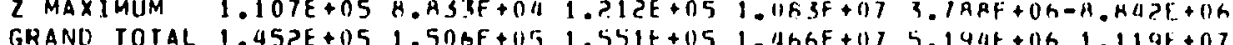

ELEMENT TYPE $(3 / 1)$ P P F. $, 1,1$ ELLMENI NUMHEH $(A H)$ 


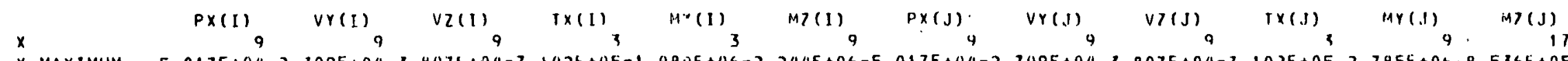

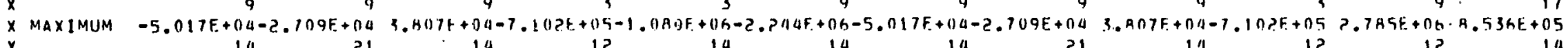

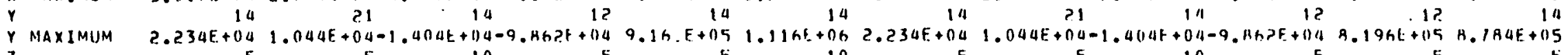

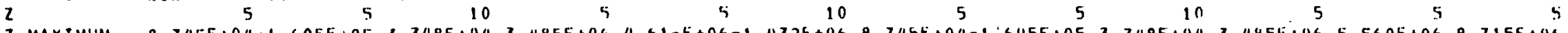

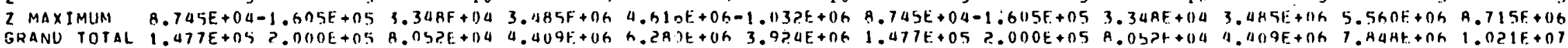

ELEMENT TYPE (3/0 P I PE. ), , LIEHENT NUMHER $($ hY)

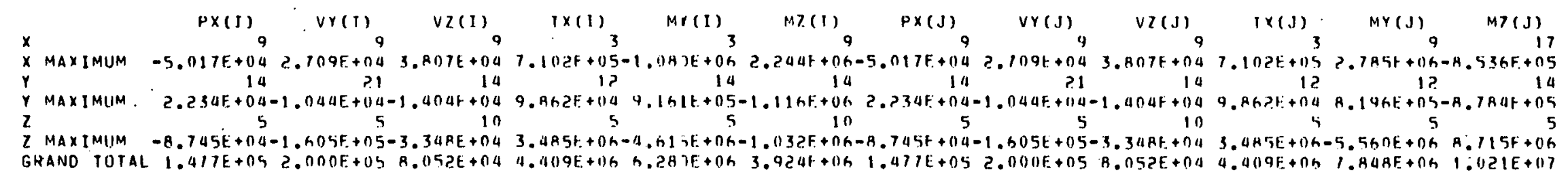
ELEMENT TYPE $(3 / 0)$ P I P E

) 1, F.LE:MENI NIMMREN ( 70$)$

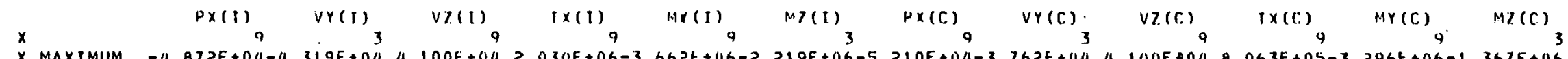
$\times$ MAXIMUM $-4.872 E+04-4.319 E+044.100 F+042.030 E+06-3.662 E+06-2.219 E+06-5.210 E+04-3.762 E+1144.100 E+04$. $0.063 E+05-3.296 E+06-1.367 E+06$ $Y$ MAXIMUM $\quad 1.360 E+04$
$z$

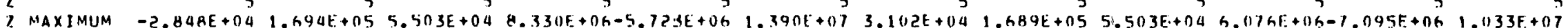




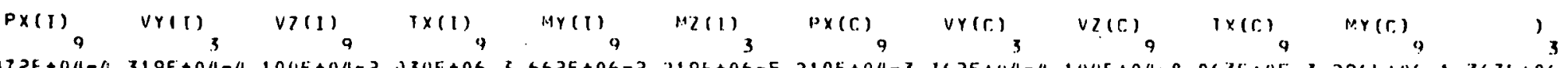

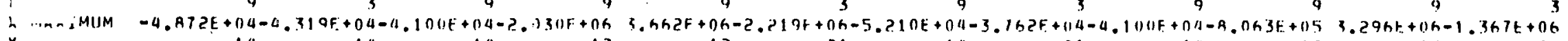

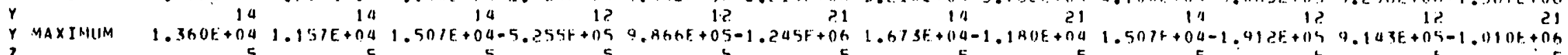

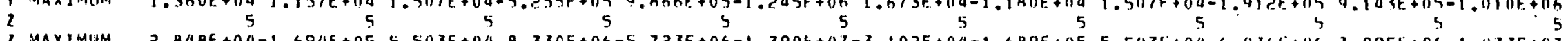

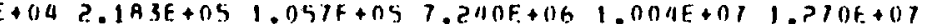

ELEMENT TYPF $(3,0) P=P E, 1$, FLEMENT NIPAHER $(72)$

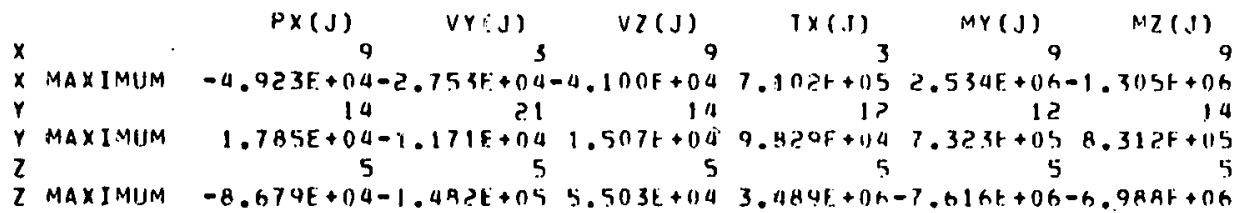

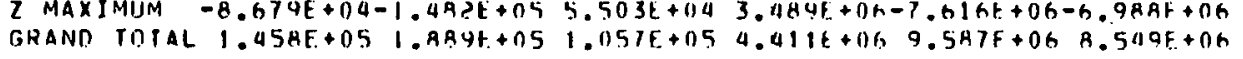

ELEMENT TYPE (3/N P I PE ), , FLFMENT NUHALE $(73)$

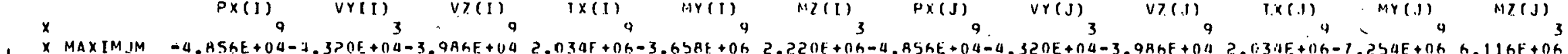

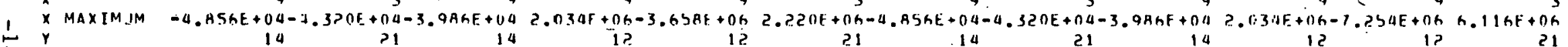

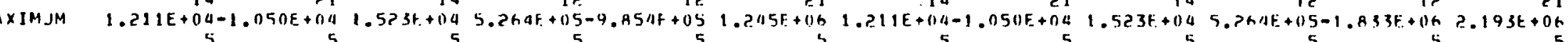

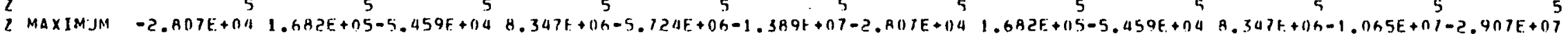

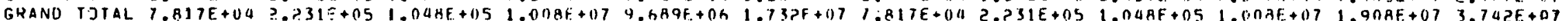

ELEMENT TYPE (3/1) P I P $, 1,1$ ELENFNT NIJMAER ( 74)

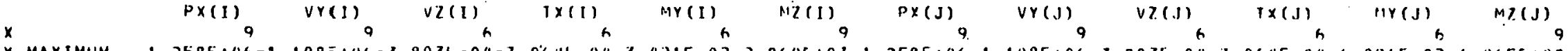

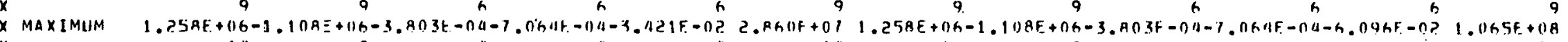

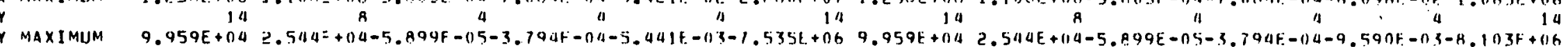

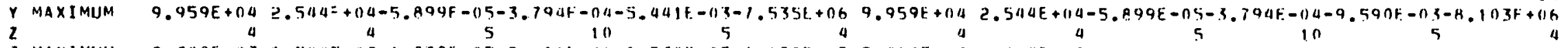

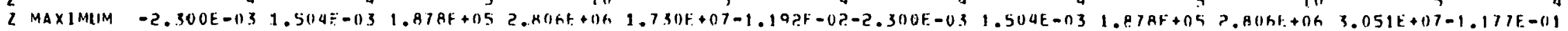

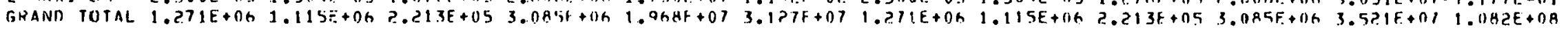

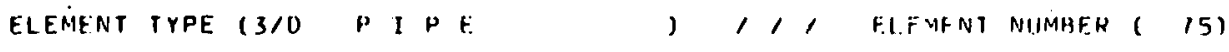

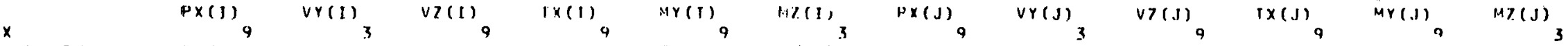

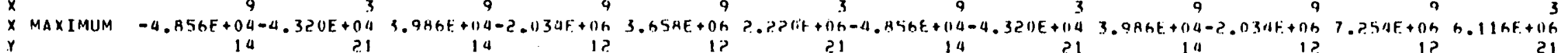

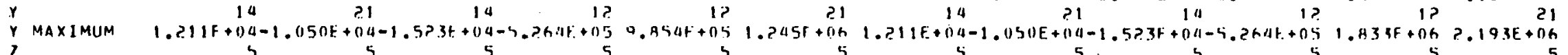

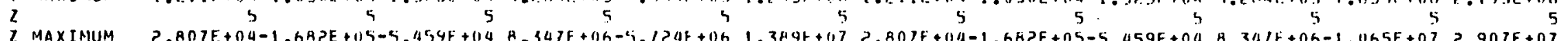

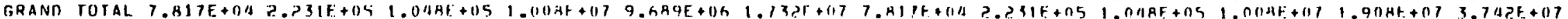
ELEMENT TYPE (3/0) P JPE , 1,1 H.FMFNT NUHHAL 1 7h)

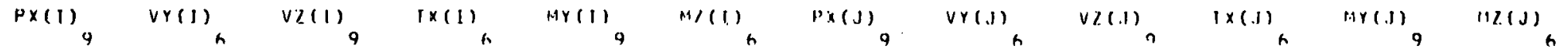

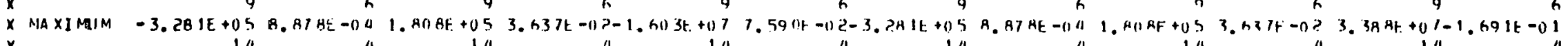




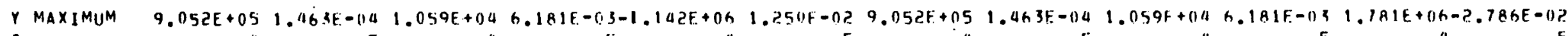

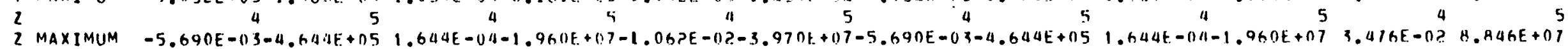

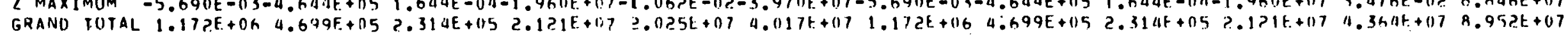
ELEMENT TYPE $(3 / \pi$ P I PEE , 1,1 ELENENT NIIPHER 177$)$

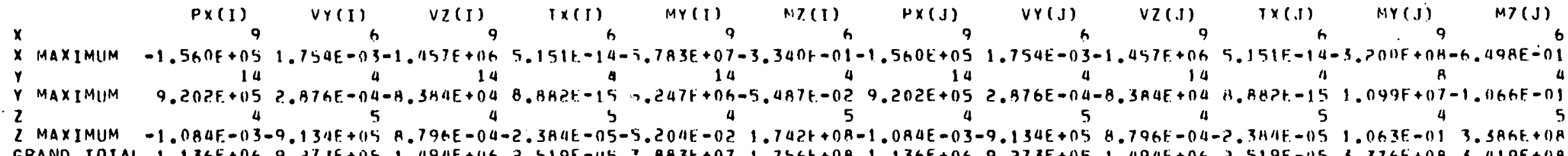

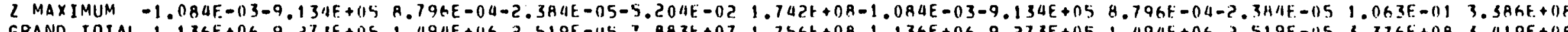
ELEMENT IYPE (3/D) $P$ I P F , 1,1 ELCMENT NUMHER $(7 A)$

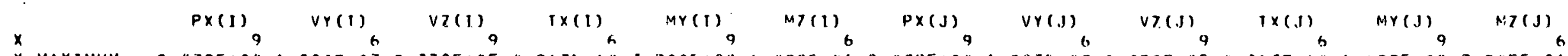

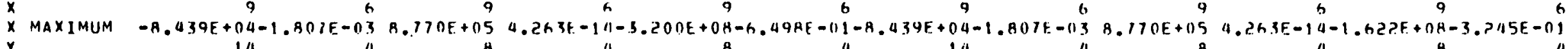

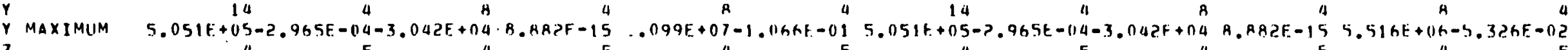

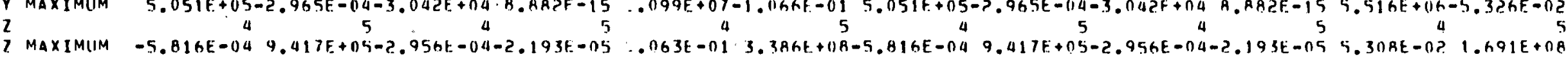

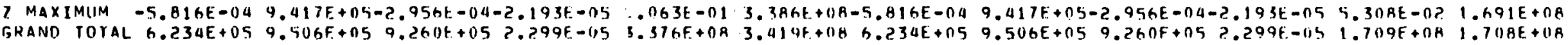
ELEMENT TYPE (3/1) PIPE , 1,1 FLEMENT NUMAER $(74)$

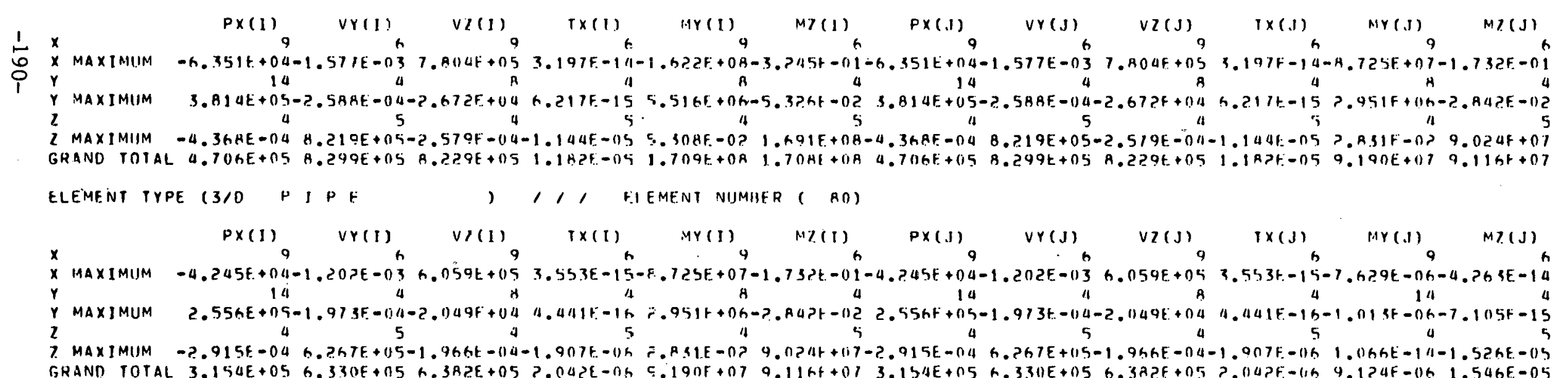

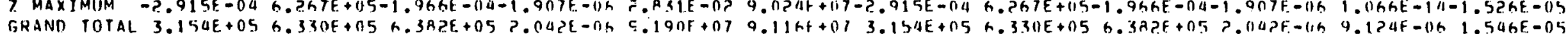


Solution $4 \mathrm{~b}$

Intermodal/Interspatial Combination

$-191-$ 


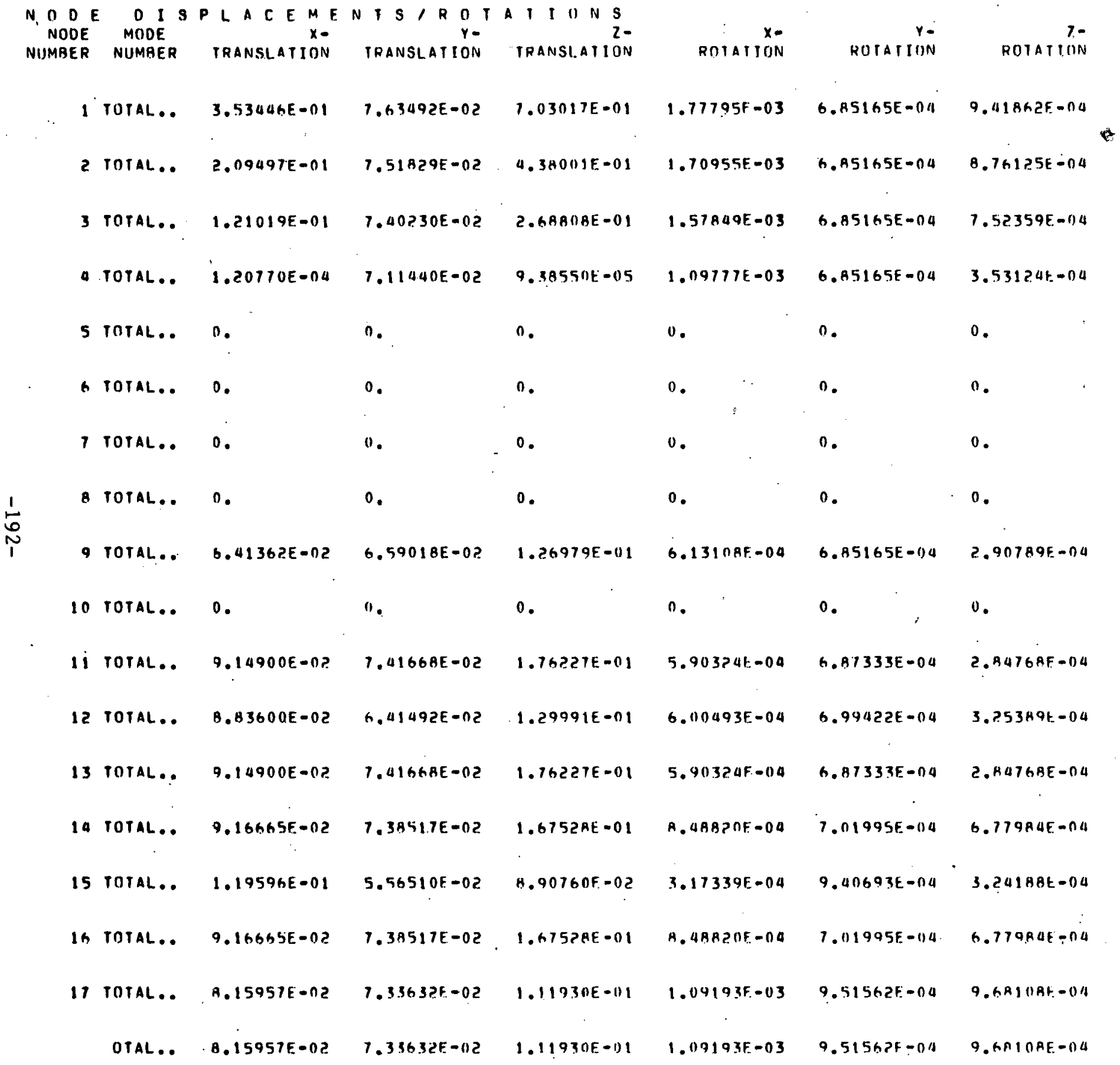




\begin{tabular}{|c|c|c|c|c|c|c|c|c|}
\hline & & ITAL... & $9.0802 a E-02$ & $5.60525 E=02$ & $7.81006 E-02$ & $1.46356 E-03$ & $5.83959 E-04$ & $1.15319 E=03$ \\
\hline & 22 & TOTAL.. & $9.080 ? 4 \mathrm{E}-02$ & $5.60525 E-n 2$ & 7.A1ODGE-O2 & $1.46356 \mathrm{~F}-03$ & $5.83959 E-04$ & $1.15319 F=03$ \\
\hline & 23 & TOTAL.. & $9.73477 E-02$ & $1.886,65 E-02$ & $1.59172 E-01$ & $3.73320 E=03$ & $1.3137 i E-03$ & 1. . 9525E-03 \\
\hline & 24 & TOTAL... & $9.73477 E-02$ & $1.88665 E-02$ & $1 . n 9172 E-01$ & $3.73320 E=03$ & $1.31377 E-n 3$ & 1.695 ? BE $=03$ \\
\hline & 25 & TORAL.. & $1.95776 E-01$ & $1.88665 E-02$ & $4.3543 A E-01$ & $3.711694 E-03$ & $1.4 n S 57 E-03$ & $1.75531 F-0.3$ \\
\hline . & 26 & TOTAL.. & $1.95776 E-01$ & $1.88665 E-02$ & $4.3643 B E-01$ & $3.74694 F-0.3$ & $1.40557 E-03$ & $1.75531 E=n 3$ \\
\hline & 27 & TOTAL... & 0. & 0 . & 0 . & 0 . & 0. & 0 . \\
\hline & 28 & TOTAL... & 0. & $n$. & 0. & 0 . & D. & 0. \\
\hline & 29 & TOIAL... & 4.547 BGE-DI & $1.96721 E-02$ & $9.54820 E-01$ & $4.1121115=03$ & $1.40557 E-03$ & 2.138 ? AF -03 \\
\hline & 30 & TOTAL.. & $0.547 A 6 F=01$ & $1.96721 E-02$ & $9.5482 \cap E-01$ & $4.112115-03$ & $1.00557 E-n 3$ & $2.13 B 2 A E-03$ \\
\hline & 31 & TOTAL... & $1.86658 E-01$ & $4.32067 E-02$ & 3.857 ख QE - 01 & $3.68470 F=03$ & $1.09033 E-n 3$ & $1.67333 \mathrm{~F}-03$ \\
\hline & 32 & TOTAL.. & $1.86658 E-01$ & $4.32067 E-02$ & $3.85729 E-01$ & $3.68470 F .-13$ & $1.49 \cap 33 F_{2}-103$ & $1.673335-03$ \\
\hline & 33 & TOTAL... & $1.59851 E-01$ & 6.87124E- 02 & 2. $40570 F=01$ & $2.19055 F .03$ & $2.521 B A E-113$ & $6.35214 E-04$ \\
\hline & 34 & TOTAL.. & $1.26530 E-01$ & $2.52152 F-02$ & B. A189.3t-(1)? & 2.2916 IIF-04 & $6.7 \mathrm{~h} 240 \mathrm{E}-1) 4$ & $3.49981 E-114$ \\
\hline & 35 & TOTAL.. & $1.59851 E-01$ & $6.8712 .4 \mathrm{E}-02$ & 2.AnS7OE-01 & ?.19055F-03 & $2.521 \mathrm{ABE}-03$ & $6.35 ? 1 \mathrm{at}=0 \mathrm{at}$ \\
\hline & 36 & TOTAL... & 1.391 A St -01 & $2.9073 \mathrm{BE}-02$ & $1.14210 E-01$ & $8.01721 F-04$ & $2.05732 E-03$ & $5.35704 E-04$ \\
\hline & 37 & TOTAL.. & $1.39185 E-01$ & P. $90738 E-112$ & 1.1421 of -101 & A.01721E-04 & 2. . $5732 E-\cap 3$ & 5.357 OIIE-OI4 \\
\hline & 38 & TOTAL.. & $1.32899 \mathrm{~F}-01$ & $1.46234 E-02$ & 1. IJA I? 3F-(1) & $1.65572 t-04$ & $? .16950 \mathrm{~F} .05$ & $4.30535 t-05$ \\
\hline & 39 & TOTAL.. & $1.344 P 0 E-01$ & $7.18797+-03$ & $1.76>235-01$ & $1.701555-114$ & $1.52306 F-115$ & $3.976011-05$ \\
\hline . & 40 & TOIAL... & $1.32899 E-01$ & $1.4 \mathrm{~h}+34 \mathrm{E}-0) ?$ & I. $|R| 2<F=01$ & $1.4547>F-114$ & ?.1nOG(lf -1) & $4.10535 F-115$ \\
\hline & 41 & TOTAL.. & 0 . & 0 & 0. & 0. & D. & 0 . \\
\hline
\end{tabular}


a) TOTAL.. O.

0.

0 .

o.

42 TOTAL.. 0.

43 TOTAL.. 0.

A4 TOTAL.. 0.

45 TOTAL.. 0.

46 TOTAL.. 0.

47 TOTAL.. $1.35475 E-01$

48 TOTAL.. 1.55439E-01

49 TOTAL.. 1.37775E-01

50 TOTAL.. 1.38582E-01

51 TUTAL.. 1.39343E-DI

S2 TOTAL.. 0.

53 TOTAL.. 0.

54 TOTAL... 0.

55 TOTAL.. 0.

56 TOTAL.. 0.

57 TOTAL.. 0.

S8 TOTAL.. 1.32899E-OI

59 TOTAL.. 1.34020E-01

OTAL.. $1.32899 E_{-01}$
0

$n$.

n.

n.

3.13?.14E-03

$9.97273 f-0$

$8.53109 E-03$

8.61402F.-05

$8.78733 E=0$

0.

a.

0.

0.

D.

$n$.

$1.46234 E-02$

T. $18797 E-03$

$1.0673456-08$
0.

0 .

0.

$1.01344 E-0$

0.

0

61 TOTAL.: $1.39185 F-01$
0 .

0.

$1.119535-01$

$1.5 h 9 h / 1 E-01$

1. DGURIE-O

$9.372906-02$

0 .

$n$.

0 .

0.
0.

).

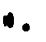

$\bullet$

$.70003 F-04$

$2.186945=04$

$011455-04$

$.5 A 973 E-04$

•.

$\bullet$

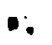

$\bullet$

$\bullet$

$\bullet$

:.65512E-04
$1.081 \geq 3 E-01 \cdot .55572 t-04$

n.

n.

$n$.

0 .

0 .

$4.01216 f .099$

4. ก1216E-09

4.01216E-09

$4.01>16 E-09$

$4.01216 E-09 \quad 3.344075-115$

0.

0 .

$n$.

0 .

0 .

0.

$2.1695010-05$

$1.06723 E-01$
$3.29880 E-015$

0.

0

0 .

0.

0.

n.

0.

4.001A2F-015

$.04163 E-05$

$.29628 F=05$

0.

0.

o.

$4.30535 E-0.5$

$3.17601 E-05$

4. 30535E-05 


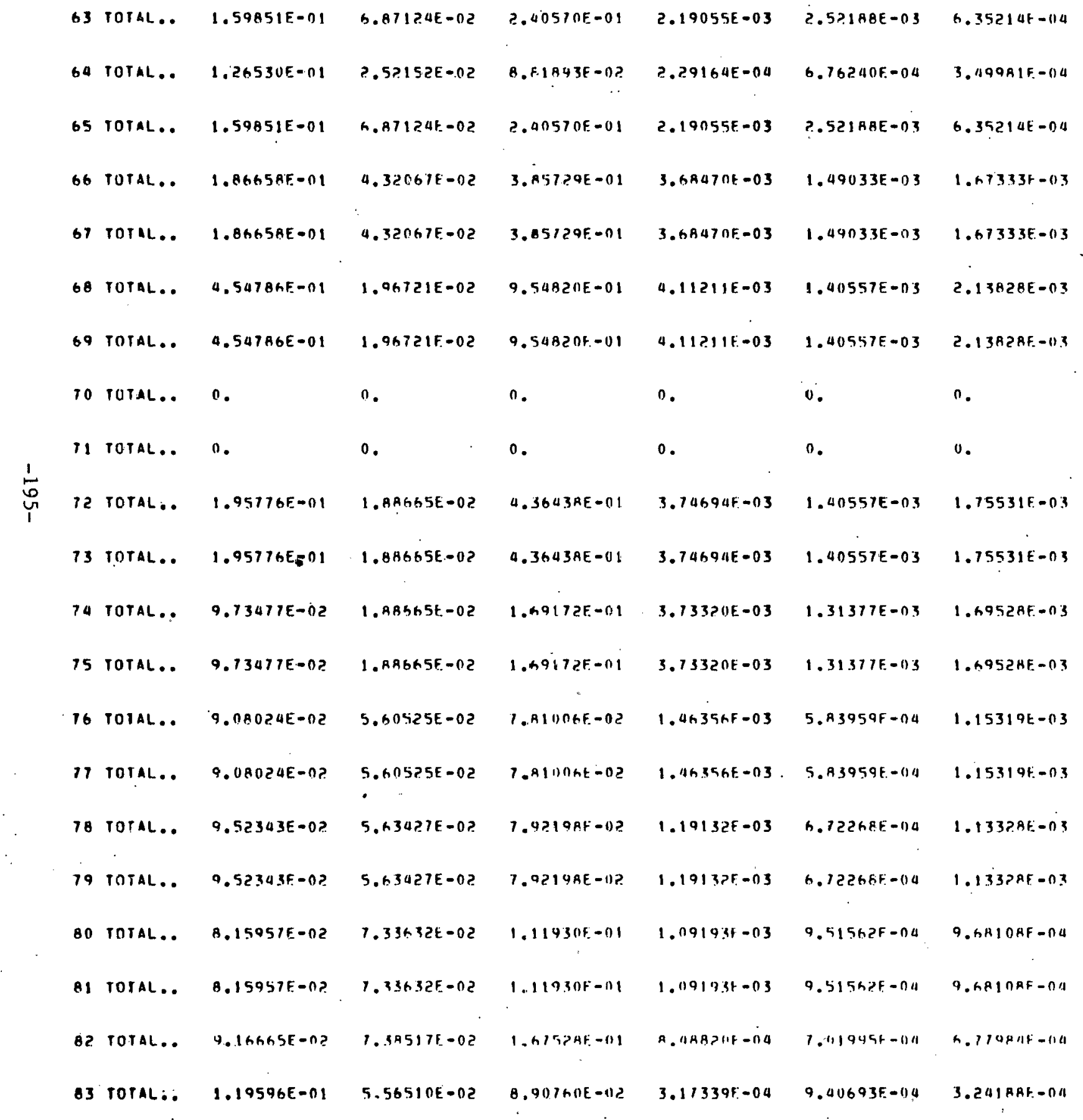




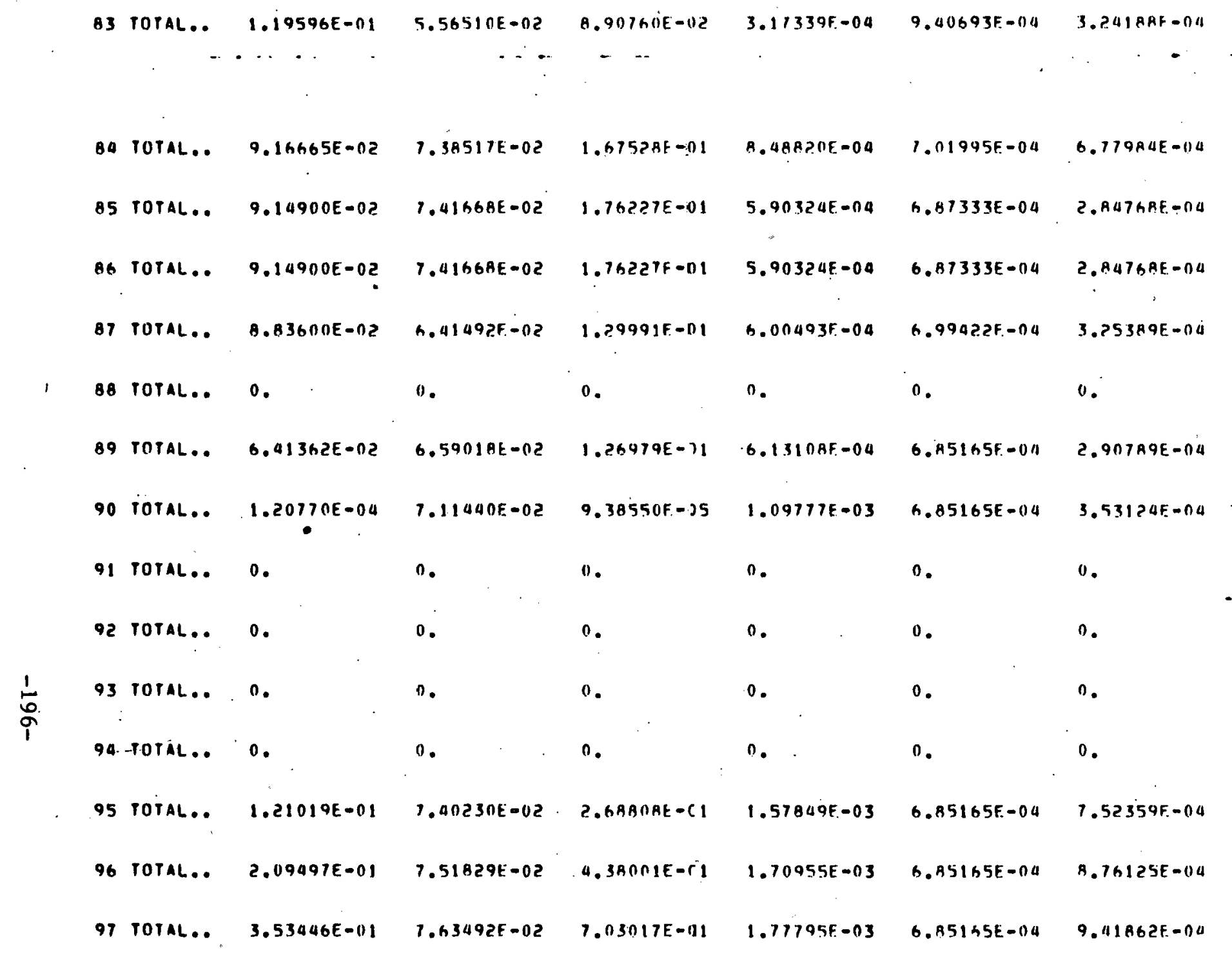




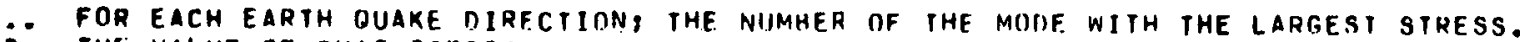

2. THE VALUE OF THAT BIRFSS.

3. IF REQUESTEO: THE MONE HY MONE STRESSES FIIR FACH EARTH DIIAKE DIRECIION.

4. THE RESULTANT FOR EACH EARTH DUAKE DIRECTION.

5: THE GRAND TUTAL OF THE THREE FARTH DUAKE MIREC IIONS.
(NOTE: THE $x$, Y, UR 2 REFERS IO THE EARTH DIIAKF OIRECTION,)

ELEMENT TYPE (B D) UI N D A R Y

BORYOF BORYAM

$x$ MAXIMUM $-1.167 E+060$

$X$ MAXIMUM -1.167E+O6 0

$Y$ MAXIMUM $5.373 E+040$ 20

2 MAXIMUM -5.077E-03 0.

GRAND TOTAL $1.20 A E+060$.

ELEMENT TYPE (B O U N D A R Y

) 1,1 ELFMFNT NUMHER $(2)$

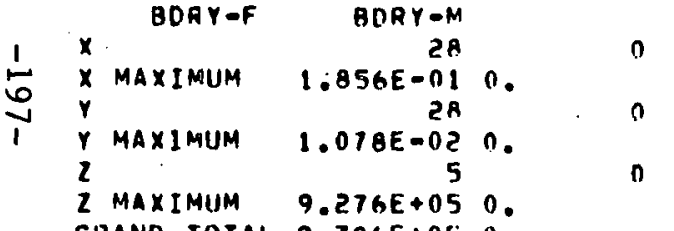

GRAND TOTAL $9.386 E+050^{\circ}$

ELEMENT TYPE (B O UN N D A

\begin{tabular}{|c|c|c|c|}
\hline Bnar & BORY $-M$ & & n \\
\hline X MAXIMUM & $1.167 E \leftrightarrow 06$ & 0. & \\
\hline$y$ & 14 & & 0 \\
\hline Y MAXIMUM & $-5.373 E+04$ & 0. & \\
\hline 2 MAXIHUM & $\begin{array}{r}20 \\
7 F-03\end{array}$ & & n \\
\hline GHAND TOTAL & $1.208 E+06$ & 0. & \\
\hline
\end{tabular}

ELEMENT TYPE (B O U N D) A.R $r$

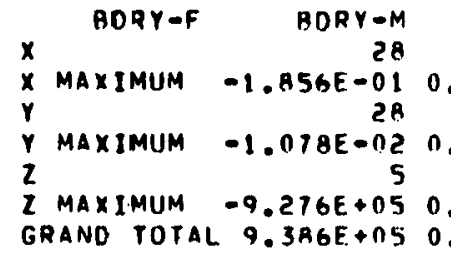

ELEMENT TYPE IB D UNDAR $Y$ 
$Y$ MAXIMUM $-1.694 \mathrm{E}+050$.

2 MAXIMUM $5.614 E+050$. GRAND TOTAL $7.312 E+050$.

ELEMENT TYPE IB 0 U N N OA A

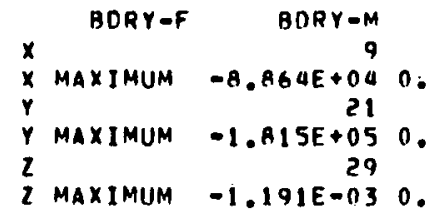

GAAND TOTAL $3.594 E+050$.

ELEMENT TYPE (B $O U N D A B Y$

HDRY-F BDRY-M

$X$ MAXIMUM $-1.841 E+050$.

$Y$ MAXIMUM 21

$\checkmark$ MAXIMUM $-1.694 E+050$.

2 MAXIMUM $-5.614 E+050$.

GRAND TOTAL $7.312 E+050$.

ELEMENT TYPE IB 0 U U N D A R Y

1,1, EleneNi nimafer $(8)$

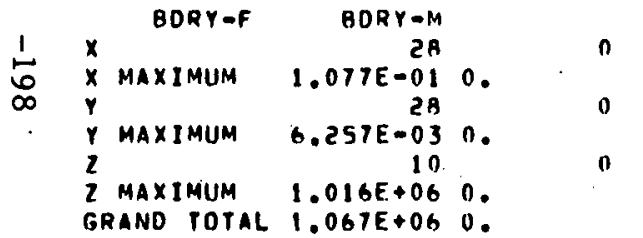

ELEMENT TYPE IBOUNOAR

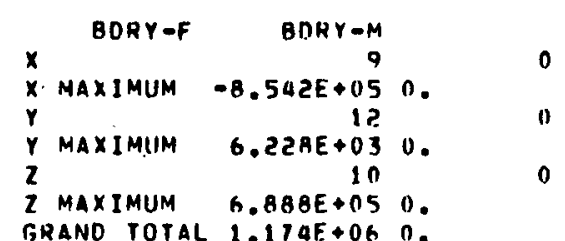

ELEMENT TYPE (A $O$ O $N$ N D A H $Y$

, 1,1 FLEMENI NIJMRER ( 10$)$

\footnotetext{
BORY-F BDRY-M -

$X$ MAXIMUM
$Y$
$Y$

$Y$ MAXIMUM $-6.228 E+03$ 0.

2 MAXIMUM $-6.228 E+03$

2 MAXIMUM $6.888 E+050$.

2 MAXIMUM $6.888 E+05$
GRAND TOTAL $1.174 E+O G$ O.
}

T TYPE LB O U N O A R

1, LIEMFON WHMMER $(11)$

RYAF BDRY-M 
2 MAXIMUM $1.016 E+060$.

GRAND TOTAL $1.067 E+0 \% 0$.

ELEMENT TYPE

1,1, element NIJMAer $(13)$

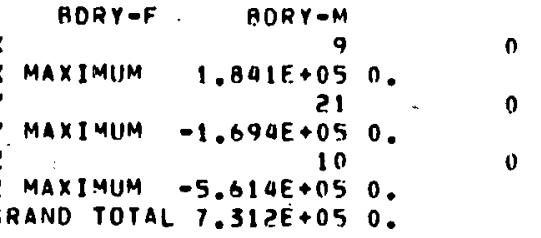

BORYAF HURY-N

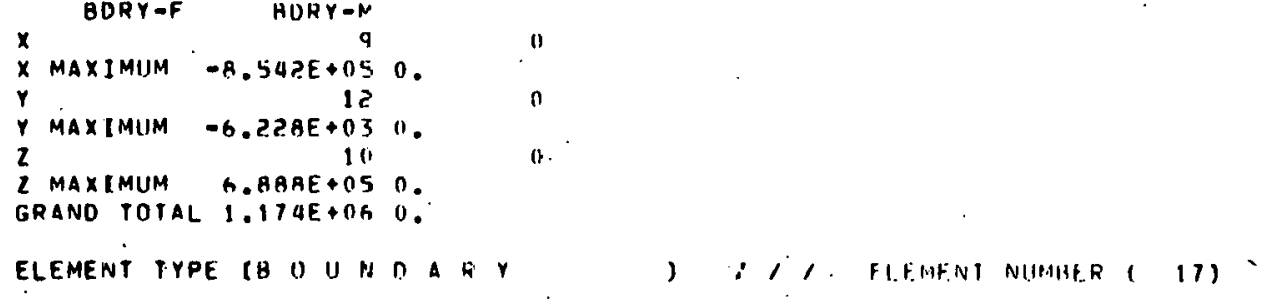




\begin{tabular}{|c|c|c|}
\hline BDRY =F & RDRY $-M$ & \\
\hline X MAXIMUM & $1.167 E+06$ & 0. \\
\hline r & 14 & \\
\hline & $\begin{array}{r}5.373 E+04 \\
20\end{array}$ & 0. \\
\hline $\begin{array}{l}2 \text { MAXIMUM } \\
\text { GRAND TOIAL }\end{array}$ & $\begin{array}{r}-1.485 E-03 \\
1.208 E+06\end{array}$ & 0 \\
\hline
\end{tabular}

ELEMENT TYPE ( $Q 0$ U N N DA A $P$

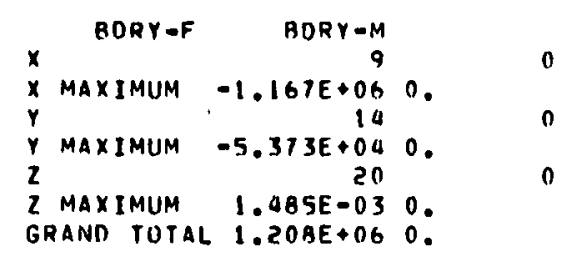

ELEMENT TYPE ( $B$ O U N D A $R$

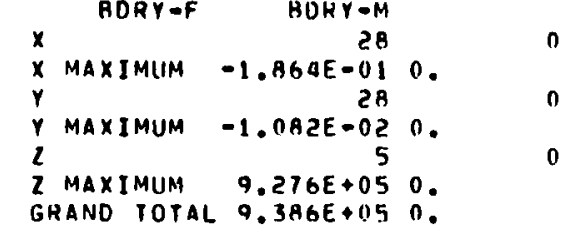


ELEMENT TYPE $13 / 0$ P I P E

1,1, ELEMENT :NUMHER $(3)$

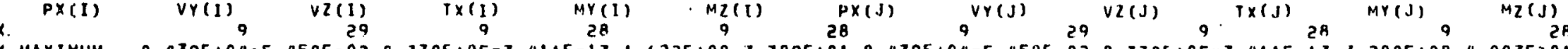

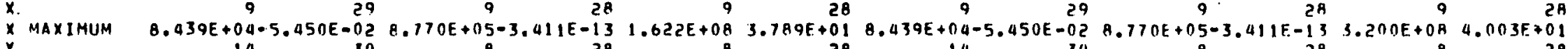

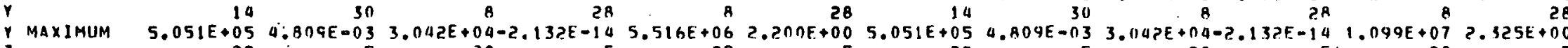

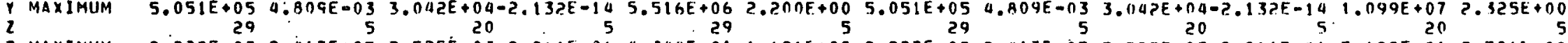

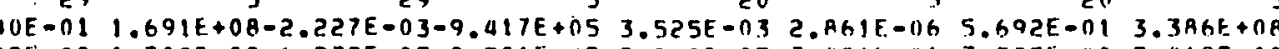

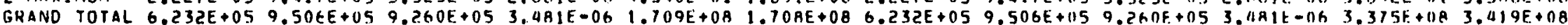
ELEMENT TYPE (3/0 P I P E. , , , ELEMENT 'NUMHER $(4)$

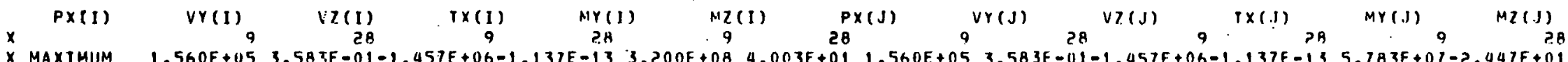

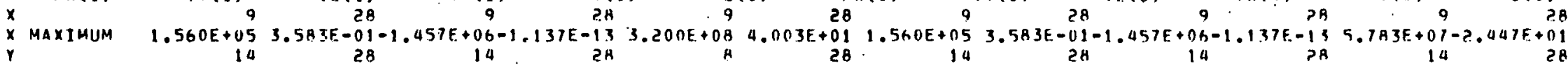

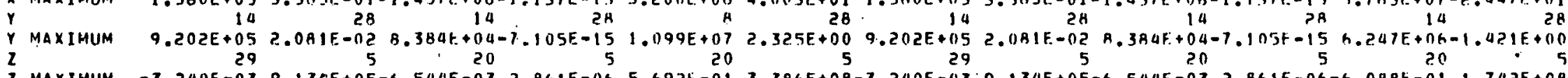
2 MAXIMUM -3.240E-03 $9.134 E+05-6.544 E-03 \quad 2.861 E-06 \quad 5.692 E-013.386 E+08-3.240 E-03-9.134 E+05-6.544 F-03 \quad 2.861 E-06-6.0 A B E-01 \quad 1.742 E+0 B$ GRAND TOTAL $1.136 E+069.273 E+051.494 E+062.816 E-063.315 E+0 A 3.419 F+0 B 1.136 E+069.273 E+051.494 F+062.876 E-06 \quad 7 . A A 1 E+071.756 E+0 B$ ELEMEN TYPE (3/0 P I P E , , , ELEMENT NUMHER $(5)$

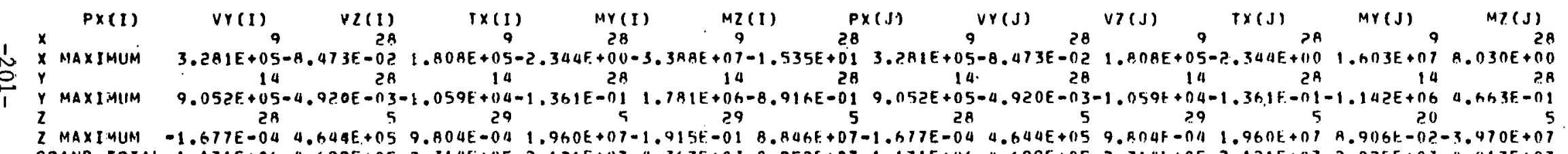
GRAND ELEMENT TYPE (3/0 P I P E , 1,1 ELEMENI NIPTAFR $(6)$

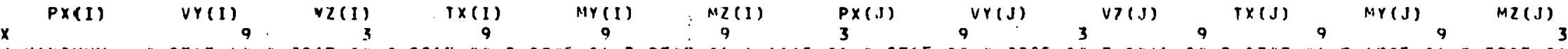
$x$ MAXIMUM $0.856 E+04-4.32 D E+04-3.986 E+04$
$y$

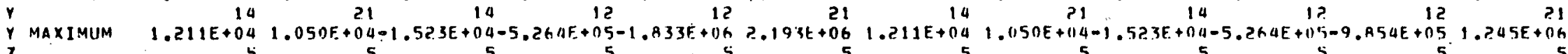

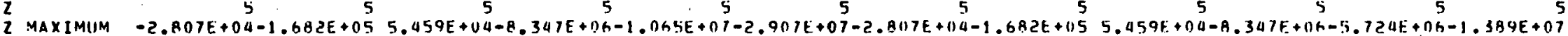

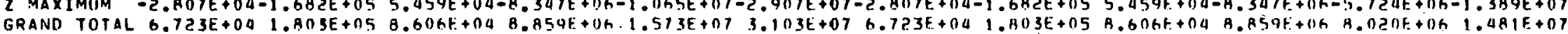
ELEMENT TYPE (3/0 P I PE , , , FLEMENT NUMHER 11 )

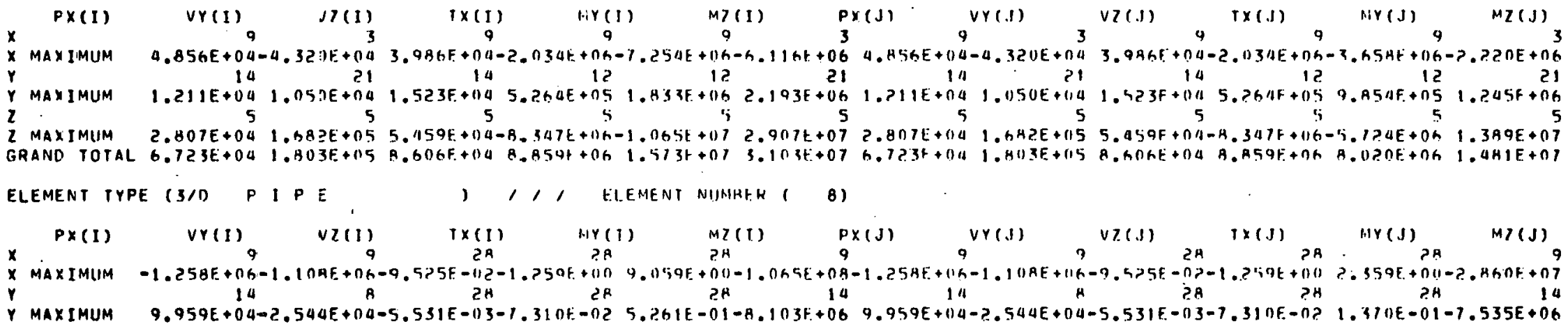




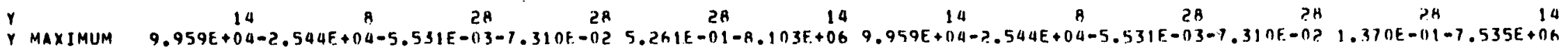

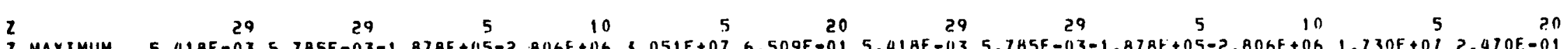

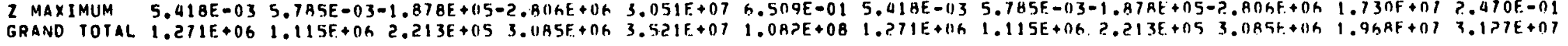
ELEMENT IYPE ( $3 / 0$ PI $P$, , 1, ELEMENT NUMAER 19 )

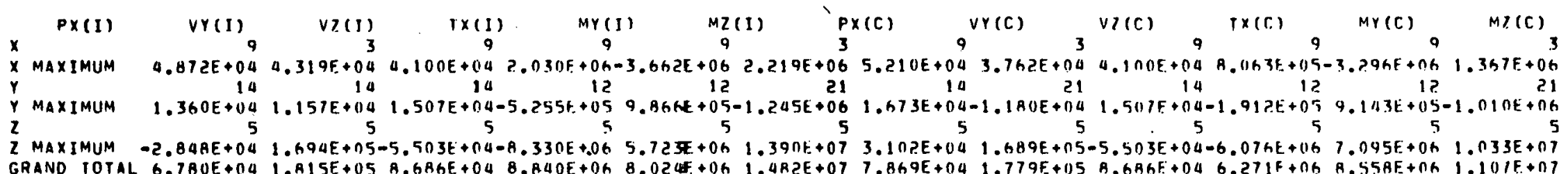

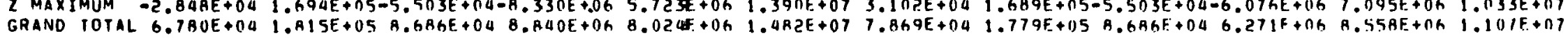

ELEMENT TYPE (3/0 P I PE , , , ELEMFNT NUMRER ( 9$)$

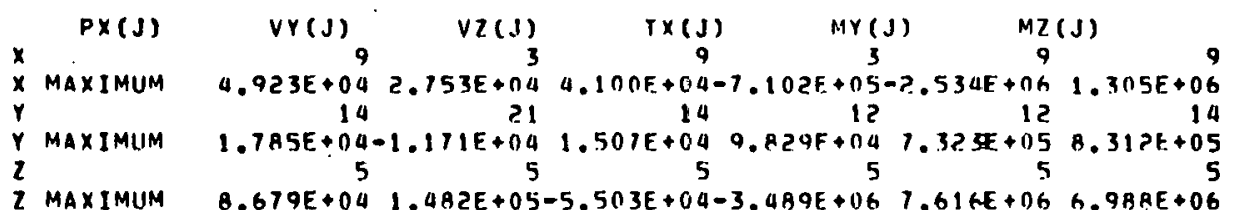

2 MAXIMUM $8.679 E+04 \quad 1.482 E+05-5.503 E+04-3.489 E+06 \quad 7.616 E+06 \quad 6.9 B A E+06$
GRAND TOTAL $1.164 E+05 \quad 1.559 E+1558.696 E+04 \quad 3.672 E+06 \quad 8.430 E+06 \quad 7.689 E+06$

ELEMENT TYPE (3/D P 1 PE , , , ELEMENT NIMHER $(10)$

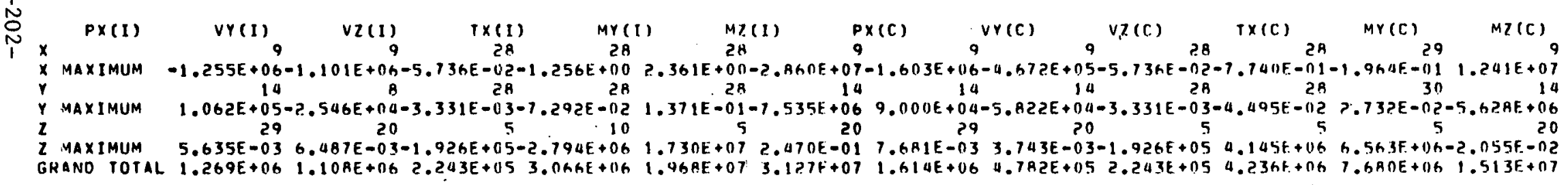

ELEMENT TYPE (3/0 P 1 PE, $1 /$, FLEMENT NIMABER $(10)$

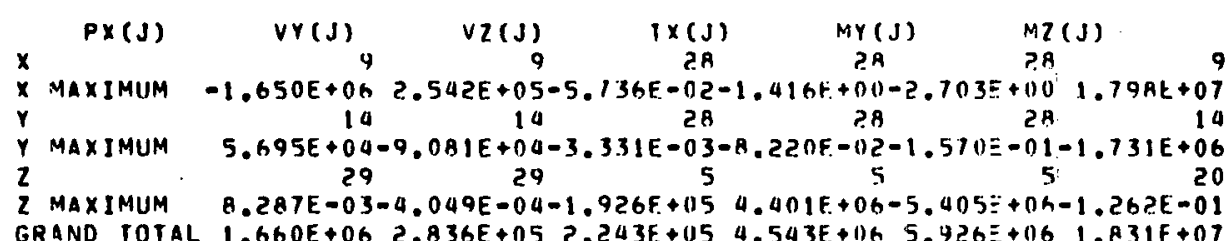

ELEMENT TYPE (3/0 P I PE ; , , ELEMEVT NUMAER ( 1 i)

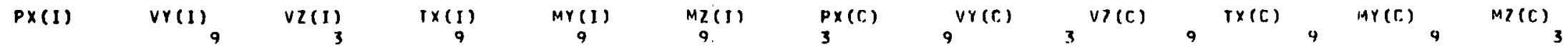

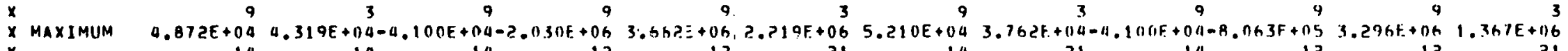

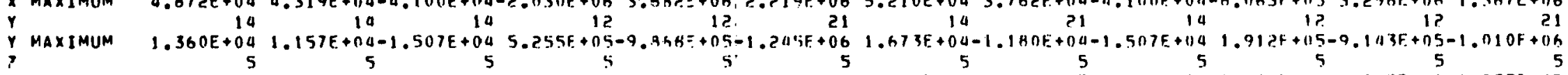

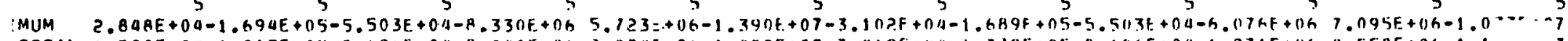

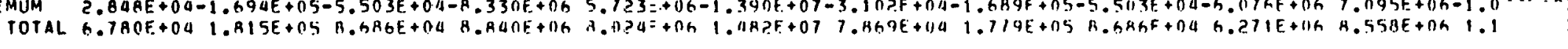


AUM $\quad 4.923 E+042.753 E+04-4.100 E+04 \quad 7.102 E+05 \geq .5345+06 \quad 1.305 E+06$ $Y$
$z$ 2 MAXIMUM $-0.679 E+04-1.482 E+05-5.503 E+04-3.489 E+06 \quad 1.616 E+106-6.98 B E+06$

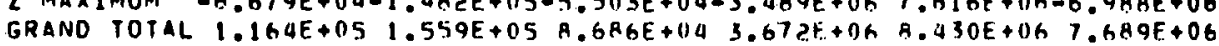

ELEMENT TYPE $13 / 0$ P I PE 1,1, ELEMENT NUMGer 1 (2)

\begin{tabular}{|c|c|c|c|c|c|c|c|c|c|c|c|c|}
\hline$P X(I)$ & Vr(1) & $v 2(1)$ & $T \times(1)$ & MYCI & $M Z C$ & (I) & $(J)$ & $\operatorname{Vr}(J)$ & $v>(J)$ & $T \times(j)$ & $\operatorname{MY}(. I)$ & $M Z(J)$ \\
\hline МА Х ТмИМ & & & & 3 & 9 & 17 & 9 & 9 & 9 & 3 & 3 & 9 \\
\hline MAXIMLIM & $5.017 E+04-$ & $-2.709 E+04$ & 3. $007 E+04=$ & $-7.102 F+05-$ & $-2.185 E+06-$ & $-A .536 E+05$ & $5.017 E+04-$ & $-2.709 E_{-}+04$ & $3.8075+04=$ & $-7.10 ? F+115$ & 1.OROE + OR & $? . ? 44 E+06$ \\
\hline Y & 14 & 21 & 14 & 12 & 12 & 10 & 14 & 21 & 14 & 12 & 14 & 14 \\
\hline MAXIMUM & $2.234 E+04=$ & $E+04$ & $1.4 \cap 4 F+014$ & 9.eh2F+104 & H.196F. US & A. $7 \mathrm{~B}$ & $2.234 E+04-$ & $-1.044 E+114$ & $1.404 E+04$ & - $P$ SE $+\cap 4$ & $9.161 E+05$ & $.11 \mathrm{bE}+0 \mathrm{G}$ \\
\hline$z$ & 5 & 5 & 10 & 5 & 5 & 5 & 5 & 5 & 10 & 5 & 5 & 10 \\
\hline $\begin{array}{l}2 \text { MAXIMUM } \\
\text { GRANO TOTAL }\end{array}$ & $\begin{array}{l}8.745 E+04 \\
1.187 E+05\end{array}$ & $\begin{array}{l}+05 \\
+05\end{array}$ & $\begin{array}{r}-3.348 E+04= \\
6.910 F+00\end{array}$ & $\begin{array}{l}-3.485 E+0 h \\
3.664 F+17 h\end{array}$ & $\begin{array}{l}5.560 E+116 \\
6.819 E+116\end{array}$ & $\begin{array}{l}8.715 E+06 \\
9.134 E+06\end{array}$ & $\begin{array}{l}8.745 E+04 \\
1.182 E+05\end{array}$ & $\begin{array}{l}1 . h \mathrm{~h} \\
1 . \mathrm{hP}\end{array}$ & $\begin{array}{l}-3.3 / 1 A E+04= \\
6.91 \cap E+04\end{array}$ & $\begin{array}{r}-3.495 E+06 \\
3.669 F+06\end{array}$ & $\begin{array}{l}4.61 \mathrm{hE}+06- \\
5.011 \mathrm{H}+10 \mathrm{~h}\end{array}$ & $\begin{array}{l}E+0 h \\
E+06\end{array}$ \\
\hline
\end{tabular}
ELEMENT TYPE (3/0 PI PE , $1 ;,$ ELEMENT NUMHER $(13)$

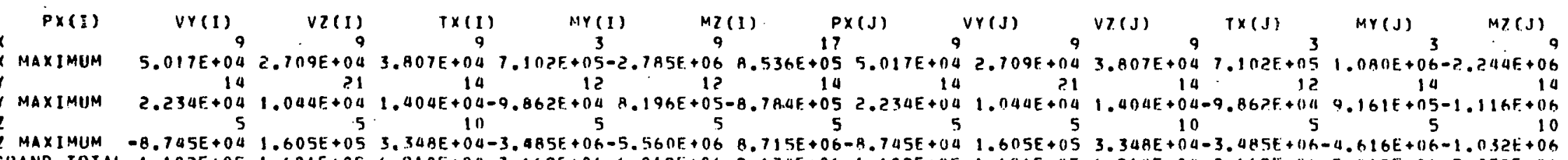

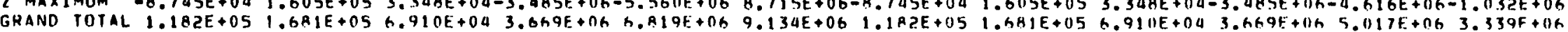

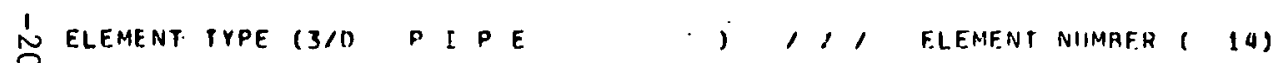

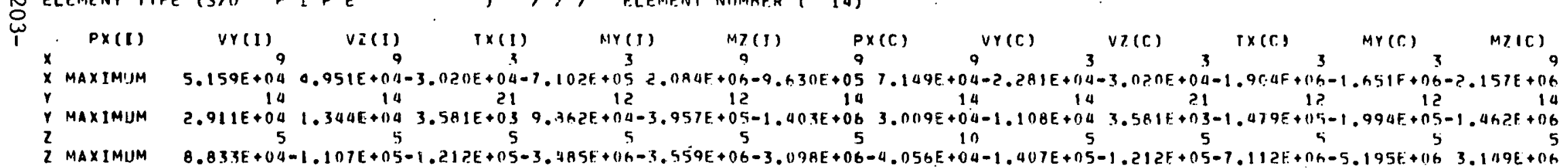

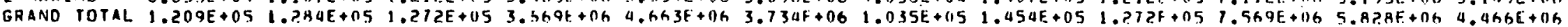
ELEMENT TYPE (3/D P P

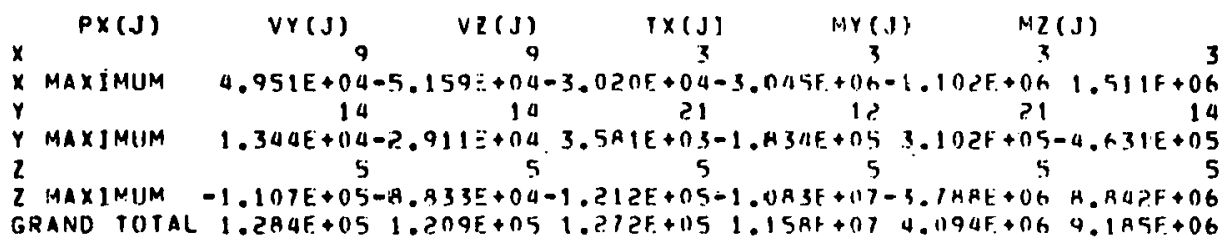

ELEMENT TYPE (3/1) P I.PF, , , ELF.HF.N1 NIJMHFR ( 15)

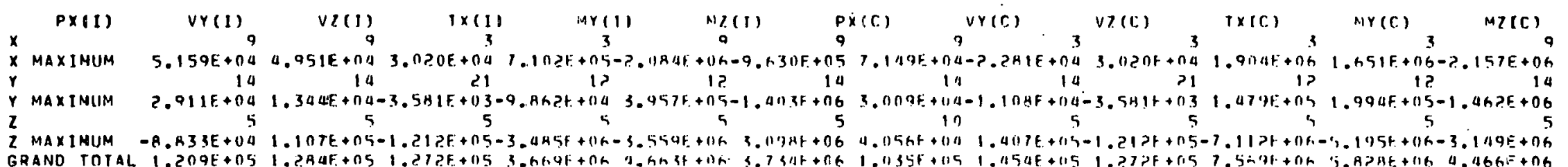

ELEMENT TYPE (3/0 P I P E , , , FLEMENT NIJHAER 1 15) 


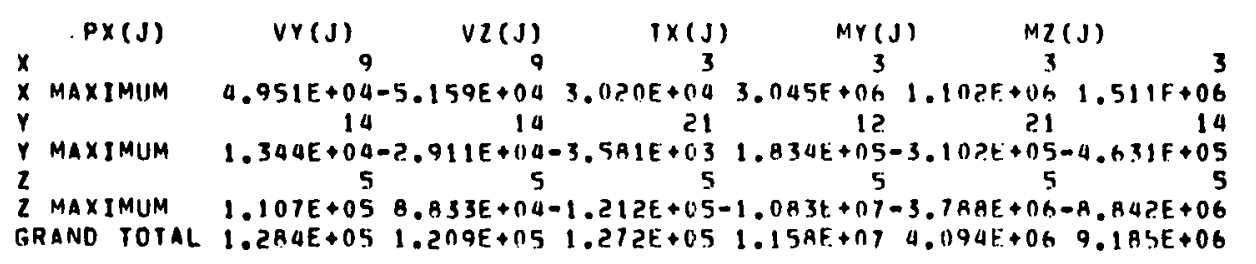
ELEMENT TYPE (3/0 P 1 P E , 1,1 ELEMENT NUMHER $(16)$

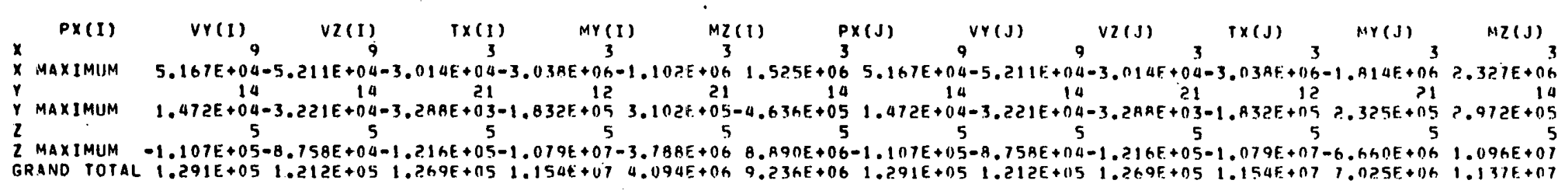

\begin{abstract}
ELEMENT TYPE $(3 / 0$ P I P E , , , ELEMENT NIJMAER $(17)$
\end{abstract}

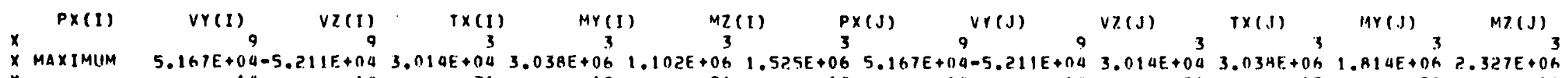

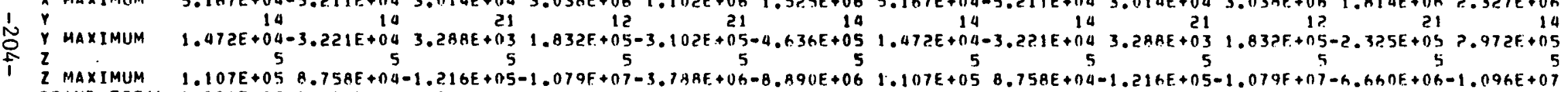

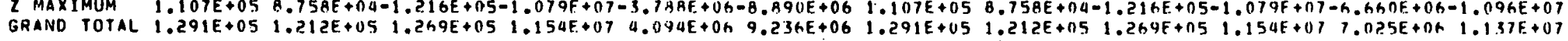
ELEMENT TYPE (3/0 P 1 PE , , , ELEMENT NUMAER $(18)$

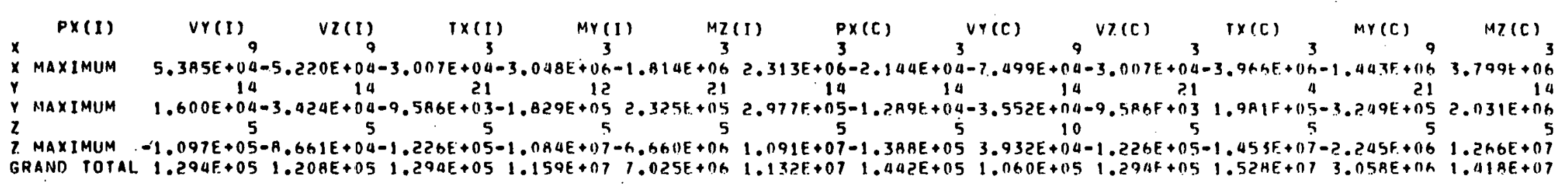

ELEMENT TYPE (3/1) P 1 PE , , , ELFMENT NUMRER $(.18)$

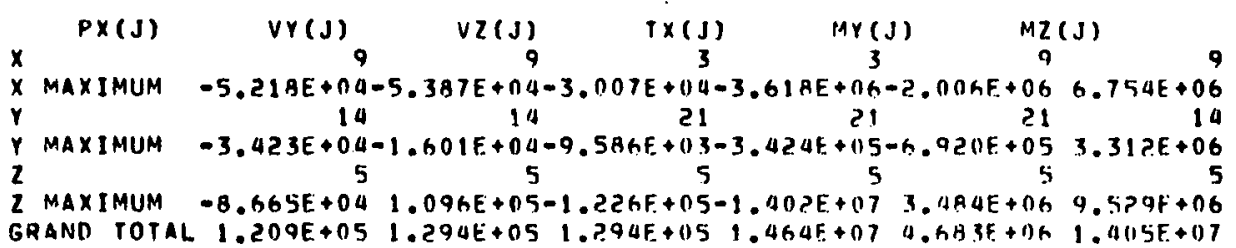

ELEMENT TYPE $13 / 0$ P 1 PE 1,1, ELEMEHT NUMHER. 19$)$

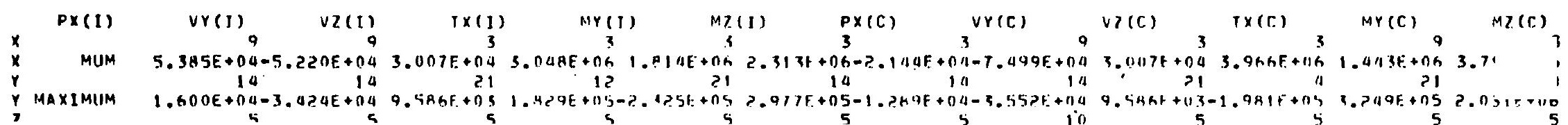


GA PTAL $1.294 E+05 \quad 1.208 E+05 \quad 1.294 E+05 \quad 1.159 E+07 \quad 7.025 F+061.132 E+071.442 E+05 \quad 1.050 E+051.294 F+05 \quad 1.52 A F+177 \quad 3.05 A E+0 \mathrm{~A} \quad 1.411$ ELEMENT TYPE (3/D P I P E $, 1,1$ ELEMENT NIMMHER $(19)^{\circ}$

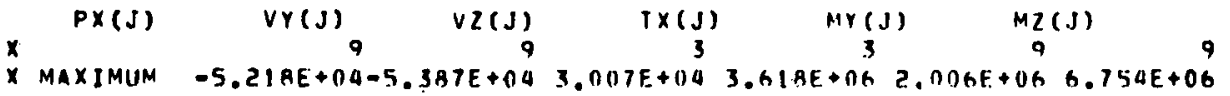

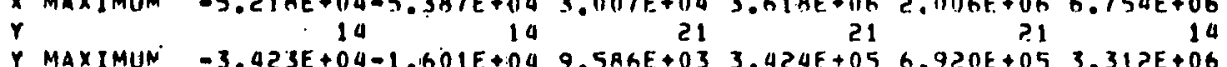

2 MAXIMUN $-3.423 E+04-1.601 E+04 \quad 9.5 A 6 E+03 \quad 3.424 F+05 \quad 6.920 E+05 \quad 3.312 E+06$

2 MAXIMUM $8.665 E+04-1.096 E+05-1.226 E+05-1.402 F+1173.184 F+06-9.529 E+06$

ERAND TOIAL $1.209 E+051.294 E+051.294 E+051.414 F+011.593 F+061.405 F+07$

ELEMENT TYPE 1310 P I P E , , , ELEMENT NUMAER $(20)$

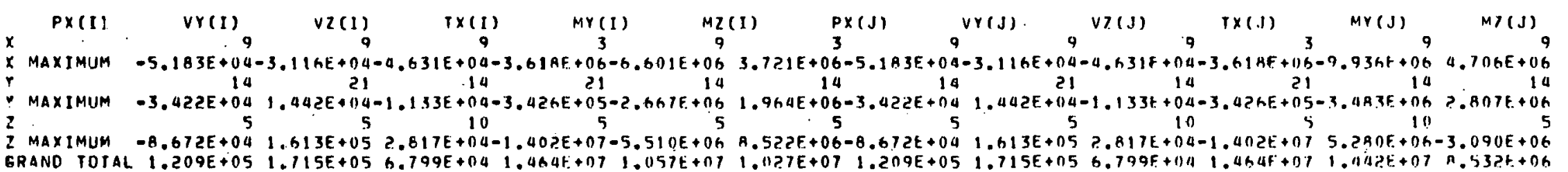

ELEMENT IYPE (3/D P I P E , , , ELEMENT NUMHER $(21)$

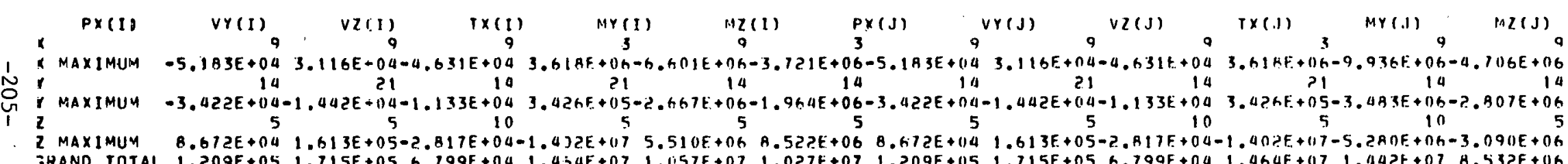
ELEMENT TYPE (3/0 P I D , , , ELENENT NIMAER ( 22$)$

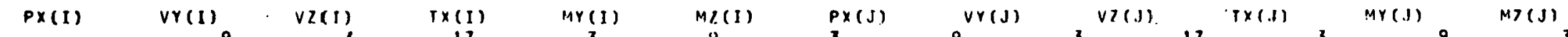

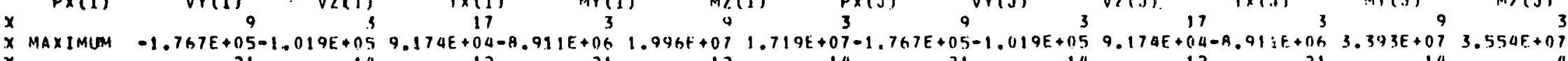

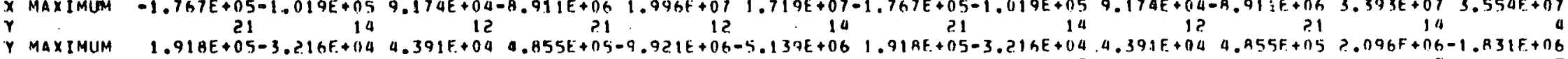
$Y$ MAXIMUM $1.918 E+05-3.216 F+114$
2

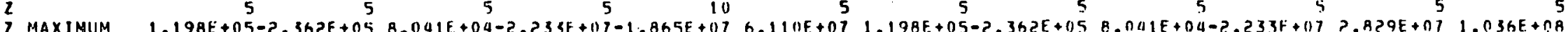

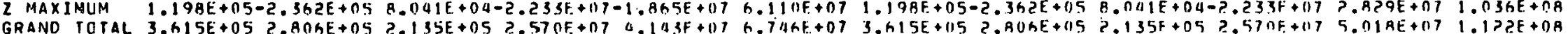
ELEMENT TYPE (3/0 P 1 PE ,, 1, ELEMENT NIJMHER 1 23)

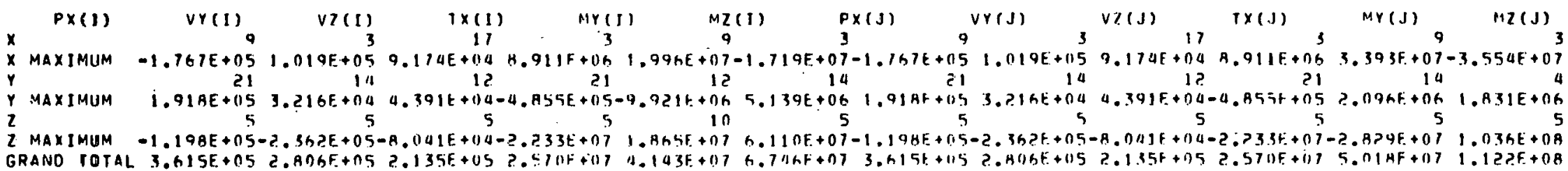
ELEMENT IYPE (3/0 P IPE 1,1, FI.EMFiNT NHMMILE 1 24)

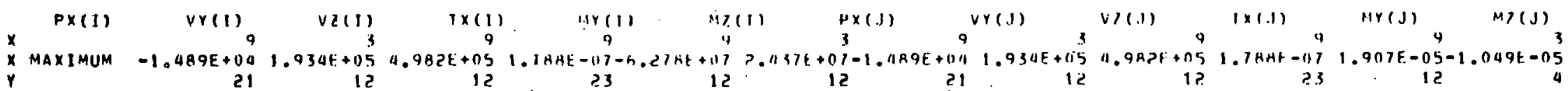




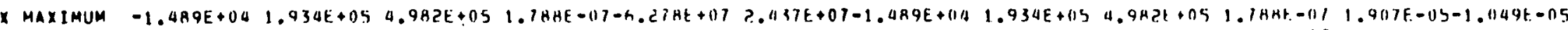

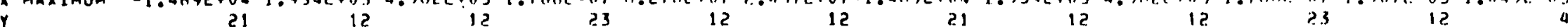

$Y$ MAXIMUM 1.43AE+05 4.107E+04-1.412E+05-3.125F-1)A $1.779 E+07 \quad 5.175 F+06 \quad 1.438 E+05 \quad 4.101 E+(14-1.41>E+05-3.7>5 F-08-1.9197 F-06$ A.911E-07

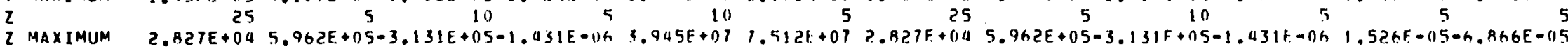

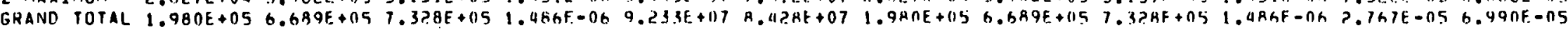
ELEMENT TYPE (3/D P I PE , , , ELEMENI NIJMHFR ( 25)

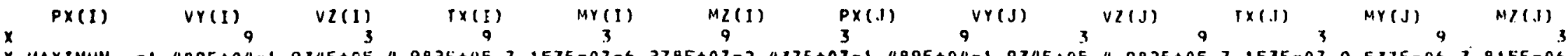

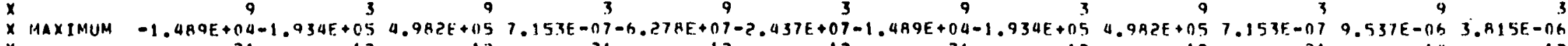
$Y$ MAXIMUM $1.438 E+05-4.107 E+04-1.412 E+05-7.451 E-0 R$
$1.179 E+07-5.175 E+06$

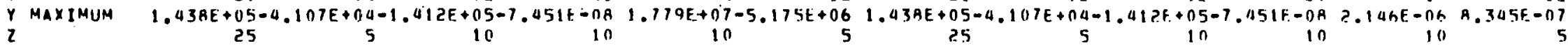

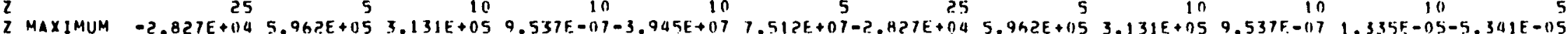

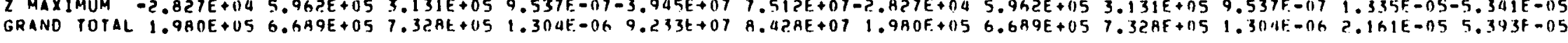
ELEMENT IYPE (3/D P I P F , 1,1 ELEMENT NIJMHER $(26)$

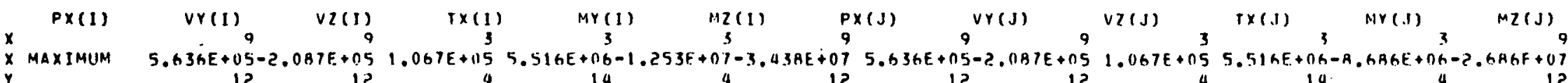

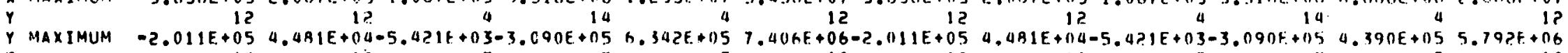

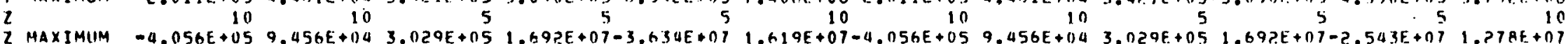
GRAND TOTAL B.959E+05 $2.599 E+053.356 E+051.81$ Q $+074.001 E+014.301 E+078.959 E+052.599 E+053.356 F+051.8196+072.794 E+073.366 E+01$

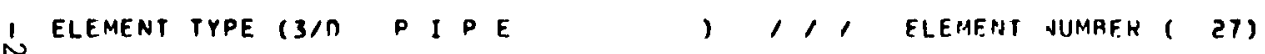

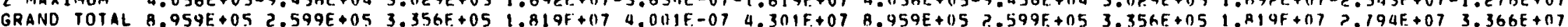

ELEMENT TYPE (3/0 P I P E , , , ELEMENT MUMHER $(28)$

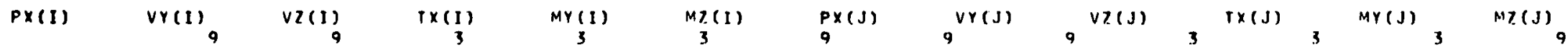

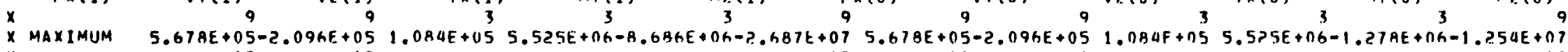

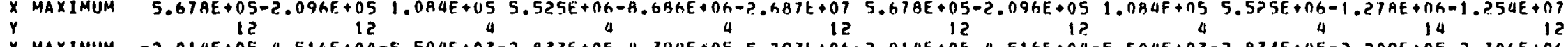

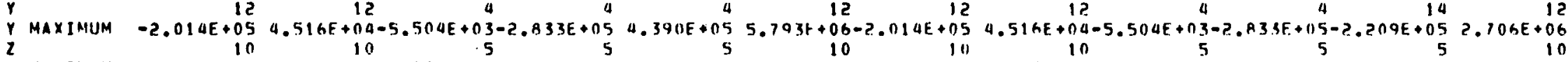

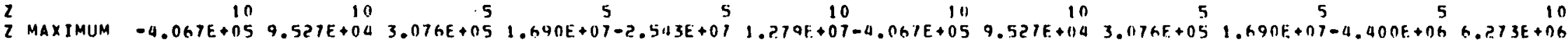

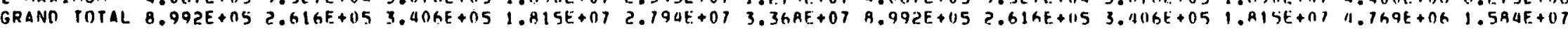

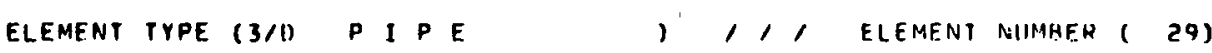

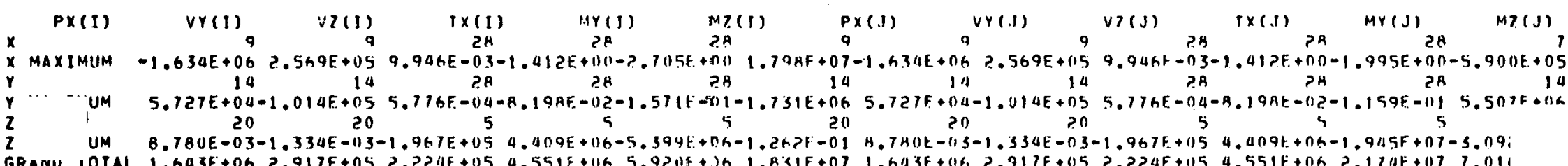


作

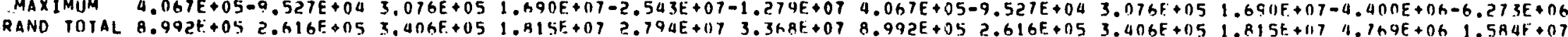

ELFMENT TYPE (3/D P I PE ,,$\quad$, ELEMENT NUMAER ( 31$)$

PX(I)

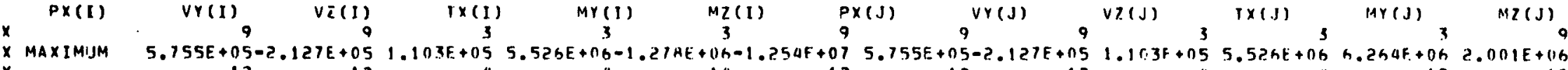

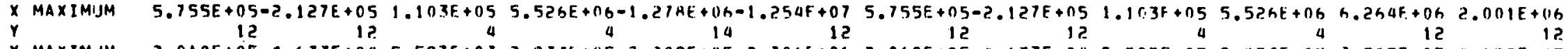

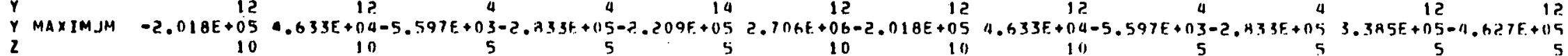

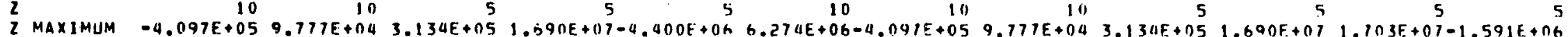
GAANO TDTAL $9.052 E+052.669 E+053.458 E+051.315 F+074.169 F+06 \quad 1.5 A 4 F+079.052 E+052.669 E+053.45 A F+051.815 F+071.904 E+072.971 E+06$ ELEMENT TYPE (3/0 PIPE , , , ELEMENT NIJMAER $(32)$

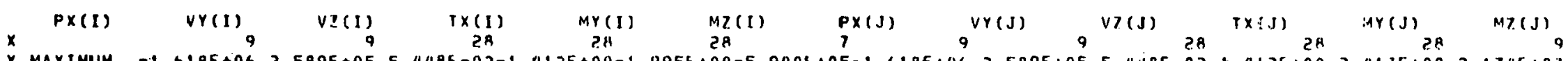
$X$ MAXIMLIM $-1.618 E+06$
$Y$

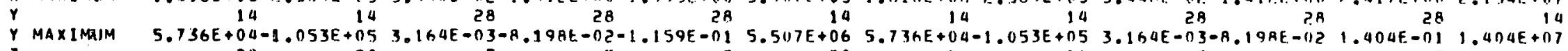

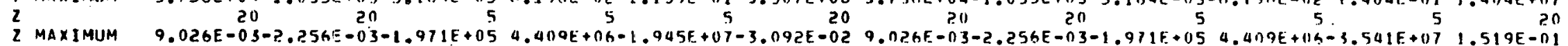

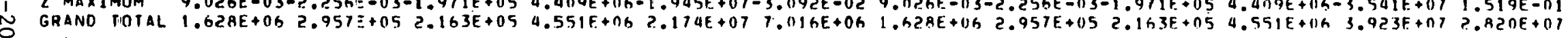
ELEMENT TYPE (3/0 P I PE ), 1 , ELEMENT NIIMHEH ( 33)

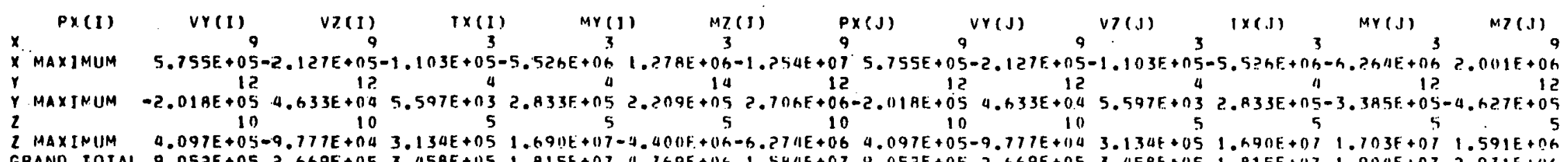

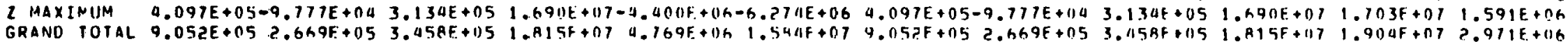
ELEMENT TYPE (3/1) $P$ I

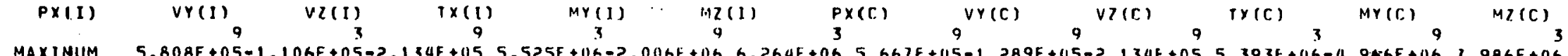

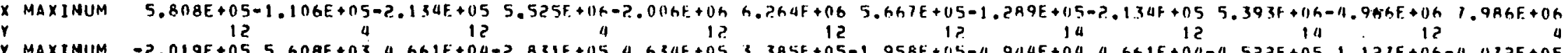

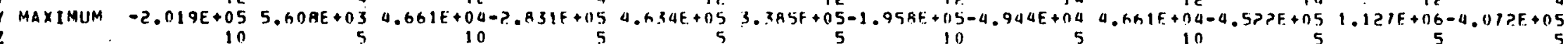

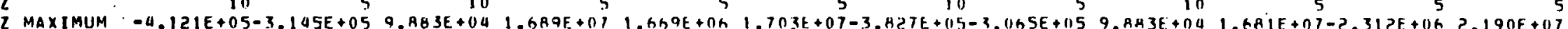

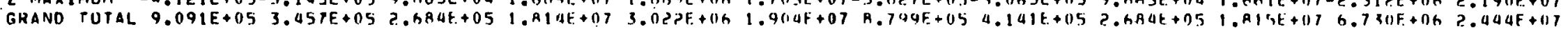
ELEMENT TYPE (3/0 P 1 PE $, 1,1$ TLLFEMT NIMHER $(34)$

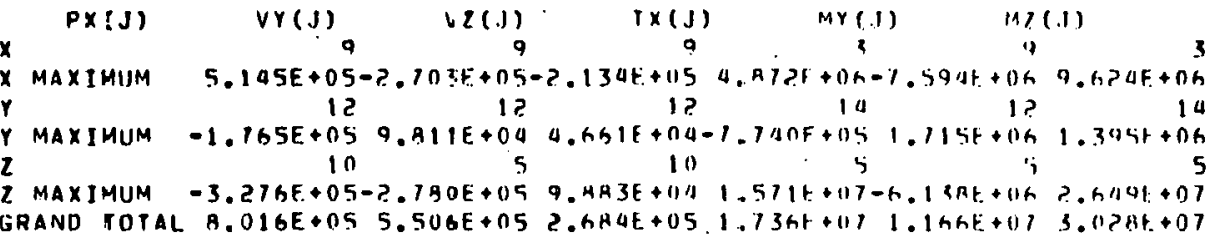


ELEMENT TYPE ( $3 / 0$ P I PE , 1, ELENENT NIJMRER $(35)$

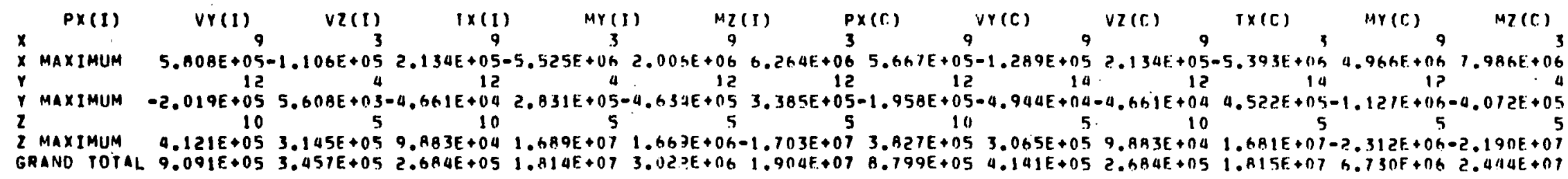

ELEMENT TYPE (3/0 P 1 P E , 1,1 ELEMENI NIJMHER 135$)$

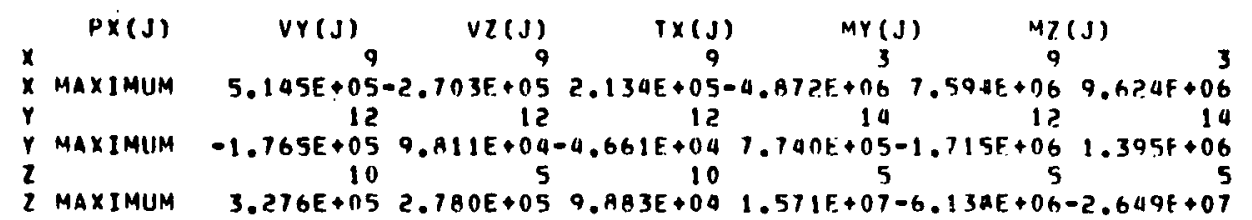

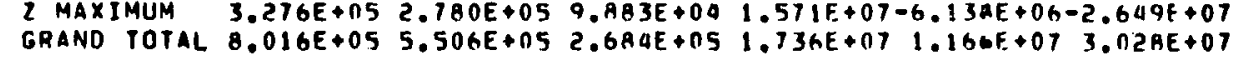

ELEMENT TYPE (3/D P I P E , , , ELEMEN ${ }^{-}$NUMAER $(36)$

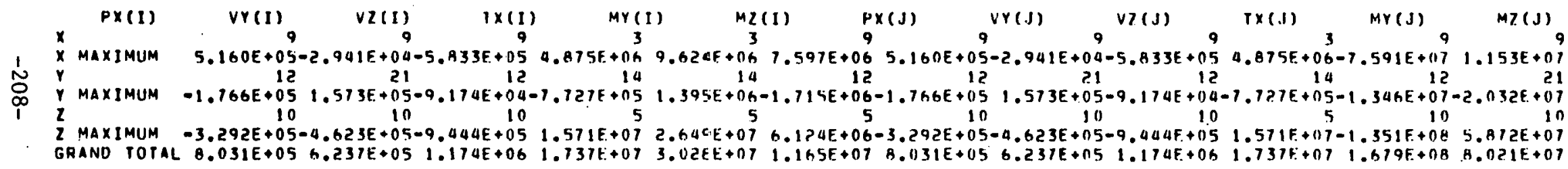
ELEMENT TYPE (3\%0 P I P E. J, , ELEMENI NUMAFR $(37)$

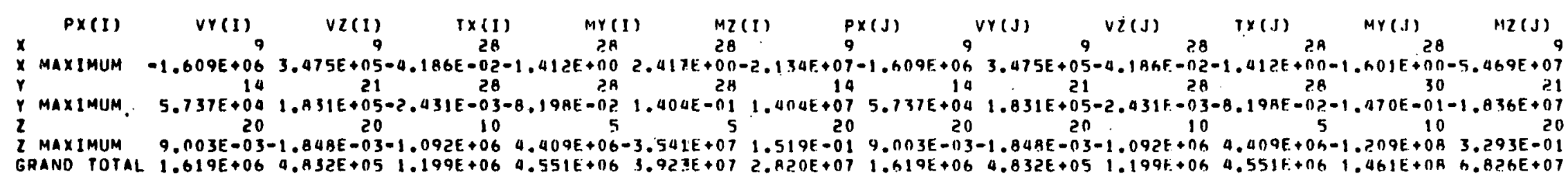
ELEMENT TYPE (3/D P I PE ) 1,1 ELEMENT NUMBER $(38)$

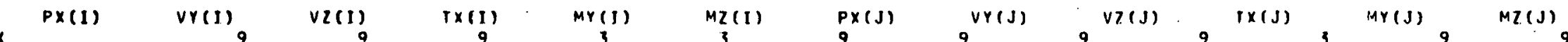
$\begin{aligned} & x \text { MAXIMUM } 5.160 E+05-2.941 E+04 \\ & Y\end{aligned} 5.833 E+05-4.875 E+06-9.624 F+06 \quad 1.597 F+06 \quad 5.16(1 E+05-2.941 E+045.8335 .+05-4 . A 75 E+06 \quad 7.591 F+07 \quad 1.153 E+07$

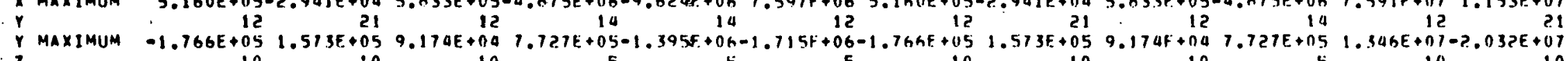

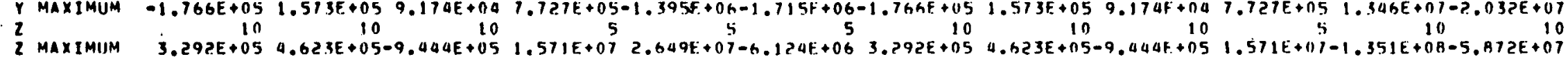

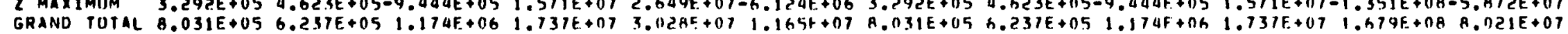
FI CMENT TYPE (3/0 PIPE , , , ELEMENT NIMMHER $(39)$

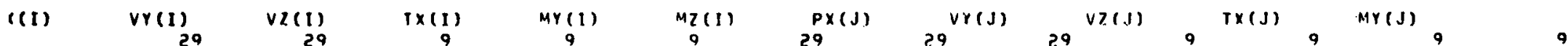

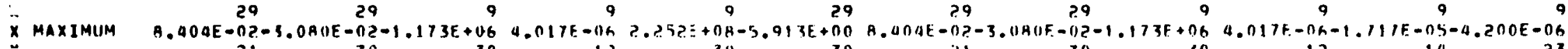


$2 \quad U M \quad 1.042 E-03 \quad 1.628 E+062.344 E-02-4.604 E-07-4.500 E+00 \quad 3.125 E+08 \quad 1.042 E-03 \quad 1.628 E+062.344 E-02-4.604 E-07-1.172 E-06-7.622$

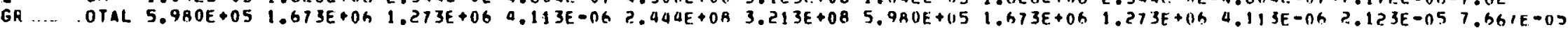
ELEMENT TYPE (3/D P I P E

, 1, ELEMENT NUMBER $(40)$

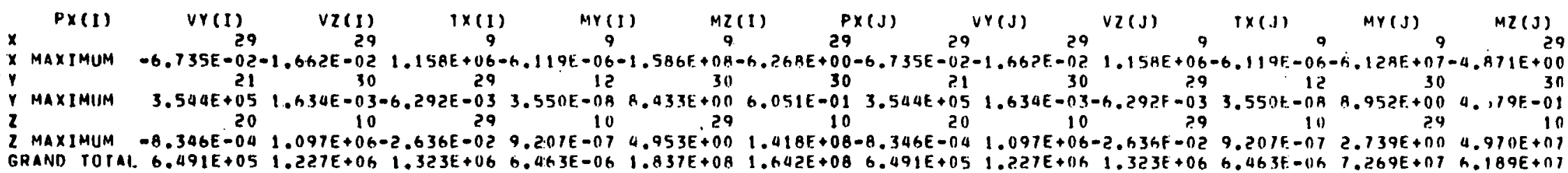

ELEMENT TYPE (3/D P I PE , 1,1 ELEMENT NUMHER ( 41$)$

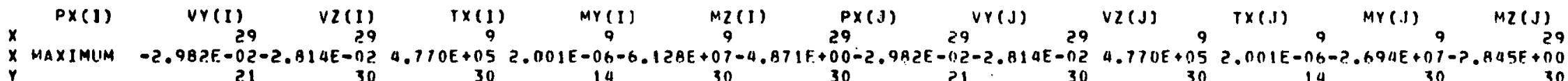

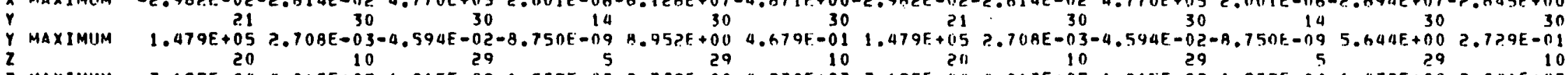
I MAXIMUM $-3.695 E-04 \quad 4.067 E+05-1.815 E-02 \quad 1.575 F-07 \quad 2.739 E+00 \quad 4.970 E+07-3.695 E-104 \quad 4.047 E+05-1.815 E-02 \quad 1.975 E-01 \quad 1.432 E+002.041 E+07$

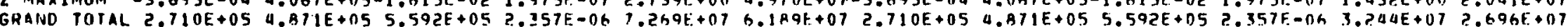
ELEMENT TYPE (3,0 P I P E, 1, ELEMENT NUMHER ( 42)

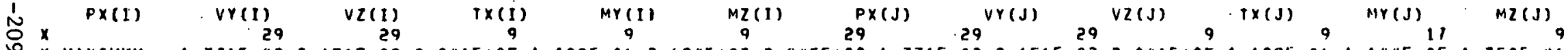
$X$ MAXIHIJM -1.331E-02-2.156E-02 2.041E+05-1.189E-06-2.694E+07-2.845E+00-1.331E-02-2.156E-02 2.041E+05-1.189E-06 1.144E-05 1.750E-06

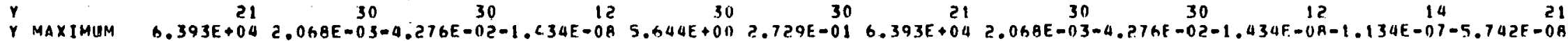
$\begin{array}{lccccc}Y & & \end{array}$ 2 MAXIMUM -1.650E-04 1.546E+05-1.085E-02-1.256E-07 1.032E+00 2.041E+07-1.650E-04 1.546E+05-1.085E-02-1.25hF-07 1.820E-06-1.907E-05

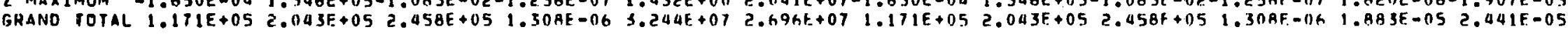
ELEMENT TYPE (3/0 P 1 PE $), i$, ELEMENT NUMHER $(43)$

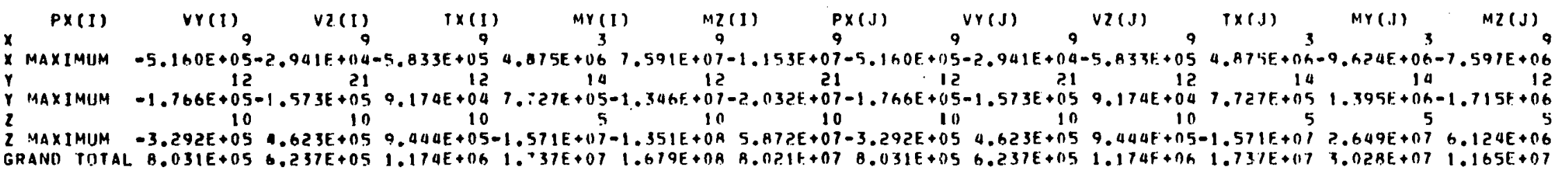
ELEMENT TYPE (3/1) P : PE , $:$, ELEMENI NUMAER ( 44)

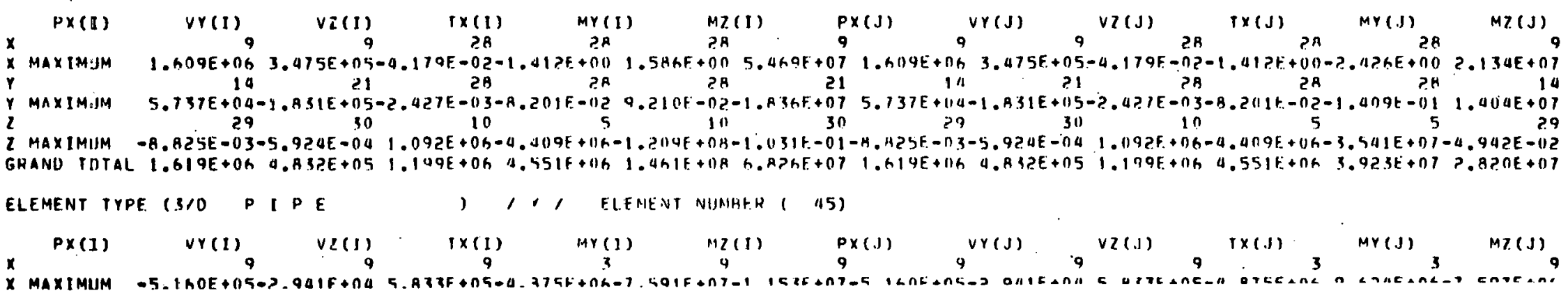




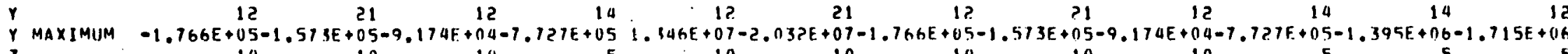

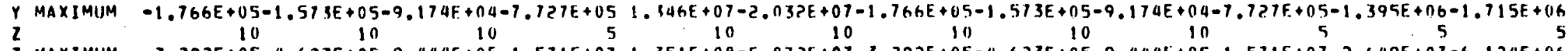
2 MAXIMUM $3.292 E+05-4.623 E+059.444 E+05-1.571 E+07-1.351 E+0 B-5.872 E+073.292 E+05-4.623 E+05-9.444 E+05-1.571 E+072.649 E+07-6.124 E+06$ GRAND TOTAL B.031E+05 $6.237 E+051.174 E+06 \quad 1.737 E+07 \quad 1.679 E+0 B \quad B .021 E+078.031 E+05 \quad 6.231 E+051.174 E+061.737 E+073.02 A F+071.165 E+07$ ELEMENT TYPE $13 / 0$ P I PE , 1, ELEMENT NUMBER (46)

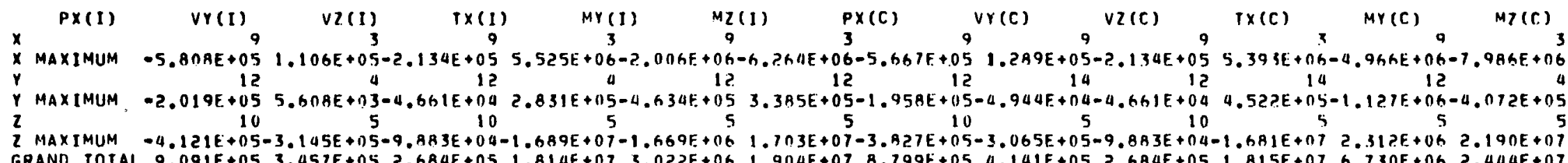
GRAND TOTAL $9.091 E+053.457 E+052.6 A 4 E+051.814 E+073.022 E+061.904 E+078.799 E+05 \quad 4.141 E+052.6 A 4 E+051.815 E+07 \quad 6.730 F+062.444 F+07$ ELEMENT TYPE (3/1) PIPE , 1,1 ELEMEMT NUMHER $(46)$

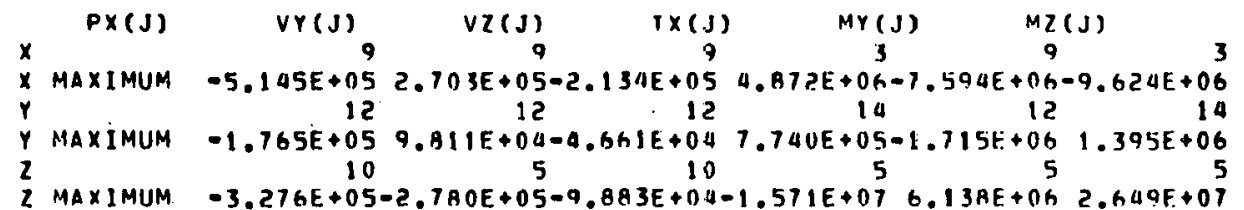

GRAND TOTAL $-3.276 E+05-2.7 A 0 E+05-9.8 A 3 E+04-1.571 E+076.13 A E+06 \quad 2.649 E+07$

'́ ELEMENT TYPE $(3 / 0$ P I P

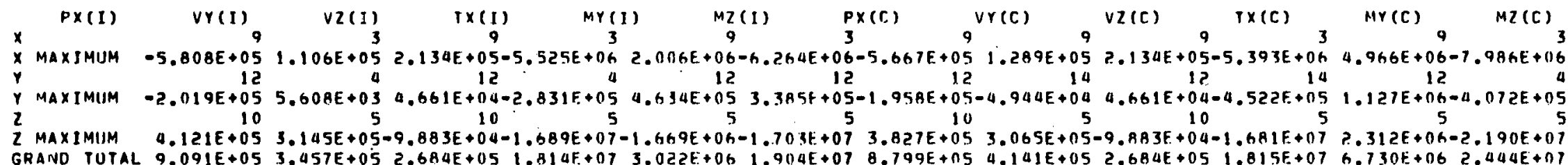

ELEMENT TYPE (3/0 P I P E , , , ELEMENT NIJMBER 147 )

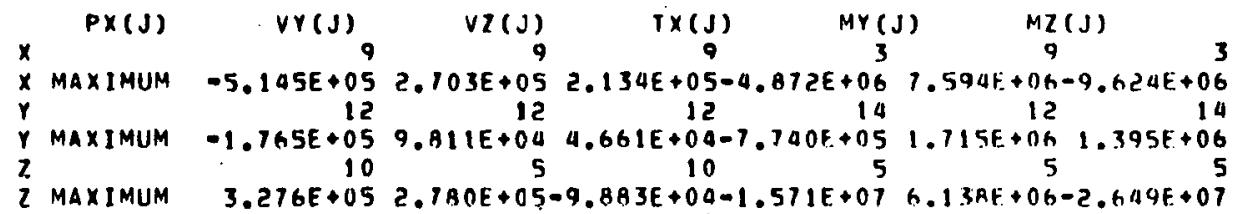

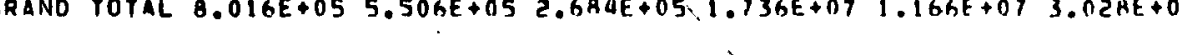

ELEMENT TYPE (3/0 P I PE , , $Q$, ELEMENT NUMAER $(48)$

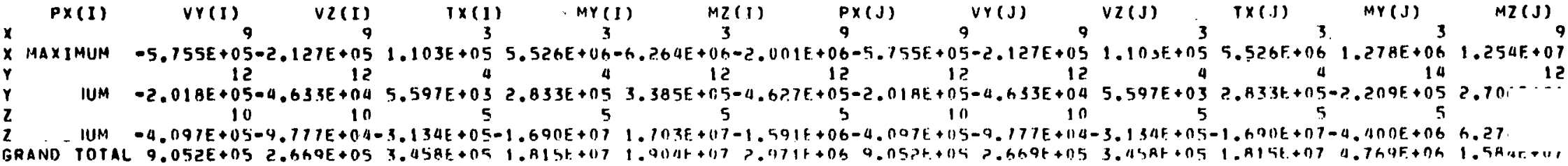



$Y$ MAXIMUM $5.136 E+041.053 E+053.170 E-03-8.201 E-02-1.409 E-011.404 E+075.736 E+04 \quad 1.053 E+053.170 E-03-8.201 F-021.159 E-015.507 E+06$ 2 MAXIMUM $-8.779 E-03-8.781 E-041.971 E+05-4.409 E+06-3.541 E+0.7-4.942 E-02-8.779 E-0.3-8.787 E-041.971 E+05-4.409 E+06-1.945 E+07 \quad 2.175 E-02$ GRANU TCTAL 1.628E+06 2.957E+05 2.163E+05 4.551F+06 3.923E+07 2.8?0E+07 $1.628 E+0162.951 E+052.1 \mathrm{~h} 3 E+05.4 .551 E+06 ? .174 E+071.016 E+06$ FLEMENT TYPE (3/0 P I P E , , , ELEMENT NUMHER $(50)$

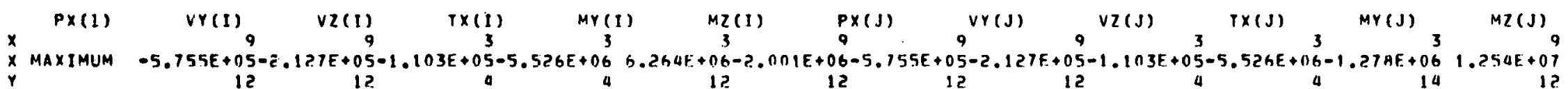
$Y$
$Y$

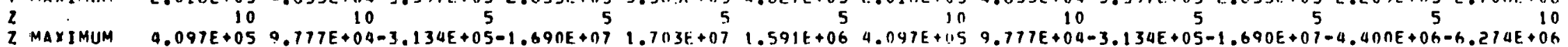
GRAND TOTAL $9.052 E+052.669 E+053.458 E+051.815 E+071.904 F+072.971 E+069.052 E+052.669 E+053.45 A E+051.815 E+074.769 E+061.584 E+07$ ELEMENT TYPE $13 / 0$ P I

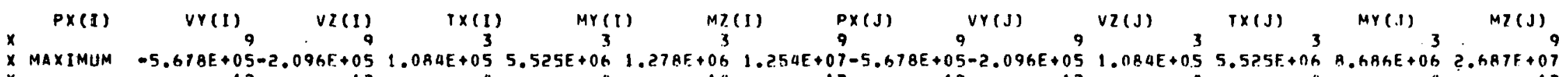

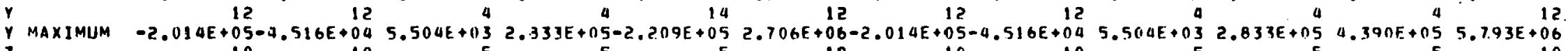

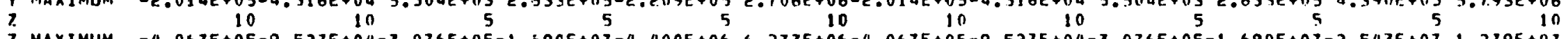
12 MAXIMUM $-4.067 E+05-9.527 E+04-3.076 E+05-1.390 E+07-0.400 E+06 \quad 6.273 E+06-4.067 E+05-9.527 E+04-3.076 E+05-1.690 E+07-2.543 E+07 \quad 1.279 E+07$ $\sim$ GRAND TDTAL $8.992 E+05$ 2.616E+05 3.406E+05 $1.915 E+074.169 E+061.5 A 4 E+07 B .992 E+052.616 E+053.406 E+051 . A 15 E+072.794 E+013.36 A E+07$

1 ELEMENT TYPE (3/0 P I P , , , ELENENT NUMHER 1 S2)

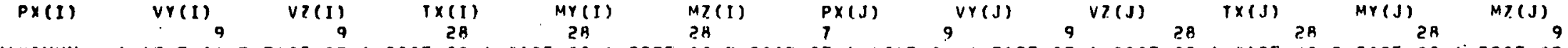
$x$ MAXJMUM $1.639 E+06$
$\times$

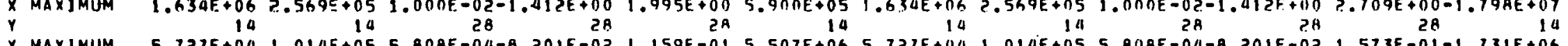

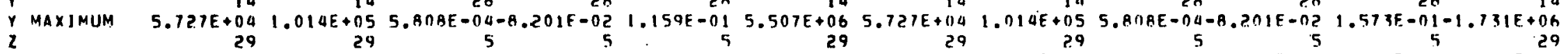
2 MAXINUM $-8.594 E-03 m 6 . A 67 E-04 \quad 1.967 E+05-4.409 E+06-1.945 E+07 \quad 2.175 F-02-8.594 E-03-6.867 E-04 \quad 1.967 E+05-4.409 E+06-5.399 E+06 \quad 7.078 E-02 ?$

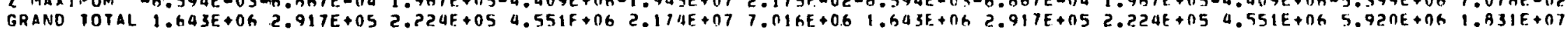
ELEMENT TYPE (3/0 P 1 PE , , , ELEMENT NUMAER ( 53)

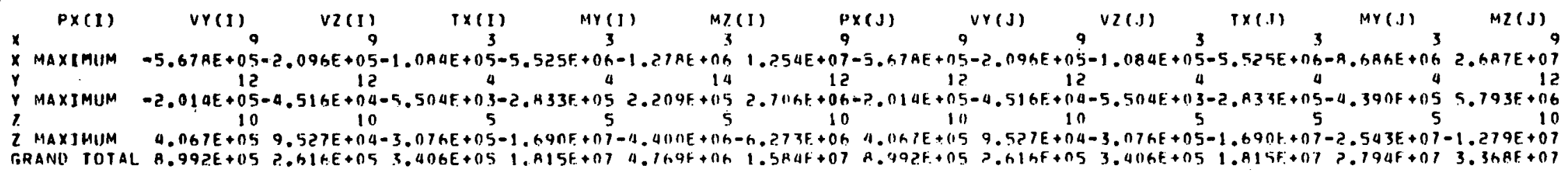

ELEMENT TYPE (3/1) PIPE, , , ELFHFNI N(BAHER 1 S4)

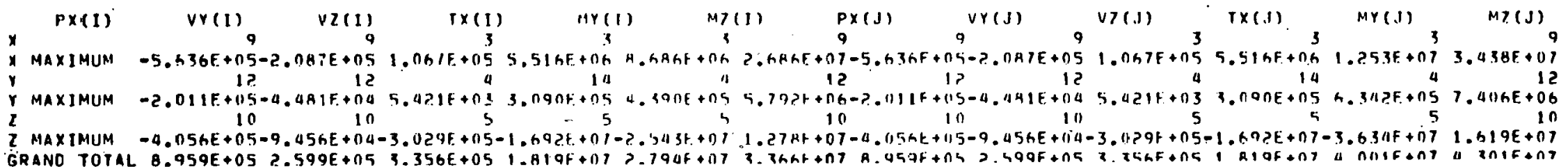


ELEMENT TYPE (3/D P I PE ), 1, ELEMENT NUMHER ( 55)

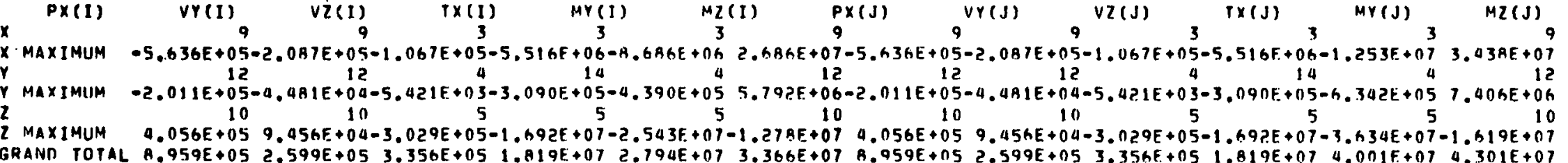
GRAND TOTAL A.959E+05 2.599E+05 $3.356 E+051.819 E+072.794 E+073.366 E+07 A .959 E+052.599 E+053.356 E+051.819 E+074.001 E+074.301 E+07$ ELEMENT TYPE (3/D P I PE , 1, ELEMENT NIJMHER ( 56)

\begin{tabular}{|c|c|c|c|}
\hline$P X$ & Vr(1) & $v 2(1)$ & $T \times(I)$ \\
\hline MAX IMII & 9 & & 9 \\
\hline & 21 & 2 & $\begin{array}{l}12 \\
12 \\
05\end{array}$ \\
\hline $\operatorname{MAXIML}$ & Z.R2TE & $\begin{array}{r}4.107 E+04 \\
5 \\
5.962 E+05\end{array}$ & $\begin{array}{r}1.412 E+105 \\
10 \\
3.131 E+105\end{array}$ \\
\hline
\end{tabular}

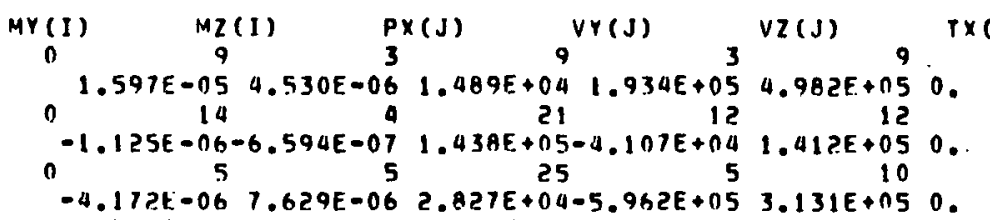

$$
\begin{aligned}
& -4.172 E-06 \quad 7.629 E-062.827 E+04-5.962 E+05 \quad 3.131 E+050 . \\
& 1.789 F-051.112 E-051.980 E+05 \text { G.6B9E+05 } 7.32 A E+050 \text {. }
\end{aligned}
$$

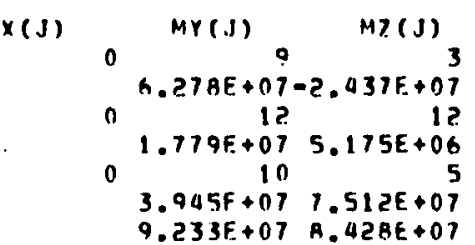

ELEMENT TYPE $13 / 0$ P I P E

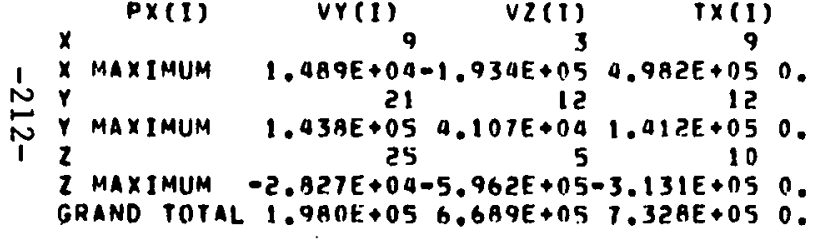

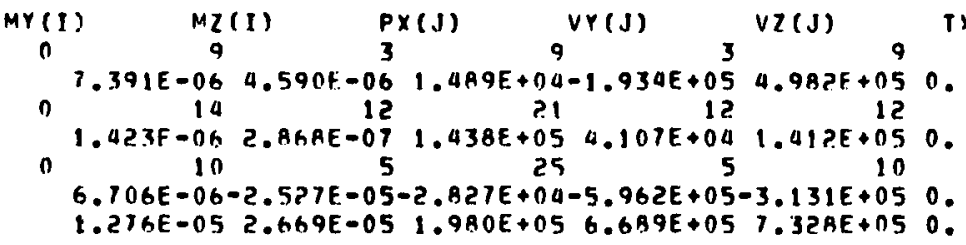

$1.2 T 6 E-05$ 2.669E-05 $1.980 E+056.6 B 9 E+057.32 A E+050$.

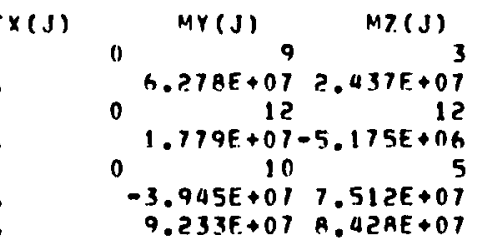

ELEMENT TYPE (3/0 P I PE $), 1$, ELEMENT NUMAER $($ 58)

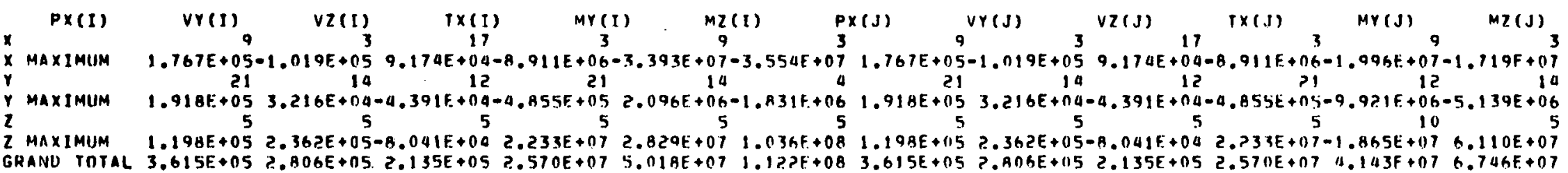
ELEMENT TYPE (3/D P I PE ) 1,1 ELEHENT NUMHER $(59)$

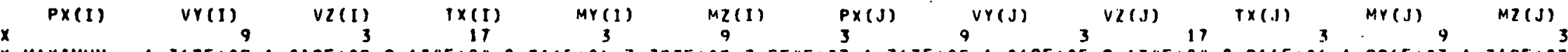

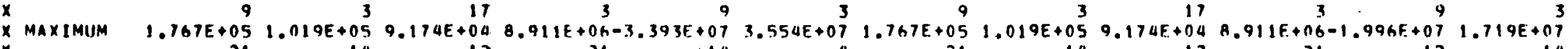

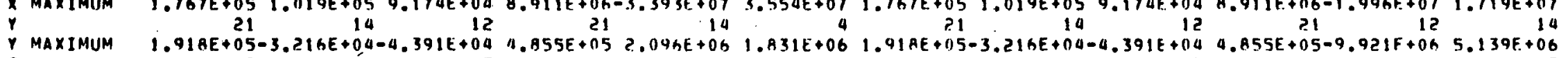

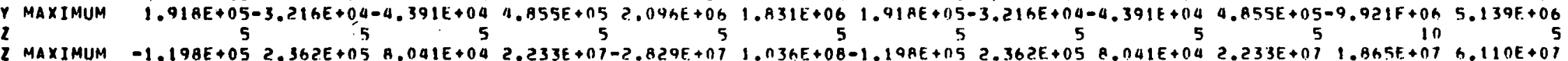

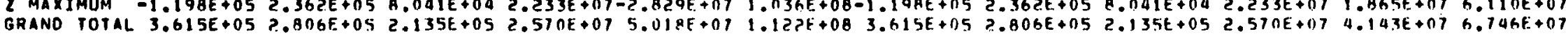
ELEMENT TYPE $(3 / 0$ P I P E

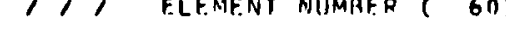

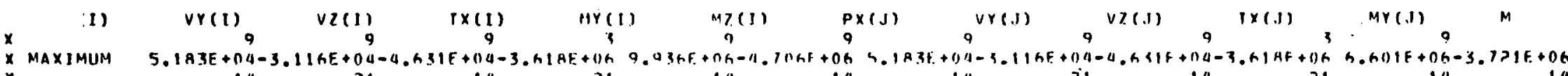




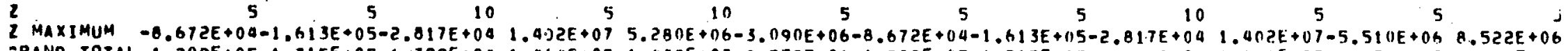

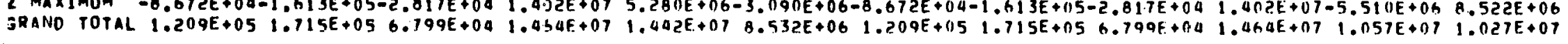
ELEMENT TYPE (3/0 P IPE ), $;$ ELEMENT NIMHER $($ G1)

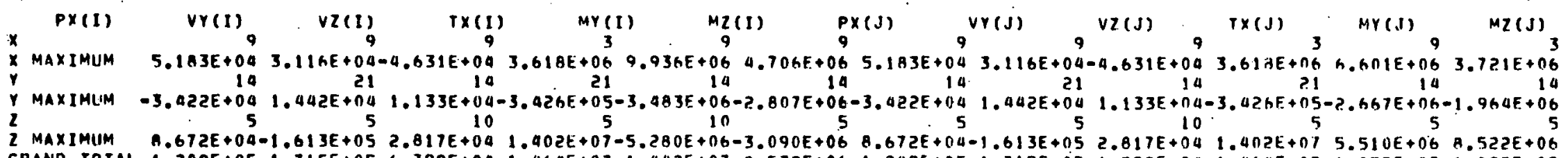

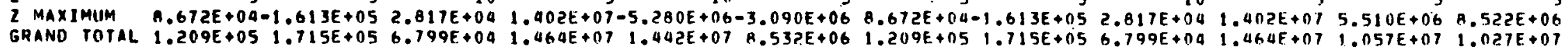
ELEMENT TYPE (3/D P I PE , , , ELEMENT NUMBER 1 G 6 \}

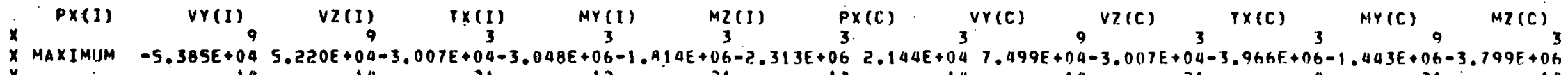

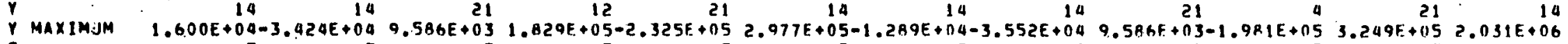

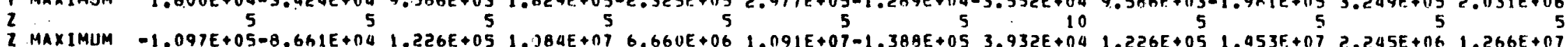
GRAND TOTAL $1.29 \Delta E+051.20 A E+051.294 E+051.159 E+01,1.025 E+061.132 E+071.442 E+051.060 E+051.294 E+051.529 E+073.05 A E+061.41 A E+07$

ELEMENT TYPE (3/0 P I PE , 1, ELEMENT NUMAER 1 62)

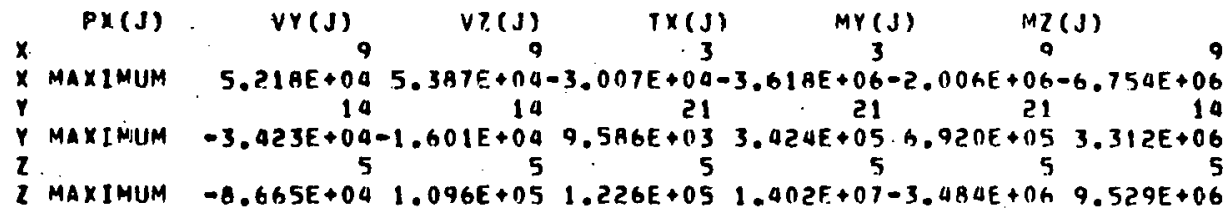
GRAND TOTAL $1.209 E+05 \quad 1.299 E+05$ 1.?9AE+05 $1.064 E+074.683 E+061.005 E+07$

ELEMENT TYPE (3/R P I PE , , , ELENENT NUMBER $(63)$

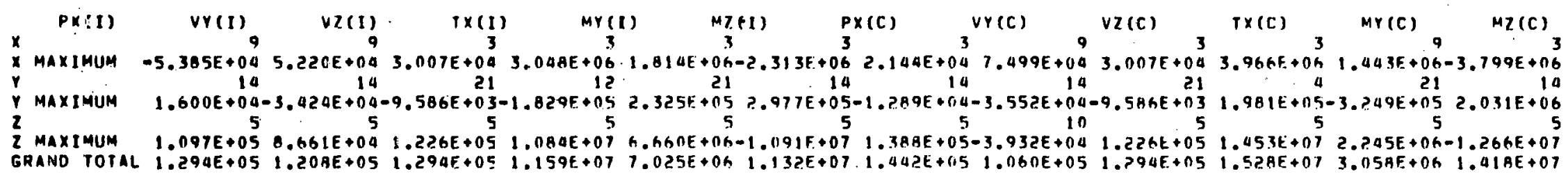
ELEMENT TYPE (3/D P I P E , , , ELEMENT NIIMAER $($ क3)

\begin{tabular}{|c|c|c|c|c|c|c|}
\hline$P \times(J)$ & $\operatorname{Vr}(J)$ & $1 / 2(J)$ & $T \times(3)$ & $\operatorname{Mir}(3)$ & $M Z 1$ & (J) \\
\hline & 9 & & 3 & 3 & 9 & \\
\hline MAXIMUM & $5.21 A E+04$ & 5.3A7E+04 & $3.007 E+04$ & $3.619 E+06$ & $2.006 F+06-$ & $=h .754 t+06$ \\
\hline 1 & 14 & 14 & 24 & 21 & 21 & 14 \\
\hline$y$ MAXJAUM & $23 E+04=$ & E & br. +0 & $\Delta F$ & h. $920 E+n 5$ & $3.3125+06$ \\
\hline 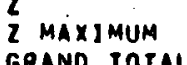 & $65 \mathrm{E}$ & $E+05$ & $\begin{array}{r}5 \\
+05 \\
.115\end{array}$ & $2 E+07$ & on- & \\
\hline
\end{tabular}

ELEMENT TYPE $13 / 0$ FIPE

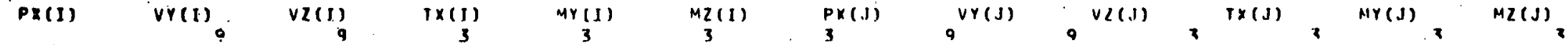


$X$ MAXIMUM -5.167E+04-5.211E+04-3.014E+04-3.038E+06 1.814E+06-2.32.7F+06-5.167E+04-5.211E+04-3.014E+04-3.038F+06 1.102F. 06-1.525E+06

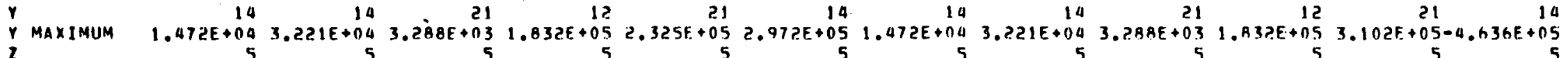

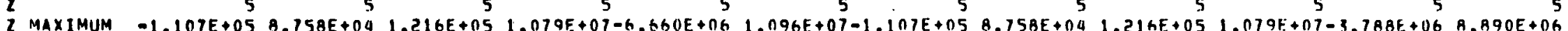
GRAND TOTAL $1.291 E+051.212 E+051.269 E+051.154 E+077.025 E+061.137 E+071.241 E+051.212 E+051.269 F+051.1511 E+074.094 E+069.234 E+06$ ELEMENT TYPE (3/0 P I P E , , , ELEMENT NUMAER $($ 65)

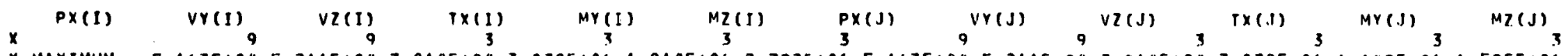
$X$ MAXIMUM -5.167E+04-5.211E+04 3.014E+04 3.038E+06-1.814E+06-2.327E+06-5.167E+04-5.211E+04 3.014E+04 3.03AE+ OB-1.102E+06-1.525E+06

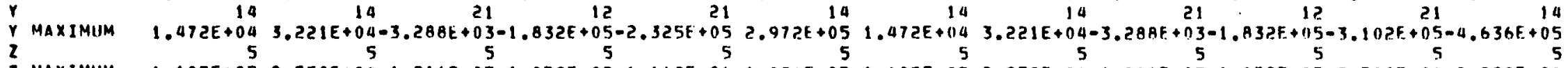
2 MAXIMUM $1.107 E+05-8.758 E+04 \quad 1.216 E+05 \quad 1.079 E+07-6.660 E+06-1.096 E+071.107 E+05-8.758 E+04 \quad 1.216 E+05 \quad 1.079 E+07-3.788 E+06-8.890 E+06$ GAAND TOTAL $1.291 E+051.212 E+051.269 E+051.154 E+077.025 E+061.137 E+071.291 E+051.212 E+051.269 E+051.1514+074.094 E+069.236 E+06$

ELEMENT TYPE (3/0 P I P E.

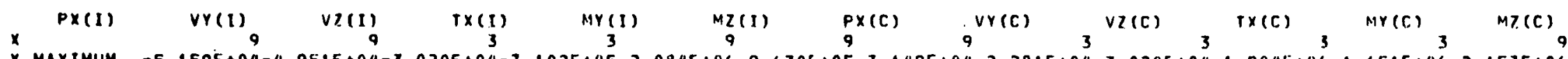
$\times$ MAXIMUM -5.159E+04-4.951E+04-3.020E+04-7.102E+05 2.084E+06 $9.630 E+05-7.149 E+042.281 E+04-3.020 E+04-1.904 E+016-1.651 E+06 \quad 2.157 E+06$ $Y$ MAXIMUM $2.911 E+041.344 E+04-3.5 B 1 E+03-9.862 E+043.957 E+05-1.403 E+063.009 E+04-1.108 E+04-3.5 B 1 E+031.479 E+051.994 E+05-1.462 F+06$

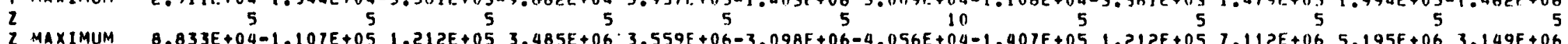
1 GRANO TOTAL $1.209 E+051.2 .244 E+051.272 E+05 \quad 3.669 E+06 \quad 4.663 E+06 \quad 3.734 E+06 \quad 1.035 E+051.454 E+051.272 E+05 \quad 7.569 E+06 \quad 5 . A 28 E+06 \quad 4.466 E+06$ ELEMENT TYPE (3/0 P I PE , , , ELEMENT NIMMAER 1 66)

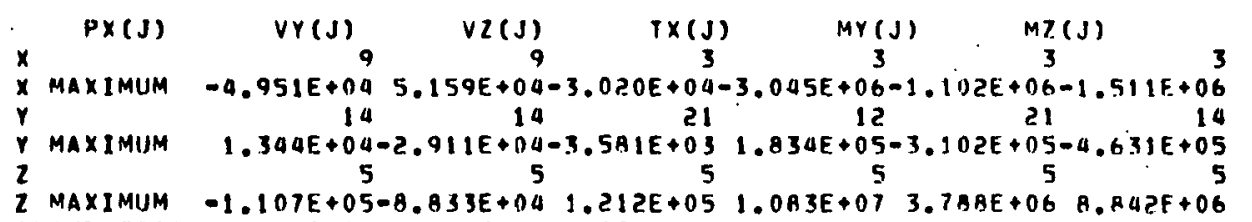
GRAND TOTAL $1.284 E+051.209 E+05,1.272 E+051.15 B E+070.094 E+069.185 E+06$

ELEMENT TYPE (3/0 P I PE 1,1, ELEMEMT NIJMBER 167$)$

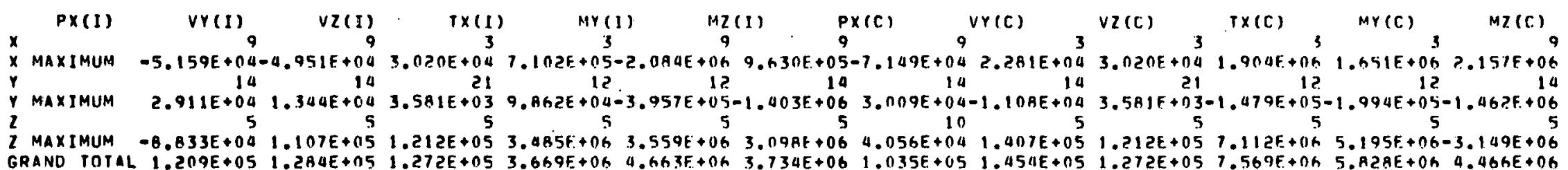

ELEMENT TYPE (3/D P I PE , , , ELEMENT VUMBER 1 67)

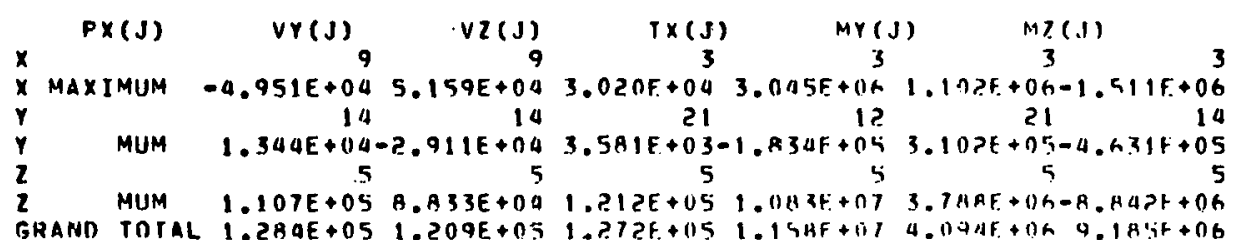




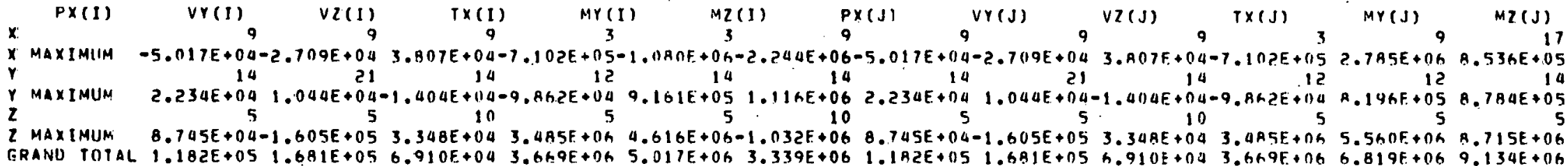
ELEMENT TYPE $(3 / D$ P I P E

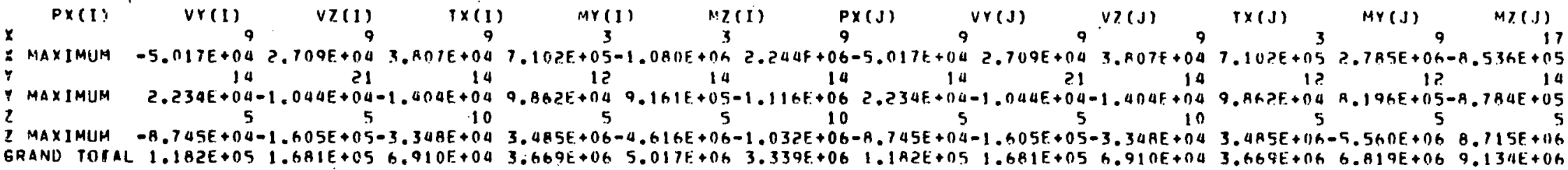
ELEMENT TYPE (3/D P I PE ),, 1 ELEMENT NIJMAER $(70)$

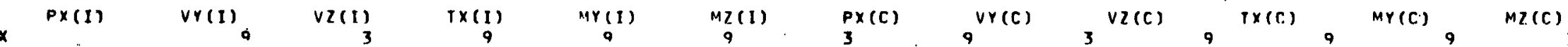

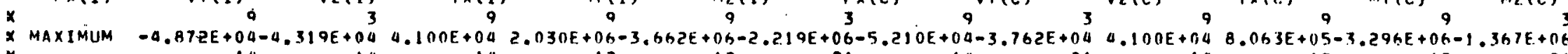

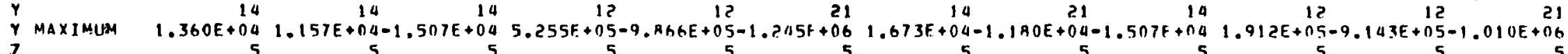
$\dot{N} 2$
$\sim$

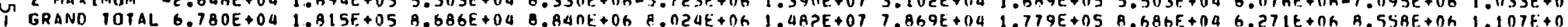

ELEMENT TYPE (3/D P I P E , , , FLEMENT NUMHER 1 T0)

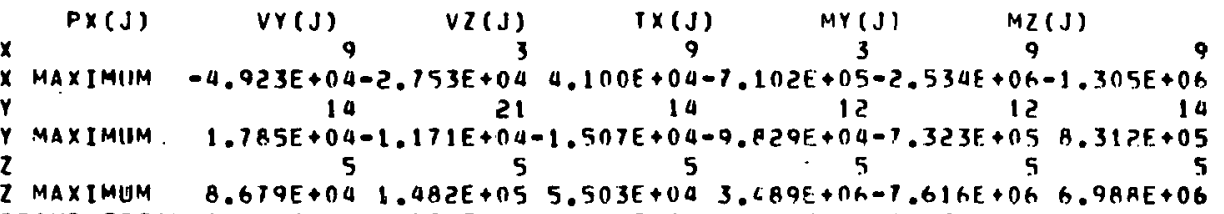

GRAND TOTAL $1.164 E+05 \quad 1.559 E+05$ Q.6BGE+04 $3.6 .12 E+06 \quad 8.430 F+06 \quad 7.689 E+06$

ELEMENT TYPE (3/0 P I PE ),, 1 ELEMENT NIJMHER $(11)$

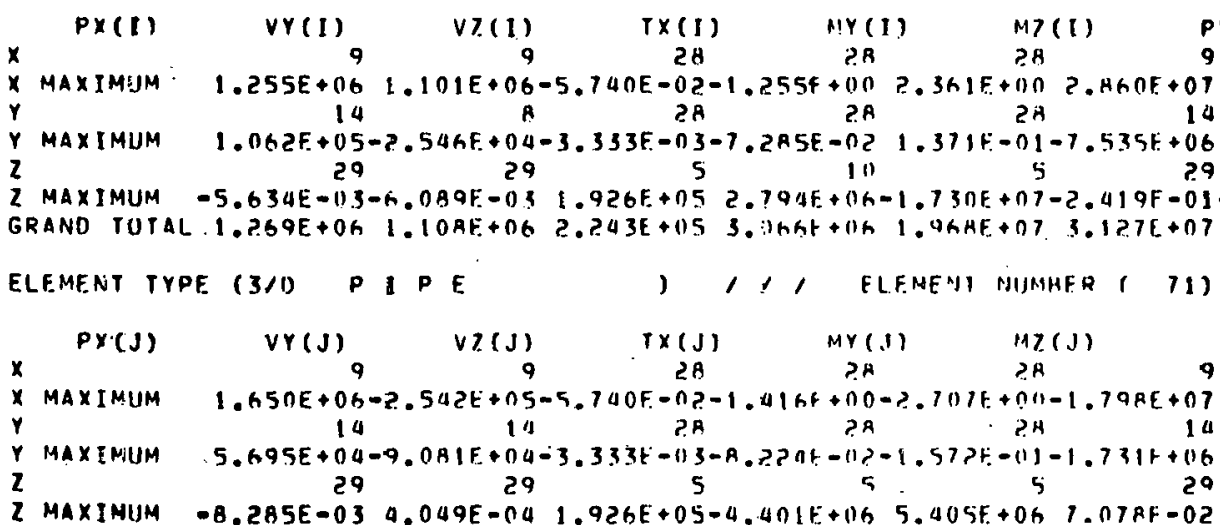


GRAND TOTAL $1.660 E+06 \quad 2.836 E+05 \quad 2.243 E+05 \quad 4.543 E+06 \quad 5.926 E+06 \quad 1.831 E+07$

ELEMENT TYPE (3/0 P I PE ,, 1 , ELEMENT NIMAER $(72)$

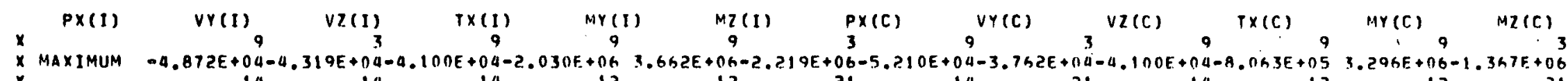

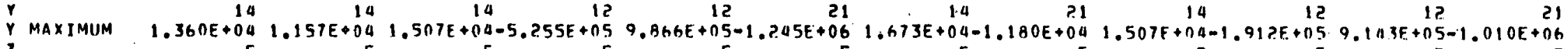

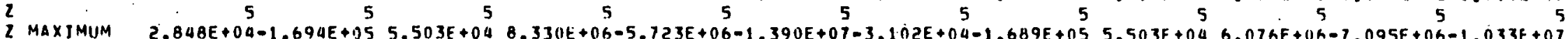

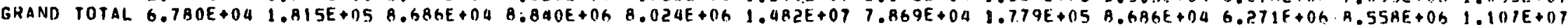

ELEMENT TYPE (3/D PI PE , 1,1 ELEMENT NIJMBER $(12)$

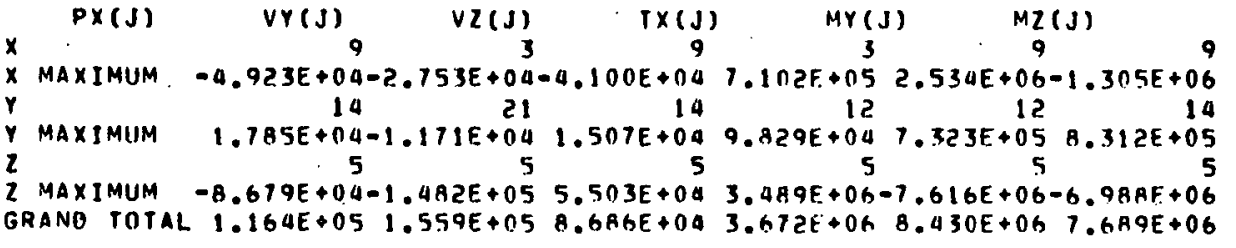

ELEMENT TYPE (3/0 P I PE 1,1, ELEMENT NUMRER ( 73).

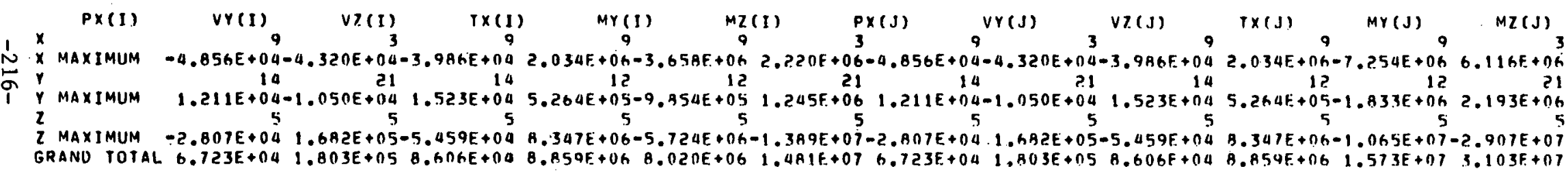
ELEMENT TYPE (3iס P I PE, , 1,1 element NUMHER 1 74)

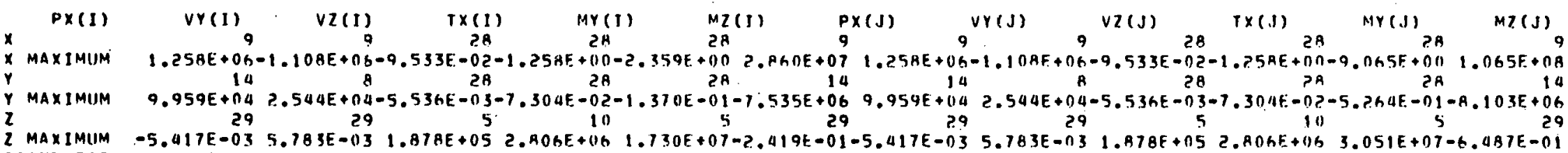
GRAND TOTAL $1.271 E+061.115 E+06$ 2.213E+05 3.0BSE+06 $1.96 A E+073.127 E+071.271 E+061.115 E+062.213 E+053.0 A S E+06 \quad 3.521 E+071.0 A 2 E+08$

ELEMENT TYPE (3/0 P 1 PE , , , ELEMENT NUMMER, 75)

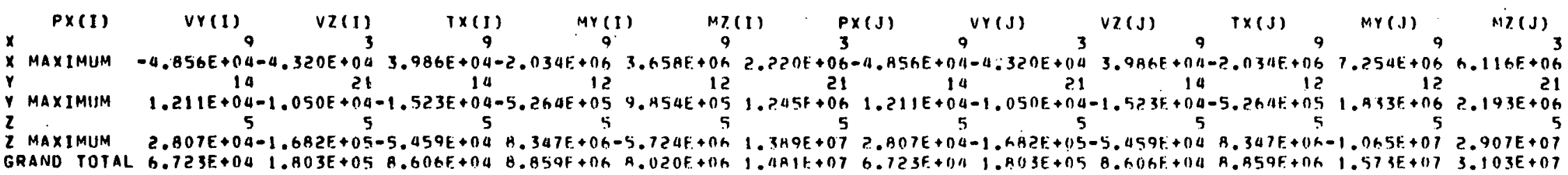
EL TYPE (3/0 P I PE 1,1, ELEMENT NIMHER 1 76)

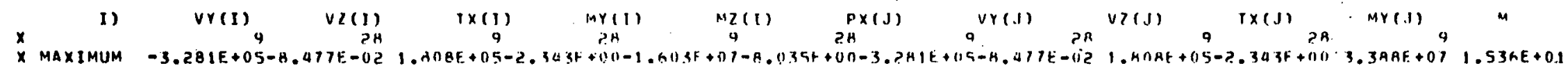




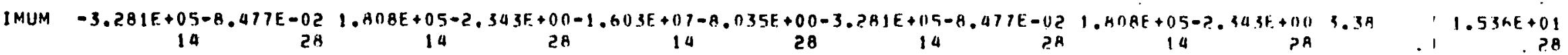

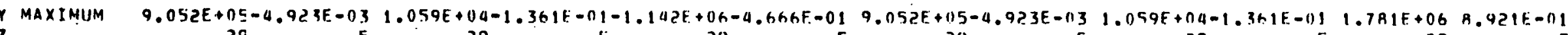

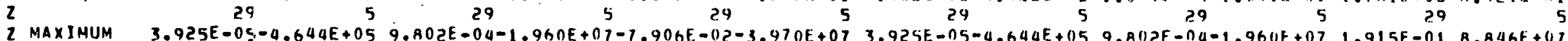

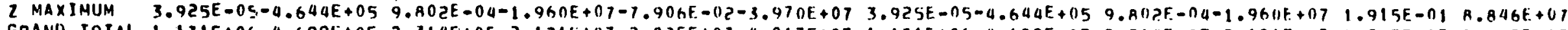

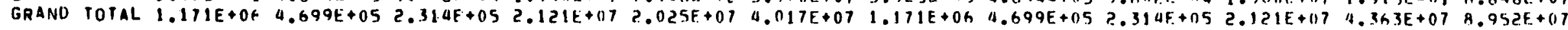
ELEMENT TYPE (3/0 P I P E , , , ELEMENT NUMHER 177$)$

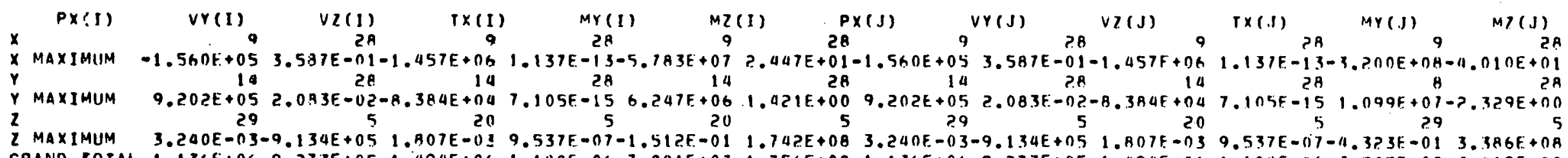

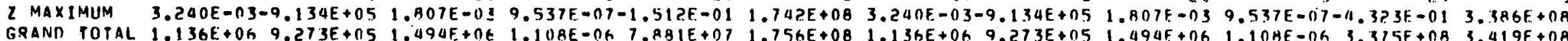
ELEMENT TYPE (3/0 P I P E , , , ELEMENT NUMHER 1 18)

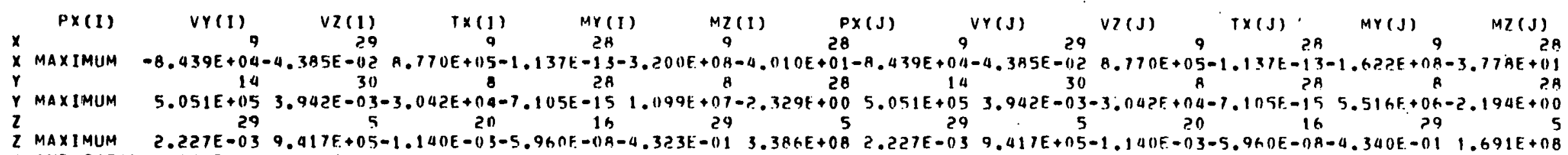

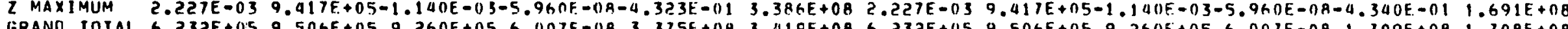

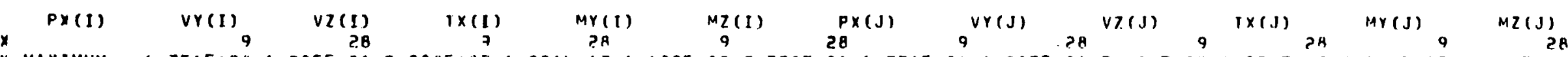

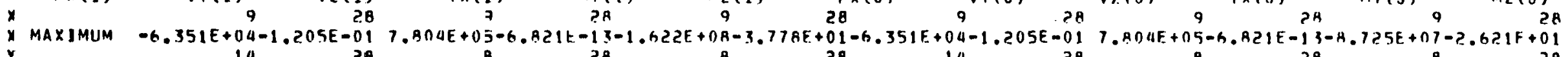

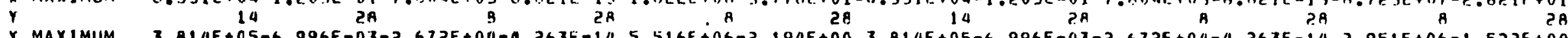

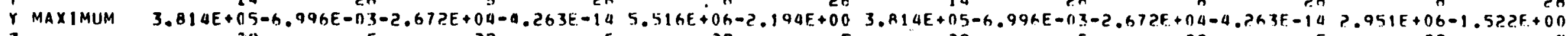

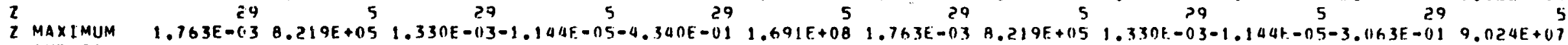

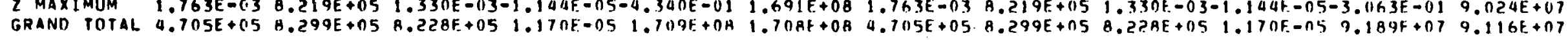
ELEMENT TYPE (3/0 P I PE $), 1$, ELEMENI NUAHER $($ RO)

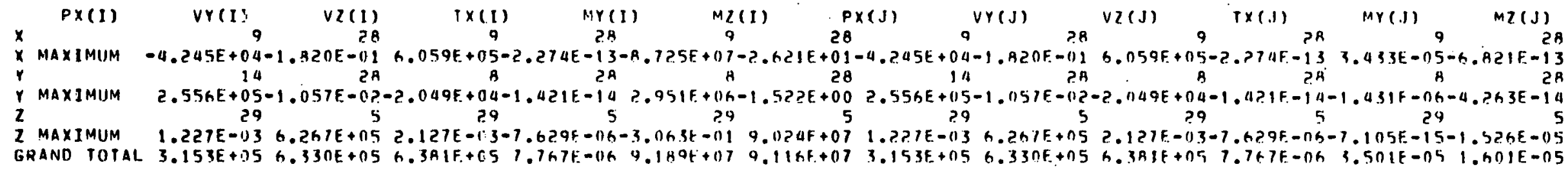



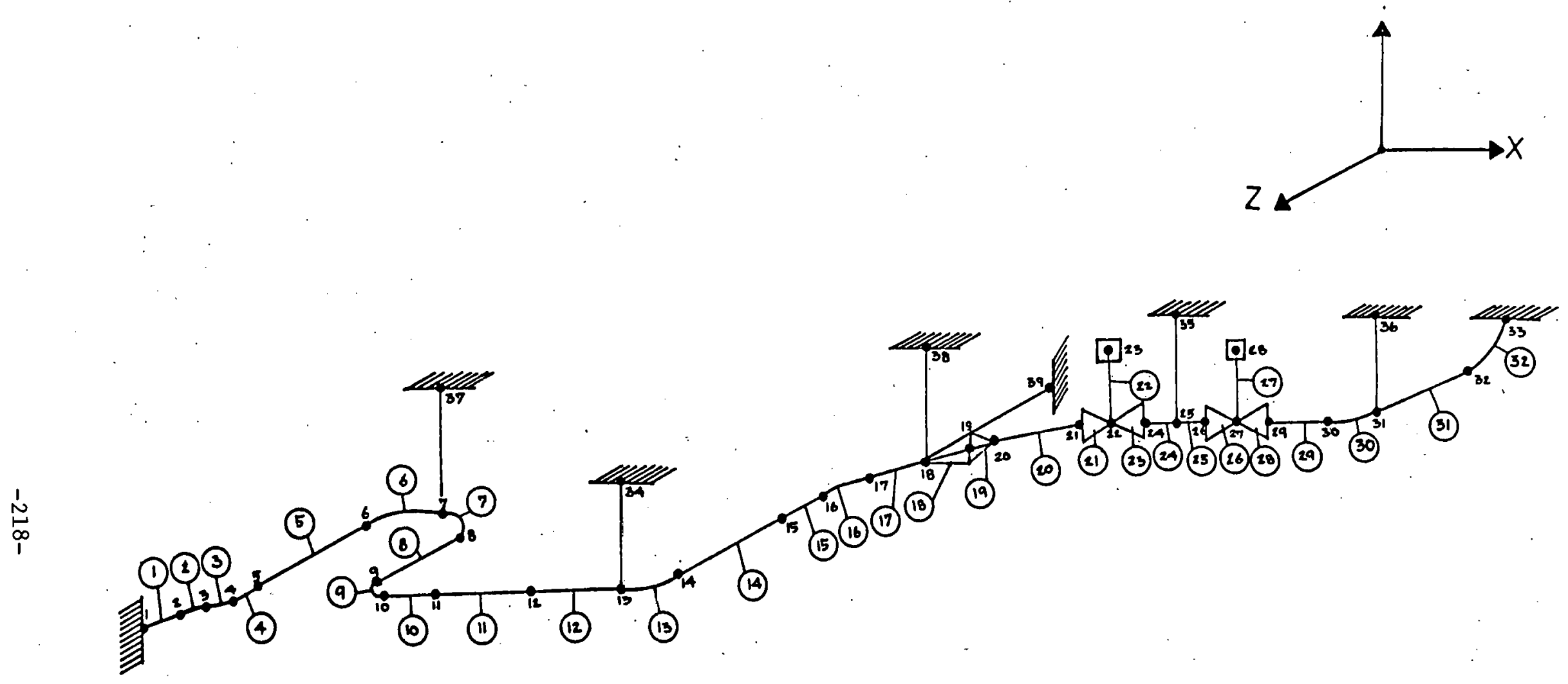

BENCHMARK PROBLEM NO. 5

Figure 5.1 
5000

1000

500

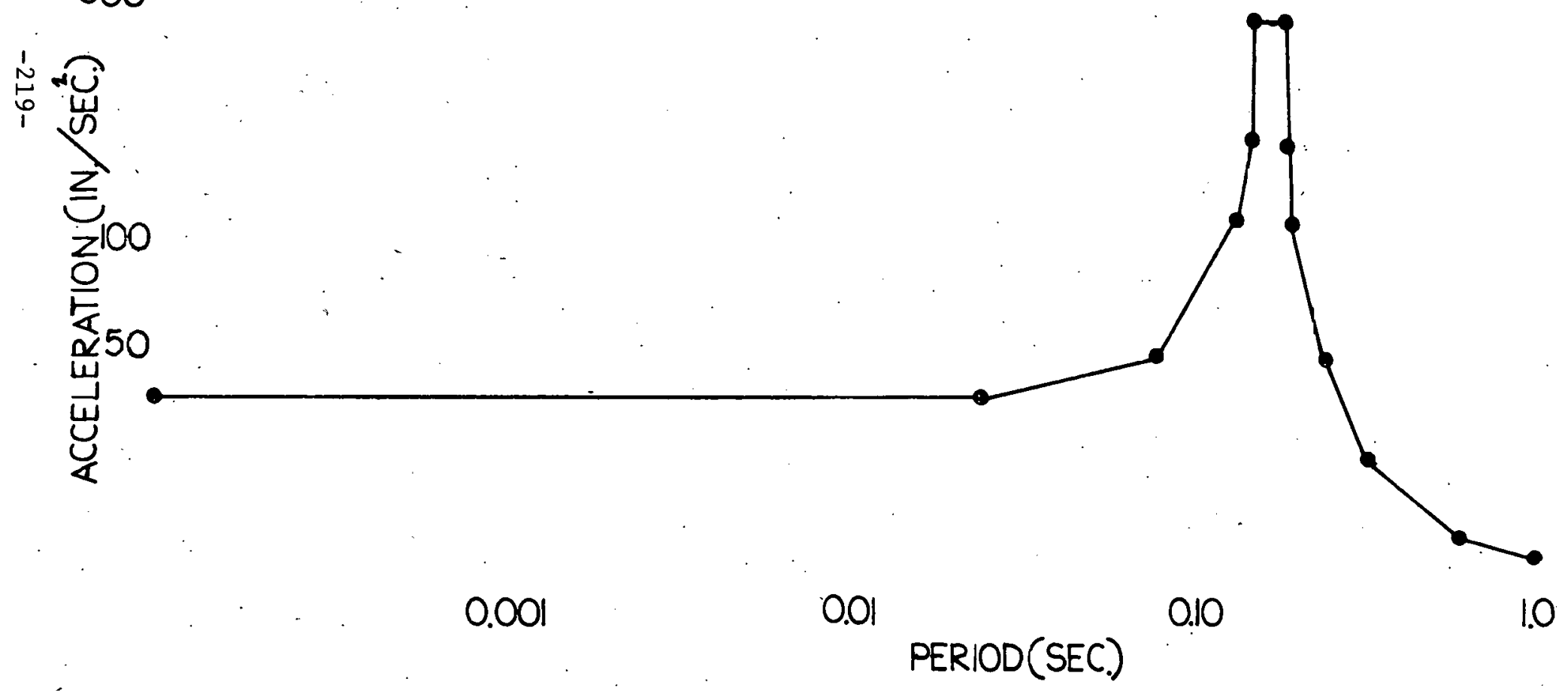

10.0

Figure 5.2 


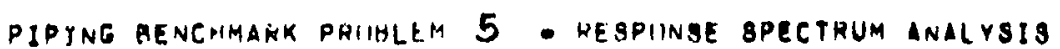

C I.I N T U U L INFUKMATIUN

NIIMBER UF N(IOAL PIIINTS

MIIMAER OF ELEMENT TYHFS a ?

NIIMEER OF LIJAL CASE

NIJMBER OF FHEWUENCIES

MNALYSIS CIIIE (NUYN)

E.0.0, STATIC

Q.1. MLIOAL EXTKACTIIIN

Q.2. FULCED HESPUINSE

Q.3, RESPIINSE SPECTRUM

D.G, UIRECT IVIIEGRATI!IN

SILUTIUN MUDE (MIIH)E $X$ )

EQ.n, EXECUTJ!JU

Q.1. DATA CHECK

ITERATIUN VECTURS (NAD) -

FOUATIUNS PER GLUCK

TAPEIO SAVE FLAG (NIOSV):

NIIIAL POIVT JHPUT DATA

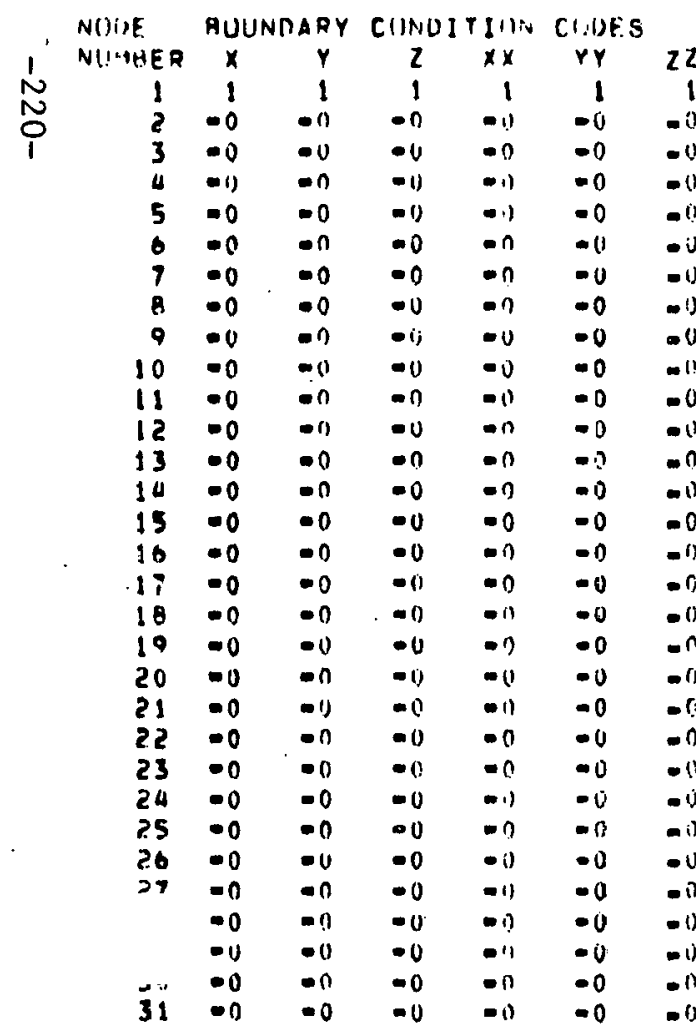

NTUAL PUINT CMURDINATES

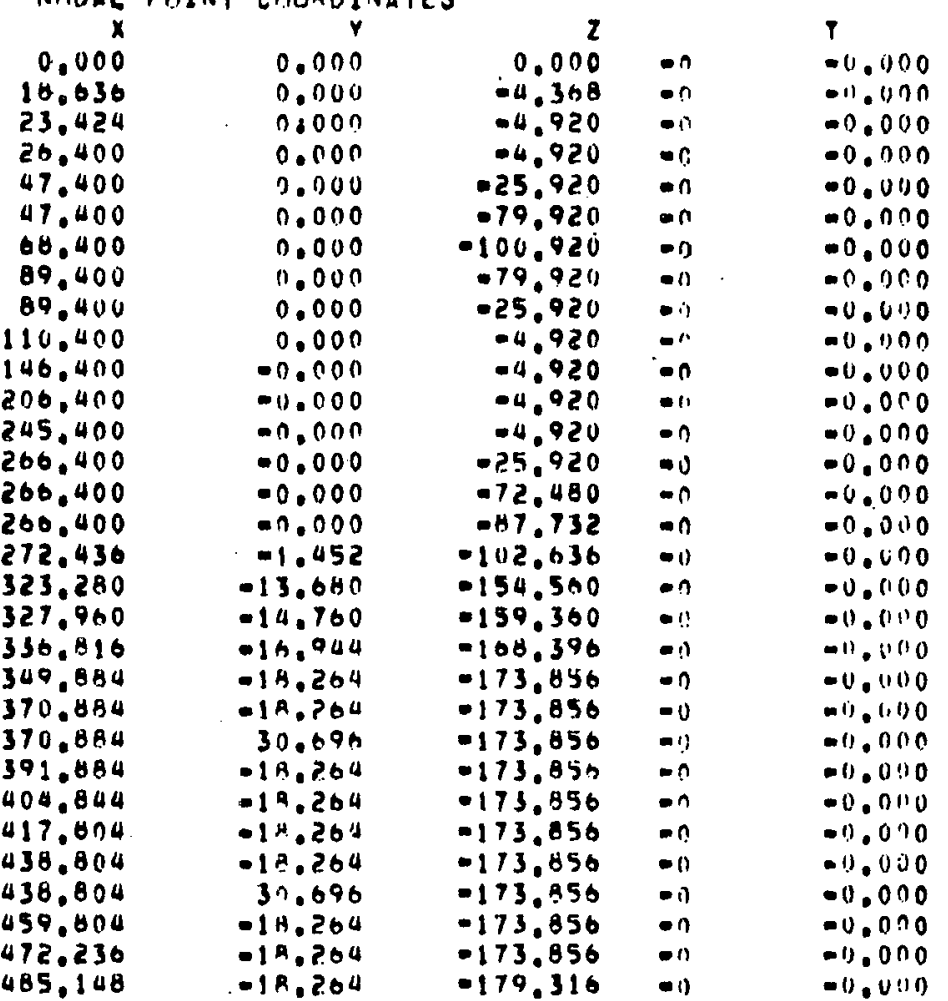


$\begin{array}{rr} & 0 \\ 35 & 1 \\ 30 & 1 \\ 37 & -1 \\ 30 & 1 \\ 39 & 1\end{array}$

507.300

519,840

249.400

$404,5.44$

485.148

63.400

-18.264
-.264
12.000
12.000
12.000
12.000
12.000
-13.080

$-202.128$

.215 .040
-4.920

$\rightarrow n$

$-0.0110$

173.656 -n $\quad-0.1110$

179.376 on $\quad=0.0110$

-100.920 on $\quad-0.000$

-154.560 on

$\begin{array}{lll}-166.960 & -0 & -0.000\end{array}$

GEHERATEU NIJUAL DATA

\begin{tabular}{|c|c|c|c|c|c|c|}
\hline$N(M 1) E$ & But & DAHY & corse & אוחנז & CIILES & \\
\hline NUMAER & $x$ & Y & 2 & $x x$ & Yy & \\
\hline 1 & $\hat{i}$ & 1 & $i$ & 1 & 1 & \\
\hline 2 & $=0$ & -0 & -0 & -0 & -0 & \\
\hline 3 & $=0$ & -0 & -0 & -0 & -0 & \\
\hline 4 & $=0$ & -0 & -0 & -0 & -0 & \\
\hline 5 & -0 & $-n$ & -0 & $\bullet 0$ & -0 & \\
\hline 6 & -0 & -0 & -11 & $-r$ & -0 & \\
\hline 7 & .0 & -0 & -11 & 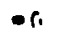 & - 1) & \\
\hline A & -0 & $-n$ & -11 & $=0$ & $=0$ & \\
\hline 9 & $=0$ & -0 & -0 & -0 & -0 & \\
\hline 10 & -0 & -0 & $=0$ & -10 & -0 & \\
\hline 11 & -0 & -0 & -0 & $=n$ & -0 & \\
\hline 12 & -0 & -1 & -0 & $=0$ & -0 & \\
\hline 13 & $=0$ & $-n$ & -0 & -0 & -0 & \\
\hline 14 & -0 & $-1)$ & $-u$ & -0 & -0 & \\
\hline 15 & -0 & -11 & -0 & -.0 & $\bullet 0$ & \\
\hline 16 & -0 & $-n$ & -0 & -1 & -0 & \\
\hline 17 & -0 & -0 & -0 & -0 & -0 & \\
\hline ia & $=0$ & $=10$ & -0 & $=0$ & -0 & \\
\hline 19 & .0 & $-1)$ & -0 & $-1)$ & -0 & \\
\hline 20 & $=0$ & -0 & -0 & -0 & -0 & \\
\hline 21 & $-a$ & -0 & -0 & -0 & .0 & \\
\hline 22 & -0 & -11 & -0 & -0 & -0 & \\
\hline 23 & -0 & -0 & .0 & -11 & -0 & \\
\hline 24 & $\bullet 0$ & -0 & -0 & $-n$ & -0 & \\
\hline 25 & $\bullet \mathfrak{l}$ & $=n$ & -0 & $-n$ & -0 & \\
\hline 26 & $-c$ & -0 & $-n$ & - & -0 & \\
\hline 27 & $-c$ & -11 & -0 & -0 & -0 & \\
\hline $2 A$ & $-c$ & -11 & -1 & -0 & -0 & \\
\hline 20 & -0 & -0 & -0 & $=0$ & -0 & \\
\hline 30 & -0 & -9 & -0 & $-1)$ & -0 & \\
\hline 31 & $\omega$ & .0 & -0 & -0 & -0 & \\
\hline 32 & $\bullet$ & $=0$ & $=0$ & $=0$ & -0 & \\
\hline 33 & 1 & 1 & 1 & 1 & 1 & \\
\hline 34 & 1 & 1 & 1 & 1 & 1 & \\
\hline $35^{\circ}$ & I & 1 & 1 & 1 & $\mathfrak{l}$ & \\
\hline $3 n$ & I & 1 & 1 & 1 & 1 & \\
\hline 37 & I & 1 & 1 & 1 & 1 & \\
\hline $3 A$ & 1 & 1 & 1 & 1 & 1 & \\
\hline 39 & 1 & $!$ & 1 & 1 & 1 & \\
\hline
\end{tabular}

NUDAL PIINT CIIUROINATES

\begin{tabular}{|c|c|c|c|}
\hline 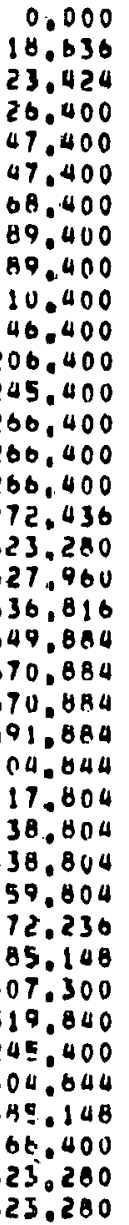 & 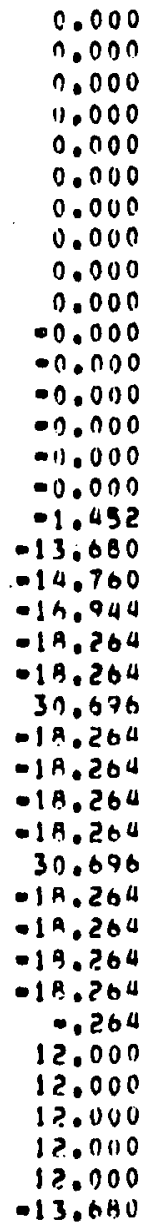 & $\begin{array}{r}0.000 \\
-4.368 \\
-4.920 \\
-4.920 \\
-25.920 \\
-79.920 \\
-100.920 \\
-79.920 \\
-175.920 \\
-4.920 \\
-4.920 \\
-4.920 \\
-4.920 \\
-25.920 \\
-12.440 \\
-17.732 \\
-102.636 \\
-154.960 \\
-159.360 \\
-168.396 \\
-173.856 \\
-173.856 \\
-173.856 \\
-173.456 \\
-173.856 \\
-173.856 \\
-173.856 \\
-173.856 \\
-173.856 \\
-173.856 \\
-179.316 \\
-2112.126 \\
-215.0110 \\
-4.920 \\
173.830 \\
179.316 \\
-100.920 \\
-154.560 \\
-106.560\end{array}$ & 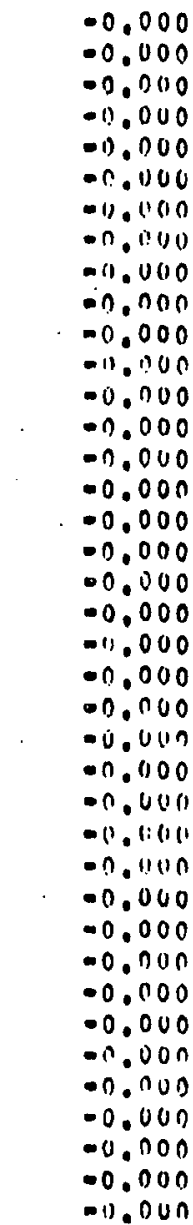 \\
\hline
\end{tabular}


PIPE ELEMENTIINPUTTUATA

CUNTAUL INFIKMATIUN

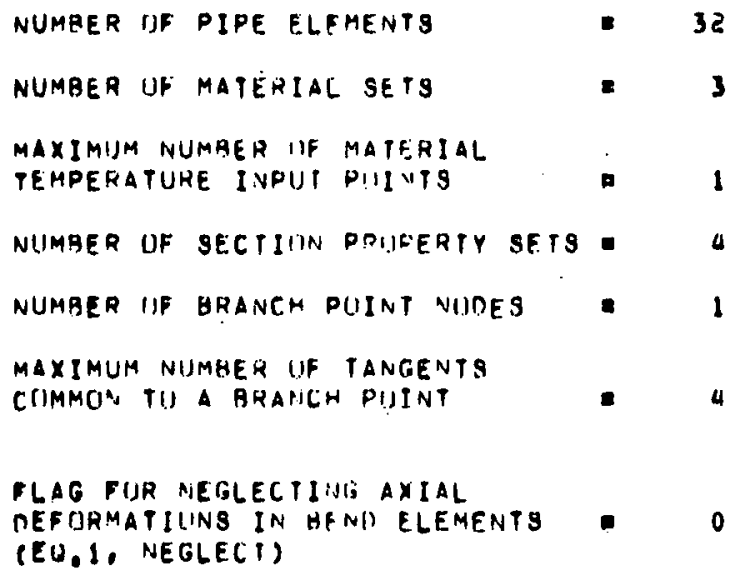

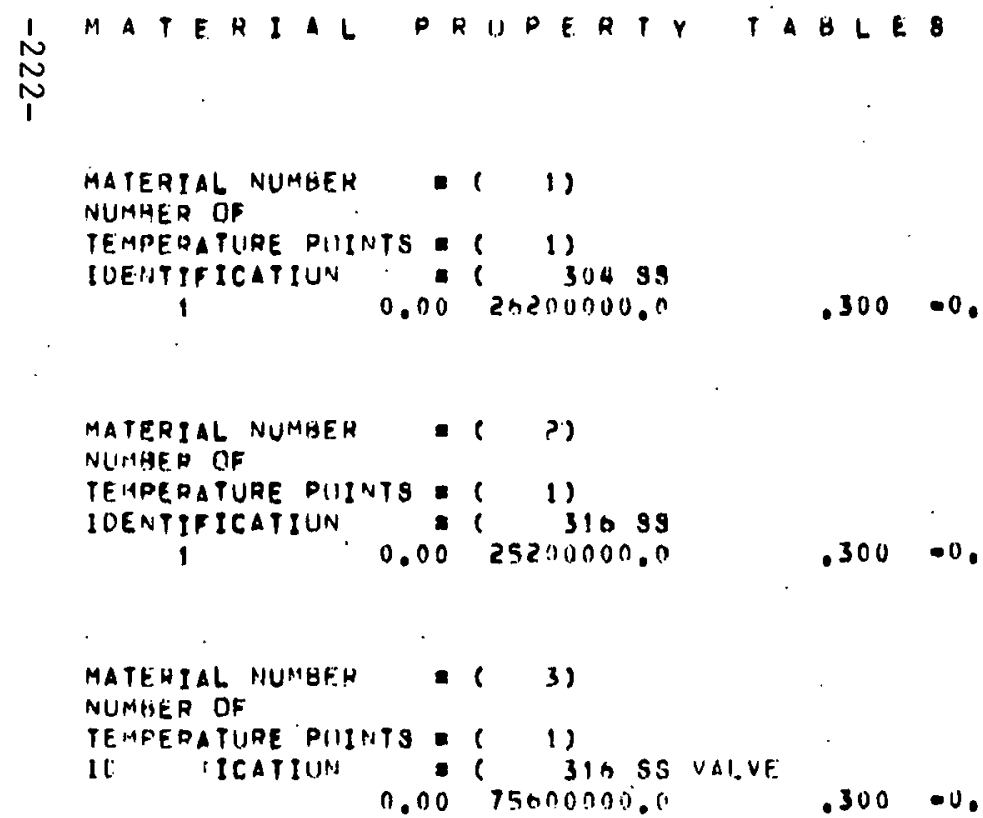




.4381
.3750
1.3120
2.01100

$-0.0000$

$-0,0000$

-0.
-0.

2.01100

$-0.0000$

0.
0.
0.

IA IN. PIPE
IS IN. PIPE
I2 IN. PIPE
VALVE OTEM

E LE M N T

L If A L

C $\triangle S F$

M U L T I PLIERS

x-DIRECTIONA GHAVITY

YODIRECTIUN GRAVITY

CAgE CABE H CABE C

$1.0100 \quad 1.000 \quad 1.000$

11000 1.000

1 inon 1.000

ZOOIRECTION GRAVITY

$1.00 n$

$\$ .020$

THERMAL DISTLIATIIIN

1.1100

1.020

1.000

1.00 n

1.000

CASE U

1.000

1.000

1.000

PRE SBURE DISTURTIIIN

1.000

1.000

1.000
1.000 
PI PE ELEMENTIIVPUT UATA

ELEMENT ELEMENT NIJUE NIIDE MATL. SECTION

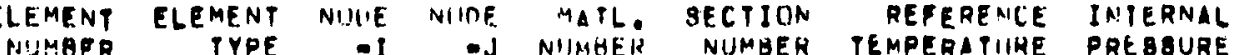

REPERENCE TNIIRE PRE 8BURE

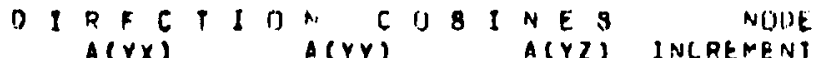

INPUT

(BENO (THIHD

$A(r x)$

A(YZ) INCREMENI

IAG

RADIUS) PUINTI

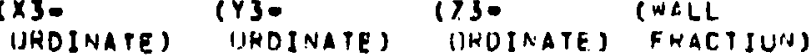

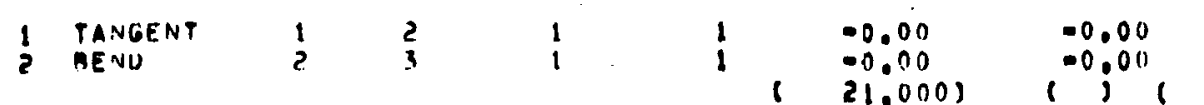

$-0.0000$

$-0.0 .005$

$-0.0000 \quad 1$

11

3 TANGENT

$\begin{array}{ll}1 & 00.00 \\ 1 \quad 0.00\end{array}$

$i^{-0,00} 1$

21. 0 (1) 1

$0.00 n)($

$-4.920)(.1000)$

0.00
0.000

0.0000

0.11000

$.0 .0000 \quad 1$

$47.400)($

0.10016

$-4.920)(.1000)$

5 TANGENT

$i \quad 0.00$

.0 .00

$-0.0000$

$-0.0000$

$-0.0001$

1

BENU

$(21.0003$

$i^{-0.00}$

$47.400)($

$0.000)(-100.020)(.1000)$

$1, \quad 0.0000$

$i^{-0,00} 1$

A9.400)

$0.000)(-100.92(1)(.1001)$

$1 \quad 0.000$

$-0.00$

TANGENT

0.000
$\quad 21.000)$

$i^{-0,00} \mathrm{c}$

$-0.0000$

0.00001

Q9.4no)

$0.000)($

$-0.020)(.1000)$

\section{$-0.00$}

0,00

.0 .00

$\begin{array}{lll}\text { TANGENT } & 10 & 11 \\ \text { TANGENT } & 11 & 12 \\ \text { TANGENT } & 12 & 13\end{array}$

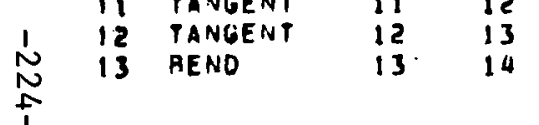

-0.00
0.000

$=0.00$

$-0.0000$

$=0.0000$

0.0000

0.0000

$-0.0000$

$-0.000 n$

$.0,00$

$260.400)($

0.00016

$-4.9202$

1

0.00

0.00000

0.00000

$.1000 s$

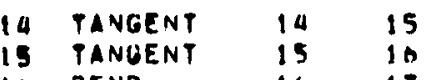

16 REND

1

-0.00
-0.00

.0 .00

$-0.0000$

$-0.0001$

-0.0000
.0 .0000

1

$266.400)($

0.00011

$.96 .480)(.100119$

$\begin{array}{lll}\text { TANGENT } & 17 & 18 \\ \text { TANGENT } & 18 & 19 \\ \text { TANGENT } & 19 & 20 \\ \text { REND } & 20 & 21\end{array}$

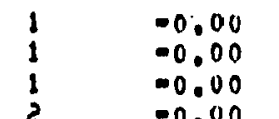

0.000

$-0.0000$

$-0.0000$

$-0.0000$

-0, nona

.0 .0000

.0 .0000

1

$(18.000)$

$-0.6000$

$-0.0000$

$-173.856)$

.1000

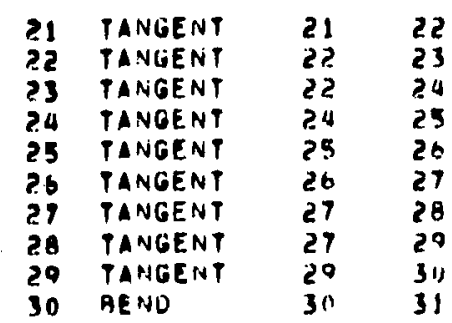

3
4
3
3
3
3
4
3
3
3

$-0.00$

$=0.00$

$-0.00$

-0.000 ir

$-18.2643$

.0 .0000

$\begin{array}{ll}0.0000 & 00.0000 \\ 0.00011 & =0.0001\end{array}$

$-11.00013 \quad-0.01100$

0.0 .0000

$-0.091) n$

$-0 \operatorname{son} 00$

$=0.00 n \pi$

$-0.0 n 00$

- 0.017$) 0$

$-0,0100$
$-0.0 n 00$

$-11.0003$

-0.011001
0.0000

$.0,0000$

.0 .0000

$=0.0000$

-

$-6.11000$

$-0.0000$

.0 .00

$-0.00$

$-0.00$

$-6,110011$

$-0.0000$

.0 .010011

1
1
1
1
1
1
1

$\begin{array}{llll}\text { TANGENT } & 31 & 32 & 2 \\ \text { RENO } & 32 & 33 & 2\end{array}$

-0.00
18.0005

$(; 06$

$490.814)($

$-18.2643(-173.856)(.1000)$

II

IC

I!

$1 \mathrm{I}$

11
1

ic

$=0.00 \quad-0.00$

- o. nnno

$-0.000 n$

$0.0000 \quad 1$

( 18.000$)$

519.84016

$-18.24(4)(-215.04(1), \quad .100(i)$ 


\section{EL TYPE TELENENTS:}

ELEMENT GUAD CASE MULTIOLIERS

$\begin{array}{cccc}\text { CASECA) } & \text { CASECA) } & \text { CASECC: } & \text { CASECO) } \\ 1.0000 & -O .0000 & -0.000 O & -0.0000\end{array}$

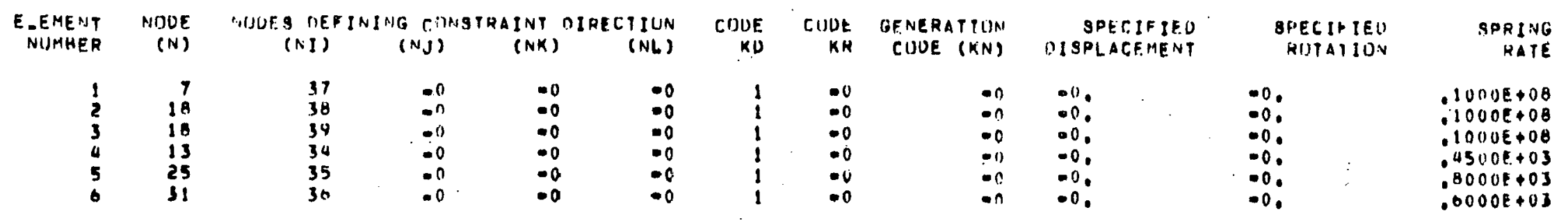

N 
NUDAL LUADS (STAIIC) URMASSES (DYNAMIC)

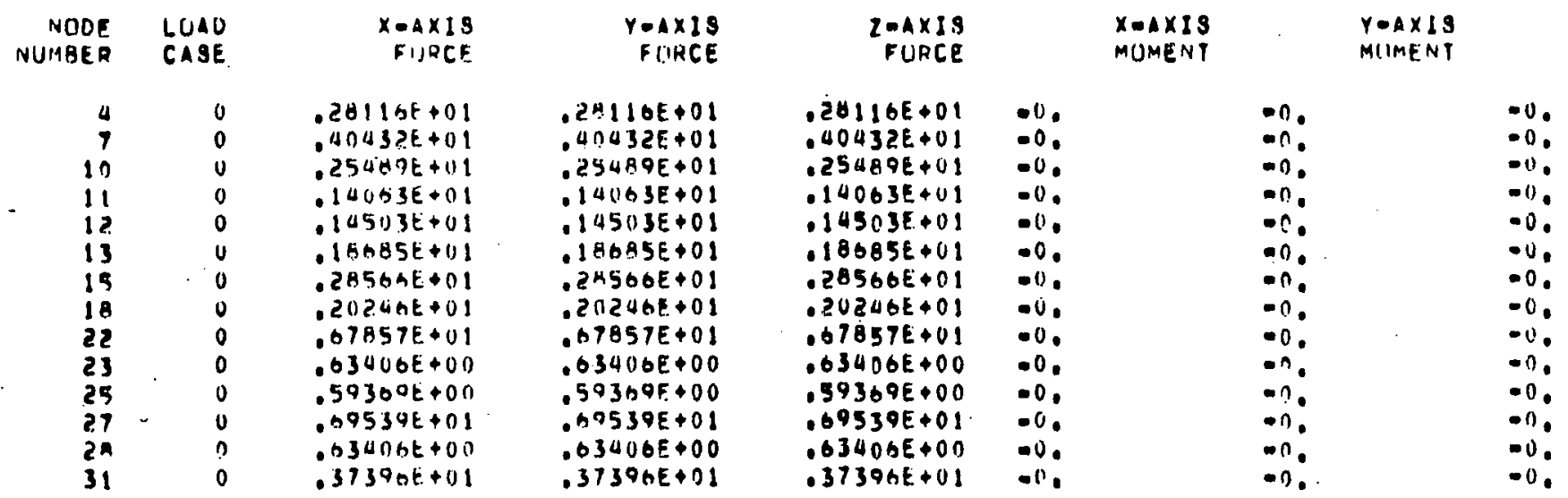

STQUCTURE

LIIAII CASE

1

ELEMENT LUAN MULIIPLIERS

$0.000 \quad-0.000 \quad-0.000 \quad-0.000$ 
PFINT OF FREUUENCIES

\begin{tabular}{|c|c|c|c|}
\hline 1 & $.2536 E+02$ & . $\triangle \cap 3 \cap E+01$ & $.2478 E+00$ \\
\hline 2 & $.2075 E+02$ & $.4251 E+01$ & $.2340 E+00$ \\
\hline 3 & $.572 B E+02$ & $.0116 E+01$ & $.1007 E+0.0$ \\
\hline 4 & $.7029 E \leftrightarrow 112$ & $.1119 E \bullet 02$ & $.0938 E=01$ \\
\hline$\dot{5}$ & $.1075 t+03$ & $.1711 E+02$ & $.5846 E=01$ \\
\hline$b$ & $.1142 E+03$ & $.1 A 17 E+1) 2$ & $.5503 E=01$ \\
\hline 7 & $.140 .0 E \div 03$ & . 2?3AE+02 & $.44 O B E=01$ \\
\hline 8 & $.1709 E \$ 03$ & $.2719 E+02$ & $.3677 E \cdot 01$ \\
\hline 9 & $.1760 E+03$ & $.2801 E+02$ & $.3570 E=C 1$ \\
\hline 10 & $.2307 E+U S$ & $.379 A E \nrightarrow 02$ & $.2633 E=01$ \\
\hline 11 & $.2575 E+03$ & $.419 G 7 E+02$ & $.2441 E=01$ \\
\hline
\end{tabular}


MODE NUMBER 1

PREQUENCY 4.03554 HZ

EIGENVECTURS NUIHMALIZE" TU A UHIT MASS MATRIX

UISPLACEME:17 S/RUTATIURS OPF UNRESTHAINEO NUDES

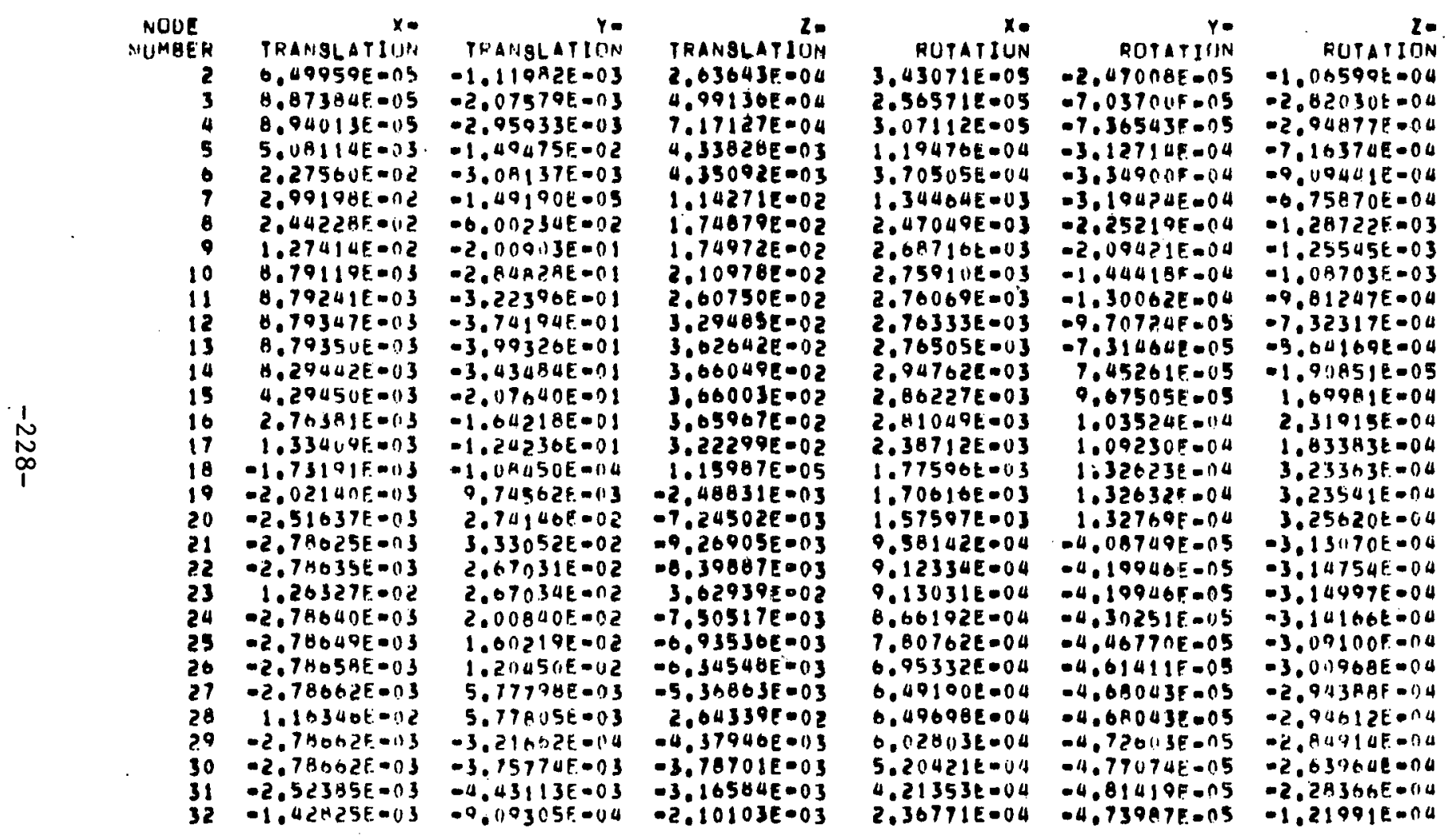


MTIDE NUMHEH

FIGE JVECTIJHS NIJEMALILEN TII 1, DUO

DISPLACEMEPTSIRUTATIIINS OF UNHESTRAINED NUDES

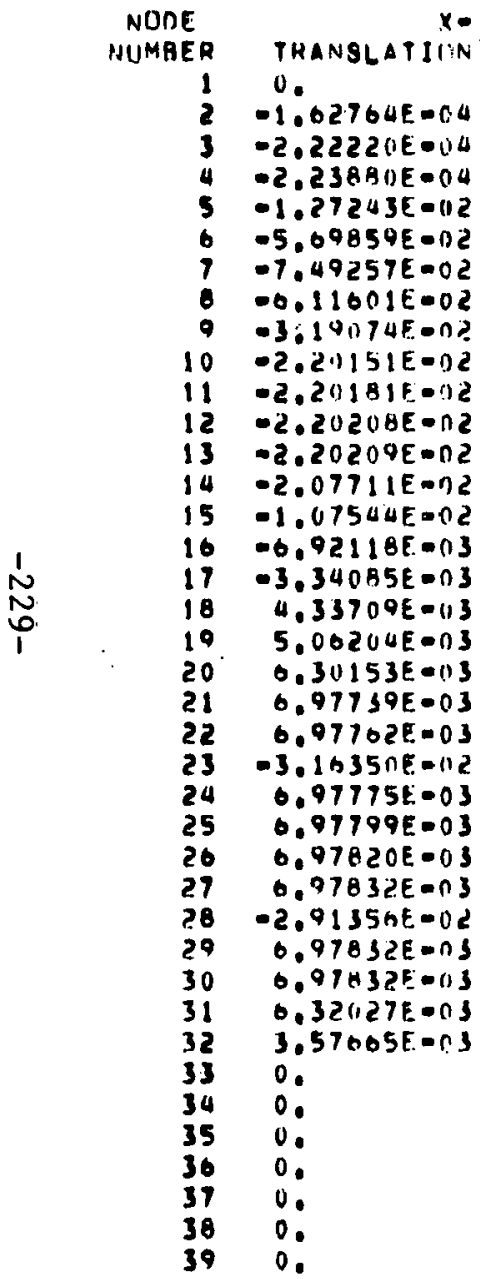

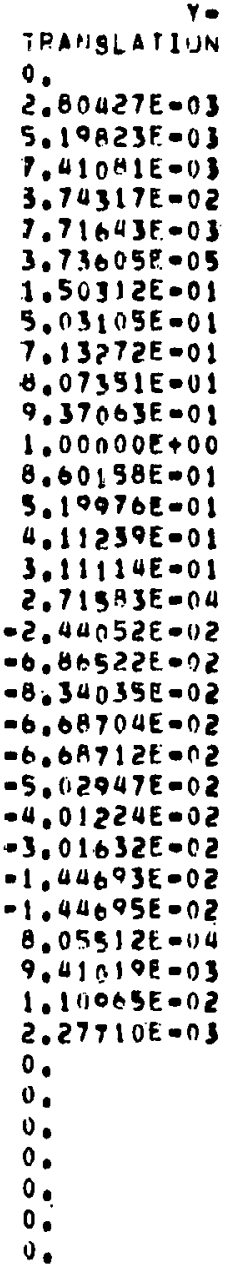

TRANSLATIIIN 0.

$-0.00221 E \oplus 04$

$1.79584 E-03 \quad 0.42509 E-05$

$-1.080408=02$ - 2099194000

- 1 00050E-0

- $1.089562002-9.272$ 2SE-0

$-4.37935 E-112 \quad-0.18665 t-03$

- 4.301098002

$-4.3 B 109 \mathrm{E}-02$

$-5.28335 E-02$

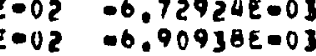

- 5297 deloz $\quad 0.013368003$

$-6.25102 E-02 \quad-6.91999 E-03$

$-9.08134 E-02 \quad-6.92430 E-03$

$x=05$

$-9.16553 \mathrm{E}=02$

$-9,16463 E=0$ ?

$-8,07106 E-02$
$-2,90457 E 003$

$-2,90457 E 005$
0,231205003

$0.23126 E 003$

1.014318002
3.321178002

0.03 a $=02$

$-0.04880 E-0 z$

$1.89940 E-02$

$1.73677 E=02$

$1.58905 E-02$

$1.38905 E=02$

$-6.61902 E-02$

$1.09671 \mathrm{E} 00$ ?

9. $483512=03$

$.92795 E-03$

6.

6.

0.

0

0.

$-7.16774 E-03$

$-5.977806=03$

0.447396003

0.272618003

$2.399408-03$

$-2.284698003$

$-2.28643 E-13$

$-2.16913 E-03$

$-1.95520 E-03$

$-1.74260003$

$1.62699 \mathrm{E}-03$

$-1.50955 t-03$

$1.303258 \cdot 03$

$-1.05916 E-03$

$0.92920 E=0$

0 .

0.

0.

0.

0.
- aOtation

0 .

$6.15503 E-05$

$1.762225-04$

1.844078 .04

$7.831045-04$

6.3BGAUE-nL

$7.90909 E-04$

$5.63928 \mathrm{~F}-04$

$3.240368-14$

3.616548 .04

$3.25705 E=04$

$2.43000 F=04$

$1.831751-04$

$-1.86630 \mathrm{~T} .04$

$-2.422 A U E-04$

$-7.03807 E-0$

$-1.629712003$

2.592478 .04

$2.73530 E=04$

3.3211 OE $=04$

$-3.321395-00$

$-3.324 \mathrm{E} 2 \mathrm{COO}$

$1.02360 E-04$

$1.05164 E-04$

1.051648 .04

$1.07144 E-04$

1.118 1E.04

1.195475000

1.172085 .00

$1.172085-04$

1.1 A 35 OF 0

1.1947 UE -00

$1.18607 \% 00$

0.

0.

0 .

0.

0.

0.

$7.00265 E-114$

T.34436E-04

$1.70396 E=03$

$2.27744 E=03$

1.20

$1.69253 E 0$

列

$3.103 ? 10003$

$2.72217 E-03$

$2.45726 E-03$

$1.6338 B E-03$

$1.412 B 0 E-03$

$4.77933 E-05$

$-4.25660 E-04$

- 592315004

4.5931 .004

$0.09792 E-1) 4$

- $.10218 E-04$

$0.15425 E-04$

$83996 E=04$

TBRB?

7 BnTUIE-OA

? TUOSJEDOU

E 04

IECOU

7.3777 CE-114

7.134 HEOAC

$5.07078+00$

$3.05495 t-104$

0 .

0 .

0.

0.

0. 
MODE NUMRER

PREQUENCY 4.25689 HZ

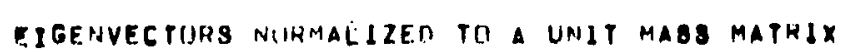

DISPLACEAEWTSIPUTATIUNS OF UNRESTRAINED NUNES

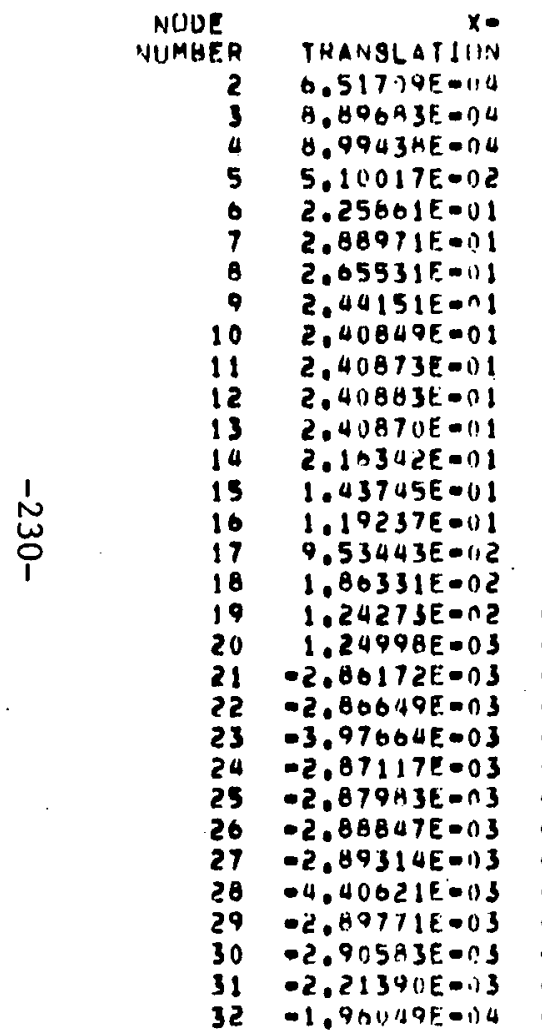

TRANGLATION TRANSLATION

2.10087E-0

$3.112577 E-04$

$1.52255 E-03$

$1.05093 E=04$

$5.07214 E-00$

b. $20805 E-03$

$1.98196 E=02$

$1.98196 E=02$
$3.13117 E=0$.

$3,02066 E-05$
3.

$3.02066 E=02$
$3.34805 E-02$

3. 348OSE-O2
3. $41270 E=02$

$2.80 \triangle \cap 4 E=02$

2. ORI3UE 02

$1.63246 E-02$

$1.51041 E-02$

1.51941E-02

$1.12 A 01 E=0$
$-1.25002 E=03$

$-1.25062 E=03$
$-3.53232 E=03$

- $4.72 b 55 E-03$

$-4.27 b 20 E-03$

$-4.27631 E-03$

$-3.77663 E=03$

$-3.43280 \mathrm{E}-03$

$-3.03434 E=03$

$-2.41220 E-03$

$-2.41232 \mathrm{E}-03$

- 1.75 A $4 E-03$

$-1.37137 E-03$

$-1.02898 k=03$
2.54743E-03

$4.05628 E=03$
$7.00127 E=03$

$4.32286 E=02$

$4.33246 E 002$

1.002422001

$1.33570 E-09$

$1.33654 \mathrm{E}=0$

$1.34931 E=01$
$1.30029 E=01$

$1.30029 E=0 !$
$1.10155 E=01$

$9.95700 E=02$

$6.05441 E=0$

$0.08392 E-O$

$8.08324 E-02$

$7.15422 E-02$

$8.26100 E-05$

$-5.064558-01$

$-1.00300 E=0$

$-2.22774 E-02$

$-2.00940 E-02$

$-2.25505 E-02$

- 1.75241E-02

$-1.35073 E-02$

$-1.00564 E-0$

$-1.00504 E=0$

$-1.13551 E-02$

$-0.50132 E-03$
$-4.44882 E=03$

$-2,006562.03$

$=0,00429 E=00$

\section{RUTATIUN}

$3.05968 E-06$
$.28847 E-00$

$-3.86655 E=06$

$-1.418070-05$

$-3.97760 E-05$

$-1.34682 \mathrm{~B}=04$

$-2.40975 E-U A$

$-2.548912004$

$-2.192768004$

$-2.00265 E=n 4$

- I. Busaienó

$-1.70487 E 004$

$-1.82200 E=0 A$

$-1.65753 E=04$

$-7.265405=0$

$.6 .71665 k-05$

0.716656005

-5.5aryie.0s

$.5 .03048 E-05$

$-4.57068 E-05$

$-3.80862 E=05$

$-3.04050 E-05$

$-2.63495 E-05$

$-2.05922 k-1) 5$

$-2.21164 E-115$

$-1.45997 E-05$

$-5.35791 E-U 6$
$9.64270 E=00$ botarion 2. 419 QDE-OL $6.91950 E=04$ $6.949078=04$
$.7 .277265-04$ $-7.277205-04$ $-3.12012 E=03$ $-3.25290 \mathrm{~F}-0 \mathrm{~s}$ $2.10052 F-03$ $.5 .23421 F=04$ $-3.197346=114$ 7.00878P-05 $1.56793 \mathrm{E}=04$ $3.507445-04$ $5.0327 B R=04$ $1.4988 G=03$

$1.59391)=0.03$

$1.00100 F=03$

$1.52539 E-03$

$1.5040=0$

$1.22479 F-03$

$1.147505=03$ $-9.23898 F=05$ $-1.130408004$ $-1.130405 .04$ $-1.28743 E 004$ $-1.498028000$ $-1.647715-00$ 1.685 45.-0 1

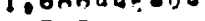
- 1.55703 Fona $-1.11943 \mathrm{f}=114$ -6.700045 - 5

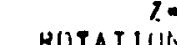
1.119435E-05 $2.91755 E-115$ $3.05405 t=115$ 7.4B527E-115 9.0067 GE-0S 7.777ARE-US $1.37753 E-1) 4$ $1.32706 E 004$ - ThUbaE-OS - 7n8baEang 1.22969t-09 3.211H7E-05 $5.69616 E-07$ $-1.5362 A E=04$ $-1.69306 E=114$ - $1.74966 E-114$ - I. 95004E=na - 950 ena 2.000 द. 00 तुbe-0s $2.2612 \Delta E-05$ $2.20974 E-1) 5$ 2. 4667 at -115 $2.76515 E-115$ 3.01107OE-OS $3.04354 E-115$ . UB - O टDEO 3. INCA GE-05 3. $045130 E-05$ $2.94263 E-115$ 2.073? $18=05$ 
MOQE NIIMAER

TIGENYECTURS NIIRUALIZTE TO 1.000

DI BPLACEMENISIRUTATIUISS LIF UNRE 8TRAINED NODES

NUDE

TRANSLATIUNA

0.

$2.25528 E=05$

$3.07880 E 005$

$3.11250 E \oplus n 3$

$1.76494 E \cdot n:$

$7.810055=0$

$1.00000 E+1) 0$

8.4 $48898-0$

Q.33471E०U

8.33554 UE $\cap$ I

$0.33589 E=11$

$0.33543 E=0$

$7.48606 E-1)$ ?

$4.97434 E-01$

$4.12 .625 E-111$

$3.299456=01$

$6.49810 E \bullet 12$

4.3005025

$-4,903170^{-113}$

$-9.91907 E-0$

$-1.37014 E-0.2$

$-9.43585 E-i\} 3$

$-9.99572 E-1) J$

$-1.00119 E-n 2$

$-1.52479 E-n$

$-1.00277 E=0$

$-1.0055 B E-11$
$-7.00134 E-115$

$-7.06134 E-11$
$-6.784 \triangle D F=1$

0 .

0.

0.

0.

0.

0.

0.
TRANGLATION 0.

$3.90517 E-0 Q$ $7.30133 E-04$ $1.04605 E-03$ 5. 26AETE-03 $0.78245 E=04$ $1.75524 E-09$ 2.1485UE-02 $0.85720 E-02$ $0.05137 E-0$ a $1.00670 E-01$ $1.1580 .18-n 1$ 1.1ROQQE-OI $1.00150 E=01$ $1.20262 E-02$ $6.34134 E-122$ $5.25800 E-02$ $3.90631 E=05$ C. 327 ARE 03 $-1.22238 E-02$ - $1.03565 E-02$ - $1.47982 E 002$ $-1.47064 E-02$ $-1.30693 E 002$ - 1. (A7OUE-1) $-1.05697 E-02$ - $A .347 A D E-03$ $-6.34707 E-03$ $-0.080^{\circ} 2 E-03$ - $470570 E=03$ $-3.36086 E-03$
$-1.37512 \varepsilon-03$ 0. 0 .

9.

0 .

0.

0 .
TRANGLATUN 0.

$8.81554 E-O 3$ $1.68054 E-02$ $2.42283 \mathrm{E}=02$ $1.49595 E-01$ 1. $49927 E-01$ $3.46094 E-01$ 4.022208001 4.02448600 $4.06935 E-01$ 4.5311895001 $4.01960 E=01$ 3.6457UE-01 $2.797600=01$ 2. T9749E-01 2.T9725E-0 I $2.47570 E-01$ 2.859008004 1.060 . $7.709236-02$ $0.7364=0$ ? $-7.00374 E .02$ $.6 .06432 E 0.02$ $-3.42347 E-02$ - $4.10190 E=02$ $-3.48007 E-02$ $-3.92951 E-02$ $-2.24942 E 002$ $-1.53954 E=02$ $0.22779 E-03$ $2,30622 E-03$ 0 0 0 0 .
Rutatgun

1. 10088000 O. $-1.004886005$ $-1.344962005$ 0.007332005 $1.370498=04$ $0.600750-04$ $0.339082=04$ $-8.02065 E-1) 4$ $-7.58817 E=04$ $-7.13794 E-04$ $-6.38755 E-04$ -5. $89980 E=04$ $-6.30846 E-04$ $-5.73599 \varepsilon=04$ $0.50477 E 004$ 0.485715004 $-2.514268=04$ - 1.093050 cou $-1.724158004$ $-1.74083 E 004$ $1.98171 E=00$ $-1.317998004$ $-1.05428 E=04$ $-9.11841 E-05$ $0.20239 E-O S$ $-5.05232 E-05$ 1.8541 UE 05 3.35078E-05 0. 0 0. 0. 0
RUTATIDN $-2.40477 E-13$ $-2.518345 .03$ $-1.07074 \mathrm{~V}-1) 2$ $-1.12571 E .02$ $-7.61150 E-03$ $-1.01133 E-03$ 3.7330 5. $1.21317=03$ $1.74102 \%-03$ S.186A9E - 03 $5.51441 E-03$ $5.34039 E=03$ $5.278605-01$ 4.37650003 4.385500 1.071296-03 3. $-3.19714 E-04$ - 3.911B3E-OL $-3.91183 E=04$ $-4.05523 E .0$ $-5.13309 \mathrm{~F}=0$ . 0.83321 - $2.31890 E$ ० 14 0 0. 0 0. $1.01033 E-00$ $1.05 \cap R 7 E=04$ $2.59032 E=04$ $3.3244 \mathrm{AE}-04$ $2.69155 E=04$ $4.767 n A E-C A$ $4.592375=04$ 3.034458004 . $-3,06022 E=04$ $0.034 B 1 E-04$ $-0.84110 E-C A$ $-0.17212500$ -6.00800 E-0 $9.568965-05$ $1.03844 E-110$ $1.06708 E-04$ 1.0703 UE $=04$ $1.07376 t=04$ $1.05391 E=04$ $1.01831 E=04$ $7.243 O A E=O S$ 0 .

0. 
MOVE NIJMHER I

PREDIIENCY 4.11574 HZ

EIGEHVECTUHS MHGHALIZE TH A UNIT MASS MATRIX

1)ISPLACEMENTSIRITATIINOS IIF UNRESTRAINEN MUDES

NQUE
NIIMBER

5 TRANSLATIIINA

-3.

$0.10125(1 E-3$

$1.5110 U E-01$

$2.12741 E=n 1$

5. 1 (2मस2E-1)

2. $12047 E-71$

2. $1294(1)-111$

$2.21433 E-1) 1$

$1.660635-01$

$1.40549 E=0$

$1.19963 E=01$

$2.81302 E-02$

$2.01973 E-112$

$5.35073 E-1) 3$

-3.13556 E O IA

$-3.32 \cap 32 E=1) 4$

$-1.07247 E-03$

$-3.50450 E=04$

$-3.84551 E-00$

- A. IHGULE-OA

$-4.370585 .-n a$

$-3.71710 E-13$

$-4.5530 H E-04$

$0.878875-104$

$1.49 A 2 B E=00$

1. 1 ? $704 E-1) 3$

$3,175+5 E-03$

$4.695008-00$

-5.6 म $10 \mathrm{E}-1) 3$

-3.200 HOE-GO

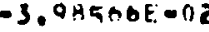

$-2.93 n 76 E=02$

$-1.05103 E=0$

$1.10691 \mathrm{~L}=03$

$1.17573 E-02$

$2.633 B 3 F=02$

$3.03873 E \bullet 02$

2.975 ? $4 \mathrm{E}-0 \mathrm{C}$

$-2.10590 E-05$

$-2.85574 \mathrm{E}-03$

$-8.07324 E-113$

$-1.1494 B E=02$

$-1.12736 E=02$

$-1.12743 E-02$

$-1.0 A 493 F=0$

$-1.030751 .02$

$-9.73054 E-\cap 3$

$-8.40567 E-113$

- $-40,17 F-113$

$\left.-6.927170_{-10}-1\right)$

$-5.960 R \Delta E=03$

$-4.65 \$ 7(1)-113$

$-1.57555 E-0.3$
traHsLATION $-2.25031 E=03$

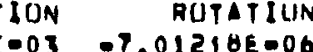
TRANBLATION -5.

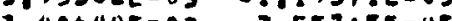
3. $-3.42257 E-02 \quad 1.50070 E-05$ $-1.20441 E-01$ 2.71014E-04 $-2.29683 E-01 \quad 4.67751 E-04$ $-2.29608 E-01 \quad 5,19131 E=04$ - 2.54278E-01 4.09204E-114 $-1.91776 E-01 \quad 5.05461)=04$ $04.167008=02 \quad 5.150798004$ 0.0157000 2 50220520004 9. cos $9.10585 E 002$ $9.19230 E-02$ 0.200490002 $-1.510596004$ $-7.30598 E-03$ $-2.03929 E-02$ $-2.85412 E=02$ $-2.53797 \mathrm{E}-02$ $-2.04429 E-02$ $-2.16388 \mathrm{E}=0$ $-1.69950 E \cdot 02$ . $.1 .11363 E-0$. $-1.07379 E-02$ $-6.28690 E-03$ $-3.00558 E-03$ $-1,71183 E-03$ $-6,40532 \mathrm{E}=04$
$5.22052 E-04$

$3.30769 E=U 4$

$2.78537 E=04$

$2.50614 E=04$ $-4.262456-05$ $-0.28673 E-05$ $0.05253 E 005$ $-8.37 \cup 32 E-05$ 0 -1.98234E-05 ?. 241 485005 - 2.2470 $3,90131 \mathrm{e}=06$ $8.90605 E=06$ $1.44963 E=05$ $2.43536 E-05$ $2.7336 E=05$ $4.795496=05$

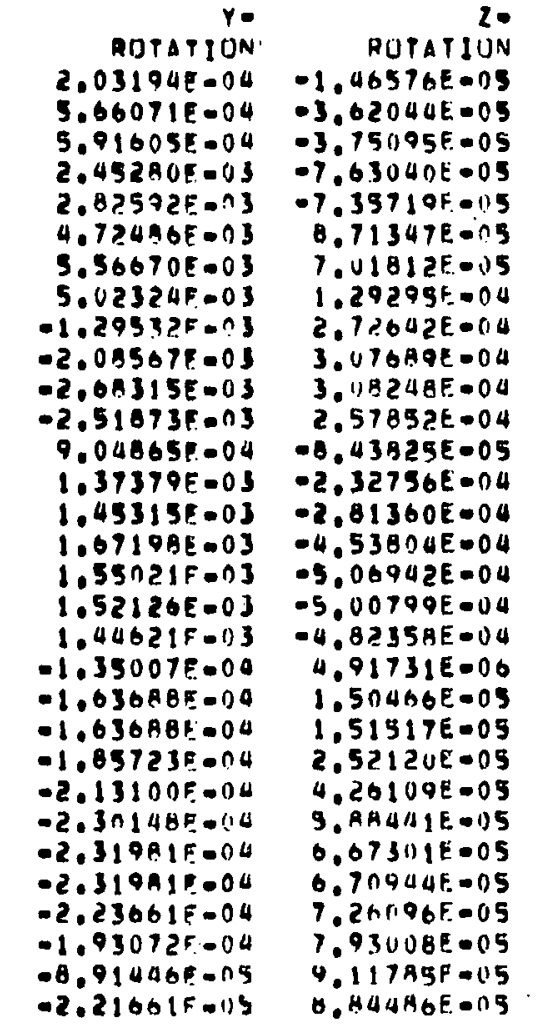


AIDE NIIMAEN 3

EIGEINVECTUIRS NUIHIALLIZE) TII 1.0190

IISPLACEMENTS/HIITATIIINS DF UNRESTHAINER NUDES

NIIDE

NIIDE
NUMBER

THANSLATIIIN

0.

$1.9 B O H I E=0 S$

$2.670 B 2 E=03$

$2.06993 E=03$

$1.527526 .-111$
$7.07277 E-11$
$1.00100 E+10$

$1.001100 E+110$

$5.51558 E-r$

$-5,90 \cup 35 E-1)$

$-8,30719 E-0$

$-B, 312 t 9 E=11$

$-8,317160=n$

$-8.31691 E=01$
$-8.64659 E=01$

$-6.50791 E-11$

$-5.60440 \mathrm{E}-\mathrm{n} 1$

$-4.68434 E=n$

$-1.098 \Delta 4 E=11$

$-7.84376 E=0$

$-2, U H 9371-112$

$1.22430 \mathrm{E}=03$

$1.2905 \mathrm{JE}-\mathrm{nS}$

4.187 2E 03

$1.36845 E-03$

$1.03473 E=03$

$1.700645=03$

$1.49150 E-02$

1.7781 UE $=03$

$1,90512 E-03$

$-5.85043 E-04$

$-4.40401 F-13$

0.

0.

0.

0.

0.

0 .
TRAMGLATIUN 0 .

$8.35420 E-04$

$1.38770 E=13$

$1.81626 E-03$

$9.03450 E=03$

$1.23900 E 02$

$-1.093321 .05$

$2.22150 E=02$

$1.27505 E-01$

$1.55633 E=01$

1. 14 4.41E-01

3.9 A9 $5 E-12$

$4.32035 E-03$

$-4.59104 E-02$

1. 1.2847E-01

I. 1 ROSTE-01

$8.574 A 4 E=05$

1.115125002

1.115121002

4. 4 BI SUE-O2

$4.20214 E-02$

प. LnZULIE-02

$4.234^{\circ} 2 E=02$

$4.00000 E 002$

$3.79961 E-1) 2$

3. 5AVSTE-nZ

3. $28247 E-02$

2. $10494 E-02$

2.

1. A $1836 E-02$

$0.15227 E-n]$

0.

0 .

0.

0 .

0.

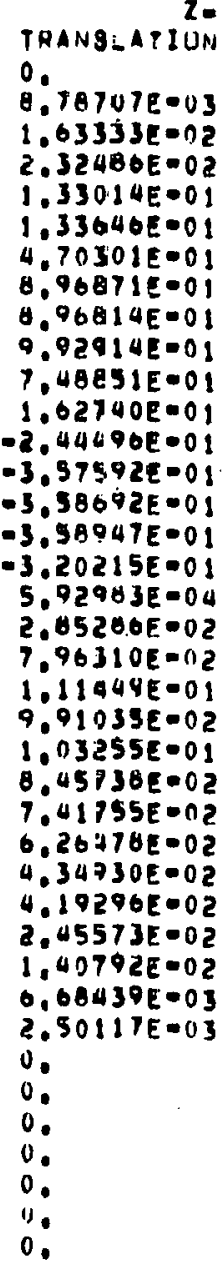

TRANS:ATIUN

0

RUTATIUN

0.

$2.73 H 14 E=03$

$0.25873 E-05$

$4.66122 E=05$

$9.98523 E=05$

$-1.060228005$

$-1.82649 E=03$

$-2.027122-03$

$.1 .94932 E-03$

$-1.07374 E=03$

$-2.01442 E-03$

$-2.04087 E-03$

$-1.29160 E-03$

$-1.08764 E-03$

$.9 .75607 E=00$

$1.664418=04$

3.026318004

$3.53446 E=04$

9.2604

$9.22445 E-115$

7.700696005

8.752605005

$5,770810-05$

$2.12371 E-05$

$-1.52339 \mathrm{E}-05$

$-3.49328 E-05$

$-3.08236 E=05$

-5.000 7 10ug

- .60057 -

$-1.47765 E=0 A$

$-1.87250 E 00 \mathrm{~A}$

0 .

0

0.

0.

0 re Rutarint 9.934395000 $-2.21041 \mathrm{~F}-03$ $-2.31012 E-03$ -9.9777 AF -03 $-1.10347 E-02$ 1.844 TBF. 02 2. $11370 F-02$ $-1.96140 E-02$ $5.058015-03$ . $1.04773 E-02$ $9.83520 \mathrm{~F}-03$ $-3.533345 .03$ $-5.36441 E .0$ $-5.67431 \mathrm{E}-113$ $6.52881 \mathrm{~F}-0 \mathrm{~J}$ $0.053308-03$ 19E-OS 5. 2717 RF 00 Te 政 . Bol75E - तa $1.25215 E .04$ . $32121 E 00 \mathrm{a}$ $0.986 B 6 F=04$ $9.05805 \mathrm{~F}$. 9.058455 .00 R.T3357E-00 $7.53914 E-O C$ 3.4809SE-C.4 $0.65547 F=n 5$ 0 .

0.

0.

0
Z-

$2355 E-09$ $1.41372 E .04$ $1.4646 B E=04$ $2.97954 E .04$ 2.872865004 $-3.411246 E=04$ $-2.74446 E=0 A$ $-9.04874 E-0 L$ - 1. volh $-1.20147 E-1) 3$ $-1.20366 E 003$ $-1.00 \triangle B T E-O 3$ 3. $29499 E=n A$ $9.08873 E-1) a$ 1.098 SGE 013 $1.772 \cap 3 E-03$ $1.47952 k-n 3$ $1.95553 E-U 3$ $1.62353 E=0 \mathrm{~S}$ $-1.92013 E=05$ $-5.47505 F=09$ $-5.41640 E=05$ $-9.844 A 7 E-05$ -1.663 HAF. ON $-2.29776 E-04$ -2.6057 OF -04 $-2.61992 E-10$ $-3.560398 .04$ $-3.453775000$ 0.

0

0.

0.

0. 
MOOE NIJMBER

FREQUENEY 11.187H HZ

FIGENVECTURS NIJFMALIZED TII A UNIT HASS MATRIX

DISPLACE. "ENTS/GLITATIUNS UIF UNRESTKAINED HUDES

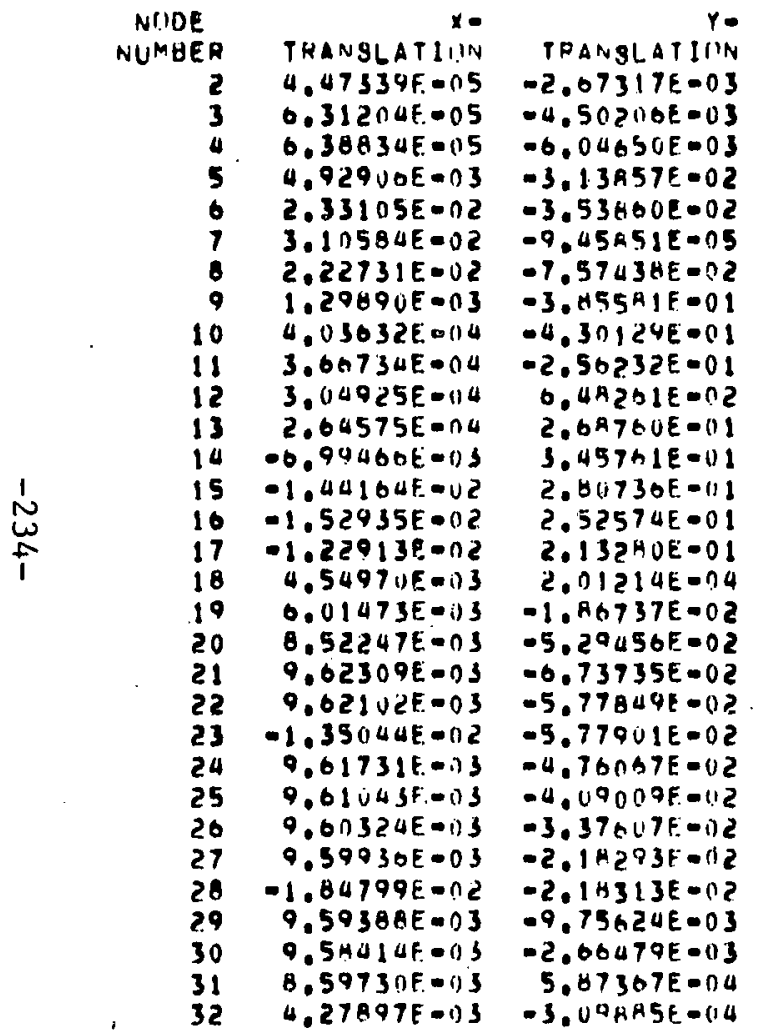

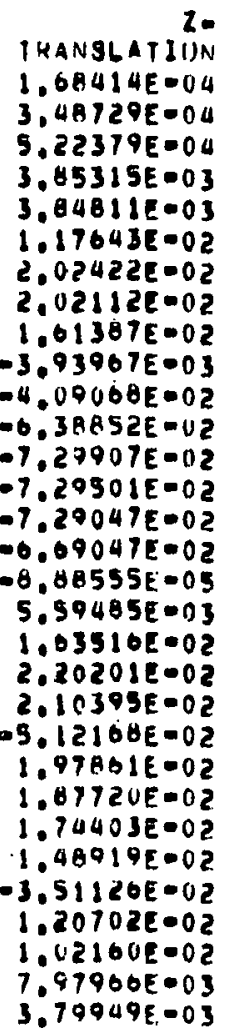

TRANSLATIUN
$1.68414 E=04$

$3.48720 E-04$

$3.45315 E=03$

3,048112003

$1.176430-02$

$1.01387 E-02$

$-14.000005-05$

$-6,3$ BR52E-U

$-7.57907 E-1) 2$

$-7.20047=02$

$-0.69047 E-02$

$5.59485 E=03$

$1.03516 E .02$
5.302018002

$2.10305 E-05$

$3.15168 E=02$

$1.877200=02$

$1.74403 E=02$

$3.51120 E-02$

$1.02160 \mathrm{CO}-02$

$3.79049 E=03$

\begin{tabular}{|c|}
\hline 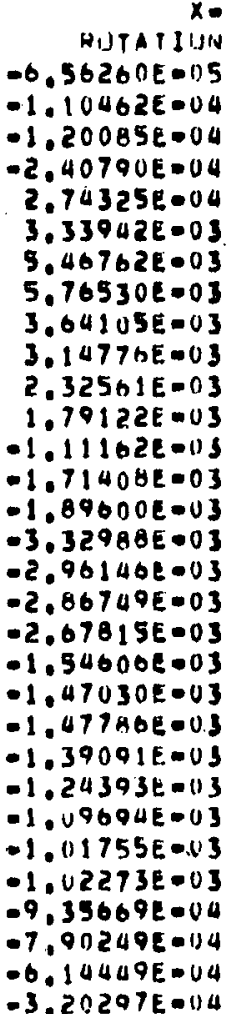 \\
\hline
\end{tabular}

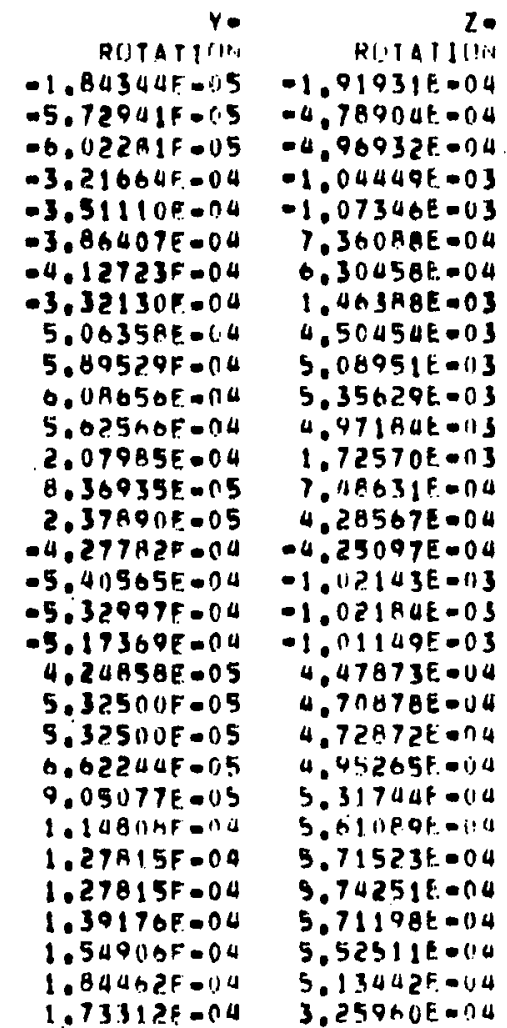


MODE NIJMREH

EIGENVECTU:S NUAMALIZE!) TU 1.0190

DI SPLACEMENTS/RUTATIURS UF LINKESTKAINED NUDEO

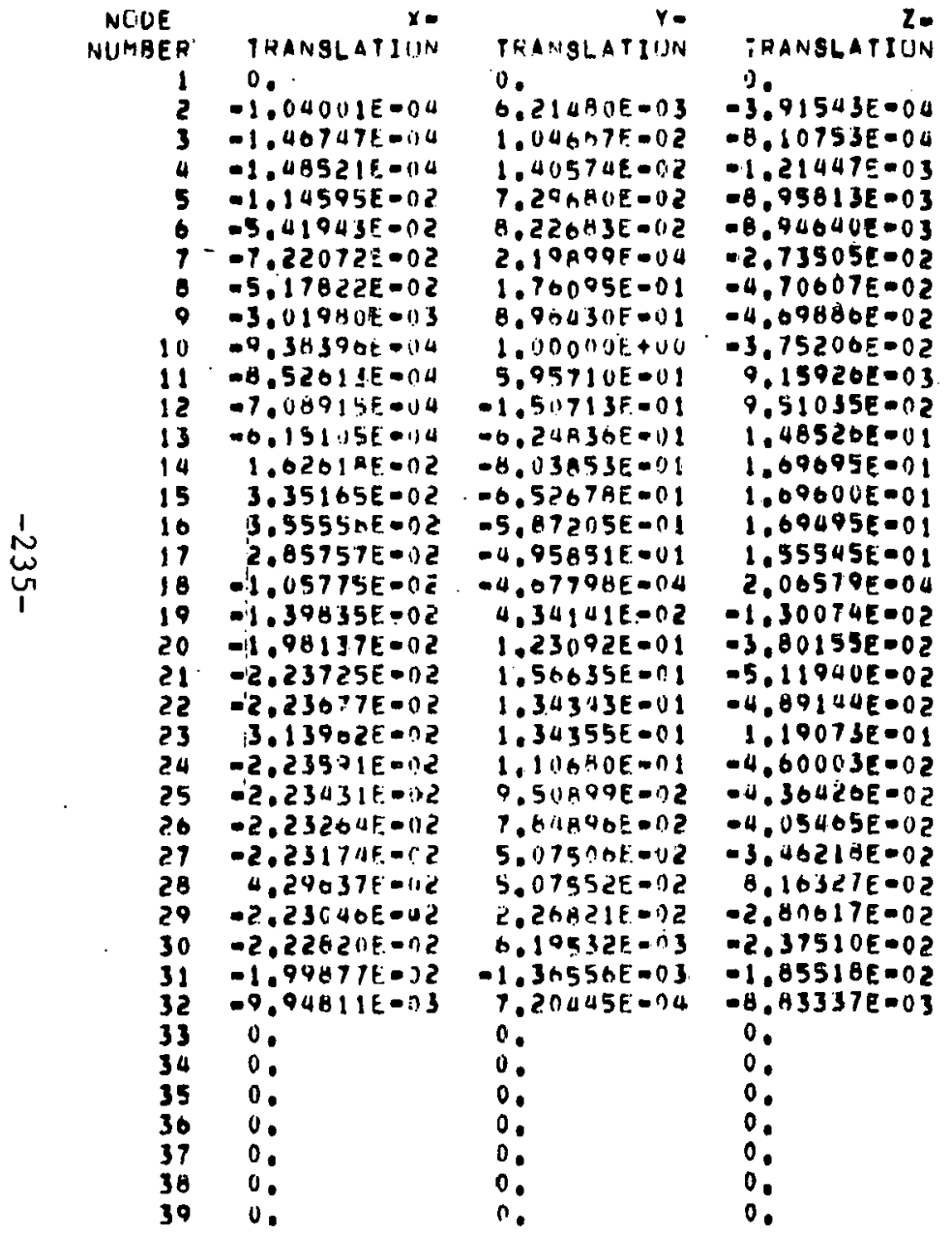

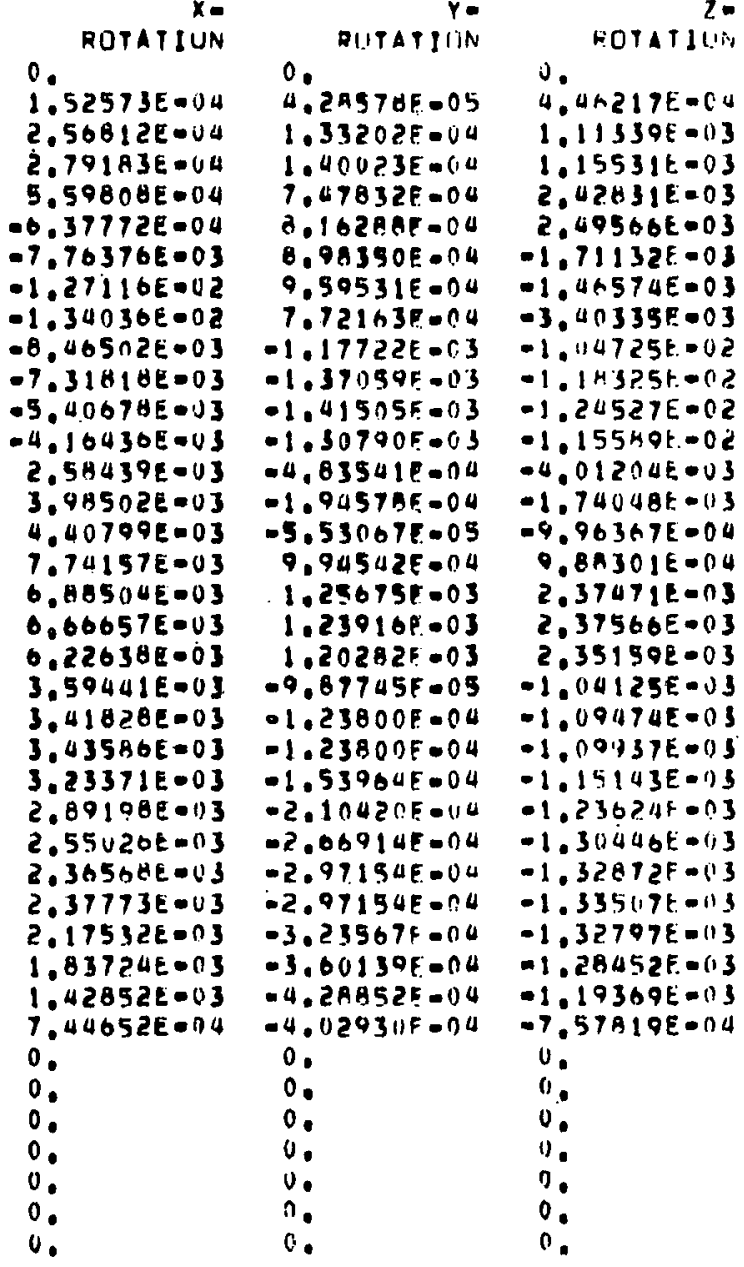



MODE NUMAER
PRE 5 UIENCY $17.1070 \quad$ HZ

EIGENVECTURS NOMMALIZE! TU A UPIT MASS MATKIX

DISPLACEMKAST/RUTATILIVS OF UNRESTRAINED NUDES

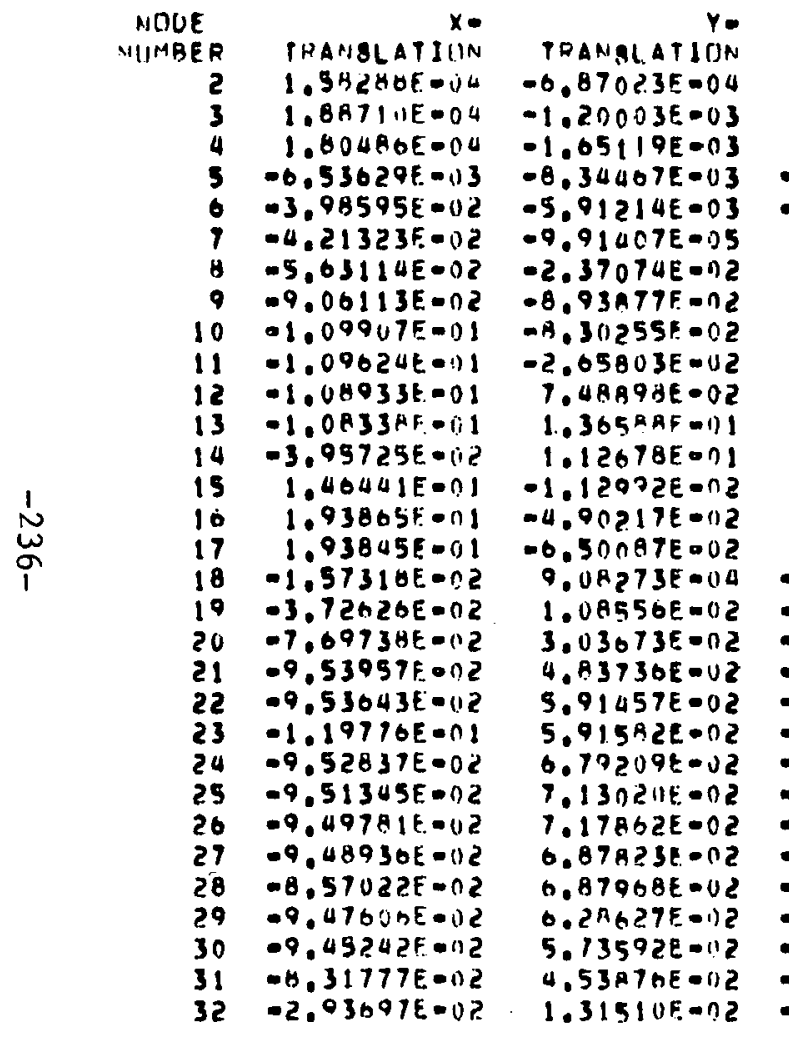

trAnBLATION 20 TRANBLATION 9.9035E-0A $-6.69853 E 007$ $1.91828 E-03 \quad-9.55076 E=06$ $1.70582 E-03 \quad-9.64783 t=06$ $-4.43055 E \cdot 04 \quad-4.19479 E-06$ $-1.86189 E=04 \quad 1.33072 E-04$ $4.73364 E=03 \quad 0.14545 E=04$ $1.91431 E=02 \quad 1.21013 E-03$ . $10235 E=02$ 0.7955002 $1.43871 E=01$ $1.82894 E 001$ $2.31297 E-01$ $2,30646 E=01$ $2.30190 E=01$ $2.236925=01$ $-4.60140 .05$ $-2.35353 E-02$ $0.76503 E=02$ $-1.08717 E-01$ - $.08717 e=01$ - $1.20370 E-01$ $-1.52529 E-01$ $-1.39425 E-01$ $-1.43077 E-01$ $-1.40371 E-01$ $-1.27505 E-01$ $-1.58488 E=01$ $0.05250=05$ $0.05350=0$ $-6.02359 E-02$
$-1.63625 E-02$ 
MUDE RIIMHER

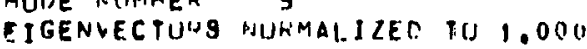

1)ISPLACEMENTSIFUPATIIINS UHF UNRESIRAINED NUDES

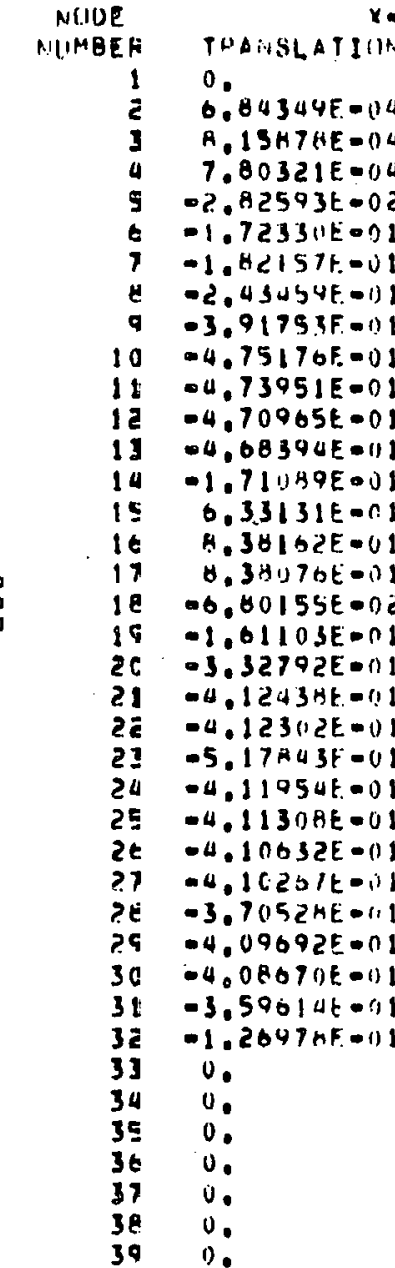

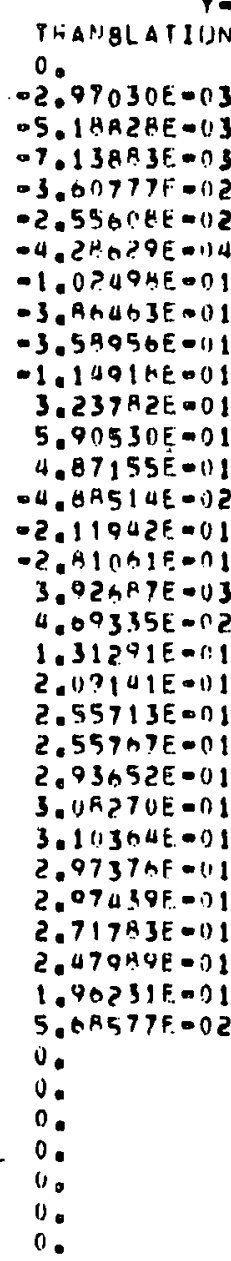

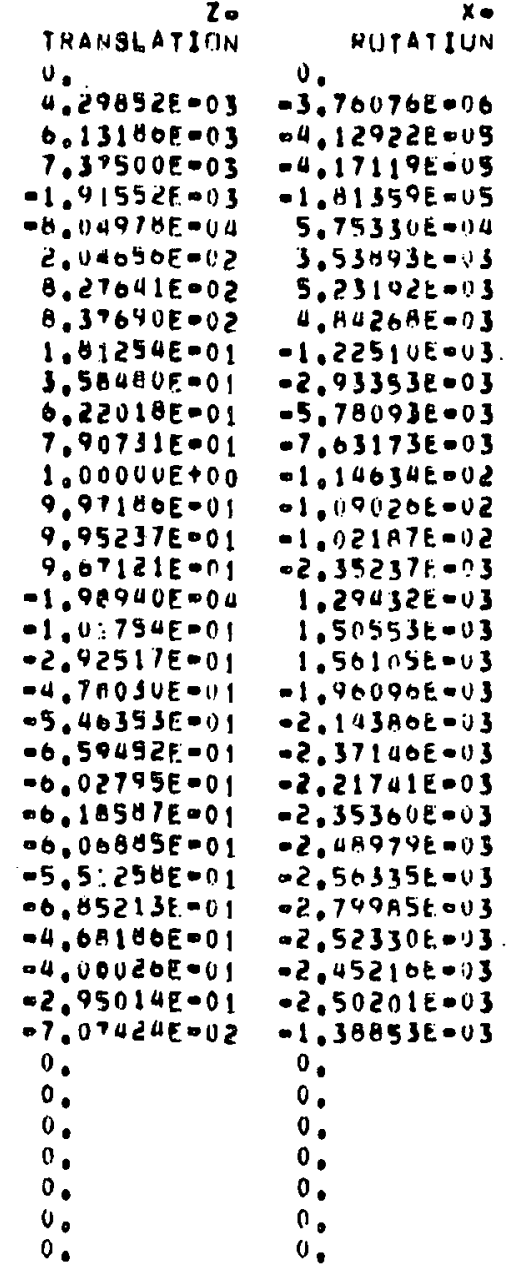

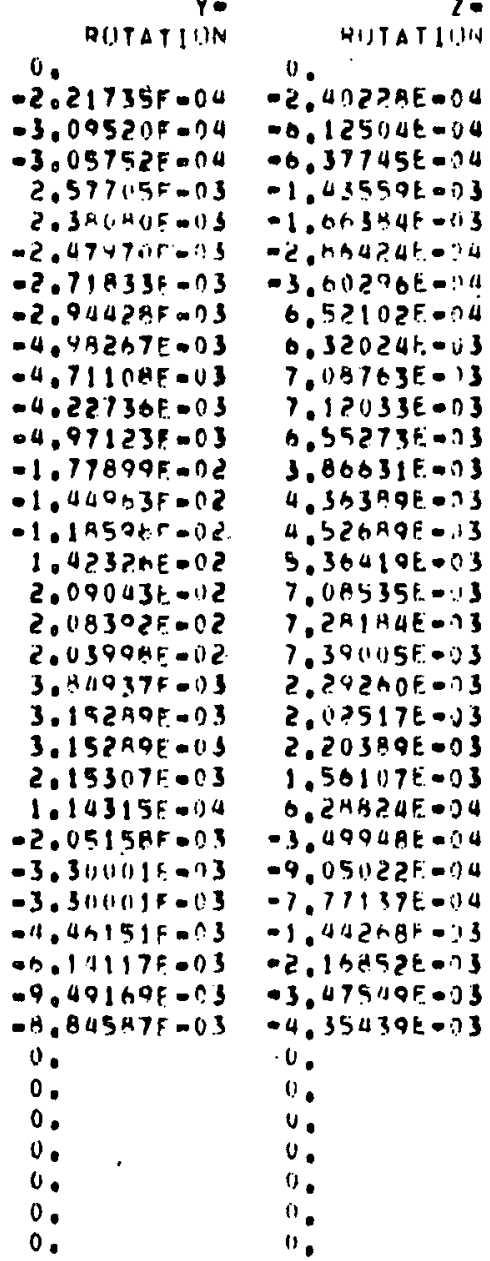


MOOE NIJMREP

FREQUENCY IR.1128 HZ

FigenVECTURS nUHMALILE TU a IINIT MASS matRIX

UISPLACENENTS/RUTATIIINS UIF UNFEBTHAINED NUDES

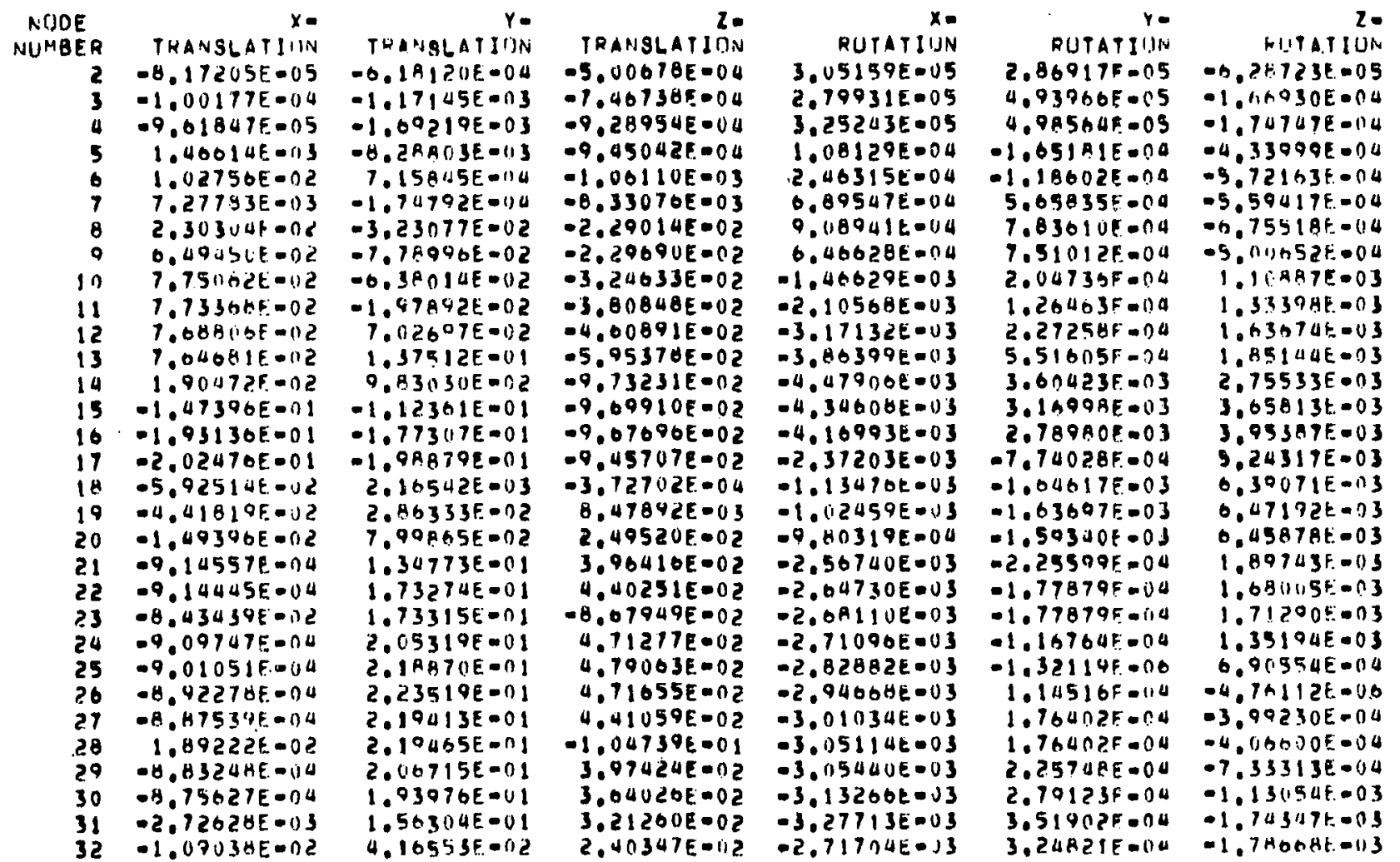


MOVE NUMBER

EIGENVECTURS NIJHMALIZED TU1 1.000

DIGPLACEMEATSIRUTATIIINS UF UNREBTKAINEO NOUES

NODE

NUMBER

$$
10.0
$$

$-3.65007 E-n L$ -4. HA I AIEECAL

$-0.303211 E-114$

$0.5593 b E=03$

$4.59721 E-12$

$3.25602 E=02$

1.0303 SE $=01$
$2.91557 F=01$

$3.46754 E \rightarrow 0$

3. $45997 E-01$

$3.43055 E-?$

$3.45110 \mathrm{~B}=0$

3. 2 दा $10 \mathrm{E} 0$

$-6.59432 E-01$

$-8.64060 E=11$

$\underset{\omega}{1}$
TEAMBLATION 0.

$0.7654 U E-13$ $5.24093 F=03$

$7.57068 E-1)$

$3,7070 \mathrm{HE}=0$

$3.20261 E-113$

-7,82002E-0I

$-1,0 B 3 T 4 E=0$ I

$-2.85441 E-01$

-8.5535 UE-OR

$3.14379 E-0$

$0.15215 E-01$

$4.39797 E-01$

$-5.02602 E=0$

$-7.93251 E=1$

$-8.69705 E-n$

$9.08745 E-03$

1.281028001

3.57A51E-01

$0.02 .059 \mathrm{E}-0$

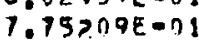

$7.75373 E-U$

$9.18570 E=01$

$9.70201 E-0$

$1.00 O \cap D E+$ IO

$9.81631 E=01$

9.81 RGUE-O

9.24 A $9 E-i)$

Q.67A27E-0

$0.902 B 7 E-01$

$1.8 n 361 E-01$

0

0 .

i).

0.

0.

(1).
IRANSLATION 0 .

0. $23090 \mathrm{0}=03$ $3.340 B 3 E=03$ - $150045=0$ - J560UE-103

- T TrJE-O

- 3. 7 Z709E-0

$-1.02458 E=0$

$1.02701 E-0$

$-1.70367 E=0$

$-2.06198 E-0$

$-2.06360 E=0$

- 4 न $35413 E-0$

$-4.33021 \mathrm{E}=0$

-4.3293 TE-O

$-4.23 \cup 99 \mathrm{E}=0$

$-1.06743 \mathrm{E}=03$

$3,7033 \mathrm{HE}-02$

$1.11035 \mathrm{se}-01$

$1.77352 E=0$

1.96963E-O

3.6A 11 IE- 0

2.14328E-01

$2.11013 \mathrm{E}=0$

$1.97325 E=0$

$-4.6 B 587 E=0$

$1.77803 \mathrm{E}=0$

$1.62861 E=01$

$1.0752 \triangle E=01$

0 .

0 .

0.

0.

0.
RUTATIUN 1.2523 HEDI:14 $1.455106-04$

$1.10199 \mathrm{E}=03$

$3.08496 E-03$

$4.06650 t=113$

$2.80294 E=03$

6.50002E-IJ

$-1.41861 E=02$

$1.728718-02$

-

- $10040 \mathrm{C}$

- $1.90439 E=02$

$1.06122 E=02$
$-5.07681 E-03$

$-4.5 H 300 E=03$

$-4.385 A \Delta E=03$

$-1.14803 E=02$

$-1.18437 E-112$

$-1.19950 E=02$

$-1.21285 E-112$
$-1.26558 E=02$

$-1.31831 E-02$

$-1.34679 k=02$

$-1.36505 E-02$

$-1.360512=112$

1.401522002

$-1.21558 E=02$

0.

0.

0.

0.

0.
PIJTAT IUIN 0. . 209055004

.

7. 300035004

$5,310145-04$

$2.53149 F-03$

3.5157 पF 013

$3.35905 E-C 3$

, 1590DE-04

5.657 A2E-04

$1.01673 E=03$

2. 467 त2E-103

1.612496 .02

1.41 स 2F-02

$1.24813 E-02$

$-3.46202 F=03$

$0.364771-03$

$0.3236 .35=113$

$-7.12871 F=03$

$1.00931=03$

$-7.058135=04$

$-7.05813 E-04$

$-7.92813 E-04$

-5.99118 b -00

$5.12330 E=04$

$7.892 \pi$ TFEA

7.892015-04

$1,24 B 775003$

$1.57437 E-03$

$1.45321 E=03$

0.

0 .

0.

0.

RUTATION

$40=04$

7. uhazTE-ol

A) TOBE-114

941ate-Us

- .557 HUE $=03$

-?. SI2 277E-03

$-3.112219 E=03$

$2.23046 E-03$

$.90090 E-\cap 3$

5.9nUIITE=03

$7.32258 E-03$

$8.20313 E-03$

1.232705002

1.03th

. 7 गु

- 7659 a

$2.34574 E=n 2$

2. . 599 प UF. 02

$2.89347 E .02$

$2.889591-02$

8. 498 ABE-O3

$7516105=03$

$7.00333 E=03$

$0.0435 E-03$

- 1 . A HOLE=0j

-3.2 . $076 \mathrm{E}=03$

$-5.03703 t=03$

$-7.80009[-113$

$-7.99330 t-03$

0 .

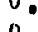

0.

0

0. 
MDDE NUMAER 1

PREQUENCY 22.3795 HZ

EIGENVECTURS NURMALIZED TU A UNIT MASS MATRIX

DIBPLACEMENTS/RUTATIUNS IIF UNHESIRAINED NUDES YOE TRANSLATIUN ZO ROTATIUN $\begin{array}{ll}-2.95110 E-03 & -4.03138 E-06 \\ -4.47050 E-03 & -8.33646 E-07\end{array}$ $-5.62 .46 U E-03 \quad-1.5936 U E=116$

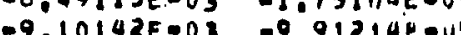
- $007457 E=02$ - $4033520-110$ $-3.92799 E=02 \quad-5.44859 E=04$ $-5.42828 E=02 \quad 5.16758 E=04$ $-7.90353 E-02 \quad 0.26977 E 004$ $-1.07704 E-01 \quad 1.34067 E 003$ $-1.20810 \mathrm{E}-01 \quad 1.67458 \mathrm{E}-03$ $-1.78169 E=01 \quad 2.15504 E-193$ $-1.773278=01 \quad 1.034828-03$ - 1.767305001 1.014070E003

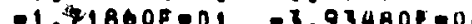

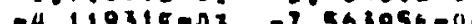

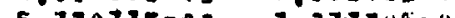
2. 10999802 2. $110252-04$ $2.47137 \mathrm{E}=0$ 2. $28533 E=04$ $-8.07688 E-02 \quad 2.91785 E=04$ $-1.12750 E-01 \quad 3.74449 E=04$ $-1.28586 E=01 \quad 4.57113 E=04$ $-1.41259 E-01 \quad 5.01762 E-04$ $-1.192098-01 \quad 4.31348 E=0$ $-1.43000 E-01 \quad 5.80238 E 000$ $-1.35709 E-01$ 1.19610E-0 $9.19610 E=00$
$8.60849 E=00$ $1.02151 E=03$

\begin{tabular}{|c|c|}
\hline 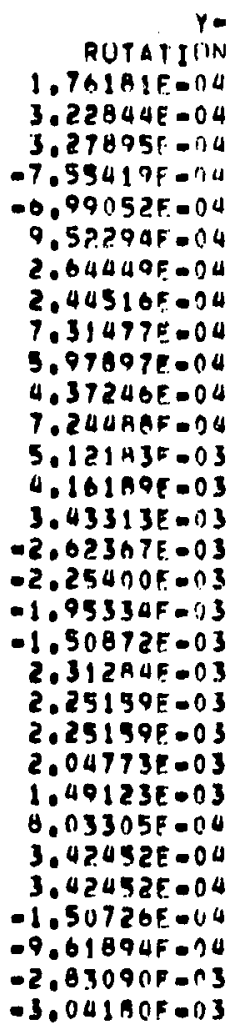 & $\begin{array}{l}E=04 \\
E=04 \\
E=04 \\
F=04 \\
=07 \\
=004 \\
E=04 \\
E=04 \\
E=04 \\
E=04 \\
E=04 \\
E=04 \\
E=04 \\
=01 \\
E=03 \\
E=03 \\
E=04 \\
E=04 \\
E=05 \\
E=05 \\
E=05 \\
E=04 \\
E=04 \\
=00 \\
E=04 \\
E=04 \\
E=05\end{array}$ \\
\hline
\end{tabular}


MODE NUMAER

F. IGENVECTURS NITRMLLIZEN TO 1.000

WISPLACEMEHTS/ROTATILINS OF LNRESTRAINED NUDES

NODE
NUMBER

$$
\text { TRANGLATIUN }
$$

$2.26436 E-13$

$2.21508 E-n 3$

$-1.44430 E-12$

$-1.00764 E-01$

$-1.58871 \varepsilon=0$

- 3 . $41553 E=01$

- 5748745001

-

$-2,7428 \mathrm{HE}=01$

$-2,71567 E-11$

$1,893: 3 E-1) 4$

$7.82190, E-1)$

$1.00000 E+00$

$4.99668 E=01$

$4.71098 E-1)$

$4.29712 E-01$

$4.40070 E=01$

$4.30901 E=01$

$4.28401 E=0$

$4.39464 E-U 1$

$4.34543 E=01$

$0.37565 F .01$

. 3703 BE= II

$4.87130 E=01$

$4.36120 E=01$

$4.34490 E=01$

$3.89042 F=01$

1.3011 HE-O

0.

0.

1.

390 .
TRANSLATION TRANOLATION

0.0

$1.30794 E-03 \quad 9.92079 E-03$

$-2.43\{42 E=1)\}$

$-3.3 A 3 h 2 E-n\}$

$-1.67101 \mathrm{0} 02$

$-7.926915=03$

$2.69410 E=04$

5.1 hO55E=0

1.0618

- 2008 TE-0

.

.775s

. 79921 and

$-2.02230=0$

$-2.70620 E=0$

2. 5964 पE 0

3.26917E-O

3. $66917 E-02$

$1.31250 E-01$

$1.43121 E=01$

$1.45172 E-0$

$1.49943 E=01$

$1.50394 E=0$

$1.45502 E-01$

$1.30 A 3 B E-01$

$1.30885 E 001$

1.123 ABE-01

$1.01908 E=0$

$7.81802 E-12$

$1.05976 E=02$

0 .

0.

0.

0.
$1.502008 \cdot 02$

$1.89083 E=02$

$8.45449 \mathrm{E}-0 \mathrm{Z}$

$3.05965 E=02$

$1.30012 E=0$

$1.320468=0$

1.82 पU UE $=0$

$2.65695 E-01$

$3.020712=0$

$4.26302 E-01$

$5.98954 E=01$

$5.96124 E 00$

5.94147E-0

$5.77748 E \cdot 01$

$1.38400 E 002$

$1.939968=02$

$1.09100 \mathrm{E}=02$

$2.00340 E 002$

$1.443198-08$

1.0587000

$2.984105=01$

$3.70033 E=0$

4.32271E-0

$0.74873 E=01$

$4.00749 E-01$

$4.00740 E=0$

$4.56217 E-0$

$3.70575 E-0$

$1.22078 E=0$

0 .

0.

0.

0.

:.
RUTAT XUN 0.

O. ROTATIUN 0.

$5.35724 E-06$

.8865IE-05

$1.537600=03$

$2.172928=03$

1.831675003

$1.74302 E=03$
$-2.70007 E-03$

$-4.90098 \mathrm{E}-0 \mathrm{I}$

$-5.62947 k=1)$

$-7.24467 E=1$

$-6.168168003$

1.32277600

$2.54279 E-0$

2.44656800

$7.10753 E=04$

$0.30800 E=04$

$7.692668=0$

$-9.80903 E .0$

$1.25880 E-U J$

$-1.53669 E 003$

$1.68679 E$. 03

$-1.05007 E-03$

$-2.41913 E-01$

$2.80304 \dot{E}-0$

3. $43404 E-0$

0.

0.

$0:$

0

0
$5.92271 E-04$

- $.0 B 531 E-0 J$

$-1.10220 E-03$

2.530518 .03

$3.20135 E-03$

$8.890065-04$

- $210945-04$

$-2.45902 \mathrm{E}-0$

$-2.00996 E-03$

$-1.409908 .03$

$-2.435538 .03$

-1.721 वरद-02

-1.39911 E02

$1.15412 E-02$

$0.82007 E-03$

1.577317 .03

6.566598 .03

5.071BQE-03

$7.79515 E-03$

$-7.569225-03$

$-7.569222001$

$-6.68390 E-03$

$-5.013107 .03$

$-2.700495-03$

$-1.15123 \mathrm{~F}=03$

1.151235 .03

5.0660950 .04

$3.233028-03$

$9.51671 E-03$

$1.02257 \mathrm{~F}-02$

0.

0.

0

0 .
.17136E-OA

$3.00403 E=014$

$-3.13274 E-10$

$7.23640 E=04$

$.78704 E 004$

$1,30250 E .06$

$3.05906 E-03$

$3.36003 E=03$

2. $94312 E-03$

$2.362-4 E=03$

$9.01418 E-04$

$1.0400 Q E-1) 3$

$.01578 t .03$

$.30022 E=03$

$58672 E=03$

3.69771003

3.69714003

3

$1.65952 E=0 \mathrm{U}$

1.741625004

-5. ABGA7E-OL

$0.13182 E \cdot 04$

$-1.10092 E-03$

$-8.65938 E=04$

70 TO

- .00715ecou

$1.27992 E-13$

0.

0

0.

0 
MODE NUMBER

PREQUENCY 27.1930 $H Z$

F. OOENVECTURS NUHALLILED TO UNIT MASB MATHJX

UISPLACEMEUTSRLUTATIINE UF UNKESTRAINET NUIJES
TRANGLATIIN TRANGLATIIIN RO RUTATIUN $\begin{array}{lll}3.35795 E-04 & -3.24773 E-02 & -7.20160 E-06 \\ 6.02563 E-04 & -4.997208-02 & -4.47293 E-00\end{array}$ $A . \triangle 3 A \triangle B E-00 \quad-6.35657 E-02 \quad-5.57596 E-06$ $4.07225 E-05 \quad-1.24729 E-01 \quad-2.61862 E-05$ $1.24700 E-03 \quad-1.30796 E-11 \quad-9.27727 E-05$ $1.00802 E-05$ 1. $99002 E-15$ $1.32897 E-02$
$3.83618 E .02$ $3.83618 E .02$
$2.92482 E-02$ $2.84648 E-04$ $-4.03 A 27 E-02$ $-0.25719 E-02$ $6.60525 E-n .2$ B. $840 R 7 E-12$ $8.07604 E-02$ 4.020425004 $-7.155178-113$ $-7.155172-113$
$-2.10574 F=02$ $-2.10574 F=02$
$-2.46219 E=02$ $-2.46219 E-02$
$-7.20655 E-03$ $-7.21030 E-03$ $1.015 B 2 E-112$ $2.02429 E-02$ $3.07341 E-02$ $3.90025 E-112$ $3.90025 E-112$ $3.96437 E-02$
$4.79903 E-02$ 4. $19903 E-02$
$5.12103 E-02$ $4.39031 E-02$ $1.03 A 13 E-02$

$-2.15291 E-01 \quad-3.00503 E-04$

-370904001 - $1.800448=04$ $-2.00201800$ $-1.57332 E=0$ $-2.65182 E 002$ $5.05020 E 002$ $5.73309 E=02$ $5.74532 E .02$ $5.64642 E=02$ $5.64642 E-02$ - . $03501 E-0$ $-2.00607 E=02$ $-3.16841 E=02$ $-2.54394 E-02$ $-1.03302 E-01$ $-1.76920 E=02$ $-1.20340 E-02$ $-9.06765 E-03$ $3.91404 E=03$ $-7.41024 \mathrm{~F}=02$ $1.30500 E=02$ $1.74720 \mathrm{E}-02$ $1.82603 E-02$
$1.11541 E-02$

- 4.869 aUE 3.783 ก LE 0 $0.09457 E=0$ 9.003395000 $1.35431 E 00$ $1.69889 E=0$ $2.01696 E 013$ $1.56216 E-03$ $1.30086 E-03$ $4.561128=04$ - $1.50303 E-0$ $-1.20393 E=0$

$-1.24009 E 0 u 3$ - $1.33970 E 00$ $-1.07063 E-03$ -1.5257 UE $=03$ -1.615 A $9 E-03$ $-1.529558003$ $-1.530611003$ $-1.54367 \mathrm{E}=03$ $-1.54748600$ - 1 SU - .612162003 - $1.520222=03$ $-1.43655 E=03$ -2.141 TIE-02 $-1.47180 E=03$ 1.219
RUTATION RUTAPIJN $2.01139 E=03$
$3.88521 E=03$ $3.865215-03$
$3.964085-03$ $4.902600-03$ $0.84278 E-13$ $5.02374 E-03$ $-4.90468 E-03$ $-5.39012 E-113$ 1.57600 CnA 6.131360 .04 $-2.79078 E-03$ $-3.62090 E=03$ $-2.69973 E=113$ $-1.76053 E-13$ $-1.40100 E-03$ $8.53025 E .04$ $1.75966 E-03$ $1.804908-03$ $1.00403 F=03$ $-2.73391 E-04$ $-3.33023 E=04$ $-3.330236 .04$ $-3.879408-104$ $-4.43000 E=04$ $-4.64362 E-11$ $-4.4951350 .04$ $-4.405138-0$ $-4.00531 \mathrm{E}=0$ . .02234 प. $3.52948 F=04$
LURATIN $2.97365 E 005$ $7.63 \cup 5 B E=05$ $7.94137 F=05$ $1.64212 E=04$ 2.32Uर1E-04 1.532238004 1.667962004 SIDSBE-05 $7.53114 E=04$ The - O $7696 \mathrm{e}$-0. $3.70950 E-04$ $2.82437 t-05$ 1. SHARCE 04 - 2.2ก169E-0O $-9.8123 B E=04$ $4.66544 E-04$ $-3.78277 E-04$ - 2. $2170 B E-04$ $6.15493 E-04$ $0.295711=04$ $0.5435 B E-D 4$ $6.0421 A E-14$ $7.03225 E-04$ $5.624 H 3 E-04$ $4.59395 E-64$ $4.0 A 175 E-B A$ $3.52393 E=04$ $2.077928-04$ $-0.32946 E=05$ $-1.73 A 1 \mathrm{AE}-04$ 
MODE NUMBER

EIGENVECTUAS NURMALIZED TIJ 1.000

DISPLACEMENTS/RLITATIIINS OF UNRESTRAINED NUDES

NODE

PUMGE

10

$-2.63072 \mathrm{E}-\mathrm{C} 2$

$-2,59057 E-C 2$

$-7.8526 \mathrm{BE}-1.3$

$1.00000 E+C .0$

$9.60924 E-6$.

$9.37219 E-C 1$

$-7.49395 E-C 2$

$-2.6 \$ 43 H E-C 1$

$-2.72240 E-C 1$
10.03

$3-2.72534 \mathrm{E}-\mathrm{Cl}$

$-1.58113 E-C .2$

$-1.58113 E-C .2$

$4.2320 \mathrm{NE}-\mathrm{C}$

$4.31121 E-G$

$1.59251 E=01$

$1.27024 E-01$

$6.70870 E-12$

$4.3976 U E-12$

$4.38160 E-C 2$

$-9.51764 \mathrm{E}-\mathrm{Cl}$

$4.36164 E=02$

$4.32457 E-0$.

$4.26668 E-32$

$4.20622 E=02$

$-3.37160 E=02$

$4.24102 E=C .2$

$4.19027 E-02$

$4.08576 E-12$

$1.47987 E-02$

0.

0

0.

0.

0 .
TRANGLATIIIN

$12461 E-03$

. $01804 E-03$

$2.02612 E-03$

1.363 B 3002

. $17650 E=0 \mathrm{~J}$

2.57A13E.0O

$4.45045 E-02$

$1.28477 E-01$

$9.79550 E=02$

$9.53314 E=04$

$-1.48642 E-01$

$-2.09559 E-01$

$-7,17112 E-02$

$2.24230 E-0$

$2.96300 E=0$

$3.00636 E-0.1$

$0.39633 E=0$

$-7.052311 .02$

$-8.24611 E-02$

$-2.41354 E-02$

$-2.414 B 3 E-02$

$3.40208 E=02$

$6.77952 E=02$

$9.02403 E-02$

$1.32633 E=01$

$1.32904 E-01$

$1.00754 E=01$

$1.71521 E=0$

$1.47337 E-01$

$3.47678 E=02$

0 .

0 .

0.

0 .

0 .
TRANBLATIUN

$1.08769 E=01$

$1.07361 E=01$

$-2.12887 \mathrm{E}-0$

$-4.17730 E-0$

$4.380408=0$

T. $21027 E-0$

- $.20878 E-O$

$9.31795 E-01$

$0.74290 E=0$

$8.71457 E=01$

$5.26987 E-01$

B.B8113E-O2

$1 . B 9230 E=01$

$1.92000 E=0$

1.9201 I OEOO

1. B91 0 UE-O

$-2.58911 E-03$

$.7 .38033 E-02$
-.700135001

$-1.06113 E=0$

$-8.51969 E=02$

$-3.46108 E-0$

$-4.03029 \mathrm{E}-02$

$1.76513 E-02$

$1.31085 E-02$

$-2,48377 E-0$

$3.35154 \mathrm{E}=02$

$0.11755 E=02$

$3.73500 E=02$

0 .

0

0.

0.

0.

0 .
RUTATIUN 1.490028005

I.86744E-0

. 770015005

$3,10704 E=04$

I. $22075 E=03$

$-1.63082 E=03$

$-1.26698 E=03$

$2.04280 E=03$

$3, n 1532 E-113$

$4.63617 E 003$

$5.68073 E=03$

$0.754978=03$

$5.23183 E=03$
$4.35671 E-03$

1.527568003

$-4.03200 \mathrm{E}-03$

$-4.15317 E-03$

0.486796003

$4.95205 E=03$

$-5.109 B 5 E 003$

$-5.41176 E-0$

-5.122622003
$-5.14625 E-03$

$-5.16080 E-03$

$-5.102645=0$

$-5.39926 E-03$

$-4.92920 E=03$

$-4.81113 E-03$

$0.01930 E=03$

0.

0 .

0.

0.

0.
RUTETINON

0.

1.301196002

1.327615 .02

$1.67207 E 002$

$1.621 \mathrm{BQE}-0$ ?

$1.88344 \mathrm{~F}-132$

$1.042 n 2 F-112$

OOTABF. IL

. 2A1) 1 त्व 04

$-2.05345 \mathrm{~F}=113$

9.279601003

$1.21267 E 002$

. 0A105E-03

$0.099119 \mathrm{Q}-n_{3}$

. 896A6E-03

$5,803275 \cdot n 3$

$6.044765=03$

$6.0449 T E .03$

$-0.16282 E-04$

$-1.11532 \mathrm{~F}-03$

$-1.11532 \mathrm{~F}=03$

$-1.29025 \mathrm{P}-03$

$-1.436960 .03$

$-1.55519 E=03$

$-1.50540 E-03$

$-1.50546 E=03$

$-1.3414\left\{\begin{array}{l}0.03 \\ 0\end{array}\right.$

$9.692108-04$

1.182068 .03

0.

0 .

0.

0.

0.

0.

TATION

$0015-05$

$2.95555 \mathrm{C}-0 \mathrm{~A}$

$2.66633 E=04$

$6.23642 E=04$

$7.78400 E=04$

$5.13156 E 004$

. SR500t.0a

. B LS5UE-04

$1.24234 E-113$

4.475 LEEUS

. 3 ? $042 E-U 4$

$-7,37367 E-04$

$0,2 A 625 E=03$

$1.56250 E=03$

$1.2608 B E-03$

$42519 E-04$

$2.778316-03$

$2,00131 E=03$

2. 03 SEE

2.69350003

2.

$1.88381 E=03$

$1.53836 E=03$

. $56796 E-03$

$1.180208-03$

$95913 E-04$

$.44997 E=04$

$-5, B 21325-1) 4$

.

0.

0 .

0.

v. 
MOOE NIMARE

PREQUENCY 28.011

$\mathrm{Hz}$

EJOENVECTURB NURMALIZED TU A UNIT MASS MATRIX

DISPLACEMENTS/RUTATIINNS OF UNREBTRAINED NUDES

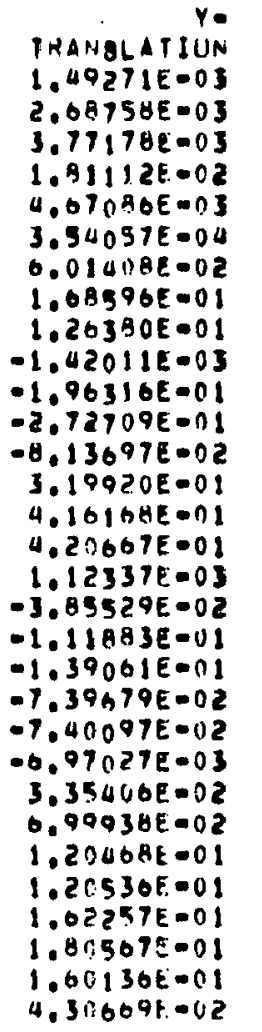

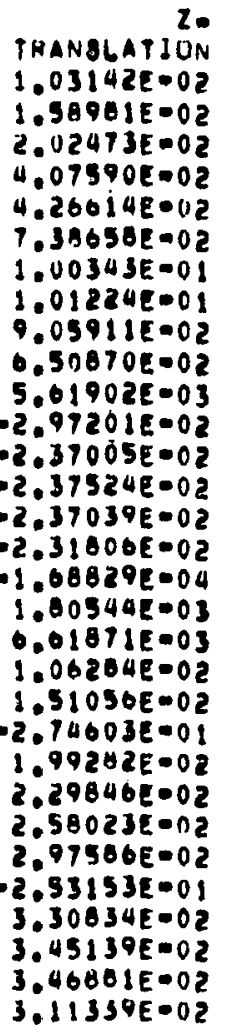
RUTATIUN $-3,61100 E=05$ $-2,47804 E=05$ $-3.03002 E 005$ $-1.31527 E=04$ $-4.275200=04$ $-2.602526003$ $-2.119016003$ $-2.119816003$ 1.610476003 2.897248003 $4.22036 \mathrm{E}=0.03$ 6.441616003 7.801516003 $9.191 \mathrm{BQC}=03$ $7.03110 \mathrm{AE}=03$ 5.010576003 2. 43047500 $-2.43047 E=03$
-5.80742600 $-5.0070936003$ 5.96993e-03 -6.2920 OE 03 $-5.031476003$ $-5.73174 E=03$ $-5.98585 E-03$ $-5,70994 E-03$ $-5.66997 E-03$ $-5.629218003$ $-5.607400-0$ $-5.00700603$ $-5.84166 E-03$ -5. $473062-03$ $-5.23448 E-03$ $-5.07705 E=1)$ $-3,73249 E-113$

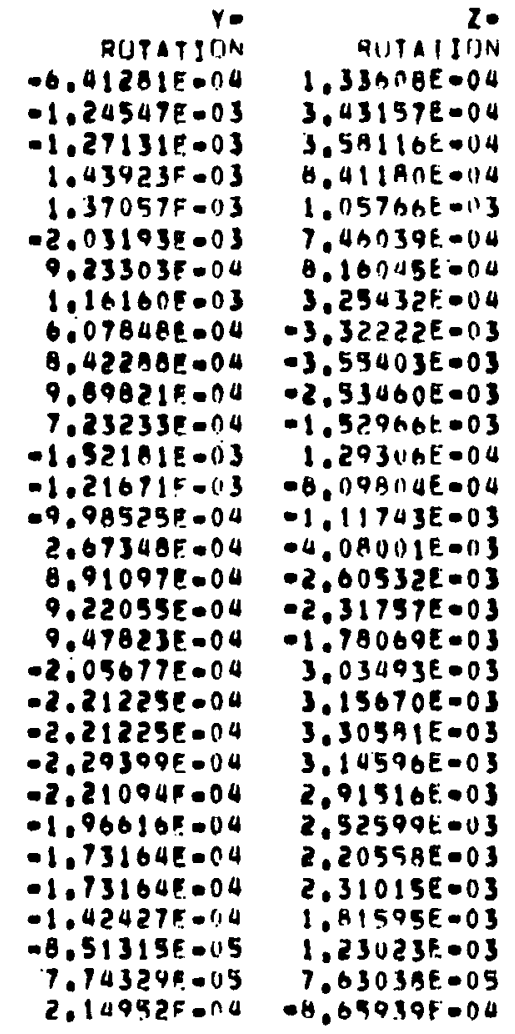


MODE NUMBER 9

FigenVECTURO NUHMLLIZEO TI) 1.000

UISPLACEMENTS/RUTATIONS OF UNHESTHAIMEN NUDES

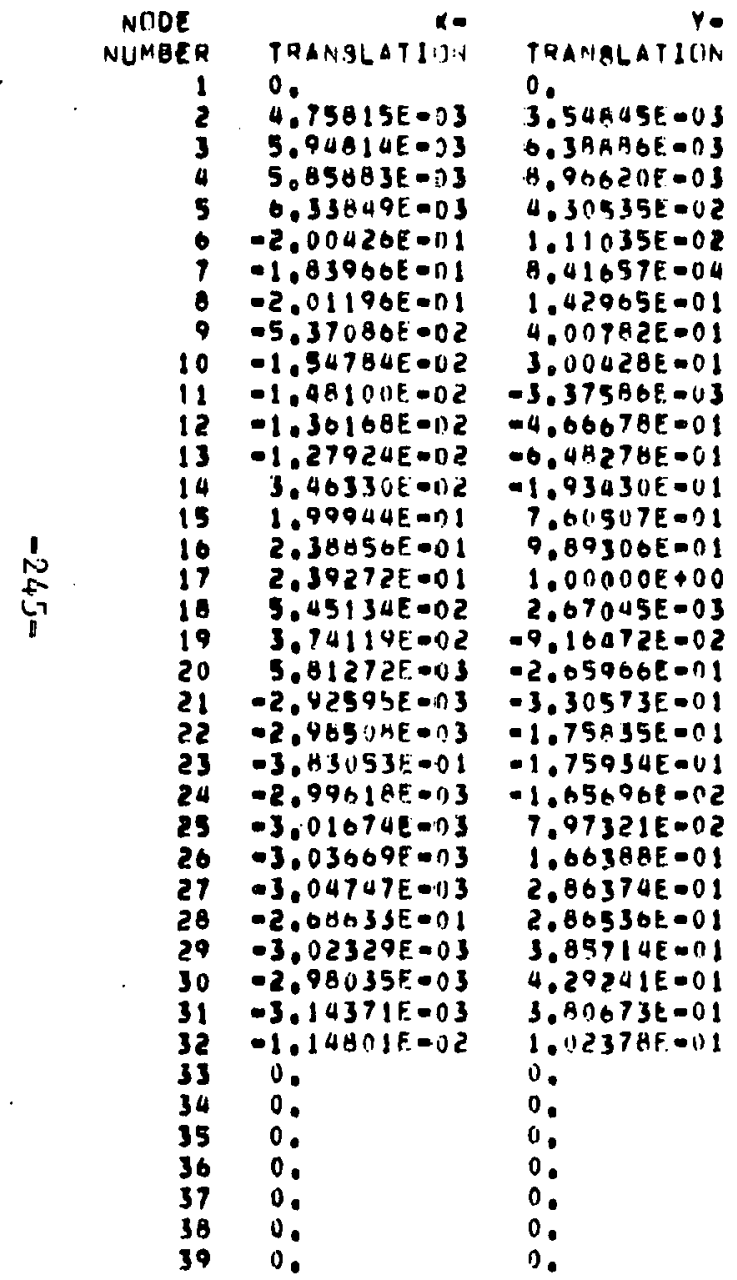

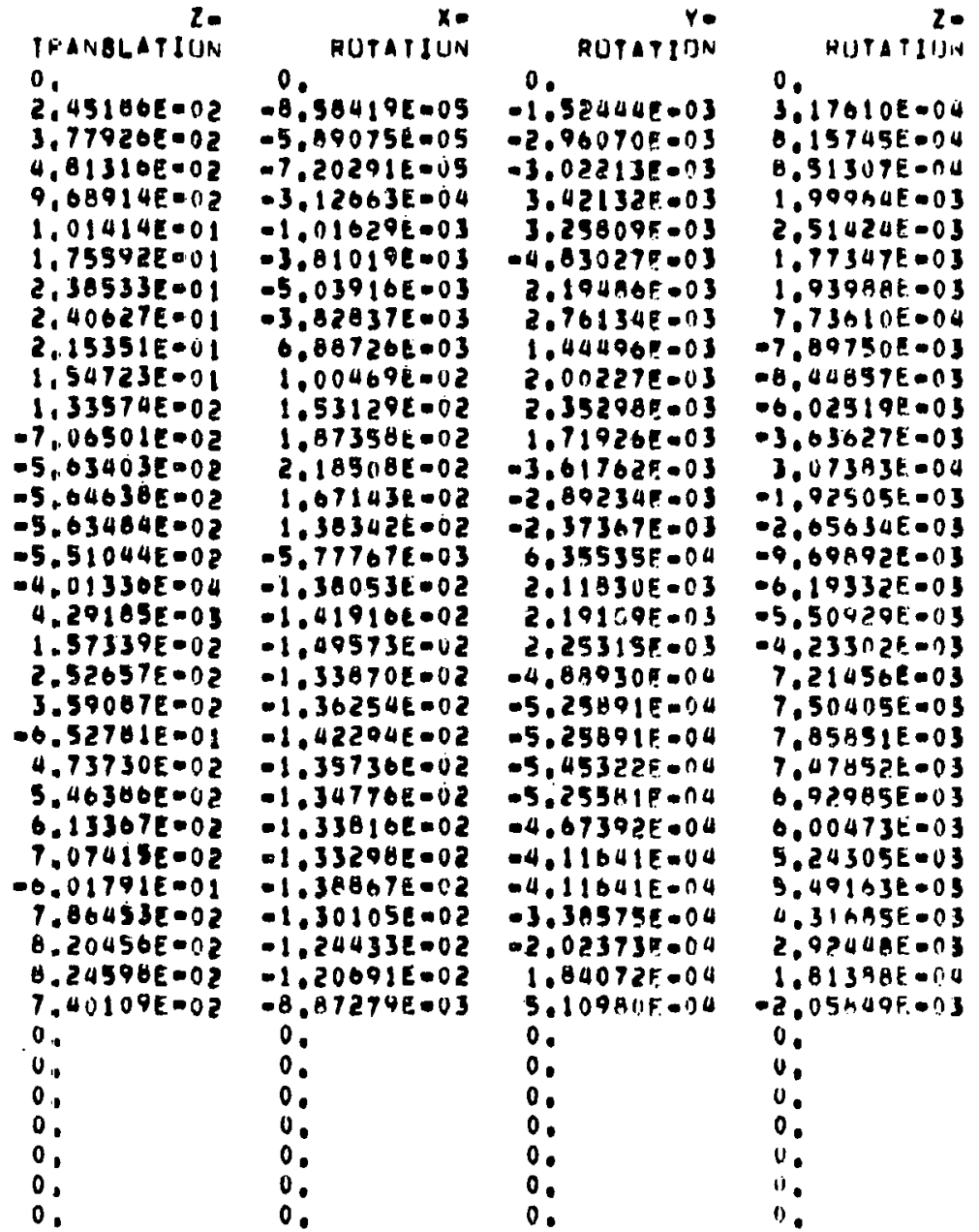


ETGENVECTURS NURMALIZED TO A UNIT HASS MATHIX

DISPLACEMENTS/RUTATIUNS UF UNRESTRAINED NUDES

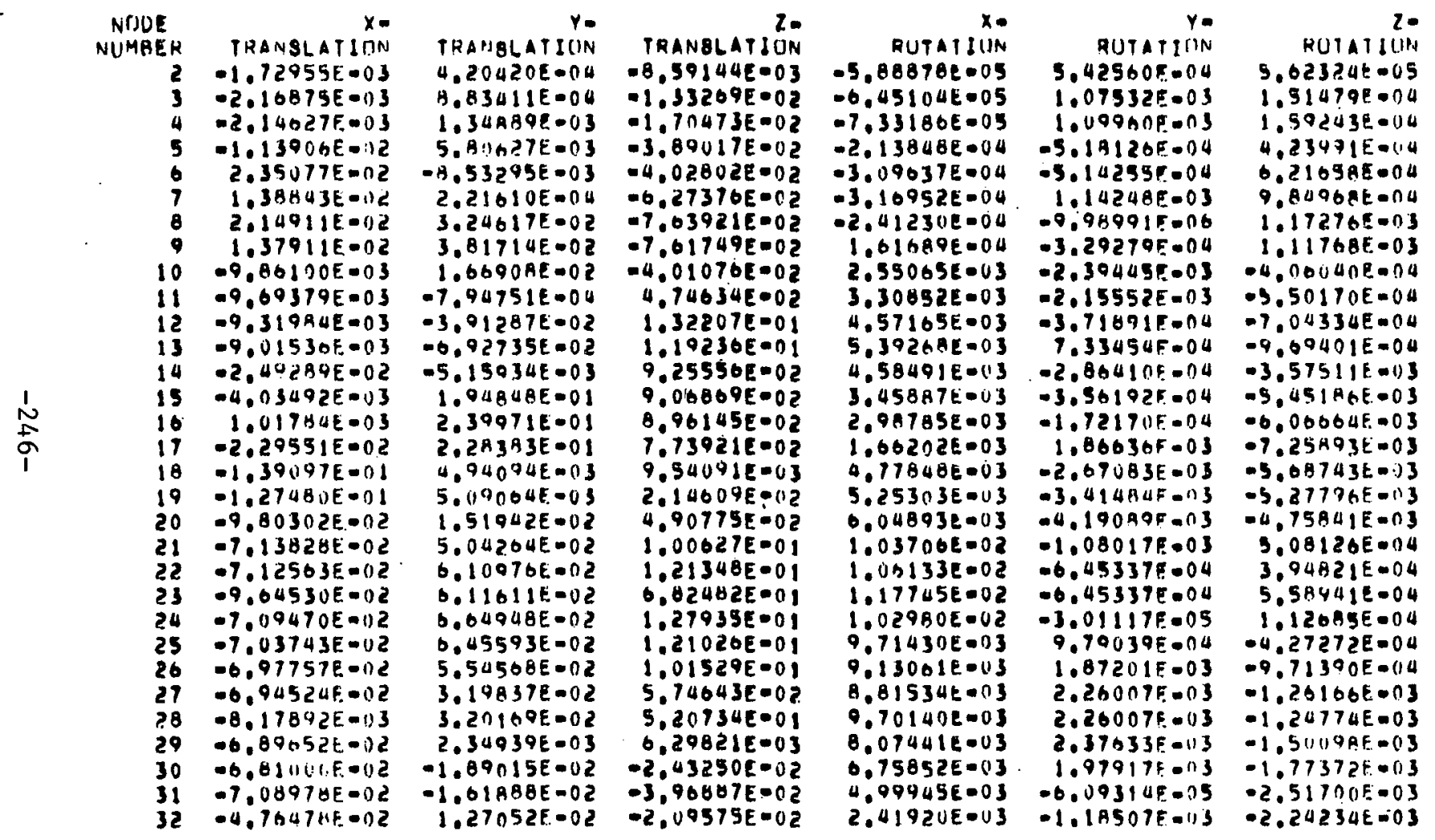


MUDE NJMBER

10

EgENVECTUAS NUHMALIIED TJ 1.000

UISPLACEMEHTSIRUTATIUNS IIF UIAESTRAINED NUDES

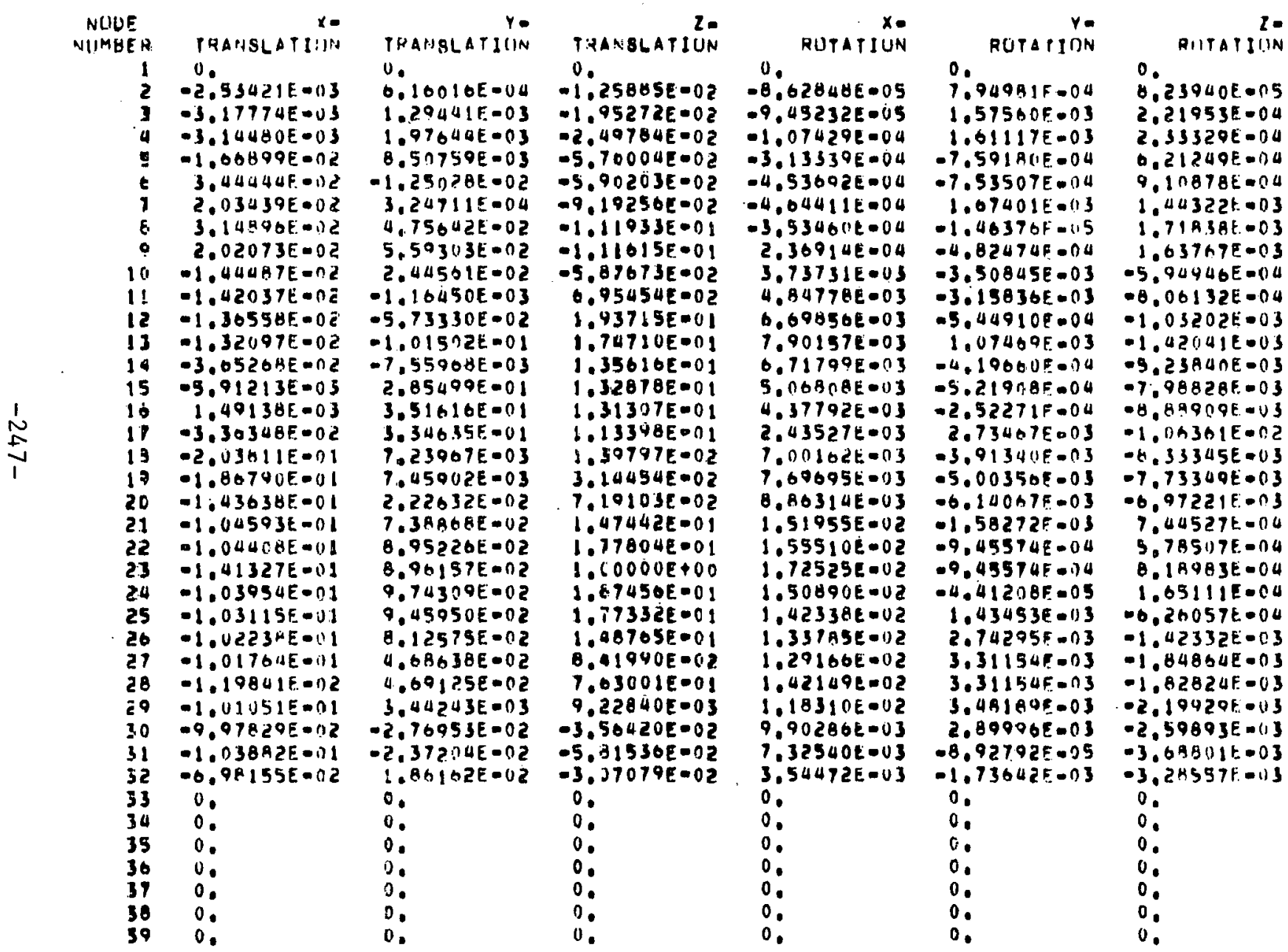


MODE NUMAER II

FREIJUENCY WO. BTUV

EgENVECTLHS HHHLLLIZE TU A UHIT HASS MATHIX

11 ISPLACEMEPITS/HUTATIINA UF UNRESTRAINED NUDES

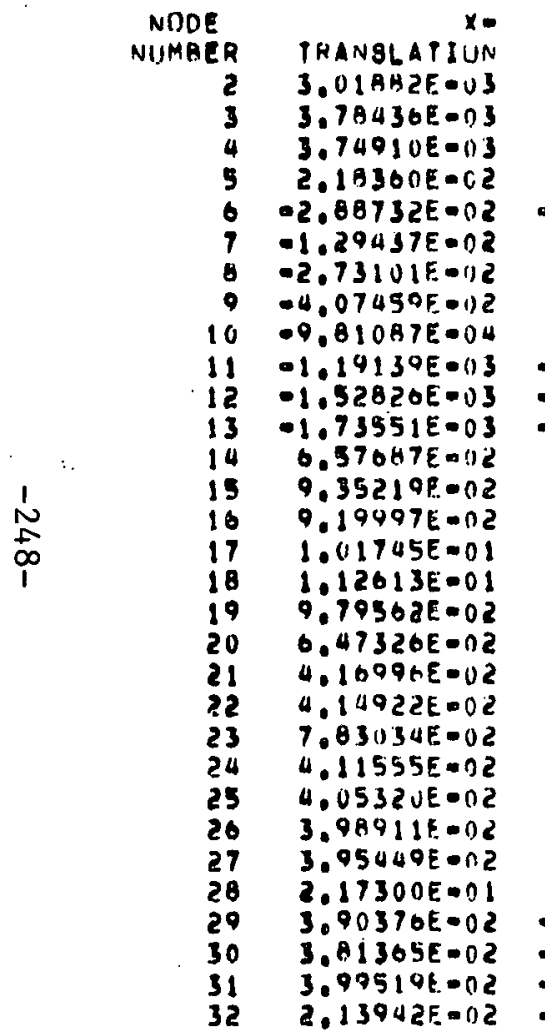

TRAHSLATIIIN

TRANBLATIUN

1.48744EDOZ -3.09285EOUS

$5.16535 E-04$ 0.792116004

$1.41852 E-03$

$0.17940 E-03$

$03.71421 E-03$

$2,94907 E=02 \quad-4,353858-05$

$6.809410-0,2$

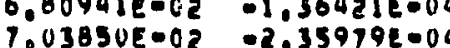

$1.94086 E=04$

$2.6905 \cup E=02$

T.0

1.246445001

.001 DEE $=n 2$

$2.53943 E 002$

$1,24010 E-01$

$-4.33535 E 004$

0.00669004

1.477608004
1.953958003

$1.95395 E=03$

3,680612003

$-5.37272 E-02$

$6.59748 F-02$

$1.21213 E=02$

$1.02575 E-n 1$

- 1.05767 CO

4.382076003

$3.66587 E-03$

$2.17505 E-03$

1.516720003

$.87125 E-01 \quad-7,79360 E-02$

-

$.76373 E 003$

3.31उA2E-Uट

$9.377119 E-02$

- .

- $8.96040 E-02$

- 01097003

$4.52229 E=03$

5. $404936-03$

$6.45220 E=03$

$8.41120 E-03$

$1.02333 E-01$

$-1.54475 E-1$

$.62530 E=(1) 1$

1. पद2 प2E-U1

$0.46190 E-U 3$

0.015516003

$8.24064 E 003$

$7.848108-03$

$7.84010 e-03$

8.50007E-02

- $1.30022 E=01$

$1.81373 E-02$

OSE. 02

$7.23031<003$

$7.79587 E 003$

$0.74920 E=03$

$5.88787 k=03$

- 0.0316

-9.42874E-02 -2.03758E-02

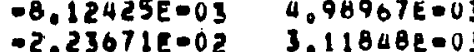

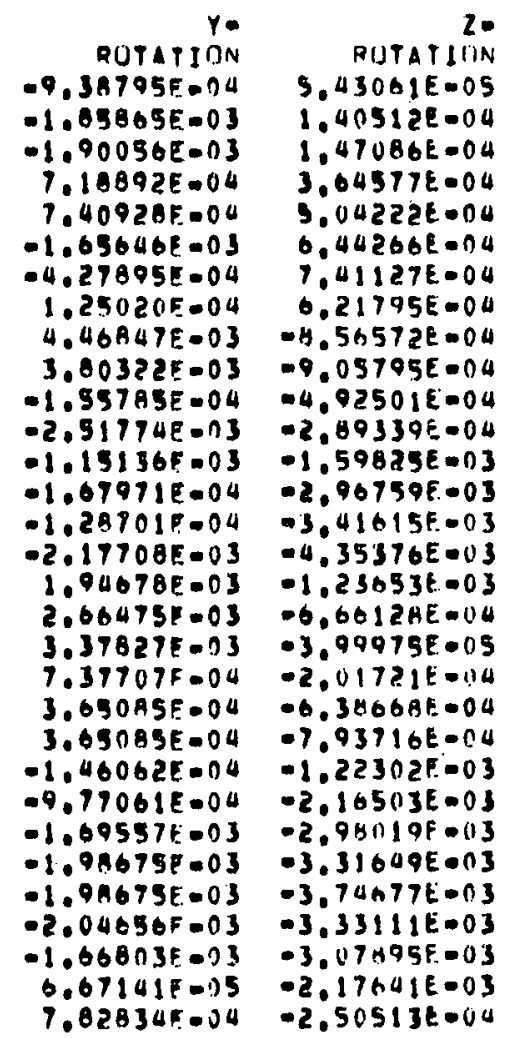


MODE NUMBER 11

? GENVECTUAS NUTMALIZEO TU 1.000

CISPL OCENENTS/RUTATIONS OF UNRESTHAINED NUNES

\begin{tabular}{|c|c|c|c|c|c|c|}
\hline $\begin{array}{r}\text { NODE } \\
\text { AUMBER } \\
1 \\
2 \\
3 \\
4 \\
5 \\
5 \\
6 \\
7 \\
6 \\
9 \\
10 \\
11 \\
12 \\
13 \\
14 \\
15 \\
10 \\
17 \\
10 \\
10 \\
20 \\
21 \\
22 \\
23 \\
24 \\
25 \\
20 \\
27 \\
28 \\
29 \\
30 \\
31 \\
32 \\
33 \\
34 \\
35 \\
36 \\
37 \\
38 \\
39\end{array}$ & 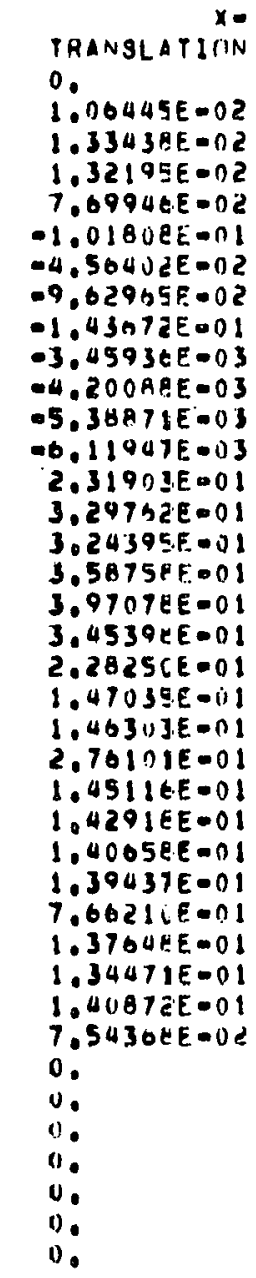 & 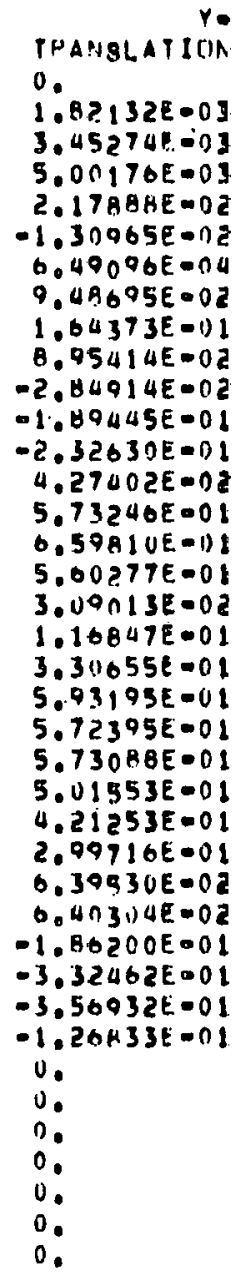 & 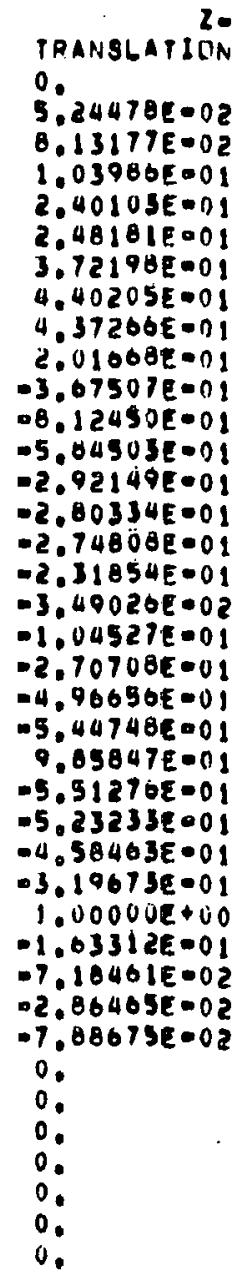 & 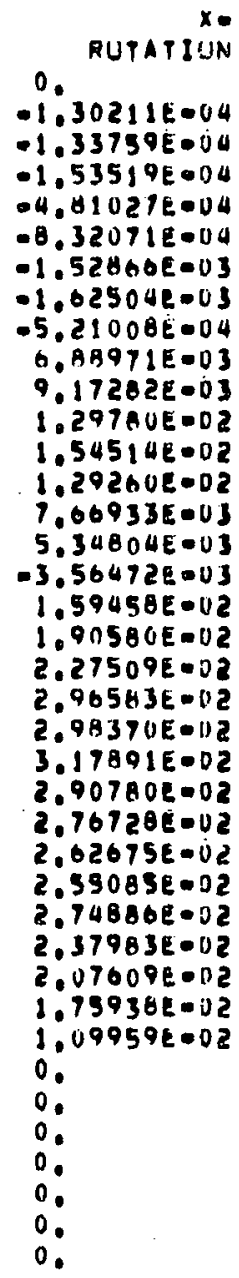 & 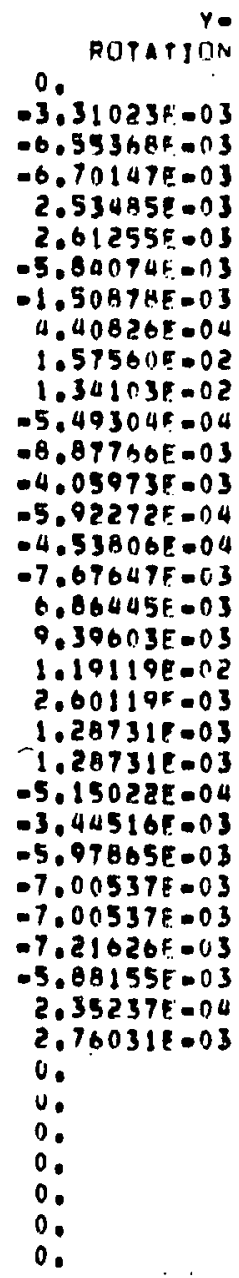 & 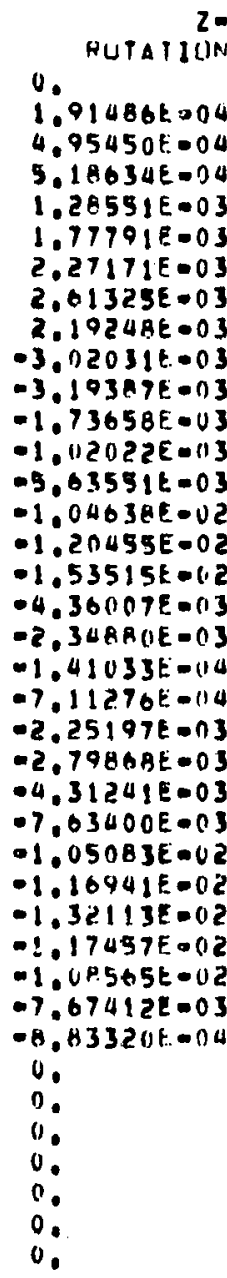 \\
\hline
\end{tabular}

IGEN OLUTIIN TIME LU U

E.gGENSOLUTIION:
POTNTING 
CASE 1

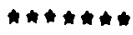

DIRECTION FACTUHS

$x=1.0000 \quad y=.0607 \quad z=1.0000$

INOICATUR FUR DISPLACEMENT IRR ACCELEHATTUN OPECTHUM E

EQ.0 DIBPLACEMENT

EU.1 ICCELERATIUN IN IN. A SEC, ?

EO.? ACCELERATIIIN IN GS

I SPECTRA aRE ENIEREO FIIR CASE 1. KINDE 2

Cluster Factur, CF. $=.00001$

No 


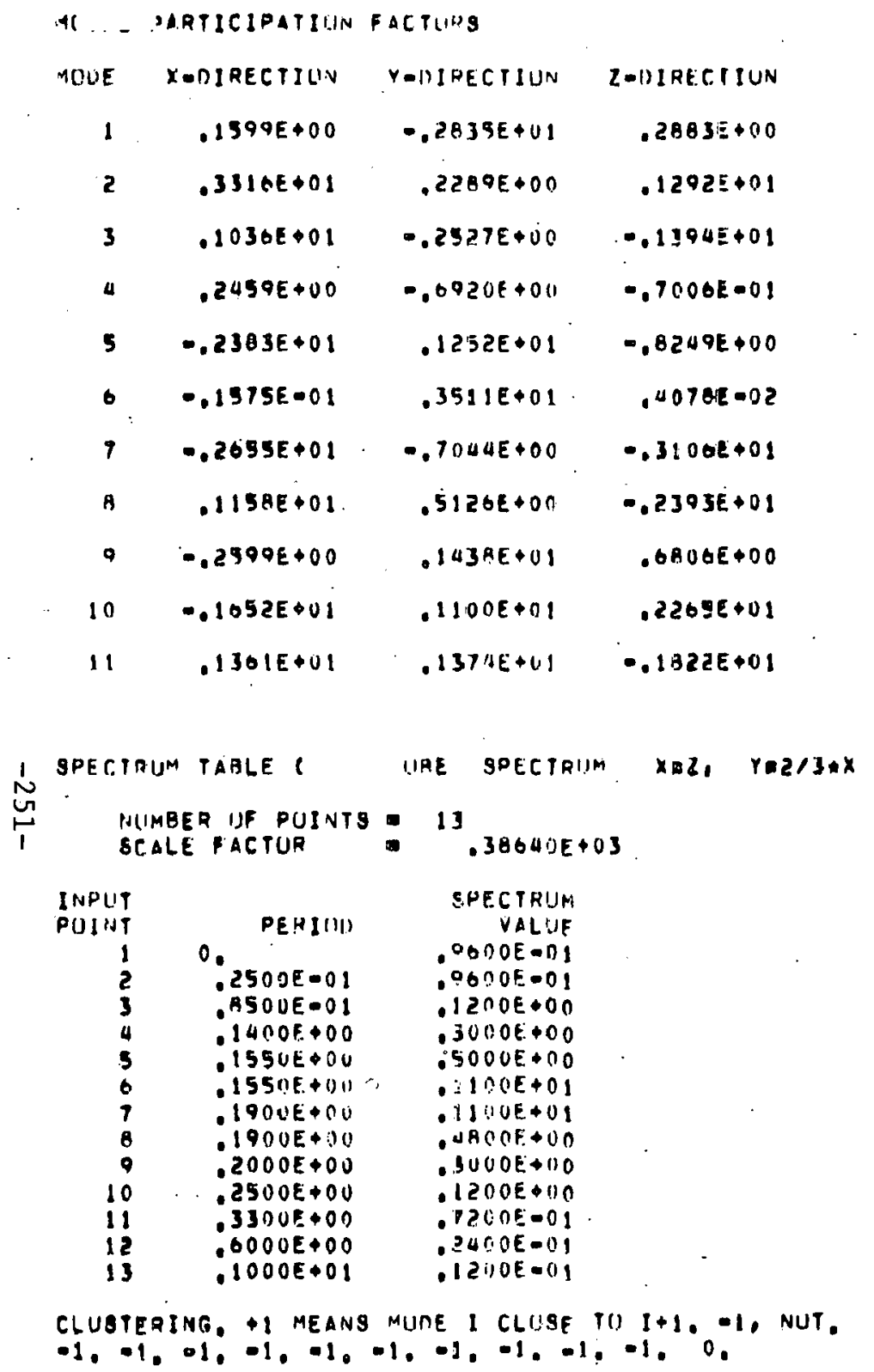




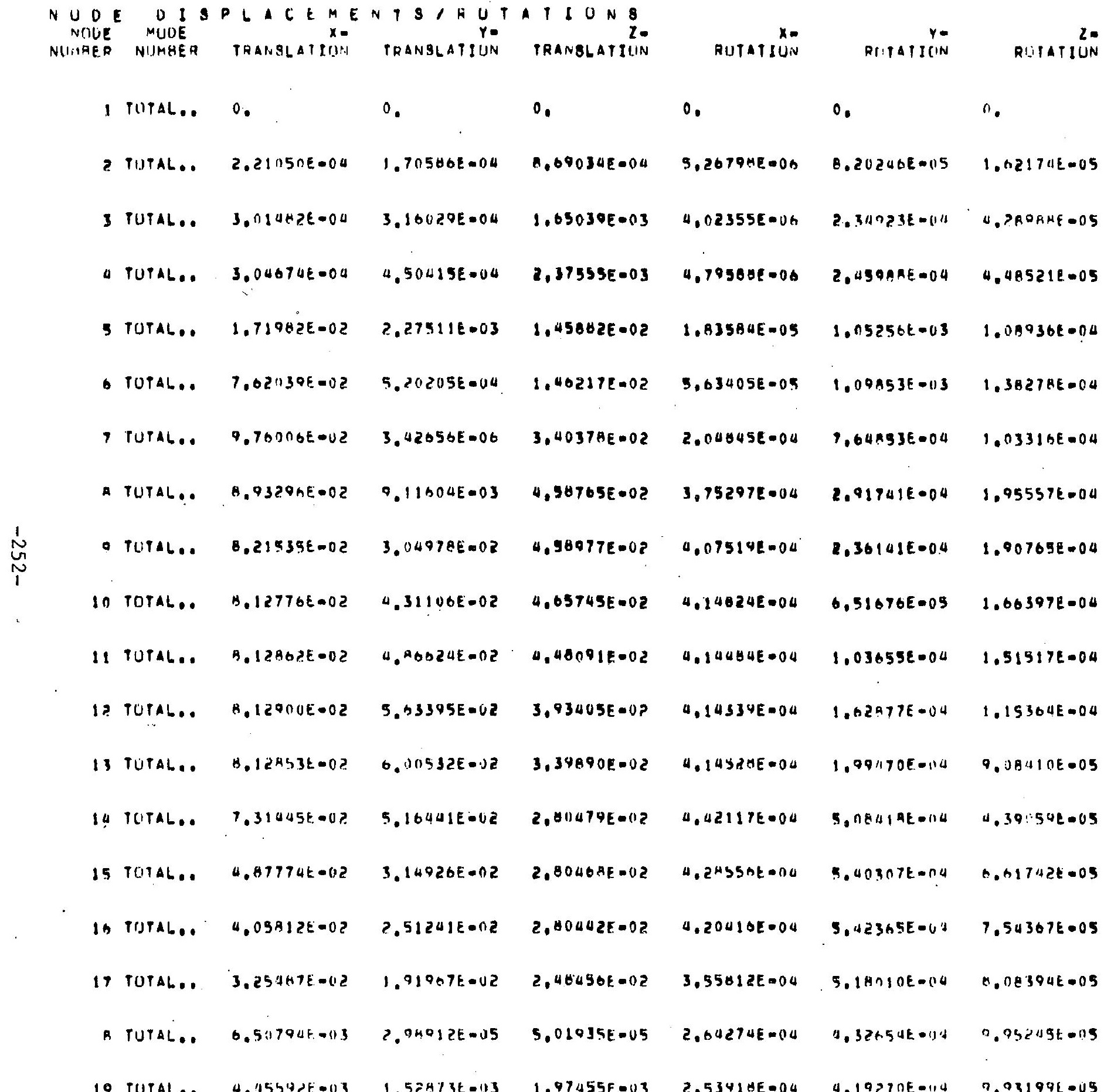




\begin{tabular}{|c|c|c|c|c|c|c|c|}
\hline $2 n$ & rotal.. & $1.45711 E=0.3$ & $4.3 i 115 t-03$ & $5.50370 E=03$ & $2,34014 E=04$ & 3. $33 ? 20 \mathrm{O}=0 \mathrm{~d}$ & $0.21462 E-05$ \\
\hline & 'vial.. & 1. TMOEREDO13 & $9.33602 E=0.3$ & $7.77122 E=03$ & $1.46242 E-114$ & $3.95286 E=0.5$ & $4.94522 E=05$ \\
\hline 22 & POTAL... & 1.7B11RE-03 & $4.484396-03$ & $1.00520 E=03$ & $1.307235 \cdot 04$ & $4.3933 \cup E=05$ & $4.932 H 9 E=05$ \\
\hline 23 & TUTAL.. & $2.87304 \mathrm{E}-\mathrm{U} 3$ & $4.18 \triangle 5 A E=03$ & $0,58944 E=03$ & $1.40334 E-04$ & $4.39334 E=05$ & $4.04477 E-09$ \\
\hline 20 & POTAL.: & $1.75080 E=03$ & $3.69432 E=03$ & $6.20105 E=03$ & $1.32955 E-04$ & $0.17723 t=09$ & $\triangle .87179 E-05$ \\
\hline 25 & TOTAL.. & $1.78 ?: 2 E=03$ & $3.2412 A E=03$ & $5.68741 E=03$ & $1.20501 E=04$ & $5.29035 E-1.5$ & $4.73 .357 E=05$ \\
\hline 20 & THTAL.. & $1.17331 E 003$ & 2. $.05638-03$ & $3.02989 E-03$ & $1.08175 E 0.04$ & $5.72 b 10 E=05$ & $4.61427 E-05$ \\
\hline 27 & TIJTAL.. & 1.17ABAE-03 & $2.10753 t=03$ & $3.90974 E-03$ & $1.01587 E-00$ & $5.95610 E=1.5$ & 4.547078 .05 \\
\hline $2 n$ & TIJAL... & $2.7312 R E=0.3$ & $2.19791 E=03$ & $5.93835 E-03$ & $1.02154 E=04$ & $5 . A 5+19 E=05$ & 4.561406 .05 \\
\hline 20 & TUTAL.. & $1.17757 t-03$ & $1.03040 \mathrm{E}=03$ & $2.00007 E=113$ & $9.47955 E=05$ & 5.431 ATE-OS & $4.44031 E=0.9$ \\
\hline 30 & TUTALE. & $1.17525 E-03$ & $1.7 H A 5 \triangle E-U 3$ & $2.17710 E-U 3$ & $8.29114 E=05$ & $5.5 A \cap 15 E-1.5$ & $4.21030 E=05$ \\
\hline 31 & TUTAL.. & $1.51934 t=03$ & $1.51347 E=03$ & $1.54340 E=03$ & $6.91994 E=05$ & $4.995 n A F=119$ & $3.80650 \varepsilon=05$ \\
\hline 32 & Total... & $5.13331 A E-\dot{U} 4$ & $a .0379 h E-O A$ & $5.30544 E=04$ & $4.20654 E-05$ & $4.01 ? 21 F-05$ & $? .436508-05$ \\
\hline 33 & TDTAL.. & 0. & 0. & 0. & 0. & 0. & 0. \\
\hline 34 & TOTAL.. & 0. & 0. & 0. & 0. & 0. & 0. \\
\hline 35 & TOTAL.. & 0. & 0. & 0. & 0. & 0. & 0. \\
\hline 36. & Tutal... & 0. & $n$. & 0. & 0. & 0. & 0. \\
\hline 39 & TUTAL... & u. & $\therefore$ & 0. & $n$. & $n$. & u. \\
\hline 32 & TUTAL... & 0. & 1). & 0. & 0. & 0. & $n$. \\
\hline 39 & TUTAL.. & 0. & $n$ & 0. & 0. & 0. & $n_{0}$ \\
\hline
\end{tabular}


RE SPONBE SPELIRUM STRES: COMPIINENTS (KINDE Z)

UR EACH ELEMENT, IHE FIJLIINING INFUAMATIUN IS PHINTEOI

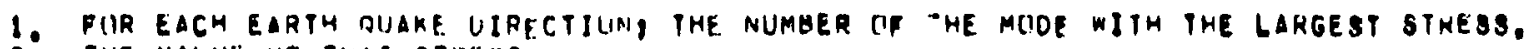

2. THE VALUE UF THAT STREYS.

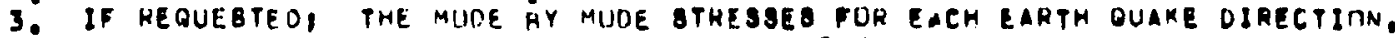

a. THE REQULTANT FIIR SACH EARTH IJUAKE DIRECTIUN.

5: THE GRAHD THTAL UF THE THPEE EIRTH DUAKE OIREC-IUNB.

(NUTE: THE $X_{1}, Y$, DR $Z$ REPEAS TU PHE EARTH WUAKE UIRECTEON.)

ELEMENT TYPE (3/U P I D , , , ELEMENT NUMHER 1 1)

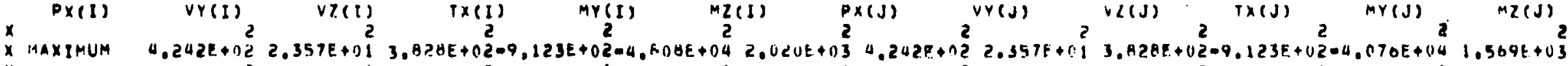

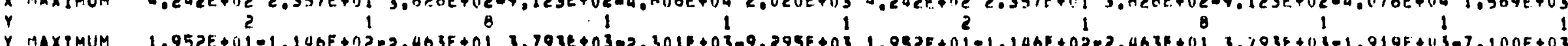

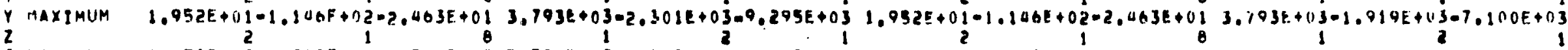

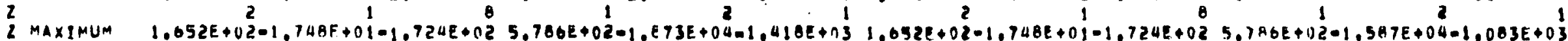

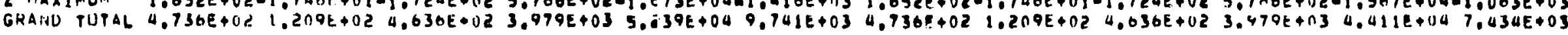
ELEMENT TYPE $13 / 0$ P I E, 11, ELEMENT NUMAER $(2)$

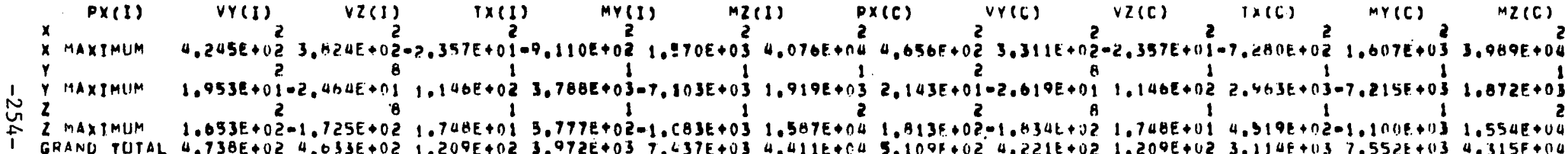
ELEMENT TYPE (3/U P I P E I 11, ELEMENT NUMGEK $(2)$

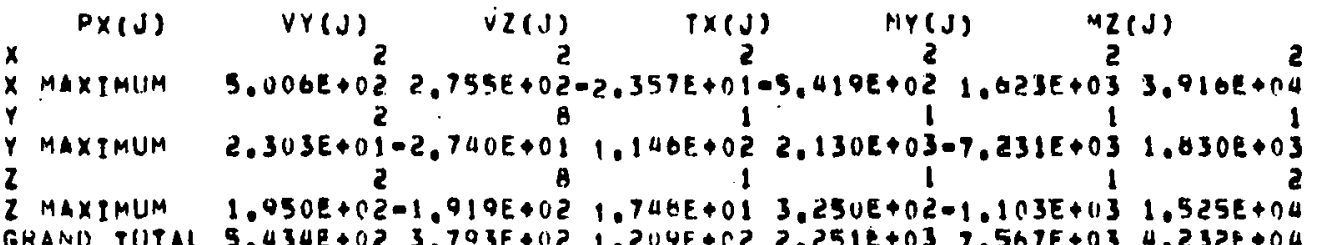
GRANO TUTAL $5.434 E+023.703 E+021.2 U 4 E+C 2 \quad 2.251 E+037.567 E+03 \quad 4.232 E+04$

ELEMENT TYPE (3/D) P I PE , , , ELEMENT NIJMHEH 13 )

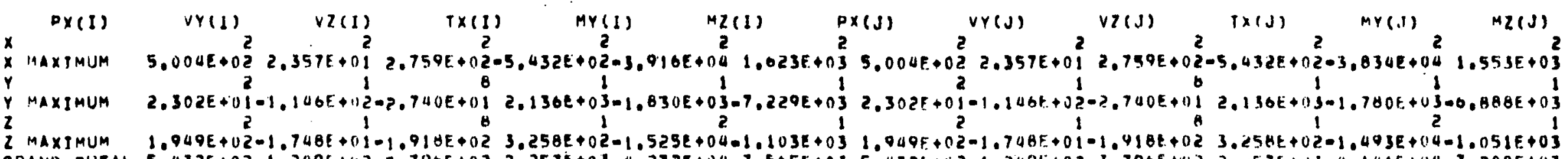

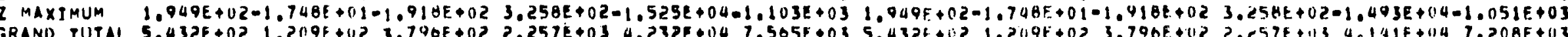
ELE TYPE (3/0 P D E , , , ELEMENT NUMAER 1 (4)

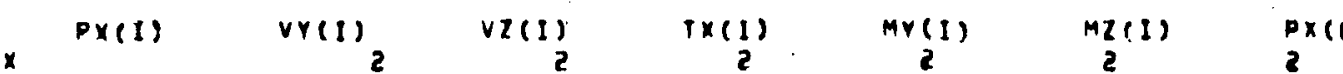




\begin{abstract}
ELEMENT TYPE (3/0 P I P E $), 1$, ELIMENT NUMAEH 1 A)
\end{abstract}

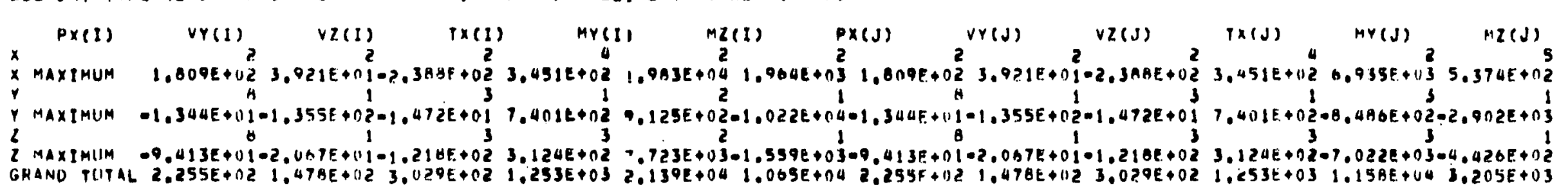
ELEMENT TYPE (3/0 P I DE $, 1,1$ ELEMENT NUMBEK 19 )

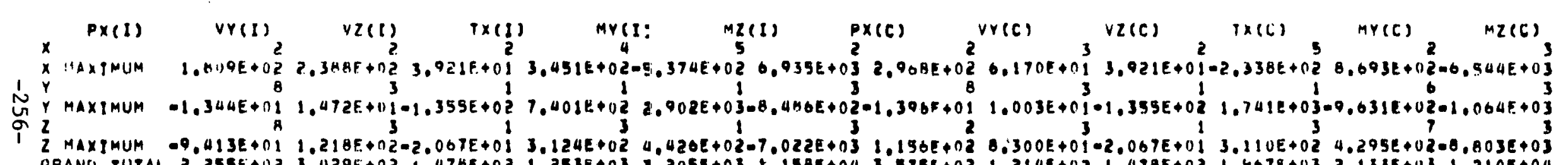

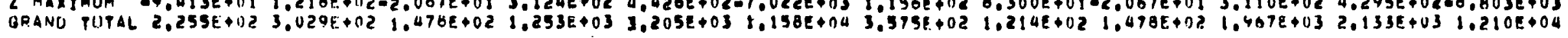
ELEMENT TYPE (3/0 D I PE $, 1,1$ ELEMENT NUMBEh 1 O)

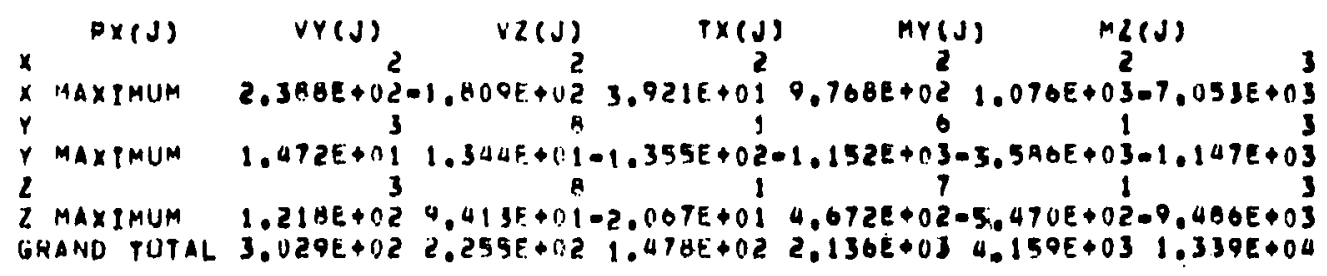

ELEAEHT TYPE (3/D P I E , 1, , ELEMENT NUMBEH 1 10)

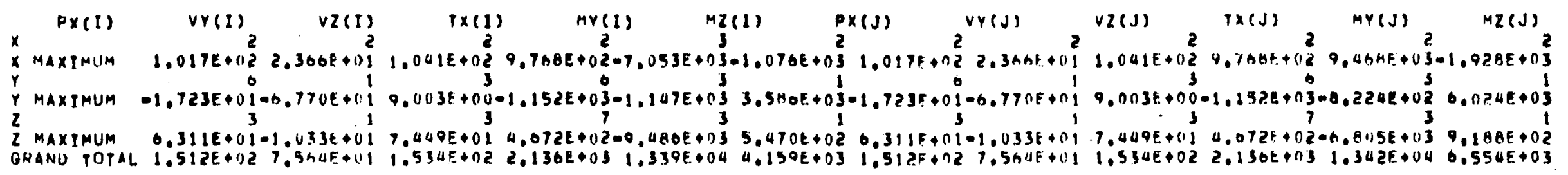

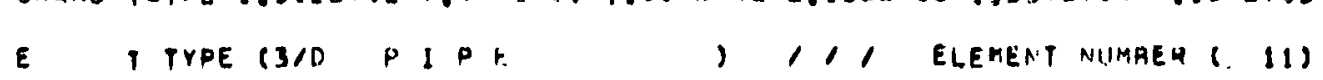

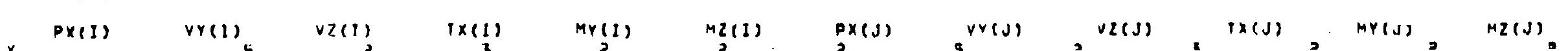




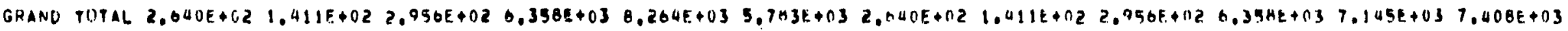
ELEMENT TYPE (3/0 P I PE , 1,1 ELEMENT NUMBER $(16)$

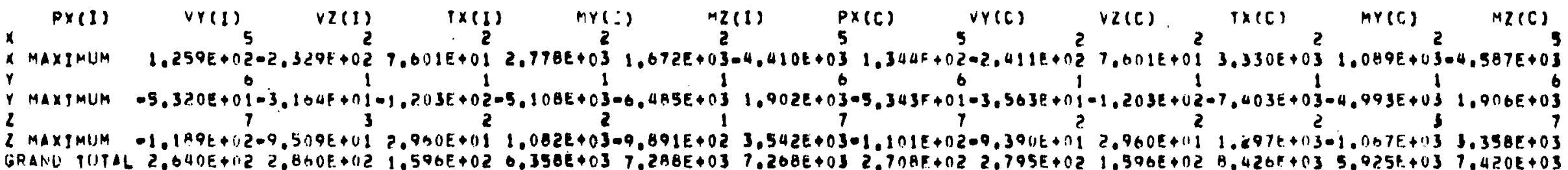
ELFRENT TYPE (3/6) I D E , 1, ELEMENT NUMBER 1 (6)

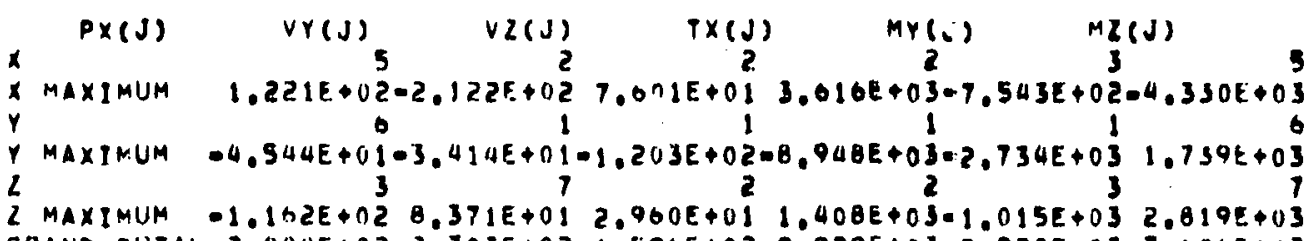

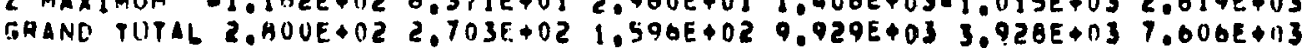

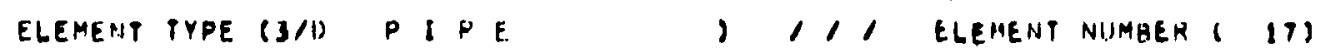

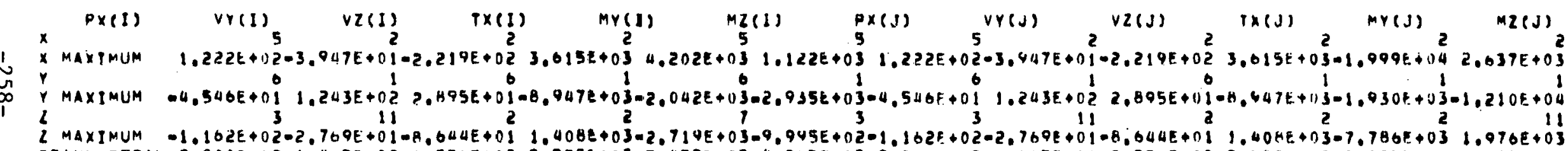

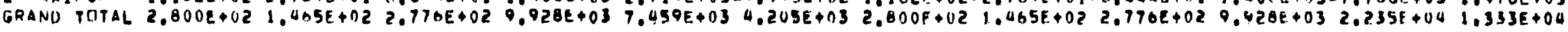
ELEMENT TYPE (3/L $P$ IP E $, 1,1$ ELEMENT NUMAEK $($ IA)

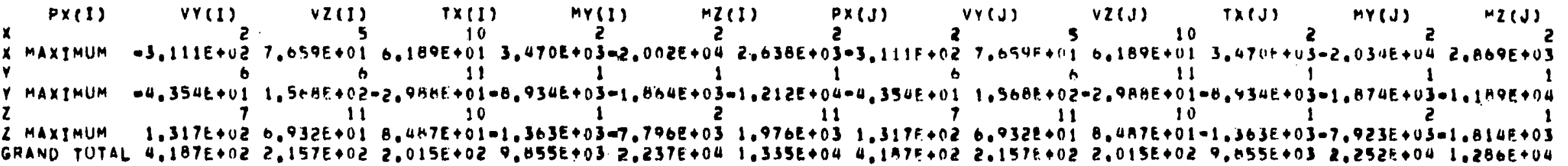
ELEMENT TYPE (3/0 P I P E , 1,1 ELEMENT NUMBEA 1 19)

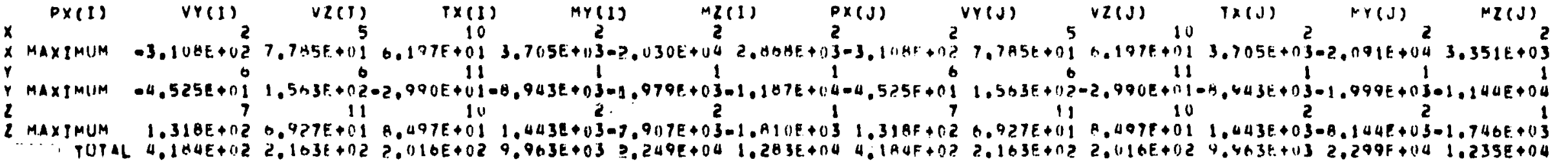
NT TYPE (3/0 P I HE , 1, ELEMENT NUMBEA $(20)$

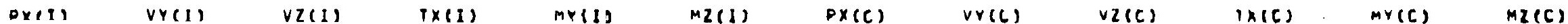




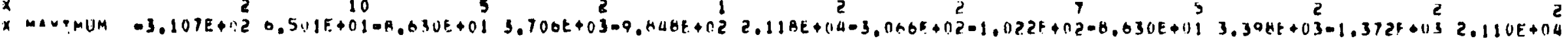

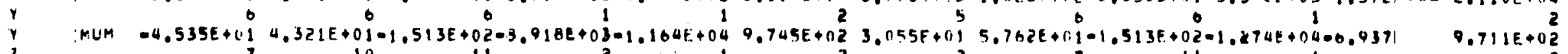

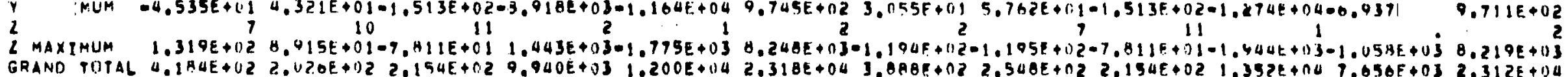

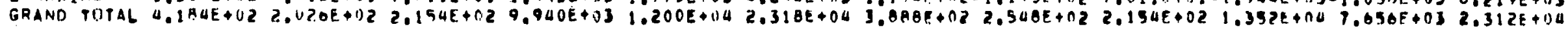
ELEMENT TYPE (3/0 P I P E $), 1 /$ ELEMENT NUMBER $(20)$

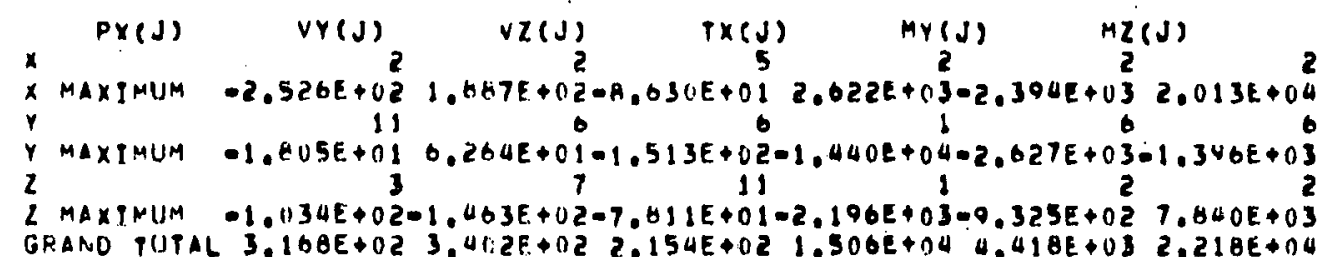

ELEMEHT IYPE (3/D P I HE , 1,1 ELEMENT NUMRER ( 21 )

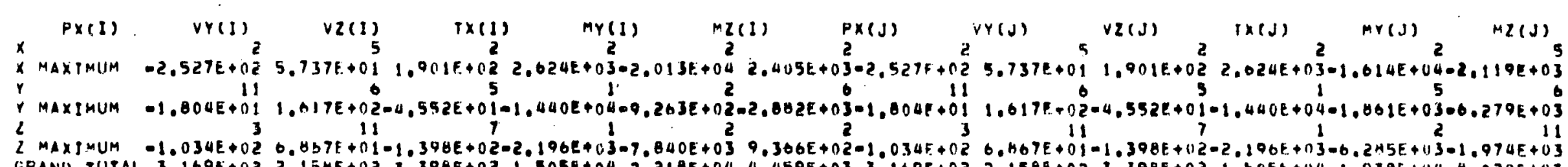
ज̃

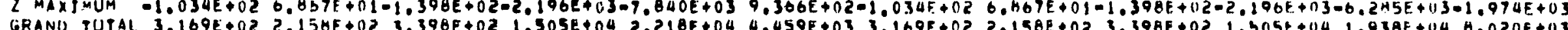
ज̈ ELEMENT TYPE (3\% P 1 PE

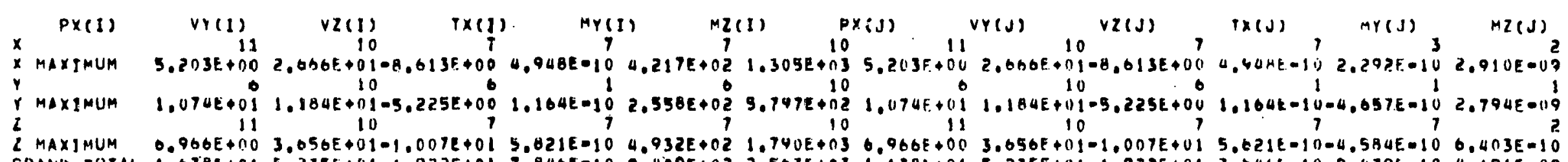

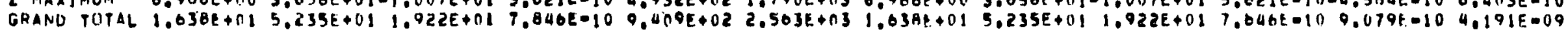
ELEMENT TYPE (3/0 P I P E , 11 . ELEMENT NUIMHER 1 23)

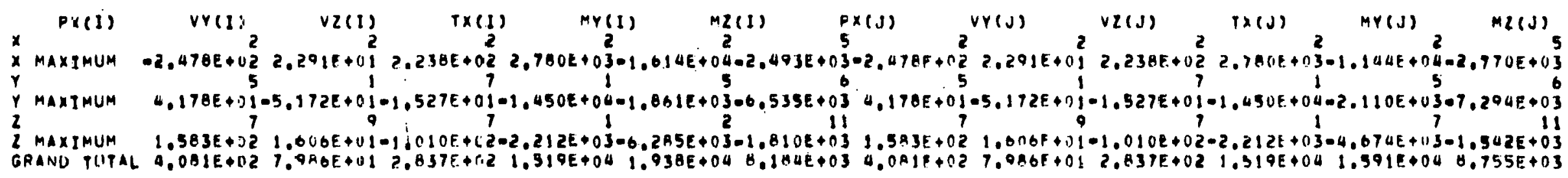
ELEMENT TYPE (3/U P I PE , 1,1 ELEMENT NUMHER 1 2a)

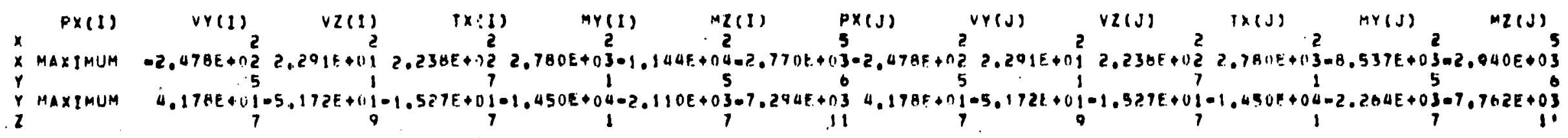




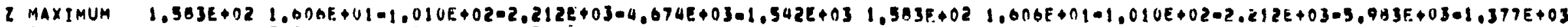

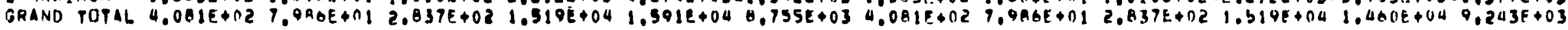
ELEMENT PYPE (3/0 P I P E , $1 / 1$ ELEMENT NUMBEK $(25)$

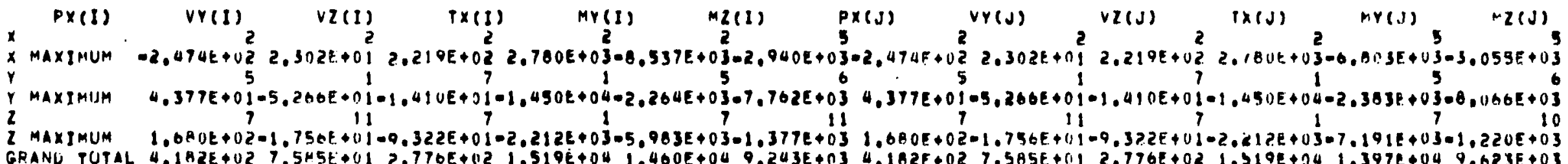
ELEMENT TYPE (3/0 P I PE I, / ELEMENT NUMEEA $(26)$

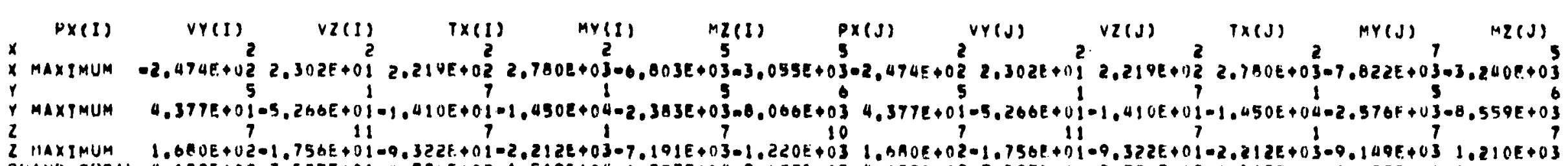

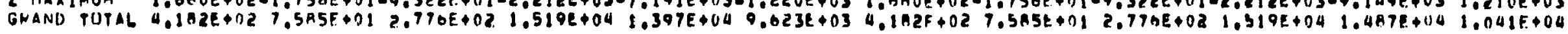
ELEMENT IYPE (3/D P I D E , 1,1 ELEMENT NIMAER 6 (27)

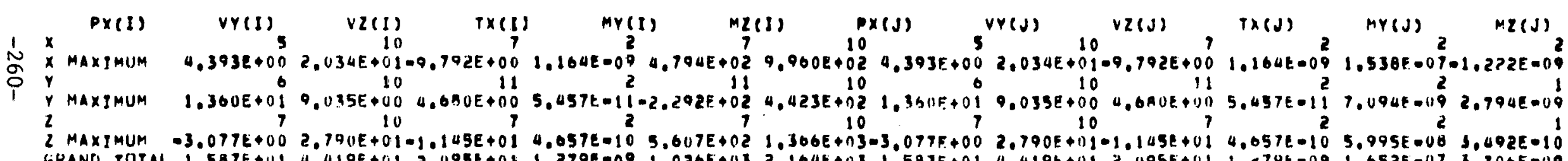

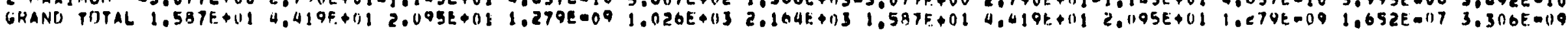
ELEMENT TYPE (3/U P I D E $1,1 /$ ELEMENT NIIMBEN $($ 2B)

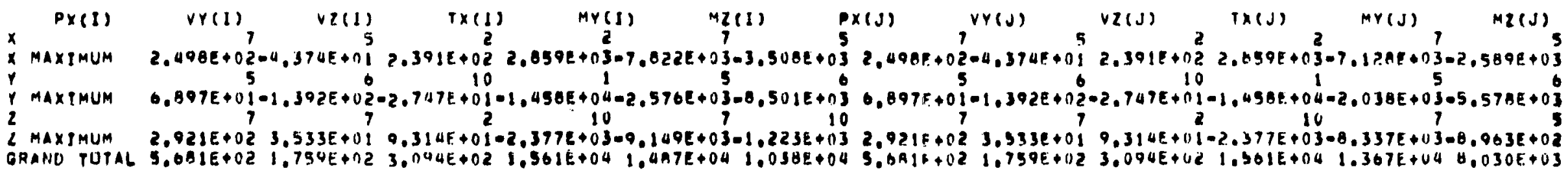
ELEMENT TYPE (3/0 P I D E ) 1,1 ELEMENT NUMBEH 1 20)

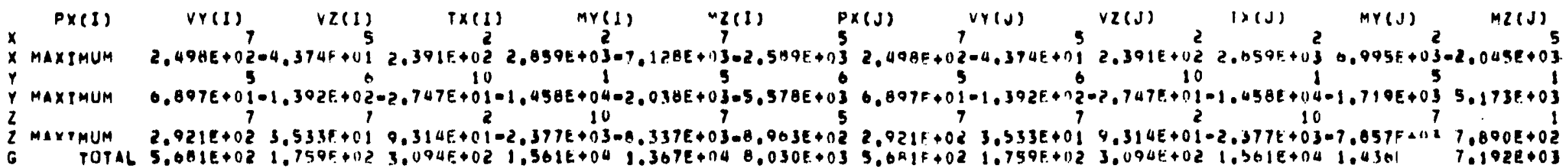


GRAND TOTAL 3, U27E+01 O.

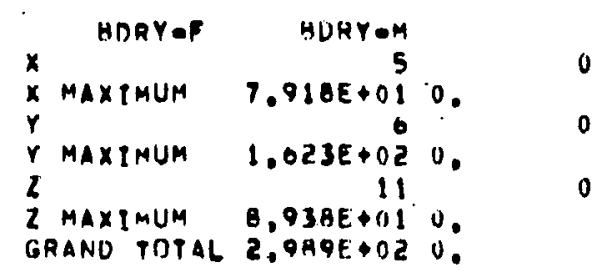

ELEMENT TYPE IB UUNDAR $Y$

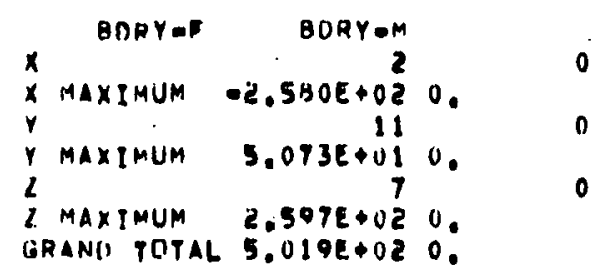

ELEMENT IYPE CB OUNDA $Y$

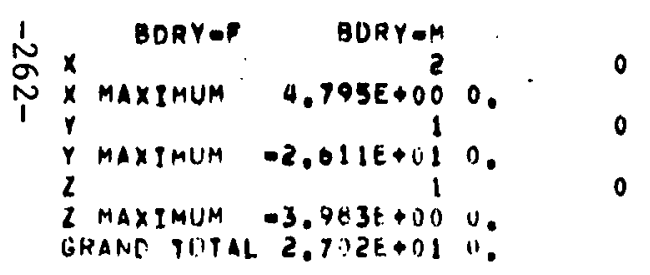

ELEMENT TYPE IH WUN U A R $Y$

\begin{tabular}{|c|c|c|c|}
\hline BARY $\oplus$ & GURYOM & & \\
\hline$x$ & 2 & & 0 \\
\hline$X$ MAXIMUM & $-3.974 E+00$ & u. & \\
\hline$Y$ & 1 & & 0 \\
\hline Y MAXIMUM & -6.4275 .01 & 0. & \\
\hline 2 MAXIMUM & & & 0 \\
\hline GRAND TUTAL & $4,586 E+1) 0$ & 0 & \\
\hline
\end{tabular}

ELEMENT TYPE (BUUNDAN Y

\begin{tabular}{|c|c|c|}
\hline EDHY $P$ & HURYAR, & \\
\hline MAXIMUM & $-5,100 E-U I$ & 0 \\
\hline & $-\quad-0$ & \\
\hline MAXTMUM & $-3.075 E-01$ & 0 \\
\hline $\begin{array}{l}\text { IMUM } \\
\text { TOTAL }\end{array}$ & $\begin{array}{r}-4.229 E-01 \\
9.524 E=01\end{array}$ & $\begin{array}{l}0 \\
0\end{array}$ \\
\hline
\end{tabular}

) 1 1 ELEMENT NUMBER ( 3)

) 1,1 Glement nUmben 1 a)

$, 1,1$ element numaen $($. S) 

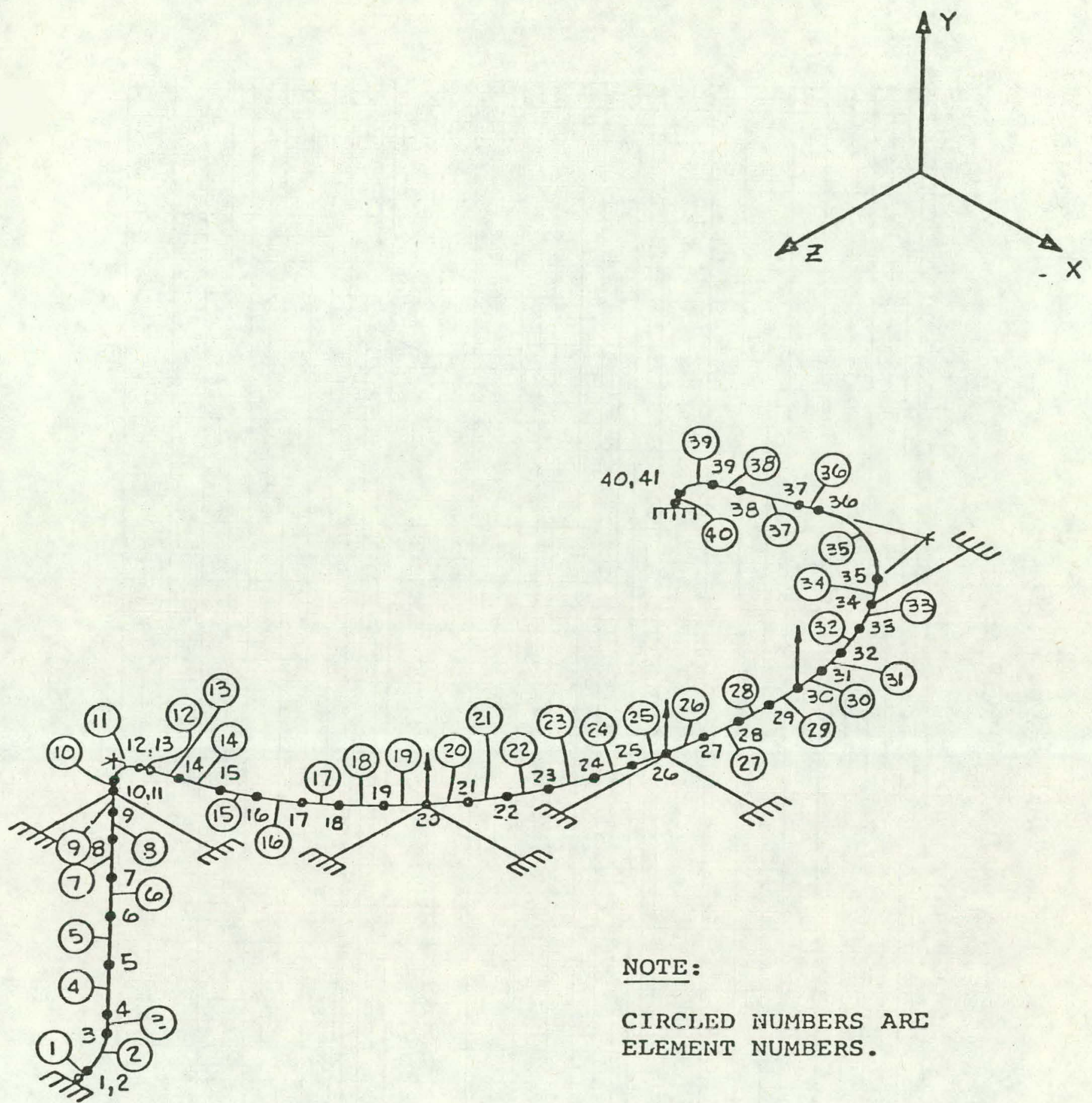

BENCHMARK PROBLEM NO. 6

Figure 6.1 


\section{$X$ AND $Z$ SPECTRUM}

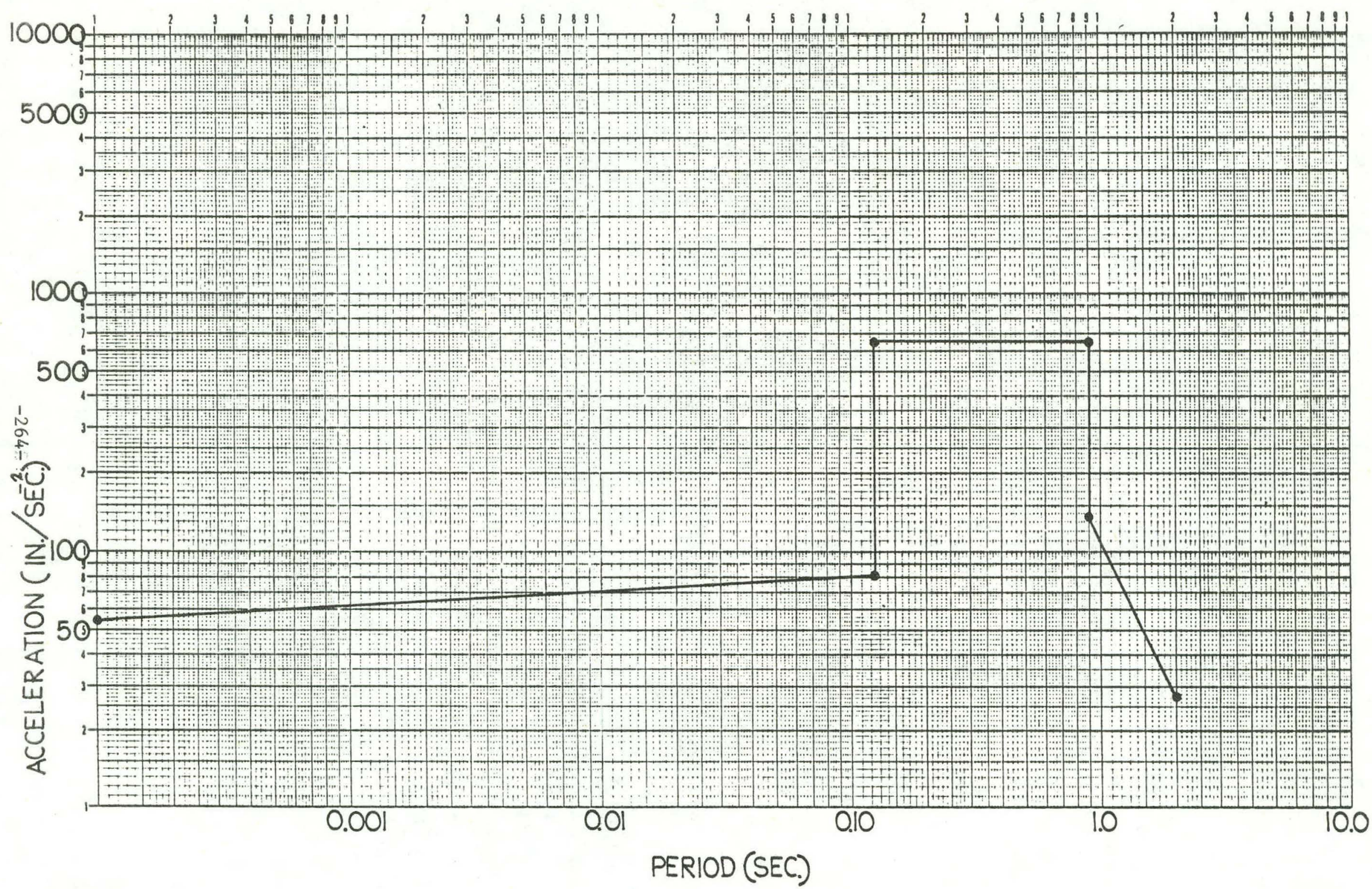

Figure, 6.2 


\section{Y SPECTRUM}

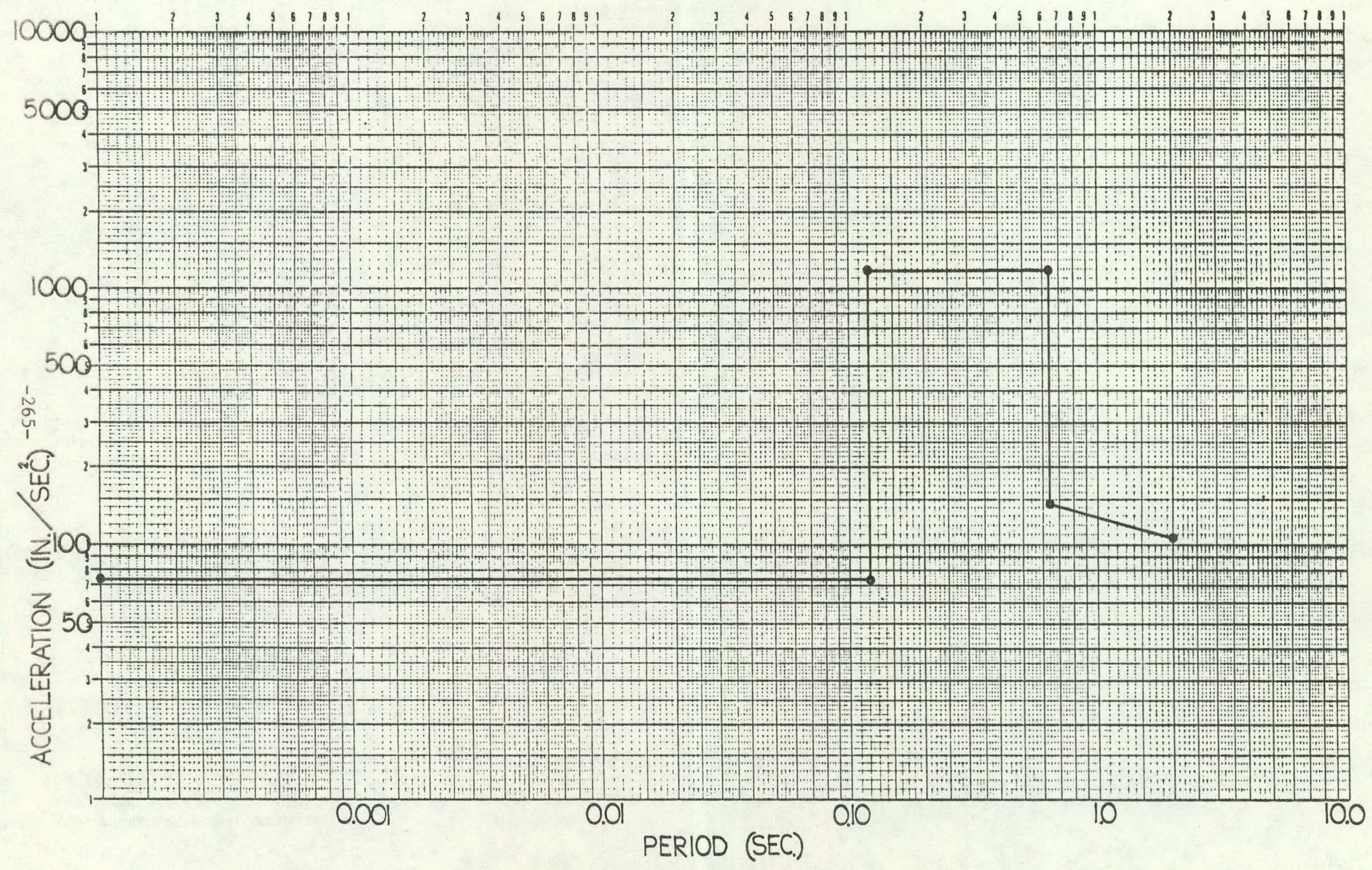

Figure 6.3 
PIPING BENCHMARK PROBLEM - ND. 6

C ONTROL INFORMATION

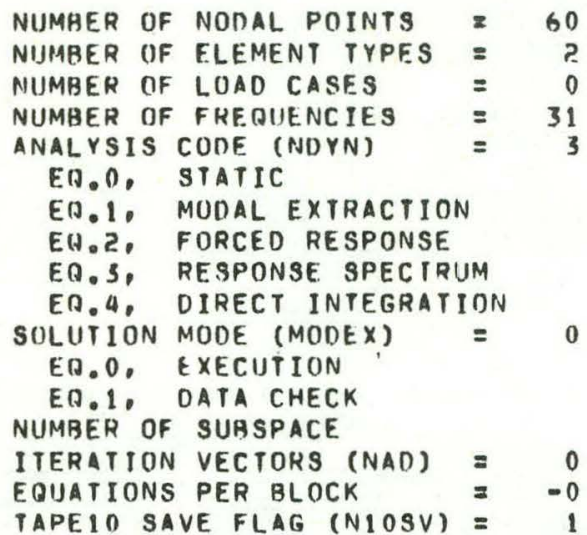

NODAL POINT INPUT DATA

NIDE BOUNDARY CONDITION CODES

\begin{tabular}{|c|c|c|c|c|c|}
\hline \multirow[b]{2}{*}{ AMBER } & & & & \\
\hline & $x$ & Y & $z$ & $x x$ & $Y Y$ \\
\hline 1 & -0 & -0 & -0 & -0 & -0 \\
\hline 2 & -0 & $=0$ & -0 & -0 & -0 \\
\hline 3 & -0 & -0 & -0 & -0 & -0 \\
\hline 4 & -0 & -0 & -0 & -0 & -0 \\
\hline 5 & -0 & $=0$ & -0 & $=0$ & -0 \\
\hline 6 & -0 & $=0$ & -0 & -0 & -0 \\
\hline 7 & $=0$ & $=0$ & -0 & -0 & $=0$ \\
\hline 8 & -0 & $=0$ & -0 & $=0$ & $=0$ \\
\hline 9 & $=0$ & .0 & -0 & -0 & -0 \\
\hline 10 & $=0$ & -0 & $=0$ & -0 & -0 \\
\hline 11 & -0 & -0 & -0 & -0 & -0 \\
\hline 1? & -0 & -0 & -0 & -0 & -0 \\
\hline 13 & -0 & $=0$ & -0 & -0 & -0 \\
\hline 14 & $=0$ & -0 & -0 & $=0$ & -0 \\
\hline 15 & -0 & -0 & -0 & -0 & -0 \\
\hline 16 & -0 & -0 & -0 & $-1)$ & -0 \\
\hline 17 & $=0$ & -0 & $=0$ & $-1)$ & -0 \\
\hline 18 & -0 & $=0$ & -0 & -0 & $-n$ \\
\hline 19 & $=0$ & $=0$ & -0 & -0 & -0 \\
\hline 20 & -0 & -0 & -0 & -0 & -0 \\
\hline 21 & $=0$ & -0 & -0 & -0 & -0 \\
\hline 22 & -0 & -0 & $=0$ & $-1)$ & -0 \\
\hline 23 & $=0$ & -0 & -0 & -0 & -0 \\
\hline 24 & -0 & -0 & $=0$ & -0 & -0 \\
\hline 25 & $=0$ & -0 & -0 & $=0$ & -0 \\
\hline 26 & -0 & -0 & $=0$ & -0 & -0 \\
\hline 27 & $=0$ & -0 & -0 & $=0$ & $=0$ \\
\hline & 0 & -0 & -0 & -0 & -0 \\
\hline & 0 & -0 & -0 & -0 & -0 \\
\hline & 0 & $=0$ & -0 & $=0$ & $=0$ \\
\hline 31 & -0 & -0 & -0 & $=0$ & $=0$ \\
\hline
\end{tabular}

NODAL POINT COORDINATES

\begin{tabular}{|c|c|c|c|c|}
\hline$x$ & r & 2 & & $T$ \\
\hline 126.000 & $\triangle B 3.996$ & 705.840 & -0 & $=0.000$ \\
\hline 126.000 & 483.996 & 704.640 & -0 & -0.000 \\
\hline 126.000 & 528.996 & 659.640 & -0 & $=0.000$ \\
\hline 126.000 & 567.996 & 659.640 & -0 & -0.000 \\
\hline 126.000 & 651.996 & 659.640 & -0 & $-0.0 \cap 0$ \\
\hline 126.000 & 735.996 & 659.640 & -0 & $=0.000$ \\
\hline 126.000 & 802.596 & 659.640 & -0 & -0.000 \\
\hline 126.000 & 869.196 & 659.640 & -0 & -0.000 \\
\hline 126.000 & 917.196 & 659.640 & $=0$ & $=0.000$ \\
\hline 126.000 & 965.196 & 659.640 & -0 & $=0.0 \cap 0$ \\
\hline 126.000 & 968.196 & 659.640 & -0 & -0.000 \\
\hline 169.860 & 1013.196 & .449 .560 & $=0$ & $=0.000$ \\
\hline 173.928 & 1013.196 & 648.624 & -0 & -0.000 \\
\hline 229.836 & 1013.196 & 630.996 & -0 & -0.000 \\
\hline 283.992 & 1013.196 & 608.556 & $=0$ & -0.000 \\
\hline 335.988 & 1013.196 & $581.4 A 4$ & $=0$ & -0.000 \\
\hline 385.428 & 1013.196 & 550.080 & -0 & -0.000 \\
\hline 431.940 & 1013.196 & 514.392 & -0 & -0.000 \\
\hline 475.164 & 1013.196 & 474.780 & -0 & -0.000 \\
\hline 514.776 & 1013.196 & 431.556 & -0 & -0.000 \\
\hline 550.464 & 1013.196 & 385.044 & -0 & -0.000 \\
\hline 581.964 & 1013.196 & 335.604 & -0 & .0 .000 \\
\hline 609.036 & 1013.196 & 283.608 & -0 & $=0.000$ \\
\hline 631.474 & 1013.196 & 229.452 & -0 & $=0.000$ \\
\hline 649.104 & 1013.196 & 173.544 & -0 & $=0.000$ \\
\hline 661.788 & 1013.196 & 116.304 & $=0$ & -0.000 \\
\hline 669.444 & 1013.196 & 58.176 & -0 & -0.000 \\
\hline 672.000 & 1013.196 & -.396 & $=0$ & -0.000 \\
\hline 669.444 & 1013.196 & -58.968 & -0 & -0.000 \\
\hline 661.788 & 1013.196 & -111.096 & -0 & -0.000 \\
\hline 649.104 & 1013.196 & -174.336 & -0 & -0.000 \\
\hline
\end{tabular}




\begin{tabular}{|c|c|c|c|c|c|c|c|c|c|c|c|}
\hline \multirow[t]{4}{*}{38} & -0 & $=0$ & -0 & -0 & -0 & $=0$ & 262.368 & 1013.196 & -407.844 & -0 & $-0,000$ \\
\hline & -0 & -0 & -0 & -0 & -0 & -0 & 221.700 & 1013.196 & -393.840 & -0 & $-0,000$ \\
\hline & -0 & -0 & -0 & -0 & -0 & -0 & 179.160 & 968.196 & $-379.1 A 8$ & $=0$ & $-0,0 \cap 0$ \\
\hline & -0 & -0 & -0 & -0 & $-n$ & -0 & 179.160 & 968.172 & -379.188 & -0 & -0.000 \\
\hline 42 & 1 & 1 & 1 & 1 & 1 & 1 & 127.000 & 965.196 & 659.640 & -0 & -0.000 \\
\hline 43 & i & 1 & i & 1 & $i$ & $i$ & 126.000 & 965.196 & 660.640 & -0 & $-0,1100$ \\
\hline 44 & 1 & 1 & 1 & 1 & 1 & 1 & $515.77 \mathrm{~h}$ & 1013.196 & 431.556 & -0 & -0.000 \\
\hline 45 & i & i & i & i & $i$ & $i$ & 514.776 & 1014.196 & 431.556 & -0 & -0.000 \\
\hline 46 & $i$ & $i$ & i & $i$ & 1 & $i$ & 514.776 & 1013.196 & 432.556 & -0 & -0.000 \\
\hline 47 & $i$ & 1 & i & $i$ & $i$ & $i$ & 662.788 & 1013.196 & 116.304 & -0 & -0.000 \\
\hline 48 & $i$ & $i$ & 1 & 1 & 1 & 1 & $661.78 B$ & 1014.196 & 116.3104 & -0 & -0.000 \\
\hline 49 & 1 & 1 & 1 & 1 & $i$ & 1 & 661.788 & 1013.196 & 117.304 & -0 & $-0,000$ \\
\hline 50 & $i$ & 1 & 1 & $i$ & $i$ & $i$ & 661.788 & 1014.196 & -117.096 & -0 & -0.000 \\
\hline 51 & $i$ & 1 & 1 & $i$ & 1 & $i$ & 582.223 & 1013.196 & -337.362 & -0 & -0.000 \\
\hline 52 & i & j & i & $i$ & $i$ & $i$ & 180.160 & 968.196 & -379.188 & -0 & -0.000 \\
\hline 53 & $i$ & 1 & i & $i$ & $i$ & i & 179.160 & 969.196 & -379.188 & -0 & -0.000 \\
\hline 54 & $i$ & 1 & i & 1 & $i$ & i & 179.160 & 968.196 & $.378 .18 B$ & -0 & -0.000 \\
\hline 55 & $i$ & $i$ & $i$ & $i$ & $i$ & i & 180.160 & 968.196 & -379.188 & -0 & -0.000 \\
\hline 56 & 1 & 1 & 1 & 1 & $i$ & i & 179.160 & 969.196 & -379.188 & -0 & $-0.0 \cap 0$ \\
\hline 57 & 1 & 1 & 1 & 1 & 1 & 1 & 179.160 & 968.196 & $-378.1 \mathrm{AB}$ & -0 & -0.000 \\
\hline $5 B$ & $i$ & 1 & i & i. & $i$ & i & 127.000 & 483.996 & 705.840 & -0 & -0.000 \\
\hline 59 & $i$ & 1 & i & $i$ & $i$ & $i$ & 126.000 & ABA.996 & 705.840 & -0 & -0.000 \\
\hline 60 & 8 & $i$ & i & $i$ & i & 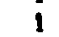 & 126.000 & 483.996 & 706.840 & $=0$ & -0.010 \\
\hline
\end{tabular}

GENERATEO NODAL DATA

\begin{tabular}{|c|c|c|c|c|c|}
\hline \multirow{2}{*}{\begin{tabular}{l}
$I$ NOOE \\
\multirow{N}{N}{ NUMBER }
\end{tabular}} & \multicolumn{2}{|c|}{ BOUNOARY } & \multicolumn{2}{|c|}{ CONDITION } & \multirow{2}{*}{$\underset{Y Y}{\text { CODES }}$} \\
\hline & $x$ & $Y$ & 2 & $x x$ & \\
\hline$\pi$ & -0 & -0 & -0 & -0 & -0 \\
\hline 2 & -0 & -0 & -0 & -0 & -0 \\
\hline 3 & -0 & -0 & -0 & -0 & -0 \\
\hline 4 & -0 & -0 & $=0$ & $-n$ & -0 \\
\hline 5 & -0 & $\infty 0$ & -0 & -0 & -0 \\
\hline 6 & -0 & $\bullet 0$ & -0 & -0 & -0 \\
\hline 7 & -0 & -0 & -0 & -0 & -0 \\
\hline 8 & $=0$ & -0 & -0 & -0 & -0 \\
\hline 9 & -0 & -0 & -0 & -0 & -0 \\
\hline 10 & -0 & -0 & -0 & -0 & -0 \\
\hline 11 & -0 & -0 & -0 & -0 & -0 \\
\hline 12 & -0 & -0 & -0 & -0 & -0 \\
\hline 13 & -0 & -0 & -0 & -0 & -0 \\
\hline 14 & $-n$ & -0 & -0 & -0 & -0 \\
\hline 15 & -0 & -0 & -0 & -0 & -0 \\
\hline 16 & -0 & -0 & -0 & -0 & -0 \\
\hline 11 & -0 & -0 & -0 & -0 & -0 \\
\hline 18 & -0 & -0 & -0 & -0 & -0 \\
\hline 19 & -0 & -0 & -0 & -0 & -0 \\
\hline 20 & -0 & -0 & -0 & -0 & -0 \\
\hline 21 & $=0$ & -0 & -0 & -0 & -0 \\
\hline 22 & -0 & -0 & -0 & -0 & -0 \\
\hline 23 & -0 & -0 & -0 & -0 & -0 \\
\hline 24 & -0 & -0 & -0 & -0 & -0 \\
\hline 25 & $\bullet 0$ & -0 & $=0$ & -0 & -0 \\
\hline 26 & -0 & -0 & -0 & $-n$ & -0 \\
\hline 27 & -0 & -0 & -0 & -0 & -0 \\
\hline 28 & -0 & -0 & -0 & -0 & -0 \\
\hline 29 & -0 & -0 & -0 & $=0$ & -0 \\
\hline 30 & -0 & -0 & -0 & -0 & -0 \\
\hline 31 & -0 & -0 & -0 & -0 & -0 \\
\hline
\end{tabular}

NODAL POINT COOROINATES

\begin{tabular}{|c|c|c|c|}
\hline$x$ & $\gamma$ & $z$ & T \\
\hline 126.000 & 483.996 & 705.840 & -0.000 \\
\hline 126.000 & 483.996 & 704.640 & -0.000 \\
\hline 126.000 & 528.996 & 659.640 & -0.1000 \\
\hline 126.000 & 567.996 & 659.640 & -0.000 \\
\hline 126.000 & 651.996 & 659.640 & -0.000 \\
\hline 126.000 & 735.996 & 659.640 & -0.000 \\
\hline 126.000 & 802.596 & 459.640 & -0.000 \\
\hline 126.000 & 869.196 & 659.640 & -0.000 \\
\hline 126.000 & $917.19 \mathrm{~h}$ & 659.640 & -0.000 \\
\hline 126.000 & 965.196 & 659.640 & -0.000 \\
\hline 126.000 & 968.196 & 659.640 & -0.000 \\
\hline 169.860 & 1013.196 & 649.560 & -0.000 \\
\hline .928 & 1013.196 & 648.624 & -0.000 \\
\hline 229.836 & 1013.196 & 630.996 & -0.000 \\
\hline 283.992 & 1013.196 & AOR.55h & -0.000 \\
\hline $335.98 R$ & 1013.196 & 581.484 & -0.000 \\
\hline $385.42 \mathrm{~A}$ & 1013.196 & 550.080 & -0.000 \\
\hline 431.940 & 1013.196 & 514.392 & -0.000 \\
\hline 475.164 & 1013.196 & $474.7 A \cap$ & -0.00 .0 \\
\hline $510.77 \mathrm{~h}$ & 1013.196 & 431.556 & -0.000 \\
\hline 550.464 & 1013.196 & 385.044 & -0.000 \\
\hline 581.964 & 1013.196 & 335.604 & -0.000 \\
\hline 609.036 & 1013.196 & $2 A 3.60 A$ & -0.000 \\
\hline 631.471 & 1013.196 & $229.45 ?$ & -0.000 \\
\hline 649.104 & 1013.196 & 173.5144 & -0.000 \\
\hline 661.788 & 1013.196 & 116.304 & -0.000 \\
\hline 669.444 & 1013.196 & 58.176 & .0 .000 \\
\hline 672.000 & 1013.196 & -.396 & -0.000 \\
\hline 669.444 & 1013.196 & $-58.9 h B$ & -0.000 \\
\hline $78 B$ & 1013.196 & $-117.09 \mathrm{~h}$ & -0.000 \\
\hline 101 & 1013.196 & $-17 a .336$ & $-1) .000$ \\
\hline
\end{tabular}




$\begin{array}{rrrrrr}32 & -0 & -0 & -0 & -0 & -0 \\ 33 & -0 & -0 & -0 & -0 & -0 \\ 34 & -0 & -0 & -0 & -0 & -0 \\ 35 & -0 & -0 & -0 & -0 & -0 \\ 36 & -0 & -0 & -0 & -0 & -0 \\ 31 & -0 & -0 & -0 & -0 & -0 \\ 38 & -0 & -0 & -0 & -0 & -0 \\ 39 & -1 & -0 & -0 & -0 & -0 \\ 40 & -0 & -0 & -0 & -0 & -0 \\ 41 & -0 & -0 & -0 & -0 & -0 \\ 42 & 1 & 1 & 1 & 1 & 1 \\ 43 & 1 & 1 & 1 & 1 & 1 \\ 44 & 1 & 1 & 1 & 1 & 1 \\ 45 & 1 & 1 & 1 & 1 & 1 \\ 46 & 1 & 1 & 1 & 1 & 1 \\ 47 & 1 & 1 & 1 & 1 & 1 \\ 48 & 1 & 1 & 1 & 1 & 1 \\ 49 & 1 & 1 & 1 & 1 & 1 \\ 50 & 1 & 1 & 1 & 1 & 1 \\ 51 & 1 & 1 & 1 & 1 & 1 \\ 52 & 1 & 1 & 1 & 1 & 1 \\ 53 & 1 & 1 & 1 & 1 & 1 \\ 54 & 1 & 1 & 1 & 1 & 1 \\ 55 & 1 & 1 & 1 & 1 & 1 \\ 56 & 1 & 1 & 1 & 1 & 1 \\ 57 & 1 & 1 & 1 & 1 & 1 \\ 58 & 1 & 1 & 1 & 1 & 1 \\ 59 & 1 & 9 & 1 & 1 & 1 \\ 60 & 1 & 1 & 1 & 1 & 1\end{array}$

$631.47 \mathrm{~h}$

1013.196 1013.196

81.964

1013.196

550.596

1013.196

1013.196

345.576

1013.196

1013.196

1013.196

221.700

$96 A .196$

968.172

965.196

127.00

965.196

126.000

013.196

515.776

514.776
514.776

1014.196

662.788

$1013.19 \mathrm{~h}$

1013.196

1014.196

661.788

1013.196

582.223

1014.196

1013.196

968.196

969.196

968.196

968.196

969.196

968.196

483.996

484.996

483.996

$-230.244$

$-0.000$

$-336.396 \quad-0.000$

$-385.512 \quad-0.000$

$-046.736 \quad-0.000$

$-436.500 \quad-0.000$

$-407.844 \quad-0.000$

$-393.840 \quad-0.000$

$-379.188 \quad-0.000$

$-379.18 \mathrm{~A} \quad-0.000$

$659.640 \quad-0.000$

$660.640=0.000$

$431.556 \quad-0.000$

431.55t -0.000

$432.556 \quad-0.000$

$116.304 \quad-0.000$

$116.304 \quad-0.000$

$111.304 \quad-0.000$

$-117.096 \quad-0.000$

.337 .362

- 379.362

-379.1 R

$-379.188$

$-0.000$

0.000

$-0.000$

$-0.000$

$-0.000$

370

$-0.000$

$-0.000$

$705.840 \quad-0.000$

$705.840 \quad-0.000$

$706.840 \quad-0.000$

N ${ }^{\prime}$ EQUATION NUMBERS

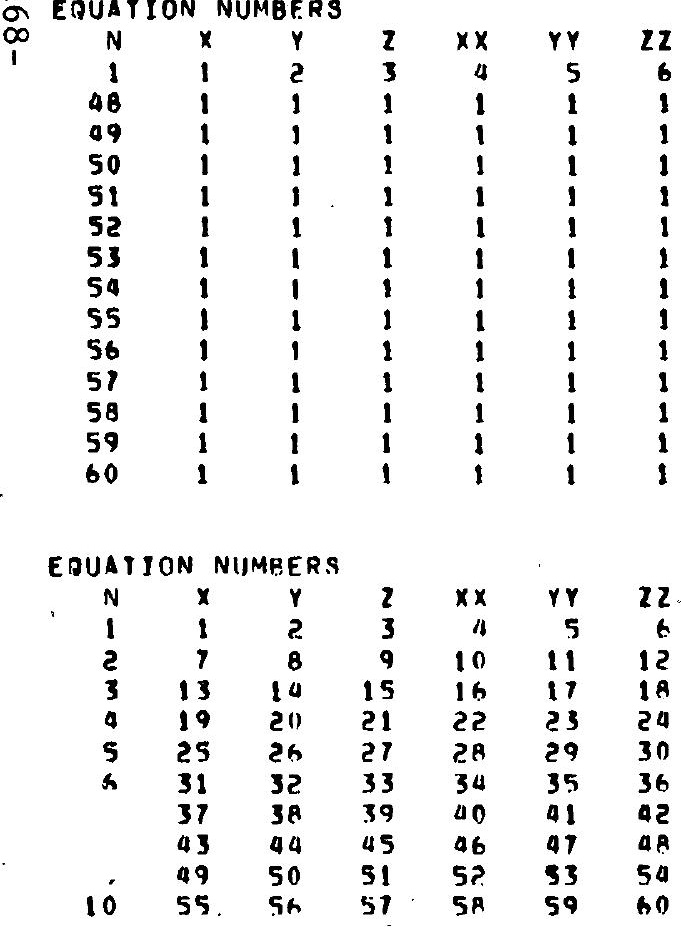

$\begin{array}{llll}661.788 & 1014.196 & 116.300 & -0.000 \\ 661.788 & 1013.196 & 117.304 & -0.000 \\ 661.188 & 1019.196 & -117.096 & -0.000 \\ 582.223 & 1013.196 & -331.362 & -0.000 \\ 180.160 & 968.196 & -379.188 & -0.000 \\ 179.160 & 969.196 & -379.188 & -0.000 \\ 179.160 & 968.196 & -378.188 & -0.000 \\ 180.160 & 968.196 & -379.188 & -0.000 \\ 119.160 & 969.196 & -379.188 & -0.000 \\ 179.160 & 968.196 & -378.188 & -0.000 \\ 127.000 & 483.996 & 705.840 & -0.000 \\ 126.000 & 484.996 & 705.840 & -0.000 \\ 126.000 & 483.996 & 106.840 & -0.000\end{array}$


BOUNDARY ELEMENTS

ELEI TYPE

NUMBER OF ELEMENTS

19

ELEMENT LOAN CASF MIILIIPLIERS

$\begin{array}{rrrr}\text { CASE (A) } & \text { CASE (B) } & \text { CASE(C) } & \text { CASE(D) } \\ 1.0 O O D & 1.0000 & 1.0090 & 1.0000\end{array}$

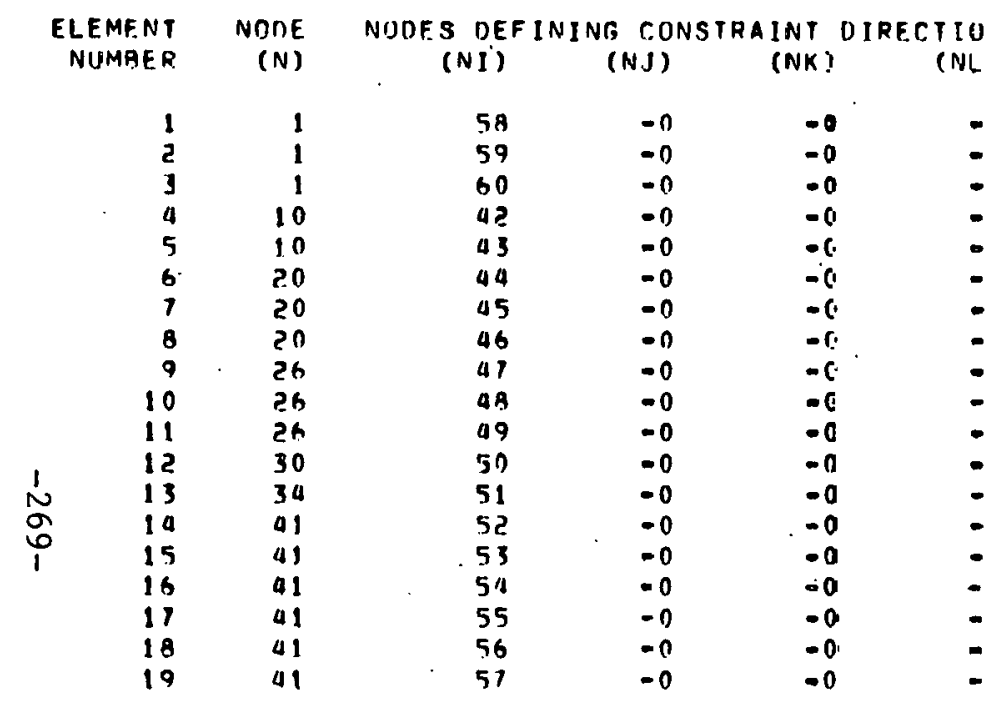

CODE

CONE GENERATION DISPLACFMENT

SPFEIFIF. SPRING

(NL)
KD I

-0
-0
-0
-0
-0
-0
$=0$
$=0$
$=0$
-0
-0
-0
-0
-0
-0
-0
-0
-0
-0
-0

1
1
1
1
1
1
1
1
1
1
1
1
1
1
1
-0
-0
-0

$\begin{array}{ll}-0 & -0 . \\ -0 & -0 . \\ -0 & -0 . \\ -0 & -0 . \\ -0 & -0 . \\ -0 & -0 . \\ -0 & -0 . \\ -0 & -0 . \\ -0 & -0 . \\ -0 & -0 . \\ -0 & -0 . \\ -0 & -0 . \\ -0 & -0 . \\ -0 & -0 . \\ -0 & -0 . \\ -0 & -0 . \\ -0 & -0 . \\ -0 & -0 . \\ -0 & -0 .\end{array}$

$-0$

-0.
-0.
-0.
-0

-0.
-0.

-0.
-0.

$-0$.

$-0$

$-0$.

$-0$

-0 .

-0.
-0.

$-0$.

$-n$.

$-0$.

RUIATION

RATE

- $1000 E+20$

$.1000 E+? 0$

$.1000 E+20$

- $1000 E+07$

- $1000 E+07$

- $25 \cap O E+0 R$

. $000 E+17$

. $2500 E+06$

$. \triangle 500 E+06$

- $2000 E+07$

$.4500 E+06$

$.2000 E \bullet 07$

AOOOE+OR

$1000 E+10$

10nOF+10

- $10 N O F+10$

$.1000 E+10$

$.1000 E+12$

$.1000 E+12$
$1000 F+1$ ? 
PIPE ELEMENT I NPUT

CONTROL INFURMATION

NUMAER OF PIPE ELEMENTS

$=.40$

NIJMAER OF MATERIAL SETS

$=2$

MAXIMUM NIJMBER OF MATERIAL

TEMPERATIJRE. INPIIT POINTS

NIJMHER OF SECTION PROPERTY SETS =

NUMAER OF BRANCH POINT NOIES

MAXIMUM NIIMBER OF IANGENTS

COMMON TO A BRANCH POINT.

FLAG FOR NEGLECTING AXIAL

DEFORMATIONS IN AEND ELEMENTS = (EA.1. NEGLECT)

\footnotetext{
MATERTAL PRUPERTY TABLES
}

$\stackrel{1}{0}$

MATERIAL NUMRER = (1)

NUMBER OF

TEMPERATURE POINTS $=(1)$

IDENTIFICATION \& 1 MAT ..........

1301.00 29900000.0.....

MATERIAL NUMAFR $=\left(\begin{array}{ll}2\end{array}\right)$

NUIABER OF

TEMPERATUAE POINTS = $(1)$

IDENTIFICATION =

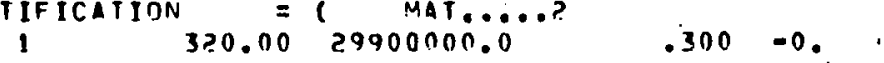


SF- I IN PREPERTY TAELE

SEI

OIITSIOE

WALL

SHAPE FACTOR

WE IGHT,

MASSI

NUMBER DIAMETER

THICKNESS

FOR SHEAR

INIT LENGTH

UNIT IFNGTH

DESCRIPTI O N

1
2
32.000

.9500
.9050

.0 .0000
.0 .0000

-0.
-0.

0 .

ELEMENT LOAO CASE MULTIPLIERS

$X$-DIRECTION GRAVITY
$Y$-DIRECTION GRAVITY

Y-DIRECTION GRAVITY
Z-DIRECTION GRAVITY

Z-DIRECTION GHAVITY

CASE A
1.000

CASE B

CASE C

$1.000 \quad-0.000$

$1.000 \quad-0.000$

$1.000 \quad-0.000$

$-0.000 \quad-0.000$

$-0.000$

CASE D

THERMAL OISTURTION

$-0.000$

$-0.000$

$-0.000$

-0.000
-0.000

$-0.000$ 
PIPE ELEMENT INPUT DAIA

ELEMENT ELEMENT NODE NOTE MATL. SECTION

REFERENCE INTERNAL TEMPERATIILE PRESSIJRE (BEND) (IHIRD

D I R E C T I O N C OS I NES NODE INPUT NUMAER TYPE -I - -J NIJMAER NUMBER

RADIUS ) PDINT)

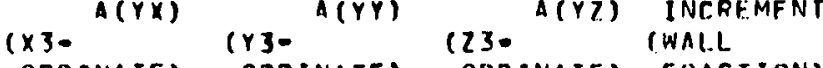

MROINATE) (Y3- ORDINATE) (Z3E OROINATF) FRACTIINN)

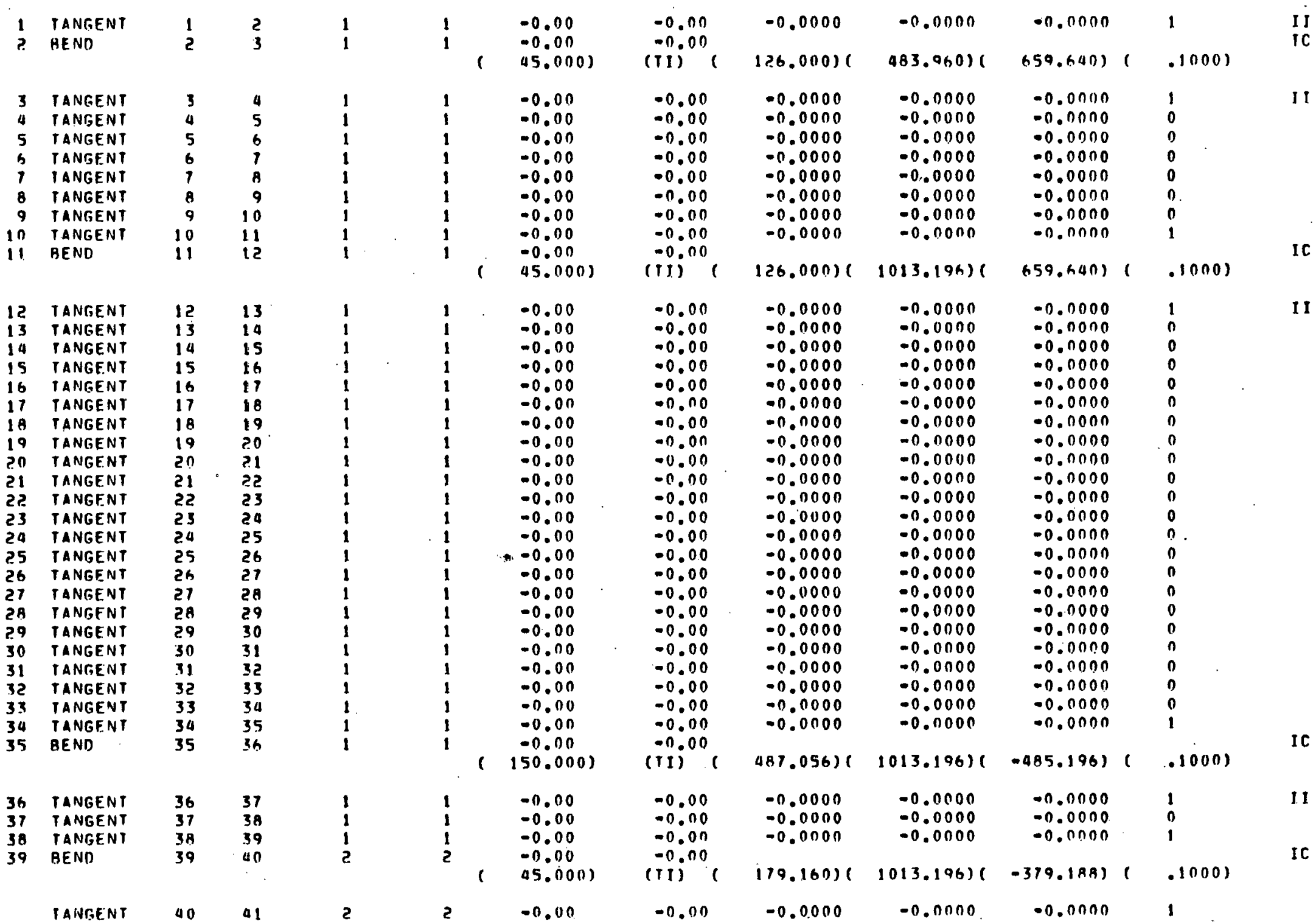




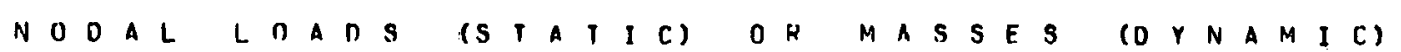

$\begin{array}{ll}\text { NUMBER } & \text { LOAD } \\ \text { CASE }\end{array}$

$x-A \times I S$
FORCE

Y-AXIS
FORCE

ZAAXIS
FORCF

$Y=A \times I S$

$2-4 \times[S$

$\begin{aligned} 4 & - \\ 5 & - \\ 6 & - \\ 7 & - \\ 9 & - \\ 11 & - \\ 17 & - \\ 13 & - \\ 15 & - \\ 17 & - \\ 19 & - \\ 21 & - \\ 23 & - \\ 25 & - \\ 21 & - \\ 29 & - \\ 31 & - \\ 33 & - \\ 35 & -0 \\ 36 & - \\ 31 & - \\ 38 & -\end{aligned}$

$9.92500 E+00$
$5.45300 E+00$
$4.89600 E+10$
$5.88600 E+00$
$5.37300 E+00$
$3.95000 E+00$
$2.43000 E+00$
$3.94100 E+00$
$7.60920 E+00$
$7.60920 E+00$
$7.61200 E+00$
$7.61100 E+00$
$7.411100 E+00$
$7.61100 E+100$
$7.61100 E+00$
$7.61100 E+00$
$7.61100 E+00$
$7.60100 E+00$
$1.02930 E+01$
$7.51800 E+00$
$3.87100 E+00$
$1.05280 E+01$

$9.92500 E+00$

$5.4530 N E+00$

4. ABAODE +00

$5.88800 E+00$

$5.37300 E+00$

3. $95000 \mathrm{OE}+00$

$2.43000 E+00$

$3.94100 \mathrm{E}+0 \mathrm{O}$ ?

$7.60920 E+00$

$7.60920 E+00$

$9.92500 E+00$

$5.45300 E+100$

-0
-0

$5.88 R O O E+00-0$.

$5.37300 E+00$

$3.95000 E+00$

$2.43000 E+00$

$3.941005+00 \quad-0$

$\begin{array}{ll}3.94100 E+00 & =0 . \\ 1.60920 E+00 & -0\end{array}$

$7.60920 E+00-0$.

$\begin{array}{lll}7.61100 E+0 N & 7.61200 E+00 & -0 . \\ 7.61100 E+00 & =0 .\end{array}$

$7.61100 E+00 \quad 7.61100 E+00 \quad-0$

$\begin{array}{lll}7.61100 E+00 & 7.61100 E+00 & -0 .\end{array}$

$7.61100 E+00$

$7.63100 E+00$

$7.61100 F+00$

$7.60100 E+00$

$7.61100 E+00$

$7.61100 E+00=0$

$7.6010 N E+D 0=0$.

$1.02930 \mathrm{~F}+01$

$1.02930 E+01$

$3.8 T 700 E+00$

7. $51 B O O E+0 O$

$3.97700 E+00$

1. OS? AOE + 01

I. 052 2. $0 \mathrm{~F}+01$

T⿱宀八 STRUCTIJKE

W LOAD CASE

ELEMENT

8

I.DAD MULIIPLIFRS

D

I

$-0.000$

$-0.000$

$-0.000$

$-0.000$ 
PRINT OF FREBUENCIES

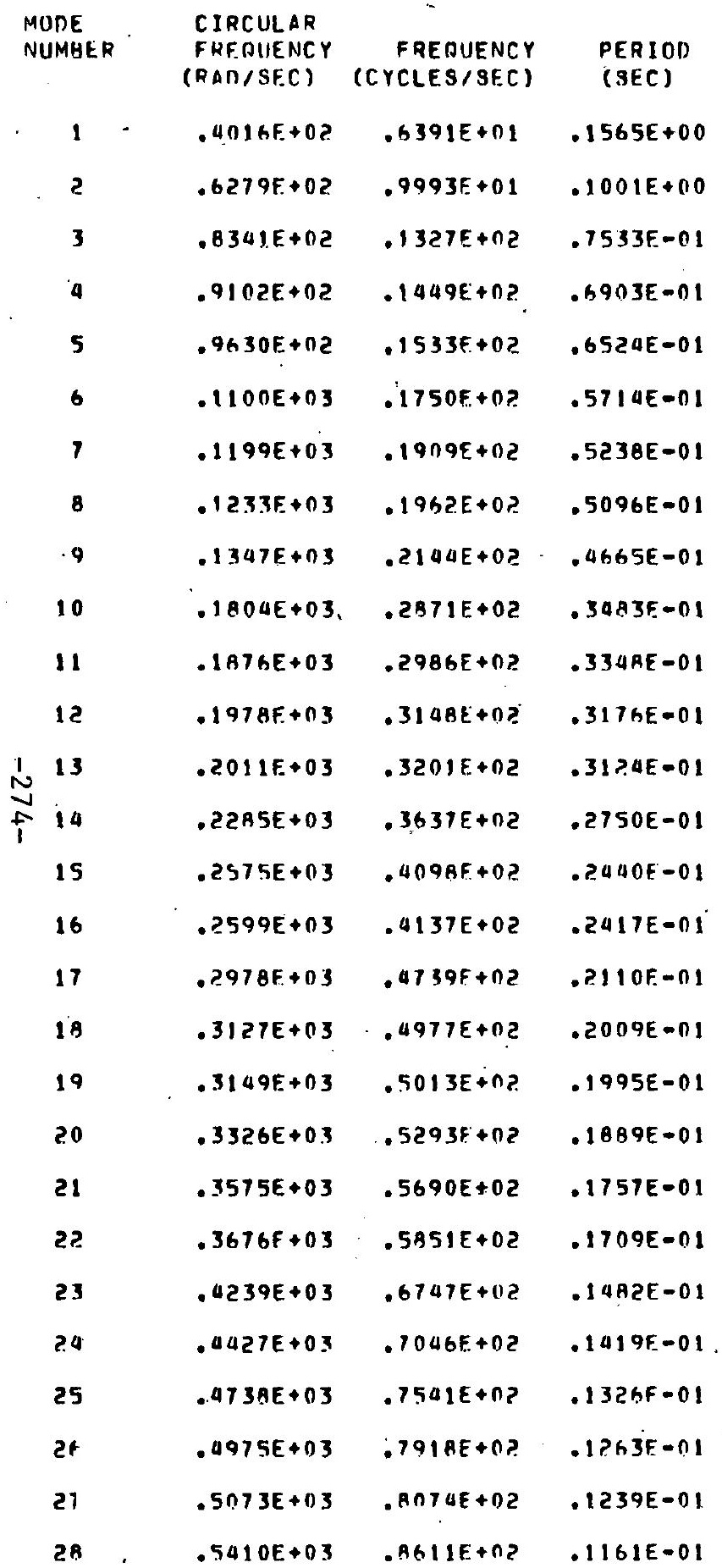


$30 \quad .5827 E+03 \quad .9274 E+02 \quad .107 A E-01$

$1.6243 E+03 \cdots .9936 E+0\rangle \quad .1006 E-01$

EIIGENSOLUTION TIME LOG

EIGENSOLUTION $=5.47$

PRINTING = 
CASE 1

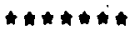

DIRECTION FACTIRS

$x=1.0000 \quad y=1.0000 \quad z=1.0000$

INDICATOR FUR DISPLACEMENT OR ACCELERATION SPECTRUH =

ER. O DISPLACEMENT

EO.1 ACCELERATIUN IN IN./SEC. 2

EQ.? ACCELERATIUN IN FS

3 SPECTRA ARE ENTEREO FOR CASE 1. KINDE?

- ClUSTER FACTOR: C.F $=.10000$

No. 
MODAL PARIICIPATION FACTORS

\begin{tabular}{|c|c|c|c|}
\hline M & $X-1)$ IRECTION & PODIRECTION & Z-OIRECTION \\
\hline - & $.6081 E+00$ & $-.5977 E+01$ & $-.1234 \mathrm{E}+00$ \\
\hline ? & $-.5761 E+01$ & $-.1995 E+00$ & $-.2996 E+01$ \\
\hline 3 & $-.55 A 3 E+00$ & $-.3156 E+01$ & $-.1951 E+01$ \\
\hline 4 & $.2036 E+01$ & $.9930 E-01$ & $-.35 \triangle 0 E+01$ \\
\hline $\mathbf{s}$ & $-.3312 E+01$ & $-.4261 E-03$ & $-.73695+01$ \\
\hline 6 & $.4790 E+01$ & $-.3029 E+01$ & $-.2998 E+01$ \\
\hline 1 & $.3712 E+01$ & $.12 B 7 E+00$ & $.3637 E+01$ \\
\hline 8 & $.1375 E+01$ & $.2015 E-01$ & $-.3465 E+00$ \\
\hline 9 & $-.5872 E+01$ & $-.1797 E+01$ & $.0262 E+01$ \\
\hline 10 & $.5580 E+00$ & $-.4298 E+00$ & $-.2992 E+01$ \\
\hline 11 & $.1568 F+00$ & $.67 \cap 0 E+01$ & $\because$. $920 E+00$ \\
\hline 12 & $.9426 E+00$ & $.0841 E+00$ & $.1059 E+01$ \\
\hline 13 & $.1052 E+01$ & -.11 TE + OO & $.1533 E+01$ \\
\hline 14 & $-.19 a 3 E+01$ & $-.1115 E+00$ & $.1564 E \cdot 01$ \\
\hline 15 & $-.3199 E+01$ & $.1091 E+01$ & . 19N1EROI \\
\hline $15^{\circ}$ & $.4581 E-02$ & $-.6178 E+00$ & $-.7525 E-03$ \\
\hline 17 & $.7430 E+00$ & $.1711 E+01$ & $-.17 T I E+N 1$ \\
\hline 18 & $-.1391 E+01$ & $-.44 .15 E+01$ & $-.3 B 22 E \times 00$ \\
\hline 19 & $.1405 E+01$ & $-.215 B E+01$ & $.15 T 7 E \cdot 00$ \\
\hline 20 & $-.2703 E-02$ & $.5116 E+00$ & $-.1092 E+01$ \\
\hline 21 & $-.8638 E+00$ & $.2137 E+01$ & $-.3519 E \cdot 00$ \\
\hline 22 & $.1718 E+O D$ & -.21?AE + 01 & $-.9082 E=01$ \\
\hline 23 & $-.6176 E+00$ & $.79 .3 \triangle E \nrightarrow 00$ & $-1388 \mathrm{1}+01$ \\
\hline 5 & $-.3312 E+01$ & $=.42 . S I E-03$ & $-.7389 E+01$ \\
\hline 6 & $.4790 E+01$ & $-.30 \geq 4 E+01$ & $-.2998 E+01$ \\
\hline 7 & $.3712 E+01$ & - IPAIE $\cap 0$ & $.3637 E+01$ \\
\hline 8 & $.1375 E+01$ & $.2015 E-01$ & $-.3465 E$ DOO \\
\hline 9 & $-.5 A 72 E+01$ & $-.17975+01$ & $.4262 E \div 01$ \\
\hline 10 & $.55 B 0 E+00$ & $\because .427 B E+00$ & $=.2992 F+01$ \\
\hline 11. & $.156 B E+00$ & $.6730 E+01$ & $=.8920 \mathrm{t}+00$ \\
\hline
\end{tabular}




\begin{tabular}{|c|c|c|c|c|}
\hline & 12 & $.947 .6 E+00$ & - $A 941 E+00$ & $.1059 E+01$ \\
\hline & 13 & $.1052 E+014$ & $-.117 .5 E+00$ & $.1533 E+01$ \\
\hline & 14 & $-.1943 E+01$ & $-.1115 \mathrm{~F}+00$ & $.1564 E+01$ \\
\hline & 15 & $-.3199 E+01$ & $.1091 E+01$ & $.1981 E+01$ \\
\hline & 16 & $.45 B 1 E=02$ & $-.6178 E+00$ & $=.7525 E-03$ \\
\hline & 17 & $.7430 E+00$ & $.1711 F .+01$ & $-.1777 E+01$ \\
\hline & IA & $-.1391 E+01$ & $-.4445 E+01$ & $-.3822 E+00$ \\
\hline & 19 & $.1405 E+01$ & $-.215 A E+01$ & $.1577 E+00$ \\
\hline & 20 & $-.2703 E-02$ & $.5116 E+00$ & $-.1092 E+01$ \\
\hline & 21 & $\because R G 3 A E+00$ & $.2137 E+01$ & $-.3519 E+00$ \\
\hline & 22 & $.1718 E+00$ & $-.2\{2 B t+01$ & $-.9082 E-01$ \\
\hline & 23 & $-.6176 E+00$ & $.7944 E+00$ & $-.1388 E+01$ \\
\hline & 24 & $-.5035 E \mapsto 00$ & $-.3020 F+00$ & $.6644 E-01$ \\
\hline & 25 & $.4893 E+00$ & $.100 R E+00$ & $-.2774 E+00$ \\
\hline & 26 &.$A 700 E \bullet 00$ & $.1094 E+01$ & $\because R 919 E+00$ \\
\hline & 27 & $=.9497 E+00$ & $-.114 I E+00$ & $=.6471 E+00$ \\
\hline & 28 & $-.7154 E+00$ & - $2176 E+00$ & $0.9148 E+100$ \\
\hline & 29 & $-.1405 E+01$ & $-.1393 \mathrm{E}+01$ & $.6969 E+00$ \\
\hline & 30 & $.4065 F+00$ & $.3429 E+100$ & $-.2216 E+00$ \\
\hline & 31 & $.7980 f+00$ & $-.1621 E+00$ & 2A62E- OI1 \\
\hline
\end{tabular}

SPECTRIJM TARLE $(\times$ DIRECTION SPECTRUM........

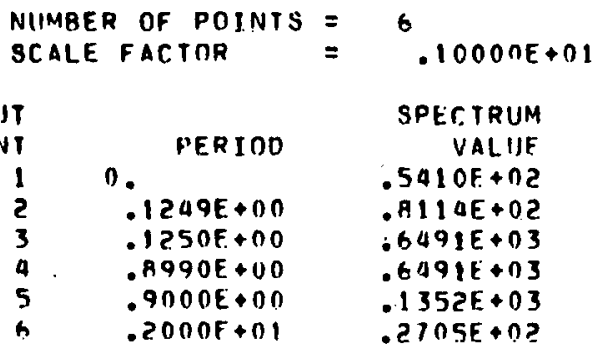


INPIIT SPECTRUM

$$
\text { PERTOD VALIIE }
$$

$0 . \quad 07325+02$

$\begin{array}{lll}5 & .2100 E+00 & .732112 E+02 \\ 3 & .2100 E+00 & .1190 E+04 \\ 4 & .6400 E+00 & .1190 E+04 \\ 5 & .6400 E+00 & .1930 E+03 \\ 6 & .2000 E+01 & .108 P E+03\end{array}$

SPECTRIJM TABLE ( 7 DIRECTION SPECIFIIM........

NUMAER OF POINTS $=6$

STALE FACTOK $=0.10000$ + $=11$

\begin{tabular}{|c|c|c|}
\hline $\begin{array}{l}\text { INPUT } \\
\text { POINT }\end{array}$ & PEKIOD & $\begin{array}{r}\text { SPECTRUM } \\
\text { VAL. 19E }\end{array}$ \\
\hline$i$ & & $.5410 E+D 2$ \\
\hline 2 & $.1249 E+.00$ & . BII U UE+ OD \\
\hline 3 & $1250 E+00$ & $.6491 E+03$ \\
\hline $\begin{array}{l}4 \\
5 \\
6\end{array}$ & $\begin{array}{l}.8990 E+00 \\
.9000 E+00 \\
.2000 E+01\end{array}$ & $\begin{array}{l}.6491 E+03 \\
.1352 E+03 \\
. E 705 E+D 2\end{array}$ \\
\hline
\end{tabular}

CLUSTEFING. +1 MEANS MONE 1 CLOSE TO $1+1,-1$, NOT. $-1,-1,1,-1,-1,1,-1,1,-1,1,1,-1,-1,-1,1,-1,1,1,1,-1,1,-1,-1,1,-1,1$. 
Solution 6(a)

Interspatial/Intermodal Combination 


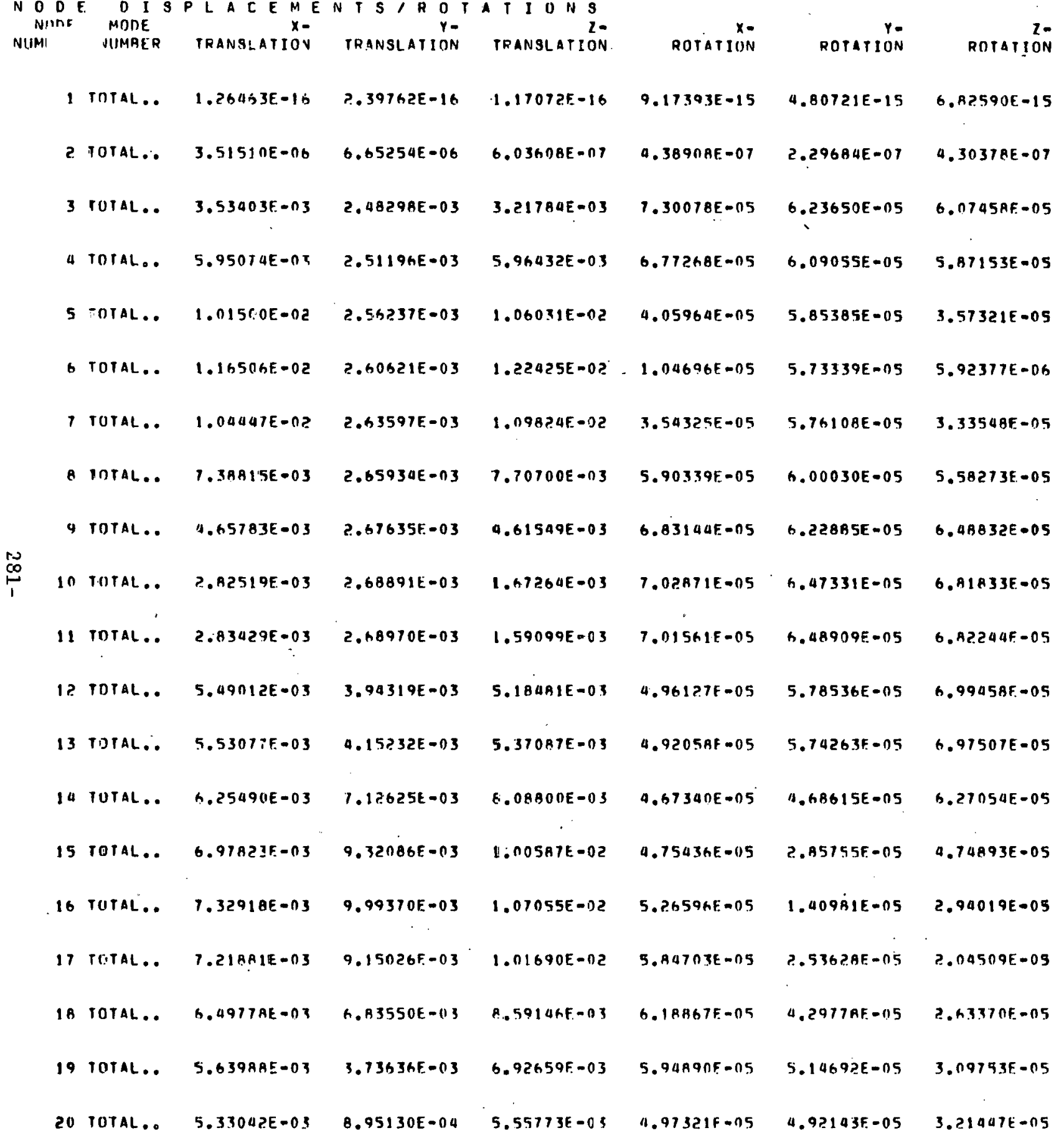




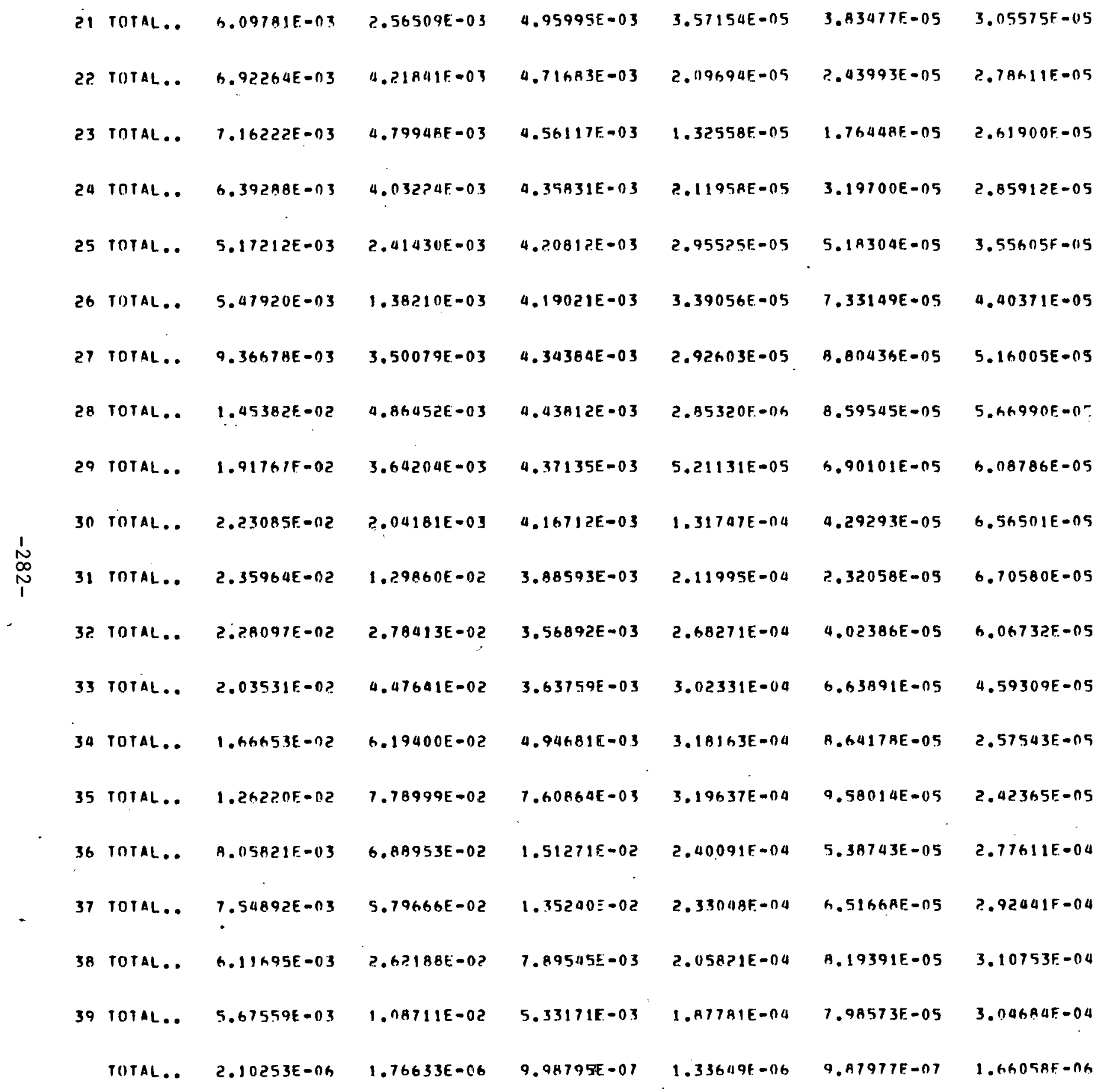


TOTAL.. 0.

43 IOTAL.. 0.0

0.

0.

0 .

A4 TOIAL.. 0.

0.

n.

$n$.

0.

45 rotal.. 0.

0.

0.

0.

$n$

0.

aG TOTAL.. 0 .

$n$

at TOTAL.. 0.

0.

0.

0.

o.

0.

UA TOTAL.. O.

o.

o.

0.

o.

ag TUTAL.. n.

0.

o.

n.

0.

0.

n. 0 .

0.

0

o.

50 TOTAL.. O.

0 .

n.

$n$

0.

\begin{tabular}{lll} 
& 51 IOTAL.. & 0. \\
1 &. & \\
$\infty$ & S? TOTAL.. & 0. \\
\multirow{N}{N}{} &.
\end{tabular}

i.

0 .

0.

n. . . .

53 TO1Al... 0.

0.

n.

$n$

0 .

SA TOTAL.. 0.

$n$.

0

0.

0 .

55 TIIAL.. 0.

$n$

0 .

0.

$n$

0.

5h intal.. 0.

$n$.

$n$.

0.

o.

57 TOIAL.. O.

0.

$n$

$n$

0

5A TIJAL.. D.

$n$.

o.

$n$

0.

59 rOTAL.: 0.

$n$

n.

0.

0.

60 TORAL.. 0 :

0.

$n$

$n$.

$n$.

$n$

n.

11. 0 .

$n$.

$n$. 
RESPONSE SPECTRUM STRES S COMPONEN TS (KTNDE. E)

DR FACH ELEMENI. THE FOLLOWING INFORMATION IS PRINTED:

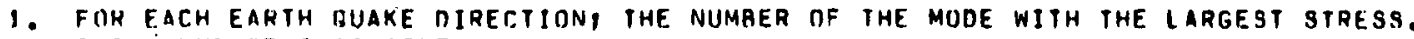

2. THF VALLIE OF TIHAT STRESS.

3. IF REDUESTED, THE MODE BY MODE STRESSF.S FOR EACH EARTH DUAKE DIRECTION.

4. THF. RESIULTANT FIIR EACH FARTH OUAKE DIRECTION.

5. THE TIRANI) TUTAL OF THE THREE EARTH (JIAKE DIRECTIONS.

5: THE TRAND TUTAL OF THF THREE EARTH (JIIAKE DIRECTIONS
(NOTE: THE $X_{\text {. }} Y$, IIR $Z$ RFFERS TO THE EARTH DUAKE DIRECTION.)

ELEMENT TYPE (H O U N D A A Y

, 1, ELEMFNT NUMBER

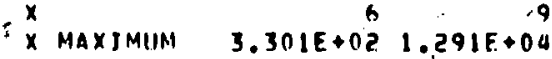

$Y$ MAXIMUM Z.RGOE+0? $0.625 E+04$

5

2. MAXIMUHA $5.793 E+022.187 E+0 Q$

GRAND TOTAL $1.265 E+039.174 E+n A$

ELFMENT TYPE TA O $\|$ N D A R Y

, 1,1 FLEMENT NUMBER $(2)$

$X$
$X$ MAXIMUJM $-5.8 B T E+02-1.453 E+04$

$Y$ MAXIMUM $1.574 E+03-6.341 E+03$

2 MAXIMUM 90

2 MAXIMUM

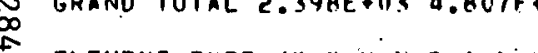

ELEMENT TYPE $B$ B $O N$ D A Y

$, 1,1$ FLEMENT NUMBER $(3)$

$\begin{array}{lcr}x & \text { HORY }-F & B D R Y-M \\ x & 4 & 4 \\ X \text { MAXIMUM } & -2.150 E+02-1.826 E+04 \\ Y & 3 & 3 \\ Y & \text { MAXIMUM } & 4.931 E+02-1.638 E+04 \\ 2 & 4 & 4 \\ 2 \text { MAXIMUM } & 3.737 E+02 & 3.174 E+04\end{array}$

GRAND TOIAL $3.737 .+023.170 E+04$

) 1,1 Rlement numaer $(4)$

$\begin{array}{lrr}x & \text { GIRRY }-F & \text { BNRY } \\ x & 9 & 0 \\ x \text { MAXIMUM } & 1.45 A E+03 & 0 .\end{array}$

ELEMENT TYPE (B O U UN $D$ A R Y

, 11, ELEMENT NUMHER ( 51

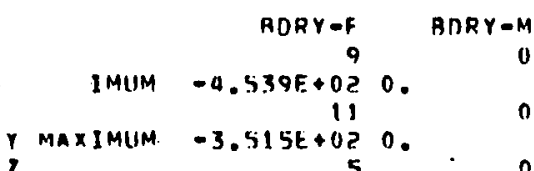


2 MaxIMUM $7.795 E+020$

GP...N TOTAL J.G73E+03 0 .

E. I TYPE IB D UNDARY

$, 1,1$ ELEMENT NUMAER $($ h)

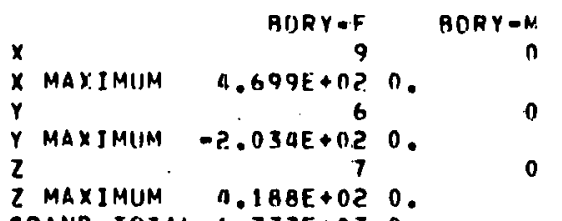

2 MAXIMUM $0.18 B E+02$
GRANO TOTAL $1.333 F+030$.

ELEMENT TYPE (B D U V D A R Y

1,1, ELEMENT NUMBer $(1)$

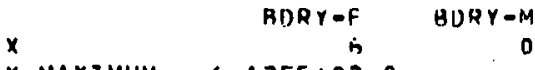

$x$ MAXIMUM $-6.125 E+n ? 0$.

$Y$ MAXIMIIM $1.079 E+030$.

2 MaximiM

2 MAXIMUA 3.A33E+OZ O.

ELEMFUT TYPE IA OOUNDARY

). , , element Numaer $($ B

X MAXIMUM N.61AE+02 0 .

i. Y MAXIMUIM $1.935 E+020$.

co 2

12 MAXIMUIM $1.030 E+030$.

GRAND TUTAL 1.3AOE+O3 0 .

ELFMENT TYPE (B $O$ IJ N D A R $Y$

$, 1,1$ element NUMBer $(9)$

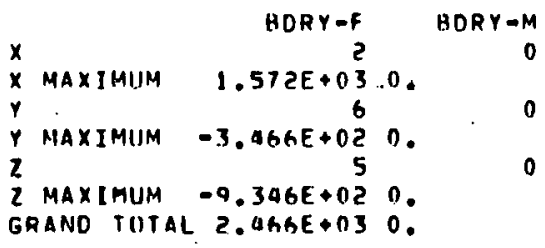

ELEMENT TYPE (H O U N D A R $Y$

1,1, element NuMBer $(10)$

$\begin{array}{rr}X & \text { RORY F } \\ X & 1\end{array}$

$x$ MAXJMIIM 1. anEE+03 0 .

$Y$ MAXIMIIM $-1.630 E+03 n$.

2 MAXIMIM $-1.6 .30 E+03 \%$.

7. MAXIMIIH $-2.974 E+020$.

GRANO. IOTAL ?.7GUE,03 0 .

ELEMENT TYPE (A O) U N O A R Y

) $1 ; 1$ fLEMENT NIMAHEP $(11)$

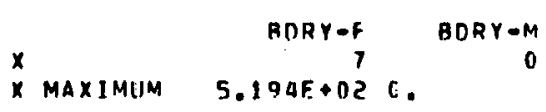




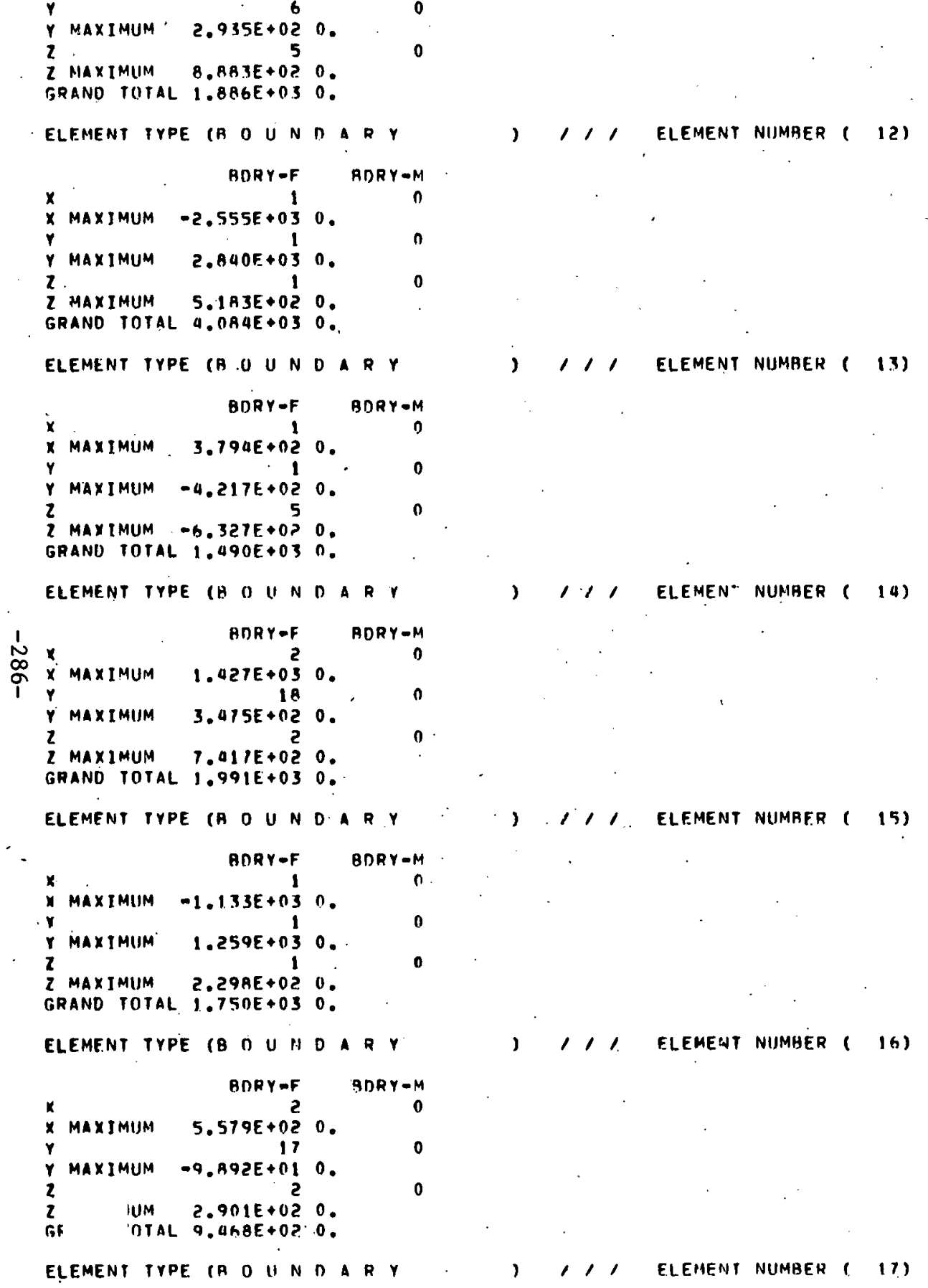




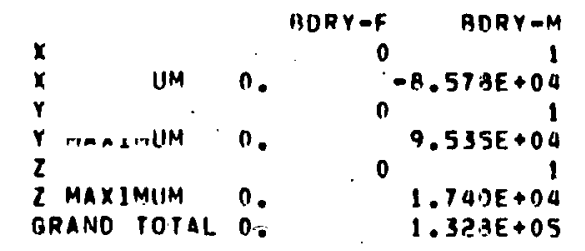

ELEMENT IYPE (A O II N O A $Y$

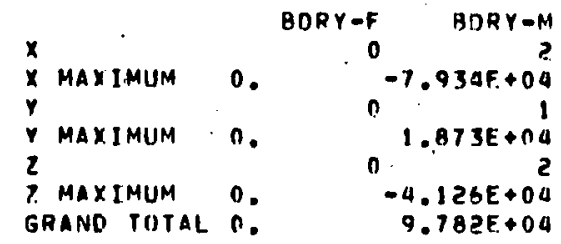

ELEMENT TYPE $C$ O 0 U N D $P$ Y

, , , element numaer ( 19)

\begin{tabular}{|c|c|c|}
\hline & RDRY -F & BDRY $-M$ \\
\hline & & 0 \\
\hline $\operatorname{MAXIMIJM}$ & $n$. & $-1.039 E+05$ \\
\hline$y$ & & D. \\
\hline Y MAXIMUM & 0. & $1.155 E+05$ \\
\hline Z MAXJMUM & 0. & $2.109 E+04$ \\
\hline GRAI & $n$. & $1.659 E+05$ \\
\hline
\end{tabular}

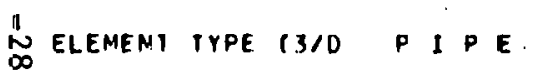

$P X(1) \quad$ Vr(1)

), 1, el.ement number ( 1$)$

$x$ MAXIMUM $2.150 E+C Z-5.8 A 7 E+023$.

VZ(I) $T \times(1)$ Mr(I) $\operatorname{Mz}(1)$

$P \times(J)$

$\operatorname{Vr}(j)$

$V Z(J)$

$\operatorname{Tx}(\mathrm{J}) \quad \operatorname{mr}(\mathrm{J})$

$M 2(J)$

$\checkmark$ MAXIMUM

$-4.931 E+05$ 1. 5 THE +03

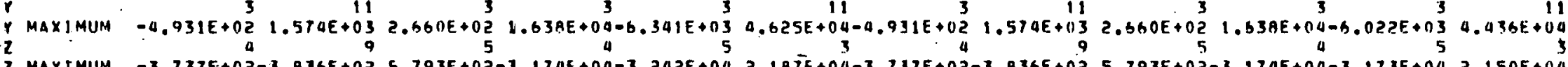

$\begin{aligned} & 5 \\ & 3 \\ & 3\end{aligned} .826 E+04-1.453 E+041.291 E+042.150 E+02-5.887 E+02 \quad 3$

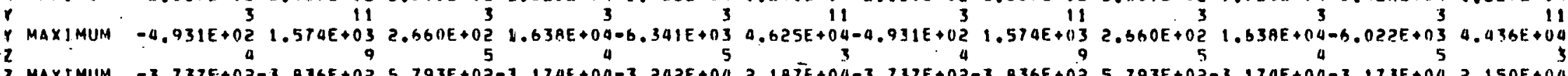

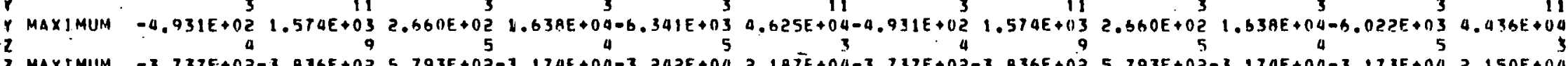

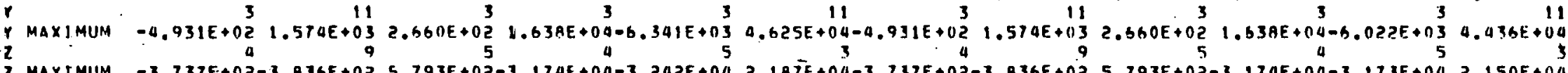

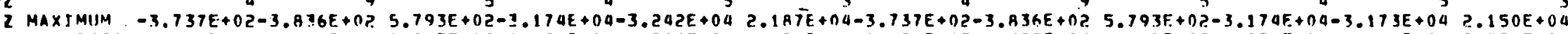

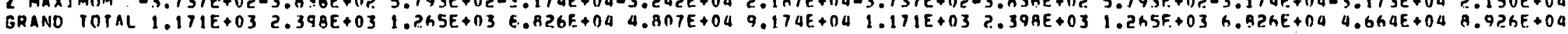
ELEMENT TYPE (3/0 P I PE

1,1, ELEMENT NUMBer $($ ?)

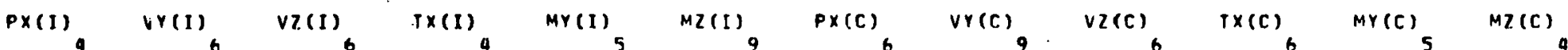

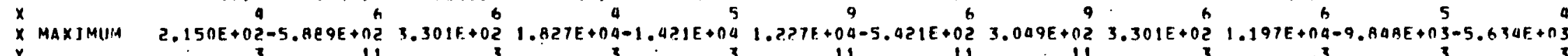

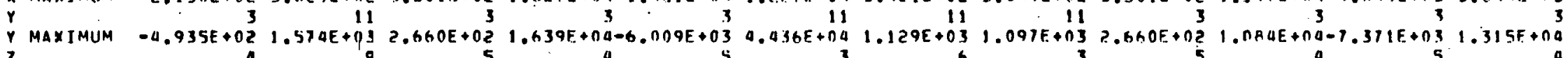
2 MAXIMUM GRAND TOTAL $1.171 E+032.39 A E+031.265 E+036.829 E+044.659 E+048.926 E+041.826 E+031.960 E+031.265 E+033, B B 5 E+043.862 E+043.086 E+04$ ELEMENT TYPE (3/D P $T$ P $F, i, 1$, ELEMENT NUMAER $(?)$

\begin{tabular}{|c|c|c|c|c|c|c|}
\hline & $P \times(1)$ & $\operatorname{Li}(J)$ & $V z(J)$ & $I \times(J)$ & $\operatorname{Mr}(\mathrm{J})$ & $M Z(J)$ \\
\hline$x$ & 6 & $a$ & 6 & h & $a$ & \\
\hline MAXIMUMM & $89 E+02-$ & $-2.150 E+112$ & $3.301 E+02$ & $i .533 E+03-$ & $-3.535 E+03$ & $1.326 E+03$ \\
\hline y & 11 & 3 & 3 & 3 & 3 & 11 \\
\hline MAXJMUM & $\Delta E+03$ & $4.935 E+02$ & $2.660 E+02$ & $5.961 E+05-$ & $h E+03-$ & $.543 F+04$ \\
\hline$A A X I M I I M$ & $7 E+02$ & $73 B E+11 ?$ & $02-$ & $015+03$ & 6.14GE.03 & $3-5.595 E+03$ \\
\hline NO TOTAL & 13 & $E+103$ & 13 & $\operatorname{lna}$ & $P A E+04$ & $7 K E+n h$ \\
\hline
\end{tabular}




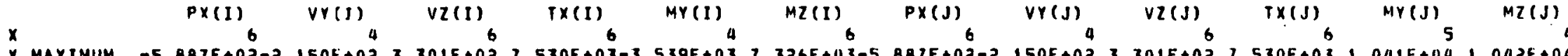

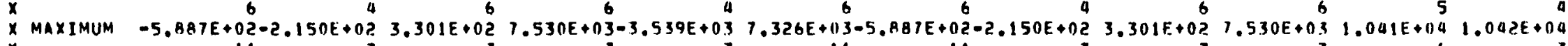

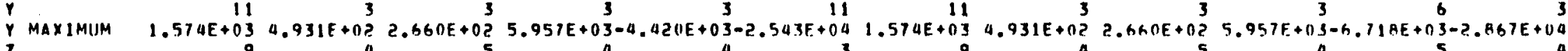

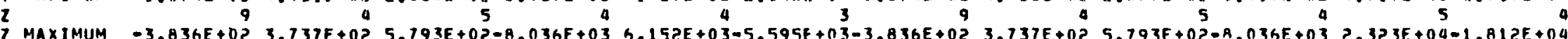
GRAND TOTAL 2.39AE+03 $1.171 E+031.265 E+032.235 E+041.429 E+043.776 E+042.39 B E+031.171 E+031.265 E+032 . ? 35 E+04 \quad 4.040 E+046.649 E+04$

ELEMENT TYPE ( $3 / D$ P I P E $;, 1$ ELEMENT NUMBER (

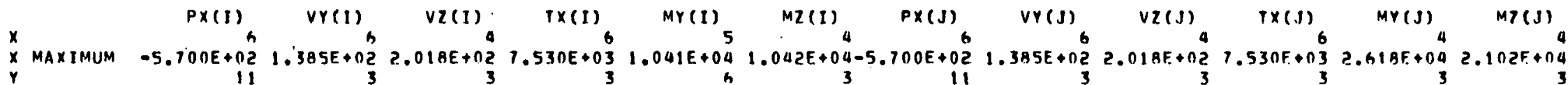

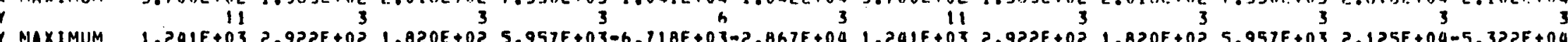
$Y$ MAXIMUM 1.241E+03 2.922E+02 $1.020 E+025.957 E+03-6.118 E+03-2.867 E+04 \quad 1.241 E+032.922 E+021.820 F+025.957 E+032.125 E+04-5.322 E+04$ 2 MAXiMUM $3.561 E+022.193 E+02-3.508 E+02-8.036 E+032.323 E+04-1 . A 12 E+043.567 E+022.193 E+02-3.508 E+02-8.036 F+03 \quad 4.753 E+04-3.654 F+04$ GRAND TOTAL $1.967 E+037.094 F+027.51 A E+022.235 E+04 \quad 4.040 F+046.649 E+041.967 E+037.494 E+027.51 A E+02 \quad ? .235 E+049.99 .3 E+041.136 E+05$ ELEMENT TYPE (3/0 P I PE, , 1, ELEMENT NUMBER $(5)$

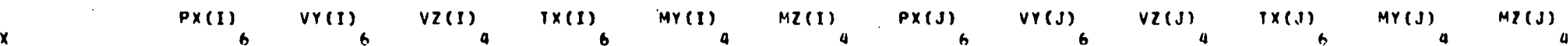

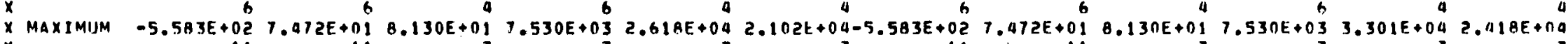

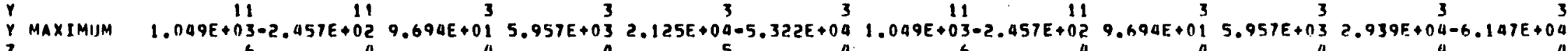
2 MAXIMUM $3.494 E+026.540 E+01-1.413 E+02-8.036 E+03 \quad 4.753 E+04-3.654 E+043.494 E+02 \quad 6.540 E+01-1.413 E+02-8.036 E+03-5.739 E+04-4.203 E+04$ $\infty$ GRAND TOTAL $1.729 E+03$ 4.202E+02 3.149E+02 $2.235 E+049.993 E+041.136 E+051.729 E+03 \quad 0.202 E+023.149 E+02$ 2. $235 F+041.222 E+051.321 E+05$

ELEMFNT TYPE $(3 / 0$ P I PE $, 1,1$ ELEMENT NUMBER $(6)$

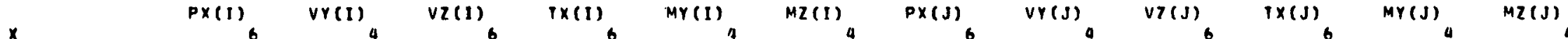

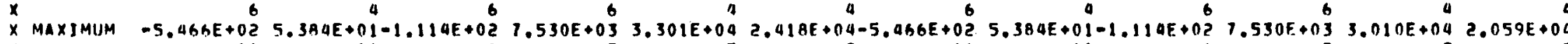

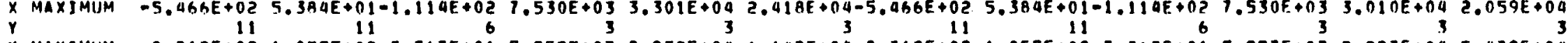
$r$ MAXIMUM A.T1PE+ 2 MAXIMUM 3. $321 E+02-1.175 E+02-2.0 R 6 E+02-8.036 E+03-5.739 E+04-4.203 E+043.421 E+02-1.175 E+02-2.046 E+0 ?-8.036 E+03-5.233 E+04-3.5 A 0 E+04$ GRAND TOTAL 1.516E+03 3.538E+02 3.130E+02 2.235E+04 1.22.2E+05 $1.321 E+051.516 E+033.538 E+023.130 E+022.235 E+041.08 A E+051.171 E+05$ ELEMENT TYHE, $13 / 0$ P I P E

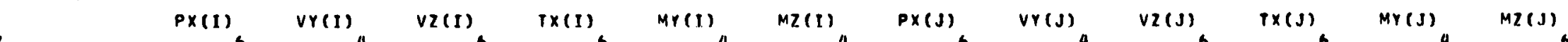

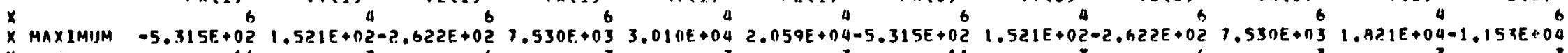

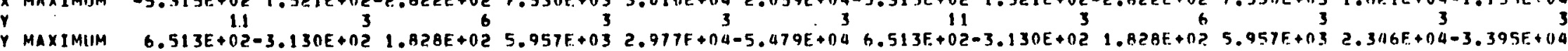
$Y$ MAXIMIIM $6.513 E+02-3.130 E+021.828 E+025.957 E+032.977 F+04-5.479 E+046.513 E+02-3.130 E+021.028 E+0 ? 5.957 E+032.3116 E+04-3.395 E+04$

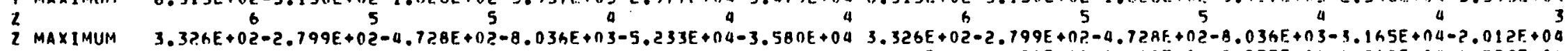
GRAND TOTAL $1.29 B E+031.92 A F+02 B .002 E+022.235 F+041.0 A B E+051.177 F+051.29 A E+03 \quad 7.924 E+028.002 F+022.235 F+046.710 E+046.9$. $3 E+04$ ELEMENT TYPE (3/D P I

$x \quad P X(I)$
6

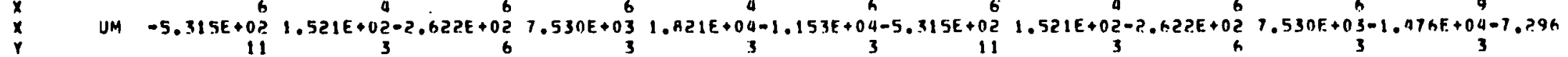

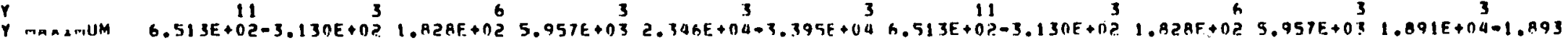


X MAXIMUMM $9.663 E+02-3.913 E+02.2 .029 E+021.117 E+04 \quad 1.115 E+041.766 E+049.663 E+02-3.913 E+022.029 E+021.117 E+042.305 E+042.966 E+04$ Y MAXIMIJM $3.381 E+02 ? .729 F+0 ?-1.061 E+112-7.791 E+03-2.804 E+03-9.0 B 3 F+033.3 A 1 E+022.0229 E+02-1.061 E+02-7.791 F+03-8.791 F+03-2.068 F+04$ 2 MAXIMUM $7.915 E+02-2.539 E+0 ?$. $0.527 E+02-6.991 E+03 \quad 2.4 A B E+04 \quad 1.332 E+047.915 E+02-2.539 E+02 \quad 1.527 E+02-6.991 E+03 \quad 5.142 E+04-1.856 E+04$

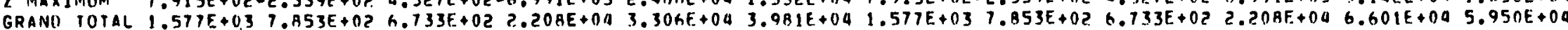
ELEMENT TYPE (3/0 P I P E , 1,1 ELEMENT NUMBER 1 14)

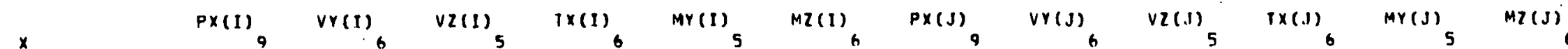
$x$ MAXIMIMM $9.5 R 0 E+02-3.913 E+02$
$Y$
$y$

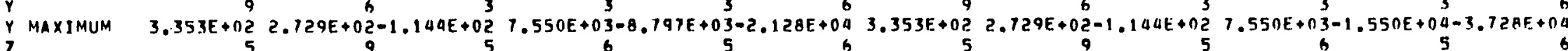

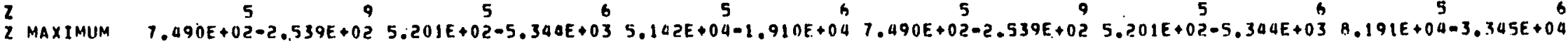

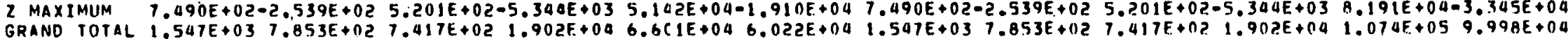
ELEMENT TYPE (3/0 P I P E , 1,1 ELEMEMT NUMAER 1 15)

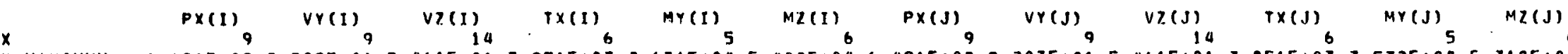
$x$ MAXIMUM $6.491 E+02$
$\times$ $Y$ MAXIMUM $2.272 E+02-1 . A 93 E+02-4.30 A E+019.532 E+03-1.530 E+04-3.766 E+042.272 E+02-1.893 E+02-4.30 A E+019.532 E+03-1.455 E+04-3.709 E+04$ 2 MAXIMIIM $6.765 E+02-6.680 E+01-5,317 E+015.651 E+038.171 E+04-3.379 E+046.765 E+02-6.684 E+01-5,317 E+015.651 E+03 \quad 7.879 E+04-3.328 E+04$ GRANO TUIAL 1.1A5E+03 3.20AE+02 ?.377E+02 1.487E+04 1.074E+051.001E+05 1.1B5E+03 3.208E+02 2.37TE+02 1.4A7E+04 1.029E+05 9.677E+00 ELEMENT TYPE $13 /$ P I P E

$, 1,1$ elemevt NUMAER $(16)$

PX(I), VY(I), VZ(I), TX(I), MY(I), MZ(I), PX(J), VY(J), VZ(J), TX(J) MY(J) $M Z(J)$ MAXIMUM $\quad . .434 E+029.207 E+019.432 E+01-2.738 E+03 \quad 3.532 E+045.331 E+046.034 E+1129.201 E+019.432 E+01-2.138 E+03 \quad 3.545 E+045.250 E+114$ Y MAXIMUM $2.252 E+02-1.093 E+02-4.289 E+011.112 E+04-1.455 E+04-3.71 A E+042.252 E+02-1 . A 93 E+02-0.2 B 9 E+01 \quad 1.112 E+014-1.40 B E+04-3,661 E+04$

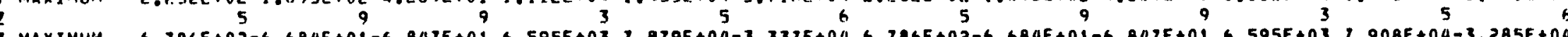
GRAND IOTAL I.181E+03 3.20BE+02 2.50BE+02 1.637E+04 1.029E+05 9.633E+04 1.181E+03 3.208E+02 $2.508 E+021.637 E+041.035 E+059.681 E+04$ ELFMENT TYPF $13 / 0$ P I PE , 1, ELEMENT NUMAER $(17)$

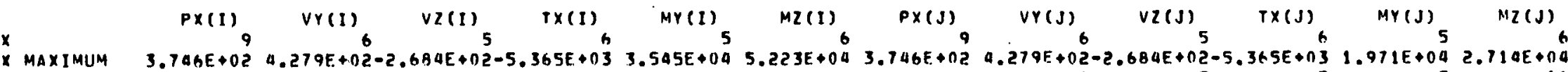

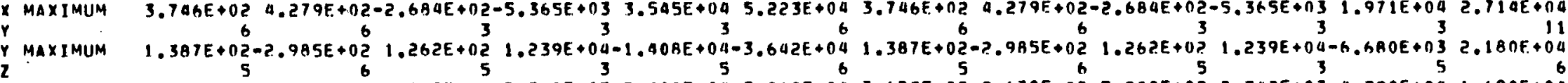
2 MAXIMUM $7.673 E+02-2.67 A E+02-5.98 A E+02 \quad 7.342 E+03 \quad 7.90 B E+04-3.268 E+04 \quad 7.673 E+02-2.678 E+02-5.9 B 8 E+02 \quad 7.342 E+03 \quad 4.39 A E+04-1.69 A E+06$

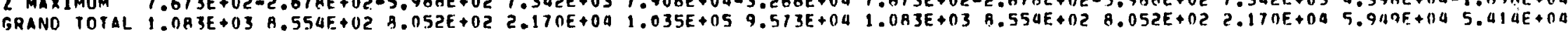
ELEMENT TYPE (3/D PI PE ; , , ELEMENT NUMHER ( 19$)$

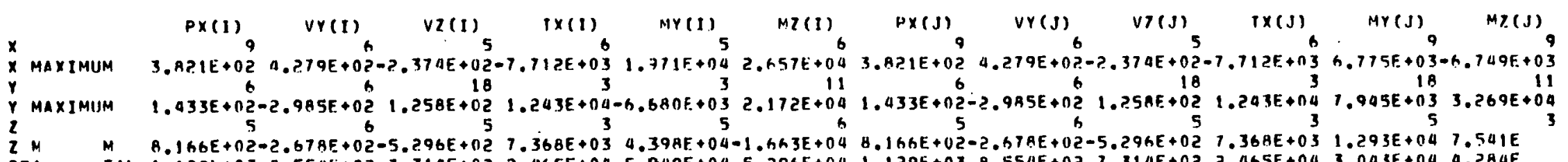

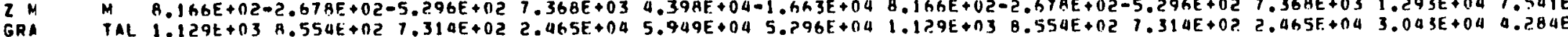

ELENENT TYPF: $(3 / 0$ P I PE , 1,1 ELEIAENT NUPAHER 1 (0) 


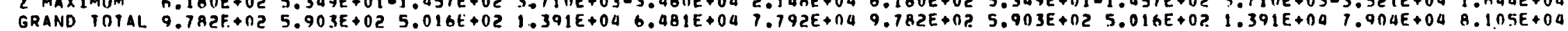


GRAND TOTAL $1.081 E+036.862 E+0 ? 6.583 E+021.713 E+0 Q 9.200 E+047.173 E+041.0 A 1 E+036.862 E+026.5 B 3 E+021.713 E+049.83 A E+046.395 F+04$ ELEMENT TYPE (3/0 P I PE $), 1$, ELEMENT NUMAER 1 25)

$P X(1) \quad V Y(1) \quad V Z(1), \quad T X(1), \quad M Y(I), M Z(1), \quad P X(J), \quad V Y(J) \quad V Z(J), \quad T \times(J), \quad M Y(J), M Z(J)$ $x$ MAXIMUM $4.163 E+02-2.719 E+02-4.418 E+02-1.264 E+04-0.20 A E+04$
5

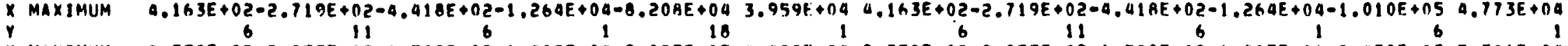
$Y$ MAXIMUM $2.330 E+02-9.033 E+02$
2

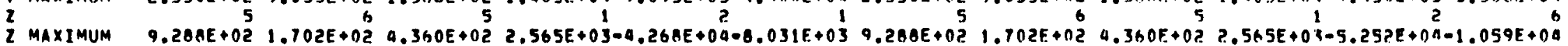
GRANO TOTAL $1.322 E+03 \quad 1.197 E+031.176 E+032.203 E+049.838 E+046.2 .3 E+041.322 E+031.197 E+031.176 E+032.203 E+041.200 E+059.1 A 6 E+04$ ELEMENT TYPE (3/D P I P E, 1, ELEMENT NUMHER $(26)$

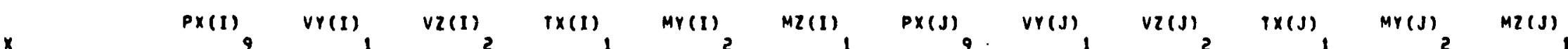

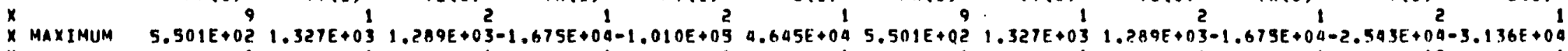
MAXIMUM $3.077 E+02-1.075 E+03-1.269 E+021.062 E+04 \quad 9.450 E+03-5.160 E+04 \quad 3.077 E+02-1.075 E+03-1.269 E+02 \quad 1.062 E+04 \quad 4.674 E+03 \quad 3.466 E+04$ ? GRANO TOIAL $1.200 E+032.13 A E+031.5 B 1 E+032.643 E+041.240 E+059.066 E+041.200 E+03 \quad 2.138 E+031.5 B 7 E+032.643 E+04 \quad 4.3 A 9 E+046 . A D 9 E+04$ ELEMENT TYPE (3/0 P I P $E, 1,1$ ELEMENT NUMBER 127 )

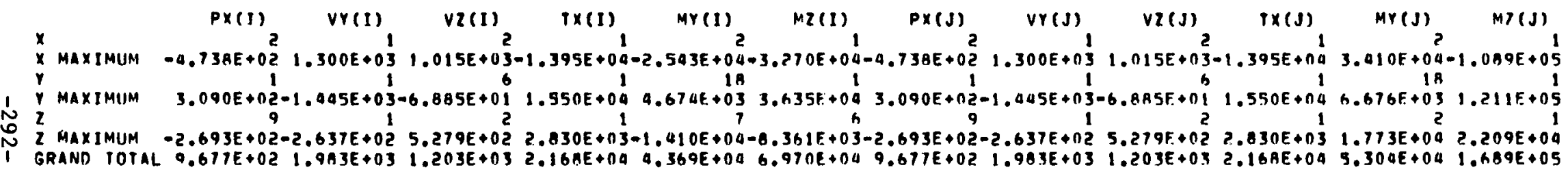
ELEMENT IYPE (3/D P I PE ,, 1, ELEMENT NUMHER $(28)$

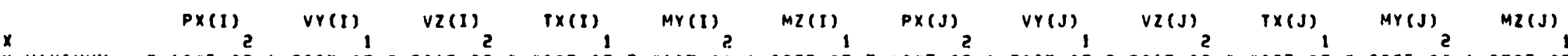

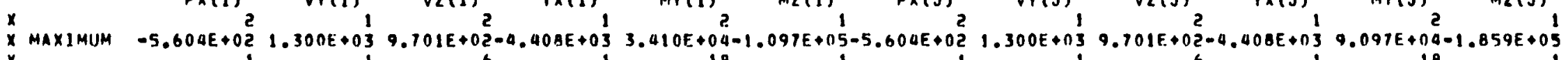

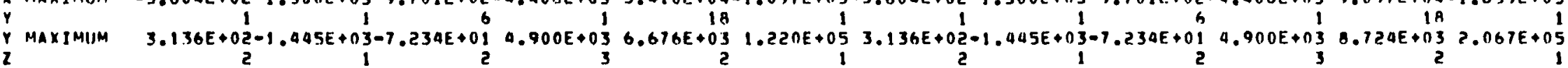

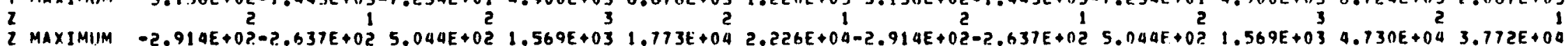
GRAND TUTAL $1.014 E+031.983 E+031.164 E+03$ B.899E+03 $5.304 E+041.701 E+051.014 E+031.9 B 3 E+031.164 E+03$ B.A99E+03 $1.1 \mathrm{hGE}+052.924 E+05$ ELEMENT TYPE (3/0 P I PE , , , FLEMENT NUMHER 1 ?9)

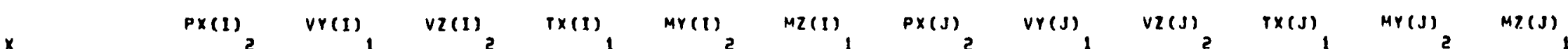

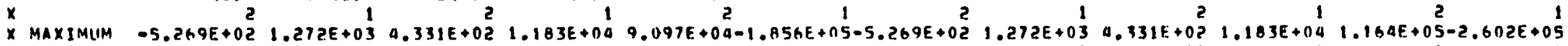

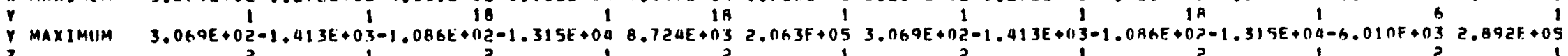

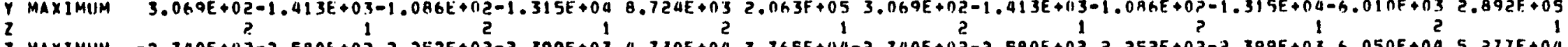

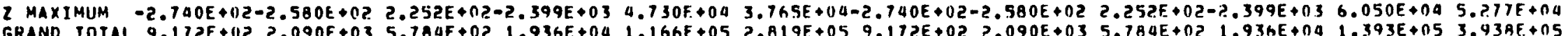
ELEMENT TYPE (3/0 P I P $, 1,1$ ELEMENT NUMAFR $(30)$

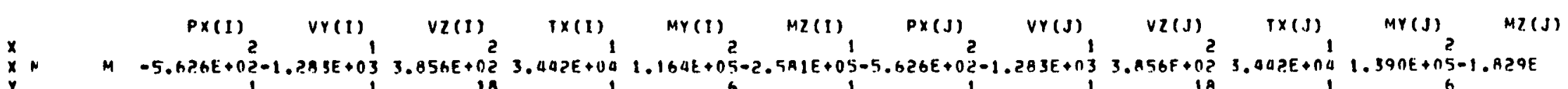


$Y$ MAXIMUM 3.055E+02 $1.426 E+03-1.067 E+02-3.826 E+04-6.010 E+032.869 E+053.055 E+021.026 E+03-1.067 E+02-3.82 .6 E+04-5.650 E+032.033 E+05$ $21 \quad I M-2.925 E+022.603 E+022.005 E+02-6.9 A 2 E+03 \quad 6.050 E+045.237 E+04-2.925 E+022.603 E+022.005 E+02-6.9 A 2 E+031.22 h E+043.1101$ GRI ITAL $9.460 E+022.046 E+035.304 E+025.251 E+041.393 E+053.908 E+059.460 E+022.046 E+0.35 .304 E+025.551 E+041.6 ? 9 E+052.1691$

ELEMENT TYPE (3/D P I PE , , , ELEMENT NUMBER ( 31$)$

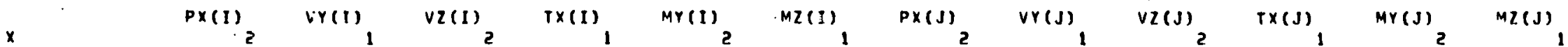
$x$ MAX:MUM $-3.765 E+0 E^{2}-1.178 E+03-2.457 E+025=024 E+0 Q 1.390 E+05-1.792 E+05-3.765 E+02-1.178 E+013-2.457 E+025.0244 E+04 \quad 1.246 E+05-1.101 E+05$ $Y$ MAXJMIIM $2 . A 94 E+021.3 n 9 E+03 \quad 7.573 E+01-5.5 A 5 E+04-5.650 E+03 \quad 1.992 E+052.894 E+02 \quad 1.309 E+03 \quad 1.573 E+01-5.5 A 5 E+04-6.495 E+03 \quad 1.224 E+05$

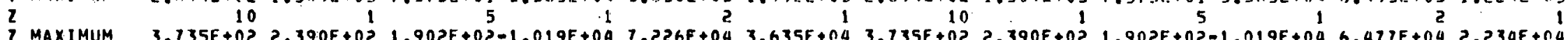
GRAND TOTAL B.6BAE+02 $1.78 R E+035.842 E+027.622 E+041.629 E+052.713 E+05 B .6 A 4 E+021.786 E+035 . A 42 E+027.622 E+041.424 E+051.6 A 4 E+05$

ELEMENT TYPE (3/0 P I PE, ) , , ELEMENT NUMBER $(3 ?)$

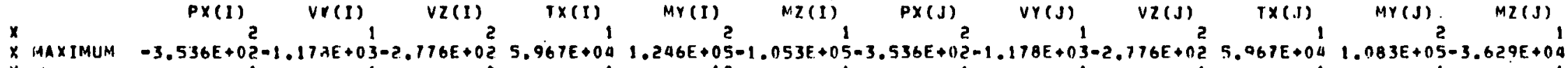

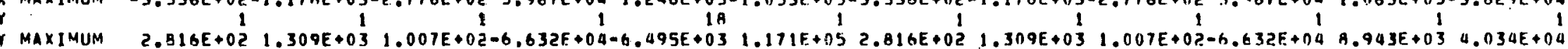
Y MAXIMUM $2.816 E+02 \quad 1.309 E+03 \quad 1.007 E+02-6.632 E+04-6.495 E+03 \quad 1.171 E+052.816 E+02 \quad 1.309 E+03 \quad 1.007 E+02-6.632 E+04 \quad 9.943 E+03 \quad 4.034 E+04$ 2 MAXIMUM 3.T77E+02 $2.390 E+022.143 E+02-1.210 E+04 \quad 6.477 E+042.137 E+043.777 E+022.390 E+022.143 E+02-1.210 E+045.631 E+047.361 E+03$

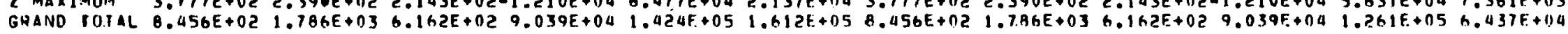
ELEMENT TYPE (3/0 P T PE , , , ELEMENT NUMBER ( 33$)$

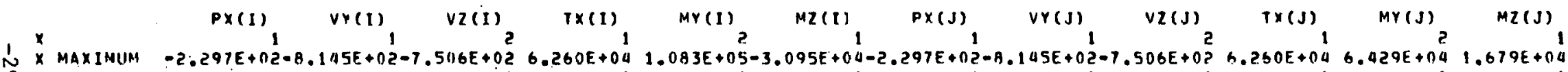

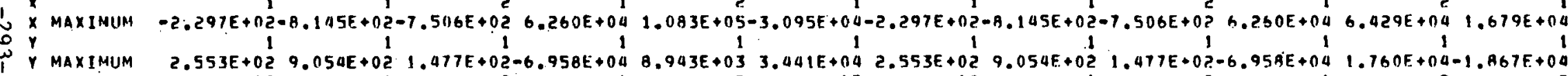
2 MAXIMIJM $4.690 E+021.652 E+02-3.903 E+112-1.270 E+04 \quad 5.631 E+04 \quad 7.190 E+03 \quad 4.690 E+02 \quad 1.652 E+02-3.903 E+02-1.270 E+04 \quad 3.343 E+04 \quad 4.579 E+03$ GRAND TOTAL A.392E+0? $1.290 E+031.11 A E+03 \quad 9.4 B 7 E+041.261 E+055.755 E+048.392 E+021.290 E+031.116 E+039.4 A 7 E+049.269 E+043.253 E+04$ ELEMENT TYPE (3/0 P I P E 1,1 ELEMENT NUMAER ( 34)

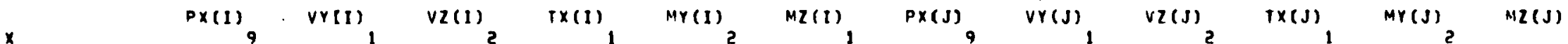

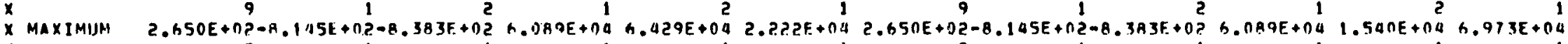

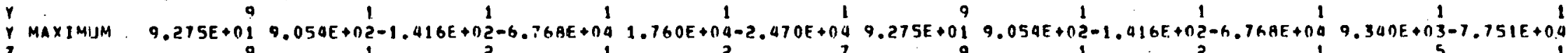
MAxIMUM -1.920E+0? 105211

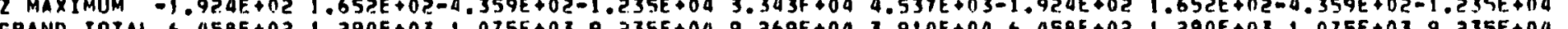
ELEMENT TYPE (3/D P I P E , , , ELEMENT NUMAER ( 35)

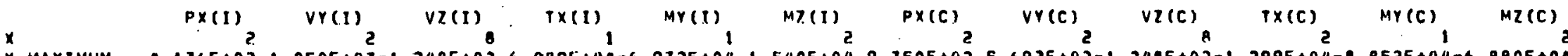
$x$ MAXIMIIM $4.136 E+02$
$Y$

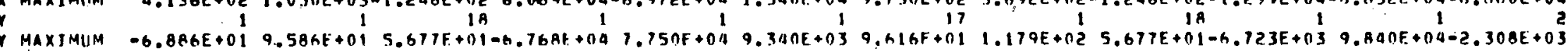

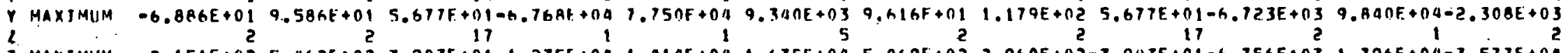
2 MAXIMLIM $2.151 E+025.462 E 202-3.903 E+01-1.235 E+04 \quad 1.910 E+041.635 F+045.069 E+02 \quad 2.960 E+02-3.903 E+01-6.756 E+03 \quad 1.796 E+04-3.577 E+04$

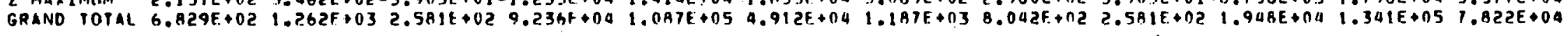

ELEMENT TYPE (3/0 PI PE . , , I ELEMENT NUMBER ( 35)

$P X(J) \quad V Y(J) \quad V Z(J) \quad T X(J) \quad \operatorname{mr}(J) \quad \operatorname{laz}(J)$


$x$ MAXIMUM $1.11 B E+03-1.675 E+02-1.24 B E+02-0.866 E+04-6.935 E+04-9.027 E+04$
$x$

$X$ MAXIMUM $1.11 \mathrm{BE}+03-1.675 E+02-1.248 E+02-0.066 E+04-6.935 F+004-9.0271 .+04$
$Y$

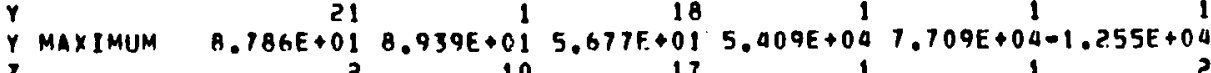

2 MAXIMUM $5.814 E+02-1.959 E+02-3.903 E+019.872 E+03 \quad 1.407 E+04-4.694 E+04$ GRAND TOTAL $1.330 E+035.369 E+0 ? 2.581 E+027.677 E+001.081 E+051.115 E+05$

ELEMENT TYPE (3/0 P I PE , 1,1 ELEMENT NUMAER $(36)$

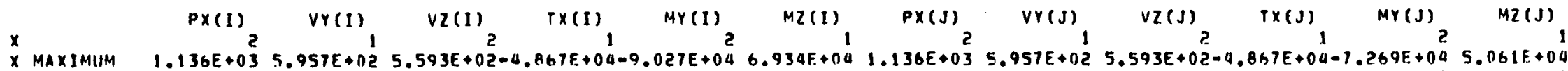
$x$ MAXIMUM $1.136 E+035.957 E+025.593 E+02-0 . A 67 E+04-9.027 E+046.934 F+041.136 E+03 \quad 5.957 E+025.593 E+02-4.867 E+04-7.269 E+045.061 E+04$

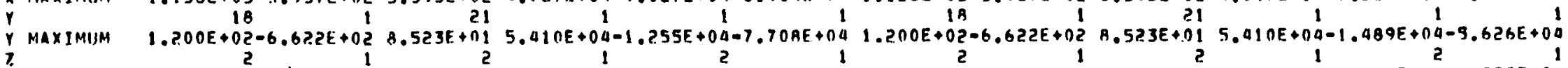

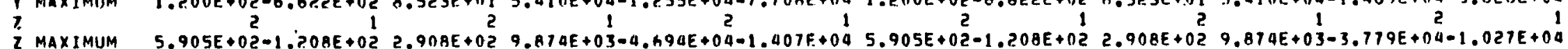
GRAND TOTAL $1.42 B E+039.290 E+n 26.733 E+02 \quad 7.679 E+041.115 E+051.091 E+051.42 A E+039.290 E+026.733 E+0 ? 7.679 E+049.960 E+04 \quad 8.076 E+04$ ELEMENT TYPE (3/D P I P E , , , ELEMENT NUMAER ( 37)

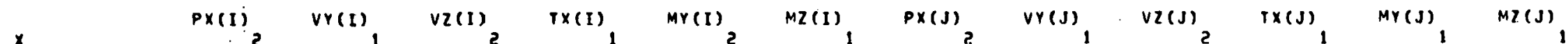

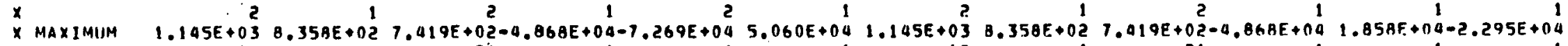

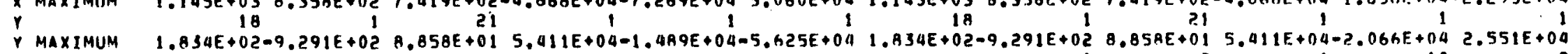

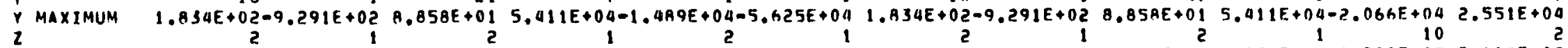

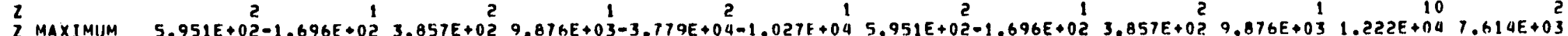

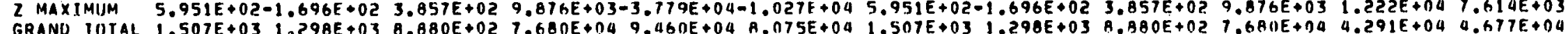
ELEMENT TYPE ( $3 / D$ P 1 P E , , ELEMENT NUMAFH $(38)$

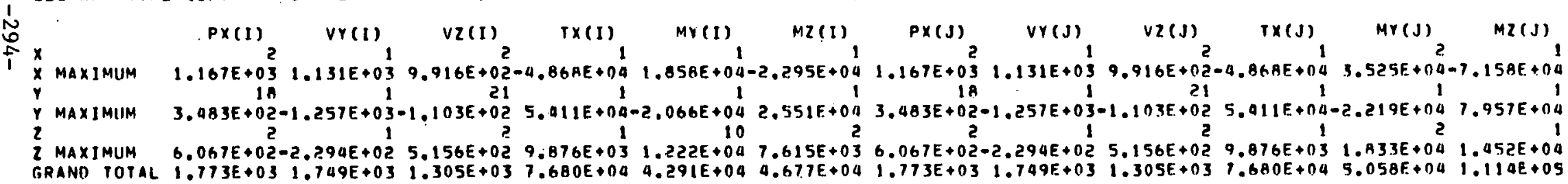
ELEMENT TYPE (3/0 P I PE ), , ELEMENT NUMHER ( 39 )

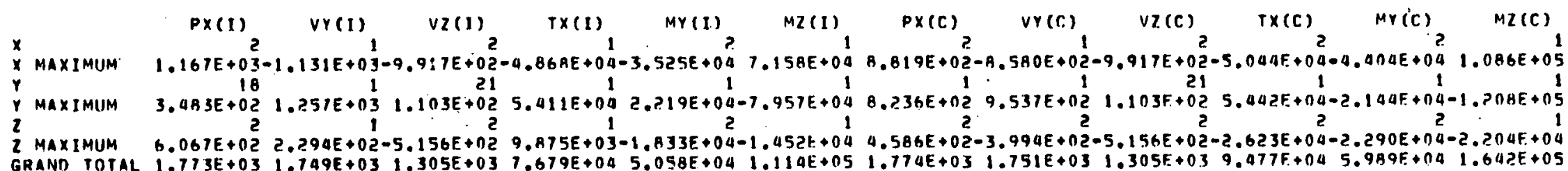
ELEMENT TYPE (3/0) P I PE ), , ELEMENT NIJMRER ( 39)

\begin{tabular}{|c|c|c|c|c|c|c|c|}
\hline & & $P \times(J)$ & $\operatorname{Vr}(J)$ & $V Z(J)$ & $T \times(J)$ & $\operatorname{Mr}(J)$ & $M Z(J)$ \\
\hline & & 1 & 2 & 2 & Z & 1 & 1 \\
\hline & MAXIMIIM & $1.131 E+03$ & $-1.167 E+03-$ & $9.917 E+02-$ & $-7.988 E+144$ & 4.1236 .04 & $1.262 f .05$ \\
\hline & & i & 1 म & 21 & 1 & 1 & 1 \\
\hline & MaximIIM & $1.257 E \bullet 03$ & $13 E+0 ?$ & $1.103 E \nrightarrow 02$ & $B O E+0 a-$ & a- & $-1.403 E+105$ \\
\hline & & & 2 & 2 & 2 & 2 & 1 \\
\hline 2 & IJM & $\begin{array}{l}290 E+02 \\
749 E+03\end{array}$ & $\begin{array}{r}-6.067 E+0 ?- \\
1.773 F+03\end{array}$ & $\begin{array}{l}-5.156 E+02- \\
1.3 n 5 E+03\end{array}$ & $\begin{array}{l}4.153 E+00- \\
1.004 E+05\end{array}$ & $\begin{array}{r}-1.40 \mathrm{BF}+14- \\
\text { B. } 11 \mathrm{DE}+\text { OA }\end{array}$ & $\begin{array}{l}-2.5608+04 \\
1.963 F+0 S\end{array}$ \\
\hline
\end{tabular}


$V Z(I)$ TX(I) Mr(I) MZ(I)

$P \times(J) \quad V Y(J)$

$V Z(J)$

$T \times(j)$

MY (J)

$M Z(J)$

X MAXIMUM

$Y$ MAXIMIM

$-1.131 E+03-5.517 E+02$

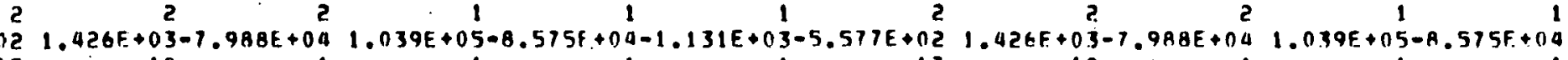

I MAXIMIIM 10.017

2 MAXIMIIM $18 \quad 1 \quad 1111$ $17 \quad 0.3-7.9 B 8 E+041.039 E+05-A .575 F_{0}+04$

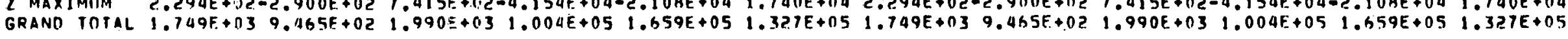


Solution $6(\mathrm{~b})$

Intermodal/Interspatial Combination 


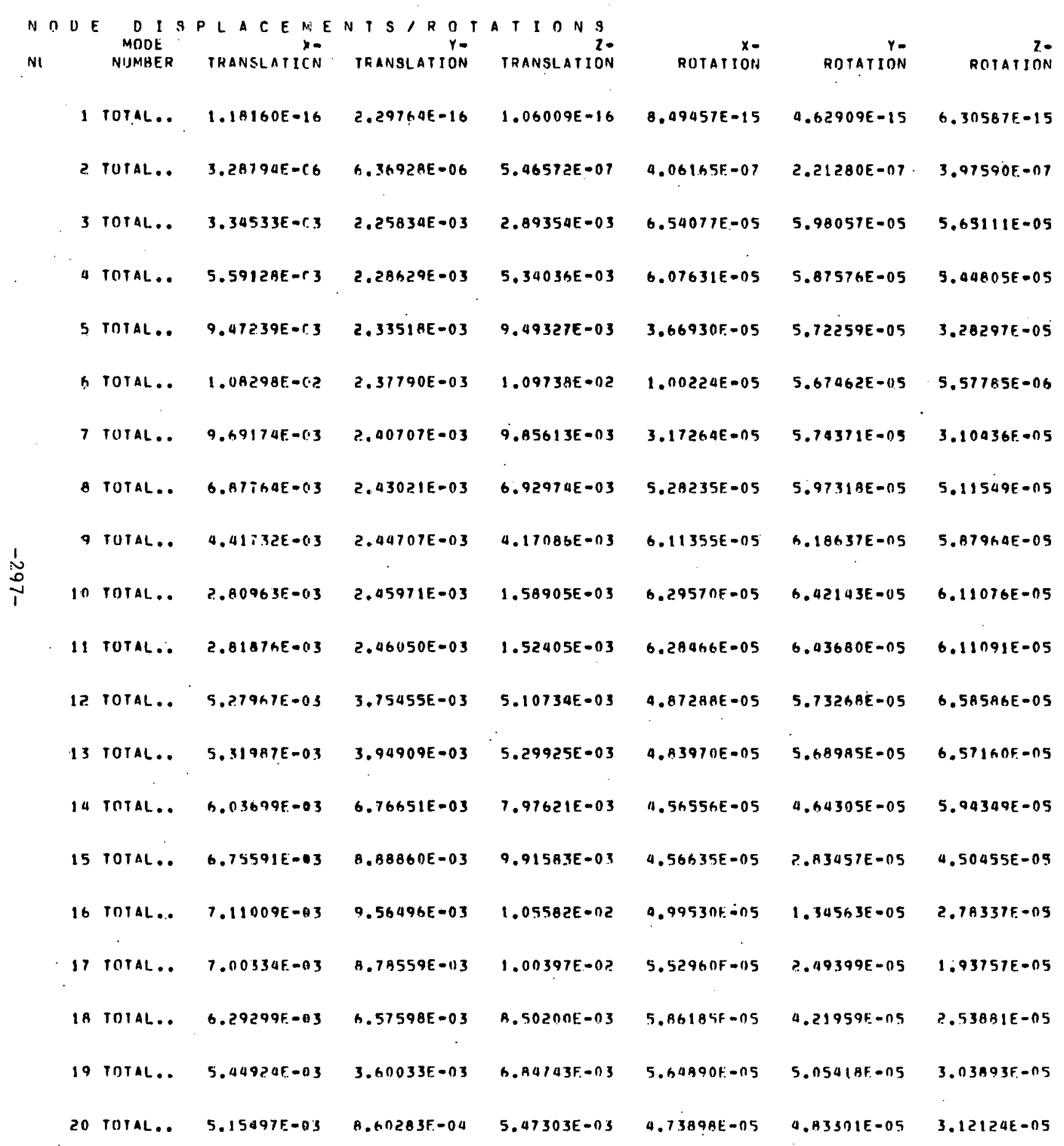




\begin{tabular}{|c|c|c|c|c|c|c|c|}
\hline 21 & TOTAL... & $5.92400 E-03$ & $2.48067 E-03$ & $4.86531 E-03$ & $3.02602 E=05$ & $3.76230 E=05$ & $2.93444 E-05$ \\
\hline ?.2 & FOTAL.. & $6.73612 E-03$ & $4.08386 E-03$ & $4.62031 E-03$ & $2.03370 E-05$ & $2.379968-05$ & $2.67391 E-05$ \\
\hline 23 & TORAL.. & $6.9655 A E-03$ & $4.64 B 64 E=03$ & $4.46465 E-03$ & 1.2BAS3E-05 & $1.71814 E-05$ & $2.55058 E-05$ \\
\hline 24 & I TUTAL.. & $6.2103 B E-03$ & 3.9058 ?E -03 & 4.26 .35 aE -03 & $2.06960 F-05$ & $3.16248 \mathrm{~F}-05$ & 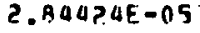 \\
\hline 25 & S TOTAL... & $5.02757 E-03$ & $2.33377 E-03$ & $4.11881 E=03$ & $2.89652 E-05$ & $5.15312 E-05$ & $3.53358 E-05$ \\
\hline 26 & TOTAL... & $5.41811 E-03$ & $1.36776 E-03$ & $4.11052 E-03$ & $3.35435 F=05$ & $7.31877 E-05$ & $4.38125 E-05$ \\
\hline 27 & TOTAL.. & $9.35674 E-0.3$ & $3.49750 E-03$ & $4.27 .229 E-03$ & $2.91597 E=05$ & $9.80150 E-05$ & $5.14207 E-05$ \\
\hline 28 & B TOTAL.. & $1.45207 E-122$ & $4.86062 E-03$ & $4.36939 E=03$ & $2.75372 E-0 h$ & A. $59385 E-05$ & $5.65464 E-05$ \\
\hline 29 & F TOTAL... & $1.915 A 9 E-02$ & $3.63618 E-03$ & $4.30022 E-03$ & $5.20125 E-05$ & 6.89558E-05 & $6.07492 E=05$ \\
\hline 30 & TOTAL.. & $2.22947 . E-02$ & $2.03375 E-03$ & $0.092 ? 1 E-03$ & $1.31710 E=04$ & $4.2 A 161 E-05$ & $6.555195-05$ \\
\hline 31 & IOTAL... & 2.358 AOE -02 & $1.29835 E-02$ & $3.81059 t-03$ & $2.11979 E-04$ & r. $27624 t-05$ & $6.69852 E-09$ \\
\hline 32 & POTAL... & $2.280675-02$ & $2.78395 E-02$ & $3.50183 E-03$ & $2.6 \mathrm{B2h} 4 \mathrm{E}=04$ & $3.98537 E-155$ & $6.06061 E-05$ \\
\hline 33 & TOTAL... & $2.03516 t=02$ & $9.47631 E-02$ & $3.59139 t-03$ & $3.02319 E=04$ & $6.61564 E-05$ & $4.58320 E-05$ \\
\hline 34 & TOTAL.. & 1.66G2?E-02 & $6.19396 E-02$ & $4.92442 E-03$ & $3.18143 E=04$ & $8.63152 E-05$ & $2.55487 E-05$ \\
\hline 35 & F TOTAL... & $1.26197 E-02$ & $7.78995 E-02$ & $7.59565 E-03$ & $3.19619 E-04$ & $9.57733 E-05$ & $2.39926 E=05$ \\
\hline 36 & D TOTAL... & A.05273E-03 & $6.88946 E-02$ & $1.510 \mathrm{RBE}-02$ & $2.400475=04$ & $5.38244 E-05$ & $2.77607 E-04$ \\
\hline 37 & TRTAL.. & $7.54350 E=03$ & $5.7965 \mathrm{AE}=02$ & $1.35002 E-02$ & $2.32999 E=04$ & $h .51232 E=05$ & $2.92438 E=04$ \\
\hline $3 A$ & TOTAL... & $6.11302 E-03$ & $2.62180 E-02$ & $7.87901 E-03$ & $2.05743 F=04$ & A.18423E-05 & $3.10744 E-0 A$ \\
\hline 39 & TUTAL.. & $5.6730 ? E=03$ & $1.08703 E-02$ & $5.32199 E-03$ & $1.87684 \mathrm{E}=04$ & $7.97301 E=05$ & $3.00672 E-04$ \\
\hline$\cdots$ & TOTAL... & $2.08363 E-06$ & $1.76376 E-06$ & $9.80421 E-07$ & $1.33501 f-06$ & 9.8537 P.E-07 & $1.65903 E=06$ \\
\hline 1 & TOTAL & $1.97471 F_{-06}$ & $1.74791 E=06$ & $9.30363 E-01$ & 1.3?5nht-06 & $9.75626 f .07$ & $1.64659 E-06$ \\
\hline
\end{tabular}




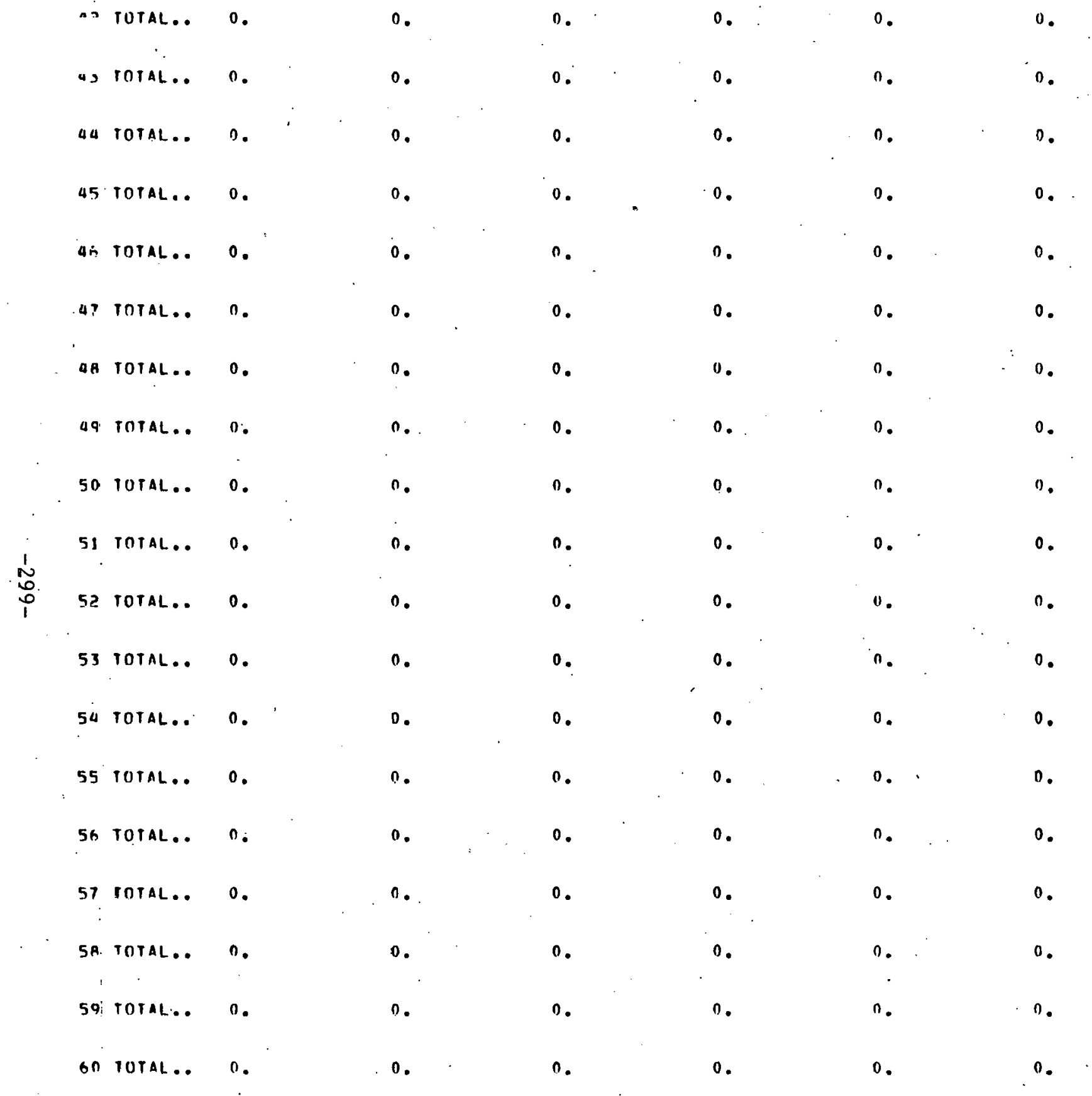


RESPONSE SPECTRUM STRESS COMPONENTS (KINR E (2)

OR EACH ELEMENT. THE FOLLOWING INFORMATION IS PRINTED?

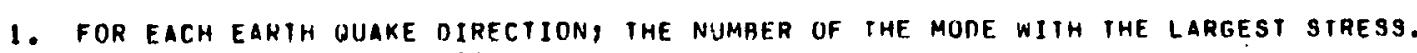

2. THE VALUE OF THAT STRESS.

3. IF REDUESTED, THE MOUE BY MDOE SIRESSES FIR EACH EARTH IIIAKF DIRECTION,

4. THE RESULTANI FOR EACH EATTH DUAKE DIRECTION.

5. THE GRAND TOTAL OF THE THREE EARTH DUAKE DIRECTIONS.

(NOTE: THE $X_{0}, y$, OR 2 REFERS TU THE EARIH DUAKE DIRECTION.)

ELEMENT TYPE IB O UN N D A R $Y$

ELEMENT NUMBER.

1)

- BITRYAF. BDRYAM

$\begin{array}{lrr}X & 6 & 9 \\ X \text { MAXIMUM } & 3.301 E+02 & 1.291 E+04 \\ Y & 3 & 11 \\ Y \text { MAXIMUJM } & 2.660 E+02 & 4.625 E+04 \\ 2 \text { MAXIMUM } & 5.793 E+02 & 2.187 E+04 \\ 2 \text { MAND TOTAL } 1.182 E+03 & B .495 E+04\end{array}$

ELEMENT TYPE. $(B$ O U N D A R $Y$

$, 1,1$ element NUMBer 1

2)

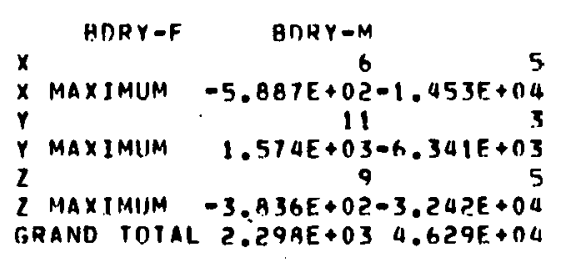

ELEMENT TYPE IB O U N D A R Y

, 1, element Number $(3)$

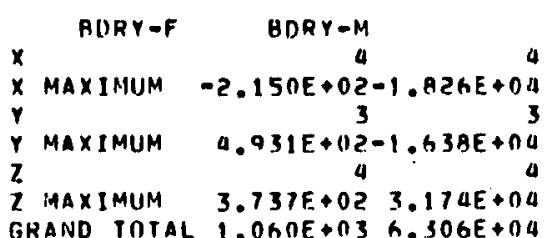

GRAND TOTAL T.OKחE + 3 h. $306 E+04$

ELEMENT TYPE (H $O U U N D A$ A $Y$

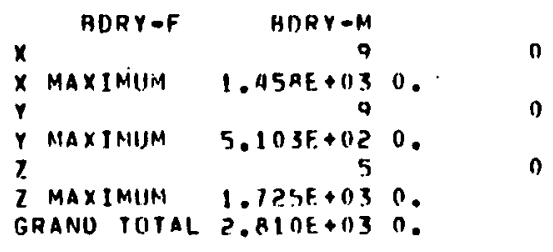

ELEMENT TYPE (A 0 U N $N$ D A R $Y$

\begin{tabular}{|c|c|c|}
\hline RDRY $-F$ & BDHY $-M$ & \\
\hline IUM & $-4.539 E+0 ?$ & $n$. \\
\hline man & $\begin{array}{r}11 \\
-3.515 F+02\end{array}$ & $n$. \\
\hline
\end{tabular}


6. MAXIMUM $7.795 E+020$.

TYPE (A O IIN N T A Y

, 1,1 element NUMBer

6)

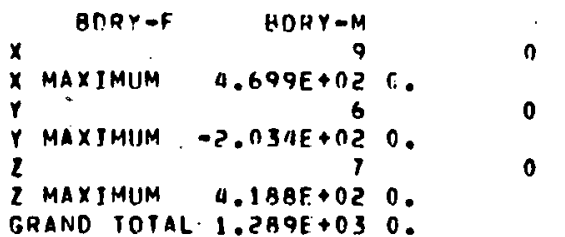

ELEMENT TYPE (B O UNNDAR $Y$.

$, 1,1$ ELEMf.NT NUMAER (

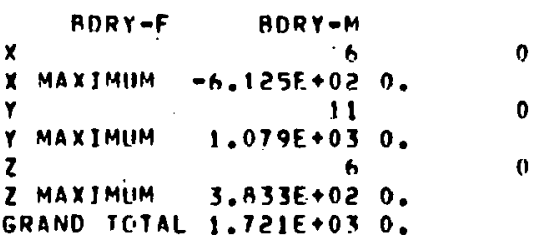

ELEMENT TYPE (H O U N D A R Y

), 1, ELEMENT NIJMHER ( R)

BDRYOF ADRY-M

$x$
$X$ MAXIMIIM

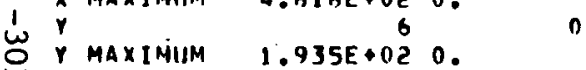

I $z$ MAXIMIIM $1.935 E+020.5$

2 MAXTMINM $1.03 n E+030$.

ELEMENT TYPE (B OU 40 A

) 1,1 ELENENT NUMHF, $(9)$

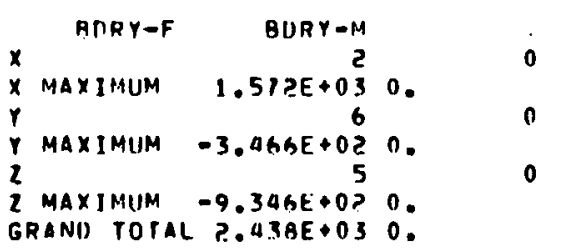

ELEMENT TYPE CB O IINOA $N$ Y

$, 1,1$ flement nUmaer $(101$

\begin{tabular}{|c|c|c|}
\hline & BOAY & \\
\hline MAXIMUM & $1.966 E+103$ & $n$. \\
\hline & 1 & \\
\hline$Y$ MAXIIAUM & $-1 . R 3 \cap E+n 3$ & n. \\
\hline MAXIMUM &, $974 E+03$ & 0 \\
\hline RANO TDTAL & $2.7365+03$ & $n$. \\
\hline
\end{tabular}

ELEMENT TYPE (A O $1 " N$ N) A R $Y$

), 1, FLEMENT NUMHER $(11)$

$\begin{array}{lc}\text { BDRY } & \text { BDRY } \\ X \text { MAXIMUH } & 5.194 F+n ? \\ & \end{array}$ 
$Y$ MAXIMIJM $2.935 E+020.0$

2 MAXIHUM $8.883 E+020$.

GRANO TOTAL $1.850 E+030$.

ELEMENT TYPE (B 0 II N

) 1,1 ELEMENT NUMBER ( 12)

\begin{tabular}{|c|c|c|}
\hline BDRY $A F$ & BDRY $=N$ & \\
\hline MAXIMUM & $-2.555 E+03$ & 0. \\
\hline & 1 & \\
\hline MAXIMUM & $2.8 \triangle O E+03$ & 0. \\
\hline 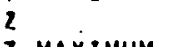 & - 1 & 0 \\
\hline
\end{tabular}

2 MAXIMIIM $5.1835+020$.

GRANO TOTAL $4.067 E+030$

ELEMENT TYPE (B O UNDAR $Y$

, 1,1 element number (13)

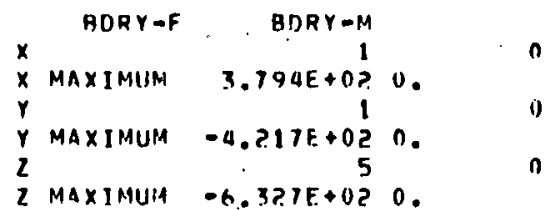

TRRAND TUTAL 1.449E +030.

ELEMENT TYPE (A $D U N$ I) A $R Y$

, 1,1 element Numaer (14)

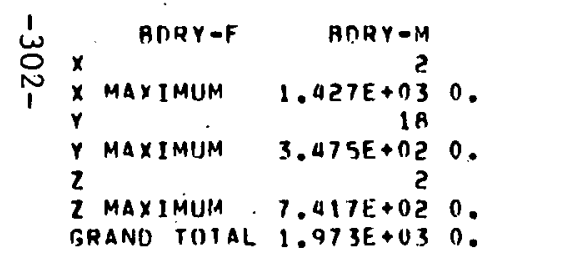

GRANO TOIAL $1.973 E+030$.

ELEMENT TYPE IA O U N DA A Y

$, 1,1$ ELEMENT NIJMIER ( 15)

\begin{tabular}{|c|c|c|c|}
\hline & BDRY $-F$ & BORY $-M$ & \\
\hline & & I. & \\
\hline & MAXIMUJM & $-1.133 E+03$ & 0 \\
\hline & & 1 & \\
\hline & MAXIMIJM & $1.259 E+03$ & 0 \\
\hline & MAXIMUJM & $2.29 A E+02$ & 0. \\
\hline
\end{tabular}

GRANO TOTAL $1.74 \mathrm{AE}+0.30$.

ELEMENT TYPE (A O UNDAR $Y$

$, 1,1$ ELEMENT NIMAER ( 16)

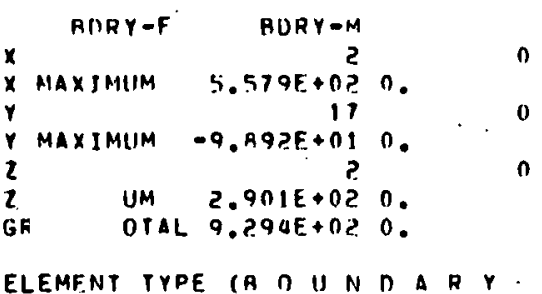

ELEMENT TYPE (A OUN $\mathrm{NA}$ A $Y$ 


\begin{tabular}{|c|c|c|}
\hline anRY-F & \multicolumn{2}{|c|}{ BORY $=$ H } \\
\hline мuм & 0 & ${ }^{0} .8$ \\
\hline & n. & \\
\hline $\begin{array}{l}2 \text { MAXIMUM } \\
\text { GRAND TOTAL }\end{array}$ & ?. & $\begin{array}{r}0 \\
1.740 E+04 \\
1.326 E+05\end{array}$ \\
\hline
\end{tabular}

ELEMENT TYPE $B$ B O UN N OA A $R$ Y

, 1, ELEMENT NUMAER $(18)$

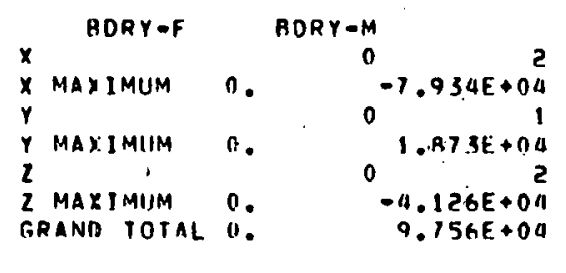

ELEMFNT TYPE (B O U N O A R Y

3,1, ELEMENT NUMRER $(19)$

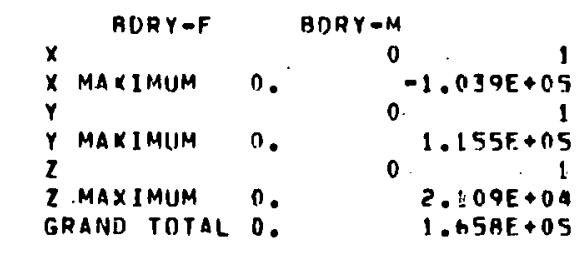

1 ELEMENT TYPE $13 / 0$ P I PE

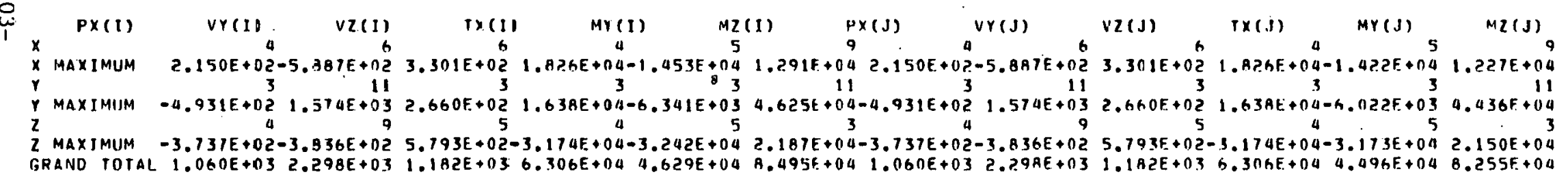

ELENENT TYPE (3/0 P I P E $1,1,1$ element NUMAER ( 2)

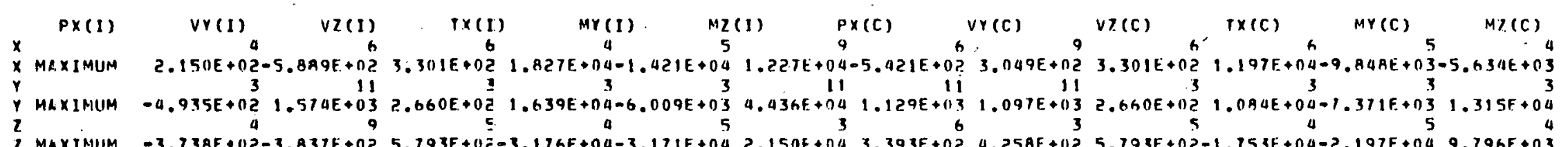

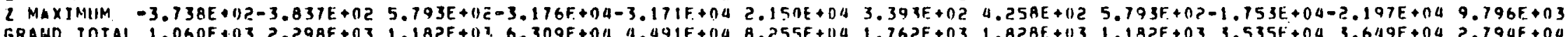
ELEMENT TYPE ( 30 P I PE $:, 1$, RELMENT NUMHFH $($ ?)

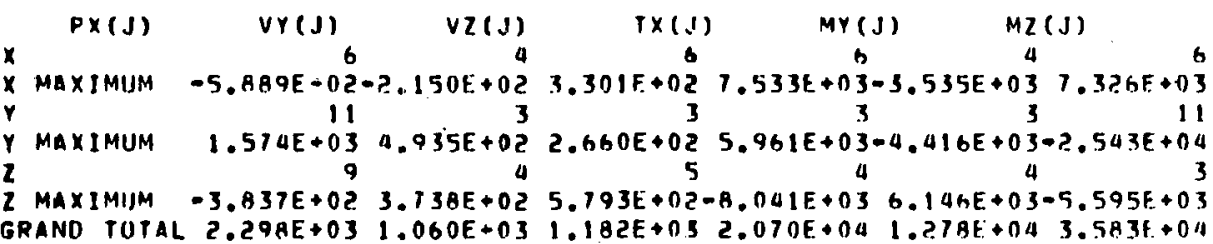




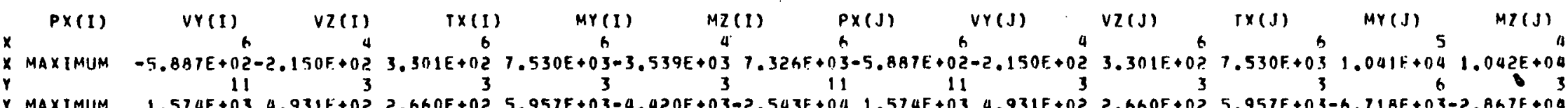
$Y$ MAXIMUM $1.574 E+03 \quad 4.931 E+022.660 E+025.957 E+03-4.420 E+03-2.543 E+0411.574 E+034.931 E+022.6 h 0 E+0 ? 5.957 E+03-6.718 E+03-2 . A h 7 F+04$ 2 MAXIMUM - 3. A36E+02 $3.737 E+025.793 E+02-8.036 E+03 \quad b .152 E+03-5.595 E+03-3.836 E+02-3.737 E+025.793 E+02-8.036 E+03 \quad 2.32 .3 F+04-1.812 E+04$ GRAND TOTAL $2.29 A E+03 \quad 1.060 E+0.31 .182 E+032.069 E+04 \quad 1.2 B 0 E+043.583 E+042.298 E+031.060 E+031.1 A 2 E+032.069 E+043.841 E+046.043 E+04$

ELEMENT IYPE (3/D P I P E

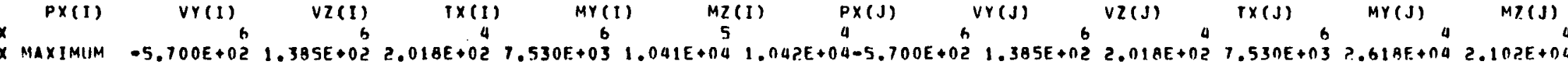

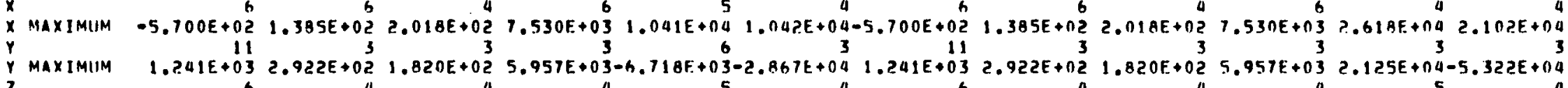

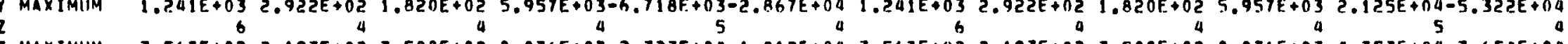
2 MAXIMUM 3.567E+02 $2.193 E+02-3.508 E+02-8.036 E+032.323 E+04-1.812 E+043.567 E+022.193 E+02-3.508 E+02-0.036 E+03 \quad 4.753 E+04-3.654 E+04$ GRAND TOTAL 1.897E+03 6.840E+ $6.937 E+022.069 E+043.801 E+046.043 E+041.897 E+036.840 E+026.937 E+0 ? 2.069 E+049.308 E+041.019 E+05$ ELEMENT TYPE (3/0 P I PE , , , ELEMENT NUMBER ( 5$)$

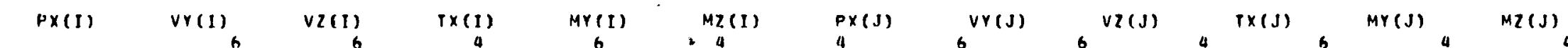

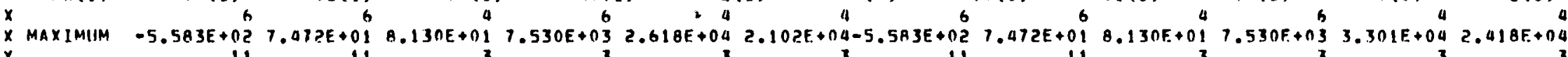
Y MAXIMUM $1.049 E+113-2.457 E+02 \quad 9.694 E+01^{3} 5.957 E+032.125 E+04-5.322 E+041.049 E+03-2.057 E+02 \quad 9.694 E+015.957 E+03 \quad 2.939 E+04-6.147 E+043$ W 2 MAXIMIJM $3.494 E+02 h .540 E+01-1.413 E+02-8.036 E+03 \quad 4.753 E+04-3.654 E+043.494 E+02 \quad 6.940 E+01-1.413 E+02-R .03 A E+03-5.739 E+04-4.203 E+04$ I GRAND TOIAL $1.675 E+03 \quad 3.940 E+022.787 E+022.069 E+049.348 E+041.019 E+051.675 E+03 \quad 3.940 E+02 \quad 2.787 E+022.069 E+041.130 E+051.186 E+05$ ELEMENT TYPE (3/D PIPE ), 1,1 ELEMENT NUMHER $(6)$

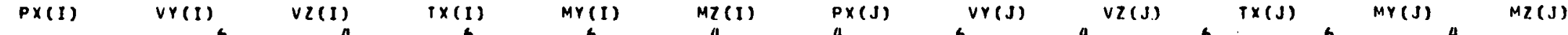
$x$ MAXIMUM $-5.466 E+02.5 .364 E+01-1.114 E+02$
$\times$ $x$ MAXIMUM -5.466E+02. $5.364 E+01-1.114 E+02$
$Y$

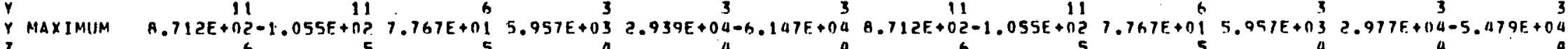
2 MAXIMUM $3.421 E+02-1.115 E+02-2.086 E+02-8.036 E+03-5.739 E+04-4.203 E+04 \quad 3.421 E+02-1.115 E+02-2.0 B A F+0 ?-2.036 E+03-5.233 E+04-3.580 E+044$

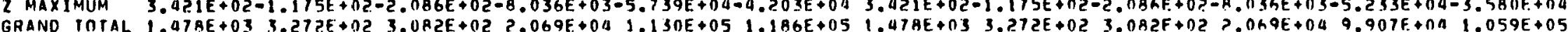
ELEMENT TYPE (3/D P I PE , , , ELEMENT NUMAER $(1)$

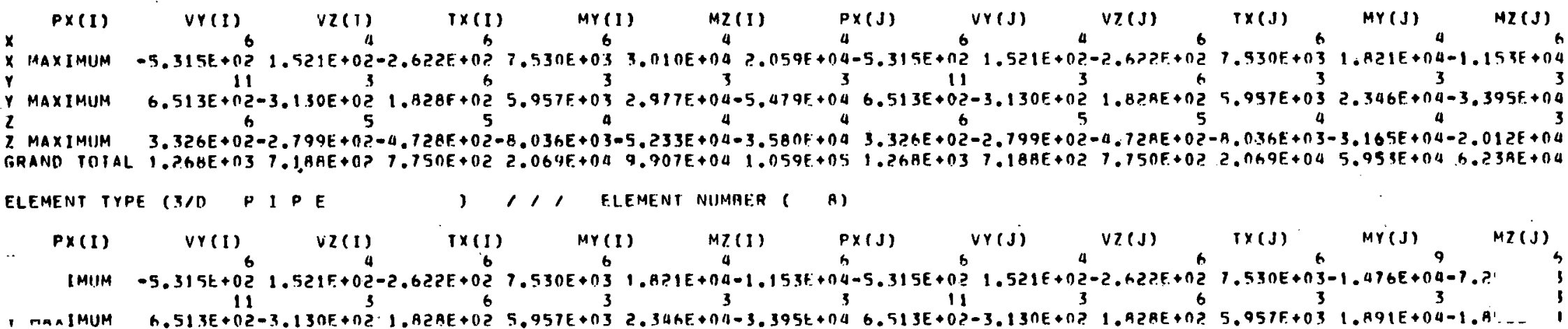




\section{ELEMENT TYPE ( $3 / 0$ P I PE , , , ELEMENT NUMAER $(9)$}

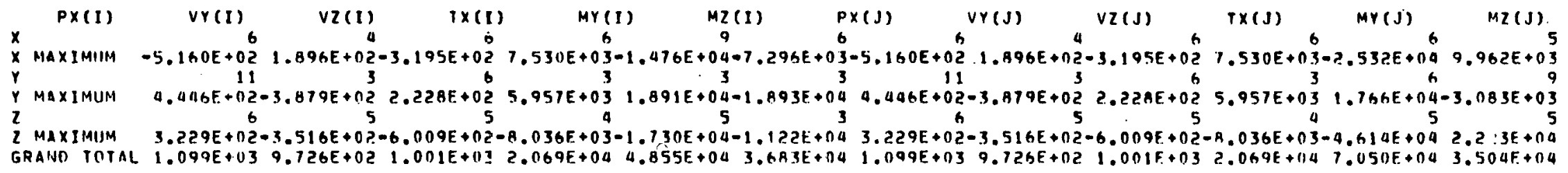
ELEMENT TYPE (3/0 P 1 PE , 1, ELEMENT NUMBER 1 (10)

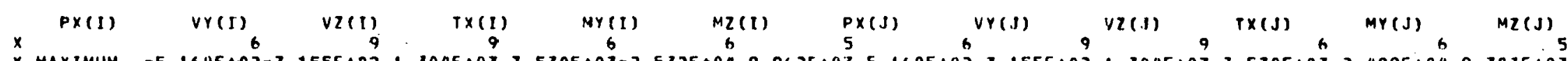

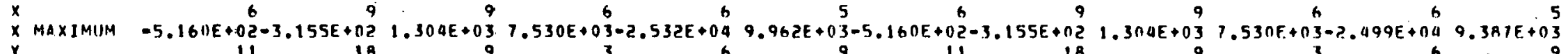

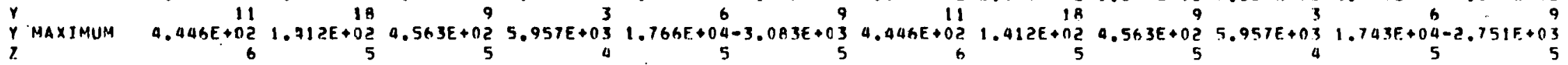
2 MAXIMUM $3.229 E+12 \quad 4.278 E+02 \quad 1.124 E+03-8.036 E+03-4.614 E+04 \quad 2.223 E+04 \quad 3.229 E+02 \quad 4.278 E+02 \quad 1.124 E+03-8.036 E+03-4.277 E+04 \quad 2.094 E+04$ GRAND TOTAL 1.099E+1) $1.002 E+032.156 E+032.069 E+04 \quad 1.050 E+043.504 E+041.099 E+031.002 E+032.156 E+03 ? .069 E+046.533 E+043.23 A E+04$ ELEMENT IYPE ( $3 / 0$ P I PE , , , ELEMENT NUMBER $(11)$

PX(I) VYCII VZC(I) TX(I: MY(I) MZ(I) PX(C) VY(C) $V Z(C) \quad$ TX(C) $M Y(C) \quad$ MZ(C)

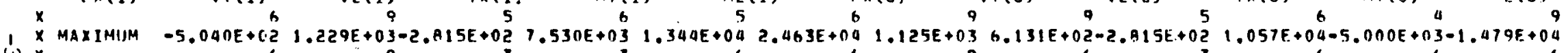

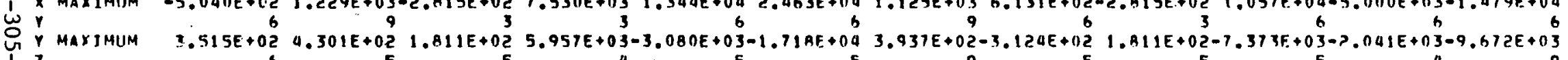

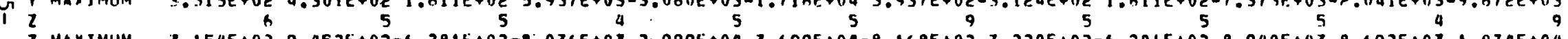
2 MAXIMUM 3.154E+02 9.452E+02-6.281E+02-0.036F+03 2.999E+04 3.699E+04-8.168E+02 $7.220 E+02-6.281 E+028.940 E+038.692 E+03 \quad 1.074 E+04$ GRANO TOTAL 1.001E+103 $1.972 E+039.377 E+022.069 E+04 \quad 4.063 E+046.2 B 1 E+041.725 E+03 \quad 1.417 E+039.377 E+02 \quad 3.097 E+04 \quad 1.325 E+043.169 E+04$ ELEMENT TYPE ( $3 / 0$ D 1 PE , , , ELEMENT NIJMAER $(11)$

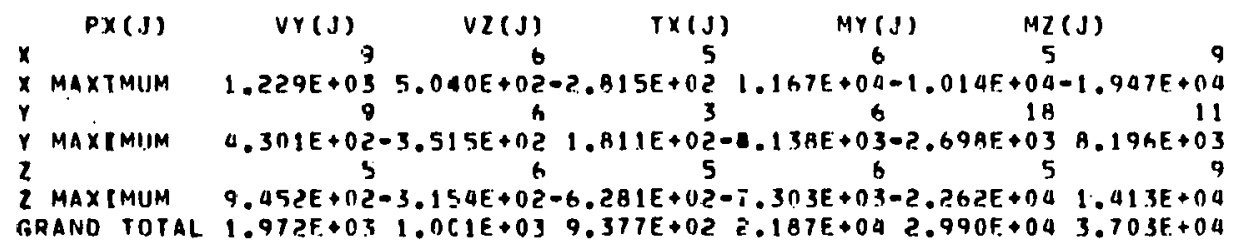
ELEMERT TYPE $(3 / 0, I P E, 1$, ELEMENT NIJMHER $($ I $)$

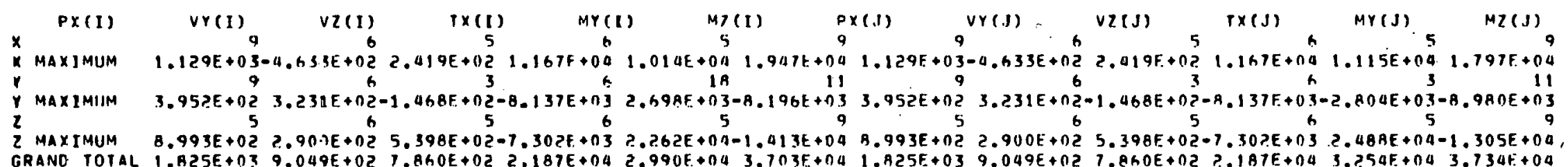
ELEMENT TYPE $(3 / \mathrm{N}$ P 1 PE , 1, ELEMENT NUMBER $(13)$

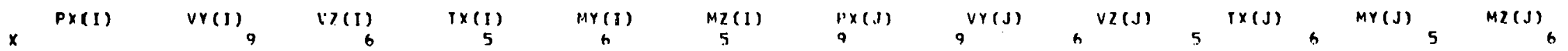


$\times$ MAXIMUM $9.663 E+02-3.913 E+02 \quad 2.029 E+02 \quad 1.117 E+04 \quad 1.115 E+04 \quad 1.766 t+049.663 E+02-3.913 E+022.029 E+02 \quad 1.117 E+042.305 E+042.966 E+04$ M MAXIMUM $3.3 B 1 E+0 ? 2.729 E+0 ?-1.061 E+02-7.791 E+03-2.004 E+03-9.083 E+033.3 B 1 E+022.729 E+02-1.061 E+02-7.791 E+03-8.797 E+03-2.06 A E+04$

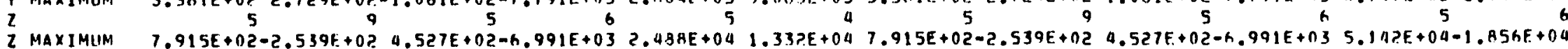
GRAND TOTAL $1.573 E+037.731 E+026.555 E+022.128 E+043.254 E+043.754 E+041.573 E+037.731 E+026.555 E+022.12 A E+146.520 E+045.639 E+04$

ELEMENT TYPE $13 \% 0$ P I PE $1 \% 1$ ELEMENT NUMBER 1 14)

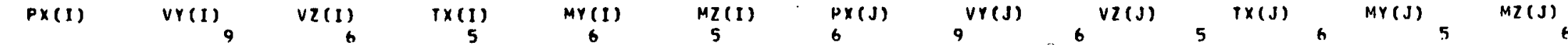

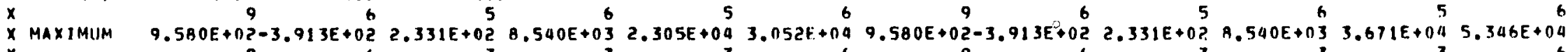

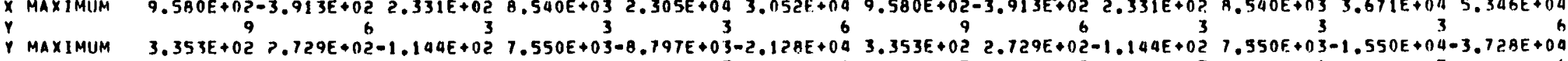
7 MAXIMUM $3.353 E+02>.729 E+02-1.144 E+02$
2 2 MAXIMUM $7.490 E+02-2.539 E+025.201 E+02-5.344 E+035.142 E+04-1.910 F+047.490 E+02-2.539 E+025.201 E+02-5.344 E+03 \quad 8.191 E+04-3.345 E+00$ GRAND TOTAL $1.542 E+037.731 E+027.250 E+021.841 E+046.520 E+045.720 E+041.542 E+03 \quad 7.731 E+027.250 E+021.841 E+041.058 E+059.649 E+04$ ELEMENT TYPE (3/0 P I P E, 1, ELEMENT NUMBER $(15)$

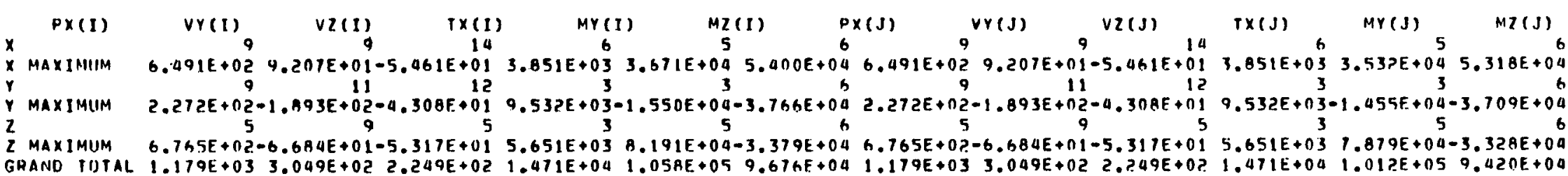
GRAND TOTAL $1.175 E+033.049 E+n 22.430 E+02 \quad 1.563 E+041.012 E+059.388 F+041.175 E+03 \quad 3.049 E+022.430 F+021.563 E+1141.01 A E+059.485 E+04$ ELEMENT TYPE (3/1) P 1 PE , 1,1 ELFMENT NUMBER $(17)$

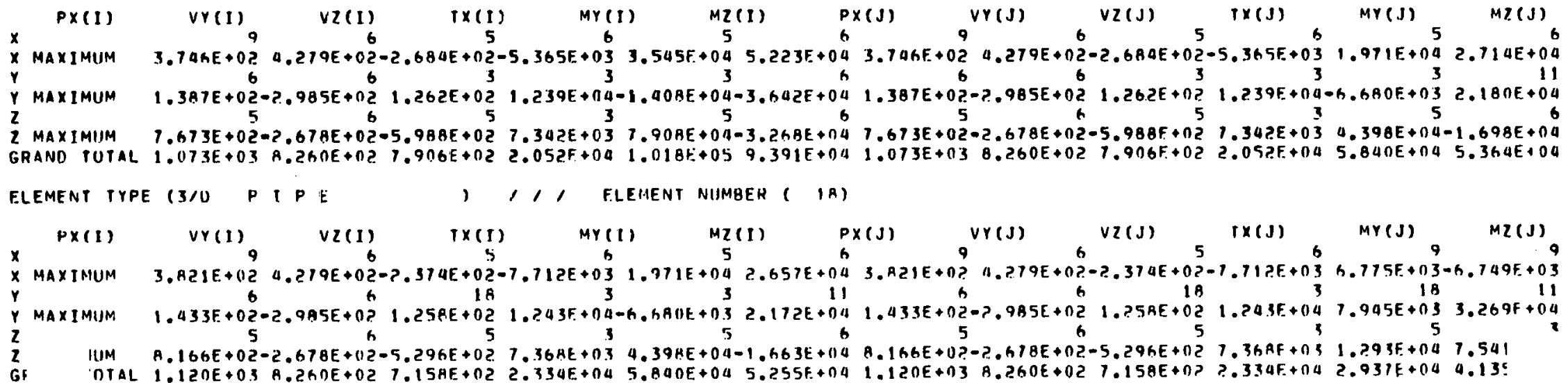

ELEMENT TYPE (3/0, $P R E, 1,1$ FLFMFNT NUMHER $($ 19) 


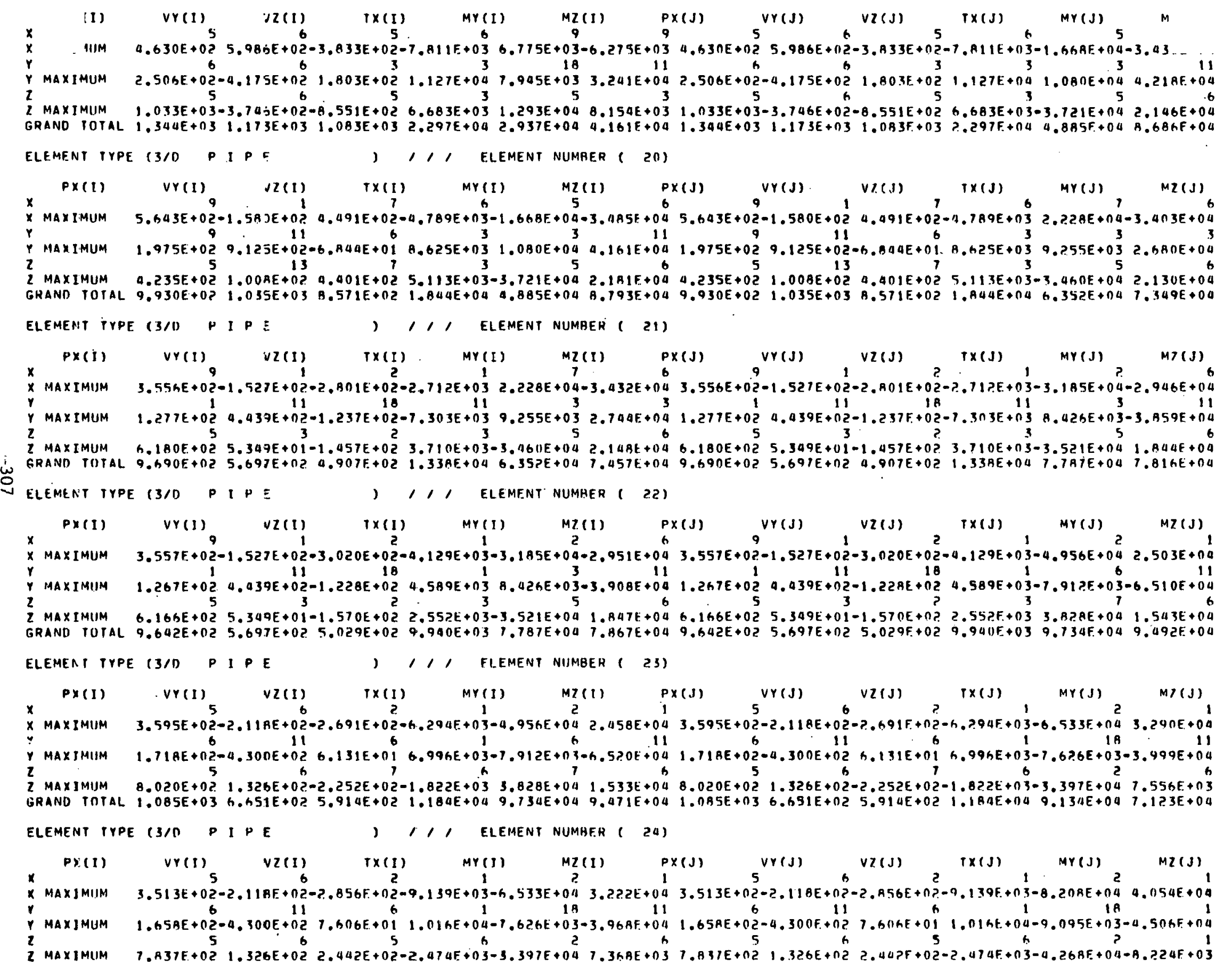


GRANO TOTAL $1.069 E+03 \quad 6.651 E+02 \quad 6.453 E+02 \quad 1.688 E+049.134 E+04 \quad 7.021 E+04 \quad 1.069 E+03 \quad 6.651 E+02 \quad 6.453 E+02 \quad 1.68 A F+049.811 E+04 \quad 6.372 E+04$ ELEMENT TYPE (3/D P I P E , , , ELEMENT NIJMAER $(25)$

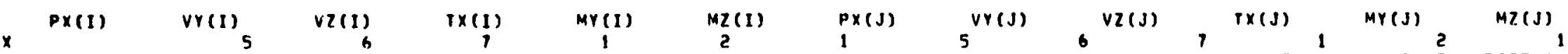

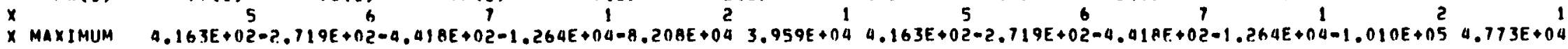

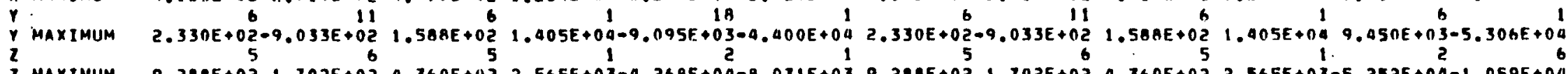
2 MAXTMUM $9.2 . A B E+021.702 E+02 \quad 9.360 E+02,2.565 E+03-4.268 E+00-8.031 E+030.284 E+021.702 E+02 \quad 4.360 E+022.565 E+03-5.252 E+04-1.059 E+04$ GRAND TOTAL $1.303 E+031.158 E+03 \quad 1.145 E+03 \quad 2.179 E+049.811 E+046.221 E+041.303 E+031.158 E+031.145 E+032.179 E+091.235 E+059.096 E+00$ ELEMENT TYPE (3/0 P I P $F, 1$, ELEMENT NUMBER $(26)$

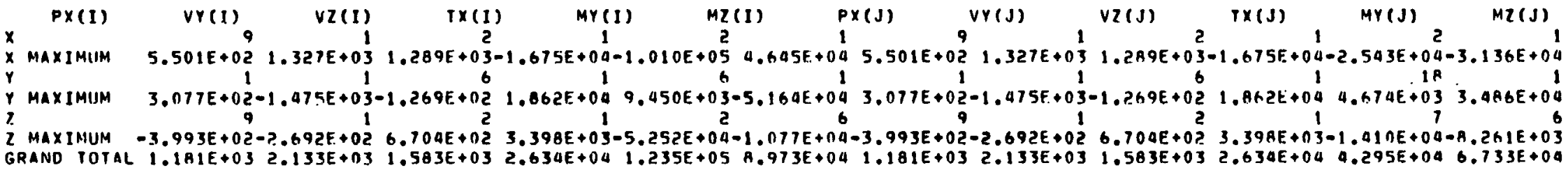
ELEMENT TYPE (3/0 PI PE $), 1$, ELEMENT NUMBER $(27)$

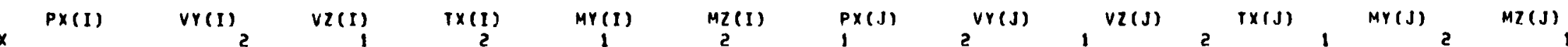

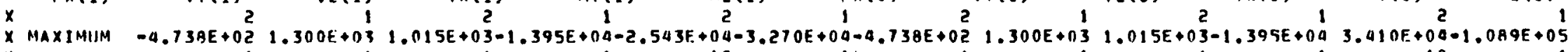
$Y$ MAXIMUM $3.090 E+02-1.445 E+03-6.8 A 5 E+01$
2

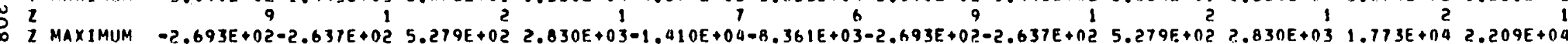
GRANO TOTAL $9.564 E+021.981 E+031.200 E+032.166 E+049.295 E+046.89 S E+049.564 E+021.9 B 1 E+031.200 E+032.166 E+045.248 E+041.687 E+05$ ELEMENT TYPE (3/0 P I PE ) 1,1 ELEMENT NUMBER $(2 B)$

$\begin{array}{llllllllll}P X(I) & V Y(I) & V Z(I) & I X(I) & M Y(I) & M Z(I) & P X(J) & V Y(J) & V Z(J) & T \times(J)\end{array}$

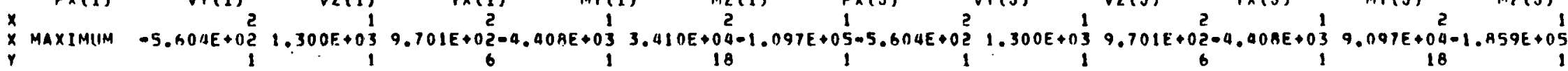

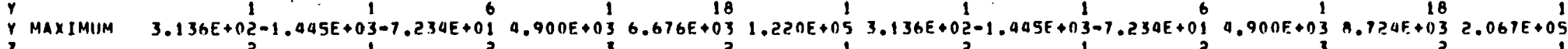
2 MAXIMIIM $-2.914 E+02-2.637 E+025.004 E+02 \quad 1.569 E+03 \quad 1.773 E+042.226 E+04-2.910 E+02-2.637 E+025.044 E+021.569 E+03 \quad 4.730 E+043.712 E+04$

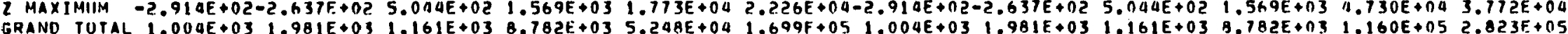
ELEMENT TYPF. ( $3 / 0$ P I PE , 1,1 ELEMENT NUMBER $(29)$

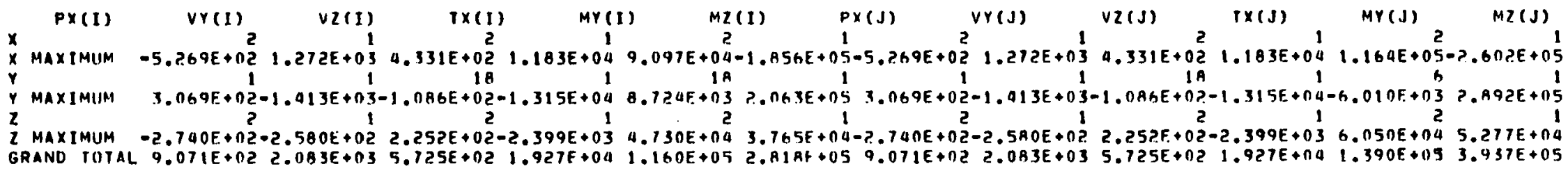
ELEMENT TYPE (3/0 P I PE ), 1, ELEMENI NIJMBSEH ( 30$)$

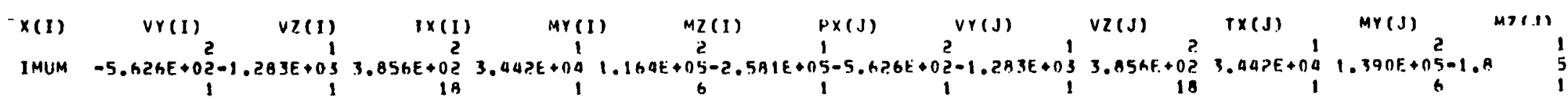


$Y$ MAXIMUIM $3.055 E+021.426 E+03-1.067 E+02-3.026 E+04-6.010 E+032.869 E+053.055 E+021.426 E+03-1.067 E+02-3.826 E+04-5.650 E+032.01 \times 5+05$

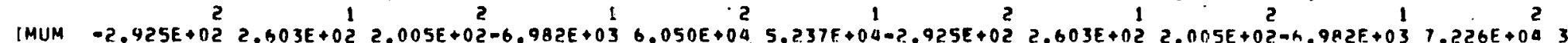

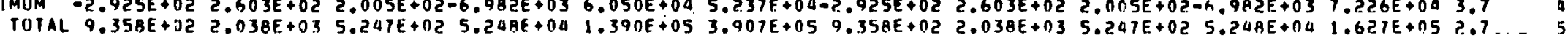
ELEMENT TYPE $(3 / 0$. P J P , , , ELEMENT NUMBER $(31)$

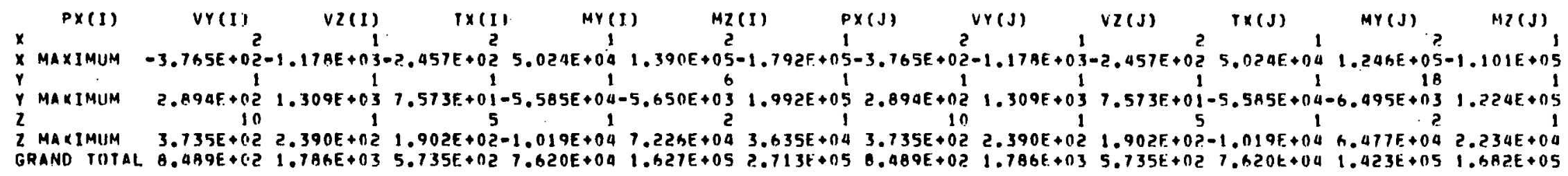
ELEMENT TYPE (3/0 P I PE ) 1,1 ELEMENT NUMBER $(32)$

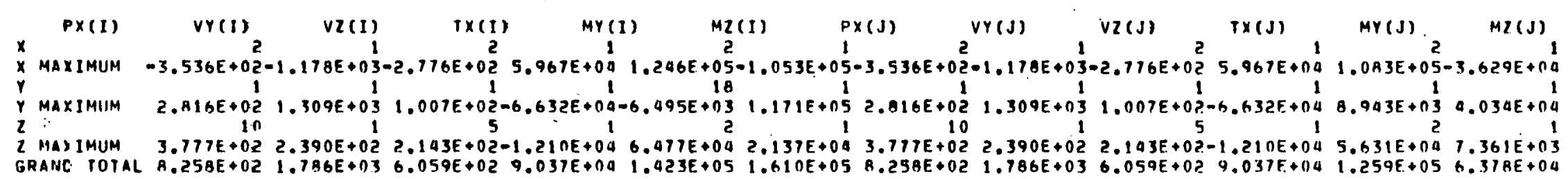
ELEMENT TYPE (3/0 P I PE ) 1,1 ELEMENT NUMBER $(33)$

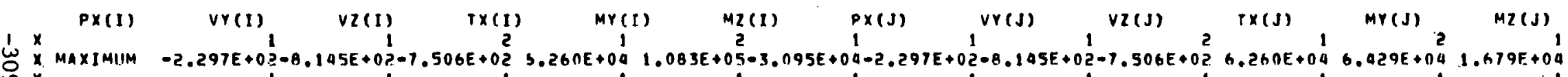
I MAXIMUM $2.553 E+029.054 E+021.477 E+0.2+6.958 E+04 \quad 0.943 E+03 \quad 3.441 E+042.553 E+02 \quad 9.054 E+021.077 E+02-6.958 E+041.760 E+04-1.067 E+04$

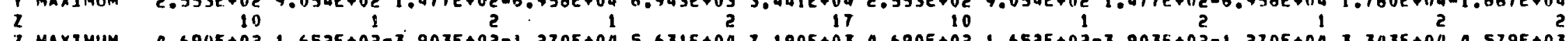

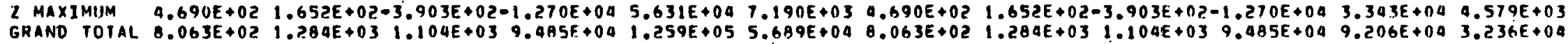
ELEMENT TYPE (3/D) $P$ I P E, 1, ELEMENT NUMBER $(34)$

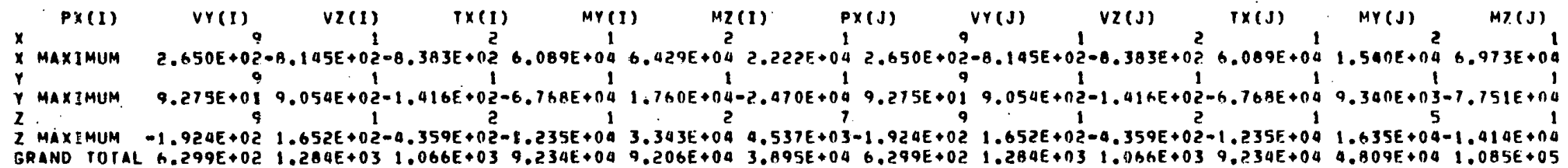

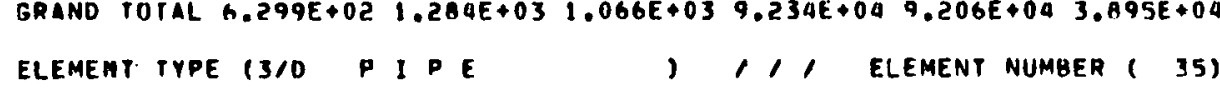

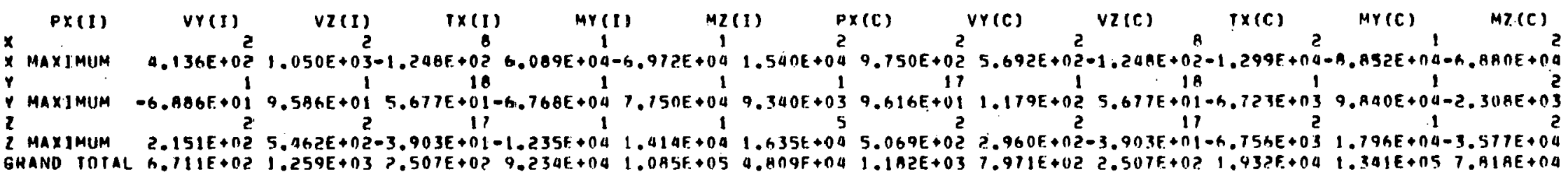
ELEMENT TYPE 1310 P 1 PE ,, 1, FLEMENT NUMRER $(35)$

$\operatorname{Px}(J) \quad \operatorname{vr}(J) \quad V Z(J) \quad \operatorname{Tx}(J) \quad \operatorname{Mr}(J) \quad M Z(J)$ 
$\times$ MAXIMUM $1.118 E+03^{2}-1.675 E+02-1.24 A E+02-4.866 E+04-6.935 E+011-9.027 E+04$

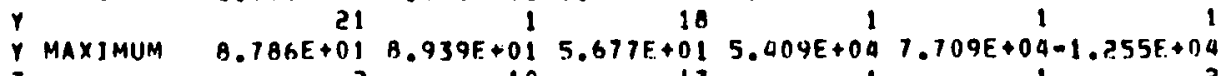

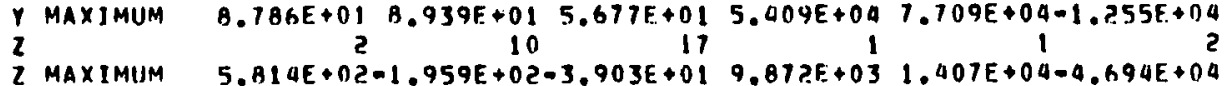
GRAND INTAL $1.32 A E+035.217 E+022.507 E+027.673 E+041.079 E+051.11 N E+05$

ELEMENT TYPE (3/D P I P E $, 1,1$ ELEMENT NUMBER $(36)$

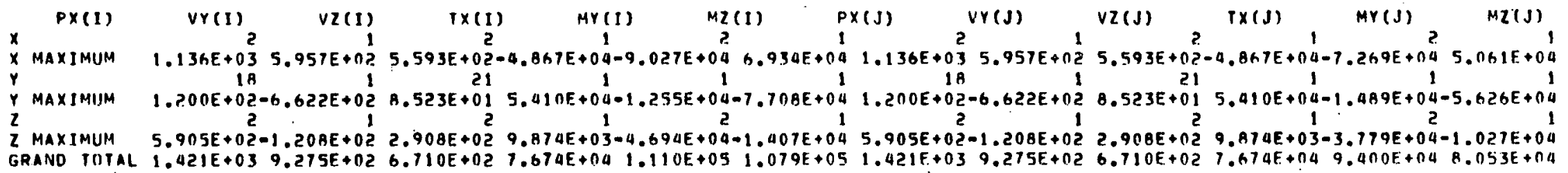

ELEMENT TYPE (3/0 P I PE , 1,1 ELEMENT NUMBER $(37)$

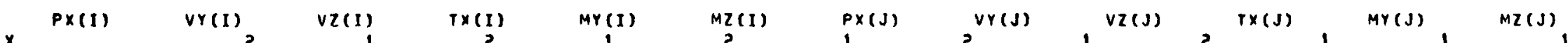

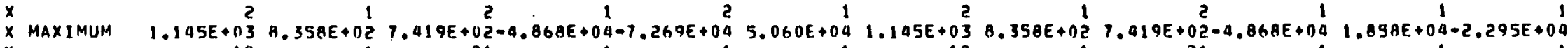

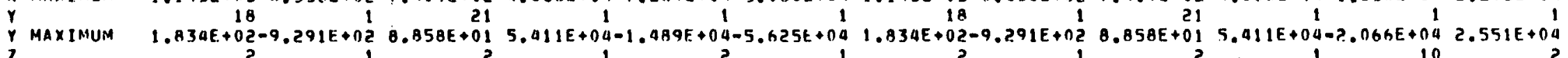

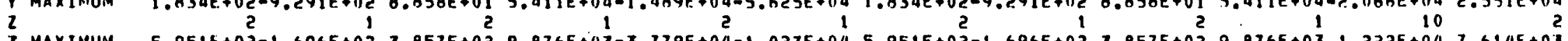
GRAND TOTAL $1.496 E+03 \quad 1.297 \mathrm{~F}+03 \quad 8.855 E+02 \quad 7.675 E+04 \quad 9.400 E+04 \quad 8.052 F+041.496 E+03 \quad 1.297 E+03 \quad 6.855 E+02 \quad 7.675 E+04 \quad 4.205 E+04 \quad 4.632 E+04$

'́ ELEMENT IYPE (3/D P I PE ), , , ELEMENT NUMBER ( 38)

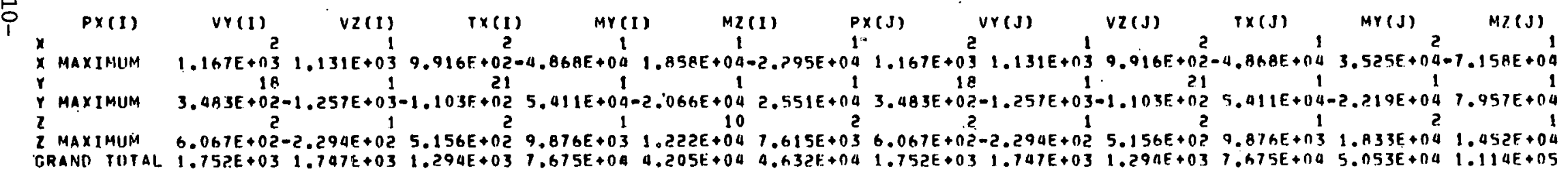
ELEMENT TYPE (3/0 PI PE , 1,1 ELEMENT NUMBER ( 39)

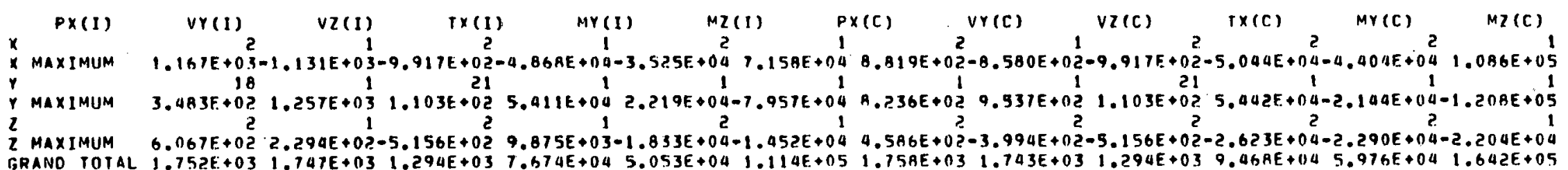

ELEMENT TYPE (3/D PI PE $)$

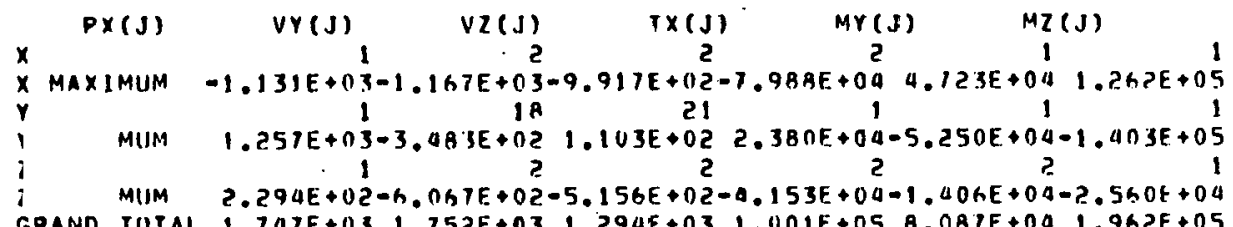


Solution 6(c)

Interspatial/Intermodal Combination

Cluster Factor $=0.0$ 


\begin{tabular}{|c|c|c|c|c|c|c|c|}
\hline NONE & $\begin{array}{l}\text { MODE } \\
\text { NUMHE H }\end{array}$ & THANSLGIION & IRANSLATIUN & IRANSIATIONN & RHTARIIII & $\begin{array}{r}r- \\
R I I A I I I N\end{array}$ & R(1) A IIIIN \\
\hline 1 & IOTAL.. & $1.07 \operatorname{sh} 2 E-16$ & $2.09226 \mathrm{t}-: 6$ & B. $84517 \mathrm{~F}-17$ & 7.97605 -13 & $4.3 t u s s t=15$ & b. 5k921t-15 \\
\hline 2 & TOTAL:. & $3.00043 t .06$ & $5.79674 E-C 6$ & $4.54624 E-07$ & $3.564415=017$ & ?.0951 DE-01 & $3.32+04 t-01$ \\
\hline 3 & TOIAL.. & S. IOABCE-OS & $1.94566 t=03$ & 2.1161 AHE $=03$ & $5.314511-03$ & S. Bsstit -0 S & $5.10+54 t-09$ \\
\hline 4 & TOTAL.. & 5.14 H QE 03 & $1.97130 t=03$ & $4.50878 E-03$ & $3.13049 t-05$ & S.S7hYGF-ios & 4. $89813 t=01$ \\
\hline 5 & TOTAL.. & $8 . t 2401 E-03$ & $2.0165 B t-03$ & A.004TAE-03 & $3.12118 F-05$ & $5.531184 t-05$ & $2.49348 E_{-}-115$ \\
\hline .6 & TOTAL.. & $9.76116 E-03$ & $2.05652 E-03$ & $9.26591 E-03$ & B. $78591 \mathrm{t}-0 \mathrm{E}$ & 3.540 ?E- 05 & S. OBABHE-OB \\
\hline 7 & TUTAL... & $B .71913 E=03$ & $2.08409 E-03$ & $8.3320 U E-03$ & $2.67006 E-05$ & $5.71030 \mathrm{E}-\mathrm{OS}$ & ?.81305t-05 \\
\hline B & TOTAL.. & $6.21097 E-03$ & $2.10634 t-03$ & $5.86444 E-03$ & $4.46511 E-05$ & $5.89079 t-05$ & $4.52103 E-05$ \\
\hline 9 & TOIAL.. & $4.09580 E=03$ & $2.12257 E=03$ & $3.5446 \triangle E-03$ & $5.16233 E-05$ & $6.05538 t-05$ & $5.09349 E-05$ \\
\hline 10 & TOTAL.. & $2.76907 E-0.3$ & $2.13310 t-03$ & $1.41829 E-03$ & $5.29050 t-05$ & $6.24634 t=05$ & $5.18679 t-05$ \\
\hline 11 & TUTAL.. & $2.77845 E-03$ & $2.13589 E-03$ & 1.3592 AE $=03$ & $5.27947 t-05$ & $6.25409 t-115$ & $5.1 A 132 E-05$ \\
\hline 12 & TOIAL.. & $5.01 d 43 E-133$ & 3.5157 ht -03 & $4.7513 \mathrm{BE}-03$ & $4.60098 E-05$ & $5.57834 t .05$ & 5. . 2 SHOE - 115 \\
\hline 13 & TUTAL.. & $5.05726 E-0 \mathrm{~s}$ & $3.69662 E-0.3$ & $4.95012 E-03$ & $4.58121 E-05$ & $5.53596 F-105$ & $5.815 B A E-05$ \\
\hline 14 & TOTAL.. & $5.75831 E-03$ & $6.23220 E-03$ & $7.57386 E=03$ & $4.15107 E-05$ & $4.52987 E-05$ & $5.27518 E-05$ \\
\hline 15 & TOTAL.. & $6.47630 E=03$ & $8.17872 t=03$ & $9.48063 E-03$ & $4.31264 E-05$ & $2.75777 t-115$ & $3.9 H 143 E=05$ \\
\hline 16 & TOTAL.. & $6.85174 E-05$ & $0.81659 t=03$ & $1.01622 E-02$ & $4.54014 \mathrm{E}=05$ & 1.18 BSE- 05 & $2.47486 E-(15$ \\
\hline . 17 & TOTAL.. & $6.71344 E-03$ & A.11842E-03 & $9.74530 E-03$. & $4: 94227 t=05$ & $2.32128 E-05$ & $1.704 A G E-105$ \\
\hline 18 & IUTAL.. & $5.93106 E-03$ & $6.08583 t-03$ & $8.36112 E-03$ & $5.22752 E=05$ & $3.95060 E-05$ & $2.27128 E-0 S$ \\
\hline 19 & TOUTAL.. & $4.91424 E-03$ & $3.33617 E-03$ & $6.64337 E-03$ & $5.05768 E-05$ & $4.756611 E-05$ & $2.83053 E-05$ \\
\hline 20 & TJIAL... & 4.3407 ㄷ $=03$ & $1.45223 \mathrm{SE}$ & $61 E-03$ & $296866-05$ & $4.52289 E=05$ & . 87219 \\
\hline
\end{tabular}




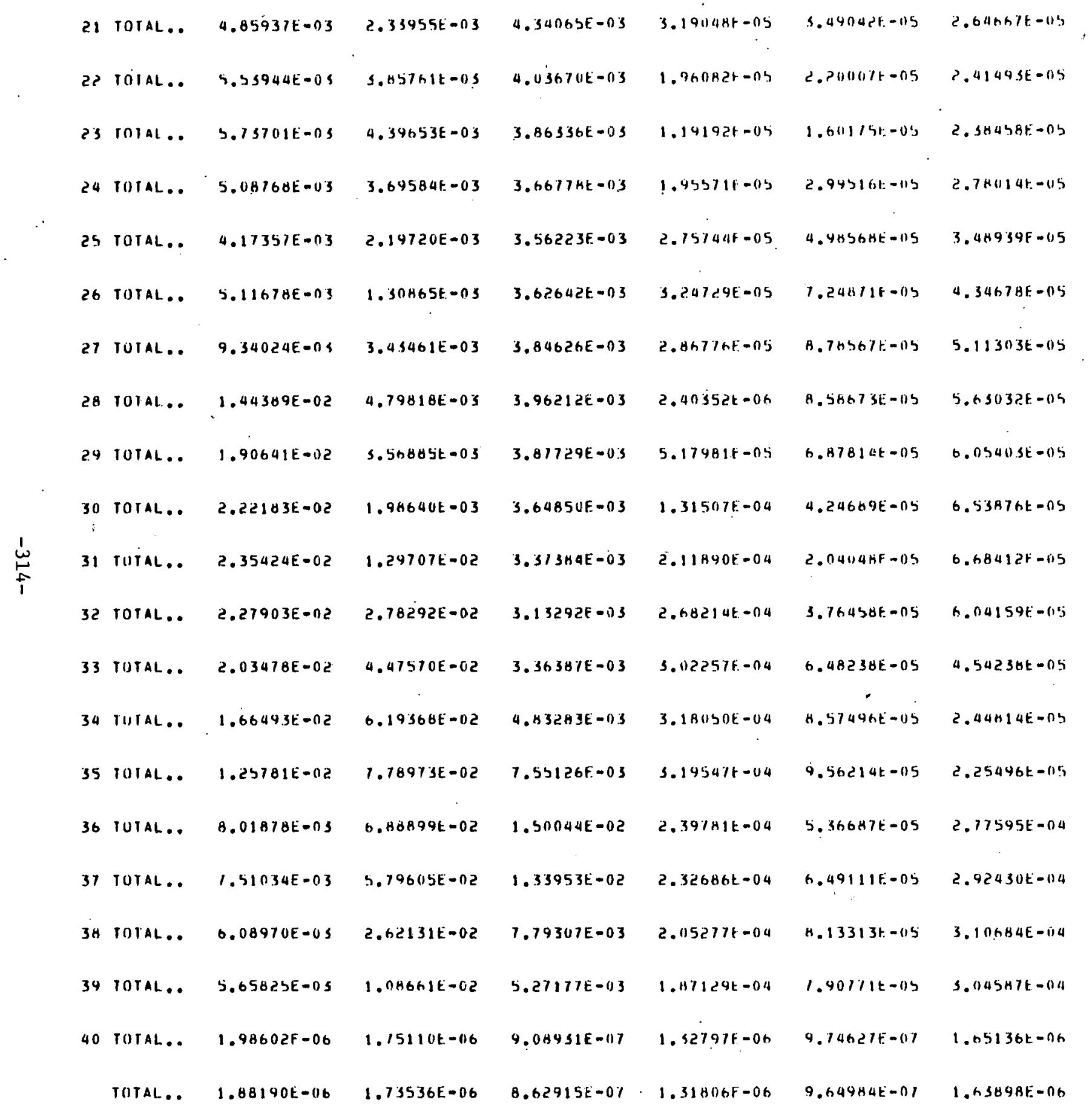




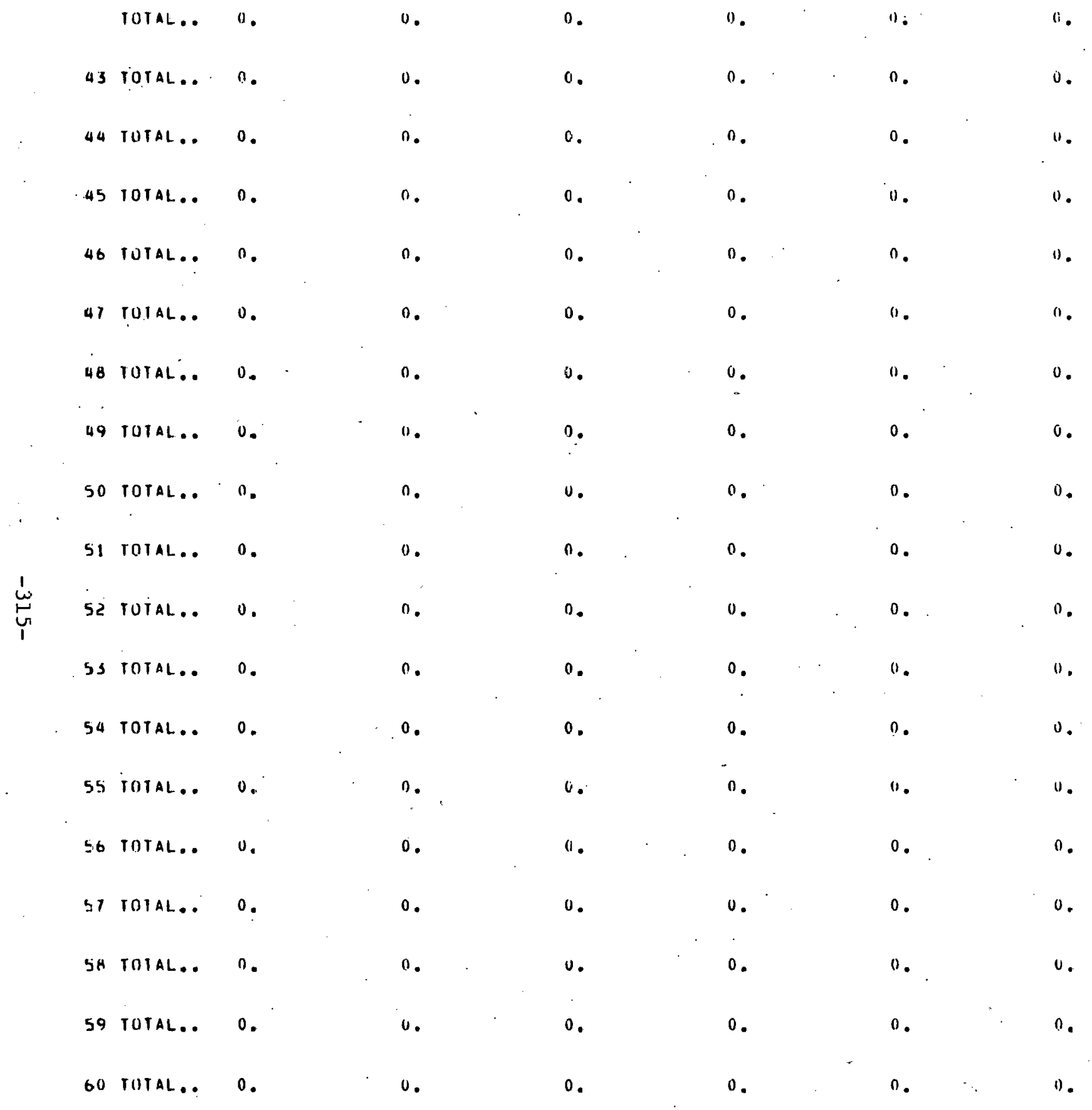




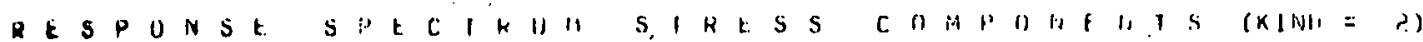

OR EACH ELENENI, THE PUH. LOWING, INGFIRMAIIIIN IS PRINIED:

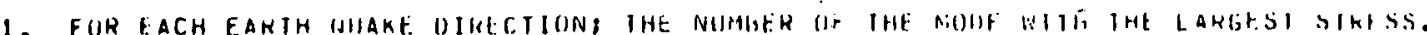

2. THe Vallie of that siniss.

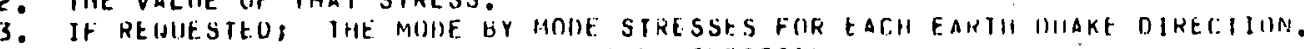

4. THE. RESIJLIAIJI FOIH EACH HARTH WIIAKE WIRECTIONN.

3. THE GRaNO TUIAL OF THE THRTE EARIH DIIAKE DIRTCIIOISS.

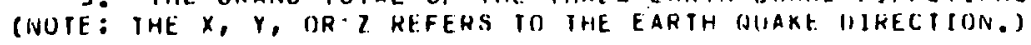

ELEMENT TYPE (B O U N D A A Y

BDRY-F BDHYM

$\begin{array}{lrr}X & 6 & 9 \\ X \text { MAXIMIIM } & 3.501 E+112 & 1.291 t+014 \\ Y & 3 & 11 \\ Y \text { MAXIMIMM } & 2.660 E+02 & 4.625 E+04 \\ Z & 5 & 3 \\ I \text { HAXIMUM } & 5.195 E+02 & 2.187 E+04\end{array}$

2 HAXIMUM $5.19 .9 E+022.187 E+00$

GHANO IOIAL $1.076 E+03 \quad 7.470 t+04$

ELEMENT TYPE $(3) U$ U N D A R Y

1,1, ELEMENT NIJMHER 1 2)

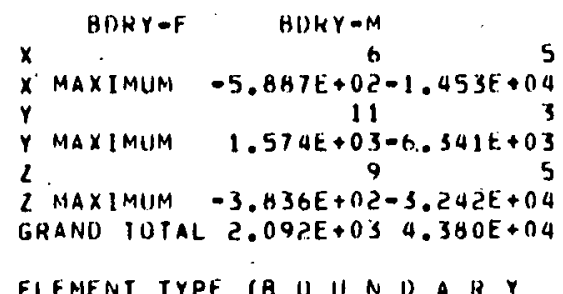
BIJRY F BURY M
$X$
$X$ MAXIMUM $-2.150 t+02-1.426 E+014$
$X$ MAXIMUM - $y$.
$X$
$Y$ MAXIMIIM $4.931 E+02-1.53 B E+04$
2 MAXIMUM $3.137 E+02.3 .174 E+04$
2 MAXIMIJM $3.137 E+02 \cdot 3.174 E+04$

GRAND TOTAL $8.895 E+025.589 E+04$

ELEMENT TYPE IB O U N

\begin{tabular}{|c|c|c|c|}
\hline & HDRY $=F$ & $B D R Y=M$ & \\
\hline$x$ & & 9 & \\
\hline$Y$ & MAXIMUIM & $1.458 E+03$ & 0. \\
\hline Y & $M A$ & $\begin{array}{l}9 \\
3\end{array}$ & \\
\hline$z$ & MA X & $\begin{array}{r}3.103 E+0.5 \\
5\end{array}$ & \\
\hline $\begin{array}{l}Z \\
G K\end{array}$ & MAXIMUMM & $1.12 \mathrm{SE}+03$ & 0. \\
\hline & & $769 E+03$ & (1) \\
\hline
\end{tabular}

ELEMENT TYPE L $O U N O A B Y$ 


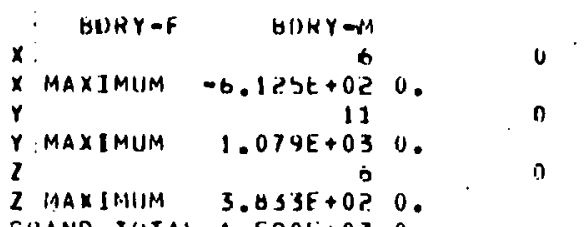

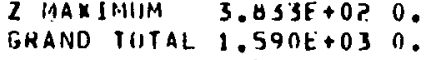

ELEMLAT TYPE (B

1,1, ELEMENT NUIHER $($ H

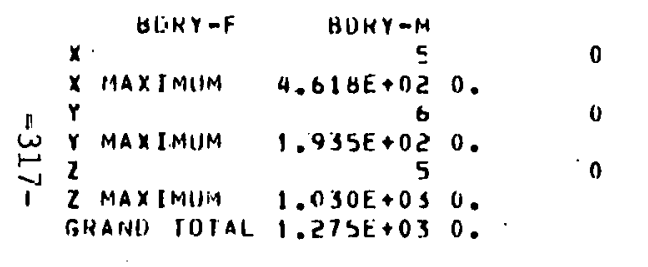

ELEMENT TYPE GO U UNOAKY

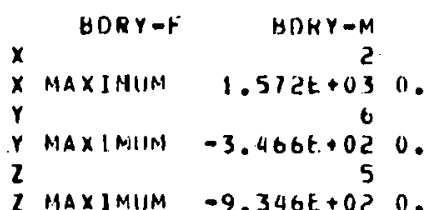

2 MAXJMIIM $-9.346 E+020$
GRAND TOIAL $2.303 E+030$

ELFMENI TYPE (B O UNDA

), 1, ELEMENT NUMHEK 1 (10)

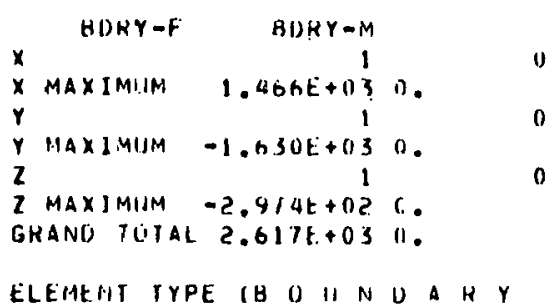




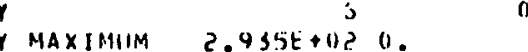

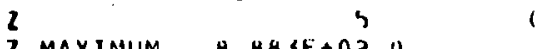

MAXIMIJM B. HASE+OZ O.

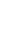
GKANO TOTAL $1.632 E+030$.

ELEMENT TYPE IB OONINUAR

\begin{tabular}{|c|c|c|}
\hline HUHY F F & HURY M & \\
\hline$x$ & 1 & \\
\hline$X$ MAXIMUM & $-2.555 E+03$ & 10. \\
\hline $\boldsymbol{r}$ & 1 & \\
\hline Y MAXIMIIM & $2.840 t+03$ & 0 . \\
\hline 2 MAXIMIIM & $5.183 E+02$ & 1). \\
\hline GRANI) TOTAL & $3.97 .3 E+03$ & 0 . \\
\hline
\end{tabular}

), 1, ELEMENT NIHAHEH $(13)$

HOHYFF BORYAM

$x$ MAXIMUM

$X$ MAXIMUM $3.794 E+0 ?$
$Y$

Y HAXIMIIN $-4.217 t+020$.

2 MAXIMUM 5

2 MAXIMUM $-6.327 t+020$.
GKAND TOIAL $1.306 E+030$.

ELEMENI TYPE (H O) II NDAB $Y$

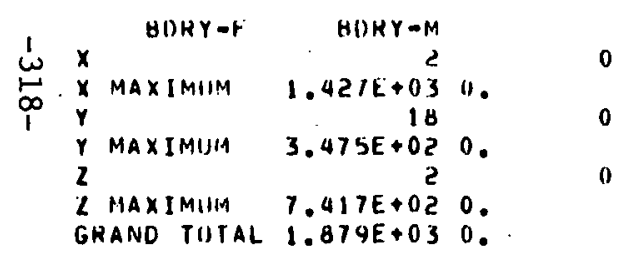

) 1,1 element indumisen $(14)$

ELFMENT TYPE $(B$ O U U N $\cap$ A $R$

), 1, ELEMEIVT NUHHER $(13)$

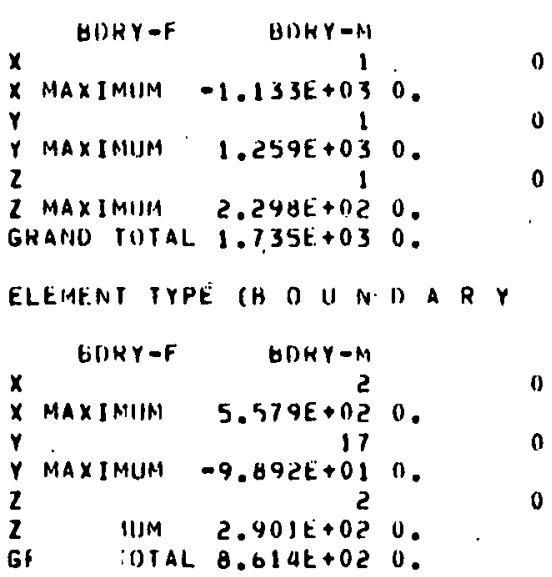

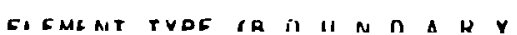

), 1, ELEMENT NUMAFR ( 16) 


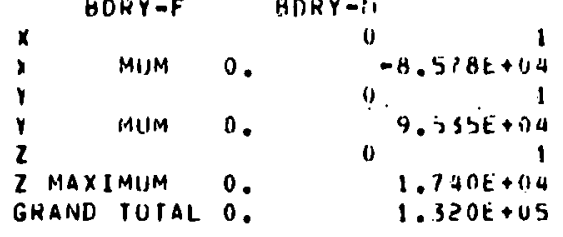

ELEMTNI TYPE (B O) U'N D A H Y

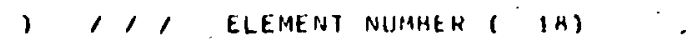

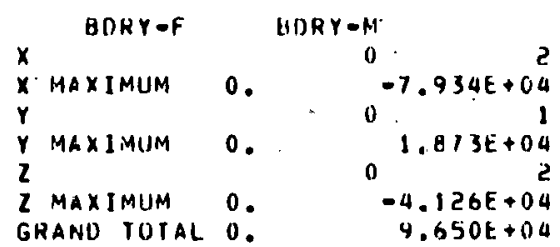

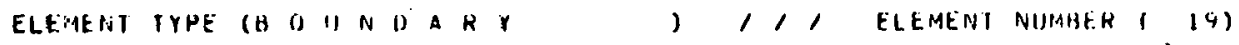

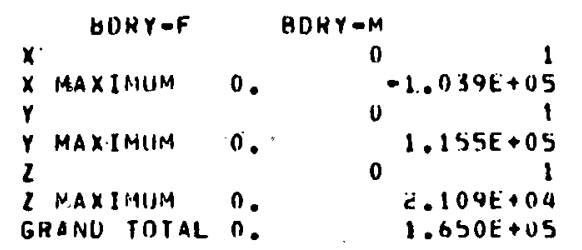

I ELEMENT TYME $13 / 0$ P 1 P E

) 1,1 ELEMENT NIIHHEK (1)

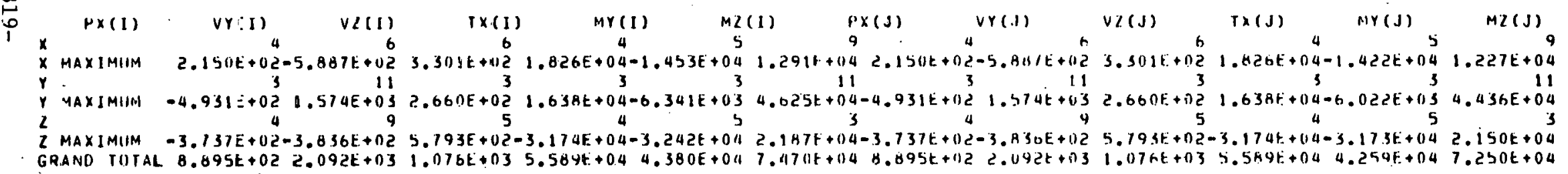

ELEMENT TYPE (3/D P I P E 1,1, ELEMENT NUMHER $($ ?)

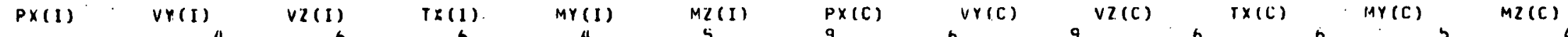
$X$
$X M A X I M U M$ $Y$
$Y$ $Y$ MAXIMIIM $-4.435 E+02 \quad 1.574 E+03 \quad 2.660 E+02 \quad 1.634 E+04-6.009 E+03 \quad 4.436 t+04 \quad 1.129 E+113 \quad 1.047 E+03 \quad 2.640 E+02 \quad 1.084 E+04-7.311 E+03 \quad 1.315 E+04$

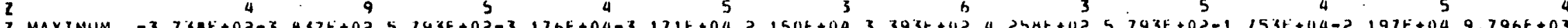

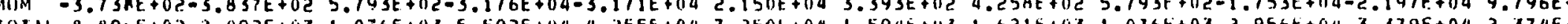
E. ELEMENT TYPE (3/0 P I PE 1,1, ELEMENI NUMHER 1 ( $)$

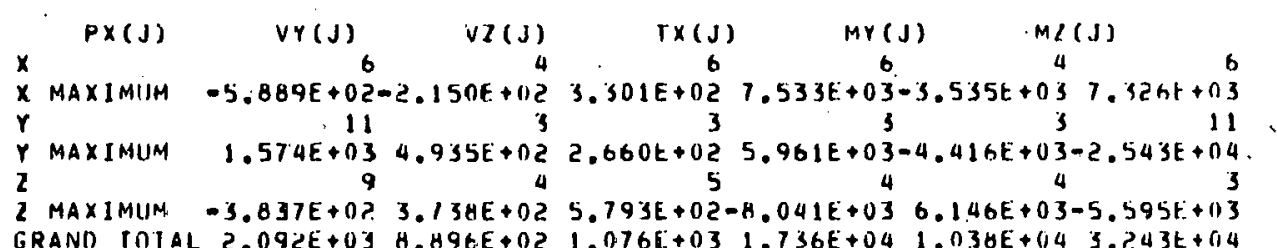




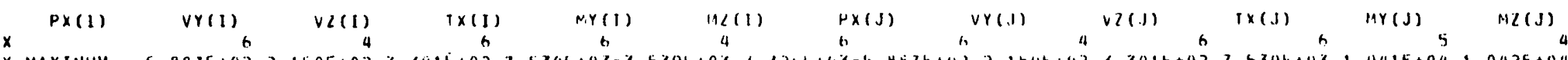

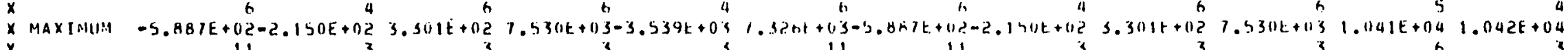

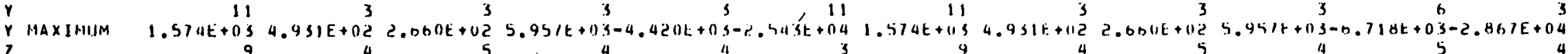

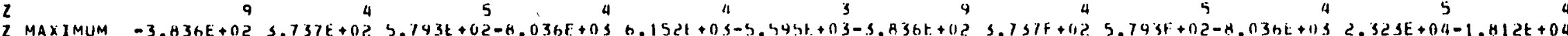
GRAND TOIAL. $2.092 E+038.895 E+021.016 E+031.735 t+1341.039 E+043.2119 t+1142.092 E+1138.495 t+021.610 t+1131.735 t+1145.579 t+045.166 E+04$

ELEMENT TYPE (3/D, JPE , 1,1 KLEMENT NUNHER 1 (1)

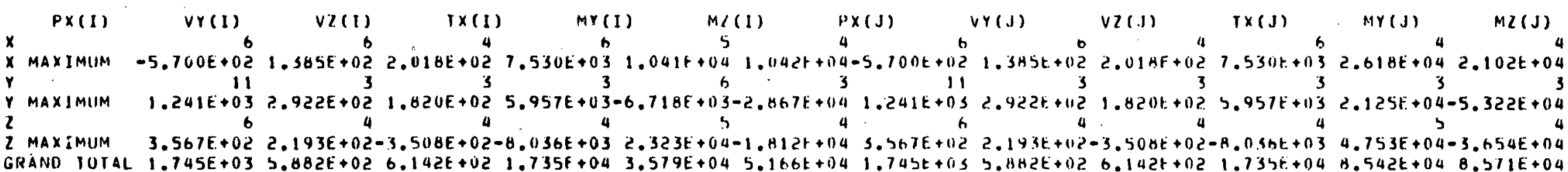
ELEMENT TYPE (3/R P I P E 1,1 ELEMENT NIPHBEH i 5 )

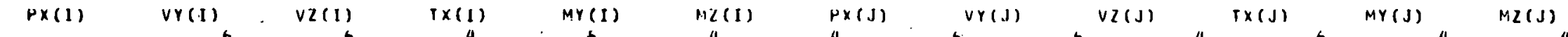
$X$ MAXIMUM $-5.583 E+02$
$X$

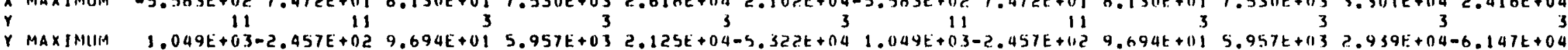

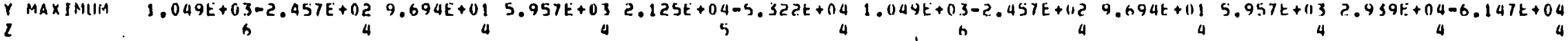

I. 2 MAXIMUM $3.494 E+026.540 E+01-1.413 E+02-8.036 t+034.753 E+011-3.654 t+043.494 E+02 \quad 0.540 E+01-1.413 t+112-8.036 t+03-5.734 t+04-4.203 E+04$ N GRAND TUTAL $1.554 E+033.417 E+022.296 t+021.735 E+048.542 E+048.371 E+041.554 t+033.411 E+022.296 t+021.735 E+041.010 E+051.004 E+05$ I ELEMENT TYPE (3/0 P I PE , 1,1 ELEMENT NUMHER $($ i)

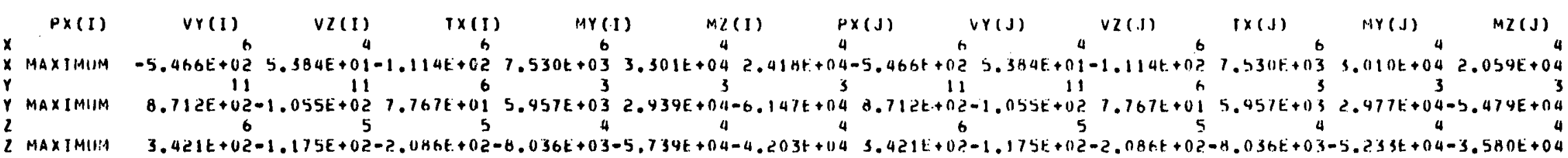
ELEMENT TYPE (3/0 P I P E , , , ELEMENT NUMHER $(1)$

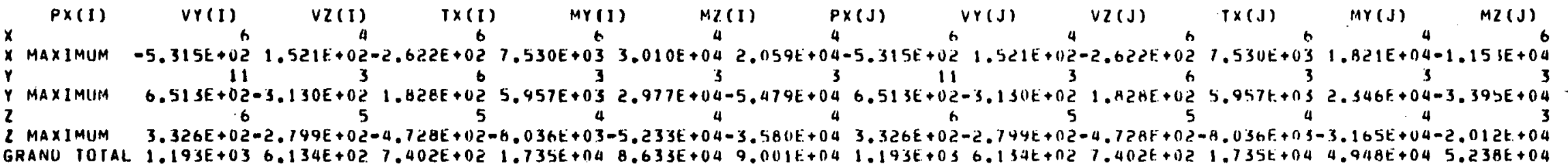
ELEMENT TYPE (3/0 P I PE , , , ELEMENT NIMHERE $($ H)

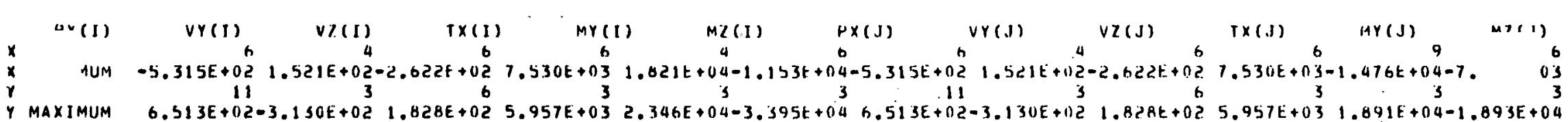




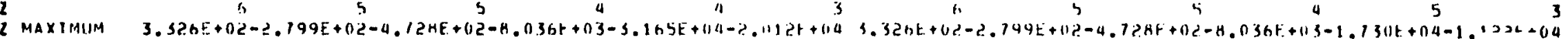

E. I TYPE (S/I) PIPE, , , ELEMENT NIIIHIH ( 9$)$

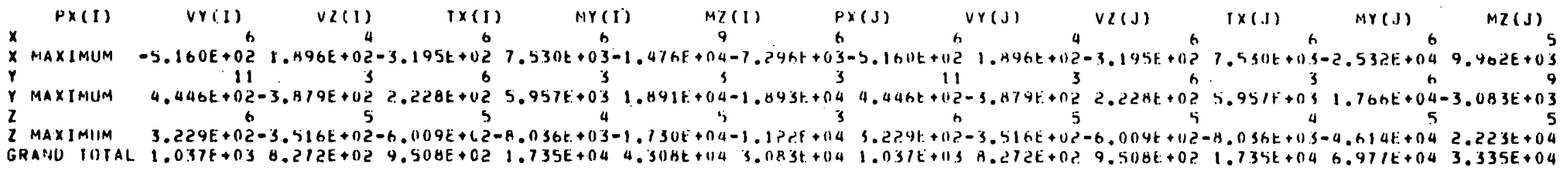
ELEMENT IYPE (3/0) PI PE , 1,1 ELEMENT NUIAHEK 1 10)

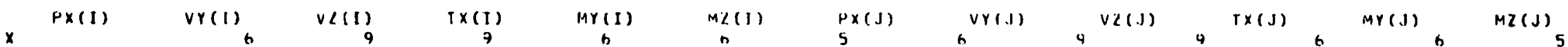

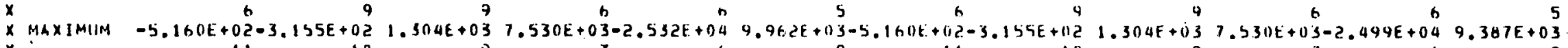

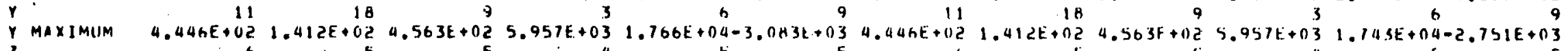

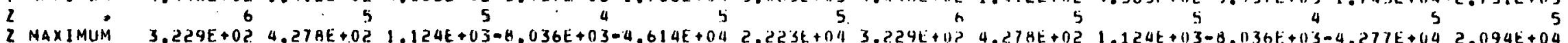
GRAND TUTAL 1.037E+03 B.622E+02 $2.131 E+031.735 E+046.471 E+043.334 E+041.037 E+039.622 E+1022.131 E+031.735 E+046.468 E+043.110 E+04$ ELENIENT TYPE (3/D P D

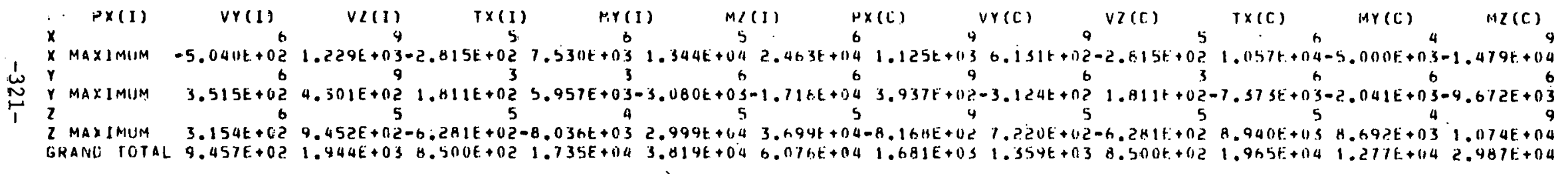
ELEMENT IYPE (3/U P I PE , 1,1 ELEMÉNT NUMHEH $(11)$

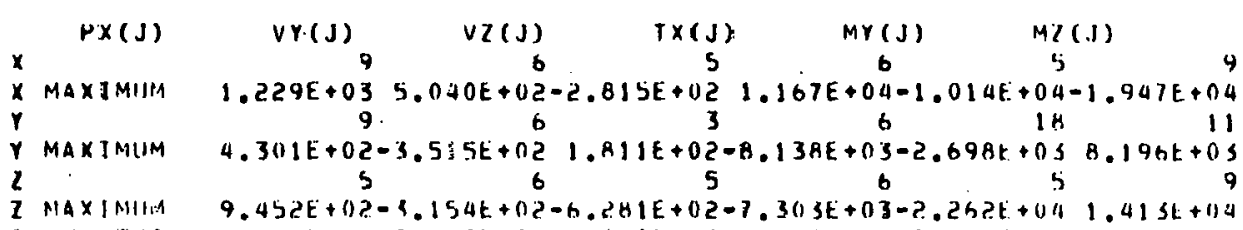

1 MAXIMIHA $9.452 E+102-1.154 t+02-h .2 H I E+02-7,303 t+03-2.252 t+041.413 t+114$

ELEMENT TYPE (S/I) P I PE $;: 1$, ELEMENT NIHHER $($ I $)$

\begin{tabular}{|c|c|c|c|c|c|c|c|c|c|c|c|c|}
\hline$\forall x(I)$ & VY (I), & $v I(1)$ & $T \times(I)$ & MYCI & & (I) & $x(n)$ & $V Y(J)$ & $V Z(J)$ & $1 \times(. J)$ & $\operatorname{Mr}(J)$ & $M Z(J)$ \\
\hline MAXIMUMM & $E+03$. & 02 & +02 & $1.167 E+04$ & $1.014 E+04$ & $1.947 t+04$ & $1.129 E+03$ & $3-4.033 t+0 \hat{2}$ & $2.419 t+02$ & $t+04$ & $t+04$ & $7 E+04$ \\
\hline & 9 & h & 3 & 6 & in & 11 & 4 & 6 & 3 & $h$ & & 3 \\
\hline $\operatorname{MAXI}$ & 02 & $02-x-3 x$ & $02-$ & 03 & & $t+03$ & $\therefore+12$ & $=02-$ & & & & 3 \\
\hline & 5 & 6 & 3 & 6 & $i$ & 9 & 5 & n & s & h & & 5 \\
\hline$A \times 111$ & $3 E+0 ?$ & +02 & b. & +03 & $E+04$ & $3 F+04$ & $3 t+112$ & $+1) 2$ & & & 04 & \\
\hline ZAND TOTAL & $.802 t+03$ & $b 6, t+U 2$ & 7 . & +04 & +114 & $t+04$ & $2 . t+103$ & $s$ & 2 & 1) $4^{\circ}$ & 14 & 04 \\
\hline
\end{tabular}

ELEMENI TYPE ( $S / O$ P I P E , 1,1 ELEHENT NUMHEK 1,13 )

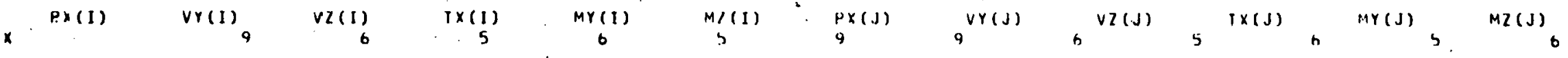


$\times$ MAXIMUJA $9.663 E+02-3.413 E+022.029 t+(121.11 / t+041.115 t+041.166,1+049.654 E+02-3.419 t+022.024 t+021.117 t+042.3115 t+042.966 E+04$

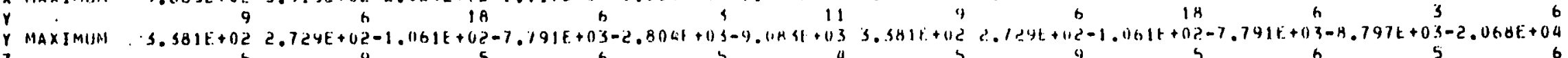

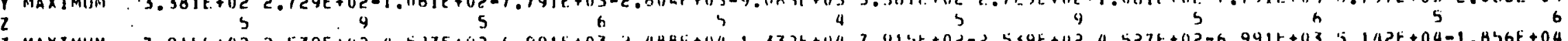

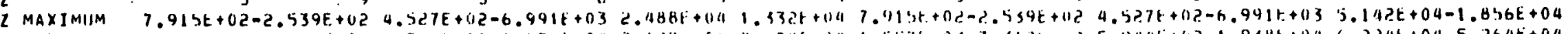

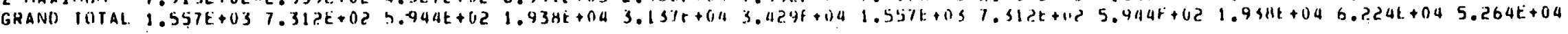

ELEMENT TYPE (SIO PIPE ), , ELEMENT NIIHHER ( 14)

$P X(1) \quad V Y(1) \quad V Z(1) \quad T \times(I) \quad M Y(I) \quad M(I) \quad P X(J) \quad V Y(J) \quad V Z(J) \quad I X(J) \quad M Y(J) \quad M Z(J)$

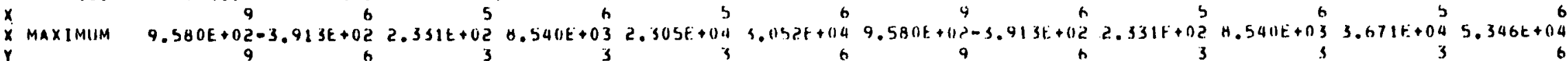
$Y$ MAXIMUM $3.353 E+02$
$z$ 2 MAXINIUM $7.490 E+02-2.539 E+025.201 E+02-5.344 E+035.142 E+04-1.910 t+047.4901+02-2.539 E+1125.201 E+02-5.344 E+03 \quad 4.141 E+04-3.345 E+04$

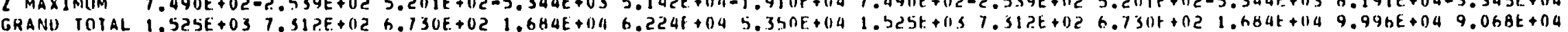
ELEMENT IYPE (310 P I P E , , ELEMENT NIJABES $(15)$

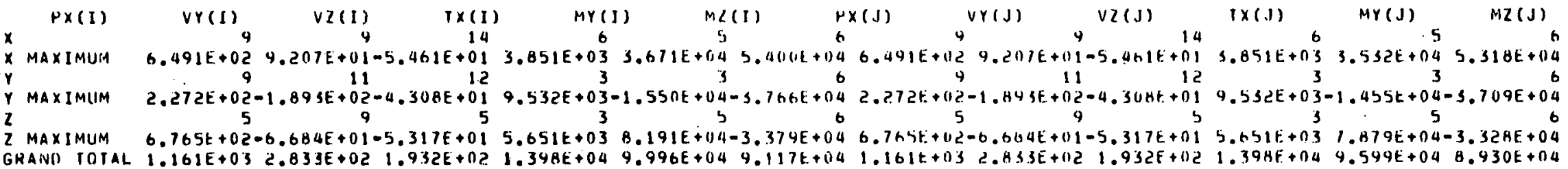
ELEMHN TYPE (3/0 P I P E , , , ELEMENT NUMHER $(16)$

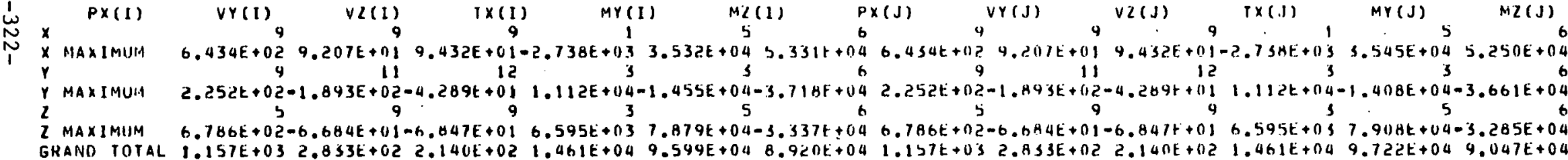
GRAND TOTAL $1.157 E+032.833 E+022.140 E+021.461 E+049.599 E+048.920 E+041.137 E+032.833 E+02 \quad 2.140 E+021.461 E+049.722 E+049.047 E+04$ ELEMENT IYPE (3/D P I P E 1,1 , ELENENT NIMHER $(17)$

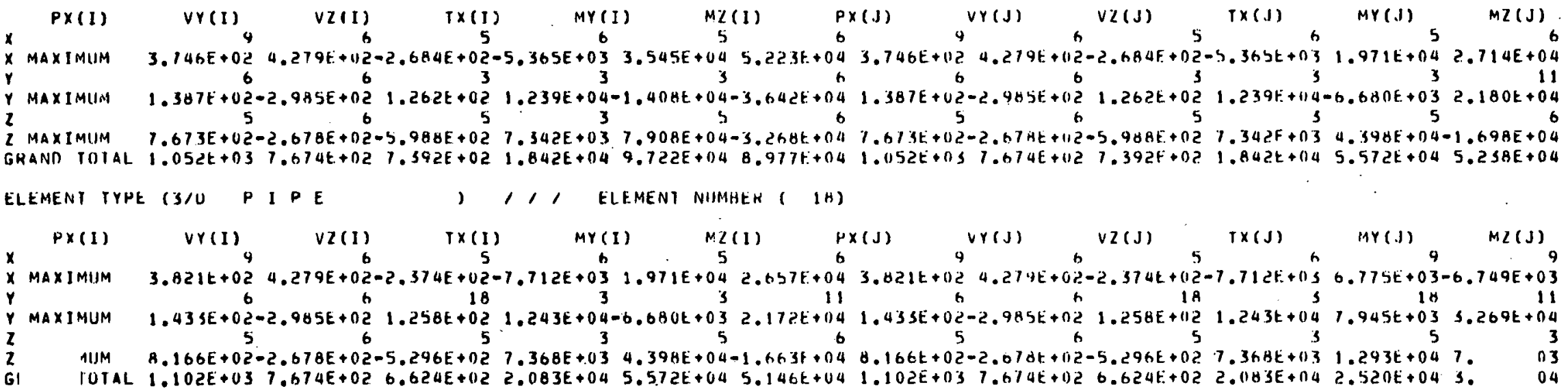




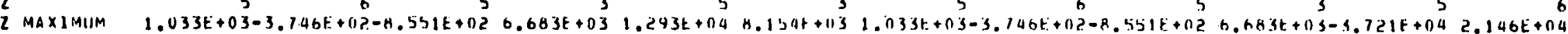
GRANO TOTAL $1.29 H E+031.091 E+1131.053 E+032.055 E+042.5201 E+043.490 E+1141.294 E+031.041 E+1131.053 E+1132.055 E+044.624 E+047.096 E+04$

ELEMENT TYPE (3/D PI PE ) 1,1 ELEMENT NUHAHEH ( 200)

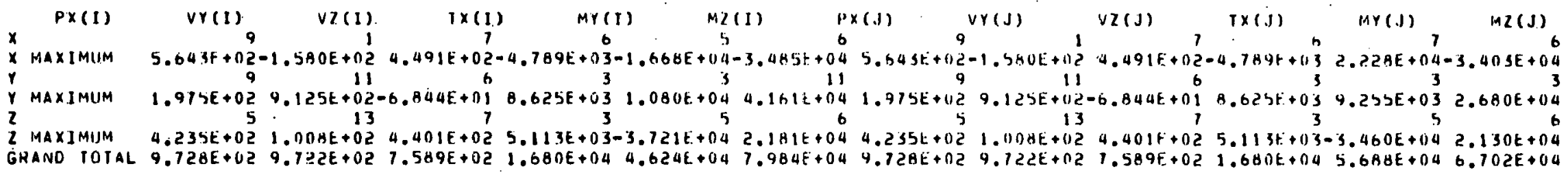

ELEMENT TYPE (3/1) $P$ I PE, , 11 , ELEMENT NUIMBER ( 21)

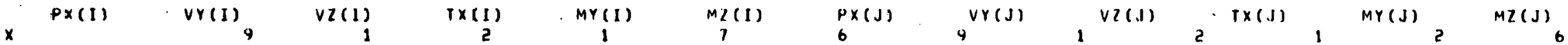

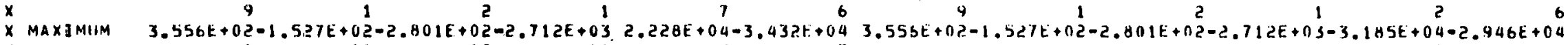

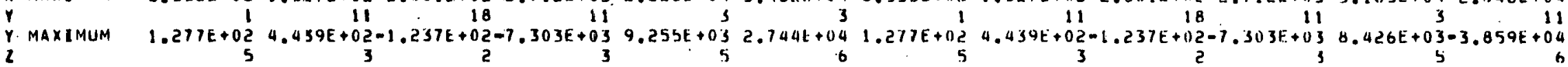
2 MAXIMIJM $6.180 E+025.349 E+01-1.457 E+02 \quad 3.710 E+03-3.460 E+04 \quad 2.14 B E+04 \quad 6.180 E+02 \quad 5.349 E+01-1.457 E+02 \quad 3.710 E+03-3.521 E+04 \quad 1.844 E+04$ GRAND TOIAL $9.373 E+025.364 E+024.274 E+021.248 E+045.688 E+046.795 E+049.373 E+1125.364 E+02 \quad 4.274 E+021.24 B E+047.10 A E+04 \quad 1.306 E+04$ ELEMENT TYPE (3/0 P I PE , 1,1 ELEMENT NUMHEK $(22)$

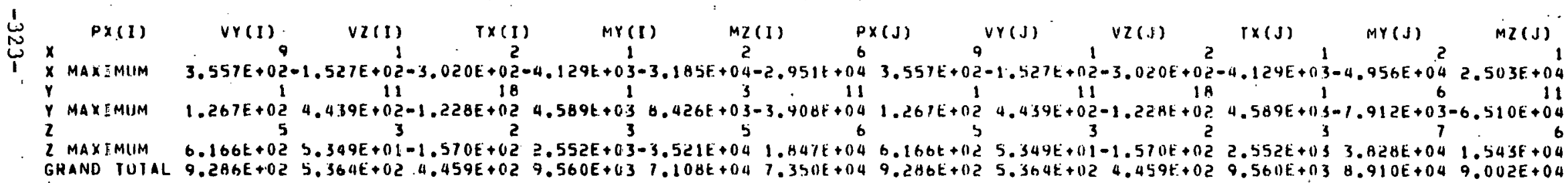

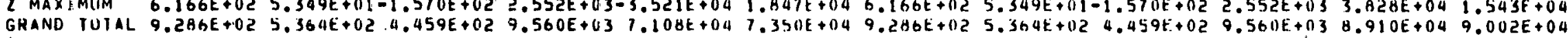
ELEMEHT TYPE ( $3 / 0$ PIPE $), 1$, ELEMENT NUMAEK ( 23 )

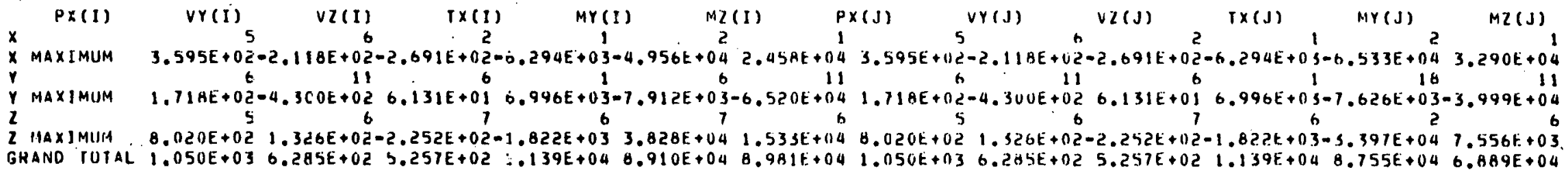
Element trPe (3/1) PI PE.,$: 1$, ELEMENT NUMHEH ( 24$)$

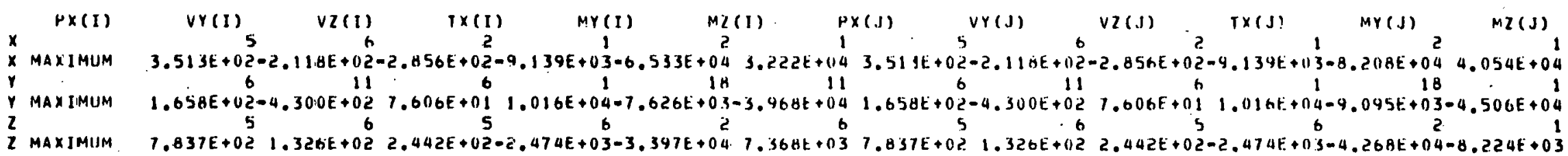




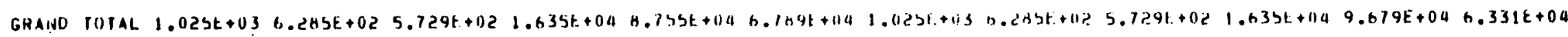
ELEMTN IYPE (3/0 P I PE , , , ELEMENI NUMHEK $($ ?

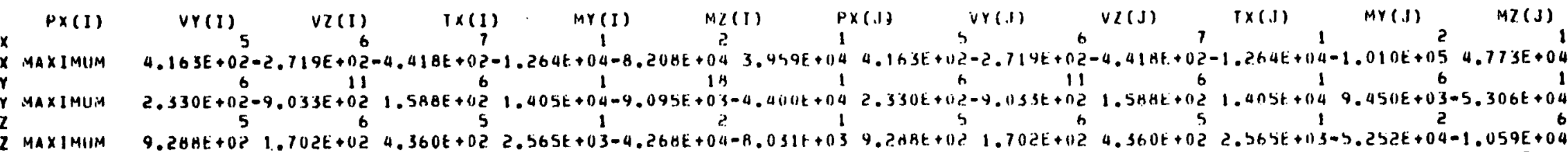

2 MAXIMIIM 9.2BAt+02 $1.702 E+024.360 t+022.565 E+03-4.264 E+04-8.031 t+039.24 A E+1) 21.702 E+1124.360 E+022.565 E+115-3.252 E+04-1.059 E+04$ GRAN( TUTAL $1.207 E+03 \quad 1.086 E+03.9 .597 E+022.134 E+049.679 E+04 \mathrm{~h} .181 E+041.207 E+1151.036 E+1) 39.597 E+1) 22.134 E+1) 41.208 E+05$ B. B SIE+04 ELEMENT TYPE (3/0 P I PE ), 1, ELEMENT NIIMHER $(2 h)$

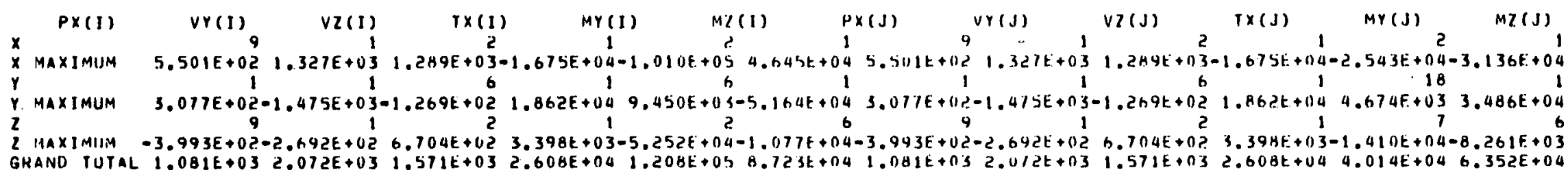
ELEMENT TYPF. (S/D PIPE , , , ELEMENT NUMHER ( 27)

$P X(I) \quad V Y(I) \quad V Z(I) \quad T X(I) \quad M Y(I) \quad M Z(I) \quad P X(J) \quad V Y(J) \quad V Z(J) \quad T \times(J) \quad M Y(J) \quad M Z(J)$ $x$ HAXIMUM $-4.738 E+02$
$\times$
$Y$

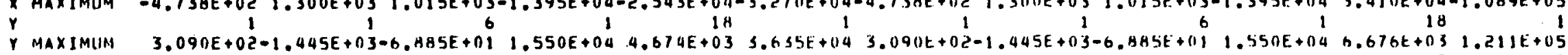

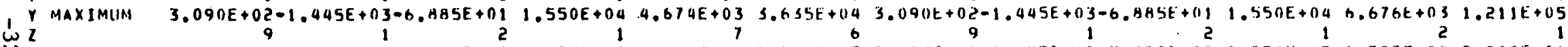
N2 MAXIHUM $-2.693 E+02-2.637 E+025.279 E+022.830 E+03-1.410 E+04-8.361 E+03-2.693 E+122-2.637 E+1125.279 E+022.830 E+1131.773 E+042.209 E+04$ I GRAND TOTAL $9.075 E+021.977 E+031.191 E+032.159 E+044.014 E+046.518 E+049.075 E+021.971 E+1131.191 E+032.159 E+044.987 E+041.673 E+05$ ELEMENT TYPE (3/0 P I E. $;, 1$, ELEMENT NUMHER $(28)$

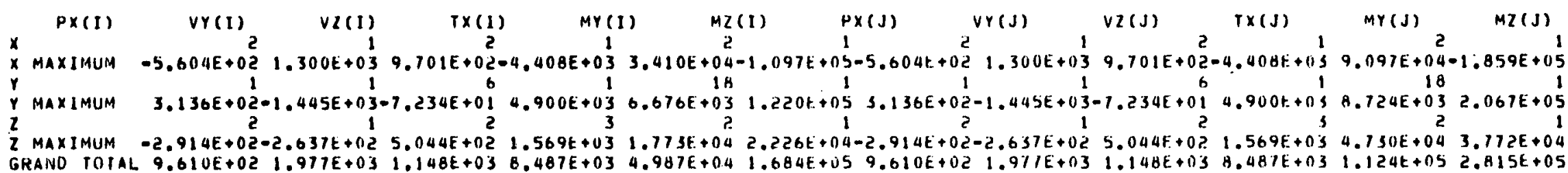
ELEIAEN TYPE (3/0 P I P E, $1 /$ ELEMENT NUHHER ( 29)

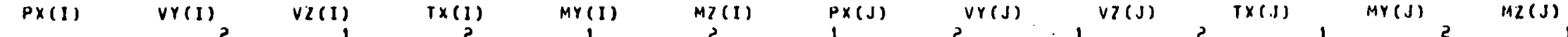

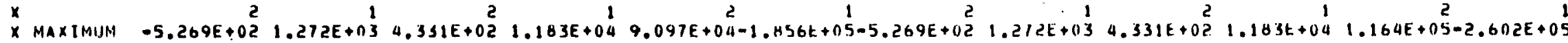
$Y$
$X$ Y MAXIMUM 3.069E+02-1.413E+03-1.086E+02-1.315E+04 $8.724 E+032.063 E+053.069 E+02-1.413 E+03-1.086 E+02-1.315 E+114-6.010 E+032.892 E+05$ 2 MAXIMIM $-2.140 E+02-2.580 E+022.252 E+02-2.399 E+03 \quad 4.730 E+04 \quad 3.765 E+04-2.740 E+02-2.5 B 0 E+1122.252 E+02-2.399 F+03 \quad 6.050 E+045.277 E+04$ GRAND TOTAL $8.722 E+02$ 2.015E+03 $5.504 E+021.896 E+041.124 E+052.810 E+058.722 E+022.015 E+035.504 E+021.896 E+041.369 E+053.933 E+05$ ELEMENT TYPE (3/D P I P E , 1,1 ELEMENT NUMHER $(30)$

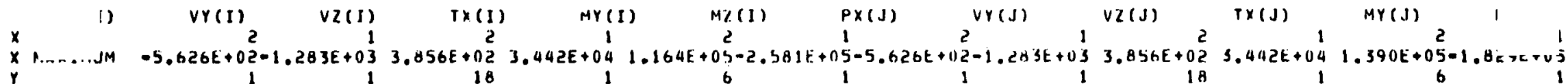


YAAXIMUM S.055E+02 $1.026 E+03-1.067 E+02-3.826 E+04-6.010 E+052.064 E+053.053 E+1) 21.426 E+113-1.067 E+02-3.826 E+04-5.650 E+032.033 E+05$

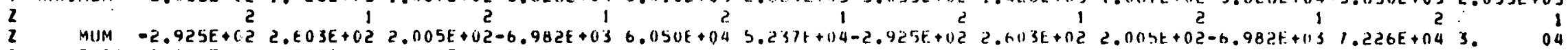
TUTAL $9.014 E+121.995 E+035.012 E+025.237 t+041.369 t+053.403 t+059.014 t+11$ ? $1.495 t+035.012 t+025.237 E+011.012 t+052$. $\quad 05$ ELEMKN IYPE (3/0.P I E ), 1, ELEMENT NIIAHER $(31)$

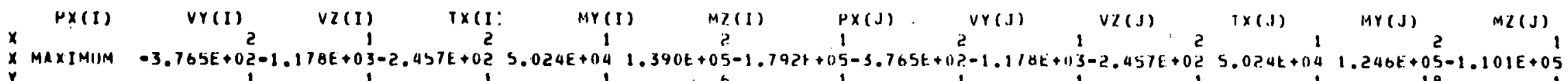
Y MAXIMUM $2.694 E+021.509 E+03 \quad 7.573 E+01-5.585 E+04-5.650 E+031.992 E+052.894 E+021.3109 E+03 \quad 7.573 t+01-5.5 H 5 E+04-6.495 E+031.224 E+05$

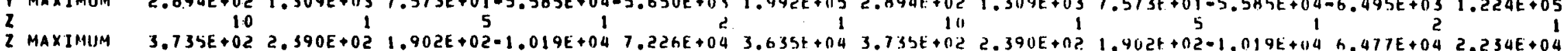

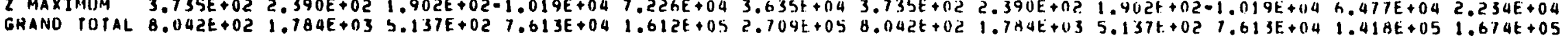
ELEMEVT TYPE (3/1) P I PE, , , ELEMENT NUMUER ( 32$)$

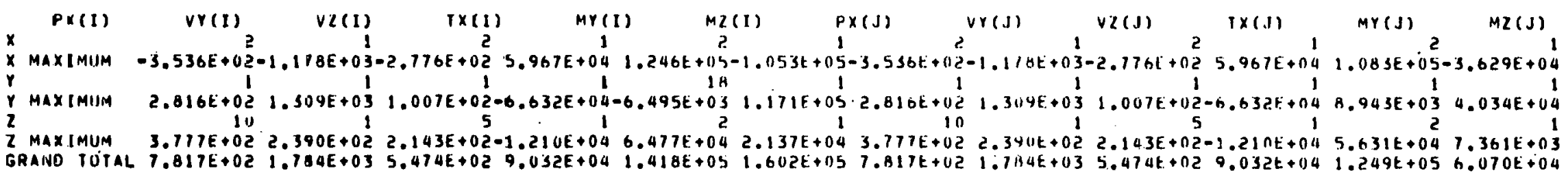
ELEMENT TYPE (3/0 I P E , , ELEMENT NUMBË ( 33)

$P X(I) \quad V Y(I) \quad V Z(I) \quad T X(I) \quad M Y(I) \quad M Z(I) \quad P X(J) \quad V Y(J) \quad V Z(J) \quad M X(J) \quad M Z(J) \quad$

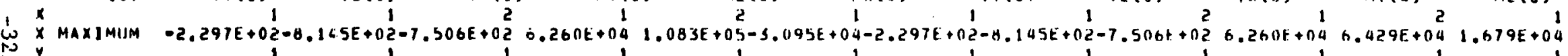

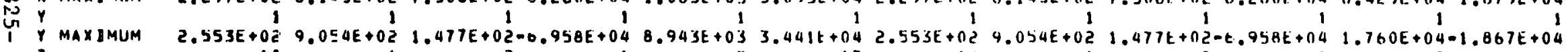

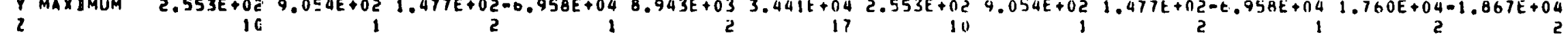
2 MAXIMUM $4.690 E+02 \quad 1.652 E+02-3.903 E+020=.270 E+04 \quad 5.631 E+04 \quad 7.190 E+03 \quad 4.690 E+02 \quad 1.652 E+02-3.903 E+02-1.270 E+043.343 E+04 \quad 4.579 E+03$

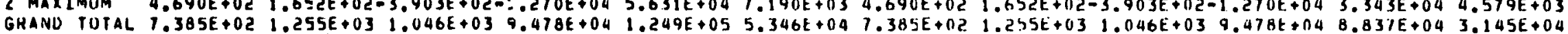
ELEMENT TYPE (3/0 P 1 PE $; 1$, ELEMENT NUMHER $(34)$

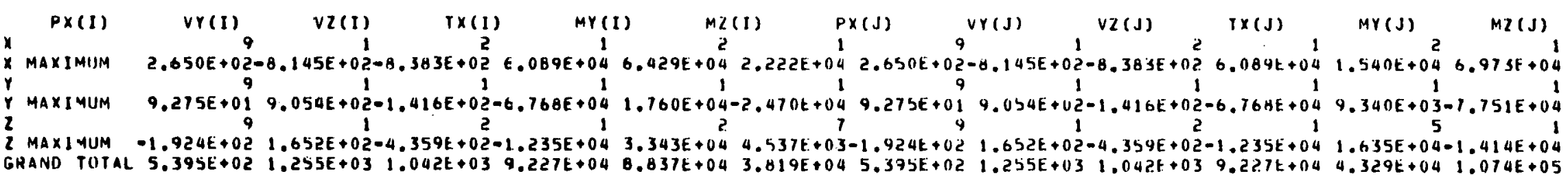
ELEMENI TYPE (3/0 PI P E ) 1,1 ELEMENT NUMBER ( 35 )

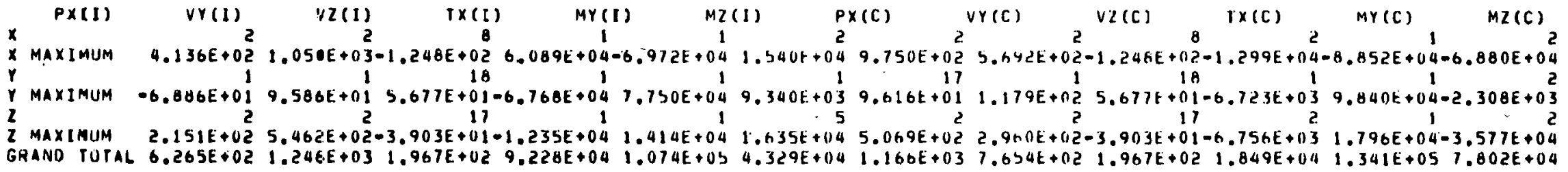
ELEMENI TYPE $13 \%$ P I P E , , , ELEMENT NUMBER ( 35)

$P X(J) \quad V Y(J) \quad V Z(J) \quad \operatorname{JX}(J) \quad \operatorname{Mr}(J) \quad M Z(J)$ 
ELEMENT TYPE (3/0 P T P E , 1,1 ELEMENT NUMAKR 136$)$

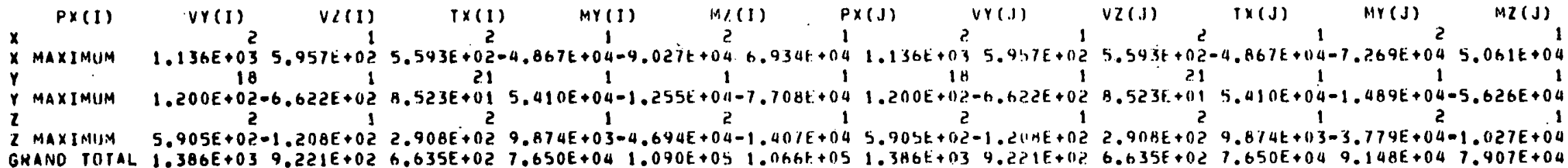

ELEMENT TYPE (3/0 PIPE, , , , ELEMENT NIIARESR i $3 /)$

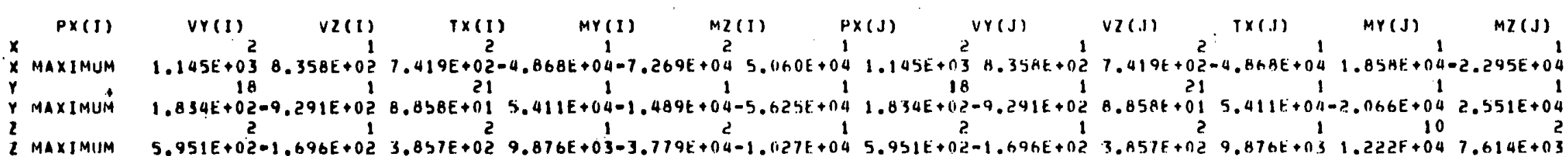

2 MAXIMIIM 5.951E+02-1.696E+02 3.857E+02 9.876E+03-3.719E+04-1.1227E+04 5.951E+02-1.69hE+02 3.A57E+ +2 $9.876 E+03 \quad 1.222 F+047.614 E+03$ I ELEMENT TYPE (3/1) PI PE , 1,1 ELEMENT NIJMIHER $(38)$

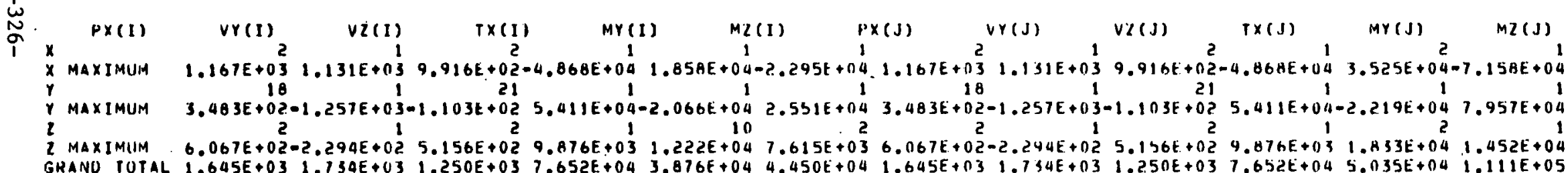

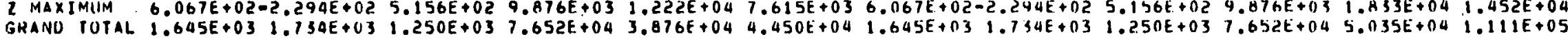
ELEMENT TYPE (3/0 PI P E , , , ELEMENT NIIMISER 139$)$

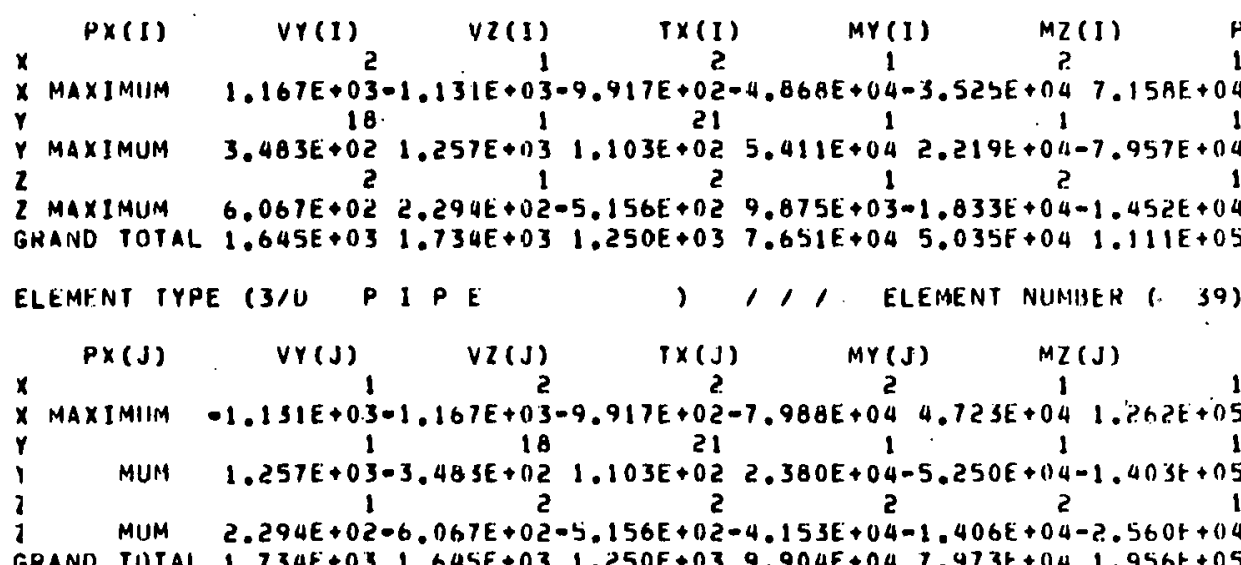




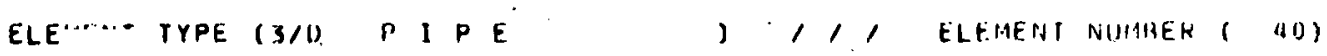

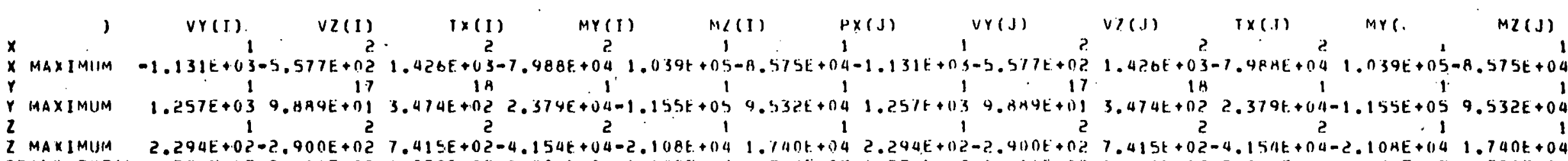

2 MAXIMUIA 2.294E+02-2.900E+02 $7.415 E+02-4.154 E+04-2.108 E+041.1110 E+042.294 E+02-2.4100 E+027.415 E+02-4.15 A E+04-2.10 H E+04 \quad 1.740 E+04$ GRAND TUTAL $1.734 E+03$ 8.611E+02 $1.878 E+039.904 E+041.649 E+051.319 E+051.734 E+038.611 E+021.818 E+039.904 E+0111.650 E+051.320 E+05$ 

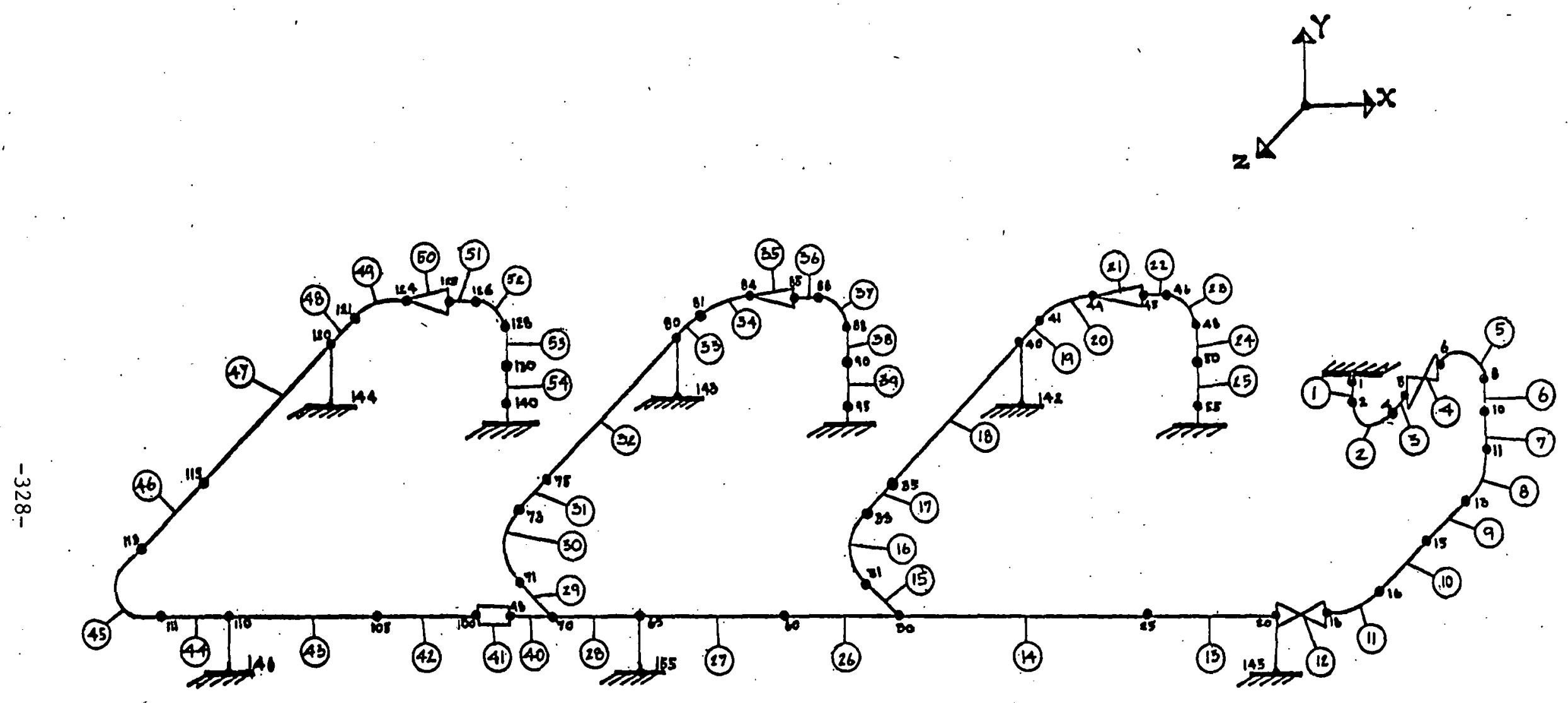

BENCHMARK PROBLEM NO. 7 


\section{BENCHMARK PROBLEM N0.7}

\section{$X$ AND $Z$ SPECTRUM}

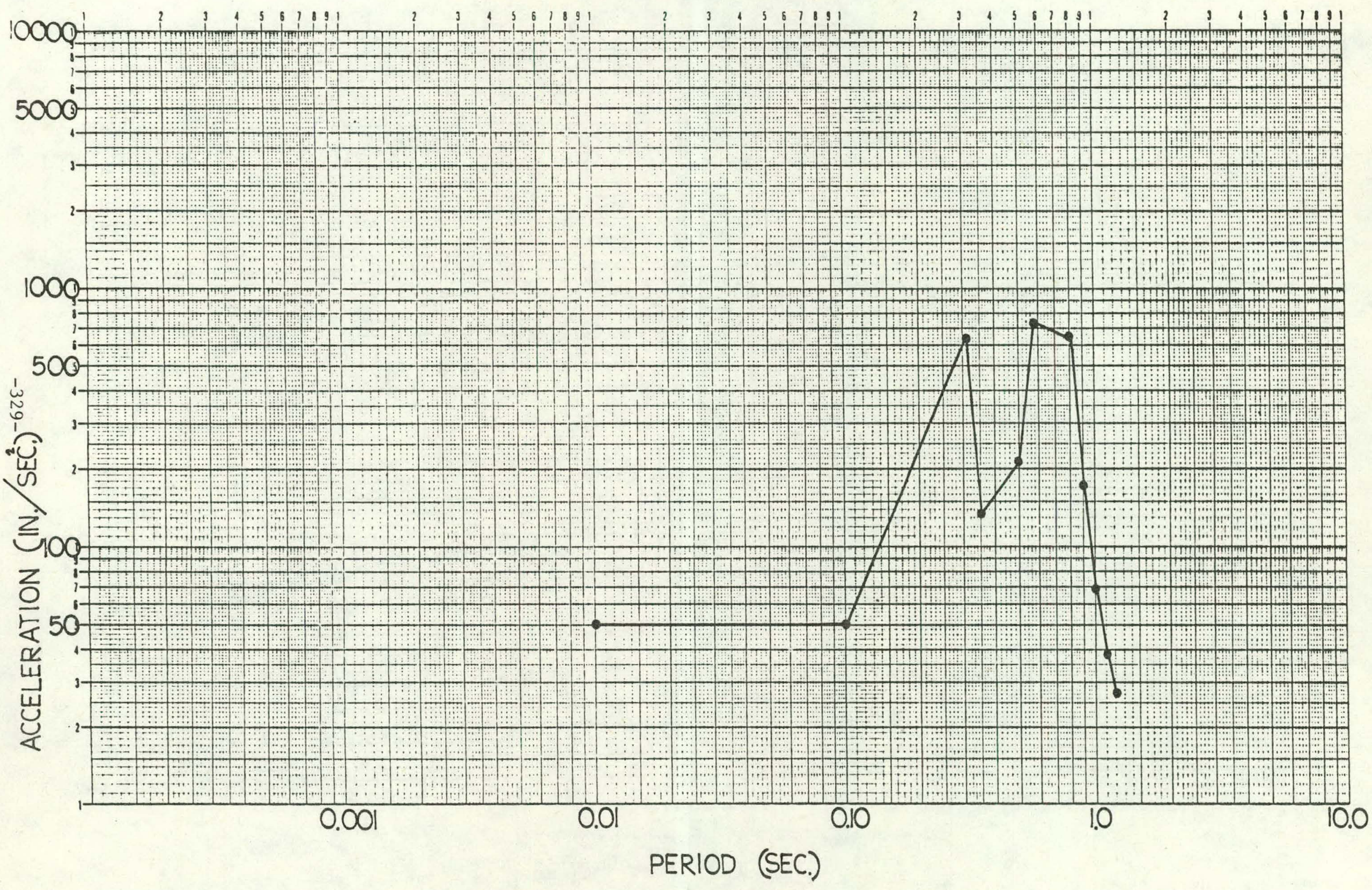

Figure, 7.2 


\section{Y SPECTRUM}

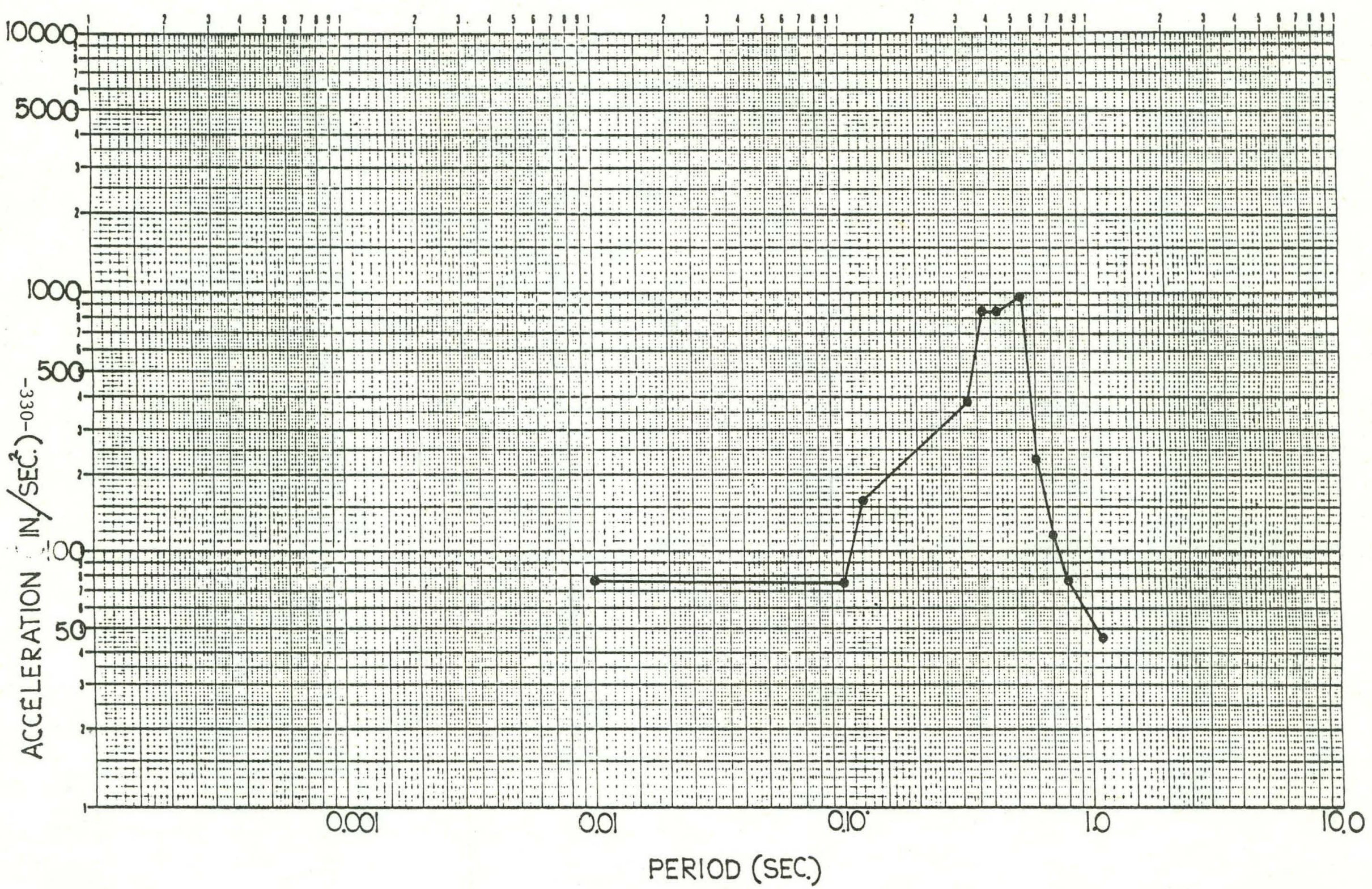

Figure 7.3 


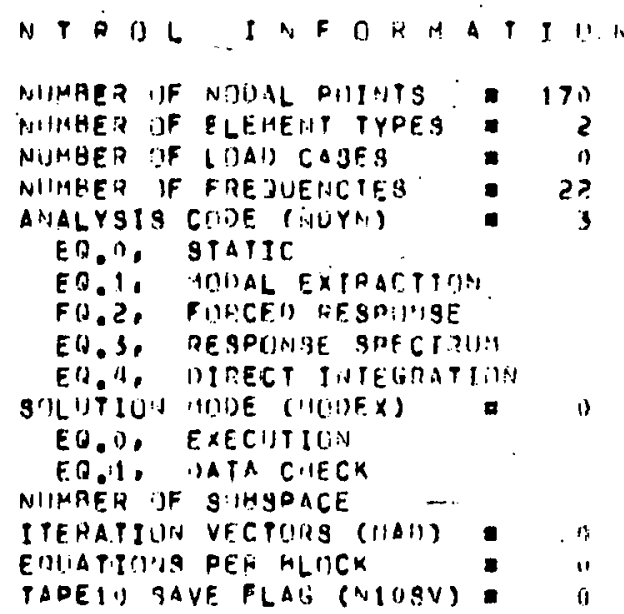

NOMAL POINT INPUT RATA

\begin{tabular}{|c|c|c|c|c|c|c|c|}
\hline & NUDE & $140^{i}$ & ADYY & C1) $\div 1$ & $7: 110$ & CU!UE: & \\
\hline & NUMAER & $x$ & r & 2 & $x x$ & $Y Y$ & 22 \\
\hline$\dot{\omega}$ & 1 & 1 & 1 & 1 & 1 & 1 & 1 \\
\hline 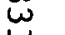 & I I $n$ & 1 & 1 & 1 & 1 & 1 & 1 \\
\hline & 2 & 0 & 1 & 0 & 11 & ؛) & $r$ \\
\hline & 4 & 0 & 0 & 0 & ) & ? & $\because$ \\
\hline & 5 & 0 & $!$ & 0 & i) & 1) & $v$ \\
\hline & 6 & $n$ & ) & :) & ) & $"$ & 0 \\
\hline & h & 0 & 1 & 0 & $n$ & 9 & 1 \\
\hline & in & 0 & ! & 0 & 9 & $v$ & " \\
\hline & 11 & 0 & i) & 11 & 0 & ) & 1) \\
\hline & 13 & 0 & 1 & 0 & :1 & 1) & i) \\
\hline & 15 & 9 & :) & $n$ & ؛) & $n$ & .1 \\
\hline & 16 & 0 & 0 & $n$ & 1 & $n$ & .) \\
\hline & $1^{4}$ & 0 & 4 & "1 & 11 & i) & $i$ \\
\hline & 20 & $n$ & ;) & 0 & $J$ & 0 & $\%$ \\
\hline & 25 & 0 & ? & $!$ & 9 & $!$ & וי \\
\hline & 30 & 0 & 1) & 0 & $"$ & 0 & $\because$ \\
\hline & 31 & 0 & !! & " & $\because$ & 1) & : \\
\hline & 3.3 & ? & 0 & i) & a & $u$ & !1 \\
\hline & 35 & ? & i) & 11 & $: 1$ & 1 & ; \\
\hline & 40 & 0 & 1 & 0 & 19 & $n$ & : \\
\hline & 41 & 0 & "y & i) & $n$ & i) & '? \\
\hline & 14 & 0 & j & I: & ') & $"$ & . \\
\hline & 45 & $n$ & .) & 11 & "! & 0 & . \\
\hline & $14 \mathrm{n}$ & 0 & 1) & " & 1 & 11 & .1 \\
\hline & 44 & 0 & $?$ & $i$ & 11 & !" & 1 \\
\hline & 3!) & 0 & ) & $n$ & ?! & 0 & $y$ \\
\hline & 55 & (1) & '1 & i) & $n$ & $n$ & 1 \\
\hline & 60 & n & $\because$ & $n$ & (. & $"$ & i) \\
\hline - & 05 & U & :) & 0) & ") & 11 &. \\
\hline & 79 & 0 & $?$ & $\Rightarrow$ & 11 & 0 & י \\
\hline & 71 & $n$ & ?) & i! & $" 1$ & 11 & ני \\
\hline
\end{tabular}

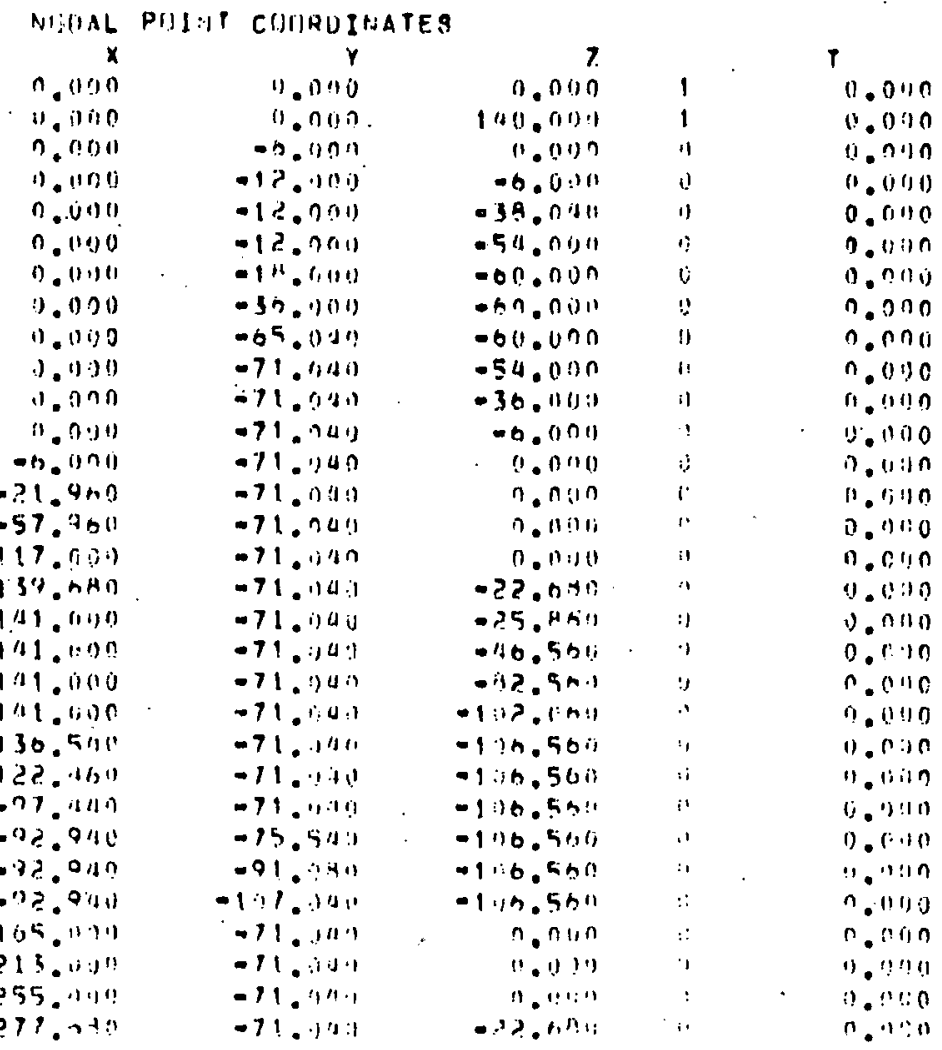




\begin{tabular}{|c|c|c|c|c|c|c|c|c|c|c|c|}
\hline 73 & 0 & ) & $v$ & ) & $1)$ & $\cdot \mathrm{J}$ & $-274.0 ! 0$ & $.71 .041)$ & -25.800 & " & 0.0100 \\
\hline 75 & 0 & ) & $n$ & 0 & v & 1 & -274.0110 & $-71.114 n$ & $-11 h .5 n 11$ & 1 & $0.00 n$ \\
\hline 80 & 0 & $\psi$ & "1 & $n$ & 0 & .1 & (2). & -71.0110 & -82.560 & '" & 0.0130 \\
\hline Bi & $n$ & 1 & $n$ & ") & 1) & ' & -279.000 & -11.11011 & $-1112.0 \mathrm{Bn}$ & " & $n .011 n$ \\
\hline 84 & 0 & $" 1$ & 11 & i) & $n$ & " & -279.500 & -71.11411 & -106.960 & : & 0.0100 \\
\hline 85 & 0 & J & 1) & 9 & 0 & 11 & .200 .460 & .71 .040 & .106 .560 & 11 & 0.0110 \\
\hline 86 & 0 & $n$ & 11 & 1 & 0 & $:$ & -235.3110 & -71 . तisis & .1116 .560 & !" & 0.000 \\
\hline AA & 0 & $\therefore$ & $"$ & " & 0 & .1 & .230 .740 & -75.540 & $-100.5 \mathrm{sil}$ & ii & 0.0110 \\
\hline 90 & 0 & J & 11 & $?$ & ") & 9 & -2310.040 & -01.0411 & - fin.560 & ? & $0 .(11) 0$ \\
\hline 99 & 0 & 1 & 0 & ה & $u$ & II & -2311.0410 & $-107.00,1)$ & -110.530 & 0 & 1.0111 \\
\hline$Q A$ & 0 & $"$ & i: & 0 & 9 & 0 & -259.941 & -71.000 & 0.000 & $\|$ & $0.011 n$ \\
\hline 100 & 0 & ? & 0 & $?$ & 0 & 9 & -263.040 & .71 .0110 & 0.000 & 11 & 11.01100 \\
\hline 105 & 0 & 1 & 0 & J & 0 & ': & -315.000 & .71 .040 & 0.000 & 11 & $0 . n \cup n$ \\
\hline 110 & 0 & 0 & " & ] & 0 & ॥ & .375 .000 & -71.1140 & $n .01)(1$. & :) & 0.0100 \\
\hline 111 & 0 & 1) & 0 & (1) & ? & ") & $.111 ? .5170$ & -71.0417 & 11.000 & $1 "$ & 0.0 .30 \\
\hline 113 & $n$ & 1 & "1 & y & 1) & !! & $-417.010 n$ & .71 .040 & $-11.5 ! 10$ & $\because$ & 0.0110 \\
\hline 115 & 1 & 1 & 0 & $\jmath$ & $n$ & $\bar{i}$ & .417 .000 & -71.154 .1 & -1.4 .1211 & $i i$ & $0.00 n$ \\
\hline 120 & ") & 0 & $n$ & $n$ & 0 & 11 & -117.1700 & $-71.04 n$ & .74 .120 & $:$ & 0.0100 \\
\hline $1 ? 1$ & 0 & " & i) & 0 & 0 & 1 & -417.9010 & $-71.041 !$ & $-137 .(81)$ & $\because$ & 0.0110 \\
\hline 124 & 0 & ") & $n$ & J) & $n$ & !! & $-41 ? .5170$ & .71 .000 & $=106.040$ & $"$ & $n$. n n \\
\hline 125 & 0 & $n$ & 0 & ? & 0 & :I & -374.460 & -71.040 & $-1116.6 A_{1 i}$ & $!$ & 10.000 \\
\hline 126 & 0 & . & 0 & $n$ & $n$ & i) & -373.440 & -71.040 & $.116 . A A 11$ & 11 & 0.0140 \\
\hline $12 A$ & 0 & j & 0 & ) & $n$ & i & -369.9110 & $.75 .94 i$ & -1116.040 & " & 0.0710 \\
\hline 130 & 0 & 1 & 0 & 0 & 0 & 0 & .364 .940 & $-91.0 A_{0}$ & $.17 n .6811$ & 0 & 0.0110 \\
\hline 140 & 0 & 1 & 1 & 11 & 0 & 0 & -368.4 .10 & -3117.1140 & $-10 n .6 A n$ & $: 1$ & 0.000 \\
\hline 141 & 1 & 1 & 1 & 1 & 1 & 1 & 0.010 & -37.1100 & .60 .0100 & 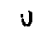 & 0.000 \\
\hline 142 & i & $i$ & $i$ & 1 & 1 & $i$ & -111.000 & .72 .040 & .82 .560 & i) & 0.000 \\
\hline 143 & 1 & 1 & 1 & 1 & 1 & 1 & -274.0110 & -72.0140 & $.92 .56 n$ & .) & 0.000 \\
\hline ial & 1 & 1 & 1 & 1 & 1 & 1 & $.017 .0 n v$ & $.72 .(141)$ & .78 .121 & .1 & $11.00 n$ \\
\hline 149 & $i$ & $i$ & 1 & $i$ & $i$ & $i$ & .21 .901 & .72 .043 & 11.000 & 18 & 0.090 \\
\hline 146 & 1 & 1 & 1 & 1 & 1 & 1 & .375 .0100 & $-72.04 ?$ & 0.000 & ?! & 11.000 \\
\hline 149 & $i$ & $i$ & 1 & $i$ & 1 & $i$ & .03 .940 & -107.041 & -170.560 & 11 & $0.02 n$ \\
\hline 149 & 1 & 1 & 1 & 1 & 1 & 1 & .93 .4120 & -107.0 .40 & $-1106.5 \mathrm{hn}$ & 1 & n. nno \\
\hline 149 & 1 & $i$ & 1 & 1 & 1 & 1 & .72 .040 & $-111^{4} .1140$ & .106 .500 & .1 & $0 . n) 0$ \\
\hline 150 & 1 & 1 & 1 & 1 & 1 & 1 & .02 .940 & $-10 ;$.nait & $-106.50,10$ & i) & 0.0110 \\
\hline 151 & 1 & $t$ & 1 & 1 & 1 & 1 & .32 .9140 & $-107.14 n$ & -1197.500 & .1 & n.non \\
\hline 152 & $i$ & $i$ & 1 & $i$ & 1 & $i$ & .93 .440 & $-107.114 n$ & -107.501 & 0 & 0.000 \\
\hline 153 & 1 & 1 & 1 & 1 & 1 & 1 & -214.020 & .71 .1140 & 0.0170 & i) & 0.000 \\
\hline 151 & 1 & 1 & 1 & 1 & 1 & 1 & -214.390 & .71 .040 & 0.000 & ، & $0.00 n$ \\
\hline 155 & 1 & 1 & 1 & 1 & 1 & $i$ & $-213.013 n$ & .72 .000 & 0.000 & i! & 0.010 \\
\hline 150 & 1 & 1 & 1 & 1 & 1 & 1 & -213.1110 & .72 .040 & 0.000 & i! & 0.000 \\
\hline 157 & $i$ & 1 & 1 & 1 & 1 & $i$ & -213.0110 & .71 .040 & $-1, n(1)$ & $n$ & 0.000 \\
\hline $15 A$ & 1 & 1. & 1 & $i$ & 1 & $i$ & -213.000 & -71.0411 & -1.000 & it & 0.000 \\
\hline 159 & i & 1 & $\mathrm{i}$ & 1 & i & $i$ & -231.9110 & -107.1140 & -100.560 & $\therefore$ & 0.0130 \\
\hline 160 & i & $i$ & 1 & $i$ & 1 & $i$ & -231.9110 & $-107 . n 4 n$ & $-106.5 n n$ & "1 & 6.0190 \\
\hline 101 & 1 & 1 & 1 & 1 & 1 & 1 & $-231 . .940$ & $-1(0 \mathrm{H} .040)$ & $-110.50 n$ & I & $n .1110$ \\
\hline 162 & 1 & 1 & 1 & 1 & 1 & 1 & -230.040 & $-10^{n} .040$ & -176.500 & u & 11.0110 \\
\hline 103 & 1 & $i$ & 1 & $i$ & 1 & $i$ & -230.941 & -107.0100 & -1107.560 & 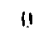 & 11.000 \\
\hline 164 & 1 & 1 & 1 & 1 & 1 & 1 & -230.940 & -1197.040 & -107.550 & $: 1$ & $n .010$ \\
\hline 169 & 1 & 1 & 1 & 1 & 1 & $i$ & -369.9411 & $-1117.140 n$ & -100.640 & $\|$ & $n .0 n 0$ \\
\hline int & 1 & 1 & 1 & 1 & 1 & 1 & -369.940 & - 107.040. & $\left.-1110.60_{0}\right)$ & $: 1$ & $0.0: 10$ \\
\hline 107 & i & $i$ & $i$ & $i$ & $i$ & $i$ & -354.940 & -1001.040 & - IIB.,AHI & 1: & 0.1130 \\
\hline 169 & 1 & 1 & 1 & 1 & 1 & 1 & $-36 \mathrm{H.010}$ & -104.0100 & $-1110.0 \mathrm{kn}$ & a & (0.01)1: \\
\hline 169 & 1 & 1 & 1 & 1 & 1 & 1 & -31.4 .9411 & $-107.014 n$ & $-1: 17.640$ & 1 & (i.) \\
\hline 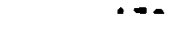 & 1 & 1 & 1 & 1 & 1 & 1 & $-31, H .414 n$ & -1117 & $-1.17 .041\}$ & if & 0.9190 \\
\hline
\end{tabular}

GENERATED NIIDNI, BITA 


\begin{tabular}{|c|c|c|c|c|c|c|c|c|c|c|}
\hline 60 & 0 & 0 & (1) & 1) & 0 & 1 & .105 .010 & -71.000 & $n, 0 \cap 0$ & 0.100 \\
\hline 61 & 1 & 1 & 1 & 1 & 1 & 1 & 0.000 & 1.000 & $60.43 ?$ & ח \\
\hline 62 & 1 & 1 & 1 & 1 & 1 & 1 & $\because 1100$ & $1.0 n !$ & D1. 430 & ). .911? \\
\hline 63 & 1 & 1. & 1 & 1 & 1 & 1 & 0.0100 & U.jon? & 62.446 & n.0n:? \\
\hline 64 & 1 & 1 & 1 & 1 & 1 & 1 & 0.0100 & 10.1300 & 63.453 & n \\
\hline 65 & 0 & I) & $n$ & $\mathrm{a}$ & 0 & $v$ & -213.130 & -71.040 & $0.00 n$ & $0 .(100)$ \\
\hline 66 & 1 & 1 & 1 & 1 & 1 & 1 & 0.070 & $\because .000$ & ด5. 1169 & 0.1100 \\
\hline 67 & 1 & 1 & 1 & 1 & 1 & 1 & 0.0110 & 1). rnn & no. 075 & 0.000 \\
\hline 69 & 1 & 1 & 1 & 1 & 1 & 1 & 0.000 & $(1.1) 00$ & 07.4 .42 & 3.000 \\
\hline 69 & 1 & 1 & 1 & 1 & 1 & 1 & 1.090 & ". (1) & $6 B .4 A Q$ & 0.000 \\
\hline 10 & 0 & 11 & 0 & .) & 0 & v & -255.1000 & .71 .040 & $0.000^{\circ}$ & 0.100 \\
\hline 71 & 0 & 1) & 0 & $?$ & 0 & $n$ & -217.6 คด & -71.000 & $=22.691$ & 0.000 \\
\hline 12 & 1 & 1 & 1 & 1 & 1 & 1 & 1). .01211 & ".000 & 71.511 & 0.000 \\
\hline 13 & $n$ & !) & 0 & 11 & $?$ & $\dot{y}$ & -279.11100 & -71.1110 & $=? 5.89 n$ & 0.100 \\
\hline 74 & 1 & 1 & 1 & 1 & 1 & 1 & (1).? & 1.016 & 73.525 & ". \\
\hline 75 & 0 & :। & () & !) & ) & $\cdot 1$ & -273.910 & .71 .040 & . & 0.000 \\
\hline 70 & 1 & 1 & 1 & 1 & 1 & 1 & $(1, n \cdot 1)$ & 1.11 (i1) & 75.540 & 9.1100 \\
\hline 77 & 1 & 1 & 1 & 1 & 1 & 1 & 19.700 & 1.19190 & $7 n .5 i 9$ & 0.101 \\
\hline 78 & 1 & 1 & 1 & 1 & 1 & 1 & 0.01 .10 & 0.000 & 71.550 & 0.6007 \\
\hline 19 & 1 & 1 & 1 & 1 & 1 & 1 & $(1.01) 11$ & $n$. rion & $7 H .561$ & ח \\
\hline so & 0 & :) & 0 & $\therefore$ & $n$ & ij & -?73.n00) & $-71.94 !$ & -82.5611 & 9. \\
\hline BI & 0 & $n$ & i) & 1) & 1) & 0 & טנית. & $-71.04 !$ & $-1: 2.0011$ & 0.000 \\
\hline ?? & 1 & 1 & 1 & 1 & 1 & 1 & 1.000 & 1). nou & $91.5 A 3$ & i). 1100 \\
\hline B3 & 1 & 1 & 1 & 1 & 1 & 1 & 1).01011 & 0.0110 & $87.5^{\circ} 1$ & 0 \\
\hline A.4 & 0 & 1) & 0 & 1) & 0 & u & -274.500 & -71.040 & -1100.501 & 10.1100 \\
\hline 85 & 0 & i) & 3 & 1) & $u$ & j) & -200.460 & -71.040 & -116.5611 & 0.000 \\
\hline 86 & ? & " & $!$ & 0 & 0 & .1 & -235.440 & -71.040 & $-1 r n . \operatorname{sinu}$ & $0 .(100)$ \\
\hline 87 & 1 & 1 & 1 & 1 & 1 & 1 & $0.10 n$ & 1.000 & 40.019 & $0.00 n$ \\
\hline $8 B$ & $n$ & ") & 0 & ) & 0 & 0 & $=2.51 .940$ & $-75.5 \mathrm{dl}$ & $-10 n .5 n n$ & 0.000 \\
\hline 10 & 1 & 1 & 1 & 1 & 1 & 1 & $n .0(n)$ & 1.0010 & 88.033 & n.000 \\
\hline$Q_{0}$ & $n$ & $n$ & $n$ & 0 & 0 & 1) & .230 .9140 & $-41 ., 1)$ O & $-\operatorname{lng} .5 b \|$ & U. $\because n n$ \\
\hline 91 & 1 & $!$ & 1 & .1 & 1 & 1 & 0.0100 & 1.0110 & $90 . n 117$ & החו:9. \\
\hline 92 & 1 & 1 & 1 & 1 & 1 & 1 & 11.000 & 11.000 & 91.055 & 0.1100 \\
\hline 93 & 1 & 1 & 1 & i & 1 & 1 & 0.000 & i). .100 & $92.0 \mathrm{~h} ?$ & 1..00n \\
\hline 94 & 1 & 1 & 1 & 1 & 1 & 1 & 0.000 & 11.0119 & 93. คhव & ". Unn \\
\hline 95 & 0 & :) & $n$ & 9 & $"$ & $i$ & $-7319.910 n$ & $-10 ? .1140$ & $-100.5 \mathrm{hu}$ & $\left.0.00_{0}\right)$ \\
\hline 96 & 1 & 1 & 1 & 1 & 1 & 1 & 9.0013 & $i .00 n$ & 95.073 & $\because .110 n$ \\
\hline 97 & 1 & 1 & 1 & 1 & 1 & 1 & 0. & $11.0110)$ & 80.091 & $9.100 n$ \\
\hline PA & 0 & $?$ & $n$ & $n$ & 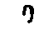 & $v$ & -759.1189 & $-71.1) 0 ?$ & $0.00 n$ & 9.000 \\
\hline 99 & 1 & $i$ & 1 & 1 & 1 & 1 & $17.010 ?$ & $\because$. nטn & पै.7:5 & 0.000 \\
\hline 100 & 0 & 11 & " & 1) & 0 & $\because$ & .261 .11111 & -71.0110 & $n .00 n$ & $1.11 n$ \\
\hline 101 & 1 & 1 & 1 & 1 & 1 & 1 & 0.1100 & 1.100 & 100.719 & n. \\
\hline 10 ? & 1 & 1 & 1 & 1 & 1 & 1 & ?.111? & 4.900 & 111.727 & 1.000 \\
\hline 103 & 1 & 1 & 1 & 1 & 1 & 1 & $(1.1 \cap n)$ & $" .0100$ & 102.7314 & 9.000 \\
\hline 104 & 1 & 1 & 1 & 1 & 1 & 1 & 1). (191) & 11.000 & 103.741 & $0.10 n$ \\
\hline 105 & $n$ & 1) & 11 & 1) & 0 & 9 & -315.1170 & .71 .9110 & 0.10110 & n. Un' \\
\hline 106 & 1 & 1 & 1 & 1 & 1 & 1 & $0.0 \cap 0$ & $n$ !n! & 115.755 & $0.110 n$ \\
\hline 107 & 1 & 1 & 1 & 1 & $t$ & 1 & 0.0011 & 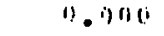 & 116.7118 & 1:.9nn \\
\hline $10 B$ & 1 & 1 & 1 & 1 & 1 & 1 & 0.001 & $9.9 \cup 0$ & $1117.77 n$ & חסו. \\
\hline 109 & 1 & 1 & 1. & 1 & 1 & 1 & ".unil & $\because .1110$ & 1.18 .777 & 0.000 \\
\hline $11:$ & 0 & 10 & $n$ & 1 & $n$ & $\therefore$ & .375 .919 ? & -71.040 & $n \cdot n$ i:n & 0.000 \\
\hline 111 & o & 1) & 11 & 11 & $\dot{v}$ & $"$ & $-1112.5: 11$ & $-71.114 n$ & 1.11010 & 11. inn \\
\hline 11 ? & 1 & 1 & 1 & 1 & 1 & 1 & 11.1111 & $\because$." & 111.790 & ". \\
\hline 113 & 0 & 1) & 0 & ! & 1 & $\because$ & ; & $-11.194 !$ & -4.5011 & ". \\
\hline 1 & 1 & 1 & 1 & 1 & 1 & 1 & 1.001 & $1.11 ! 111$ & 113.413 & $1 . .9107$ \\
\hline ; & $n$ & $!$ & " & ") & ") & 1 & $=119.16 .10$ & -71.810 & -19.120 & $0.0(01)$ \\
\hline , & 1 & $i$ & 1 & 1 & 1 & 1 & (1.01)! & i. 10011 & 115.427 & ; \\
\hline 117 & 1 & 1 & 1 & 1 & 1 & 1 & 11.390 & 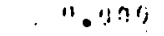 & 116.4 .35 & 7.914 \\
\hline IIA & 1 & 1 & 1 & 1 & 1 & 1 & $r_{1}, 0,10$ & 1.0106 & $|17 . \mathrm{K} \cdot 1\rangle$ & ?. \\
\hline
\end{tabular}




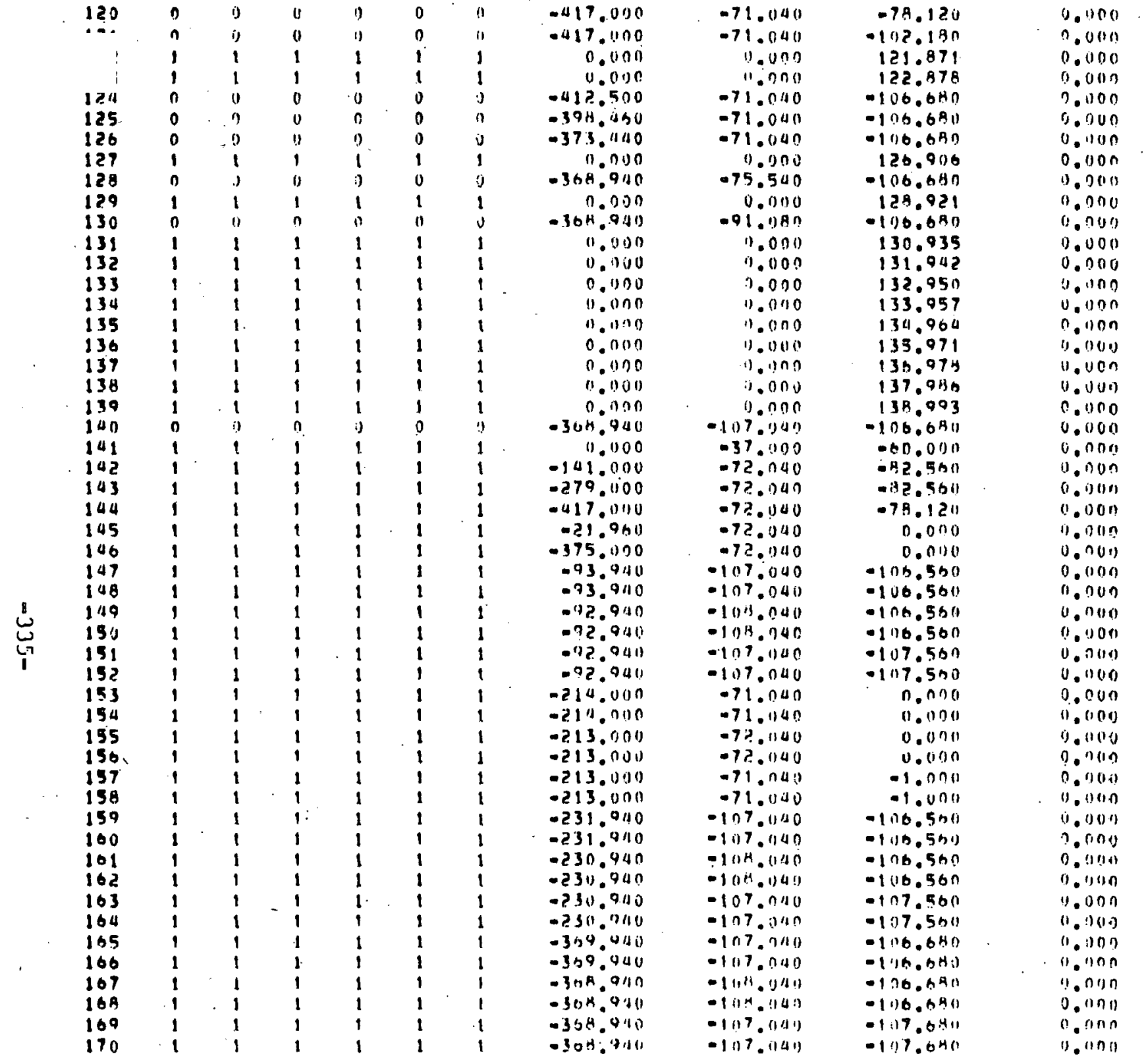

EQUATIOUN SIIIAHERG

$\begin{array}{rrrrrrr}N & X & y & 2 & x x & y y & 2 Z \\ 1 & 0 & 11 & 0 & 0 & 0 & 11 \\ 2 & 1 & 2 & 3 & 11 & 5 & 1 \\ 3 & 0 & 1 & 10 & 0 & 13 & 11 \\ 4 & 7 & 13 & 0 & 11 & 11 & 12 \\ 5 & 13 & 11 & 15 & 10 & 17 & 17\end{array}$




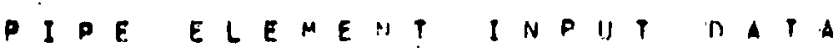

CONTRIIL I NFORAATIIIN

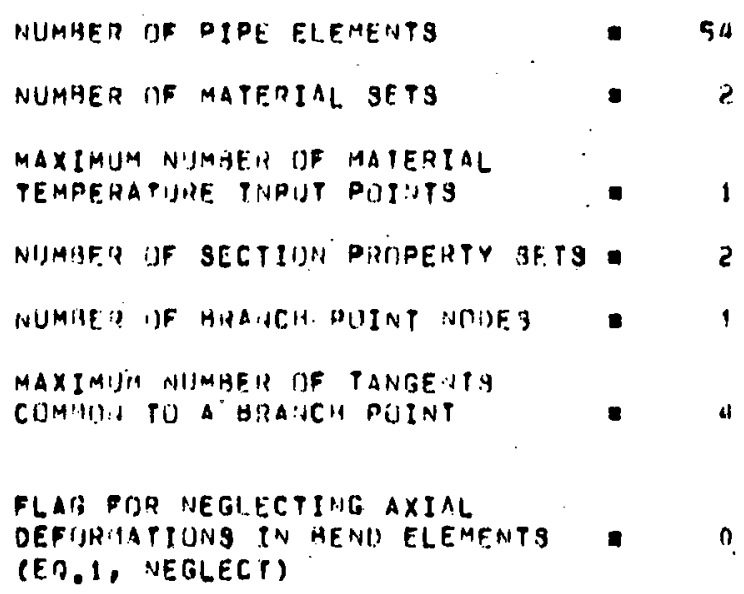

$\underset{\substack{\omega \\ i}}{i}$

MATERIALCPHIPERPYTAAELES

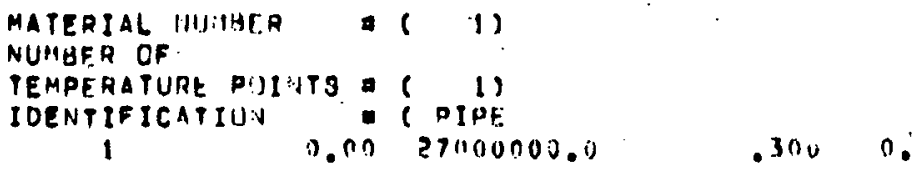

MATERIAL MUIHER $* 6$ (2)

NUMAER DF

PEMPERATUPE PIIIUTS : (1)

IDENTIFICATIOU D I VALVE

1 0.00 alououno." .3ia 0.


Ection Dacoentrital.

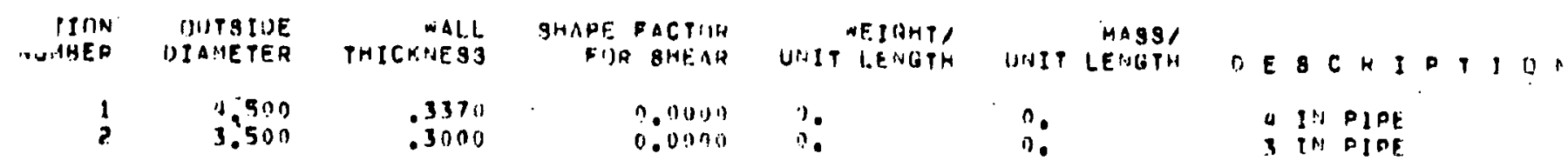

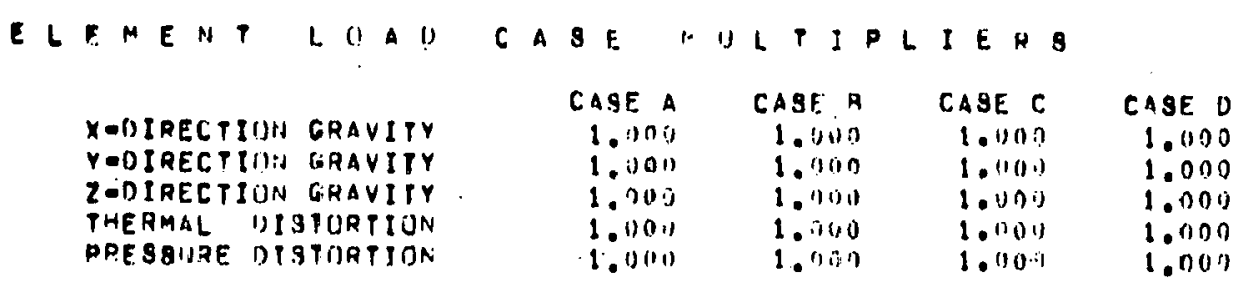




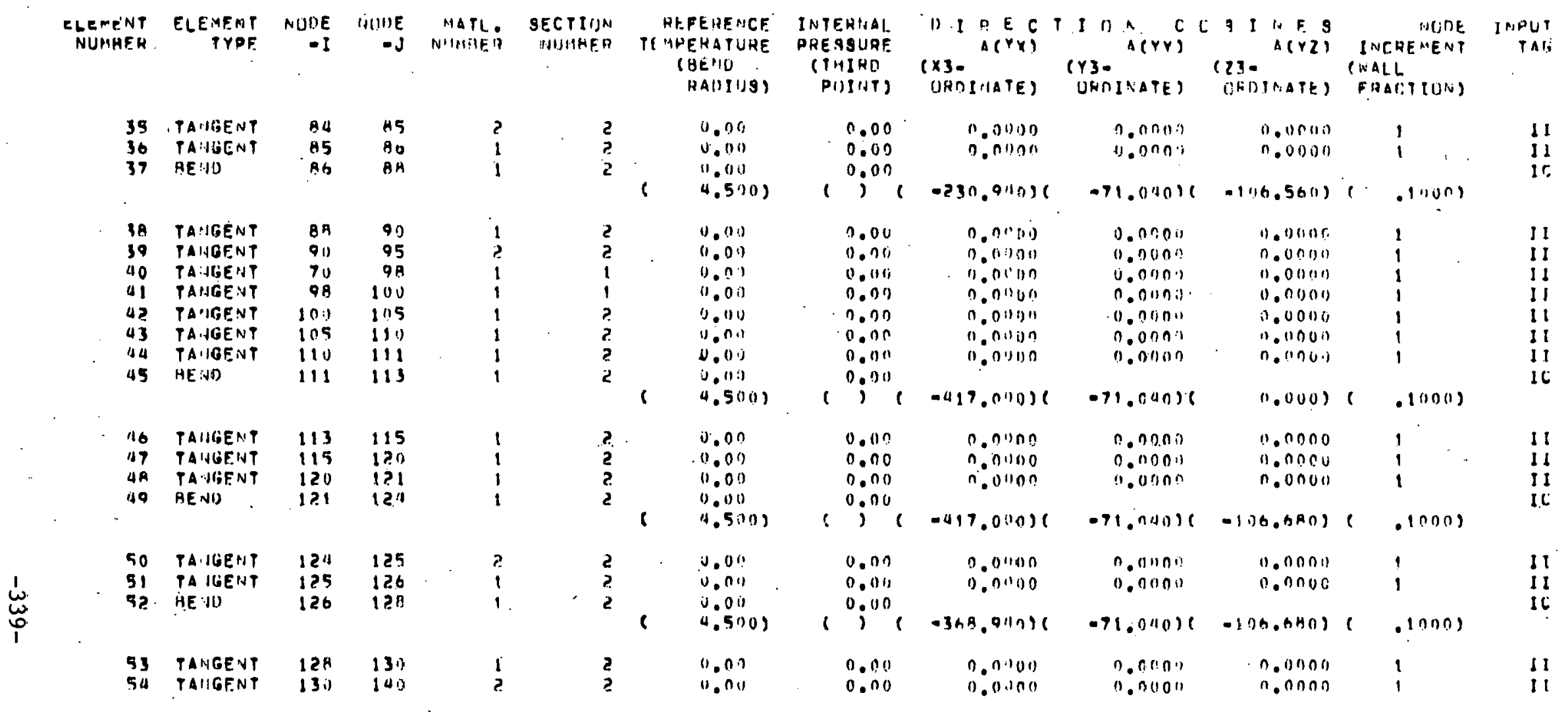


B D II N O.A R Y LEMENTE

ELEMEHT PYPE
NUMBER OF ELEMENTS: $: 3 !$

ELEMENT LOALI) CASF. MULTIOLIERS

\begin{tabular}{|c|c|}
\hline $\begin{array}{r}\text { CASE (A) } \\
\text { l.DUOA }\end{array}$ & $\begin{array}{r}\text { CABE (4) } \\
11.0000\end{array}$ \\
\hline
\end{tabular}

\begin{tabular}{|c|c|c|c|c|c|c|}
\hline & $\begin{array}{l}\text { ELEME'IT } \\
\text { NUMAEK }\end{array}$ & $\begin{array}{l}\text { Nilu) } \\
\text { (N) }\end{array}$ & $\begin{array}{l}\text { NOUF? DFF } \\
\text { (NI) }\end{array}$ & $\begin{array}{l}\text { (f } 0.19 \\
(: 1,1)\end{array}$ & $\begin{array}{l}|N T| \\
(N \mid) \mid\end{array}$ & $\begin{array}{r}\text { ILECTIIJ!) } \\
\text { (NL) }\end{array}$ \\
\hline & 1 & 1)$. & 141 & $\Rightarrow$ & (i & !) \\
\hline & 2 & 40 & 142 & 0 & 0 & $n$ \\
\hline & 3 & 80 & 143 & ) & 11 & 0 \\
\hline & 4 & 120 & $1 / 14$ & 1) & 1) & $n$ \\
\hline & 5 & 20 & 145 & ) & 11 & 1) \\
\hline & h & 111 & 1110 & 0 & 0 & 1) \\
\hline & 7 & 55 & 147 & $n$ & 11 & ) \\
\hline & A & 55 & 1149 & $?$ & 0 & i) \\
\hline & 9 & 53 & 151 & 1) & U & i) \\
\hline & 10 & 65 & 153 & ' J & 0 & 0 \\
\hline & 11 & 63 & 155 & 11 & 0 & 1) \\
\hline & 12 & 65 & 157 & 0 & 0 & n \\
\hline$\mp$ & 13 & 95 & 150 & v & 0 & 1) \\
\hline & 14 & 95 & 101 & !) & ? & (1) \\
\hline & 15 & 95 & 163 & i) & 0 & 11 \\
\hline & 10 & 140 & 165 & i) & 0 & 0 \\
\hline & 17 & $1(41)$ & 1.57 & 0 & 0 & I) \\
\hline & 12 & 1411 & $10^{\circ}$ & .) & 0 & v \\
\hline & 19 & 55 & $14 \mathrm{H}$ & 1 & 0 & ) \\
\hline & 20 & 55 & $15 n$ & 1) & ? & ) \\
\hline & ? 1 & 55 & 15? & $"$ & 0 & 1) \\
\hline & ?.2 & 65 & 154 & $?$ & 1 & $"$ \\
\hline & 23 & 65 & 150 & :) & 1 & (1) \\
\hline . & दa & 65 & 154 & 1) & 0 & i) \\
\hline & 25 & 95 & $16^{1}$ & 1) & 0 & ? \\
\hline & ?h & 99 & 162 & ") & ') & ? \\
\hline & 27 & 95 & $\ln 4$ & $?$ & J & $?$ \\
\hline & ?6 & 140 & 100 & "! & 1) & n \\
\hline & 29 & 140 & 168 & ") & ') & 0 \\
\hline & $3 n$ & 140 & 1711 & 18 & 1) & $?$ \\
\hline
\end{tabular}

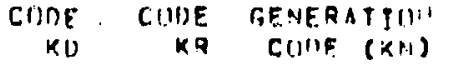

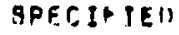

MUIA ID

SPHING 1) SPLACEN

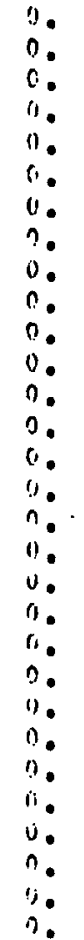

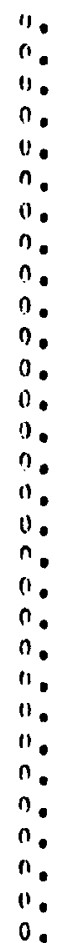

$100 n E+01$ IDINE+OI $10) n F+01$
$10 n \cap E+111$

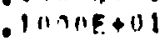
- DOIIIE+ I0 - IUNOE+ III inonetil InOnEtil - Inonet - IOnNe+lo .10OnE + III - IONOE + III - $\ln 11+10+10$ - $10 \cap 0 E+10$ $.1010 E+10$ 100ue+111 11)une +10 - Mner - InTिE+10 - Ionet - InOCE+1a - $1010 \mathrm{nE}+12$ IloOf +12 - 1 ONnE+12. $.100(1 F+12$ innoftiz ivonet - IUnet - IVnNetiz - InONE + 12 - $\ln 00 E+12$ - 1 ONOE+12 


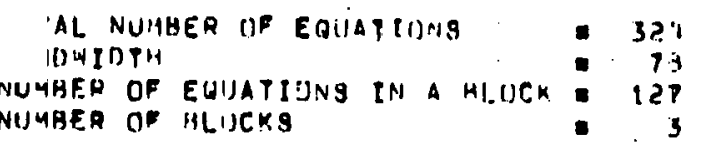




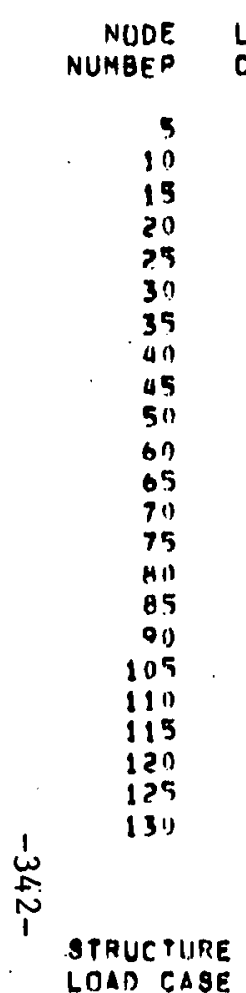

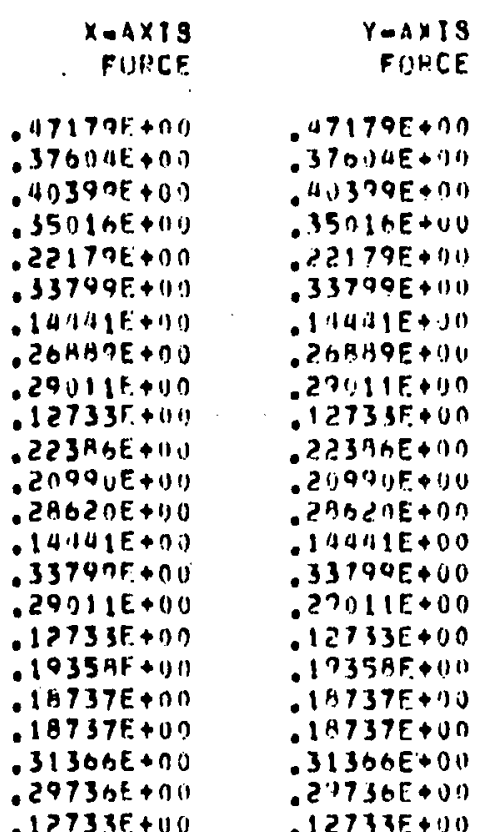

$.1>73.35+110$

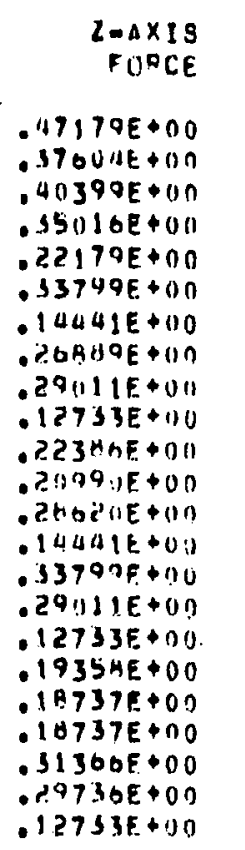

$X=4 X I S$
MOMENT

YAAXIS
MIIMENT

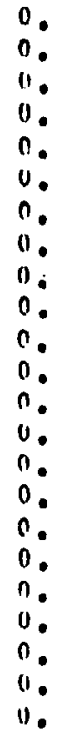

$2=4 \times I S$ 


\begin{tabular}{|c|c|c|c|c|}
\hline PR & F FREDIJENCIES & & & \\
\hline $\begin{array}{l}\text { MOLiC } \\
\text { NUMBER }\end{array}$ & $\begin{array}{l}\text { CIRCULAR } \\
\text { FHFIUIENCY } \\
\text { (RAIISSC) }\end{array}$ & $\begin{array}{r}\text { FHE OUFNCY } \\
\text { (CYCLES/SEC) }\end{array}$ & $\begin{array}{l}\text { PERIOG) } \\
\text { (SFC) }\end{array}$ & TOLERAISCE \\
\hline 1 & $.3163 E+02$ & $.5034 E+01$ & $.1987 E+n 0$ & $.254 \mathrm{AE}-13$ \\
\hline 2 & $.4909 E+0 ?$ & $.781 .35+01$ & $-12 \mathrm{AOF}+1) 0$ & $.60395-114$ \\
\hline 3 & $.51 \triangle A E+0 ?$ & $.8193 F+01$ & $.1221 E+\cdot 10$ & .109 HF -13 \\
\hline 4 & - $5 n 4 I E+0 ?$ & $.8977 F+n I$ & $.1114 E+70$ & $.1574 \mathrm{~F}-14$ \\
\hline 5 & $.5 A 51 E+02$ & $.93125+n 1$ & $.1074 E+30$ & $.4251 E-14$ \\
\hline 6 & . $6217 E+n ?$ & $.9895 F+01$ & $.1011 E+.70$ & $n$. \\
\hline 7 & $-8307 E+02$ & $-132 ? \mathrm{~F}+n ?$ & $.7553 E-.71$ & $n$. \\
\hline B & $.9398 F+0 ?$ & - IAQRE+D? & . Grant-n1 & $.131 A E-13$ \\
\hline 9 & $.9467 t+02$ & $.1507 t+n ?$ & a637E-n1 & 11. \\
\hline 10 & $.1116 E+113$ & $.1775 E+11 ?$ & $.5632 \mathrm{~F}-\mathrm{nl}$ & 1). \\
\hline 11 & $\therefore 1144 E+03$ & -1 A? IE + O? & $.549 ? E-n 1$ & $.3513 F-1 ?$ \\
\hline 12 & $.1139 E+03$ & $. ? 290 \mathrm{~F}+02$ & $.4367 F-101$ & $.7 ? 29 E-11$ \\
\hline 13 & . $15 T^{\prime} ? \mathrm{E}+03$ & $.2502 F+02$ & $.399+F_{-}-01$ & .5 A $5 F-10$ \\
\hline 14 & $.1625 t+n 3$ & $.2585 F+02$ & . 39िमEF-01 & $.1339 E-10$ \\
\hline 15 & $.1693 F+03$ & - ?hOIE+O? & $.371 P E-011$ & $.|A| I \mid E-10$ \\
\hline 16 & $.176 A E+113$ & $.2 \times 13 r+0 r$ & $.3555 E-01$ & $.3581 F-109$ \\
\hline 17 & $.190 / 1 F+0.3$ & $.3030 \mathrm{~F}+\mathrm{n} ?$ & $.33 n n E-n 1$ & $.6700 E-09$ \\
\hline 18 & $.2213 E+03$ & . 35??t+n? & .5A3(BF-n) & . RSIOF-nT \\
\hline 19 & - ?331E+0s & $.3710 E+02$ & $.26965-111$ & $.411035-117$ \\
\hline 20 & $.2677 E+13$ & - 4?61F+0? & $.2347 E-01$ & $.2 .74 A E-05$ \\
\hline 21 & $.27915+03$ & - $\triangle A \|>F+n ?$ & $.225 \mid E-01$ & $.5759 E-07$ \\
\hline $2 ?$ & . 302 RE + () 3 & . UAก9F + ?? & $.2073 \mathrm{E}-01$ & $.4401 F-115$ \\
\hline
\end{tabular}

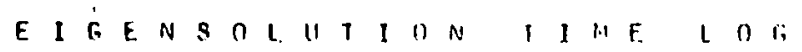

EIGENSOLUITION $=-13.01$

PHINTINT, 
CASE i

OIRECTION FACTIORS
$x=1.0000$
$r=1.0000$
$7=$
1.0000

INDICATOR FOR DISPLACEMENT OH ACCFLFRATION SPECIRUM =

EQ.0 DISPLACFMEN

ER.1 ACCELERATION: IN IN. /SF.C.?

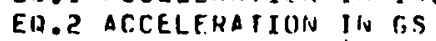

3 SPECTRA ARE FINTEHED FOH TASF 1. KIND=?

CLUSTER FACTOR, CF $=.10000$ 
MODE $X$-DIRFCTIODN

Y-DIRECTIONS Z-DIREEIION

\begin{tabular}{|c|c|c|c|}
\hline & $.176, E+00$ & $-.5959 E+00$ & .1159t: $\div 1$ \\
\hline 2 & . PA4AE $-n 1$ & $-.25096+01)$ & $.10 R+E+n 1$ \\
\hline 3 & $.1075 E+00$ & $.4332 F+110$ & .414 SE +00 \\
\hline 4 & $.8750 E+00$ & - In $P$ If +00 & $-.5389 E+00$ \\
\hline 5 & $.2 n 13 E+n 0$ & $.1108 E+01$ & . 3ha 3t-1)1 \\
\hline 6 & $.2313 E+100^{\circ}$ & $.9298 \mathrm{R}+11 n$ & -.093 IDE -02 \\
\hline 7 & $.5161 E+00$ & $.4829 F+00$ & $.79 A 2 t+00$ \\
\hline A & $.2376 E-n 1$ & $-.4: 4 a F-n 1$ & - SRAGAF +00 \\
\hline 9 & $.1017 f+110$ & $.6 A n \angle f-01$ & $.74 G 2 E-01$ \\
\hline 10 & $-.1031 E+n 0$ & $\because 3 O Q G F+\cap B$ & $.7996 E-011$ \\
\hline 11 & $.8267 E-01$ & $-.1011 F+01$ & $-.15 A B F+00$ \\
\hline 12 & $-.71194 E+00$ & $.2269 F+100$ & $.447 T E+110$ \\
\hline 1.3 & $-.577 \mathrm{AE}+00$ & $.1573 E+00$ & . $271 ? E+110$ \\
\hline 14 & $.2997 F+00$ & $-.303 ? F+00$ & $. \operatorname{lng} 35+00$ \\
\hline 15 & $-.1210 t-01$ & $-.31 .34 E-0 ?$ & . $213 F E+00$ \\
\hline 16 & $.5259 E+00$ & $.30 n S F-01$ & $-.29706-111$ \\
\hline 17 & $-. A 314 E+n O$ & -.1 Ė $3>+00$ & $-.221 P E+00$ \\
\hline $1 \mathrm{~B}^{\circ}$ & $-.5 B G 7 E-01$ & $-. P 97 A E+00$ & $-.14537+00$ \\
\hline 19 & $-.1751 E+0 \pi$ & $.2175 F+100$ & $-.190 / t .00$ \\
\hline 20 & $.271 R E+00$ & $-.1334 F+00$ & $.16145+n 0$ \\
\hline 21 & $-.1399 E-0 ?$ & $-.20 \times 1 F+0,0$ & 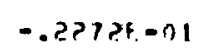 \\
\hline 22 & $.1150 E+00$ & $.1509 F+r n$ & 1 \\
\hline
\end{tabular}

SPFCIRLIM TABLE I THE SPECTRIMP X DTRFCTION

NIIMAER OF PUINIS $=11$

SCALE FACTUR $=$. 3ARAIIF+113

INPI

POINT HERIOT

$10 D \cap E-1) 1$

$-10 n(1)+00$
$-100 E+010$

$.310 N E+1011$

- $35 n N E+100$

SUECTHIII

VALIH

$.13(11++00$

- 13 (1)1 +0n

- In5nE+111

$-3500 t+110$

-550nt+00

.5ADOE+UO .19NDE+OI 


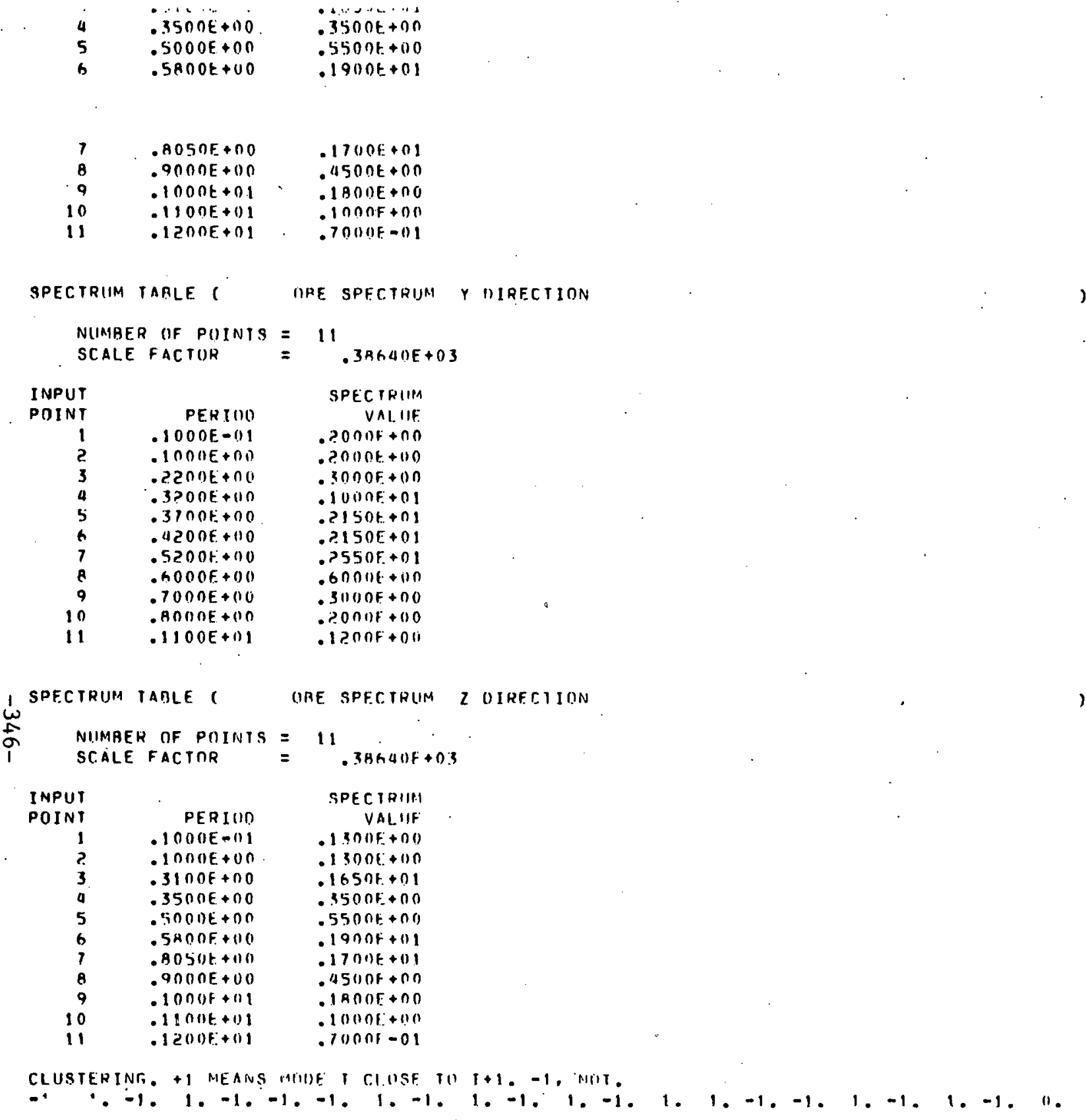


Solution 7a

Tnterspatial/Intermodal Combination 


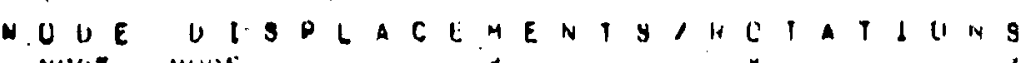

HOE ?IIII)

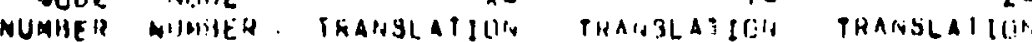

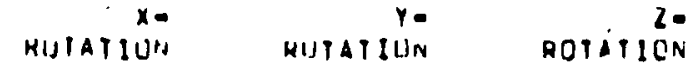

2 TOTALE 1.55.107E-UA 1.14189E-US 1.37.143E-11S

0.

$n$.

3 THYL.." "

リ.

4 TUTAL.. 3.43767E-113

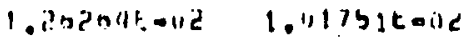

2.4432 SE $=03$

$5.96540 E-04$

5 TUTAL.. 2.0811UE-112 1.371USF-11 1.12401t-11E

$4.05103 E-03$

$9.9(1)+5 E-1) 4$

$4.81667 E-014$

a TUTAL.

$4.541) 100-02$

C. $13041 t+01$

I.1)(377t-1)2

$1.031055 E-03$

$1.021109 E-03$

$5.14153 E-114$

7 PIITAL.. U. ".

n.

$4.25000 t-1) i$

A TITAL.. S.5672AE-1I?

2. $13: 434 t-1) 1$ $-$

$\underset{\substack{\omega \\ 1}}{\infty}$

? TIITAL.. O

111 IOTAL.. 0.09409E-112

$2.1330: 05.001$

1).

$1.45+875-101$

$3.115492-111$

o.

$3.4211,16-111$

13 TOTAL.. M.11243E-11

$2.1412 ? t-11$

14 TDTAL.. U.

15 TUTAL.. 5.4007IE-112

in POTAL.. 9.74727L-13

17 PUTAL.. 1".

UTAL.. O. 1UYACF.-IS n.

$1.342555 \cdot 01$

$1.5501 \cdot 1+x ?$

).

1. 35, II) $=$-11
$3.32102 t-111$
).

$5.02345 E-113$

11.

$5.74503 E=05$

5.477 HOE $U$

0.

$4.0525 H E-115$

11.

$1.24573 E-013$

$3.04013 E-03$

1).

$3.33,5 ! t-03$ i.

$1.114 n G E \cdot U 3$

1).

$1.19029 E-03$

$0.56294 E=04$

1.27 HABE-US $0.72891 E-04$

0.

$1.367 H G E-03 \quad 4.99 A 97 E-U 4$

1 .

0.

$1.115456-113 \quad 4.194791 E-114$

$1.575191-113 \quad ? .034516-111$

1) ,

1.757 UKE-11S

$2 . \forall 414 C E-U 4$ 


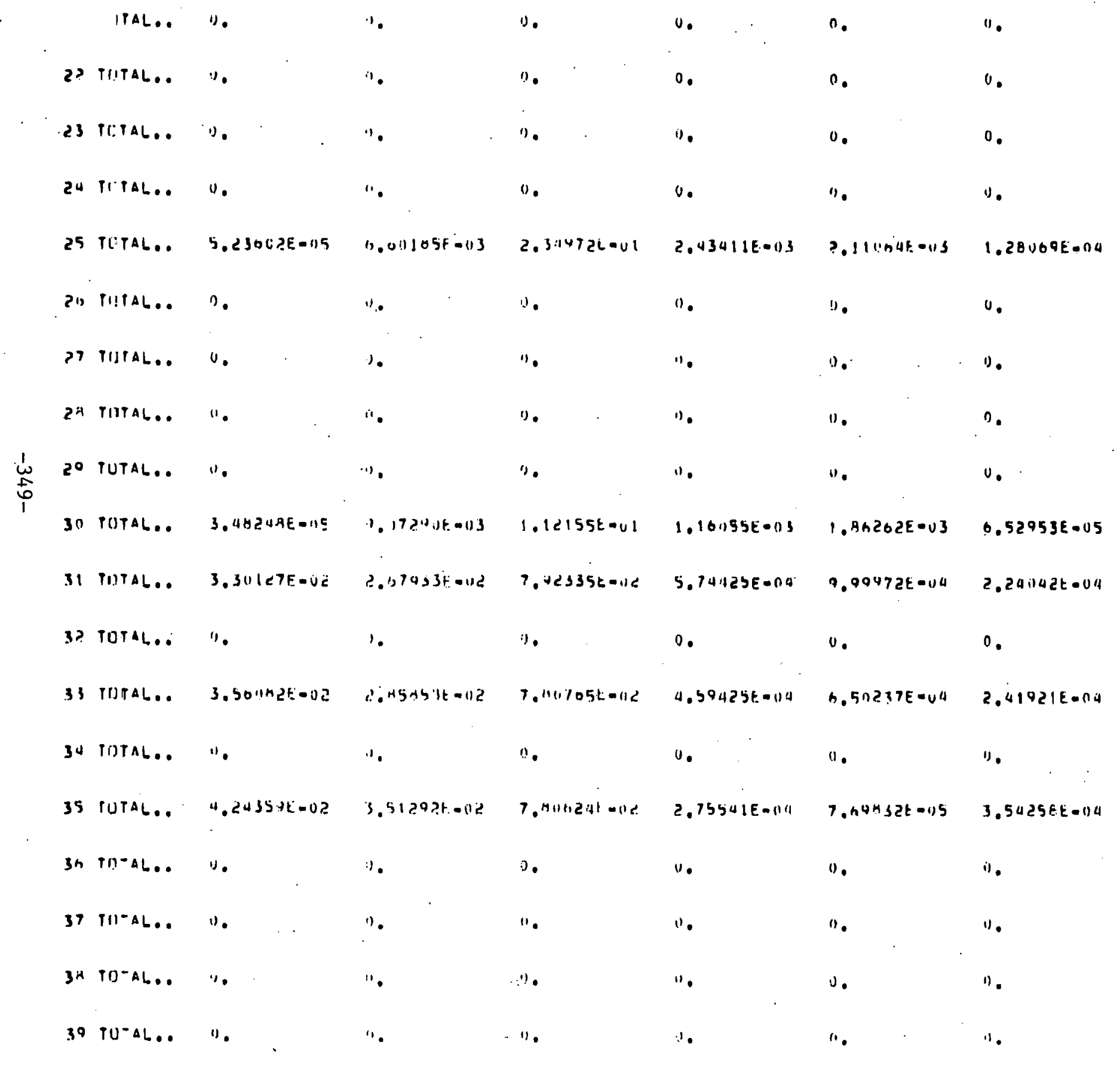




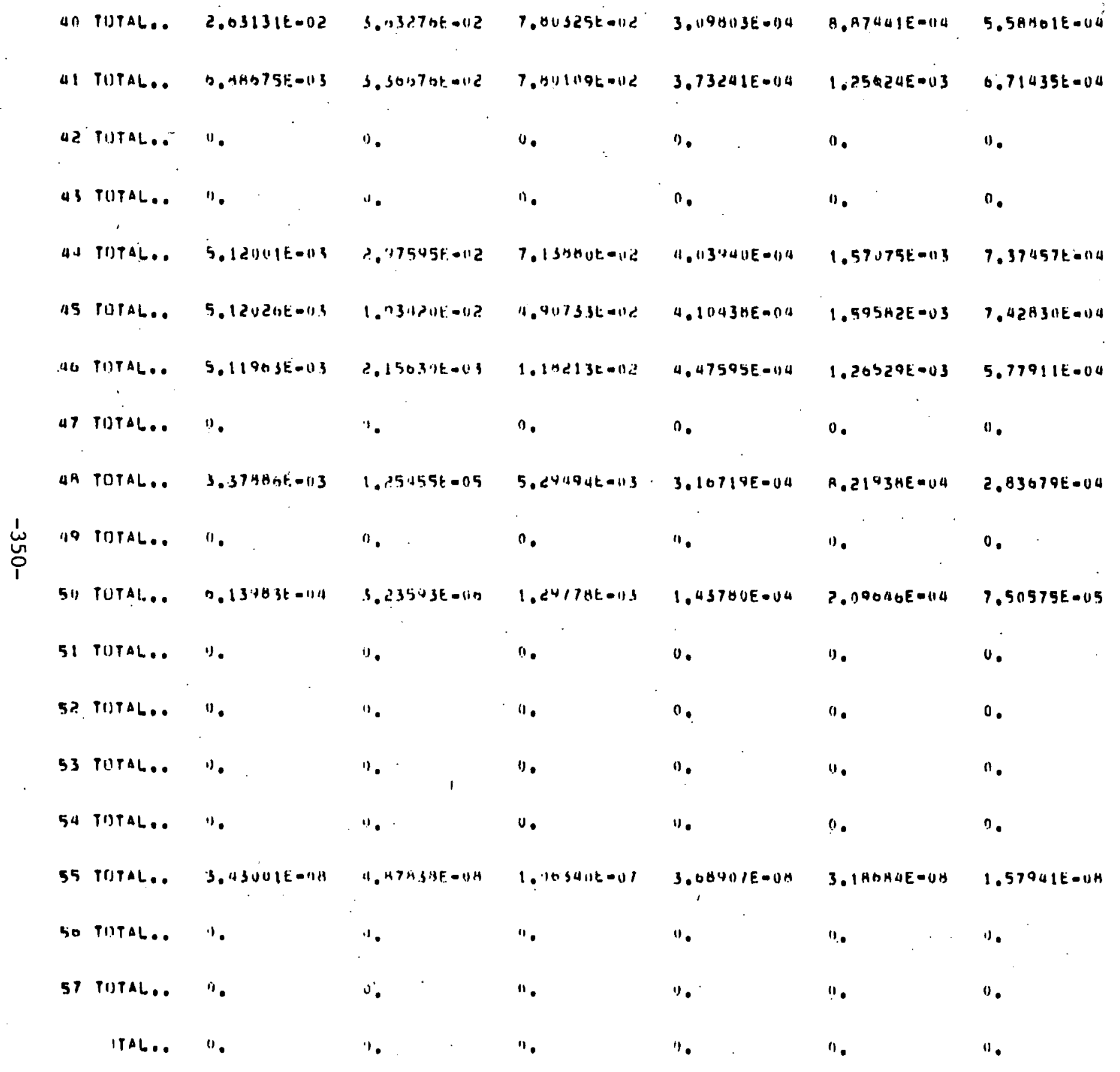




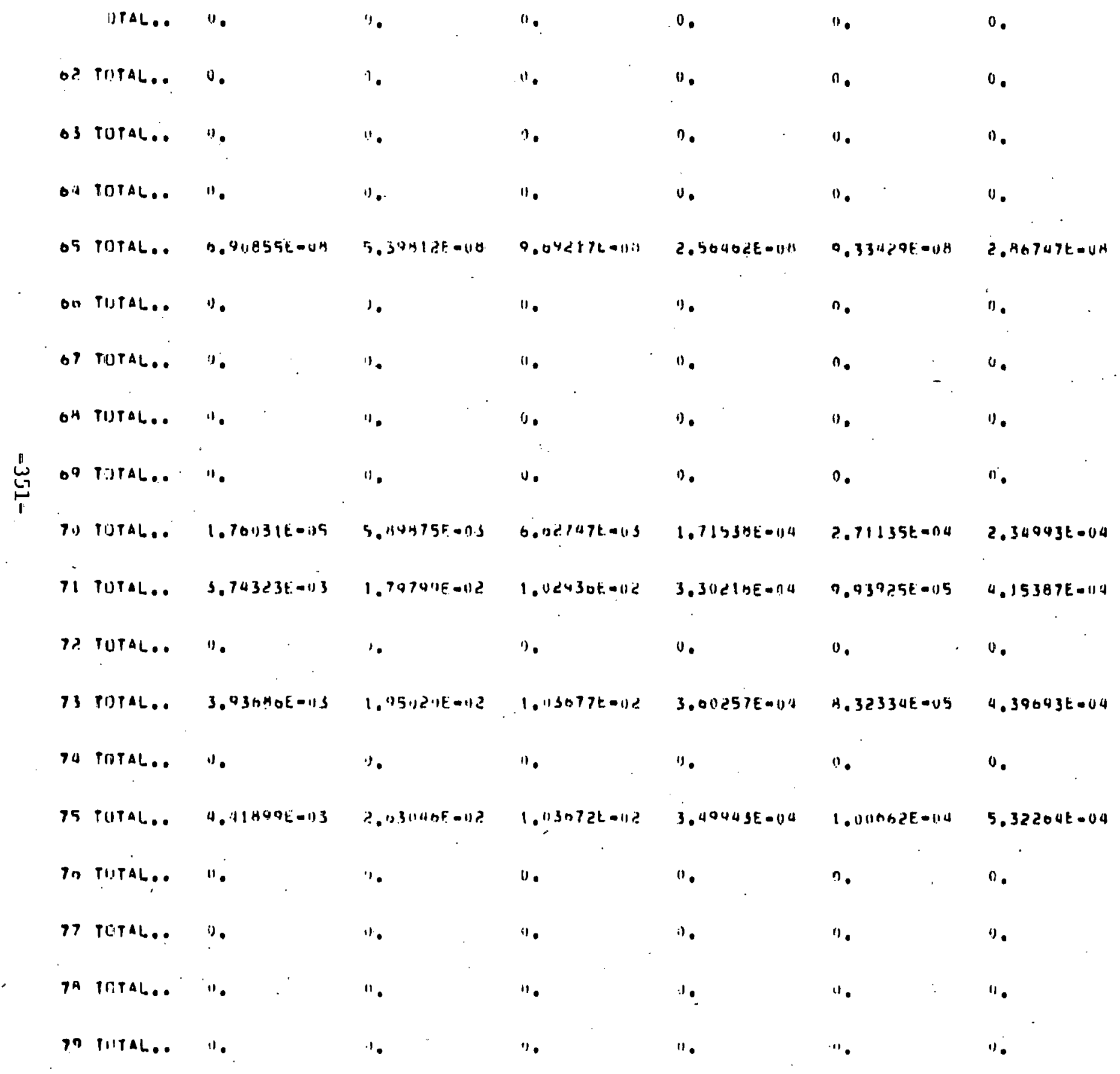




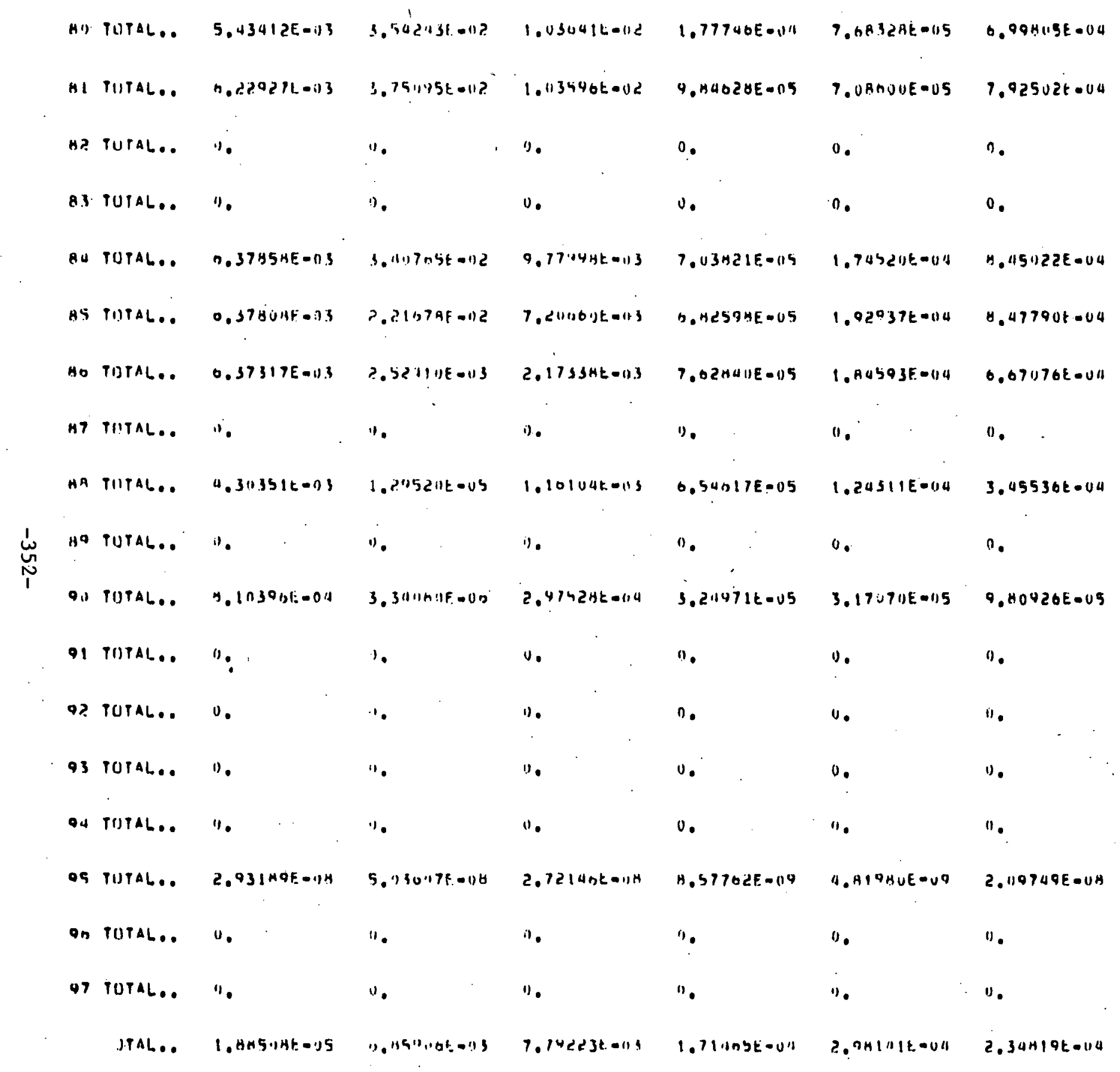




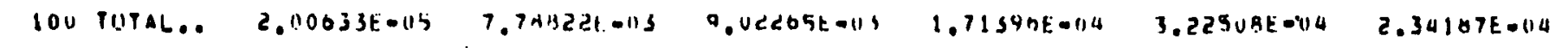

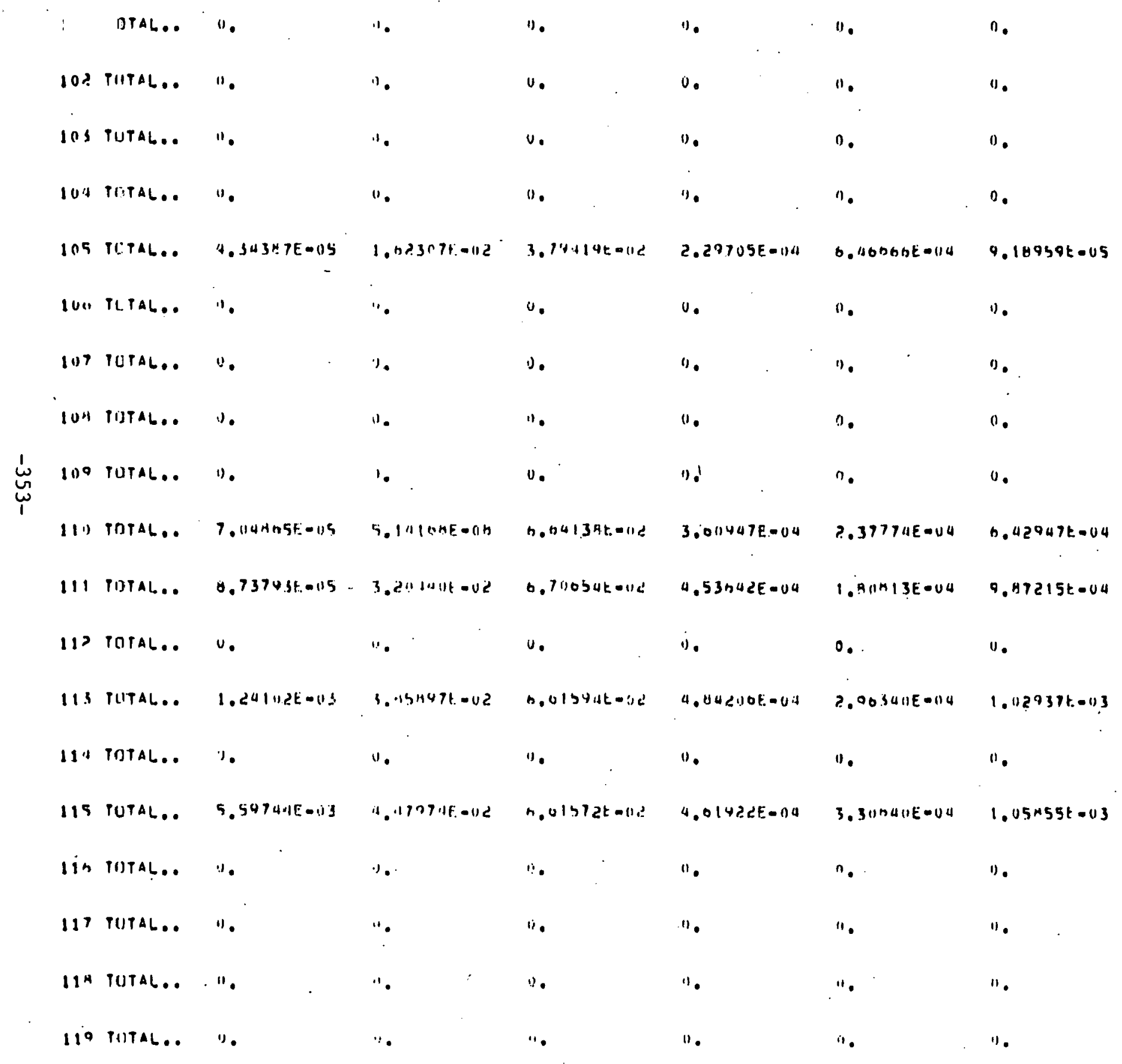




\begin{tabular}{|c|c|c|c|c|c|c|c|c|}
\hline & $(21)$ & TUTAL.. & $1.47705 t=112$ & $6.1111216-1) 2$ & $0.01252 E-132$ & $1.1143 S E=04$ & $2.284,9 E=04$ & $1.19434 E=03$ \\
\hline & 121 & TOTAL.. & $0.150 .157 f .0113$ & $5.7435,16-112$ & $n, 0 \cup 41$ ut & $2.05574 F=04$ & $7.26 H \cup 4 E=04$ & $1.2514 A E=03$ \\
\hline & 122 & TOTAL.. & ". & ". & 9. & 0. & 0. & 0. \\
\hline & 123 & TUPAL.. & .0. & i!. & $\% 0^{-}$ & 0. & $n$. & 0. \\
\hline & 124 & PUTAL.. & 9.04 S SSE-113 & $5 .(17,00.3 t-1) 2$ & $0.13312 k=1 i 2$ & $2.47327 E=04$ & $1.22^{4} 00 E=03$ & $1.280335-03$ \\
\hline & 125 & TUTAL.. & 4.63 H9 QE-1)3 & 3. P.HAOSt $=62$ & A.SSIIUE-US & $2.58435 E-014$ & $1 . ? 9 \times 3 \circ E=U 3$ & $1.27119 t-03$ \\
\hline . & $12 n$ & TOTAL.. & $4.83 .125 t-1) 3$ & 1.755ट7E-OIS & e & $3.40 S 2 i) E-04$ & $1.12 .153 E=03$ & 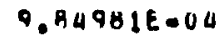 \\
\hline & 127 & TUTAL.. & .0. & נ. & 10. & 0 . & 1). & 0. \\
\hline & $12^{\lambda}$ & TITTAL... & $n, 1302111 t=n 3$ & $1.56131 E=115$ & 5.5 HZUCLE-U13 & $3.15 H 93 E-04$ & $7.42 \cup 35 E-04$ & $5.29351 E=114$ \\
\hline w & 129 & TUTALE. & $"$. & !。 & 11. & $1)$ & $n$ & 0. \\
\hline & (31) & TIITAL.. & 1.257 H5t. -113 & $1.11245 b F=11 n$ & I. $42: 24 t-11.5$ & $1.55534 t=1111$ & $1.992 .65 E-1) 4$ & $1.52200 \mathrm{E}=1114$ \\
\hline & 131 & TIITAL.. & u. & "1. & u. & 0. & 0. & $n$ \\
\hline & $13 ?$ & TUTAL.. & - ". & 1. & リ. & $v$. & 0. & 0 \\
\hline . & 133 & T!ITAL .. & "). & 1. & $!$ & 0. & i). & u. \\
\hline & 134 & TOTAL... & ". & 11. & 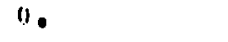 & "! & u. & 1). \\
\hline & 135 & TOTAL.. & 11. & 1. & (i) & 11. & 1. & 0. \\
\hline & 136 & TUTAL.. & $"$. & ". & ". & 0. & 0 & $n$ \\
\hline . & 137 & TOTAL.. & ". & $\because$. & ग。 & 1. & ". & 0. \\
\hline & $\cdots$ & "UTAL... & v. & .1 & 1。 & u. & $n$. & u. \\
\hline & 130 & THTAL... & ". & ". & $n$. & 11. & 11. & $n$. \\
\hline
\end{tabular}




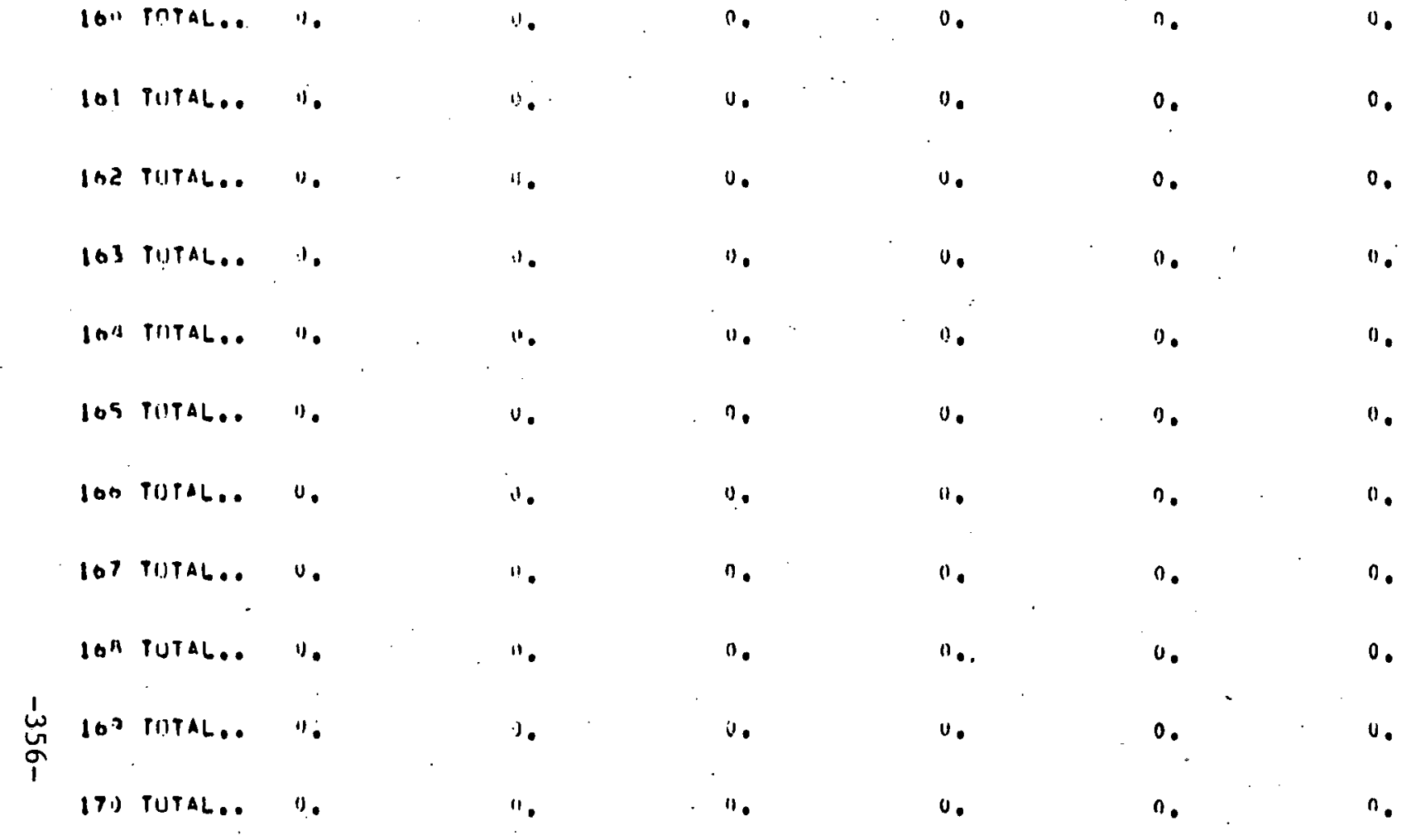


RESPONSE SFELTKUMSSTRESS CUMPONENTS (KINI) I I )

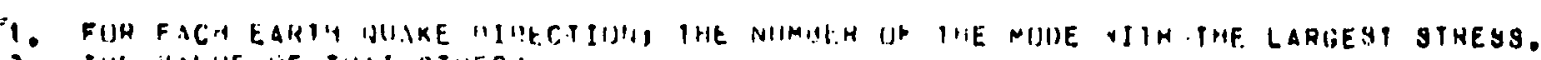

2. IHE VALIJE LIF TMAT STMESY.

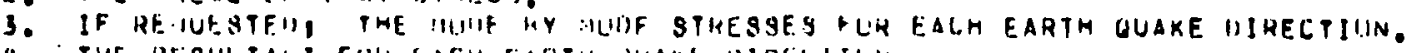

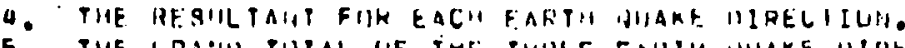

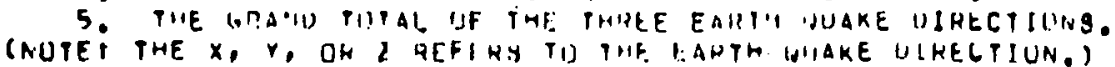

ELEME'T IYPE (3/U $I$ I E

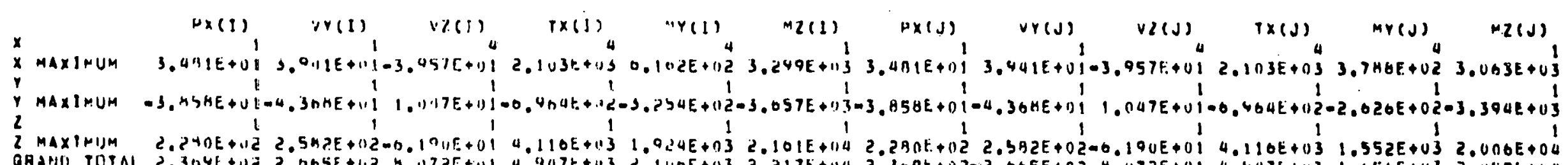

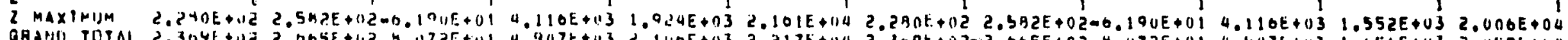

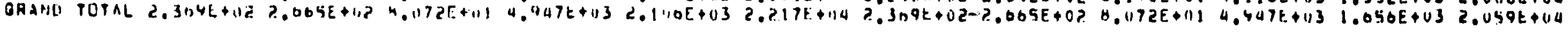

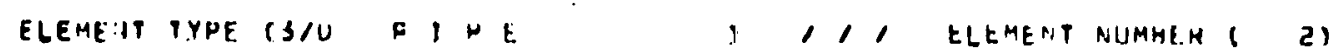

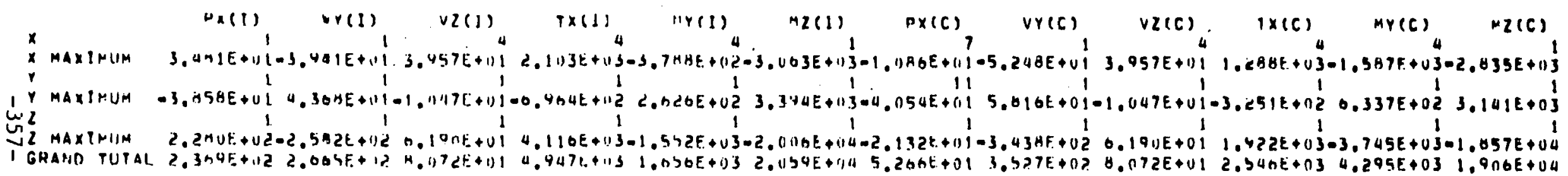

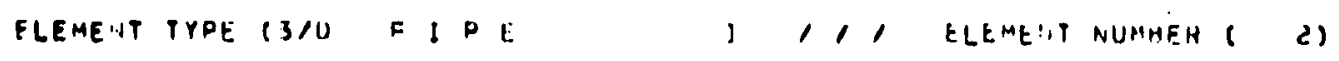

DX(J)
$\times$ MY(J)

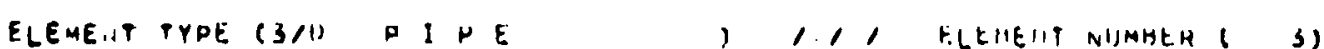

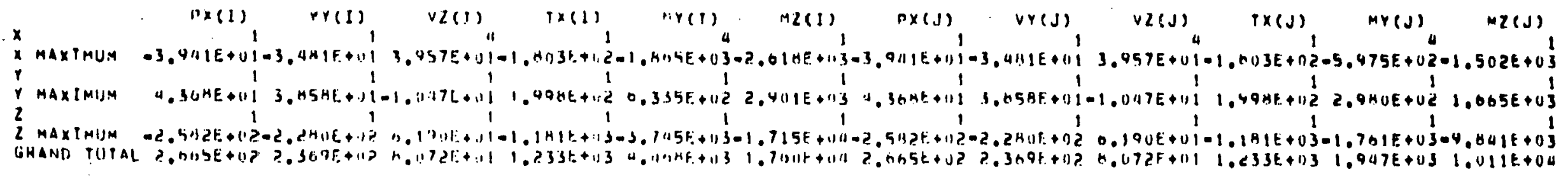

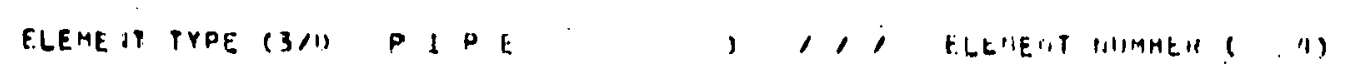

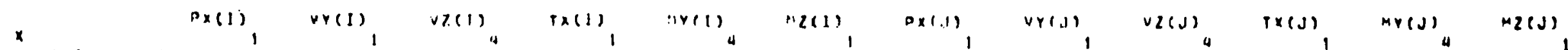

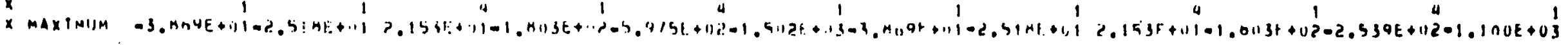




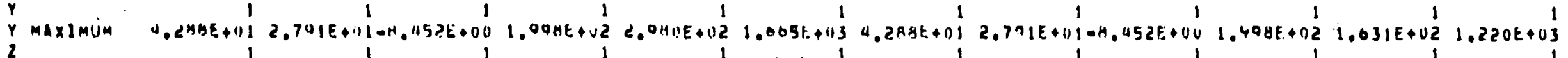

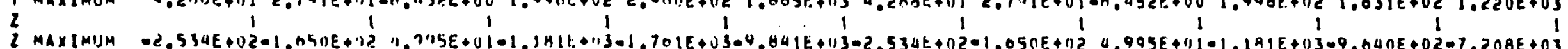

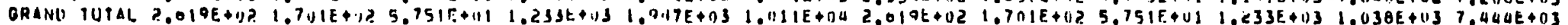

ELEMEHT TYPE (SII) P I P E , , I ELEMEAT NIJMHER ( S)

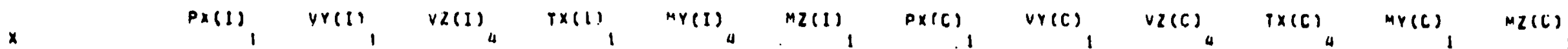

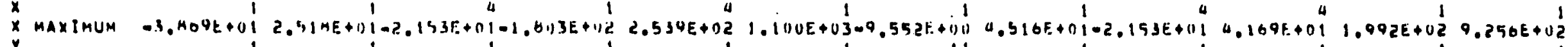

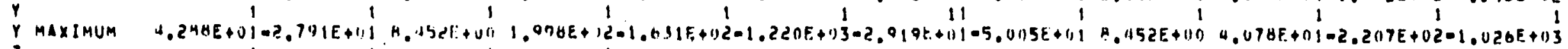

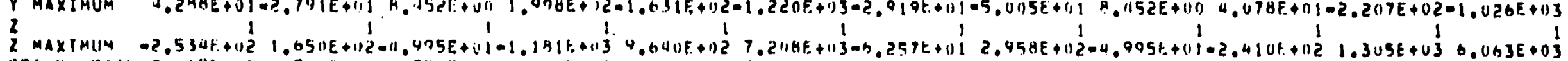

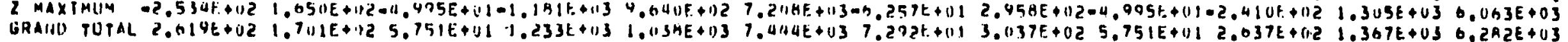
ElenE:I TYHE (3/0 D I F.

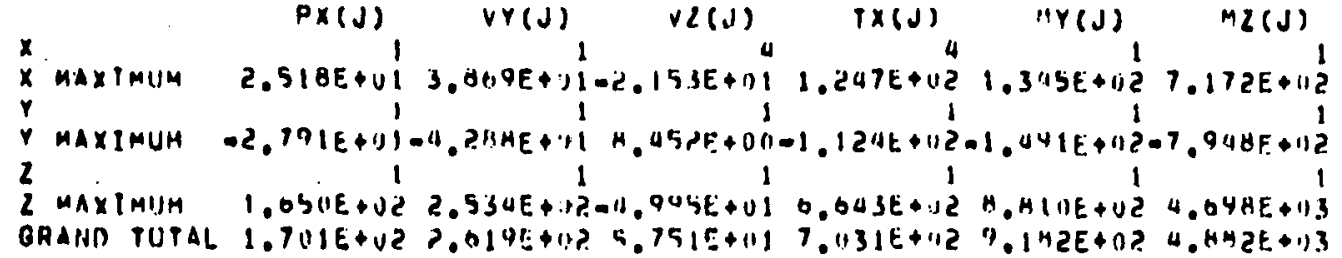

ELEMENT TYDE (3/1) D I E

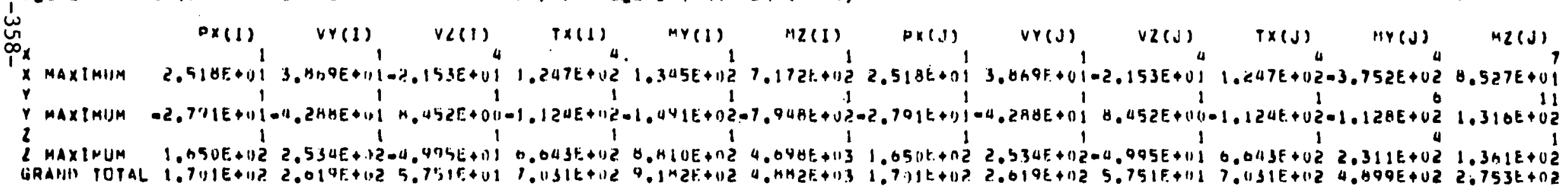

ELEMEHT PYPE (3/U P I P E ,,$/$ GLEMENT WUMHEH $(7)$

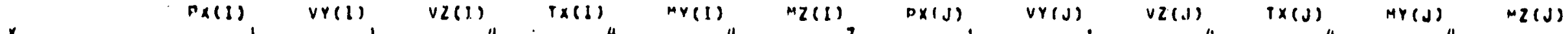

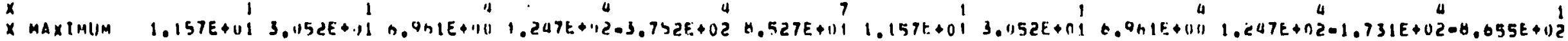

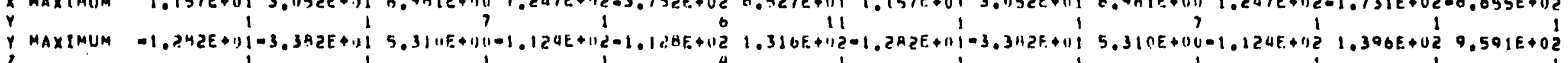

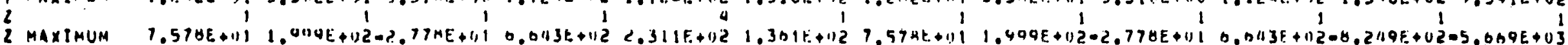

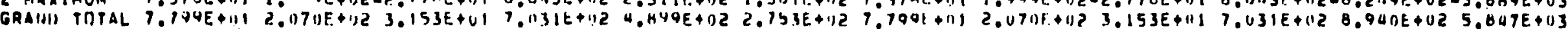

ELEHFHT PYPE (3/1) P I PE

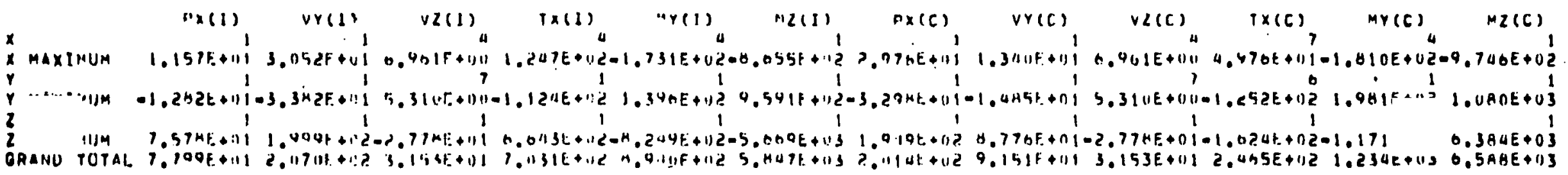




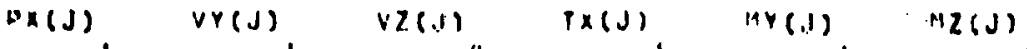
$x$ maximLIM $3.152 E+11-1.157 F+11$
$y$

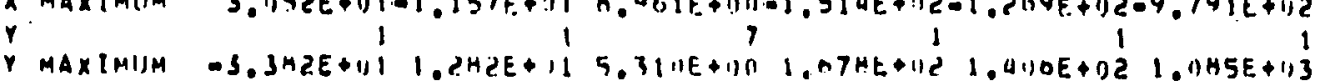

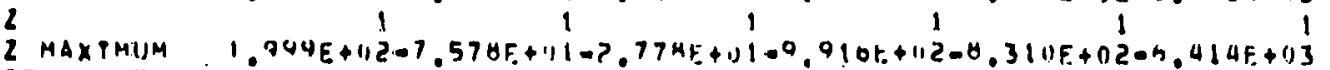

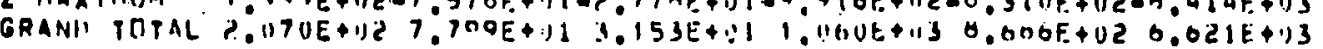

ELEHE IYT TYPE (3,0 P I H : I, 1, ELEMENT NIJMHEH 1 Q)

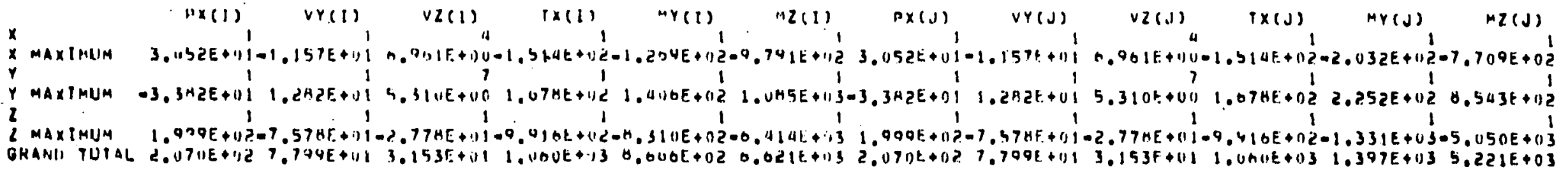

ELEMF!IP TYPE (3/1) P I P F.

, 1, ELElIETI NUMHEH $($ III)

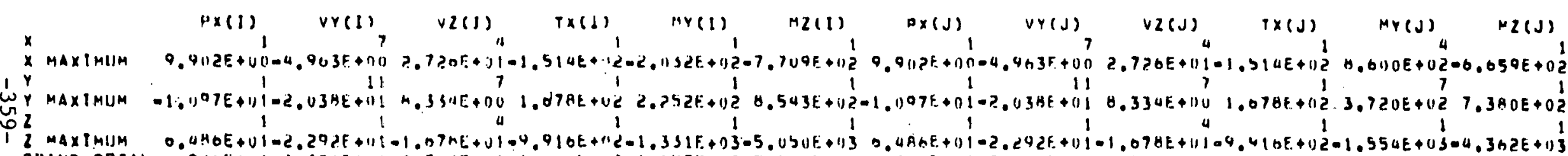

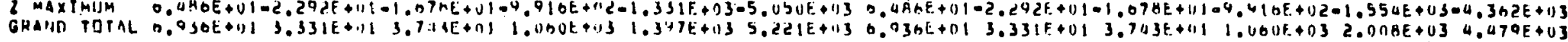

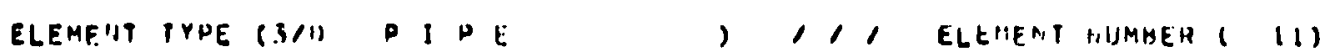

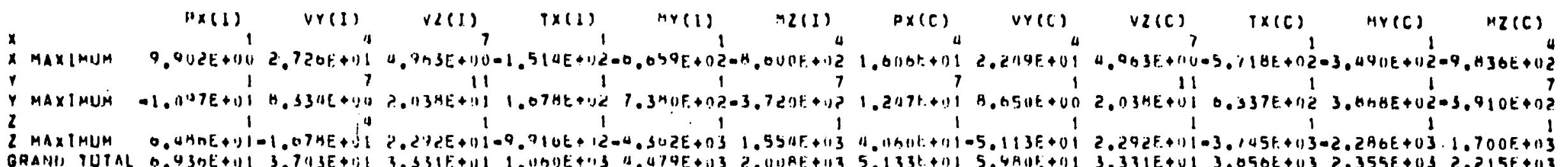

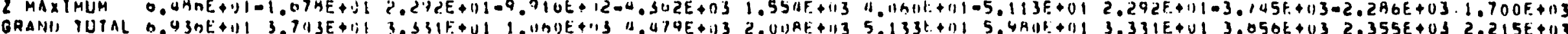

ELEMEHT PYPE (3/0 D I PE

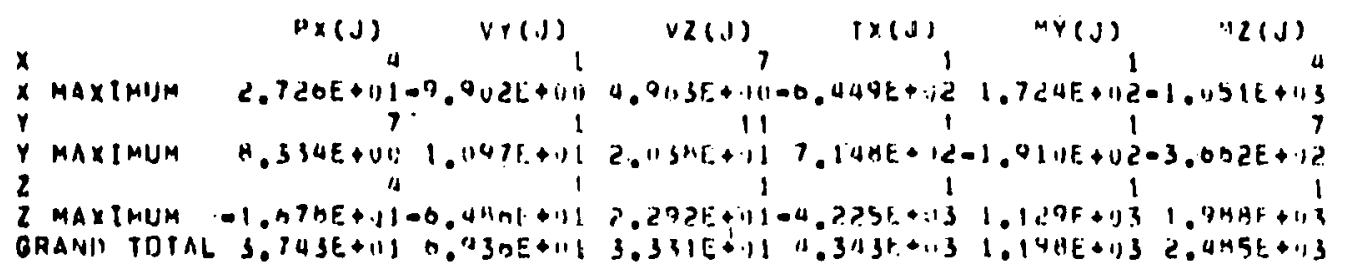

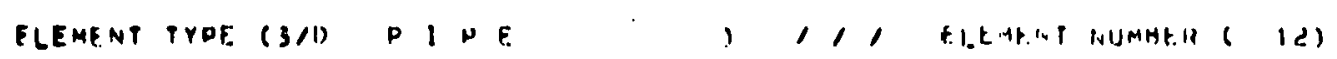

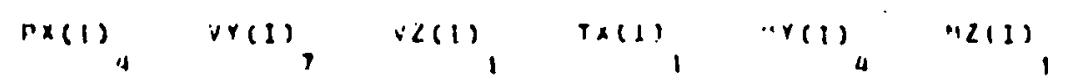

$0 \times 1.7)^{4}$ Ne(.7),

$v 2(.1)$

$1 \times(j)$

$\operatorname{MY}(J)$

$M 2(J)$ 


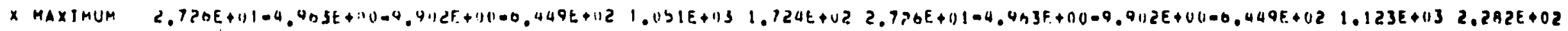

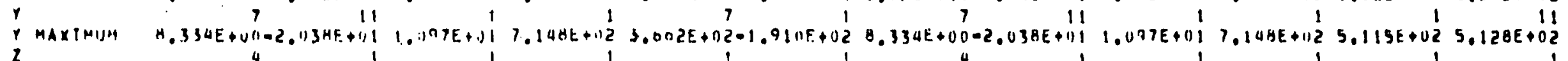

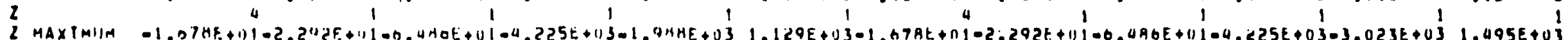

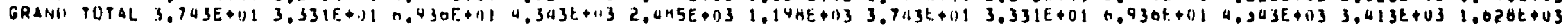

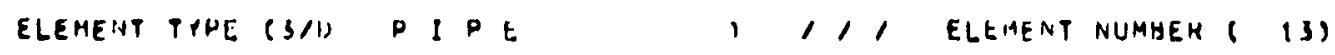

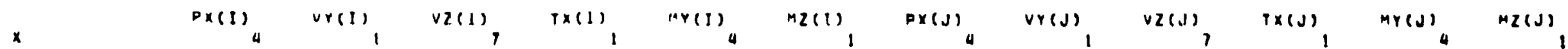

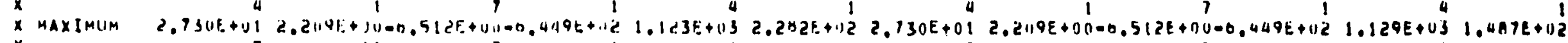

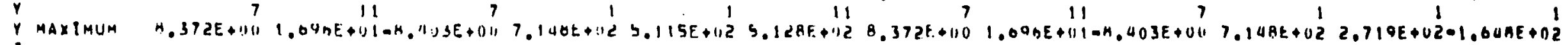

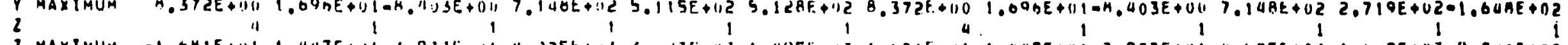

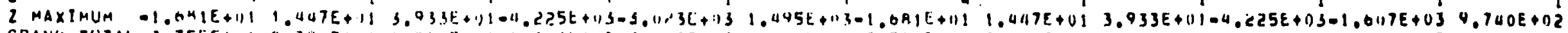

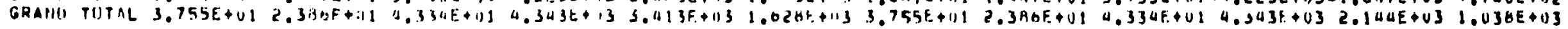
ELEMENT TYPE (3IU $I P F$, 1,1 ELEMEMT NUMAER ( 14)

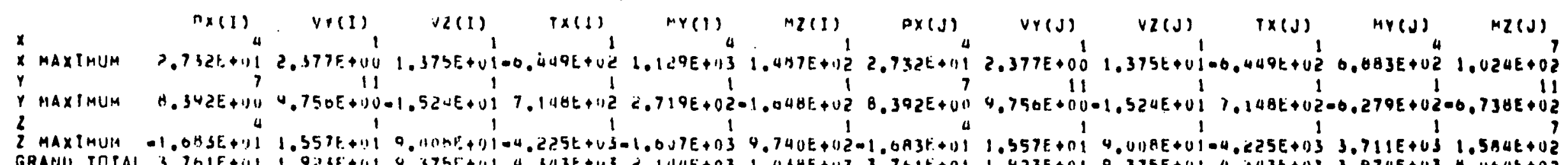

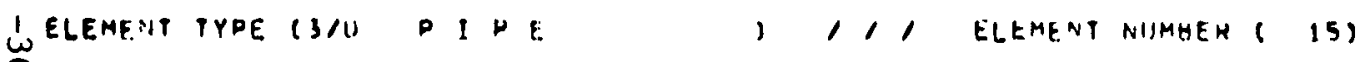

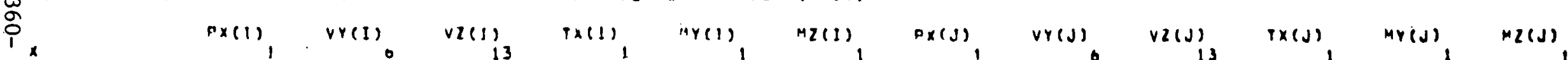

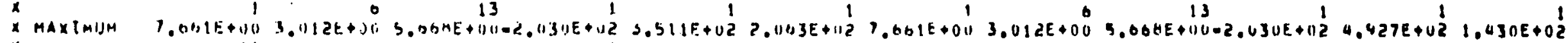

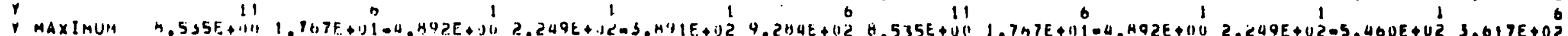

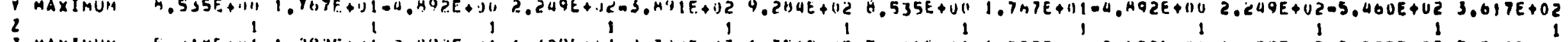

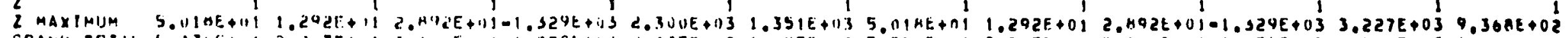

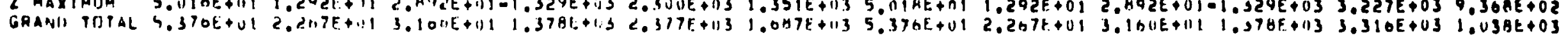

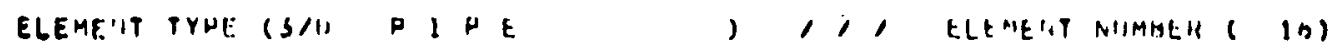

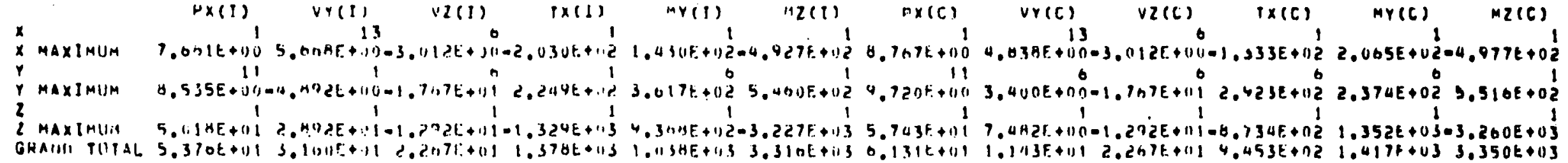

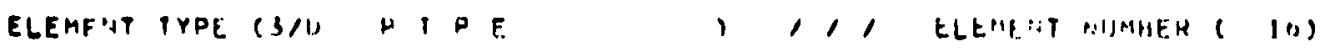

\begin{tabular}{|c|c|c|c|c|c|c|}
\hline & $1 \times(J)$ & ir (.1) & $v<(.1)$ & $9 \times 6.19$ & $\operatorname{mr}(.1)$ & ${ }^{H} Z(J)$ \\
\hline X & 1 & $\|$ & $n$ & 0 & 1 & \\
\hline WA I MUM & $10 t_{0}+110=$ & $n f+11)=1$ & $E+1 リ 11$ & $t+11$ & $1 F+02-$ & .96 \\
\hline 40 & $\begin{array}{r}1 \\
1 E+\| 1\end{array}$ & $1 t+110$ & $7 E+111$ & $\begin{array}{c}0 \\
1 \\
1 \\
0\end{array}$ & $\begin{array}{r}1 \\
+112\end{array}$ & \\
\hline & 1 & 1 & 1 & 1 & 1 & \\
\hline
\end{tabular}


EL - P POE $(B, 1)$ P I P E

1,1, ELEHE' P AIJMEER (17)

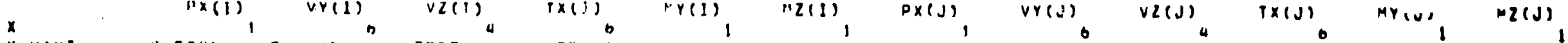

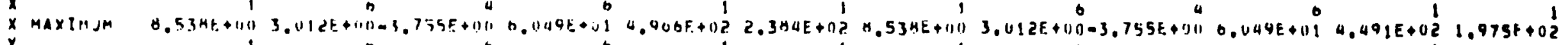

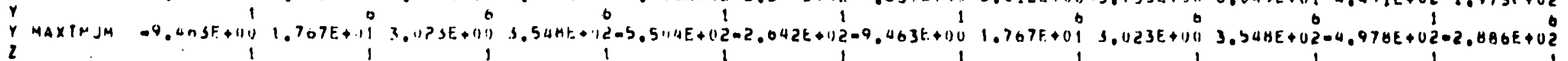

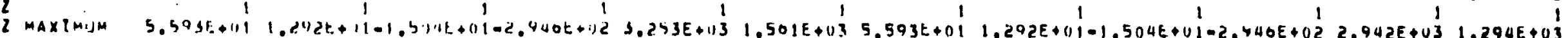

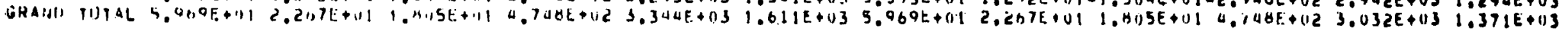

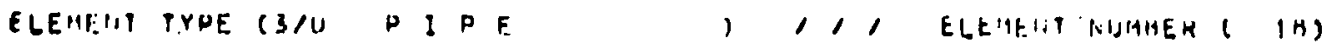

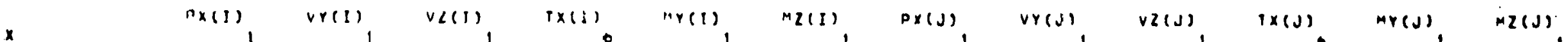

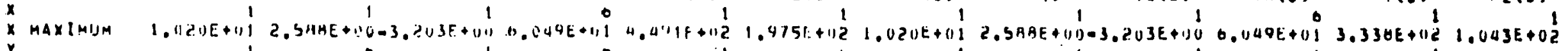

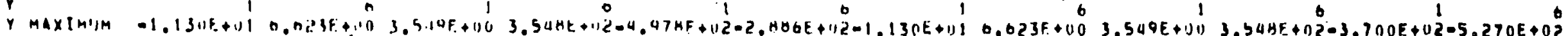

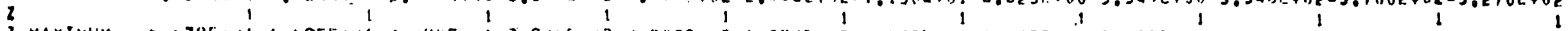

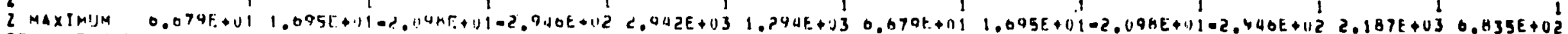

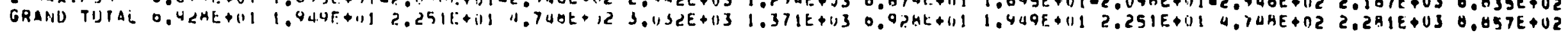
ELEME:IT TYHE (3/0 D I E

\begin{tabular}{|c|c|c|c|c|c|c|c|c|c|c|c|c|c|}
\hline & & (1) & VY(I) & $v<(1)$ & $T \times(1)$ & "Y(I) & $M 2(1)$ & $D \times(7)$ & VY (J) & $v 2(J)$ & $1 \times(2)$ & $\operatorname{Mr}(J)$ & $m 2(J)$ \\
\hline & & 1 & 1 & 13 & 0 & 1 & 1 & 1 & 1 & 13 & 6 & & \\
\hline & $M A \times I P ! I ! 4$ & $.324:+11$ & (1) & $E+v i$ & $6.11119 t+111$ & $3.35 H E+U 2$ & $1.043 t+02$ & 1.32AE + 01 & $400-$ & 00 & & & \\
\hline $\begin{array}{l}\dot{y} \\
Y y\end{array}$ & $M A X\{M I J M$ & & $=111$ & & $B B E+i) 2$ & & & & & & & & \\
\hline 2 & $\begin{array}{l}\text { MAX IMUII } \\
\text { RA!IU TI!TA }\end{array}$ & $H, 711 E+111$ & $E+11-$ & 11 & $\begin{array}{r}1 \\
16 L+112 \\
1 H L+.12\end{array}$ & $\begin{aligned} P+113 \\
F+03\end{aligned}$ & & & 11. & & 1 & & \\
\hline
\end{tabular}

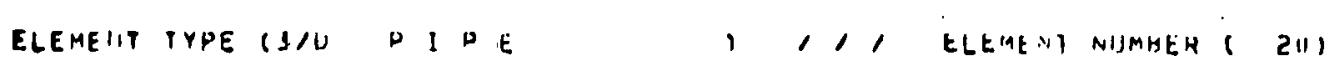

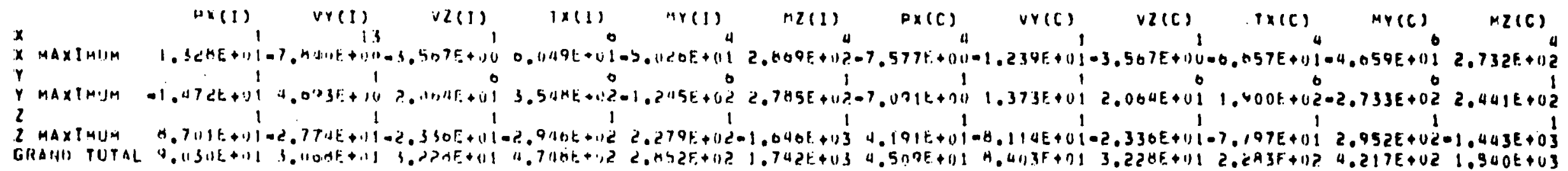

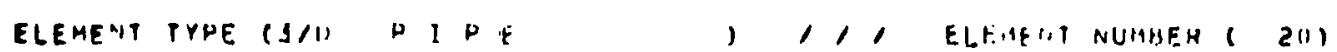

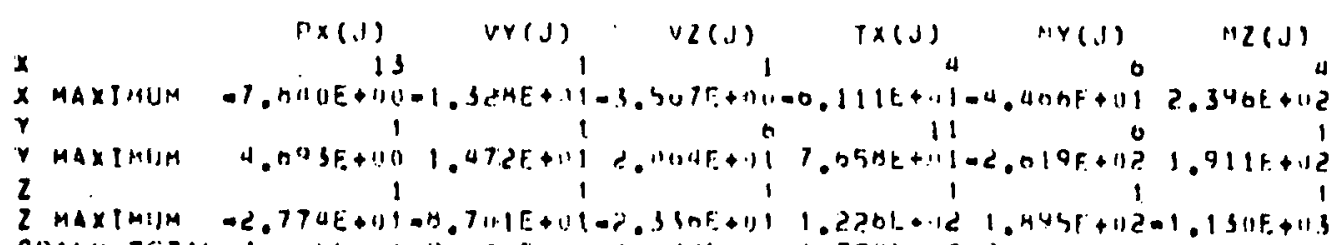

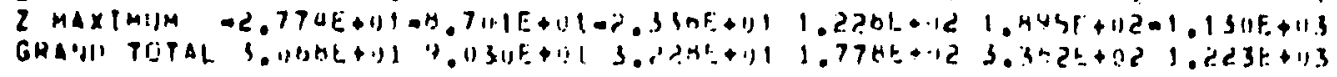

ELEMENT TYUE $(3 / 1)$ P 1 H

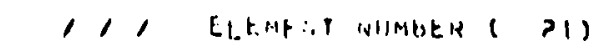

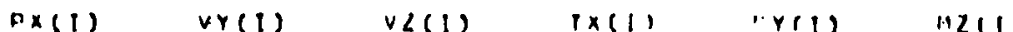




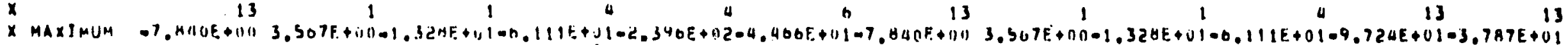

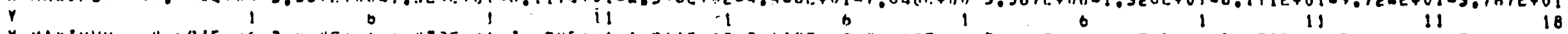

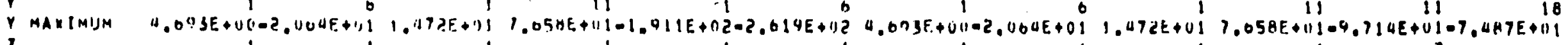

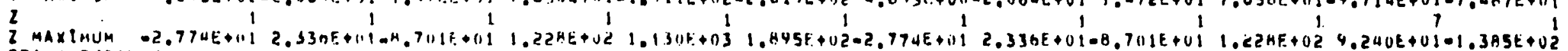

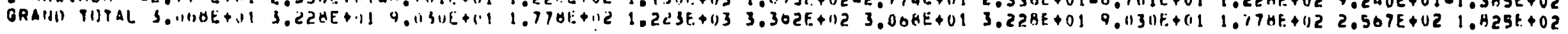

ELEMENT TYNE (3/1) P I P F 1,1, ELEAENT NUMGEH $(22)$

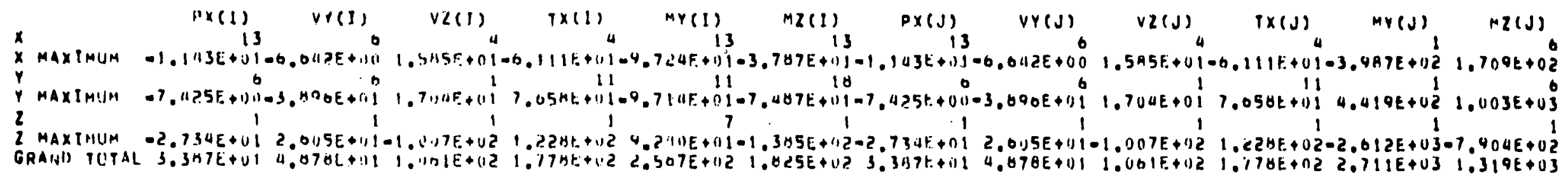

Element TYOE CB/U P I P E

1,1 ELEMENT NIJMAER 6 231

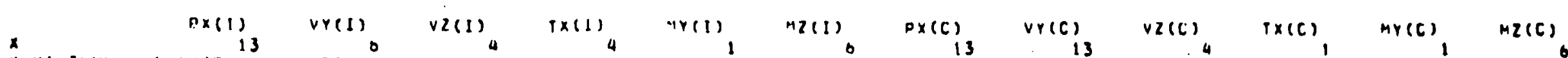

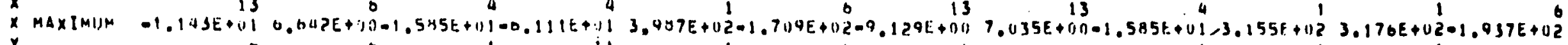

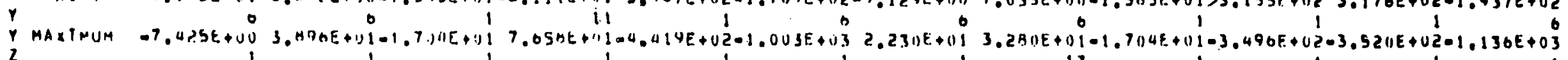

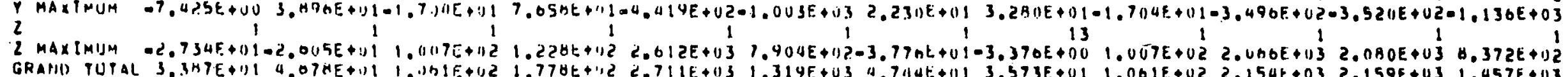

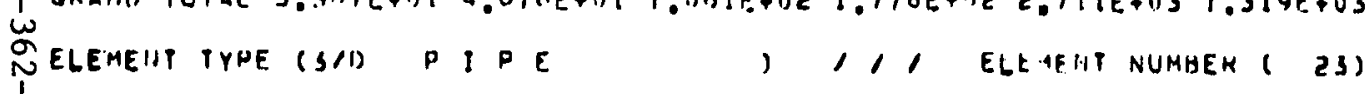

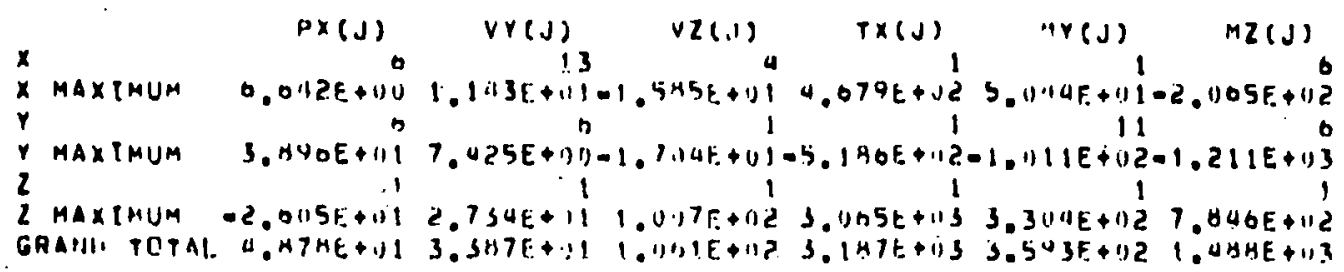

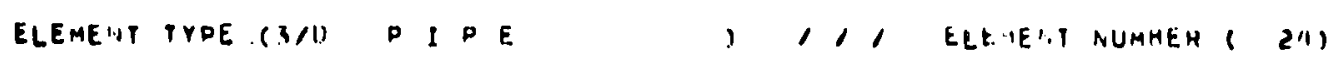

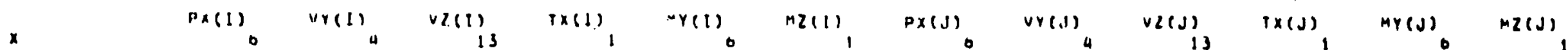

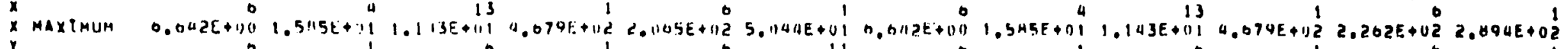

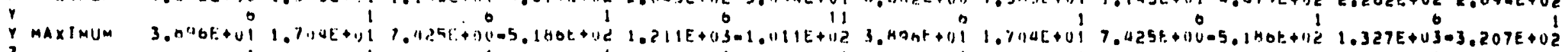

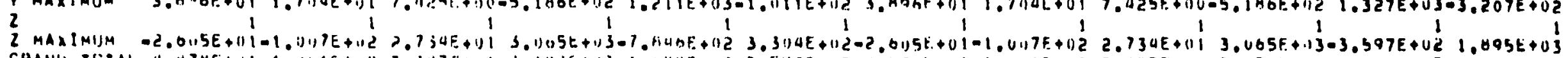

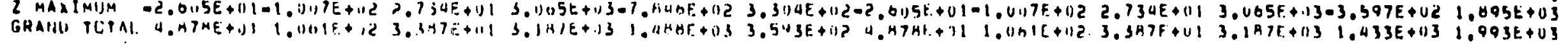

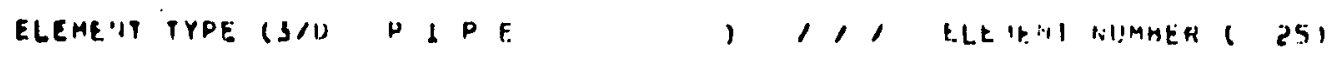
$x$
$x$
$y$
$y$

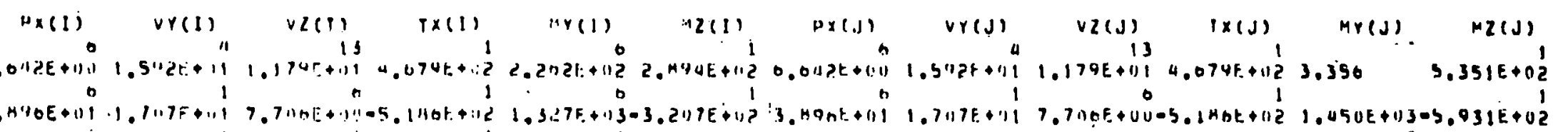




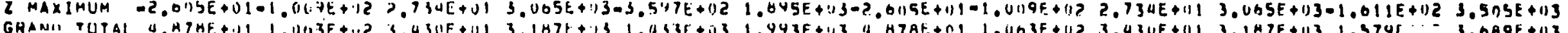

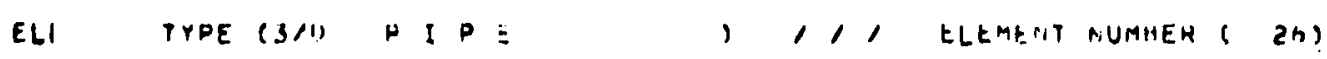

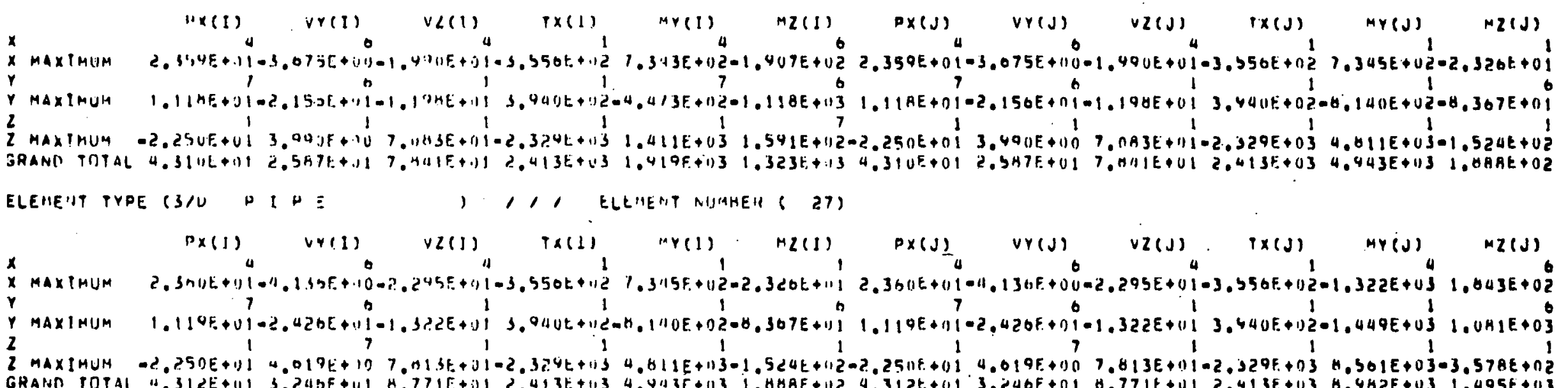

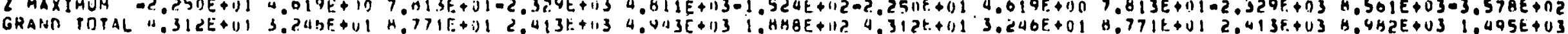
ELEMEHT TYPE (S/I P 1 PE , 1, ELEMENT NIMMHEH $($ ?h)

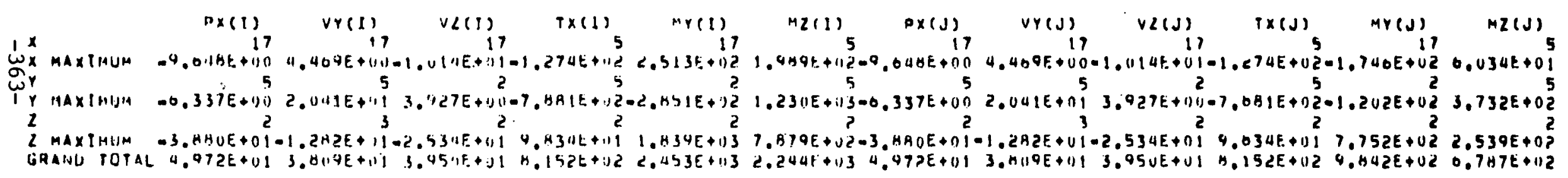
ELEMEHT TYPE (3/2 P I P E , , , ELEMEHT NIJMHEH 1 ?QY)

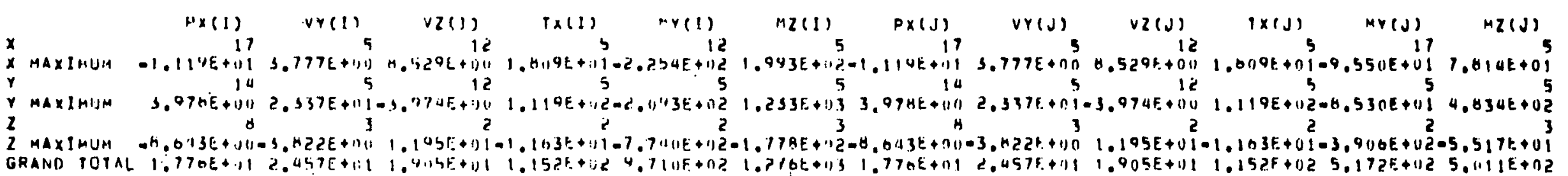

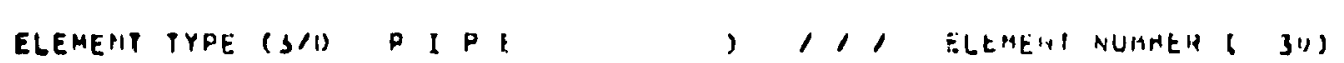

\begin{tabular}{|c|c|c|c|c|c|c|c|c|c|c|c|c|}
\hline & $P \times(1)$ & $\operatorname{vr}(t)$ & $v 2(1)$ & $1 \times(1)$ & $\operatorname{MY}(1)$ & $42(1)$ & $P x(C)$, & $v r(C)$ & $v 2(c)$ & $P \times(C)$ & $\operatorname{Mr}(C)$ & $M_{2}(C)$ \\
\hline$x$ & 17 & 12 & 5 & 5 & 5 & 17 & 17 & 12 & 5 & s & 5 & 17 \\
\hline HAXIMUM & $1.114 E+31$ & $.520 \%+1: 0-$ & $71 .+10$ & $1.4119 t+11$ & I. HIHE+ +1$)$ & Q.SSOE + $111=1$ & $1.103 t+111$ & S. BhIt: tulo & $77 E+U U$ & 4.53 & 11 & $0.7116 E \bullet \cup 1$ \\
\hline$Y$ MAXIMUH & $\begin{array}{r}111 \\
\left.3.47 M t_{1}+1\right) L_{0}\end{array}$ & $3.97 a c+110=$ & 2.S97E+ +1111 & $1.114 k+1 ?$ & $4.1154 F_{0}+02$ & $4.530 E+11^{5}$. & $\begin{array}{r}114 \\
1.910 E+1111\end{array}$ & $4 . \ln 3 t+110$. & $-2.337 F+0 I^{3}$ & $2.0110 t+112$ & $\begin{array}{r}5 \\
3.633 E+\cup 2\end{array}$ & $1, B I U E+U 1$ \\
\hline $\begin{array}{l}2 \\
2 \text { MAXINIM } \\
\text { GRANU TOTAL }\end{array}$ & $\begin{array}{r}-5.61135+116 \\
1.7701:+111\end{array}$ & $\begin{array}{l}\text { ? } \\
1.195 t+111 \\
1.9115 t+.11\end{array}$ & 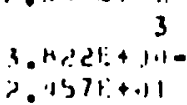 & $\begin{array}{l}1 . \ln 3 t+\cdots 1 \\
1.152 t+\ldots 2\end{array}$ & $\begin{array}{r}3 \\
-3.5175+111 \\
3.11115+11 ?\end{array}$ & $\begin{array}{c}3.4100++112=9 \\
5.172 f+112 ?\end{array}$ & $\begin{array}{r}4 \\
? .40 n t .+11 n \\
? .0725+111\end{array}$ & $\begin{array}{l}? \\
1.1051+111 \\
1.0411+111\end{array}$ & $\begin{array}{l}3.422 E+110- \\
2.457 E+111\end{array}$ & $\begin{array}{l}3 \\
2.12 A E+111- \\
2.0 H I L+112\end{array}$ & $\begin{array}{r}3 \\
-4.377 E+01 \\
3.8110 F+02\end{array}$ & $\begin{array}{r}\text { } \\
3.092 E+02 \\
4.9 H 9 E+02\end{array}$ \\
\hline
\end{tabular}

ELEMENT IYPE (3/1) D. 10 H. 


\begin{tabular}{|c|c|c|c|c|c|c|c|}
\hline & & $P \times(J)$ & $\operatorname{vr}(J)$ & $v<(.1)$ & $T \times(7)$ & $14 Y(J)$ & $M Z(J)$ \\
\hline & & 17 & 17. & 5 & 5 & 5 & 17 \\
\hline & MAXIMUM & $E+11$ & $+.10=3$ & $E+: 1 ?$ & "1 & 01 & $.144 E+111$ \\
\hline & & 10 & 5 & 5 & 5 & 5 & \\
\hline & $M A X[M I / M$ & $5.158 E+110$ & $\therefore+., 0=$ & $E+v 1$ & $3.403 t+112$ & 1.HIHE + O2 & $7.04 \Delta E+U 1$ \\
\hline & $M A X I M$ & & $\begin{array}{l}2 \\
1\end{array}$ & & & & \\
\hline
\end{tabular}

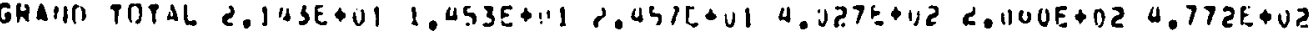

ELEMFiN TYPE (3/1) $P$ P E , , , ELEMENT NUMBEH $(31)$

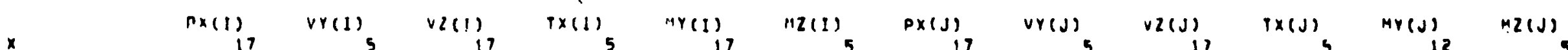

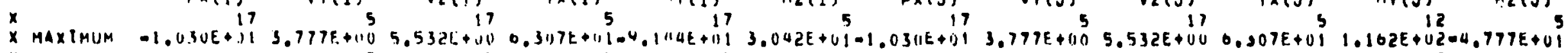

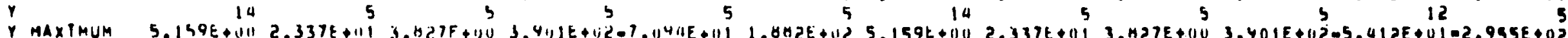

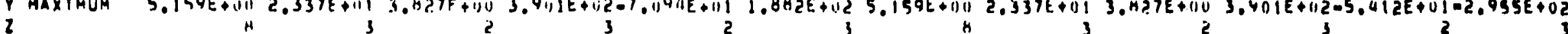

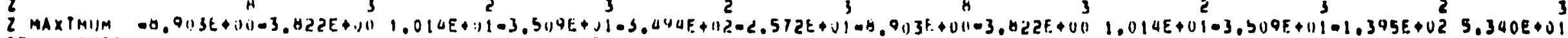

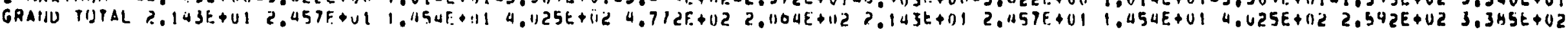

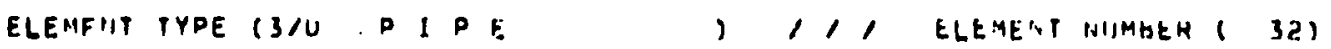

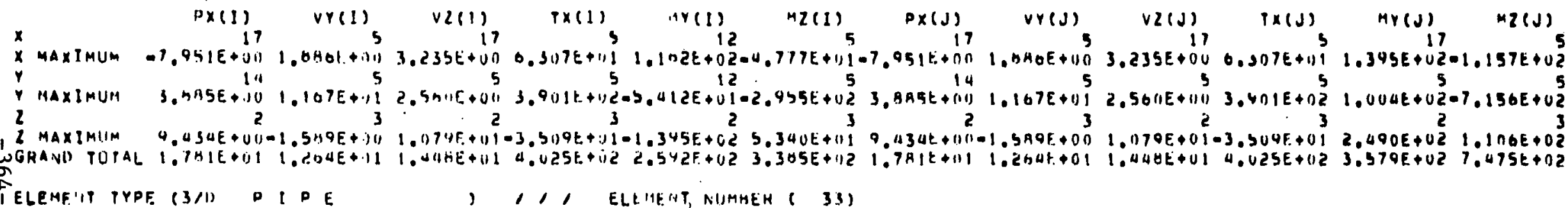

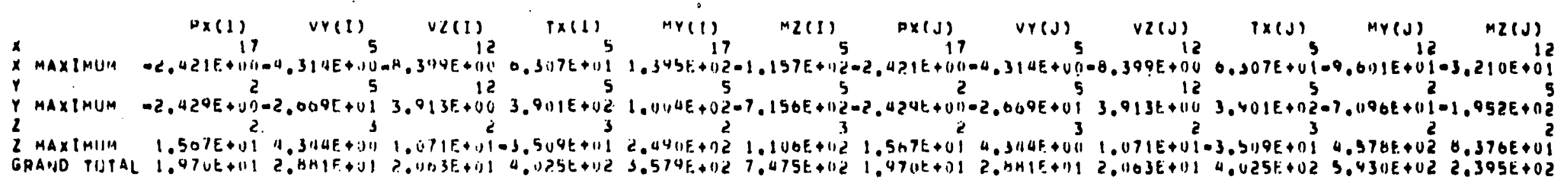

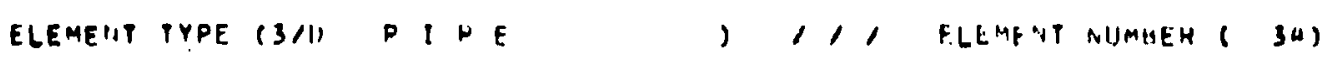

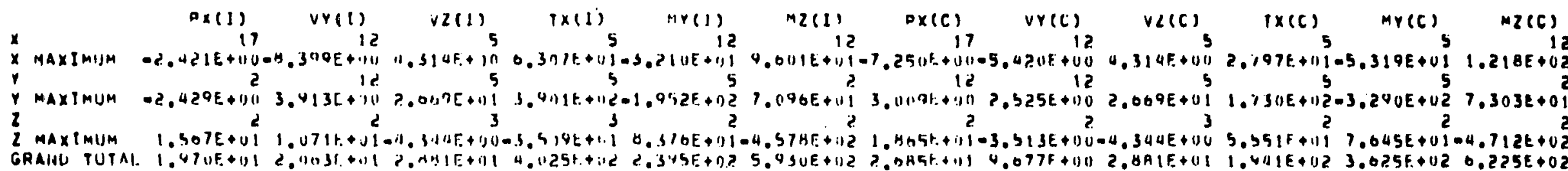

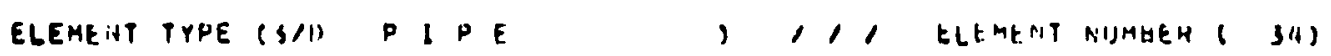

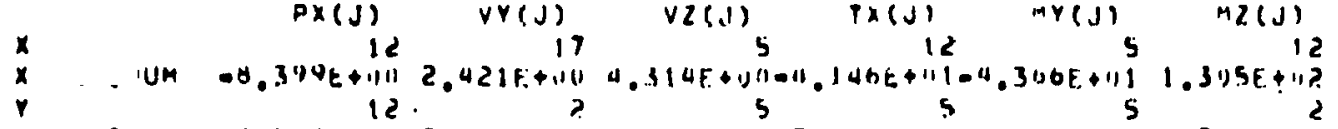


L MAXIMUM $1.071 E+111-1.507[+11-4,344 E+1109.726 E+1112.435 E+01-4.354 E+02$

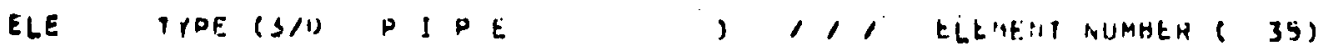

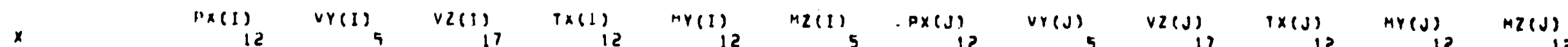

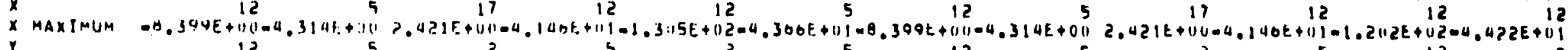

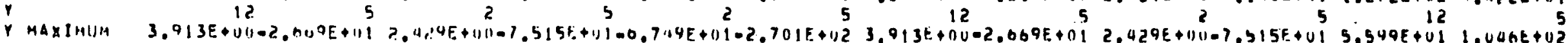

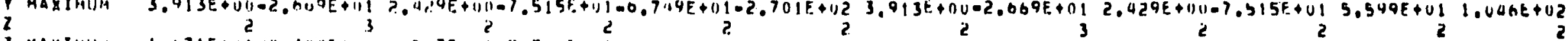

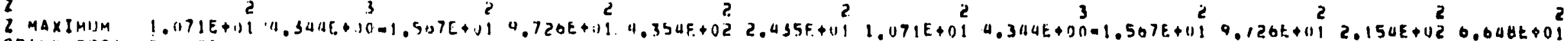

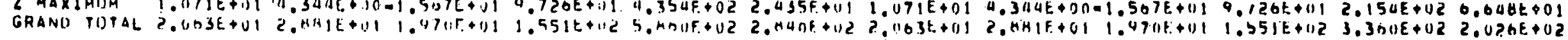

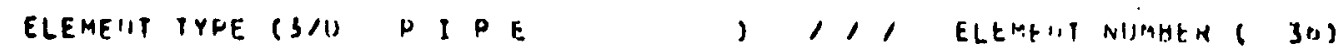

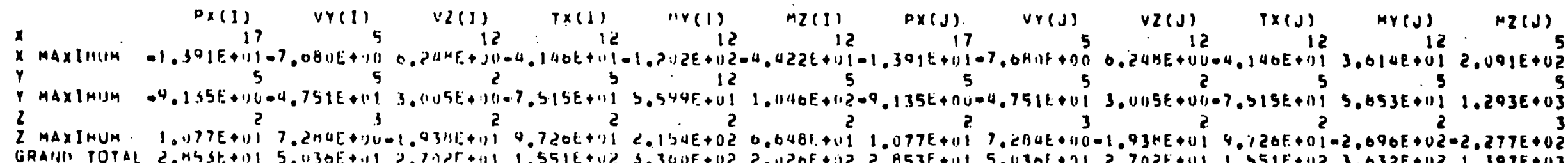

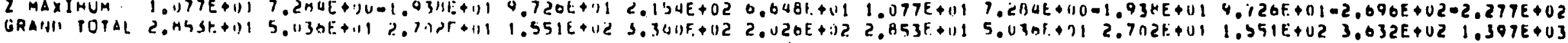
ELEME"I TYPE (3/1) 1 HE , 1,1 ELTME.'T NUMHER 6 37)

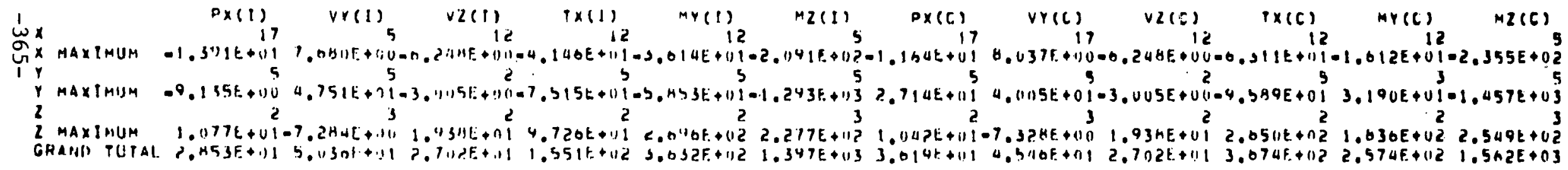
ELEHEIIT IYPE (3/W PID I: , 1, ELEMENT HIJMHEY 6 37)

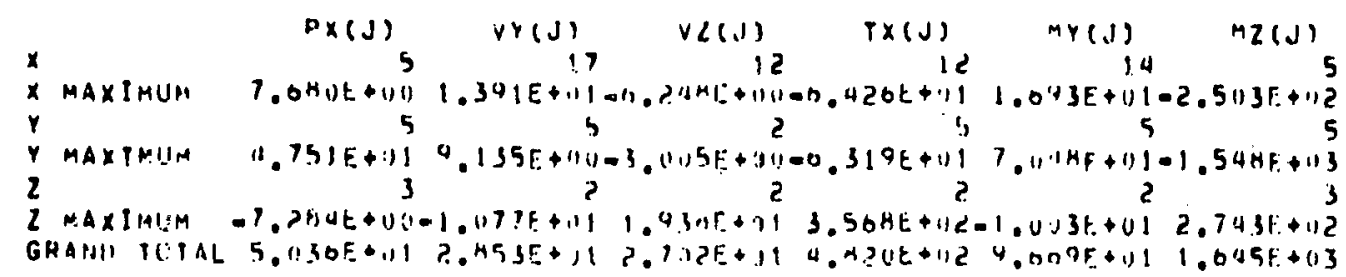

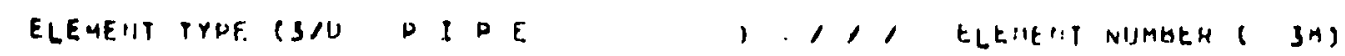

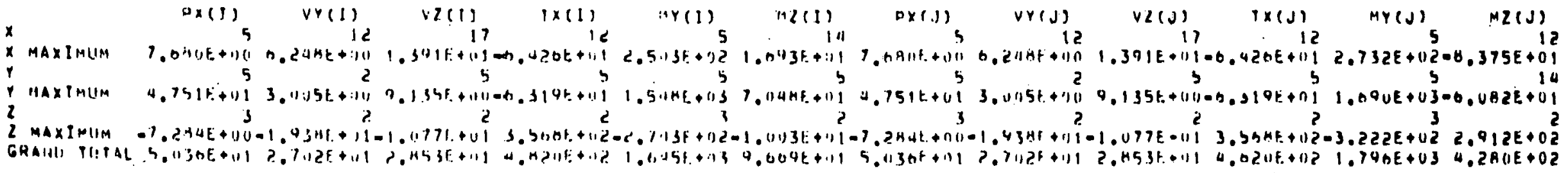

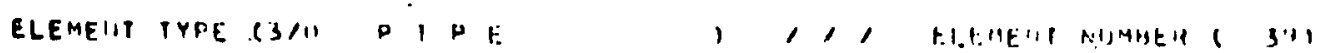




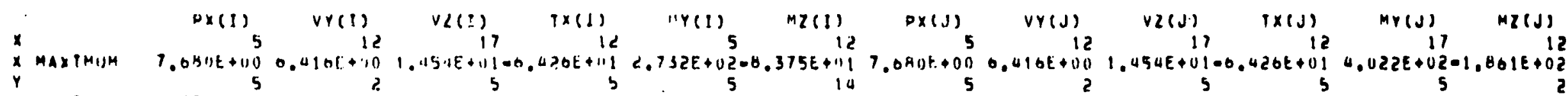

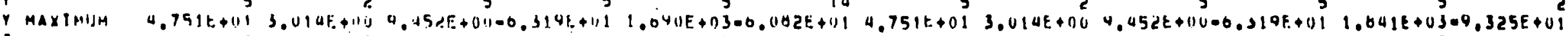

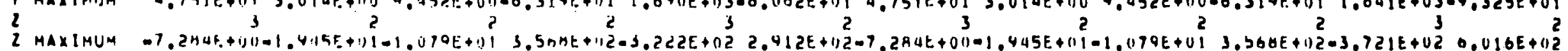

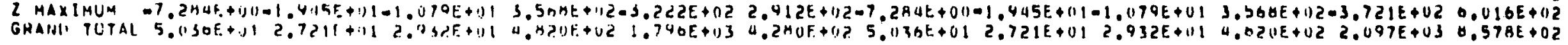

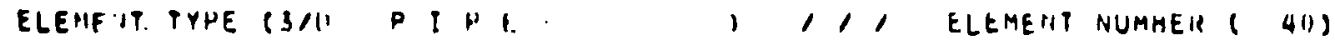

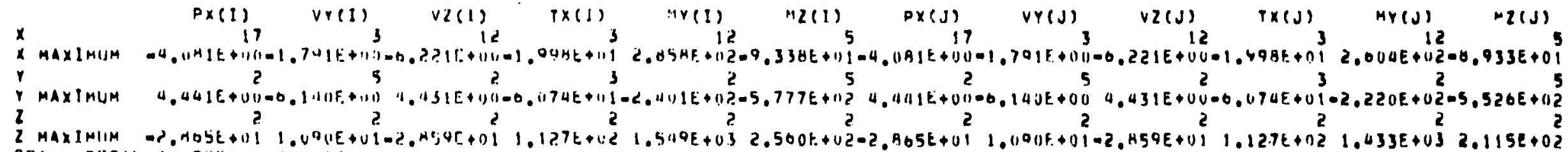

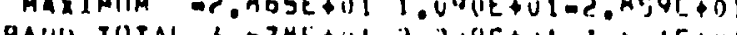

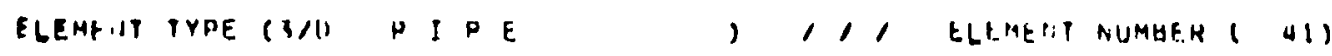

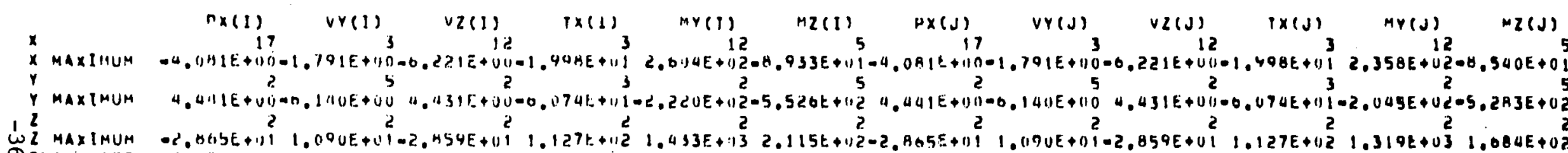

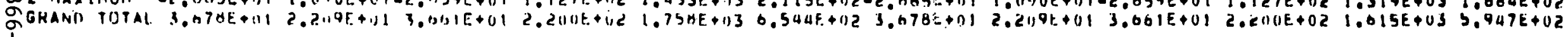

ELEMENT PYHE (3/0 P I H E 1,1, ELEMENT PNUMHER $(42)$

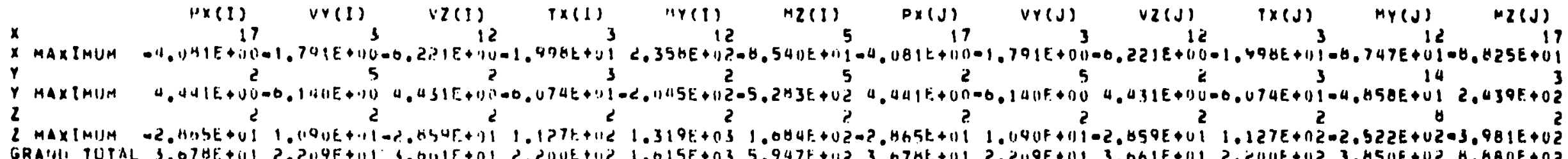

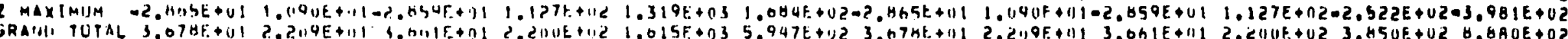
ELEMF IT TYPE (3/1 P I P F , 1,1 ELEIH.NT NUMHEH $($ IS)

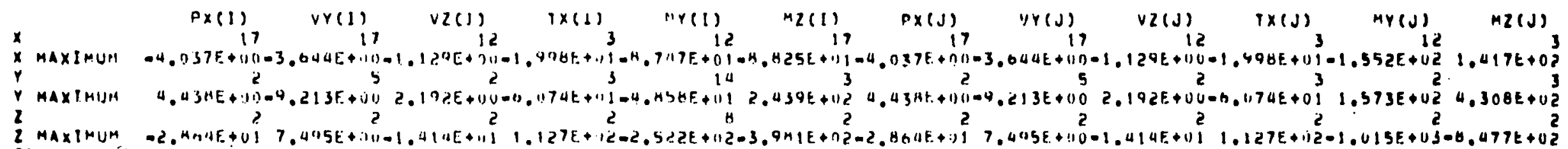

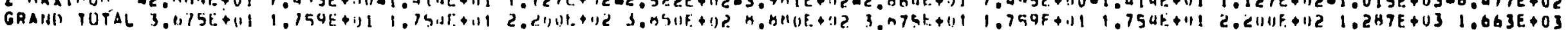

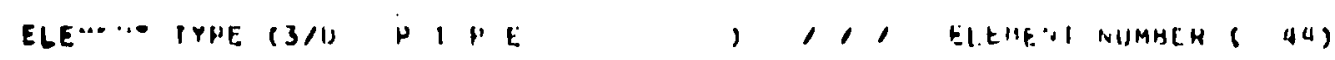

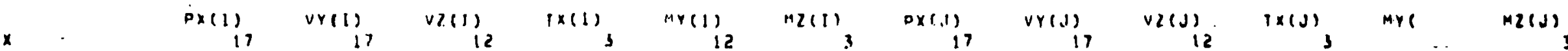

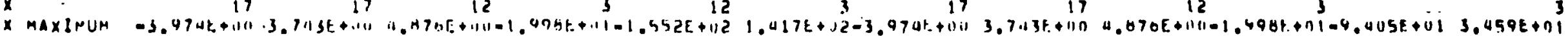


ELEMEHT TYPE (3/J P I DE 1,11 ELEME I.T HUMGEH 1 US)

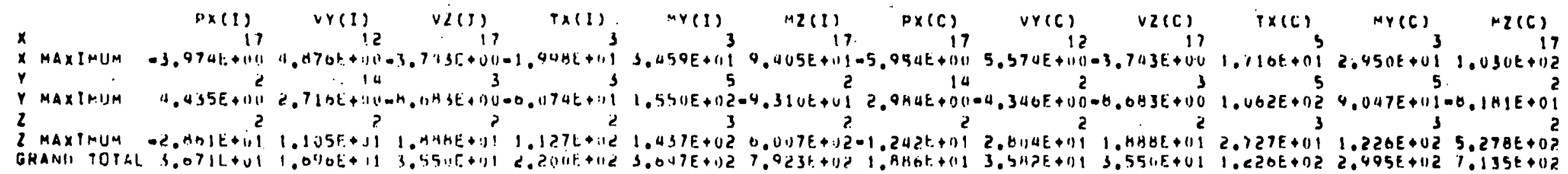

ELEHENT TYPE (3/1) PIPE , 1, ELEHEWI NUMHEH ( WS)

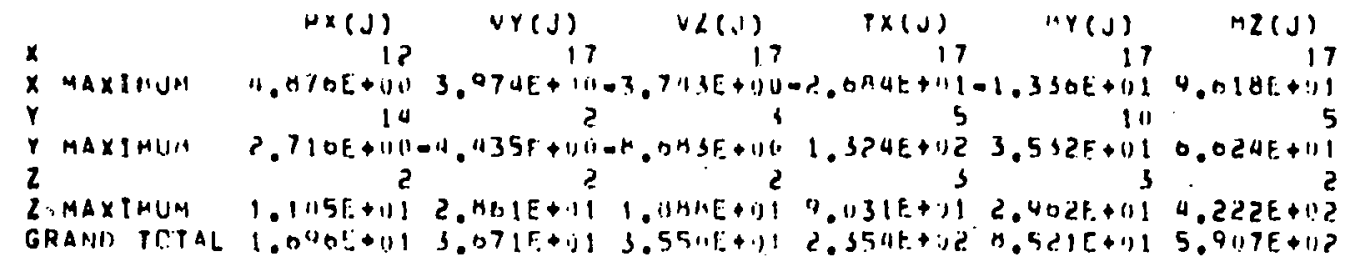

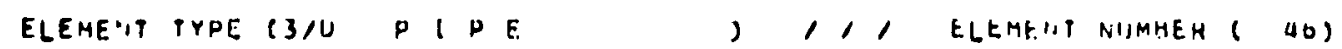

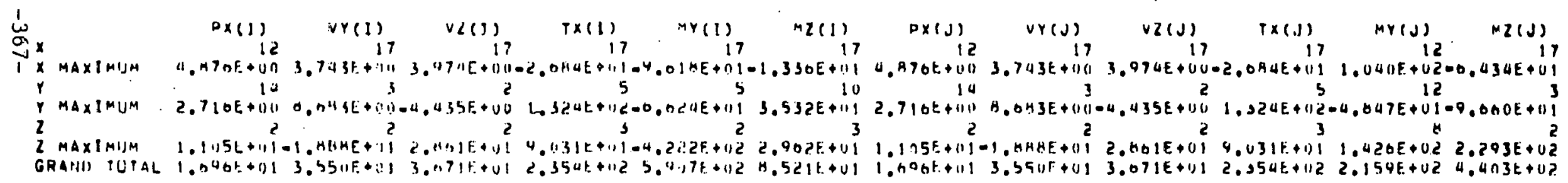

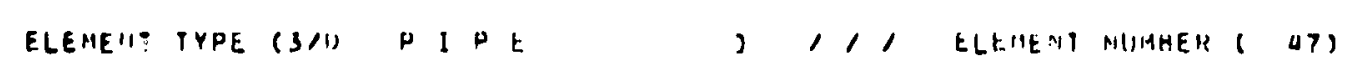

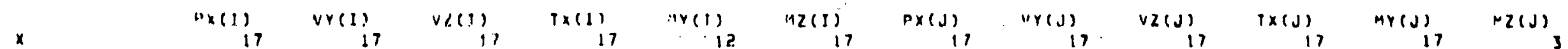

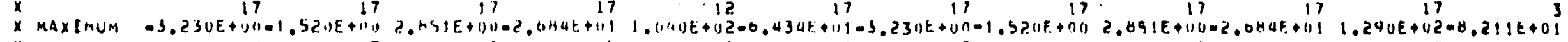

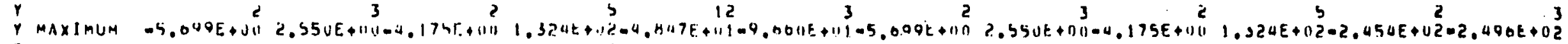

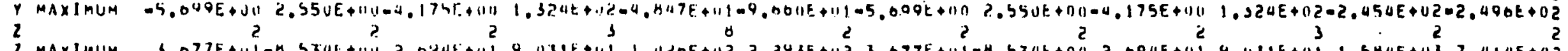

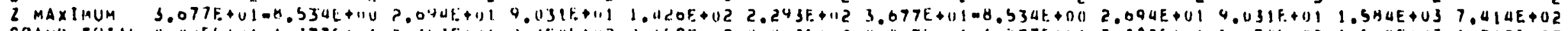

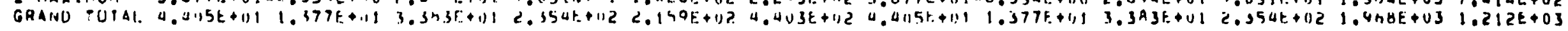

ELEMEN TYPE (3/1) $\mathrm{P}$ I E

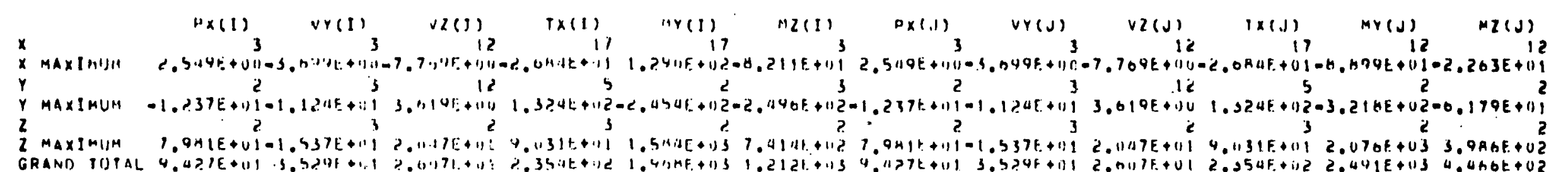

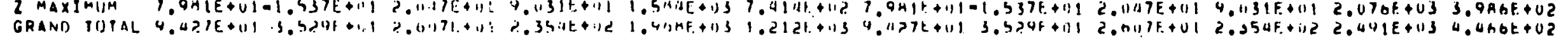


i MAXIHIJM 1.R7UE+111-5.595E+10-1.6B3

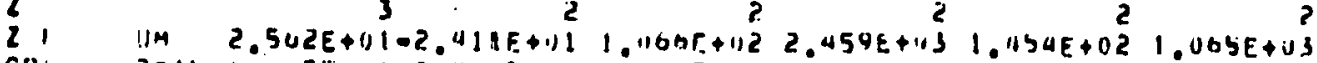

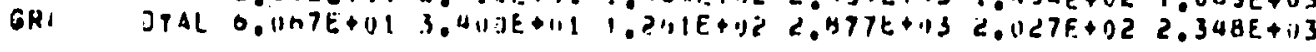

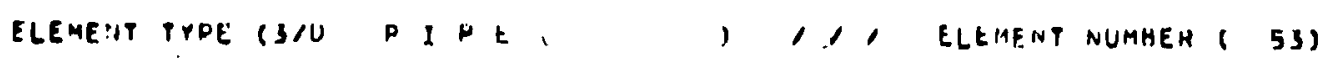

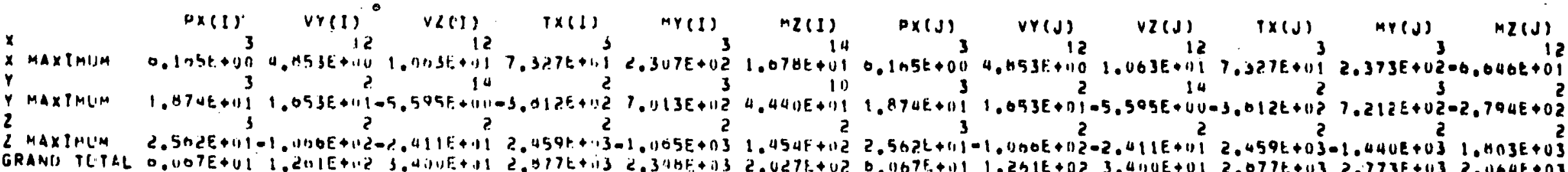

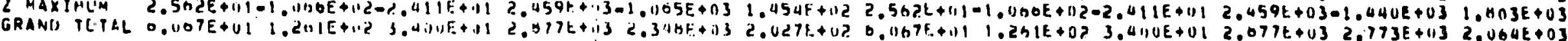

ELEMEHT TYPE (3/1) PI DE , , , ELEMEPAT NIJMHE. ( 54 )

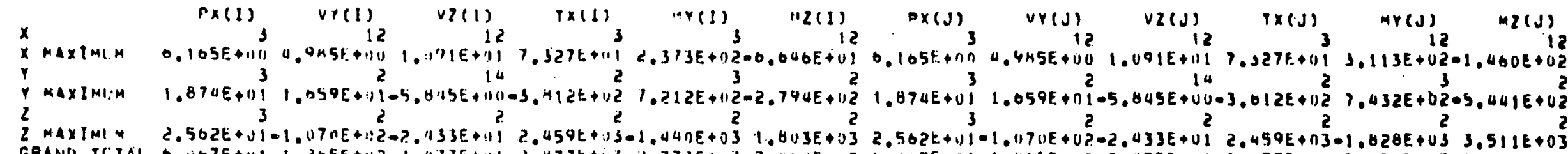

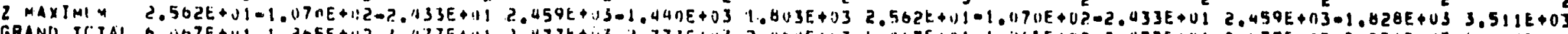

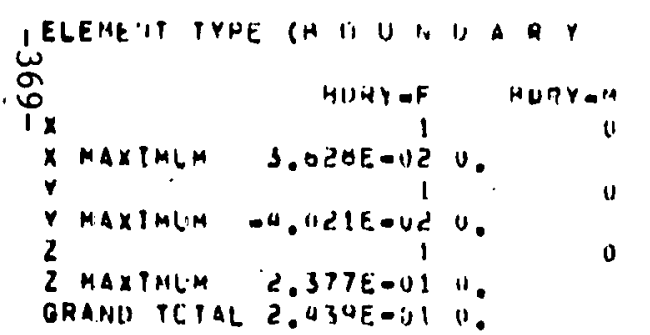

ELEMEITT TYOE (B U U N II A $Y$

1,1, ELLHE:A NUMHEH $($ R)

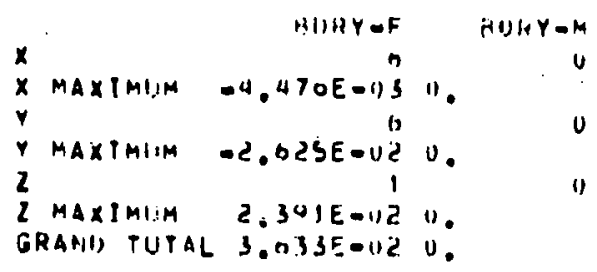

ELEMF.IT TYPE (11 1, "IN D: D A $Y$

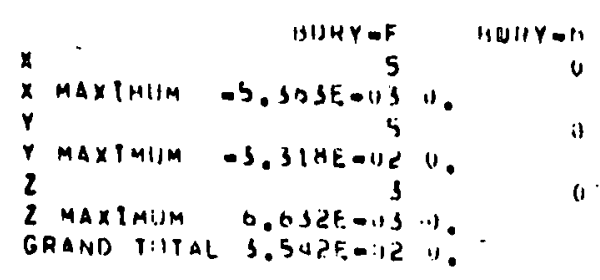

, 1, ELE!H:CT RUUAMEH ( S) 


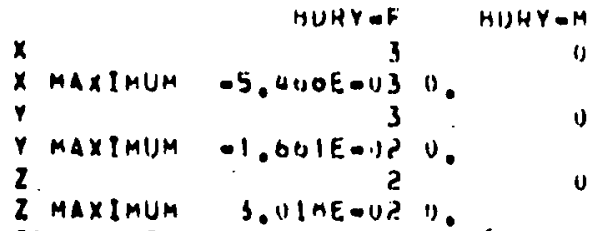


GAAN(I) TOTAL 5.1130E+111 U.

ELEMEHT TYOE III UI II N D A H Y

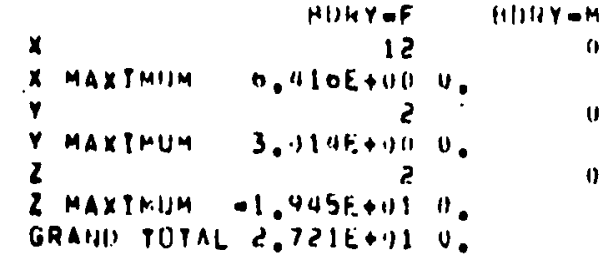

ELEMEIT PYDE ON U II N II A K Y

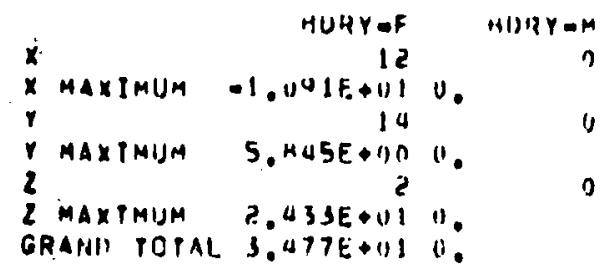

elemeit troe co U is N D A r $r$

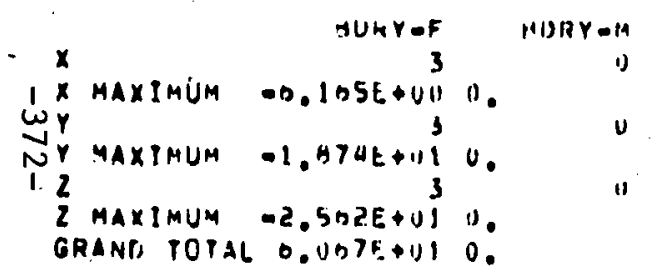

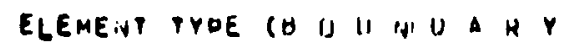

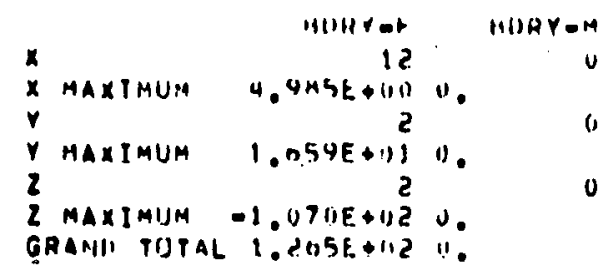

ElEme:It TrUE (N U II P. I) A H $r$

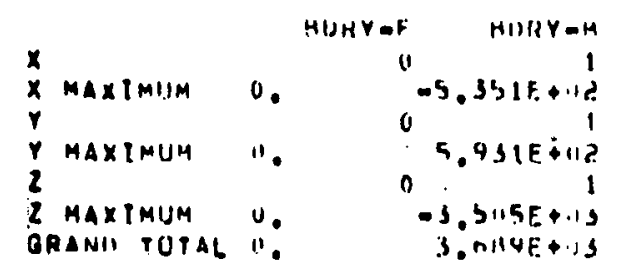

- IYPF. IH ij II li U A hr

, 1, ELK:LET NUMHER $($ in)

) 1,1 ELEMEIT NUMHEh $(17)$

1,1, ELEHENT NUMHEH $(1 \%)$

, 1, ELI:ME IA NIJMEEH ( 14)

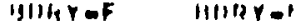




$\begin{array}{lr}2 \text { MAXIMUM 11. } & -0,1110 E+, 2 \\ \text { ORANIS TOIAL } \% & 0,57115+1 ! 2\end{array}$

ELEMEIT. TYUE ("I 1" II IN II A R Y

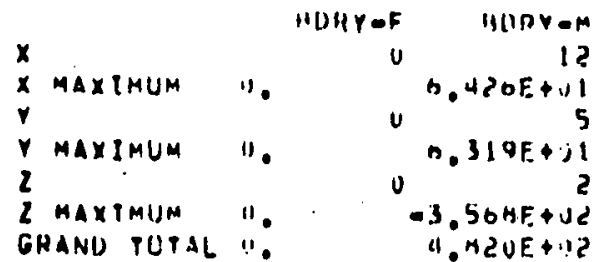

ELEMFiT IYPE (H U IJ N II A H Y

$, 1,1$ ELEME:I NIJMHEN (27)

\begin{tabular}{|c|c|c|}
\hline & NURY & $\| U H Y \rightarrow M$ \\
\hline$x$ & & 17 \\
\hline MAX $(M \|) M$ & 11. & $2 E \$ 11 ?$ \\
\hline & & 0 \\
\hline I. MAXIMUM & 1. & 1.0 \\
\hline $\begin{array}{l}\text { MAXIMUH } \\
\text { SAAND TUTAL }\end{array}$ & ". & $\begin{array}{r}-3.721 E+12 \\
2.077 F\end{array}$ \\
\hline
\end{tabular}

ELEMFNT TYPE (W I IIN

, 11, ELEHENT NUMHEL 1 2H)

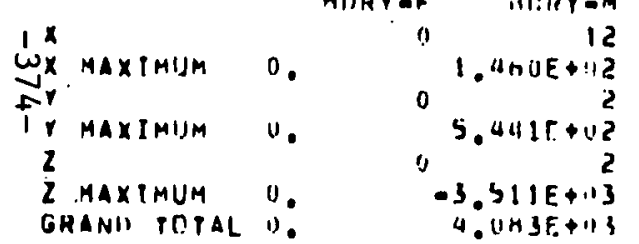

ELEME:IT TYPE (H L U UN I) A H

1,1, ELEMENT NUMGER $(29)$

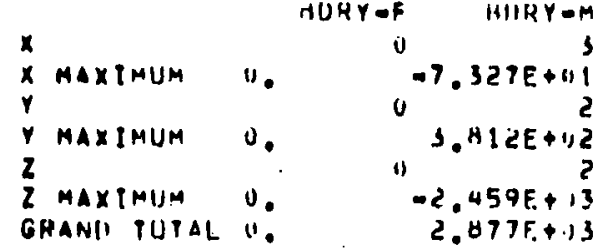

GHANII TUTAL "O

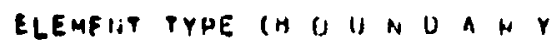


Solution $7 \mathrm{~b}$ 


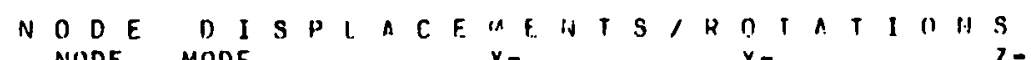
NODE MONE

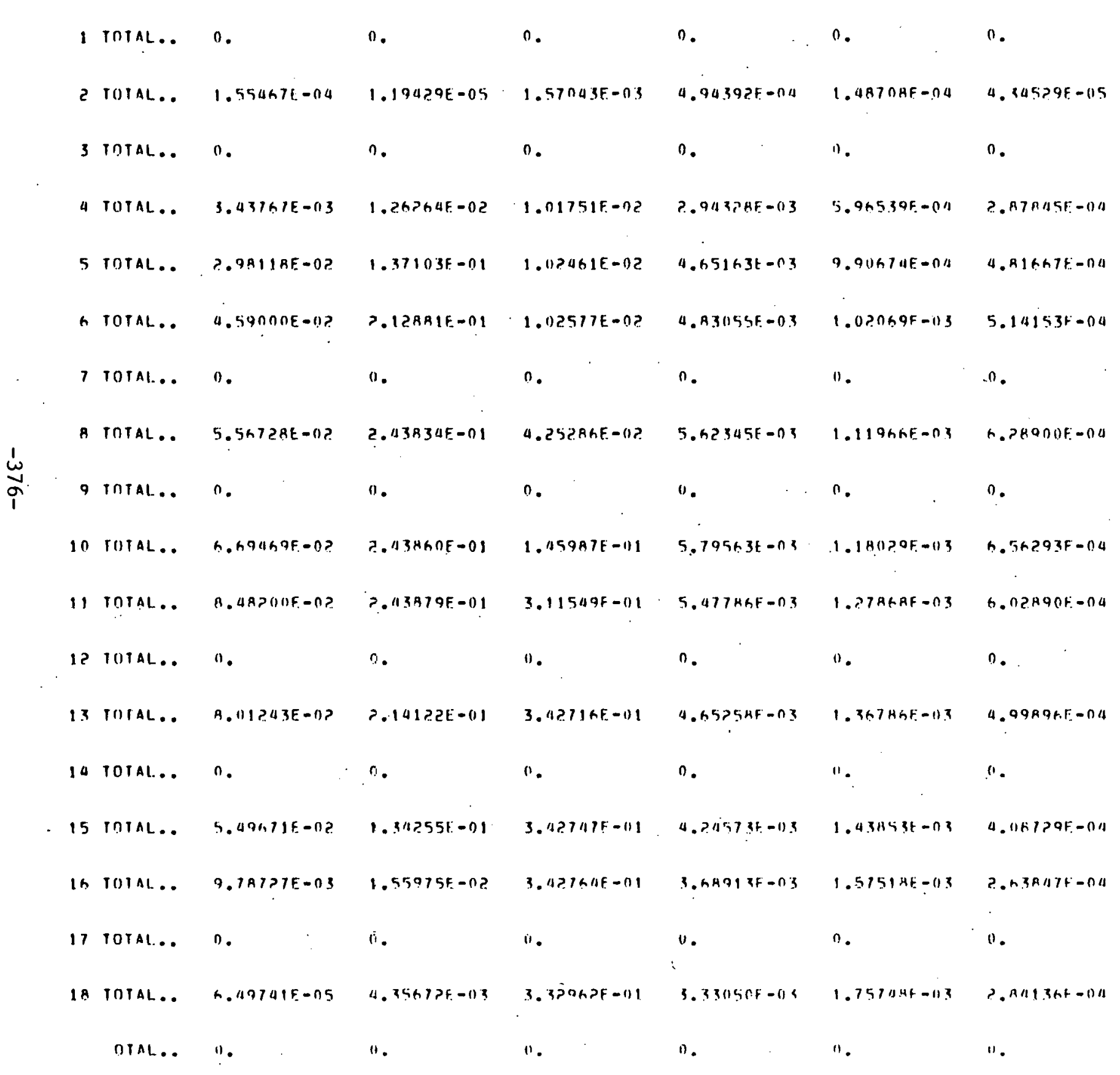




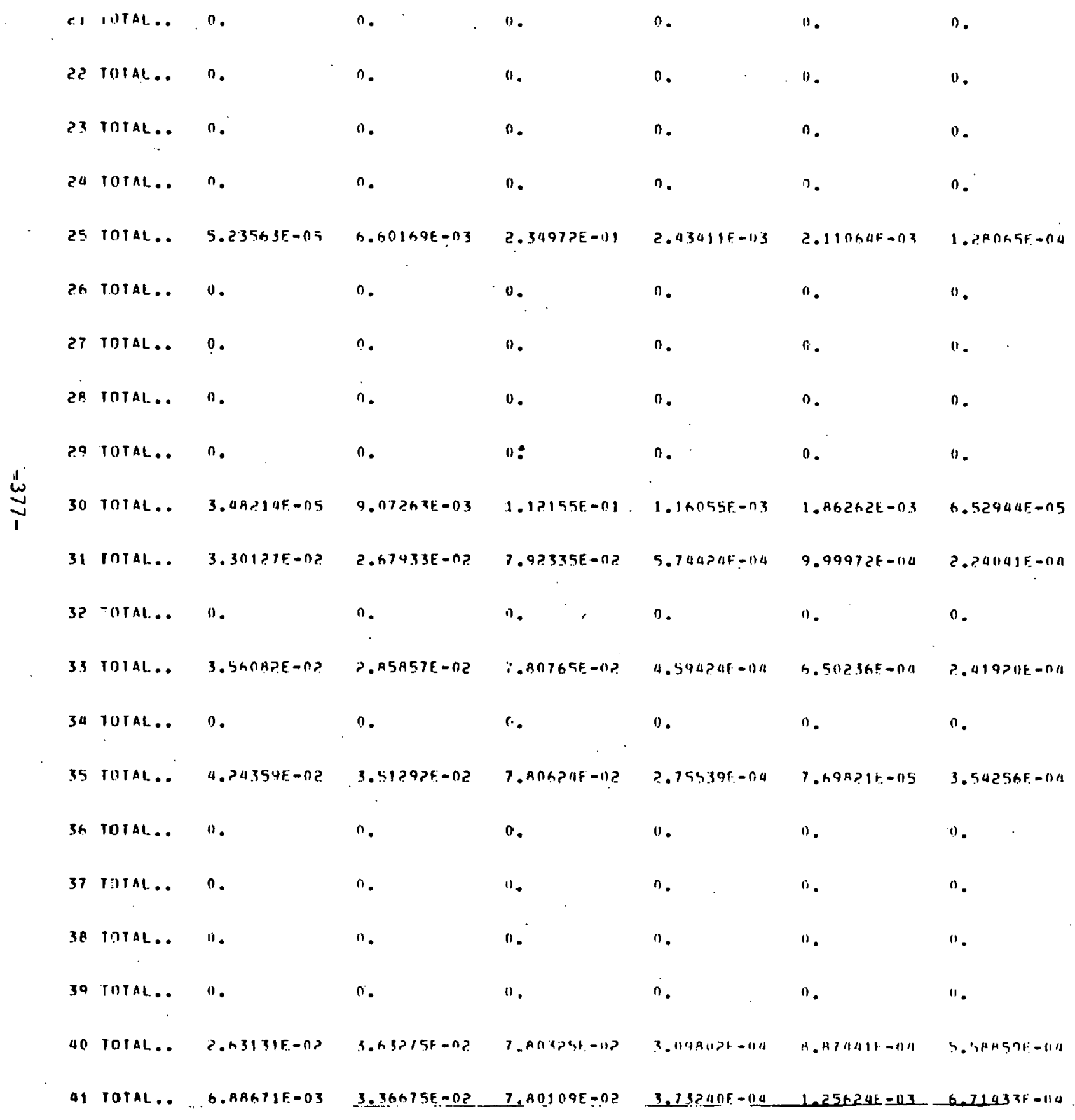




\begin{tabular}{|c|c|c|c|c|c|c|c|}
\hline a? & TOTAL.. & 0. & $n$. & 0 . & n. & 1). & $n$. \\
\hline 43 & IOTAL... & 0 . & $n$. & $n$. & r. & $n$. & $n$. \\
\hline 44 & TOTAL.. & $5.1199 B F=-13$ & 2.97594f -0 ? & 1.138AกE - $01 ?$ & $4.113939 f=04$ & $1.57075 E-113$ & $7.374555=-04$ \\
\hline 45 & TOTAL.. & $5.1 ? 02115 .=113$ & $1.931120 \mathrm{E}-112$ & $4.90733 t-11 ?$ & $4.1011375-011$ & $1.595 A_{1} t-03$ & $7.12 A \geq 7 E-114$ \\
\hline $4 h$ & IOIAL... & $5.1196 O E-03$ & $2.15 h>9 E-03$ & 1.1 1 $213 E-11 ?$ & $4.97595 f-04$ & $1 . ? 65 ? 9 F-113$ & $5.779095=04$ \\
\hline 47 & I0TAL.. & 0 . & n. & n. & $n$. & $n$. & $n$. \\
\hline 48 & TOTAL.. & $3.37+4+4 E-03$ & $1.25454 E-115$ & $5.791194 \mathrm{E}-03$ & $3.15719 E-04$ & A. $2193 \mathrm{BE}-04$ & 2. $83677 \mathrm{E}-04$ \\
\hline 49 & TOTAL.. & 1). & 0 . & 0 & $n$. & $n$. & 0 . \\
\hline 50 & IOTAL.. & h. $13479 \mathrm{~F}-(1) 4$ & 3.2959 ㄴ. -06 & 1.2977 AE -113 & $1.437 \mathrm{ROF}-04$ & 2.09h4hE-114 & $7.50570 E-05$ \\
\hline 51 & THIAL.. & $n$. & $n$. & 10. & 0 . & 0 . & $n$. \\
\hline 52 & IOTAL.. & 0. & 0 . & $n$ & n. & 0. & 0 . \\
\hline 53 & 1OTAL.. & ". & $n$. & 0. & 0 . & n. & n. \\
\hline 54 & IOTAL.. & 0 . & $n$. & $n$. & $n$ & 0. & $n$. \\
\hline 55 & IOTAL.. & $3.4 ? 997 E-1) 8$ & $A . A / H 3 \mathrm{KE}-0 \mathrm{R}$ & $1.06311 \cap E-(1) 7$ & 3. $\mathrm{AAQ}(17 \mathrm{~F}=0 \mathrm{R}$ & S. IRGHUF -1$] A$ & 1.5794 (it.-0n \\
\hline 56 & IOIAL.. & n. & ". & 0 & $n$. & n. & 0 . \\
\hline 57 & TOTAL... & 1. & (1. & $n$. & ". & n. & $n$. \\
\hline $5 \mathrm{~A}$ & TOTAL.. & ". & ". & i. & $n$. & $n$. & 0. \\
\hline 59 & TOTAL.. & $n$. & $n$. & $n$. & 0 . & $n$. & o. \\
\hline 60 & InIAL... & $1.74 \times 7$ S6. & a. $4>>610[-0 \mathrm{~s}$ & $3.30 A R 3 F-113$ & $5.411>A 5 F-111$ & $1.34 \times 27+-03$ & $1.344+5 F-04$ \\
\hline & NIAL.. & i. & . & 0 . & 10. & $n$. & $n$. \\
\hline
\end{tabular}


63 TUIAL.. 0.

GU TUTAL... n.

GS TOTAL.. G.GQ194E-OR

GA TOTAL.: 11.

h7 TOTAL.. 0.

6A TORAL.. O

ng TOIAL... 0.

70 IIITAL.. 1.13174E-05

71 TOTAL.. 3.hTEGTF-O3

72 TUTAL... 0.

73 TOTAL.. 3. SGSA7E-O

74 INTAL.. O:

75 TUTAL.. $4.355717 .-03$

76 TOTAL... 1.

7 TOIAL.. $n$.

IR TUTAL.. $n$.

79 rOIAL.. 1.

BO TOIAL.. S. S? I UF. $-0 \times$

81 TUTAL.. h.PI7PPF-1!3

A2 TITAL.. $n$.

O3 TOTAL.. 0.
11.

$5.265 B O F-O H$

i.

n.

5.1194AE-ก3

$1.7 R 217 E-11 ?$

$n$.

$1.93115 R E-02$

$n$.

?. $.1+43 E-0 \bar{E}$

n.

11

n.

0.

$3.531790-02$

4.743736 .02 n.

0 .

P. $6141 \mathrm{HE}-0 B$

$n$.

1.

n.

u.

6. 511 UhF.-1)

$1.01101 \mathrm{E}-0$ ?

$n$.

1. N1H?7E-0?

$n$.

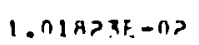

0.

1.

1.

o.

1.011935-0?

$1.011445-412$
0.

n.

2. $55 ? 34 \mathrm{~F}=0 \mathrm{~A}$

0 .

0.

$n$.

0.

$1.71361 E-0)$

$3.294216-04$

0.

$3.53916 \mathrm{ht}-119$

$n$.

3. 4วRÁวF-0N

1).

0.

n.

$1.74977+-01$

$9.100 .001-65$
0 .

$n$.

$9.30994 \mathrm{~F}-10 \mathrm{~A}$

".

$n$

$n$.

?. .66?25F-04

9. $11044 \mathrm{f}-115$

$\because$.

Q. ?HH SAt $=05$

?.

$1.000 \mathrm{OF}-01 \mathrm{~s}$

0.

0

13.

1.

$1.02135 F-145,6.07545+-04$ o.

$n$.

n.

$n$.

?.30? $11 F-$ i)

$4.119001-01$

$n$.

$19.8635 / 4-04$

1.

$5.294 ? P F-114$

n.

n.

n.

$n$.

$0.963545-115 \quad 7.304148 t-04$

1". n. i.

11 . 


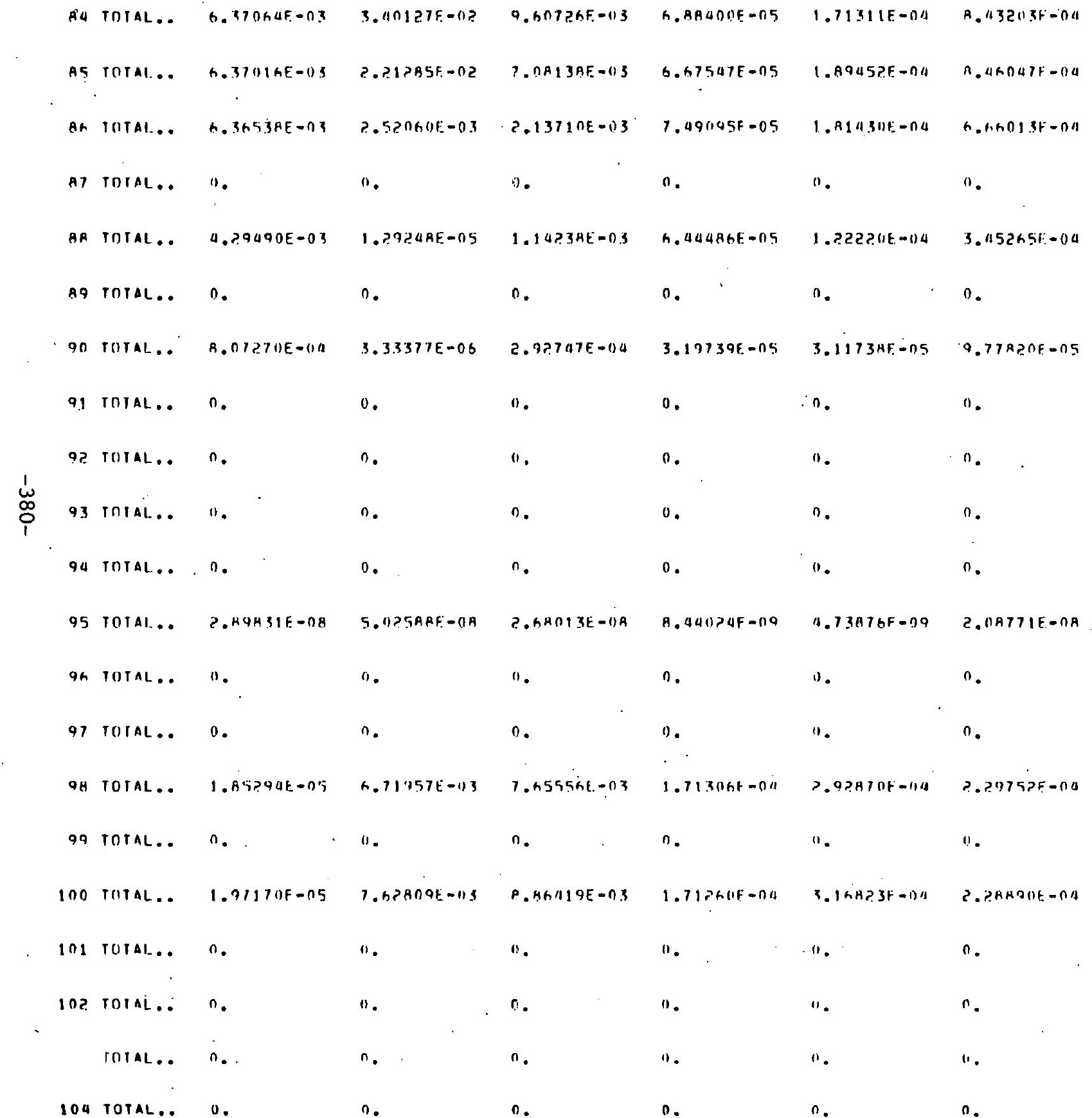




\begin{tabular}{|c|c|c|c|c|c|c|c|}
\hline 105 & TिזAl... & a. ZHISSE-DS & $1.57192 t-02$ & $3.7 ? 9 / 46 F-0 ?$ & $2 . ? 61450 F-04$ & $6.36454 E-0.04$ & $9.0754 h F=05$ \\
\hline $10 n$ & TOTAL.. & 0. & $0:$ & i. & 0. & 0. & 0. \\
\hline .107 & TOTAL.. & 0 & $n$. & 0. & 0. & 0. & $n$. \\
\hline $10 \mathrm{~A}$ & TOTAL.. & 0 & $n$. & 0 & 0. & 0. & $n$ \\
\hline 109 & InTAL.. & $n$. & $n$. & $a$. & 0. & $n$. & 0. \\
\hline 110 & ROIAL.. & R.91141E-05 & $5.0006 P E-08$ & T.S SBABGE-11? & $3.521046-04$ & P. 3679 LE-04 & $6.23037 E-00$ \\
\hline 111 & 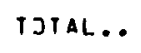 & H. ShS $45 F=05$ & $3.10439 E=02$ & B. $61158 E-0 ?$ & $4.41375 E-011$ & $1.769 ? 2 F=n a$ & $9.56425 E-04$ \\
\hline 112 & TOTAL.. & $n$ & u. & 0. & 0 & 0. & 0 \\
\hline 113 & TITAL.. & $1.2098 \div F-03$ & $3.73855 E-112$ & $6.5 ? 42$ पE $-0 ?$ & $4.70936 F-04$ & P.BAAIOE-04 & $9.972 \times 3 E-04$ \\
\hline 114 & intal... & 0 . & n. . & $n$. & 0 & 0. & 0 . \\
\hline 115 & TOTAL... & $5.45513 E-113$ & $4.34 n 40 F-n 2$ & $h .52103 E-02$ & $4.4931 A F=04$ & (.??) पGF-OA & $1.025 n 3 E-n 3$ \\
\hline 116 & TUIAL.. & 1 & 0. & 0. & 0 & $n$ & 0 \\
\hline 117 & InTAL.. & 0 & 0. & 0. & 0 & $n$. & $n$. \\
\hline 118 & TOTAL.. & 0 & 0 & $n$. & 0. & $n$ & 1). \\
\hline 119 & TOTAL... & 0. & '1). & 0. & $n$. & $n$. & 0. \\
\hline 120 & TOIAL.. & $1.43341 F=02$ & $5.8350 \mathrm{AF}-0 ?$ & ค. S? OHAF - E)? & $1.10114 F=04$ & $P .27930 \mathrm{E}=014$ & $1.157 A B E-113$ \\
\hline 12.1 & TOTAL.. & $0.7141175-133$ & $5.57 n>4 E-n ?$ & $A .51913 E-i) ?$ & ?.03P?9E-114 & $7.11912 f-114$ & $1.31365 E-03$ \\
\hline $12 ?$ & TOIAL.. & o: & n. & ". & $"$. & $n$. & $n$. \\
\hline 123 & TITAL... & 0. & $n$. & $n$. & $i$. & 11. & 0. \\
\hline 12.4 & TOTAL.. & $4.54>345=-13$ & 4. 3$\}$ \{00k - 11? & 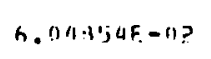 & ?.ASAHAL $=-1 A$ & $1.21+54 r-n x$ & $1.2417 G F-13 s$ \\
\hline 125 & TOTAL.. & $9.54135 E-03$ & $3.14344 E=02$ & $4.2917735-0 ?$ & $2.5 \times 3038-04$ & 1.?7R?GE-1)3 & $1.23244 E-03$ \\
\hline
\end{tabular}




\begin{tabular}{|c|c|c|c|c|c|c|c|}
\hline \multirow[t]{2}{*}{126} & \multirow{2}{*}{$\begin{array}{l}\text { TOIAL... } \\
\text { TOIAL... }\end{array}$} & 9.53 PARE $-n .3$ & $3 . n \triangle P A 3 E-03$ & $1.1354 \mathrm{RF}-1) 2$ & 3. BARDPF -114 & $1.10559 \mathrm{~F}-0.3$ & $9.5513318+011$ \\
\hline & & $n$. & $n$. & 0. & 0. & 1). & 0. \\
\hline 128 & TOTAL.. & H. 4 HOSSE-0.3 & 1.51 SP PF-05 & $5.51 \cap 2 H E-113$ & 3.12192E-กN & $7.31327 t-n 4$ & $5.13361 F-04$ \\
\hline 129 & TOIAL.. & 0. & D. & 0 & $n$. & 0 . & 0 . \\
\hline 130 & TOIAL.. & 1. PI TRUE-03 & S.9i)anZF-nh & 1. $40 \cap 95 E-03$ & $1.531 \mathrm{GOF}-04$ & $1.8 A 534 E-n .4$ & $1.176 .54 t-n 4$ \\
\hline 131 & IOIAL.. & 0. & ). & 0. & 0. & 0. & ". \\
\hline $13 ?$ & TOTAL.. & 0. & $n$. & 0. & $n$. & $n$ & 0. \\
\hline 133 & TOTAL.. & ". & $n$. & $n$. & 0. & i). & 0. \\
\hline 134 & IOIAL.. & $n$. & 0. & .0. & $n$. & $n$. & .0. \\
\hline$\stackrel{\prime}{\omega}$ & TOTAL... & $n$. & $n$. & 0. & n. & 0 . & 0. \\
\hline 136 & T01AL .. & 1). & $n$. & $n$. & 1). & n. & 0 . \\
\hline 137 & TOTAL... & 1). & $n$. & o. & $n$. & ". & 0. \\
\hline 134 & TOTAL.. & 1. & 0 . & $n$. & 11. & $n$. & n. \\
\hline 139 & TOTAL... & i. & 0. & 0 . & n. & n.. & $n$. \\
\hline $14 n$ & TOTAL.. & $3.399796-0 \mathrm{~A}$ & $5 . A 916 \cap 0-0 A$ & $1 .>46>5 E-1) 7$ & 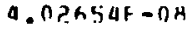 & P.A $955 P F=0$ A & $3.15 ? 71+-0 \mathrm{~A}$ \\
\hline 141 & TOIAL.. & 0. & 9. & 0. & $n$. & 0 . & ". \\
\hline $14 ?$ & TOTAl... & n. & $n$. & ". & $n$. & 1). & ". \\
\hline 143 & TOTAL.. & $n$. & 0 . & $n$ & ". & n. & $n$. \\
\hline$\cdots$ & -DTAL.. & $n$. & $n$. & $\because$ & $n$ & 0 . & n. \\
\hline 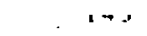 & . OTAL.. & 0. & n. & $n$ & ". & ". & ". \\
\hline
\end{tabular}




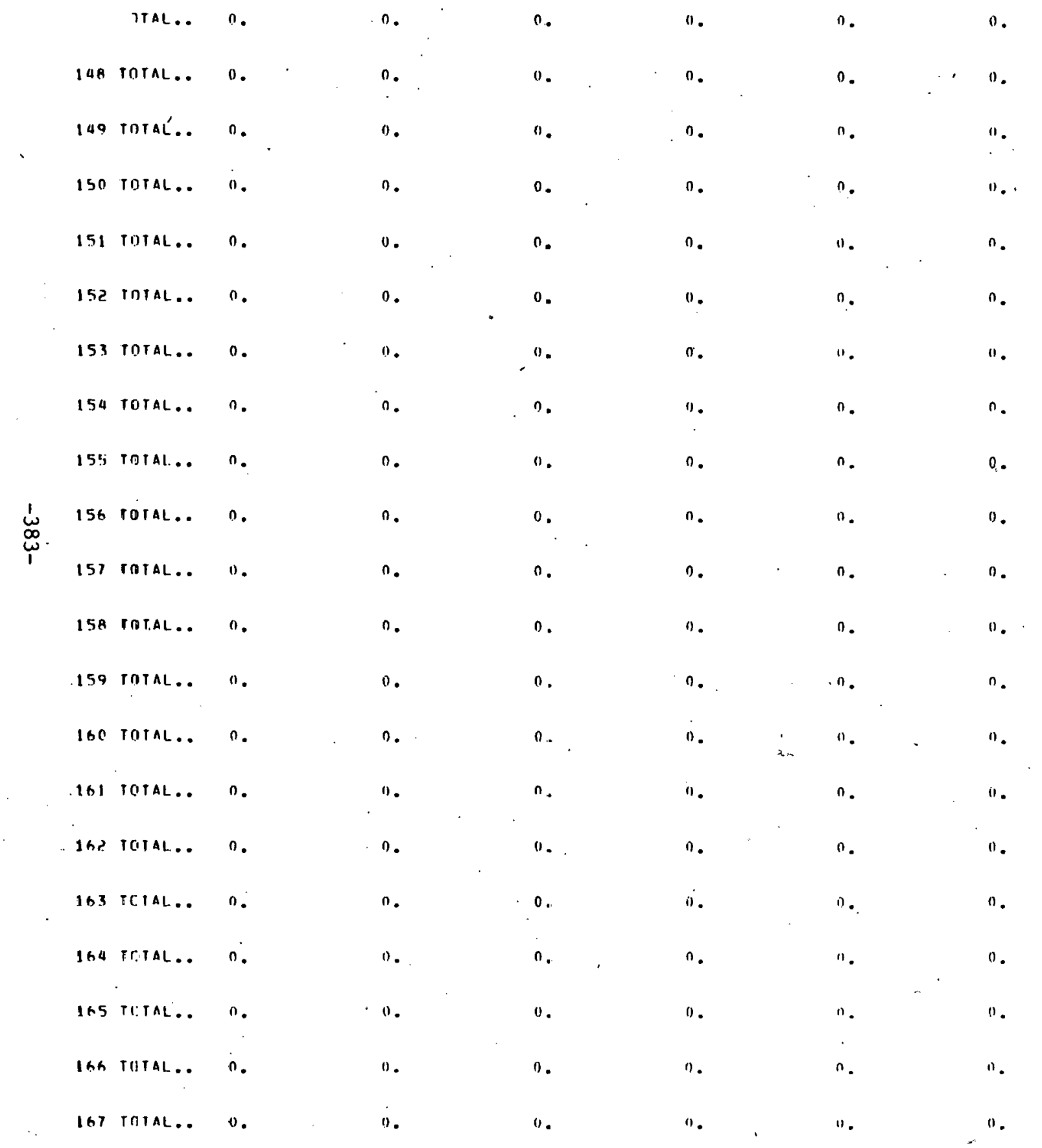


167 IOTAL.. 0.

IGE TOTAL.. O

164 T.OTAL.. ก.

170 TOTAL.. 0.

1
$\infty$
$\$$
1
0.

n.

0.
0 .

0.

0.

0.

0. $n$.

n.

0.
0.

0 .

0. $n$.

0.

0 
RESPONSE SPECTQUM STRESS COMPONFHIS (KINI = ?)

ELFMENT, THE FOLLOWIUS, INFIJRMATION IS PRINIFE:

1. FOR EACH EARTH IJJAKE UIHFCTION, THE NHMAER OF. THE MODE WITH THE LARTEST STLPSS.

c. THF VALUE DF THAT STHESS.

3. IF REDUESTED, THF MONF HY MODE STRESSE. FOR FACH EARTH IUUAKE UIRF.TION.

4. THE RFSULTANI F TR EAC.H EARTH OUIAKE OIPECTION.

(NOTE: THE GRANO TOTAL OF THE. THREE EARTH DUAKE DIRECTIONS.

ELEMENT TYPE (3/1) P I PE , , , FLEMENT NUMAER 1 (1)

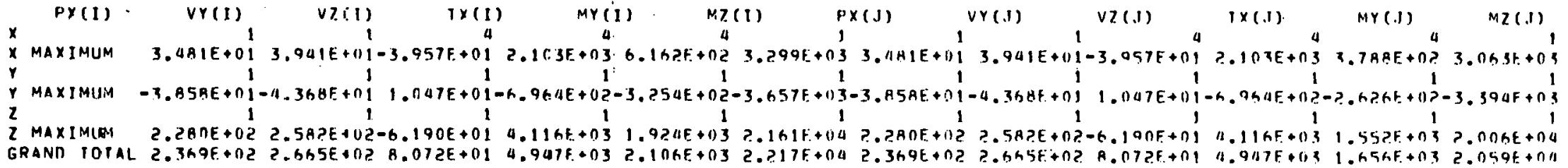

ELEMENT TYPE (3/0 P I PE , , , ELEHENT NUMHER $($ ?)

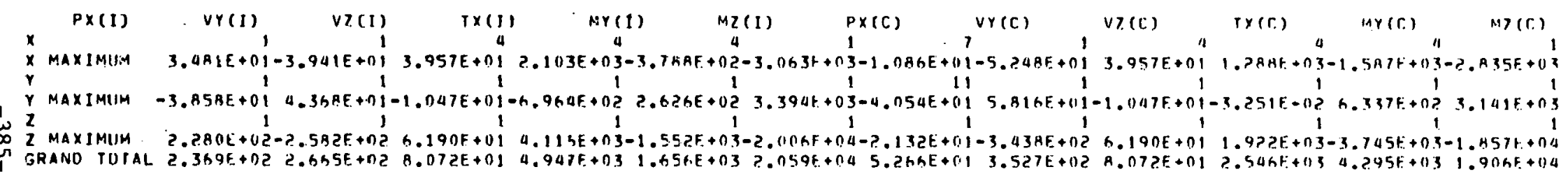
ELEMENT IYPE (3/R P I PE ) , , ELEMENT NUMAER 1 ?)

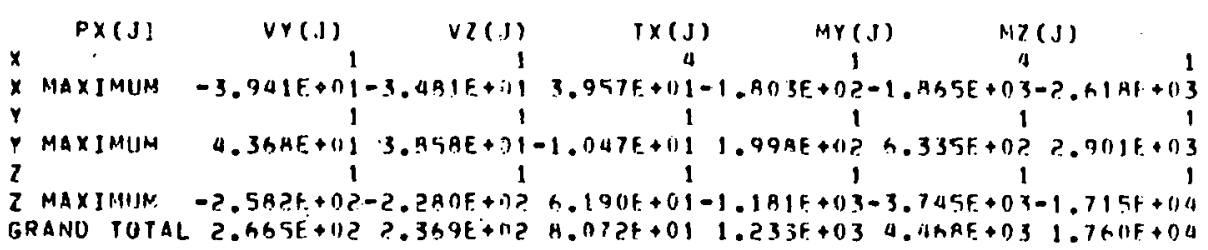

ELEMENT TYPE (3/1) P T PE , , ; ELEMENT NUIAHER ( 3)

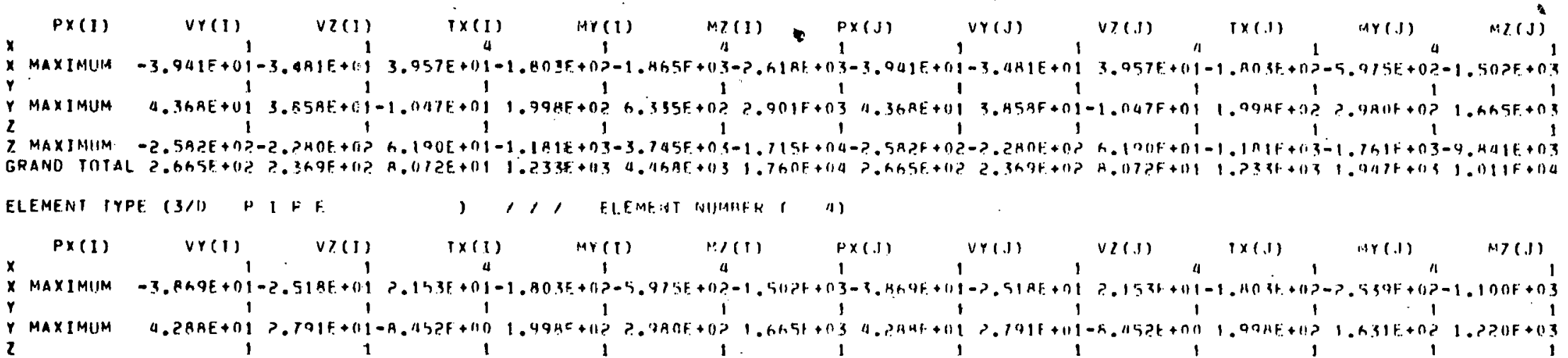




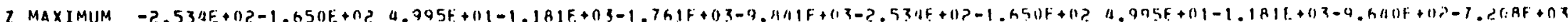

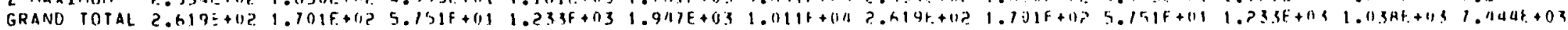
ELEMENT TYPF. (3/1) P I PE

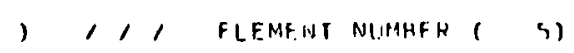

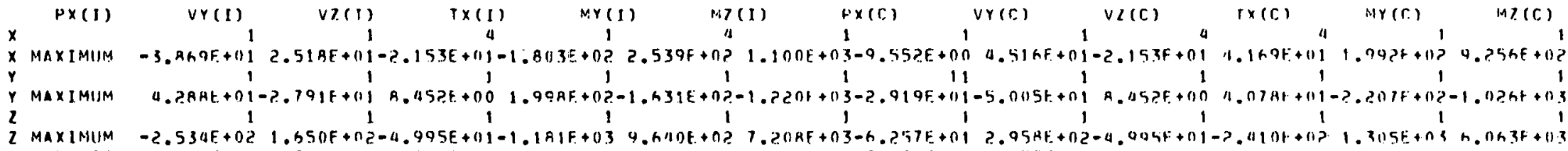

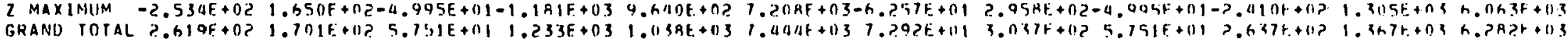

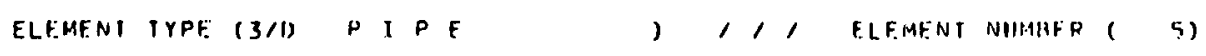

\begin{tabular}{|c|c|c|c|c|c|c|c|}
\hline & $P \times(J)$ & $V Y(J)$ & $V Z(\mathrm{~J})$ & $T \times(J)$ & $\operatorname{Ar}(J)$ & Mz. & (J) \\
\hline & & 1 & $i$ & 4 & 4 & 1 & \\
\hline & MAYIMIJM & $2.518 E+01$ & $9 F+01-$ & $.2 .153 F+01$ & $E+0 ?$ & $1 . ?$ & 7.1 \\
\hline & & $t$ & 1 & 1 & 1 & 1 & \\
\hline & MAXIMUM & $91 E+01$ & $A E+n 1$ & $8.45 P E+110-$ & af $+0 ?-1$ & & \\
\hline & & & 1 & 1 & 1 & 1 & \\
\hline & $\begin{array}{l}\text { MAXIMIIM } \\
\text { RAND TOTALI }\end{array}$ & $1.650 E+0 ?$ & 2. $.534 E+11 ?=$ & $4.995 E+11$ & 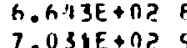 & $\begin{array}{l}8.9111 E+02 \\
9.1 H \partial E+0 ?\end{array}$ & $\begin{array}{l}\text { A. A.9AF + (1 } \\
\text { A. } 8 A 2 E+11\end{array}$ \\
\hline
\end{tabular}

ELEMENT TYPE (3/1) P 1 PE 1,1, FiLFMENT NIJMHEN $(A)$

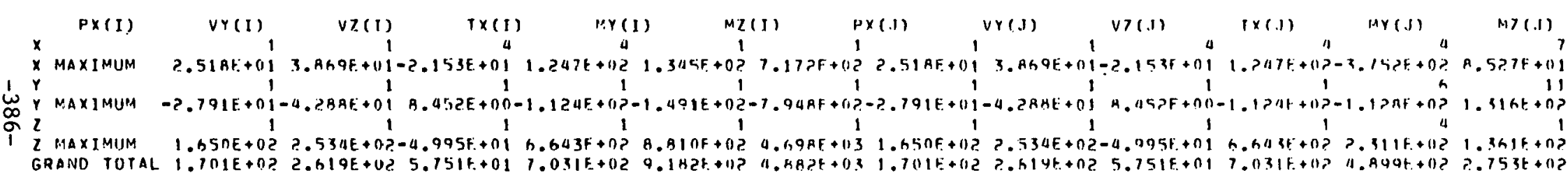
ELEMENT TYPE (3/0 PI P F , 1, ELEIAEIJT NIJMAER $(7)$

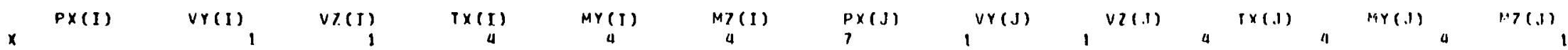

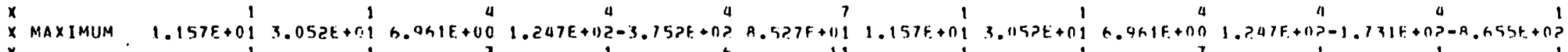

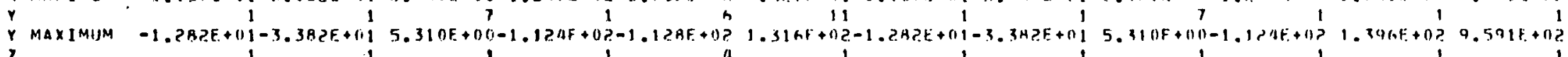

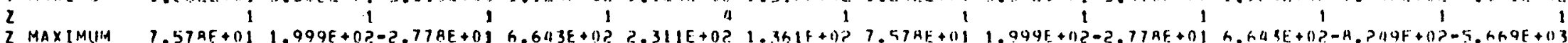

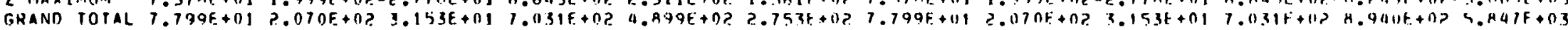
ELEMENT TYPE (3/R PIPE , , , ELEMFYI NIJAHISTR ( H)

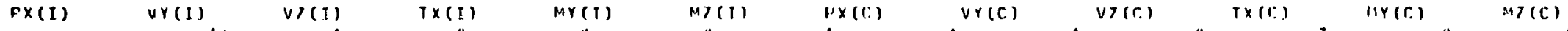

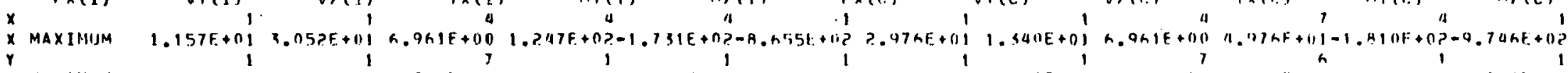

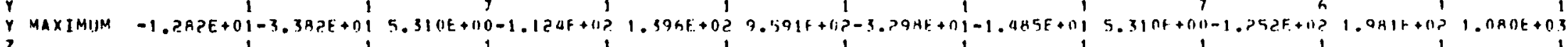

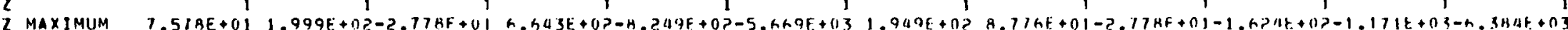

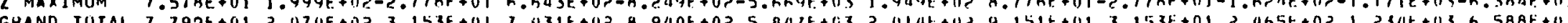

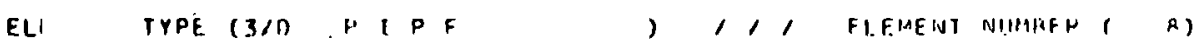

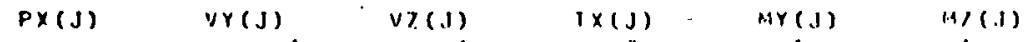




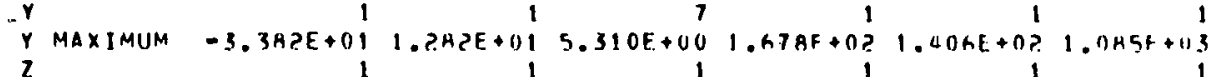

2 MAXJMUM $1.999 F+0 ?-7.57 A E+01-2.11 A E+01-0.916 E+02-A .310 E+1) ?-0.4111+01) 3$

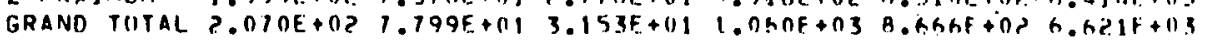

ELEMENT IYPE. (3/1) $P$ I P E , $/, \mid$ FLEMENT NUMRER $(9)$

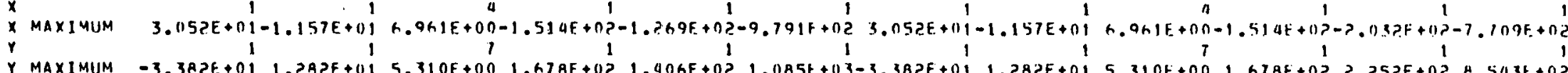

$y$

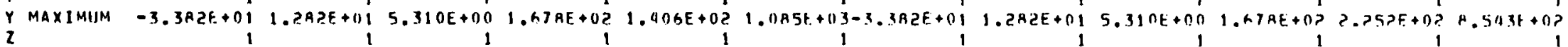
2 MAXIMIJM $1.999 E+02-7.57 A E+01-2.77 A E+01-0.916 F+02-A .310 E+02-6.414 F+031.999 E+02-7.57 A F+01-2.778 F+01-9.916 F+02-1.331 F+03-5.050 E+03$

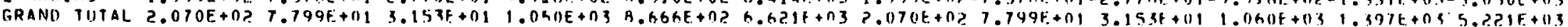

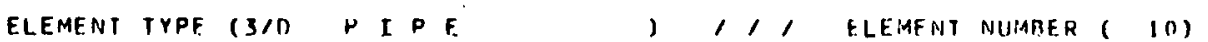

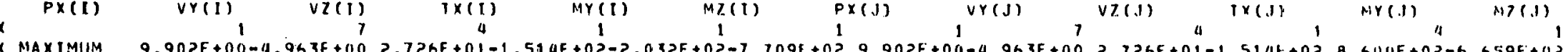
$X$ MAXIMLMM $9.902 E+00-11.963 E+00$
$Y$

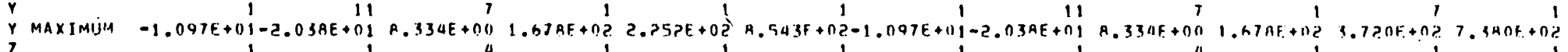

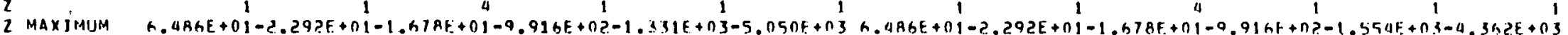

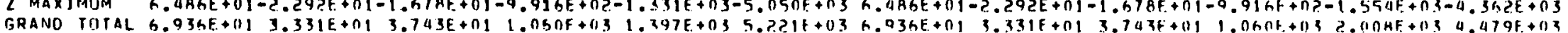
ELEMFNT TYPE $(3 / 0$ P TPE , , , FLEMFNT NUMHER $(11)$

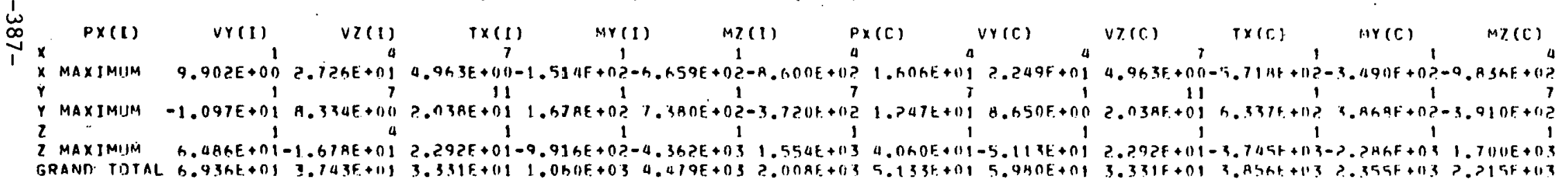

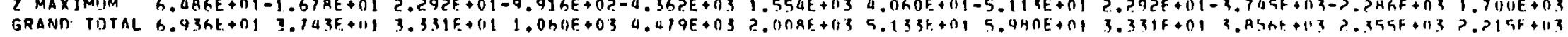

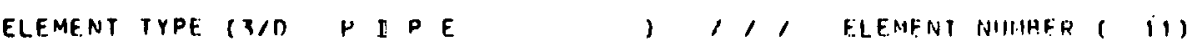

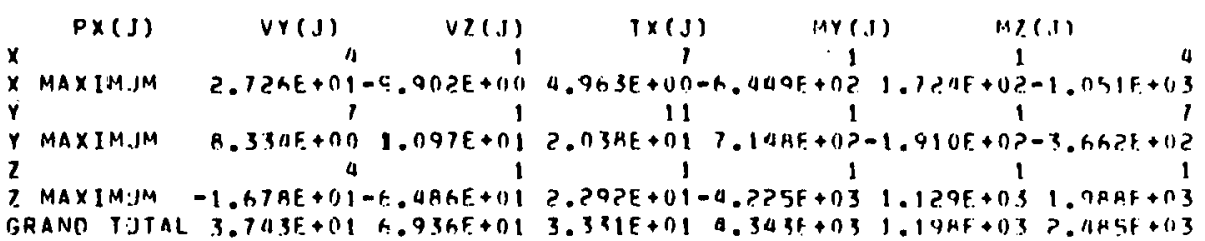

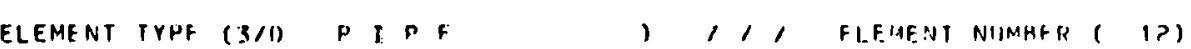

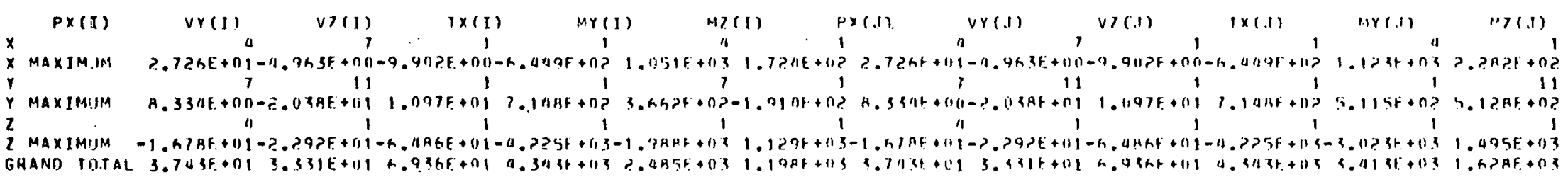
ELEMENT TYPE (3/0 P I P 


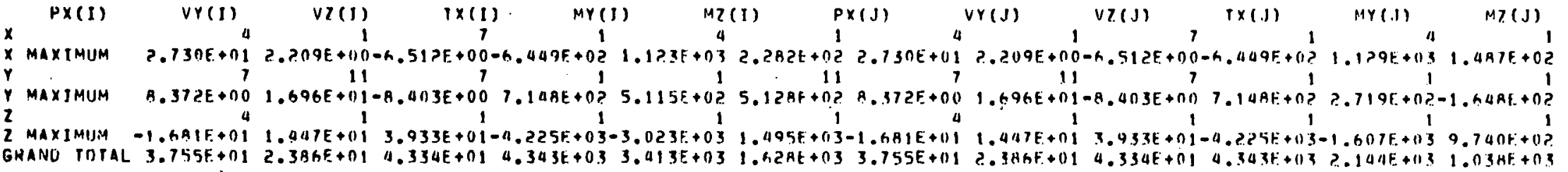

ELEMENT TYPE (3/0 PIPE , 1,1 HLEMENT NUMAER $(14)$

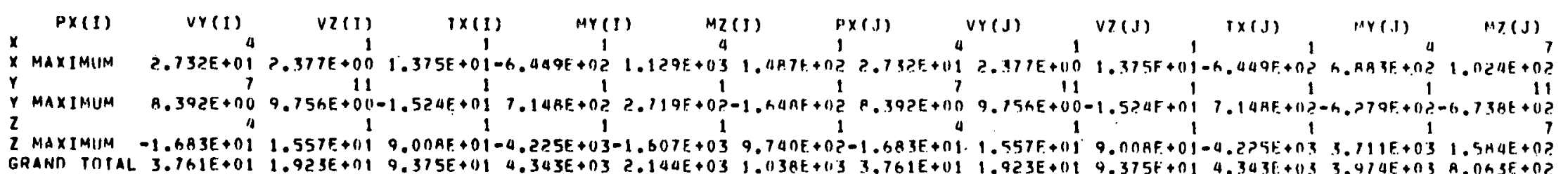
ELEMENT TYPE (3/0 P I PE , , , ELEMFNT NIJMAEP $(15)$

$P X(I) \quad V Y(I) \quad V Z(I) \quad T X(I) \quad M Y(I) \quad M Z(I) \quad P X(J) \quad V Y(J) \quad V Z(J) \quad \operatorname{TS}(J) \quad M Y(J) \quad M Z(J)$

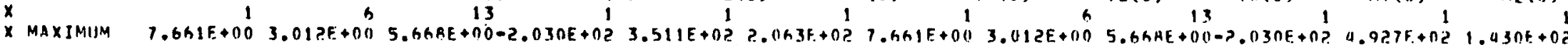

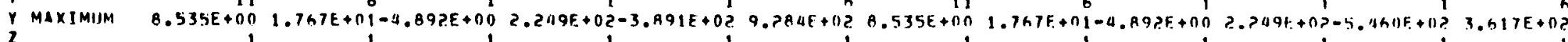

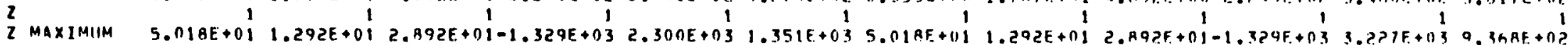

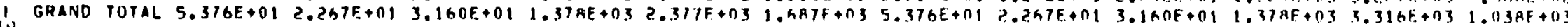
$\stackrel{1}{\infty}$ ELEMENT TYPE $(3 / 0)$ P I P E , , FLEMENT NUMAER $(16)$

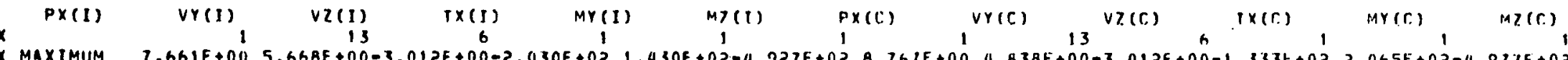

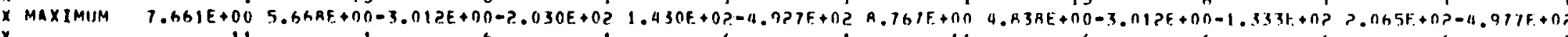

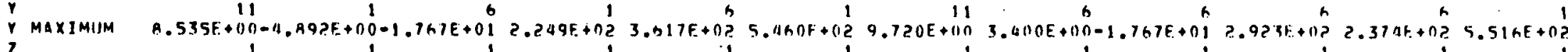

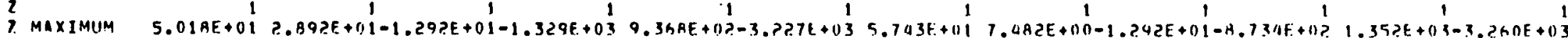

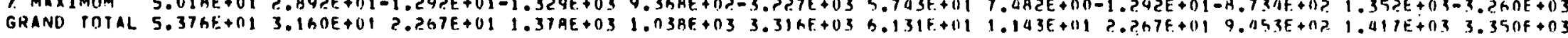

ELEMENT TYPE (3/0) 1 PE, , , ELEMEINT NUIGGEH $(16)$

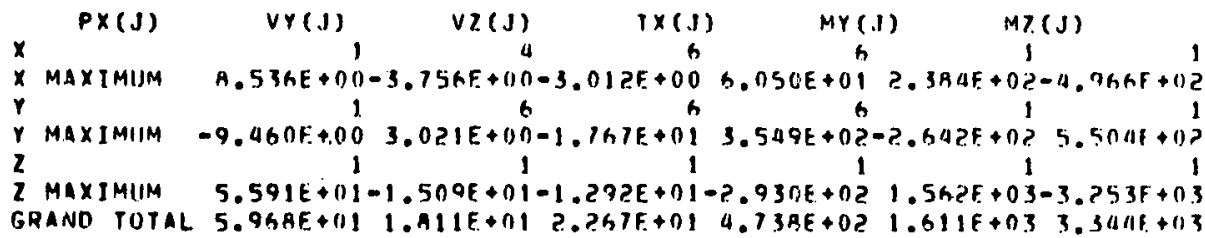

ELEMENT TYPE (3/0 P I PE ), , ELEMENT NUPHBER 1 17)

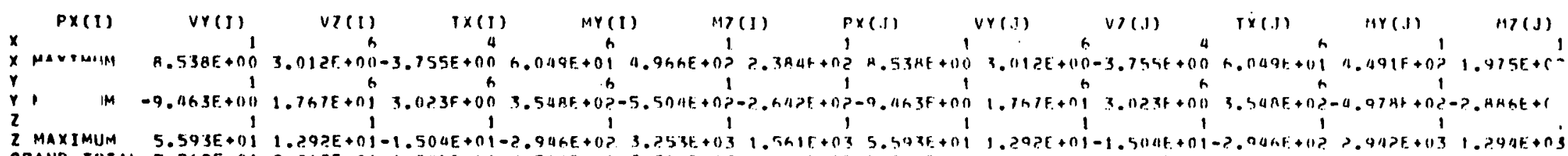


ELEMENT TYPF (3/0 P I PE , 1,1 FLEMENT NUMMEN ( IA)

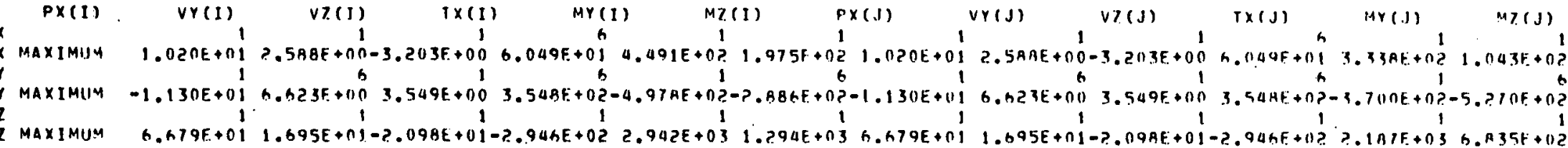

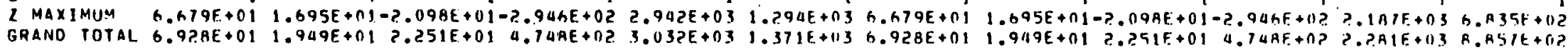
ELEMENT TYPE (3/R P I P F , 1,1 ELEMENT NINAHER 1 19)

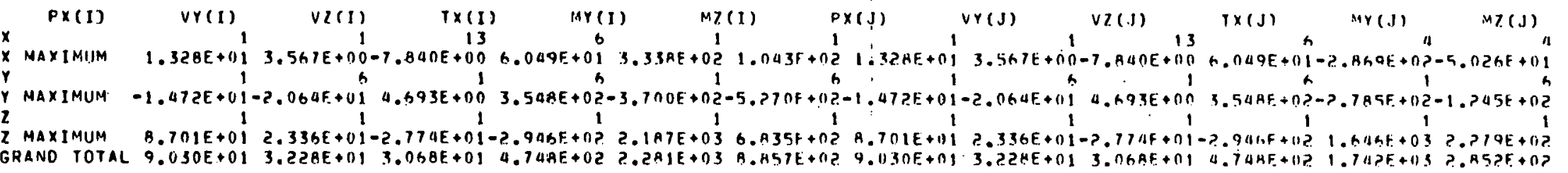
ELEMENT TYPE ( $3 / 0$ P 1 PE. ,, 1, ELEMFint NUMBER $(P O)$;

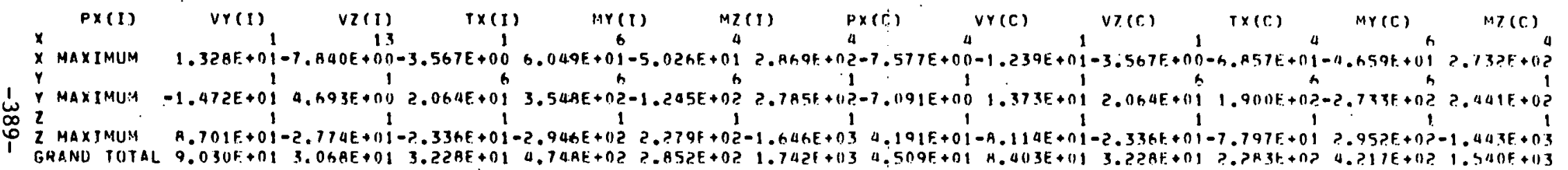
ELEMENT TYPE (3/1) P 1 PE 1,1, ELEMENT NIJMHER 1 , 0$)$

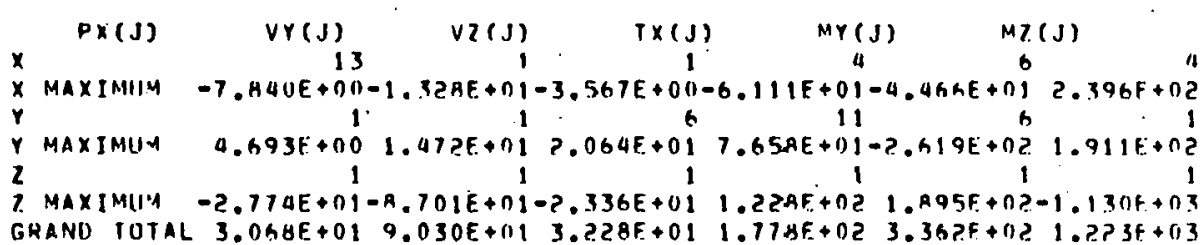

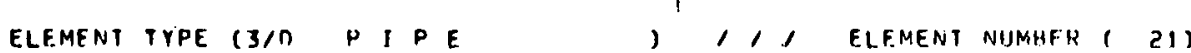

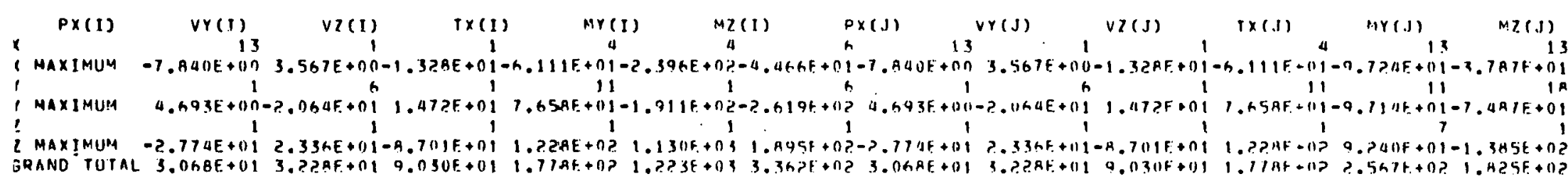
ELEMENT TYPF: (3/D P I P E , , , F.LEMEAT N(IHAFE 1 , $>$ )

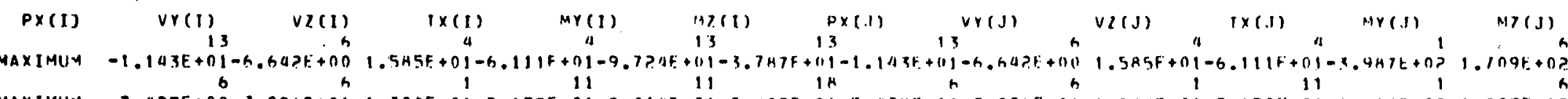

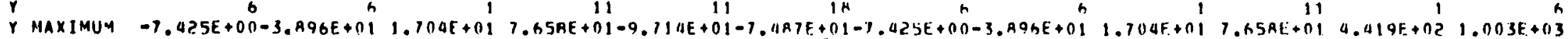




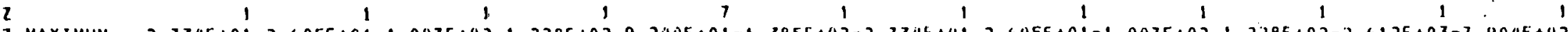

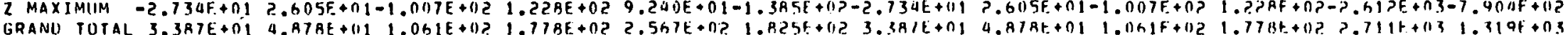
ELEMENT TYPF. (3/1) $P$ I P $E$, , , FLEMENT NIMAER $(3)$

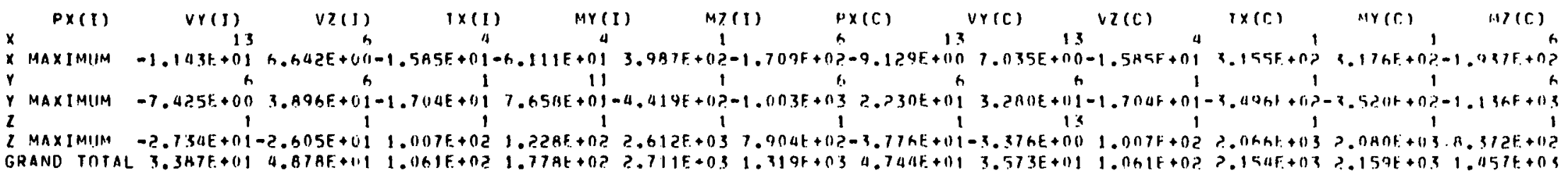
ELEMFNT TYPE (3/0 PI PE , , , ELEMENT NUJHER $($ ? 3$)$

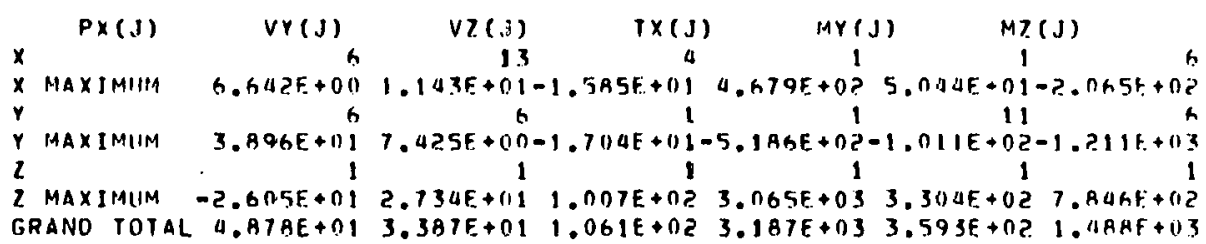

ELEMENT TYPE (3/0 P I P E, 1, ELEMFNT NUMHEH $($ 24)

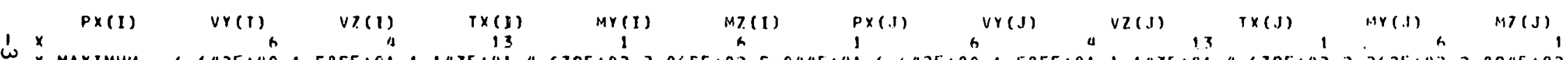

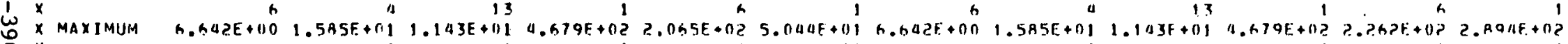

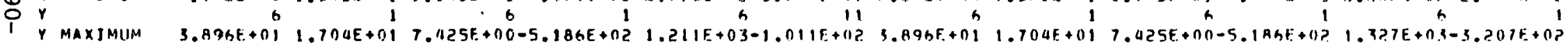
2 MAXIMUM 111011 GRAND TOTAL ELEMENT TYPE (3/0) P I. PE , , , ELEMENT NIMARER $(25)$

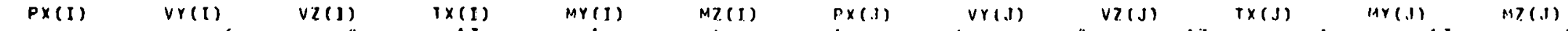

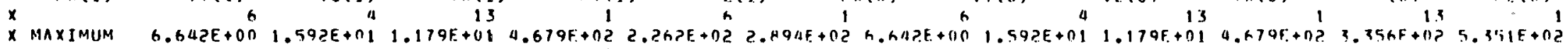
$r$ a

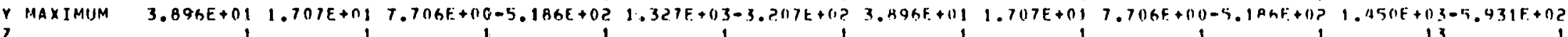

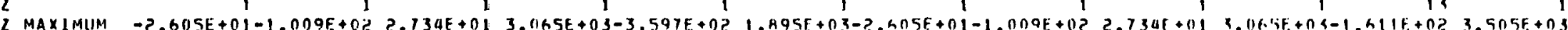

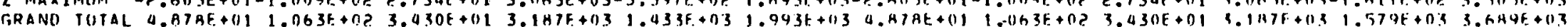

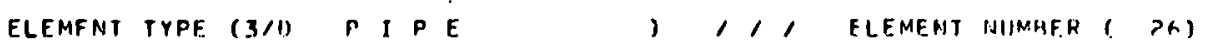

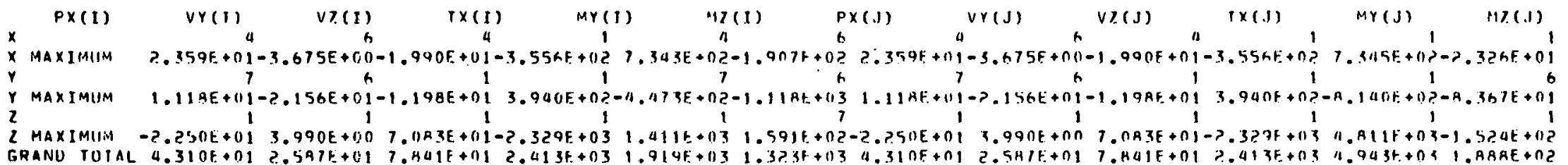

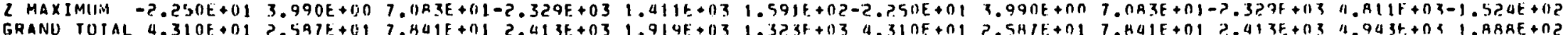
EI TYPF (YAD P I P E 


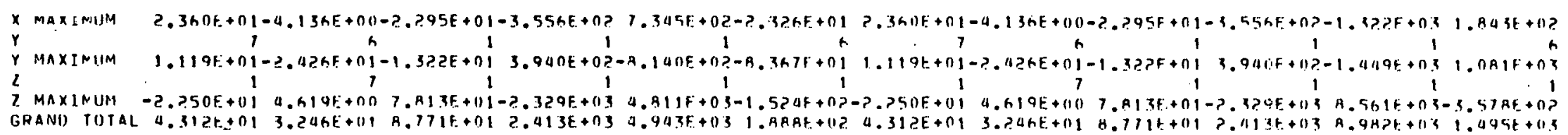
ELEMENT IYPE (3/0 PI PF, , , ELEMFNT NIJMAER ( ?a)

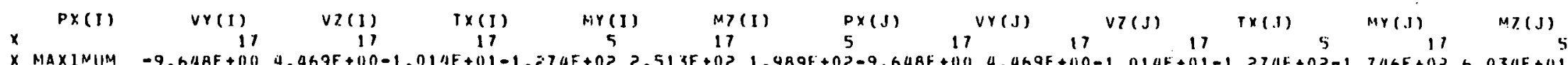
$x$ MAXINIIM $-9.6 A A E+00$
$y$

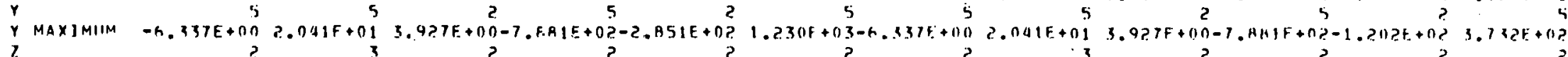

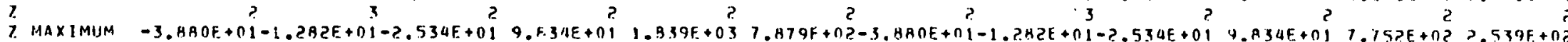

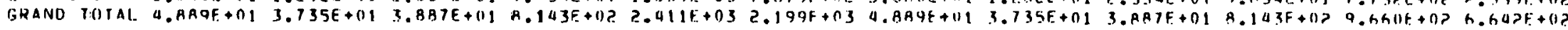

ELEMEMI TYPE (3/D P I PE $), 1$, ELFMENT NUMARER 1 ? 9 )

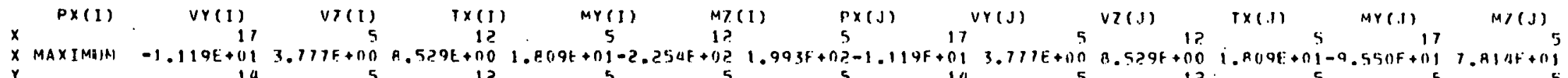

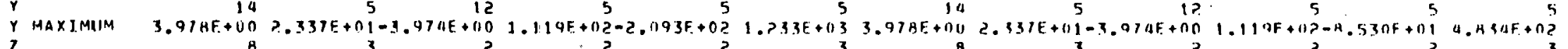

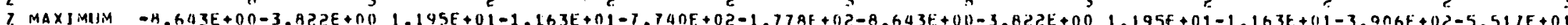

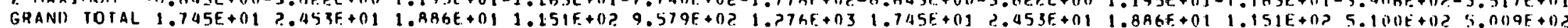

W. ELEMENT TYPF $(3 / 1)$ P I PE , , ELEMENT NUMAER $(30)$

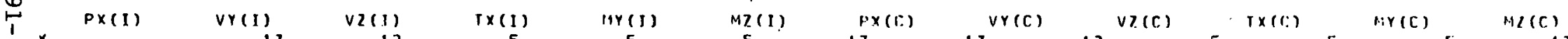

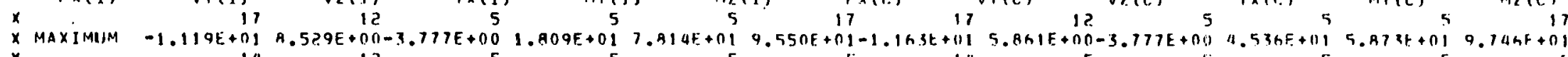

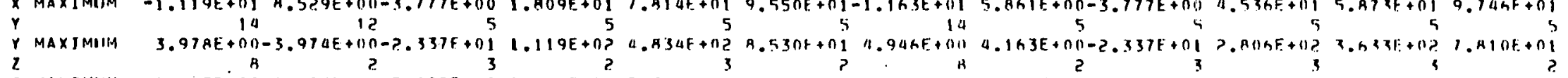

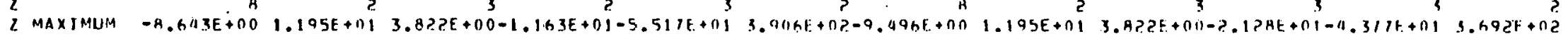

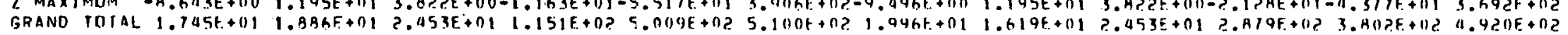

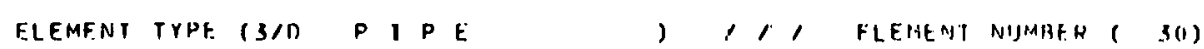

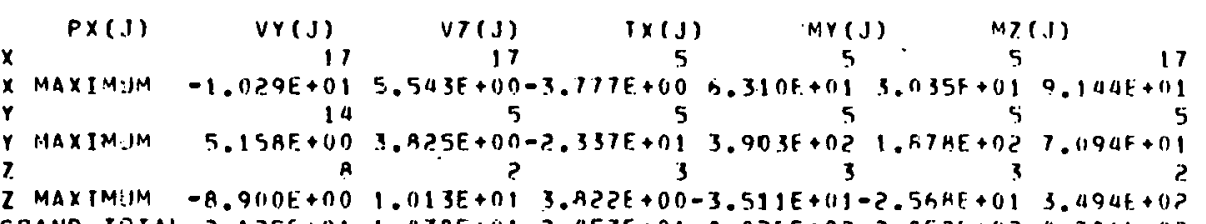

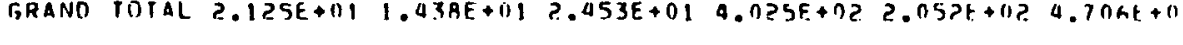

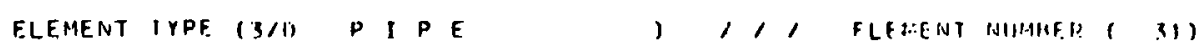

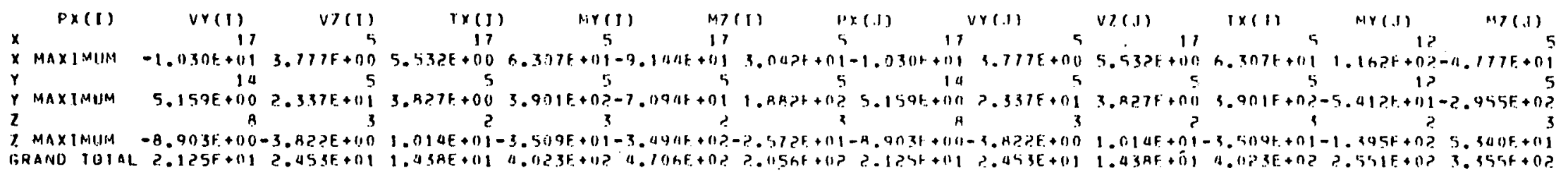

ELEMENT IYPE $(3 / 0$ P I PE ,, 1, ELEMFNT NUMHER $(3 ?)$ 


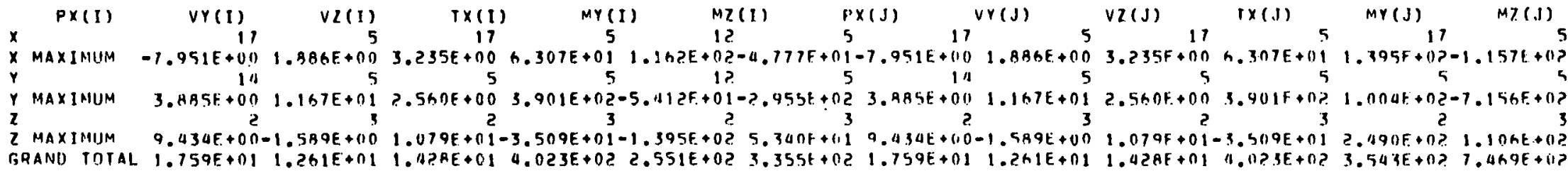
ELEMENT TYPE (3/0 PIPE ,, 1, ELEMENT NUMAEK $(33)$

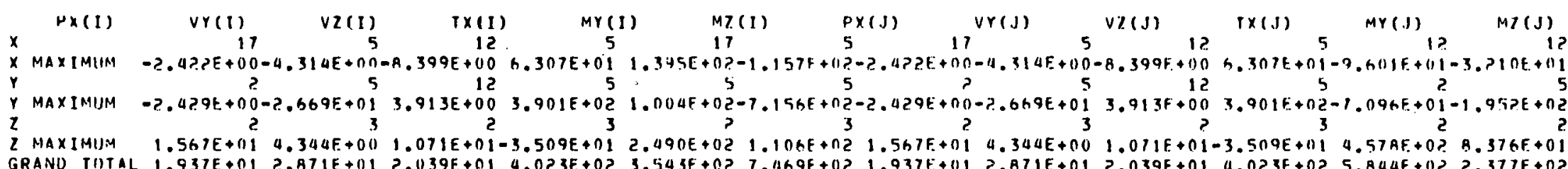

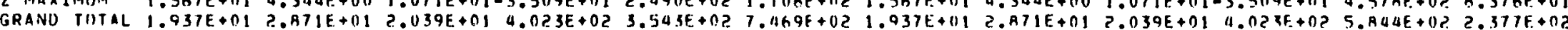

ELEMENT TYPE $13 \%$. P I P E , 1,1 , ELEMEHT NUMREK $(30)$

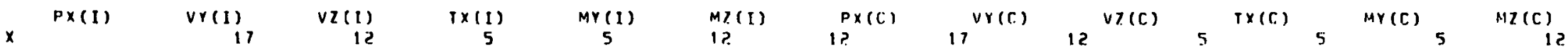

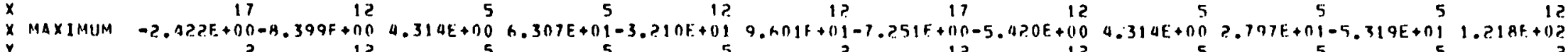

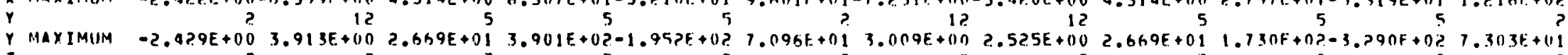

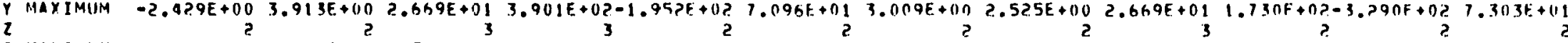
12 MAXIMUJA $1.567 E+0 i 1.071 E+0 I-4.344 E+00-3.509 E+01$ A. $376 E+01-4.57 A F+021.865 E+01-3.513 E+00-4.344 F+00 \quad 5.551 E+0 i \quad 1.545 E+01-11.71 P E+02$

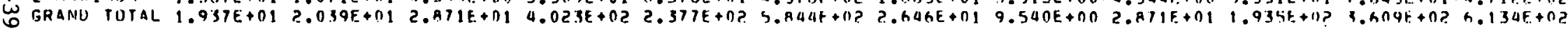
I ELEMENT TYPE $(3 / 0$ P I PE $), 1$, ELEMENT NUMHER $(34)$

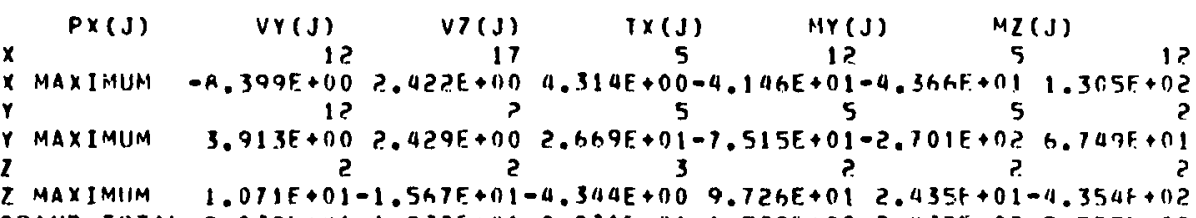

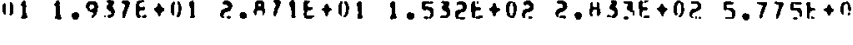

ELEMENT IYPE (3/0 P I P E ) 1, , ELEMENI NIIMMER ( 35)

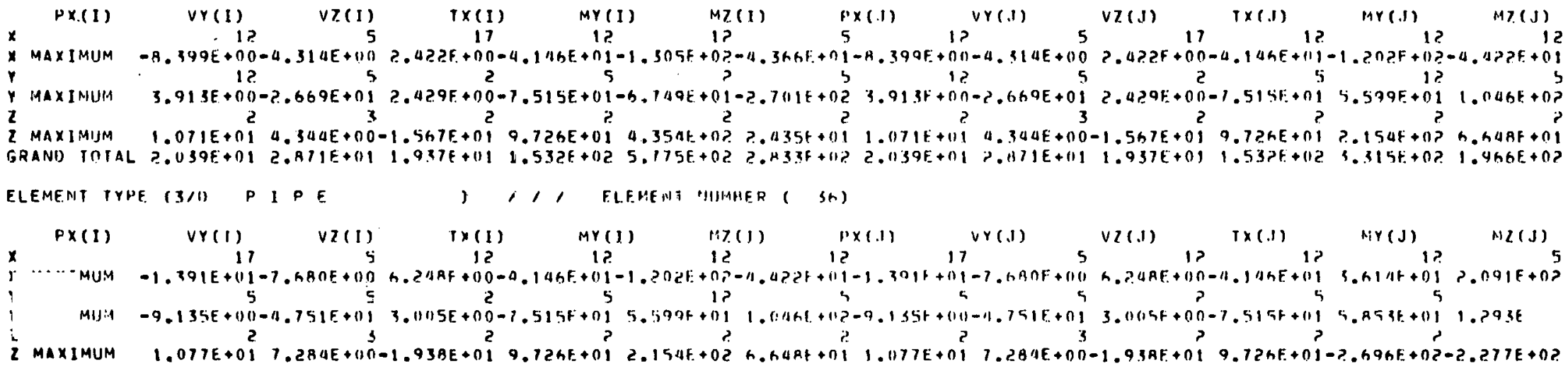




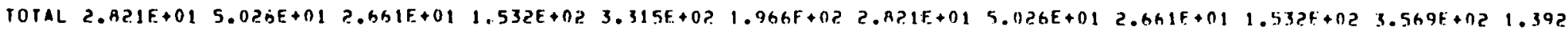
ELEMEAT TYPE (3/1) P 1 PE $), 1$, ELEMENT NUillFF ( 37)

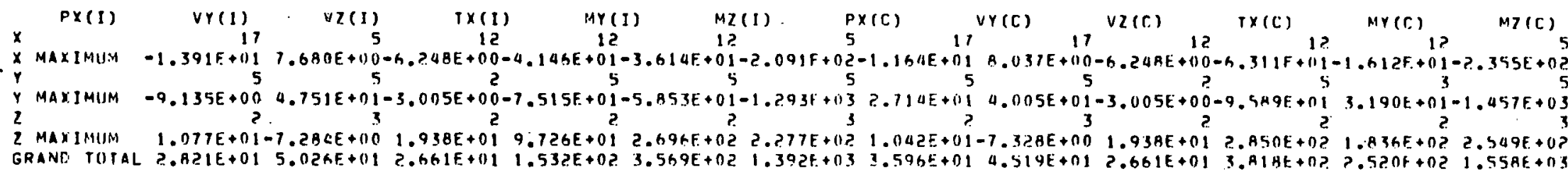
ELEMENT TYPF. (3/0 PIPE , , , ELELEENT NUMIIER $(3)$

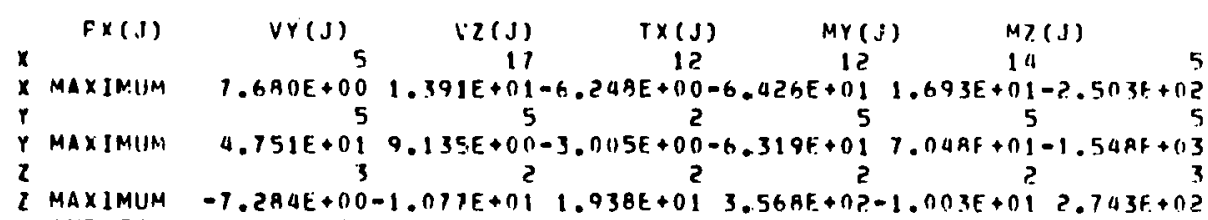

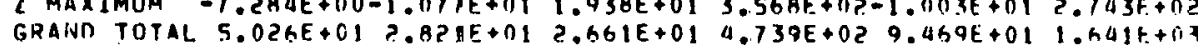

ELEMENT TYPE (3/0 PIPE , 1, ELEMENT NIMAREP ( $3 A)$

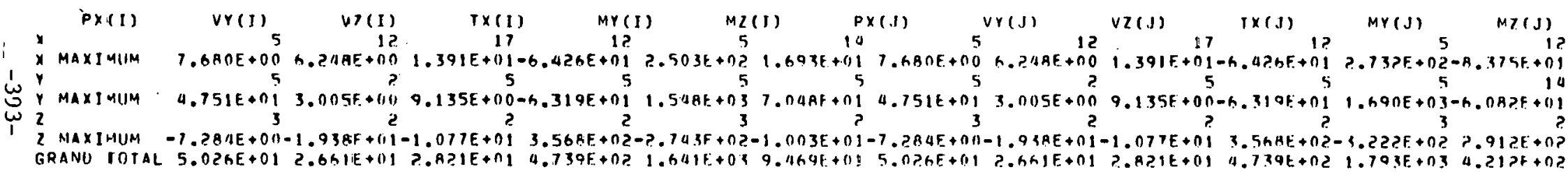
ELEMENT TYPE (3/0 P I PE , , , FLEMENT NIJMHER 139 )

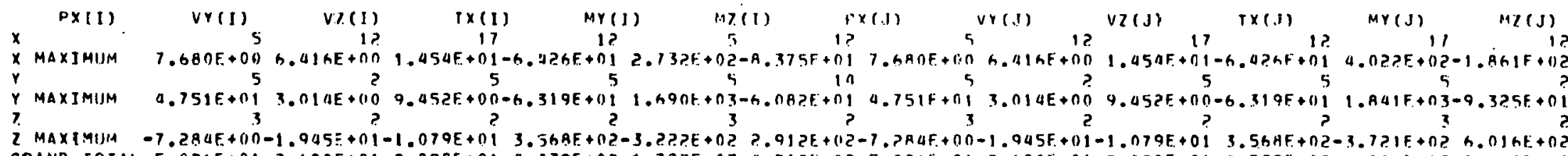

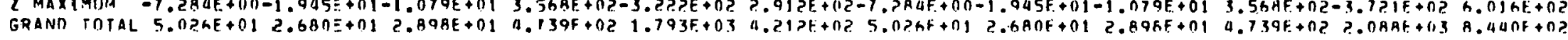

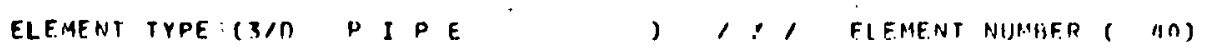

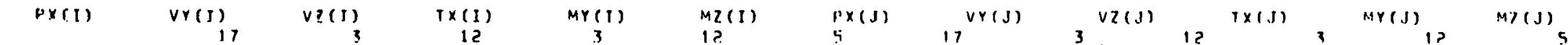

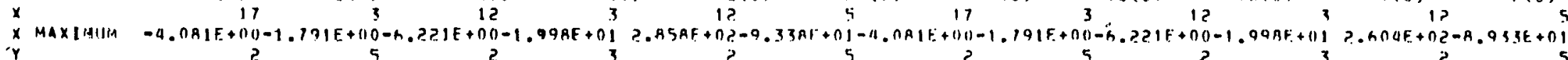

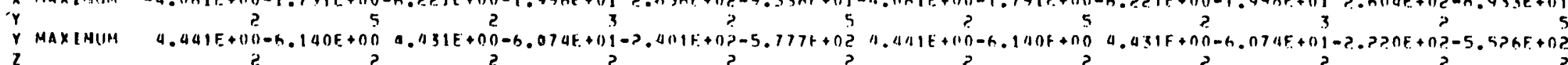

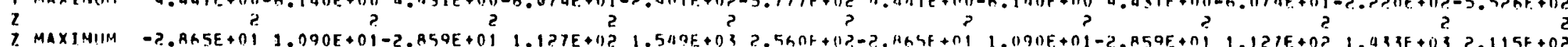

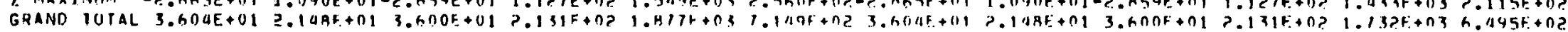
ELEMENI IYPF (3/D P I P E , , ELEMENT MIJMMFR., 11$)$

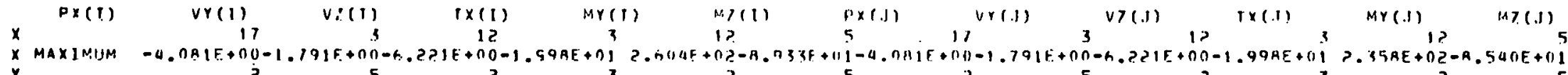




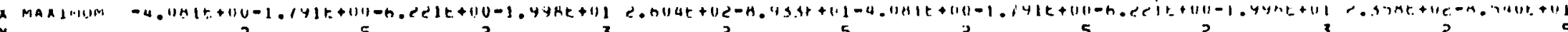

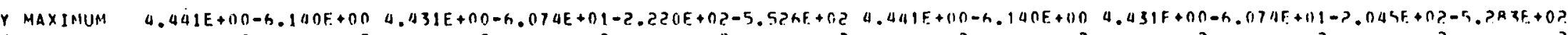

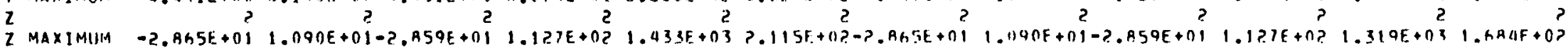

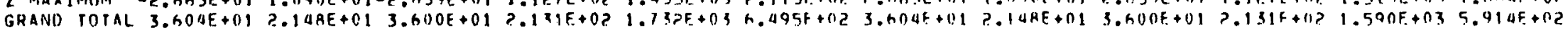
ELEIAENT TYPE $(3 / 1)$ P I P E

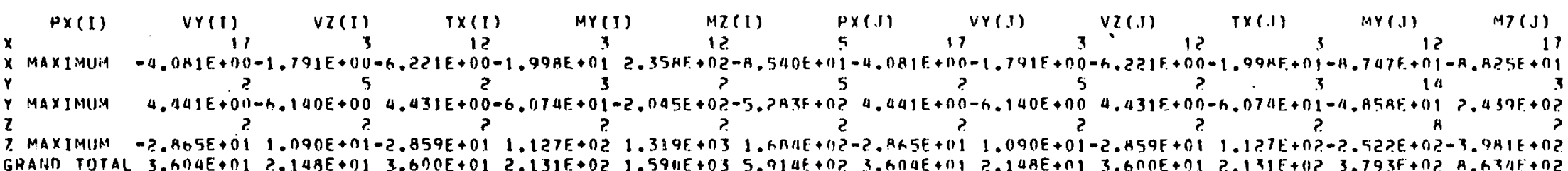
ELEMENT TYPE (3/D PI PE , , , ELEMENT NIMIREK $($, 3$)$

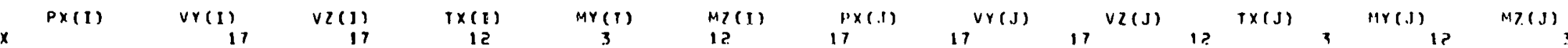

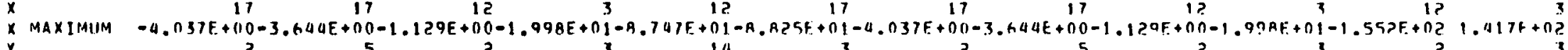

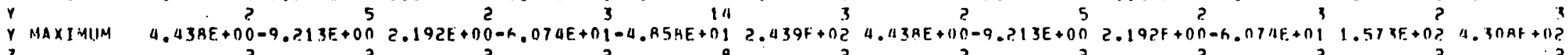

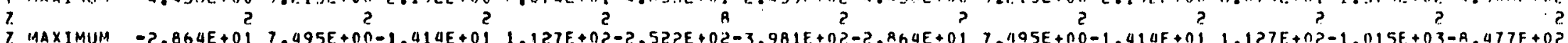

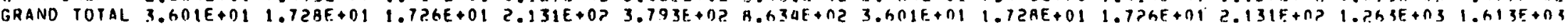

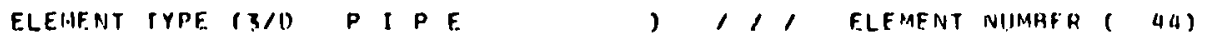

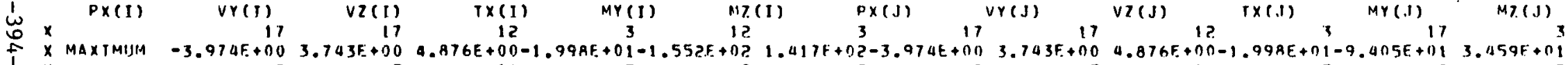
Y

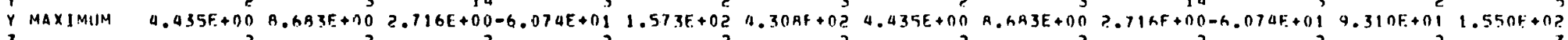

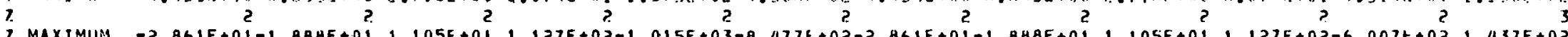

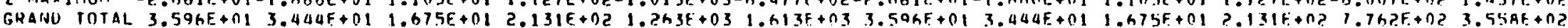
ELEMEHT TYPE (3/0 P I P E , 1,1 FLFMFNT NIJABFR ( 15)

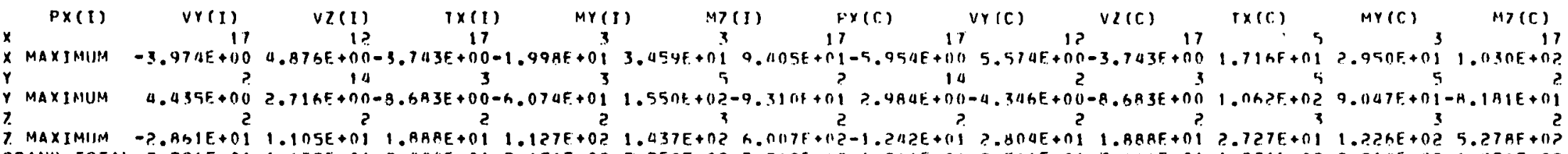

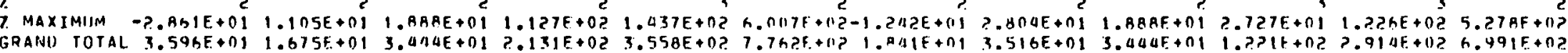
FLEMENT TYPE (3/ח P I PE , , , ELEMENT NIJMUER $(4 S)$

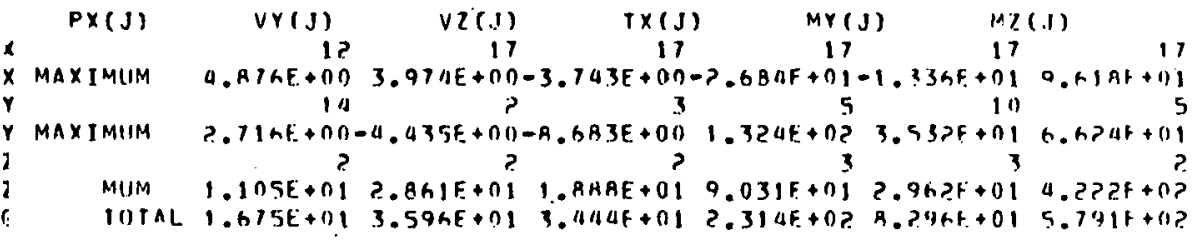




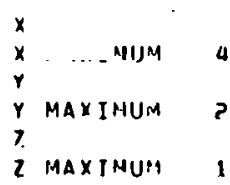

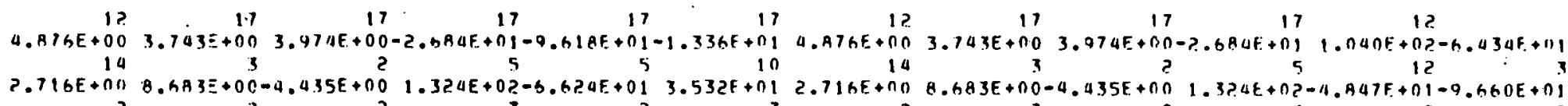

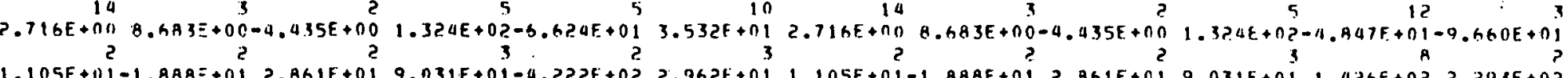

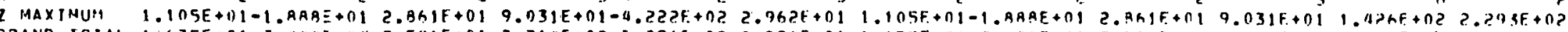

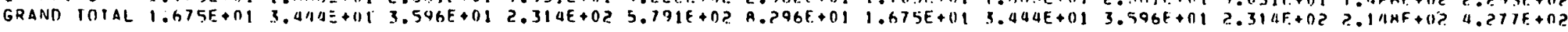
F.LFMENT TYPF $(3 / \mathrm{A}$ P I PE.,$\because$, ELEMENT NIJMBER $(47)$

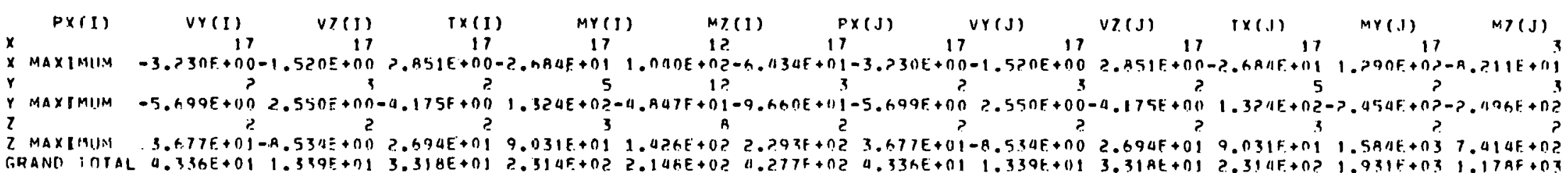

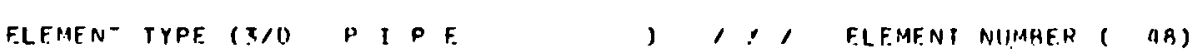

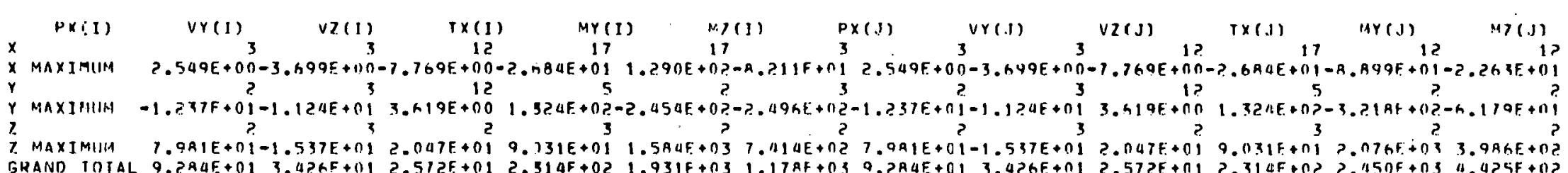

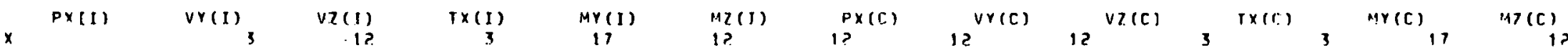

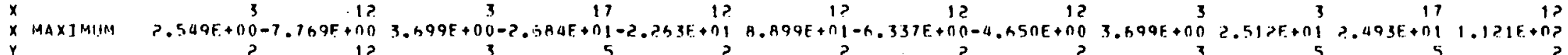

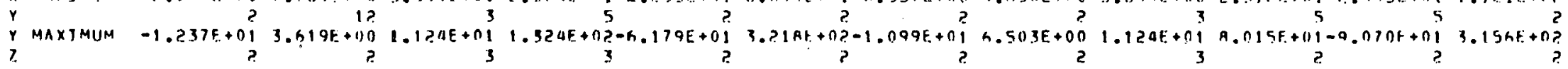

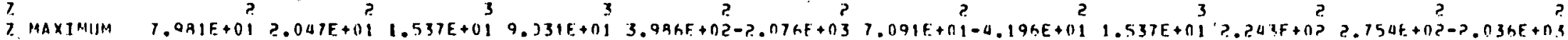

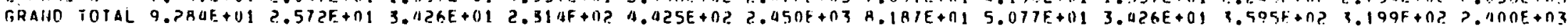

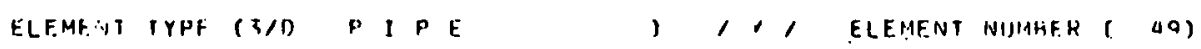

\begin{tabular}{|c|c|c|c|c|c|c|}
\hline$p \times: 3)$ & VY (N) & v $7(.1)$ & $T \times(J)$ & BY (.J & $m z$ & $(J)$ \\
\hline & 12 & & 3 & 12? & 17 & \\
\hline MAXIMUN & $.169 F+00$ & $9 E+n I)$ & $3.629 E+100-$ & $-3.379 E+01$ & $2.394 E+n 1$ & ASF \\
\hline & 1? & 2 & 3 & 3 & 5 & \\
\hline$M A X T H I I M A$ & $3 .+19 F .+n O$ & $1.231 \mathrm{E}+01$ & $1.124 E+01$ & $7.153 \mathrm{E}+111=$ & - . $27 R F+01$ & \\
\hline & $?$ & ? & 3 & 2 & 3 & \\
\hline MAXIIIUM & $? .0,47 E+191-$ & $-7.9 A \mid E+01$ & $1.5 \times 7 E+01$ & $3.545 E+0 ?-$ & $+01-2$. & \\
\hline RAND TOTAL & $2.5725+111$ & AJF $+n 1$ & $3.47 .6 F+01$ & 4. 57 HF + n? & 3 & \\
\hline
\end{tabular}

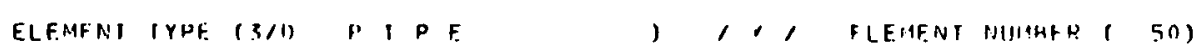

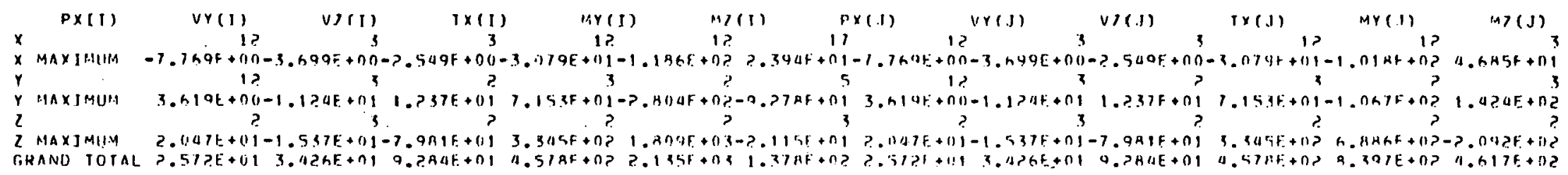




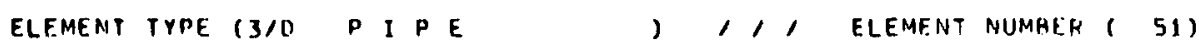

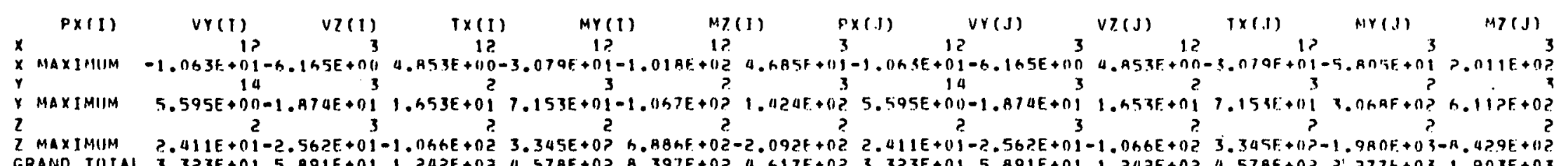

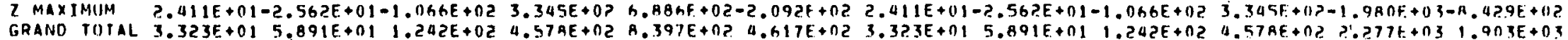
ELEMENT TYPE (3/R P I PE , 1, FLEMENT NUMHF.R ( 52$)$

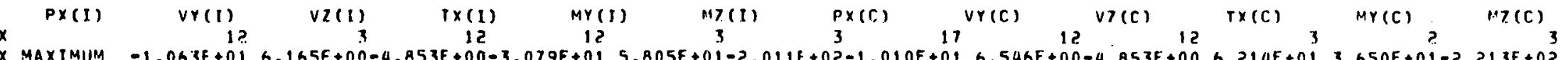

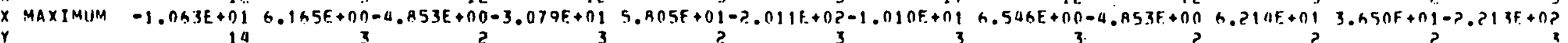

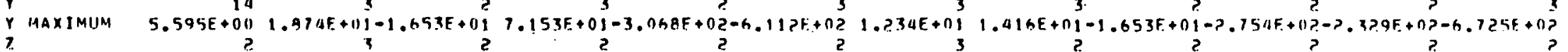

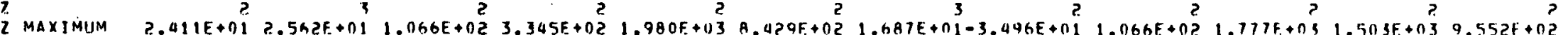

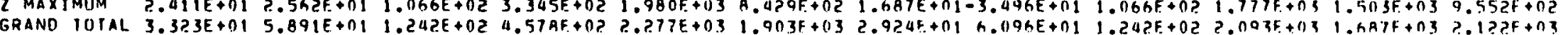

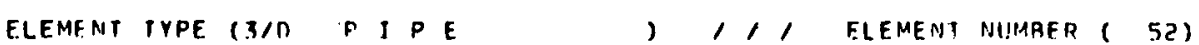

\begin{tabular}{|c|c|c|c|c|c|}
\hline PX (J & $\operatorname{vr}(1)$ & $v>(J)$ & $x(J)$ & $\operatorname{MY}(\mathrm{J})$ & $M Z(J)$ \\
\hline$M A X I M U$ & $0.1655+00^{3}$ & $3 E+n 1-$ & $\begin{array}{r}12 \\
+00\end{array}$ & $7.327 E+01^{3}$ & $\begin{array}{c}14 \\
1.67 A E+101-2 .\end{array}$ \\
\hline & & 14 & 2 & 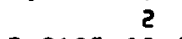 & 10 \\
\hline 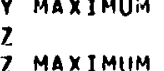 & $5 h 2 f+$ & $\begin{array}{l}95 E+100- \\
? E+0 ?\end{array}$ & $\begin{array}{r}1.653 E+01- \\
? \\
1.066 E+0 ?\end{array}$ & 159E +03 & $\begin{array}{c}+01-7.01 \\
+05 \\
+0.08\end{array}$ \\
\hline
\end{tabular}

D 2 MAXIMIIM $2.562 E+01-2.411 E+011.066 E+02 \quad 2.059 E+031.454 E+0 ?+1.065 E+0.3$

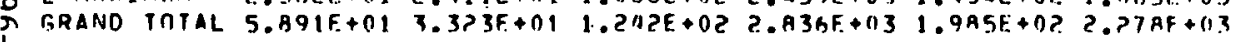

FLEMENT TYPE $(3 / 7$ P I P E , , , , ELEMENT NUIAHER ( \$3)

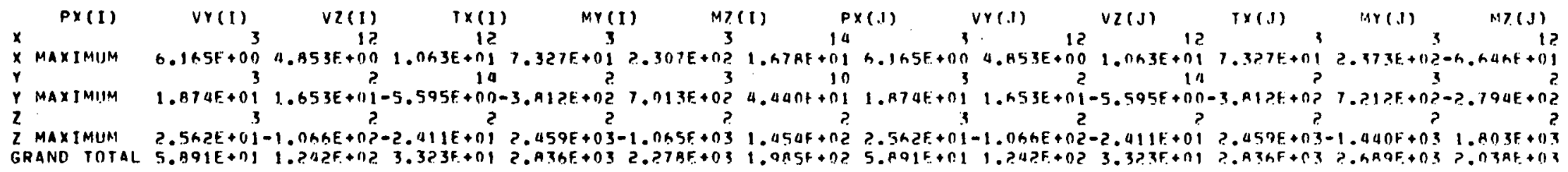
ELEMENT TYPE (3/1) $P$ I $P E$; 1,1 ELEMENT NIJMAFH $($ 51)

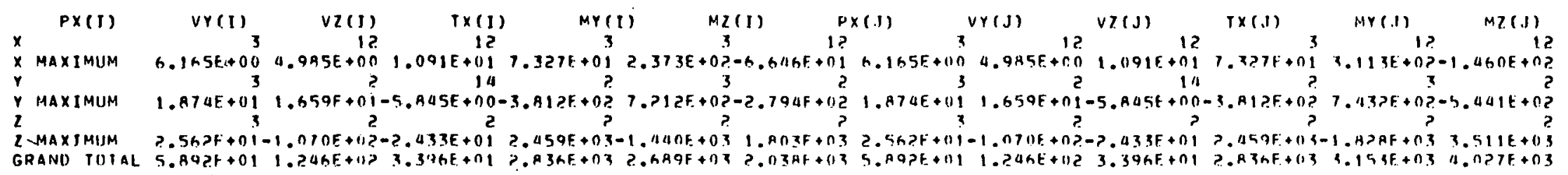

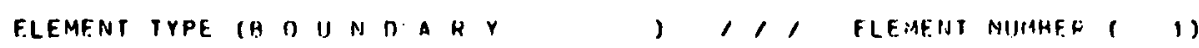

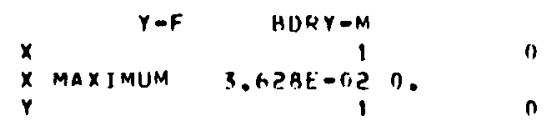


2 IUM P.377F-01 1).

GHAN! VITAL ?.439E-01 0 :

ELEMENT TYPE TH O $U$ U A D D A h

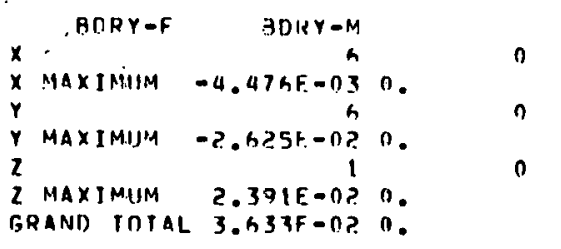

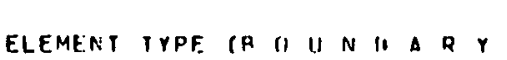

), 1, ELEMENT NIMHEF ( 3)

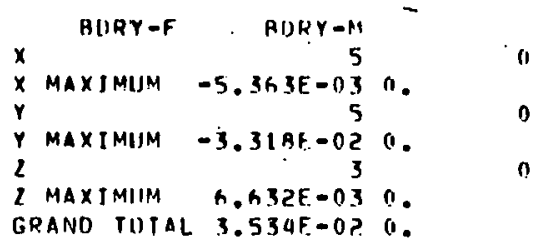

Y MAXIMIJM -1.6GIF-nZ

2 MAXJM!IM 3.01BE-0?

GHAND TBIAL 5.835F-OZ

ELEMENT TYPE: (A 0 O II N N F A R $Y$ 


\begin{tabular}{|c|c|c|c|}
\hline RY $=F$ & ADKY M M & & \\
\hline$x$ & 17 & & n \\
\hline$\times$ MAXIMIIM & $-1.454 \mathrm{~F}+01$ & 11. & \\
\hline$r$ & 5 & & 0 \\
\hline$Y \operatorname{MAX} I M I I M$ & $-9.45 ? E+00$ & $n$. & \\
\hline 2 MAXIMU:4 & 2 & & 0 \\
\hline $\begin{array}{l}2 \text { MAXJMUIH } \\
\text { GHAND TOTAL }\end{array}$ & $\begin{array}{r}1.079 E+111 \\
\text { P.ROBE +111 }\end{array}$ & $n:$ & \\
\hline
\end{tabular}

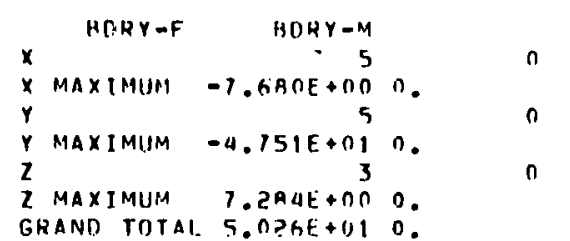

ELEMFNT TYPE I

\begin{tabular}{|c|c|c|c|}
\hline$B S_{i} H Y=F$ & $A \| R Y-M$ & & \\
\hline 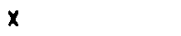 & 12 & & $n$ \\
\hline$X$ MAXIMUMA & h. $43 \mathrm{GE}+10 \mathrm{n}$ & 0. & \\
\hline r & ? & & 0 \\
\hline$Y \cdot i A A X]$ ATIIIHA & $3.09 \Delta F+00$ & 0. & \\
\hline .7 & ? & & 0 \\
\hline $\begin{array}{l}\text { 2. MAXIMIIM } \\
\text { GRAND TRITAI }\end{array}$ & $\begin{array}{r}-1.949 E+011 \\
2 ., 440 E+01\end{array}$ & $\therefore$. & \\
\hline
\end{tabular}

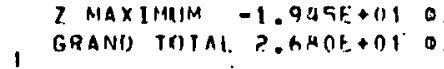

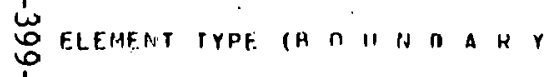

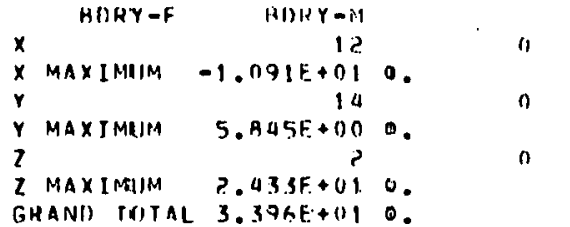

GHANI TOTAL 3.39GE+1110.

ELEMENT TYPE (H 111 N D A R Y, ,,$/$, FLEMFNT NIIMHFR 1 17)

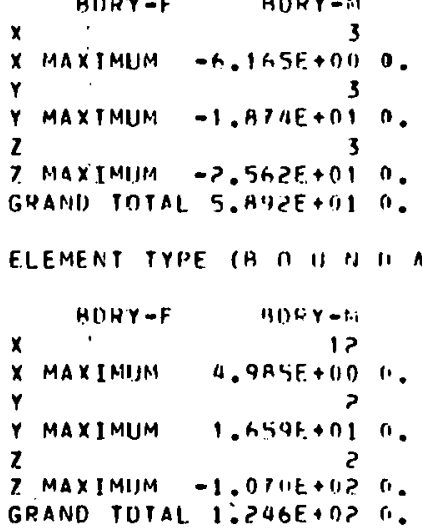




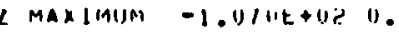

GRANO TOTAL. $1.24 G E+n ? 0$.

ELEMENT TYPE IR O II N $O A$ A $Y$

\begin{tabular}{|c|c|c|}
\hline \multirow[t]{2}{*}{ RDRY F F } & \multicolumn{2}{|c|}{ RIIRYAPA } \\
\hline & & 0 \\
\hline MAXIMUM & 0. & $-5.351 E+11 ?$ \\
\hline$M A X I M U A M$ & n. & $5.931 E+n ?$ \\
\hline & 0 & " -3. \\
\hline QANF TOTAL & 0. & 3. $5 R Q F+\cap 3$ \\
\hline
\end{tabular}

ELEMENT IYPE (H OI II $N$ D) A $R$

\begin{tabular}{|c|c|c|c|}
\hline \multirow{2}{*}{\multicolumn{2}{|c|}{ BURY F }} & \multicolumn{2}{|c|}{ RORY -M } \\
\hline$x$ & & & 0 \\
\hline & MAYIMUM & $n$. & -4 \\
\hline & $M A X I M U I M$ & 0 . & $5 . \mid$ AGF +11) \\
\hline & & & 1 \\
\hline & MAXIMIIM & $n$. & $-3.0 R 5 E+03$ \\
\hline
\end{tabular}

FLEMENT TYPE (H $1 /$ II N $N A$ A

\begin{tabular}{|c|c|c|c|}
\hline \multicolumn{2}{|r|}{ RDRY $-F$} & ADHY Y PA & \\
\hline$x$ & (l) & & 13 \\
\hline & $X$ MAXIMUM & 0 . & $3.356 E+n ?$ \\
\hline & Y MAXTMUM & 0 & $n$ arof $h$ \\
\hline & 2 & • & $0 \quad 13$ \\
\hline & $\begin{array}{l}\text { Z MAXIMIJM } \\
\text { GRANO TOTAL }\end{array}$ & 0. & $\begin{array}{r}-1.611 F+n 2 \\
1.57 D E+02\end{array}$ \\
\hline
\end{tabular}

1,1, ELEHENI NIMAHER $(10)$

$, 1,1$ FLFRENT NIMHER , 20)

$1,1$, element MHMHER ( 21$)$

$$
\begin{aligned}
& \text { RORY-F BITYYH } \\
& x \text { MaXIMUM } 0.00 \text { 3.SSAE+0? } \\
& Y \text { MAXIMUM } 0 . \quad-1 . A A I E+05 \\
& 2 \text { MAXIMIJM } 0.003 \\
& \text { GRANO TOTAL } 0.0 \text { ?. } 55 \text { ? }+03
\end{aligned}
$$

ELEMENT TYPE ( $A$ O U N O A R $Y$

1,1, FLLEMFNT MUMBGF ( ?P)

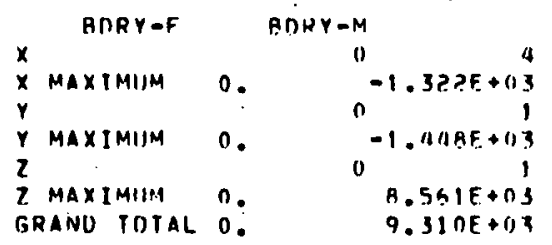

SRANO TOTAL OO

1,1, FLENENT HIMHFH $($ P.1) 


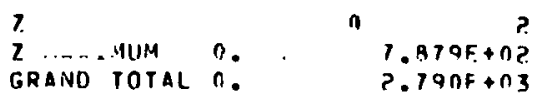

ELEMENT TYPF (A O UNA DA

1,1, ELEMENT NIJMHER $($ ?5)

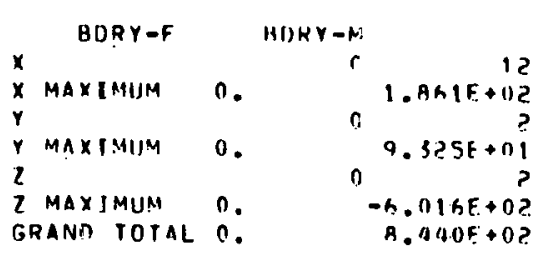

ELEMEAT TYPE IS OUN N A $Y$

1,1, ELEMENT NUMHEN ( 2h)

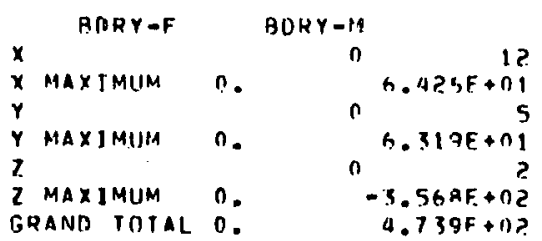

ELEMENT TYPE IA $O$ II N D A R $Y$

)$, 1$, FLEMFNT NUMAER ( 7$)$
FDRY-F HIRYTM

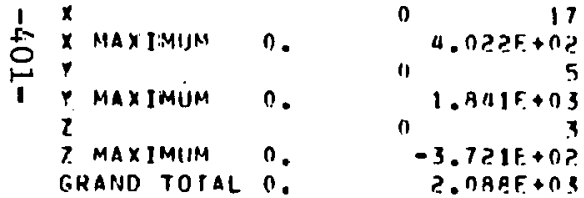

ELEMENT TYPE (a 0 "

1,1, ELEMENT NIJMAFR (. 20)

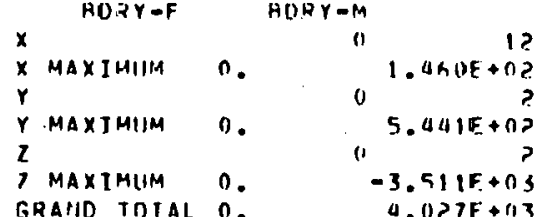

ELERFN ${ }^{-}$TYPE (H $O$ U N N I) A

$1,: 1$ FLENENT MUMARE ( Z9)

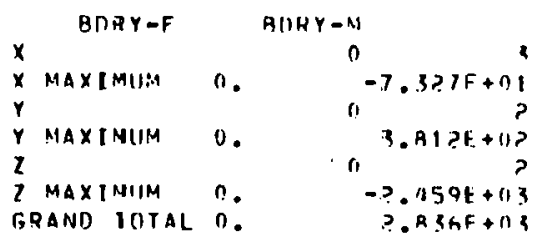

GRAND IOTAL 0 . ORSGF+II

ELEMENT TYPF (a I) "I A D a hi 4

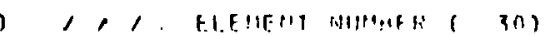





\section{REFERENCES}

Reich, M., Chang, T. Y. and Prachuktam, S. "Development of Solutions to Benchmark Piping Problems" BNL-NUREG-21241-R2, December (1977).

2. Bezler, P., Gardner, D. and Hartzman, M., "A Benchmark Solution for a Hypothetical Reactor System", BNL-NUREG 23645, July (1977).

3. Bezler, P., Hartzman,. M., and Reich, M., "EPIPE - An Elastic Piping Program for Static and Dynamic Analyses," NUREG/CR-1698, a BNL report current1y in preparation.

4. Bathe, K. J., Wilson, E. L. and Peterson, F. E. "SAP IV - A

Structural Analysis Program for Static and Dynamic Responses of Linear Systems, "Report No. EERC 73-11, University of California, Berkeley, California (1973).

5. ASME Boiler and Pressure Vesse1 Code, Section III-1971, Nuclear Power Plant Components, American Society of Mechanical Engineers, . New York, (July 1, 1971).

6. Martin, H. C. Introduction to Matrix Methods of Structural Analysis, McGraw-Hill, New York (1966).

7. Poley, S., "Mesh Analysis of Piping systems, "IBM New York Scientific Center Technical Report No. 320-2939, (March 1968).

8. Rodabough, E. C. and George, H. H., "Effect of Internal Pressure on Flexibility and Stress Intensification Factors of Curved Pipe or Welding Elbows, "Trans. ASME, Vol. 79 (1957).

9. Hovgaard, W., "Stresses in Three Dimensional Pipe Bends", Trans. ASME, Vol. 57, FSP-57-12, P401-476, (1935).

10. Hovgaard, N., "Further Studies of Three...Dimensional Pipe Bends, "Trans. ASME, FSP-50-13, Vol. 59, p. 647-650 (1937). 
11. PIPDYN II - A Computer Program for the Complete Analysis and Evaluation of Piping Systems by Zudans, Z., et al., Applied Mechantcs Lab., The Franklin Institute Research Lab., Philadelphia, PA.

12. Crede, C. E. "Shock and Vibration Concepts in Engineering Design", Englewood Cliffs, NJ, Prentice-Hall (1965).

13. Swanson, J. "A Problem on Dynamic Analysis of. Three Dimensional

Structure", Pressure Vesse1 and Piping, 1972 Computer Program

Verification, Ed. by Tuba, I. S. and Wright, W. B., American Society of Mechanical Engineers, New York (1972).

14. Tuba, I. S. and Wright, W. B. "Pressure Vessel and Piping, 1972 Computer Programs Verification", "ASME Pressure and Piping Division, New York (1972). 
4. TITLE AND SUBTITLE (Add Volume No., if appropriate)

ng Benchmark Problems

itle: Dynamic Analysis Unifürm Support Motion Response Spectrum Method

7. AUTHOR(S)

P. Bezler, M. Hartzman and M. Reich.

9. PERFORMING ORGANIZATION NAME AND MAILING ADDRESS (/nclude Zip Code)

Brookhaven National Laboratory

Building T129

Upton, New York 11973

12. SPONSORING ORGANIZATION NAME AND MAILING ADORESS (Include Zip Code)

Division of Systems Safety

Office of Nuclear Reactor Regulation

U.S. Nuclear Regulatory Commission

Washington, DC 20555

PERIOD COVERED (Inclusive dates)

13. TYPE OF REPORT

14. (Leave blank)

15. SUPPLEMENTARY NOTES

16. ABSTRACT (200 words or less)

A set of benchmark problems and solutions have been developed for verifying the adequacy of computer programs used for dynamic analysis and design of nuclear piping systems by the Response Spectrum Method. The problems range from simple to complex configurations which are assumed to experience linear elastic behavior. The dynamic loading is represented by uniform support motion, assumed to be induced by seismic excitation in three spatial directions. The solutions consist of frequencies, participation factors, nodal displacement components and internal force and moment components. Solutions to associated anchor point motion static problems are not included.

Plping Benchmark Problems

Elastic Finite Element Analysis

Response Spectrum Analysis

Seismic Analysis

7b. IDENTIFIERS/OPEN-ENDED TERMS

19. SE CLIOITY CLASS (This report) Unclassified

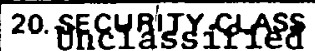

21. NO. OF PAGES

22. PRICE 
UNITED STATES

NUCLEAR REGULATORY COMMISSION

WASHINGTON, D. C. 20555

OFFICIAL BUSINESS

PENALTY FOR PRIVATE USE, $\$ 300$

POSTAGE AND FEES PAID U.S. NUCLEAR REGULATORV COMMISSION 